\title{
Summary of Water-Resources Data within the Little White River Basin, South Dakota and Nebraska
}

By Colin A. Niehus

Open-File Report 99-222

Prepared in cooperation with the Rosebud Sioux Tribe 
U.S. Department of the Interior

Bruce Babbitt, Secretary

\section{U.S. Geological Survey}

Charles G. Groat, Director

The use of firm, trade, and brand names in this report is for identification purposes only and does not constitute endorsement by the U.S. Geological Survey.

Rapid City, South Dakota: 1999

For additional information write to:

District Chief

U.S. Geological Survey

1608 Mt. View Road

Rapid City, SD 57702

Copies of this report can be purchased from:

U.S. Geological Survey

Information Services

Box 25286

Denver, CO 80225-0286 


\section{CONTENTS}

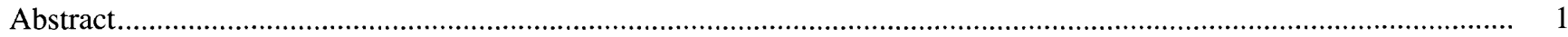

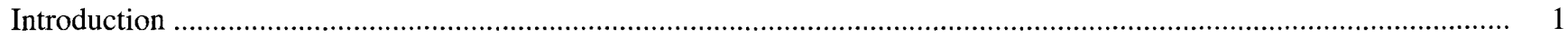

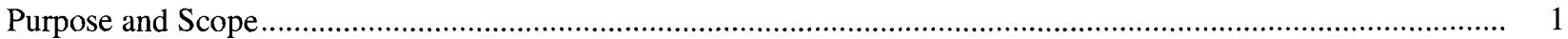

Description of the Study Area ................................................................................................................ 2

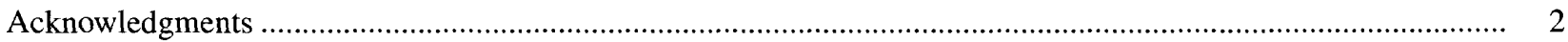

Summary of Water-Resources Data from the U.S. Geological Survey …………………………………………………... 2

Streamflow and Surface-Water-Quality Data ……………………………………………………………... 2

Ground-Water-Level and Ground-Water-Quality Data ……………………………………………………... 3

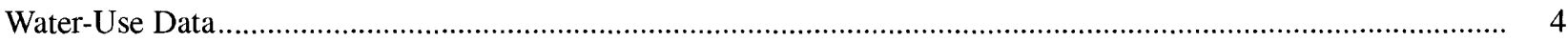

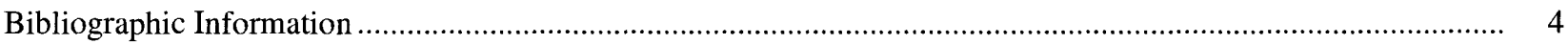

Summary of Water-Resources Data from the South Dakota Department of Environment and Natural Resources .............. $\quad 7$

Surface-Water Quality Data.................................................................................................................. 7

Ground-Water-Level and Ground-Water-Quality Data …………………………………………………. 8

Bibliographic Information ................................................................................................................ 8

Summary of Water-Resources Data from the Rosebud Sioux Tribe ................................................................................ 9

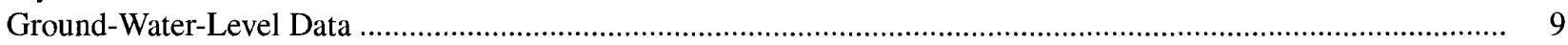

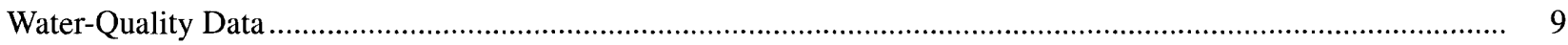

Bibliographic Information ....................................................................................................................... 9

\section{ILLUSTRATIONS}

1. Map showing location of the Little White River Basin, South Dakota and Nebraska .................................... 13

2. Map showing hillshade of the Little White River Basin, South Dakota and Nebraska ................................. $\quad 14$

3. Map showing locations of U.S. Geological Survey streamflow-gaging stations in Little White River Basin, South Dakota and Nebraska.

4-12. Graphs showing variations in annual, monthly, and daily mean flow for streamflow-gaging stations:

4. 06447500 , Little White River near Martin, South Dakota …………………………………………...... 16

5. 06449100, Little White River near Vetal, South Dakota ................................................................... 17

6. 06449300, Little White River above Rosebud, South Dakota.............................................................. 18

7. 06449500, Little White River near Rosebud, South Dakota ................................................................ 19

8. 06450500, Little White River below White River, South Dakota ........................................................ 20

9. 06448000, Lake Creek above refuge, near Tuthill, South Dakota ……………………………………. 21

10. 06449000 , Lake Creek below refuge, near Tuthill, South Dakota .......................................................... 22

11. 06449250, Spring Creek near St. Francis, South Dakota ……………………………………………... 23

12. 06449400 , Rosebud Creek at Rosebud, South Dakota ……………………………………………... 24

13-19. Maps showing location of:

13. Surface-water quality sites in the Little White River Basin, South Dakota and Nebraska, from the U.S. Geological Survey database.

14. Ground-water sites with water-level data in the Little White River Basin, South Dakota and Nebraska, from the U.S. Geological Survey database ....

15. Ground-water sites in the Little White River Basin, South Dakota and Nebraska, from the U.S. Geological Survey database.

16. Ground-water sites grouped by aquifer in the Little White River Basin, South

Dakota and Nebraska, from the U.S. Geological Survey database ...

17. Ground-water sites with water-level data in the High Plains aquifer in South Dakota from the U.S. Geological Survey database ..

18. Ground-water sites in the High Plains aquifer in South Dakota from the U.S. Geological Survey database.

19. Selected South Dakota Geological Survey ground-water-quality sites in the Little White River Basin, South Dakota and Nebraska 


\section{TABLES}

1-9. Monthly mean and annual flow, in cubic feet per second, for U.S. Geological Survey

streamflow-gaging stations:

1. 06447500, Little White River near Martin, South Dakota ............................................................ 35

2. 06449100, Little White River near Vetal, South Dakota …............................................................. 36

3. 06449300, Little White River above Rosebud, South Dakota ......................................................... 37

4. 06449500, Little White River near Rosebud, South Dakota ............................................................ 38

5. 06450500, Little White River below White River, South Dakota.................................................... 40

6. 06448000 , Lake Creek above refuge, near Tuthill, South Dakota.................................................... 42

7. 06449000 , Lake Creek below refuge, near Tuthill, South Dakota.................................................... 43

8. 06449250, Spring Creek near St. Francis, South Dakota .............................................................

9. 06449400 , Rosebud Creek at Rosebud, South Dakota .................................................................... 45

10-18. Summary of statistics on mean flow, in cubic feet per second, for U.S. Geological Survey

streamflow-gaging stations:

10. 06447500, Little White River near Martin, South Dakota, for water years 1938-40 and 1962-96 ...... 46

11. 06449100, Little White River near Vetal, South Dakota, for water years 1959-96 ........................... 47

12. 06449300, Little White River above Rosebud, South Dakota, for water years 1982-96 …............... 48

13. 06449500, Little White River near Rosebud, South Dakota, for water years 1943-96 ..................... 49

14. 06450500, Little White River below White River, South Dakota, for water years 1950-96 ............... 50

15. 06448000, Lake Creek above refuge, near Tuthill, South Dakota, for water years 1938-40, 1962-79, and 1996

16. 06449000 , Lake Creek below refuge, near Tuthill, South Dakota, for water years $1939-40$ and 1962-96.

17. 06449250, Spring Creek near St. Francis, South Dakota, for water years 1960-74

18. 06449400, Rosebud Creek at Rosebud, South Dakota, for water years 1975-96.

19-27. Correlation matrix and serial correlation for 1-year lag for monthly mean flow for

U.S. Geological Survey streamflow-gaging stations:

19. 06447500, Little White River near Martin, South Dakota, for water years 1938-40 and 1962-96

20. 06449100, Little White River near Vetal, South Dakota, for water years 1959-96

21. 06449300, Little White River above Rosebud, South Dakota, for water years 1982-96

22. 06449500, Little White River near Rosebud, South Dakota, for water years 1943-96 .

23. 06450500, Little White River below White River, South Dakota, for water years 1950-96

24. 06448000, Lake Creek above refuge, near Tuthill, South Dakota, for water years 1938-40, 1962-79, and 1996

25. 06449000, Lake Creek below refuge, near Tuthill, South Dakota, for water years $1939-40$ and $1962-96$

26. 06449250, Spring Creek near St. Francis, South Dakota, for water years 1960-74

27. 06449400, Rosebud Creek at Rosebud, South Dakota, for water years 1975-96.

28-36. Water-quality data for U.S. Geological Survey streamflow-gaging stations:

28. 06447500, Little White River near Martin, South Dakota

29. 06448000, Lake Creek above refuge, near Tuthill, South Dakota

30. 06449000, Lake Creek below refuge, near Tuthill, South Dakota...

31. Summary of selected data for 06449100 , Little White River near Vetal, South Dakota .

32. 06449250, Spring Creek near St. Francis, South Dakota

33. Summary of selected data for 06449300 , Little White River above Rosebud, South Dakota

34. 06449400, Rosebud Creek at Rosebud, South Dakota

35. 06449500, Little White River near Rosebud, South Dakota......

36. Summary of selected data for 06450500 , Little White River below White River, South Dakota

Water-quality data (from the U.S. Geological Survey database) for surface-water sites

in the Little White River Basin, South Dakota and Nebraska

in feet below land surface, for one site in the Little White River Basin, South Dakota and Nebraska 
39. Summary of periodic ground-water level data (from the U.S. Geological Survey database), in feet below land surface, for sites in the Little White River Basin, South Dakota and Nebraska

40. Selected descriptive data (from the U.S. Geological Survey database) for ground-water sites in the Little White River Basin, South Dakota and Nebraska

41. Summary of selected water-quality data (from the U.S. Geological Survey database) for ground-

water sites in the Little White River Basin, South Dakota and Nebraska

42-54. Summary of selected water-quality data (from the U.S. Geological Survey database) for ground-water sites in the:

42. 110ALVM (Quaternary alluvium) geologic unit in the Little White River Basin, South Dakota and Nebraska

43. 110AVTN (alluvium, terrace, non-glacial) geologic unit in the Little White River Basin, South Dakota and Nebraska.....

44. 111 ALVM (Holocene alluvium) geologic unit in the Little White River Basin, South Dakota and Nebraska.

45. 121ASHL (Ash Hollow Formation) geologic unit in the Little White River Basin, South Dakota and Nebraska.

46. 121OGLL (Ogallala Formation) geologic unit in the Little White River Basin, South Dakota and Nebraska.

47. 122ARKR (Arikaree Formation) geologic unit in the Little White River Basin, South Dakota and Nebraska.

48. 122MOCN (Miocene Series) geologic unit in the Little White River Basin, South Dakota and Nebraska....

49. 123CRDN (Chadron Formation) geologic unit in the Little White River Basin, South Dakota and Nebraska...

50. 123WRVR (White River Group or Formation) geologic unit in the Little White River Basin, South Dakota and Nebraska

51. 211DKOT (Dakota Sandstone or Formation) geologic unit in the Little White River Basin, South Dakota and Nebraska...

52. 211PIRR (Pierre Shale) geologic unit in the Little White River Basin, South Dakota and Nebraska.

53. 217NCSL (Newcastle Sandstone) geologic unit in the Little White River Basin, South Dakota and Nebraska.

54. 310MNLS (Minnelusa Formation) geologic unit in the Little White River Basin, South Dakota and Nebraska.

55. Summary of daily ground-water-level data (from the U.S. Geological Survey database), in feet below land surface, for two sites in the High Plains aquifer in South Dakota

56. Summary of periodic ground-water-level data (from the U.S. Geological Survey database), in feet below land surface, for sites in the High Plains aquifer in South Dakota

57. Selected descriptive data (from the U.S. Geological Survey database) for ground-water sites in the High Plains aquifer in South Dakota

58. Summary of selected water-quality data (from the U.S. Geological Survey database) for ground-water sites in the High Plains aquifer in South Dakota

59. Estimated total water use in 1995 within the Little White River Basin in South Dakota.

60. Estimated water use in $\mathbf{1 9 9 5}$ for selected categories within the Little White River Basin in South Dakota

61. Selected water-quality data (from the South Dakota Geological Survey) for ground-water sites in the Little White River Basin, South Dakota and Nebraska ...

62. Selected radionuclide data (from the South Dakota Geological Survey) for ground-water sites in the Little White River Basin, South Dakota and Nebraska .

63. Selected pesticide data (from the South Dakota Geological Survey) for ground-water sites in the Little White River Basin, South Dakota and Nebraska ....

64. Summary of annual ground-water-level data (from the Rosebud Sioux Tribe), in feet below land surface, for ground-water sites in the Little White River Basin, South Dakota and Nebraska. 
65. Summary of selected water-quality data (from the Rosebud Sioux Tribe) for sites (surface and/or ground water) in the Little White River Basin, South Dakota and Nebraska.

66. Water-quality data (from the Rosebud Sioux Tribe) for selected herbicides for sites (surface and/or ground water) in the Little White River Basin, South Dakota and Nebraska.....

67. Summary of water-quality data (from the Rosebud Sioux Tribe) for selected metals for sites (surface and/or ground water) in the Little White River Basin, South Dakota and Nebraska

68. Summary of water-quality data (from the Rosebud Sioux Tribe) for selected nonmetallic and inorganic parameters for sites (surface and/or ground water) in the Little White River Basin, South Dakota and Nebraska.

69. Water-quality data (from the Rosebud Sioux Tribe) for selected nutrients and bacteria for sites (surface and/or ground water) in the Little White River Basin, South Dakota and Nebraska

70. Water-quality data (from the Rosebud Sioux Tribe) for selected miscellaneous compounds for sites (surface and/or ground water) in the Little White River Basin, South Dakota and Nebraska.

71. Water-quality data (from the Rosebud Sioux Tribe) for PCB's for sites (surface and/or ground water) in the Little White River Basin, South Dakota and Nebraska

72. Water-quality data (from the Rosebud Sioux Tribe) for selected pesticides for sites (surface and/or ground water) in the Little White River Basin, South Dakota and Nebraska.

73. Water-quality data (from the Rosebud Sioux Tribe) for selected phenols for sites (surface and/or ground water) in the Little White River Basin, South Dakota and Nebraska.

74. Water-quality data (from the Rosebud Sioux Tribe) for selected radionuclides for sites (surface and/or ground water) in the Little White River Basin, South Dakota and Nebraska

75. Water-quality data (from the Rosebud Sioux Tribe) for selected organic compounds for sites (surface and/or ground water) in the Little White River Basin, South Dakota and Nebraska. 


\title{
Summary of Water-Resources Data within the Little White River Basin, South Dakota and Nebraska
}

\author{
By Colin A. Niehus
}

\begin{abstract}
All water-resources data for the Little White River Basin in South Dakota and Nebraska readily available from the U.S. Geological Survey, the South Dakota Department of Environment and Natural Resources, and the Rosebud Sioux Tribe's Office of Water Resources are summarized within this report. This includes streamflow and surfacewater-quality data, ground-water-level and ground-water-quality data, and water-use data for the basin. Additionally, available publications that relate to the hydrology of the Little White River Basin are listed, and all readily available groundwater data associated with the High Plains aquifer in South Dakota are summarized.
\end{abstract}

\section{INTRODUCTION}

The Bureau of Reclamation (BOR), the Rosebud Sioux Tribe, and the Natural Resources Conservation Service (NRCS) have begun a study known as the Little White River Basin Water Management Study (LWRB-WMS). The LWRB-WMS is being conducted under the Missouri River Basin Tribes Water Management Study (MRBT-WMS) in the BOR's General Investigations Program. The purpose of the MRBTWMS is to assist tribes in the Missouri River Basin in the accomplishment of water-development and management studies relative to the use of tribalreserved water rights.

The objectives of the LWRB-WMS are to develop data, information, and analysis tools necessary to assist the Rosebud Sioux Tribe in the management of water and other natural and cultural resources within the basin that are under their jurisdiction. Included in the objectives of the management study is the need to summarize the existing water-resources data for the basin. The U.S. Geological Survey (USGS) is assisting in this portion of the LWRB-WMS. As part of this assistance, the USGS and the Rosebud Sioux Tribe entered into a cooperative agreement for 1997-99 to summarize existing water-resources data for the Little White River Basin in South Dakota and Nebraska.

\section{Purpose and Scope}

The purpose of this report is to summarize all readily available water-resources data for the Little White River Basin in South Dakota and Nebraska. This includes summarizing hydrologic data (streamflow, ground-water-level, and water-use data), water-quality data (ground and surface water), and performing a literature search of publications that relate to the hydrology of the basin. Additionally, ground-water data associated with the High Plains aquifer in South Dakota are summarized.

The data that are summarized include data from the USGS, the South Dakota Department of Environment and Natural Resources (SDDENR), the South Dakota Geological Survey (SDGS), and the Rosebud Sioux Tribe's Office of Water Resources. For the literature search, primary emphasis was placed on USGS and SDDENR publications.

All results shown in the tables of this report that summarize the water-quality data by presenting selected statistics had the following substitutions made to the data prior to doing the statistics: (1) ND (not detected) values were set equal to 0 , and (2) less than some value $(<$ ?) were set equal to the value. 


\section{Description of the Study Area}

The study area is the Little White River Basin (figs. 1 and 2 in the Illustrations section at the end of the report), which occupies about 1,600 square miles within Bennett, Mellette, Shannon, and Todd Counties in south-central South Dakota, and Cherry and Sheridan Counties in north-central Nebraska. The Little White River drains about 560 square miles of the Rosebud Indian Reservation in Todd County in South Dakota.

\section{Acknowledgments}

The author would like to thank the Rosebud Sioux Tribe, the BOR, the SDDENR, and the SDGS for providing water-resources data and/or assisting in compilation of information in this report.

\section{SUMMARY OF WATER-RESOURCES DATA FROM THE U.S. GEOLOGICAL SURVEY}

All readily available water-resources data within the U.S. Geological Survey computer databases were summarized for the Little White River Basin in South Dakota and Nebraska and for the High Plains aquifer in South Dakota. The basic data were retrieved from the National Water Information System (NWIS) computerized databases maintained by the USGS. The NWIS databases include the Ground-Water Site Inventory (GWSI) system, the Automated Data Processing System (ADAPS), the Water-Quality System (QWDATA), and the Site-Specific Water-Use Data System (SWUDS).

These databases include some water-resource data collected by the SDDENR and by the SDGS. To avoid duplication later in this report, water-resources data collected by the SDDENR and SDGS and stored in the USGS databases are summarized in this section only.

The water-resources data include summaries of streamflow and surface-water-quality data, groundwater-level and ground-water-quality data, and wateruse data. A listing of USGS publications pertaining to the Little White River Basin in South Dakota and Nebraska and the High Plains aquifer in South Dakota also is included.

\section{Streamflow and Surface-Water-Quality Data}

Monthly mean and annual flow at nine USGS streamflow-gaging stations are shown in tables 1-9 in the Tables section at the end of the report. These nine streamflow-gaging stations (fig. 3 in the Illustrations section at the end of the report) include the following:

06447500, Little White River near Martin, South

Dakota

06449100, Little White River near Vetal, South Dakota

06449300, Little White River above Rosebud, South Dakota

06449500, Little White River near Rosebud,

South Dakota

06450500, Little White River below White

River, South Dakota

06448000, Lake Creek above refuge near

Tuthill, South Dakota

06449000, Lake Creek below refuge near

Tuthill, South Dakota

06449250, Spring Creek near St. Francis, South

Dakota

06449400, Rosebud Creek at Rosebud, South

Dakota

This information for two additional USGS gaging stations, 06449700 (Little Oak Creek near Mission, South Dakota) and 06449750 (West Branch Horse Creek near Mission, South Dakota) also shown in figure 3, is not included in these tables as these gaging stations had only peak-flow data collected at them.

Variations in annual, monthly, and daily mean flow for these same nine gaging stations previously listed are shown in figures 4-12 in the Illustrations section at the end of the report.

Summaries of statistics on mean streamflow are shown in tables 10-18 in the Tables section at the end of the report for the same nine gaging stations previously listed. These statistics include the following:

Number: Number of data values used to compute the statistics.

Maximum: Largest value in the group of data values.

Percentiles ["p" $=75,50$, or 25$]$ : The data value that has at most " $p$ " percent of the measurements below it and at most (100-"p") percent above it.

Minimum: Smallest value in the group of data values. 
Mean: Sum of individual values in the group divided by the total number of values in the group.

Standard deviation: Measure of the dispersion of data values about the mean; equal to the square root of the sum of the squares of deviations from the mean of all values in the group divided by the total number of values in the group minus one.

Skewness: Measure of asymmetry of a group of data values.

Coefficient of variation: Dimensionless measure of the dispersion of data values; equal to the percentage that the standard deviation is of the mean.

Variance: Sum of the squared deviations from the mean of all values in the group divided by the total number values in the group minus one.

Percent of annual flow: Dimensionless measure of the amount of streamflow that passes the measuring site; equal to the percentage that monthly streamflow is of annual streamflow.

The results of correlation analyses for the monthly mean flow at the same nine gaging stations previously listed are shown in tables 19-27 in the Tables section at the end of the report. The correlation analyses are an indication of the strength of the relation between two different variables.

Water-quality data for selected parameters from samples collected at nine USGS streamflow-gaging stations (fig. 3) are summarized in tables 28-36 in the Tables section at the end of the report. The waterquality samples were collected at the following streamflow-gaging stations:

06447500, Little White River near Martin, South Dakota

06448000, Lake Creek above refuge near Tuthill, South Dakota

06449000, Lake Creek below refuge near

Tuthill, South Dakota

06449100, Little White River near Vetal, South

Dakota

06449250, Spring Creek near St. Francis, South Dakota

06449300, Little White River above Rosebud, South Dakota

06449400, Rosebud Creek at Rosebud, South Dakota

06449500, Little White River near Rosebud, South Dakota

06450500, Little White River below White River, South Dakota
No water-quality data was available for two additional USGS gaging stations shown in figure 3: 06449700, Little Oak Creek near Mission, South Dakota, and 06449750, West Branch Horse Creek near Mission, South Dakota.

Statistical summaries for USGS streamflowgaging station 06449100, Little White River near Vetal, South Dakota, and 06450500, Little White River below White River, South Dakota, include the mean, median, standard deviation, first and third quartile, minimum, maximum, and number of valid samples used in the statistics.

Water-quality results for selected parameters from samples taken at additional USGS surface-water sites (fig. 13 in the Illustrations section at the end of the report) are summarized in table 37 in the Tables section at the end of the report. These sites are identified by a site number that is based on a 15-digit latitude-longitude numbering system. The first six digits denote the degrees, minutes, and seconds of latitude; the next seven digits denote the degrees, minutes, and seconds of longitude; and the last two digits differentiate sites within a 1 -second grid.

\section{Ground-Water-Level and Ground-Water- Quality Data}

Statistical summaries of ground-water-level data for sites (fig. 14 in the Illustrations section at the end of the report) in the Little White River Basin from the U.S. Geological Survey databases are shown in tables 38 and 39 in the Tables section at the end of the report. Table 38 shows summaries of data for one site with daily ground-water-level data, while table 39 in the Tables section at the end of the report shows summaries of data for sites with periodic ground-waterlevel data.

Descriptive data for ground-water sites (fig. 15 in the Illustrations section at the end of the report) in the Little White River Basin from the USGS databases are summarized in table 40 in the Tables section at the end of the report. Descriptive data includes site number, local identifier, county code, depth of well, altitude of land surface, and geologic unit. The associated waterquality data for these sites are shown in table 41 in the Tables section at the end of the report.

Ground-water sites are identified by a site number that is based on the same 15-digit latitudelongitude numbering system used for the surface-water sites. The first six digits denote latitude; the next seven 
digits denote longitude; and the last two digits differentiate sites within a 1-second grid.

A local identifier also has been included to correspond to each site number. The local identifier is a township-range location number that is based on the Federal system of rectangular surveys of public lands. The first number denotes the township north of a base line, the second number denotes the range west of the fifth principal meridian, and the third number denotes the section in which the site is located. The letters A, $\mathrm{B}, \mathrm{C}$, and D designate the northeast, northwest, southwest, and southeast quarter sections (160-acre tract), quarter-quarter sections (40-acre tract), quarterquarter-quarter sections (10-acre tract), and quarterquarter-quarter-quarter sections (2.5-acre tract), respectively. Consecutive terminal numbers are added if more than one site is located within a 2.5 -acre tract. An " $R$ " added to the local identifier means that it is located on a reservation.

Statistical summaries of selected water-quality data grouped by the aquifer the sample was taken from (fig. 16 in the Illustrations section at the end of the report) are presented in tables 42-54 in the Tables section at the end of the report. Statistical summaries are presented for the following aquifers:

110ALVM - Quaternary alluvium

110AVTN - Alluvium, terrace, non-glacial

111ALVM - Holocene alluvium

121ASHL - Ash Hollow Formation

121OGLL - Ogallala Formation

122ARKR - Arikaree Formation

$122 \mathrm{MOCN}$ - Miocene Series

123CRDN - Chadron Formation

123WRVR - White River Group or Formation

211DKOT - Dakota Sandstone or Formation

211PIRR - Pierre Shale

217NCSL - Newcastle Sandstone

310MNLS - Minnelusa Formation (Permian and Pennsylvanian)

As requested by the cooperator, summaries of ground-water data also were compiled for wells completed in the High Plains aquifer in South Dakota. The High Plains aquifer underlies 174,050 square miles of Colorado, Kansas, Nebraska, New Mexico, Oklahoma, South Dakota, Texas, and Wyoming. The High Plains aquifer is commonly referred to as the Ogallala aquifer but actually is made up of three main geologic units: the Ogallala Formation, the Arikaree Group, and the Brule Formation. The Ogallala Formation is generally the principal unit of the three geologic units (Gutentag and others, 1984). Nearly 37 percent of the areal extent of the High Plains aquifer and 66 percent of its volume underlies Nebraska.

Statistical summaries of ground-water-level data for wells (fig. 17 in the Illustrations section at the end of the report) completed in the High Plains aquifer in South Dakota (from the USGS databases) are shown in tables 55 and 56 in the Tables section at the end of the report. Table 55 shows summaries of data for two sites with daily ground-water level data, while table 56 shows summaries of data for sites with periodic ground-water level data.

Selected descriptive data for ground-water wells (fig. 18 in the Illustrations section at the end of the report) completed in the High Plains aquifer in South Dakota from the USGS databases are presented in table 57 in the Tables section at the end of the report. Statistical summaries of selected water-quality data for these ground-water sites are shown in table 58 in the Tables section at the end of the report.

\section{Water-Use Data}

The USGS collects and/or compiles water-use data from other Federal agencies, State agencies, local agencies, and private companies and individuals.

These water-use data are stored by the USGS in South Dakota in the SWUDS database. Many of the wateruse data are obtained from the SDDENR through water-permit applications and from annual reports from water-permit holders.

The 1995 estimated total withdrawals from surface and ground water within the Little White River Basin in South Dakota are shown in table 59 in the Tables section at the end of the report. Total withdrawals from surface and ground water in the Little White River Basin were about 7.5 million gallons per day. Over 93 percent of the total withdrawals was from ground water.

The 1995 estimated water-use withdrawals by category within the Little White River Basin in South Dakota are shown in table 60 in the Tables section at the end of the report. Water-use data are shown in this table for 11 categories.

\section{Bibliographic Information}

Water-resources data for South Dakota were first published in a series of U.S. Geological Survey Water- 
Supply Papers. Data on stream discharge and stage and on lake or reservoir contents and stage, through September 1960 , were published annually under the title "Surface-Water Supply of the United States, Parts 6A and 6B." For water years 1961-70, the data were published in two 5-year reports. Data on chemical quality, temperature, and suspended sediment for water years 1941-70 were published annually under the title "Quality of Surface Waters of the United States," and water levels for water years 1935-74 were published under the title "Ground-Water Levels in the United States."

Beginning in the 1961 water year, an annual report series was published containing only data relating to the quantities of surface water in South Dakota. In water year 1964, a similar annual report was published containing only data relating to the quality of water in South Dakota. Since water year 1975, these annual reports have been replaced by an annual publication series entitled "U.S. Geological Survey WaterData Reports," which contained stream discharge, surface- and ground-water quality, and ground-waterlevel data for South Dakota. Almost all data collected through the water-resources data program and published in these annual data reports are stored in the U.S. Geological Survey's databases in NWIS.

Additional water-resources data and other information for the Little White River Basin and the High Plains aquifer in South Dakota can be obtained from the following U.S. Geological Survey publications:

Adolphson, D.G., 1975, Test drilling at Soldier Creek, Rosebud Indian Reservation, Todd County, South Dakota: U.S. Geological Survey Open-File Report 75-355, 28 p.

Becker, L.D., 1980, Techniques for estimating flood peaks, volumes, and hydrographs on small streams in South Dakota: U.S. Geological Survey WaterResources Investigations Report 80-80, 82 p.

1982, Magnitude and frequency of floods from selected drainage basins in South Dakota: U.S. Geological Survey Water-Resources Investigations Report 82-31, 90 p.

Benson, R.D., 1988, Estimated water use in South Dakota, 1985: U.S. Geological Survey Open-File Report 88-313, 1 sheet.

Benson, R.D., Hoffman, E.B., and Wipf, V.J., 1985, Analysis of flood-flow frequency for selected gaging stations in South Dakota: U.S. Geological Survey Water-Resources Investigations Report 85-4217, 202 p.
Bradford, W.L., 1981, Water levels in bedrock aquifers in South Dakota: U.S. Geological Survey OpenFile Report 81-627, 152 p.

Burr, M.J., and Korkow, K.L., 1996, Peak-flow frequency estimates through 1994 for gaged streams in South Dakota: U.S. Geological Survey Open-File Report 96-202, 407 p.

Carter, J.M., 1997, Selected ground-water-quality data for the water-resources investigation of Mellette and Todd Counties, South Dakota, 1990-96: U.S. Geological Survey Open-File Report 97-248, 75 p. 1998, Water resources of Mellette and Todd Counties, South Dakota: U.S. Geological Survey Water-Resources Investigations Report 98-4146, $68 \mathrm{p}$.

Dugan, J.T., and Cox, D.A., 1994, Water-level changes in the High Plains aquifer-Predevelopment to 1993: U.S. Geological Survey Water-Resources Investigations Report 94-4157, 60 p.

Dugan, J.T., McGrath, T.J., and Zelt, R.B., 1994, Water-level changes in the High Plains aquiferPredevelopment to 1992: U.S. Geological Survey Water-Resources Investigations Report 94-4027, $56 \mathrm{p}$.

Dugan, J.T., and Schild, D.E., 1992, Water-level changes in the High Plains aquifer-Predevelopment to 1990: U.S. Geological Survey WaterResources Investigations Report 91-4165, 55 p.

Dugan, J.T., Schild, D.E., and Kastner, W.M., 1990, Water-level changes in the High Plains aquifer underlying parts of South Dakota, Wyoming, Nebraska, Colorado, Kansas, New Mexico, Oklahoma, and Texas_-Predevelopment through nonirrigation season 1988-89: U.S. Geological Survey Water-Resources Investigations Report 90-4153, 29 p.

Dugan, J.T., and Sharpe, J.B., 1996, Water-level changes in the High Plains aquifer-Predevelopment to 1994: U.S. Geological Survey WaterResources Investigations Report 95-4208, 1 sheet.

Ellis, M.J., and Adolphson, D.G., 1971, Hydrogeology of the Pine Ridge Indian Reservation, South Dakota: U.S. Geological Survey Hydrologic Atlas HA-357, 2 sheets.

Ellis, M.J., Ficken, J.H., and Adolphson, D.G., 1971, Hydrology of the Rosebud Indian Reservation, South Dakota: U.S. Geological Survey Hydrologic Atlas HA-355, 2 sheets. 
Feder, G.L., and Krothe, N.C., 1981, Results of a reconnaissance water-quality sampling program of the Ogallala aquifer in Colorado, Kansas, Nebraska, Oklahoma, South Dakota, and Texas: U.S. Geological Survey Water Resources Investigations Report 81-65, 7 p.

Ferrigno, C.F., 1986, Machine-readable files developed for the High Plains regional aquifer-system analysis in parts of Colorado, Kansas, Nebraska, New Mexico, Oklahoma, South Dakota, Texas, and Wyoming: U.S. Geological Water-Resources Investigations Report 86-4063, 23 p.

Gutentag, E.D., Heimes, F.J., Krothe, N.C., Luckey, R.R., and Weeks, J.B., 1984, Geohydrology of the High Plains aquifer in parts of Colorado, Kansas, Nebraska, New Mexico, Oklahoma, South Dakota, Texas, and Wyoming: U.S. Geological Survey Professional Paper 1400-B, 63 p.

Gutentag, E.D., and Weeks, J.B., 1980, Water table in the High Plains aquifer in 1978 in parts of Colorado, Kansas, Nebraska, New Mexico, Oklahoma, South Dakota, Texas, and Wyoming: U.S. Geological Survey Open-File Report 80-50, 1 sheet.

Heimes, F.J., and Luckey, R.R., 1982, Method for estimating historical irrigation requirements from ground water in the High Plains in parts of Colorado, Kansas, Nebraska, New Mexico, Oklahoma, South Dakota, Texas, and Wyoming: U.S. Geological Survey Water-Resources Investigations Report 82-40, 64 p.

1983, Estimating 1980 ground-water pumpage for irrigation on the High Plains in parts of Colorado, Kansas, Nebraska, New Mexico, Oklahoma, South Dakota, Texas, and Wyoming: U.S. Geological Survey Water-Resources Investigations Report 83-4123, 36 p.

Hoffman, E.B., Freese, M.E., and Winter, D.R., 1986, Analyses of flood-flow frequency for selected gaging stations in South Dakota through September 1985: U.S. Geological Survey OpenFile Report 86-496, 137 p.

Kastner, W.M., Schild, D.E., and Spahr, D.S., 1989, Water-level changes in the High Plains aquifer underlying parts of South Dakota, Wyoming, Nebraska, Colorado, Kansas, New Mexico, Oklahoma, and Texas-Predevelopment through nonirrigation season 1987-88: U.S. Geological Survey Water-Resources Investigations Report 89-4073, $61 \mathrm{p}$.
Kolm, K.E., and Case, H.L., III, 1983, A two-dimensional, finite-difference model of the High Plains aquifer in southern South Dakota: U.S. Geological Survey Water-Resources Investigations Report 83-4175, 34 p.

Krothe, N.C., Oliver, J.W., and Weeks, J.B., 1982, Dissolved solids and sodium in water from the High Plains in parts of Colorado, Kansas, Nebraska, New Mexico, Oklahoma, South Dakota, Texas, and Wyoming: U.S. Geological Survey Hydrologic Investigations Atlas HA-658, 2 sheets, scale 1:2,500,000.

Little, J.R., 1984, Flow-duration hydrographs for selected streamflow stations on South Dakota streams: U.S. Geological Survey Open-File Report 84-432, 129 p.

Little, J.R., and Neitzert, K.M., 1992, Water levels decline in the High Plains of South Dakota during 1989-90, in Dugan, J.T., and Schild, D.E., Waterlevel changes in the High Plains aquifer-Predevelopment to 1990: U.S. Geological Survey Water-Resources Investigations Report 91-4165, p. 45-46.

Loskot, C.L., Case, H.L., and Hern, D.G., 1984, Geologic and hydrologic data from a test-drilling program in the High Plains area of South Dakota, 1979-80: U.S. Geological Survey Open-File Report 84-148, 31 p.

Luckey, R.R., and Ferrigno, C.F., 1982, A datamanagement system for areal interpretive data for the High Plains in parts of Colorado, Kansas, Nebraska, New Mexico, Oklahoma, South Dakota, Texas, and Wyoming: U.S. Geological Survey Water-Resources Investigations Report 82-4072, 112 p.

Luckey, R.R., Gutentag, E.D., and Weeks, J.B., 1981, Water-level and saturated-thickness changes, predevelopment to 1980 in the High Plains aquifer in parts of Colorado, Kansas, Nebraska, New Mexico, Oklahoma, South Dakota, Texas, and Wyoming: U.S. Geological Survey Hydrologic Investigations Atlas HA-652, 2 sheets, scale $1: 2,500,000$.

McCabe, J.A., and Crosby, O.A., 1959, Floods in North and South Dakota, frequency and magnitude: U.S. Geological Survey Open-File Report, 132 p.

McGrath, T.J., and Dugan, J.T., 1993, Water-level changes in the High Plains aquifer-Predevelopment to 1991: U.S. Geological Survey WaterResources Investigations Report 93-4088, 53 p. 
Oltman, R.E., and others, 1951, Missouri River basin floods of April-May 1950 in North and South Dakota: U.S. Geological Survey Water-Supply Paper 1137-A, 114 p.

Reeder, H.O., 1978, Summary appraisals of the Nation's ground-water resources-Souris-RedRainy Region: U.S. Geological Survey Professional Paper 813-K, p. K1-K25.

Sando, S.K., 1991, Estimation and characterization of the natural streamflow of the White River near the Nebraska-South Dakota State line: U.S. Geological Survey Water-Resources Investigations Report 91-4096, 20 p.

Taylor, O.J., 1978, Summary appraisals of the Nation's ground-water resources-Missouri Basin Region: U.S. Geological Survey Professional Paper 813-Q, p. Q1-Q41.

U.S. Geological Survey, 1955, Floods of April 1952 in the Missouri River basin: U.S. Geological Survey Water-Supply Paper 1260-B, 302 p.

1973, Ground-water levels in the United States, 1967-71-north-central States: U.S. Geological Survey Water-Supply Paper 2090, 114 p.

1977, Ground-water levels in the United States, 1972-74, north-central States: U.S. Geological Survey Water-Supply Paper 2163, 89 p. 1978, Hydrologic unit map-1978 State of South Dakota.

Weeks, J.B., and Gutentag, E.D., 1981, Bedrock geology, altitude of base, and 1980 saturated thickness of the High Plains aquifer in parts of Colorado, Kansas, Nebraska, New Mexico, Oklahoma, South Dakota, Texas, and Wyoming: U.S. Geological Survey Hydrologic Investigations Atlas HA-648, 2 sheets, scale 1:2,500,000.

Weeks, J.B., Gutentag, E.D., Heimes, F.J., and Luckey, R.R., 1988, Summary of the High Plains regional aquifer-system analysis in parts of Colorado, Kansas, Nebraska, New Mexico, Oklahoma, South Dakota, Texas, and Wyoming: U.S. Geological Survey Professional Paper 1400-A, $30 \mathrm{p}$.

Whitehead, R.L., 1996, Ground water atlas of the United States-Segment 8-Montana, North Dakota, South Dakota, and Wyoming: U.S. Geological Survey Hydrologic Investigations Atlas 730-I, $24 \mathrm{p}$.

\section{SUMMARY OF WATER-RESOURCES DATA FROM THE SOUTH DAKOTA DEPARTMENT OF ENVIRONMENT AND NATURAL RESOURCES}

All water-resources data for the Little White River Basin in South Dakota and Nebraska and for the High Plains aquifer in South Dakota readily available from the SDDENR (including the SDGS) were summarized. The data include summaries of characteristics of the Little White River and ground-water quality. A listing of SDDENR publications pertaining to the Little White River Basin in South Dakota and Nebraska and to the High Plains aquifer in South Dakota also is included.

The USGS databases include water-resources data collected and/or analyzed by the SDDENR and SDGS, including data on ground-water levels and ground-water quality. To avoid duplication, data collected and/or analyzed by the SDDENR and SDGS and stored in the USGS databases are only included in the previous section "Summary of Water-Resources Data from the U.S. Geological Survey."

\section{Surface-Water Quality Data}

The SDDENR annually publishes 305(b) Water Quality Assessment reports to fulfill the Federal Clean Water Act reporting requirements. According to the 1996 305(b) report (South Dakota Department of Environment and Natural Resources, 1996), the Little White River does not support fishing and swimming because of high suspended solids. The high suspended solids are primarily due to natural causes, as well as to flow alteration. This report also shows that a sample taken from the LaCreek National Wildlife Refuge had a fecal coliform concentration of 320 colonies per 100 milliliters as a result of nonpoint source contamination. Furthermore, because of fecal coliform detections, the beach at this refuge was closed at one time in 1994.

The SDDENR has one surface-water quality monitoring site near the mouth of the Little White River. The location of this site is shown on figure 23 of the 1996 305(b) report. The period of record for the site is May 1968 to present. 


\section{Ground-Water-Level and Ground-Water- Quality Data}

The SDDENR has several ground-water-quality monitoring sites within the Little White River Basin and in the High Plains aquifer in South Dakota. The locations of these sites are shown in figure 20 of the 1996 305(b) report.

A summary of SDGS water-quality data at ground-water sites (fig 19 in the Illustrations section at the end of the report) are shown in tables 61-63 in the Tables section at the end of the report. Descriptive data for 14 SDGS wells and selected water-quality data for samples from the wells are shown in table 61. Selected radionuclide data for water samples from four SDGS wells are shown in table 62. Selected pesticide data for water samples from eight SDGS wells are shown in table 63. The SDGS also maintains a computer record of logs of wells and test holes. These data are available from the SDGS on request.

\section{Bibliographic Information}

Water-resources data and other information for the Little White River Basin in South Dakota and Nebraska and the High Plains aquifer in South Dakota can be obtained from the following SDDENR (including SDGS) publications:

Adolphson, D.G., and Ellis, M.J., 1969, Basic hydrogeologic data, Pine Ridge Indian Reservation, South Dakota: South Dakota Geological Survey Basic-Data Report, 75 p.

Agnew, A.F., and Collins, S.G., 1957, Areal geology of the White River quadrangle, South Dakota Geological Survey.

-1963, Geology of the Mission quadrangle South Dakota, South Dakota Geological Survey.

Baker, C.L., 1947, Deep borings of western South Dakota, South Dakota Geological Survey: South Dakota Geological Survey Report of Investigations 57, $112 \mathrm{p}$.

Barari, Assad, 1967, Ground-water supply for the city of Mission, South Dakota: South Dakota Geological Survey Special Report 41, 30 p.

1970, Ground-water study in the Little White River Valley: South Dakota Geological Survey Urban and Rural Studies 2, 13 p.
Collins, S.G., and Harksen, J.C., 1959, Geology of the Martin quadrangle South Dakota, South Dakota Geological Survey.

Collins, S.G., and Hovelsrud, E.J., 1960, Geology of the Patricia quadrangle South Dakota, South Dakota Geological Survey.

Cripe, Carl, and Barari, Assad, 1978, Ground-water study for the city of Murdo: South Dakota Geological Survey Open-File Report No. 21-UR, $13 \mathrm{p}$.

Ellis, M.J., Adolphson, D.G., and Ficken, J.H., 1972, Basic hydrogeologic data, Rosebud Indian Reservation, South Dakota: South Dakota Geological Survey Water Resources Report No. 6, 111 p.

Hammond, P.D., 1994, Ground water quality investigation in selected areas of Todd and Mellette Counties, South Dakota: South Dakota Geological Survey Open-File Report No. 68-UR, $58 \mathrm{p}$.

Harksen, J.C., 1966, The Pliocene-Pleistocene medicine root gravel of southwestern South Dakota: Bulletin of Southern California Academy of Science, v. 65, no. 4, p. 251-257.

1967, Quaternary loess in southwestern South Dakota: Proceedings of South Dakota Academy of Science, v. 46, p.32-40.

1968, Red Dog Loess named in southwestern South Dakota: South Dakota Geological Survey Report of Investigation 98, $17 \mathrm{p}$.

Harksen, J.C., and Green, Morton, 1971, Thin Elk Formation, Lower Pliocene, South Dakota: South Dakota Geological Survey Report of Investigation $100,7 \mathrm{p}$.

Harksen, J.C., and Kulick, J., 1967, Geology of the Porcupine Butte quadrangle South Dakota: South Dakota Geological Survey.

Harksen, J.C., and MacDonald, J.R., 1967, Miocene Batesland Formation named in southwestern South Dakota: South Dakota Geological Survey Report of Investigations 96, $10 \mathrm{p}$.

Harksen, J.C., and MacDonald, J.R., 1969, Guidebook to the major Cenozoic deposits of southwestern South Dakota: South Dakota Geological Survey Guidebook 2, 103 p.

MacDonald, J.R., and Harksen, J.C., 1968, Rosebud Formation in South Dakota: South Dakota Geological Survey Report of Investigation 97, $13 \mathrm{p}$. 
Petsch, B.C., 1961, Magnetometer survey of Bennett and Washabaugh Counties: South Dakota Geological Survey Oil and Gas Investigation Map 7, 1 sheet.

1962, Magnetometer survey of Todd and Mellette Counties: South Dakota Geological Survey Oil and Gas Investigation Map 8, 1 sheet.

Rothrock, E.P., 1942, A hydrologic study of the White River valley: South Dakota Geological Survey Report of Investigation 41, 32 p.

Schoon, R.A., and McGregor, D.J., 1974, Geothermal potentials in South Dakota,: South Dakota Geological Survey Report of Investigation 110, $76 \mathrm{p}$.

Sevon, W.D., Harris, C., and Hovelsrd, E.J., 1960, Geology of the Spring Creek quadrangle South Dakota: South Dakota Geological Survey.

Sevon, W.D., and Sorenson, L., 1961, Geology of the Vetal quadrangle South Dakota: South Dakota Geological Survey.

South Dakota Department of Environment and Natural Resources, 1996, 305(b) water quality assessment. Stach, R.L., and Harksen, J.C., 1974, Fused sediments near Pine Ridge, South Dakota: Proceedings of South Dakota Academy of Science, v. 52, p. 194-199.

Stach, R.L., Olson, O.E., Palmer, I.S., Helgerson, R.N., and Chadima, S.A., 1990, Selenium in South Dakota waters: South Dakota Geological Survey Report of Investigation 112, $69 \mathrm{p}$.

Tullis, E.L., 1963, Gravity survey in southwestern South Dakota: South Dakota Geological Survey Report of Investigation 94, $34 \mathrm{p}$.

\section{SUMMARY OF WATER-RESOURCES DATA FROM THE ROSEBUD SIOUX TRIBE}

Computerized water-resources data for the Little White River Basin in South Dakota available from the Rosebud Sioux Tribe Office of Water Resources were summarized. The water-resources data include summaries of selected ground-water-level and waterquality data. The ground-water-level data included summaries of annual ground-water-level data for 22 wells. The selected water-quality data included information on physical properties, herbicides, metals, nonmetalic and inorganic compounds, nutrients and bacteria, miscellaneous compounds, PCB's, pesticides, phenols, radionuclides, and organic compounds. A listing of Rosebud Sioux Tribe publications pertaining to the Little White River Basin in South Dakota and Nebraska also is included.

\section{Ground-Water-Level Data}

The Rosebud Sioux Tribe Office of Water Resources provided computerized ground-water-level data to the USGS for inclusion in this report. Table 64 in the Tables section at the end of the report summarizes annual ground-water level data for 22 wells within the Little White River Basin. Geographic locations for these sites were not provided by the Tribe.

\section{Water-Quality Data}

The Rosebud Sioux Tribe Office of Water Resources provided computerized water-quality data to the USGS for inclusion in this report. The data (tables 65-75 in the Tables section at the end of the report) included information on physical properties (table 65), herbicides (table 66), metals (table 67), nonmetals and inorganic compounds (table 68), nutrients and bacteria (table 69), miscellaneous compounds (table 70), PCB's (table 71), pesticides (table 72), phenols (table 73), radionuclides (table 74), and organic compounds (table 75).

The summaries for the physical properties, metals, and nonmetals and inorganic compounds included statistics such as mean, median, standard deviation, first and third quartile, minimum, maximum, and number of valid samples used in the statistical analysis. Geographic locations for these sites were provided by the Rosebud Sioux Tribe for most of the sites.

\section{Bibliographic Information}

Water-resources data and other information for the Little White River Basin in South Dakota and Nebraska can be obtained from the following Rosebud Sioux Tribe publications:

Bahrych, T., and Wireman, M., 1988, 8HWM-RM

Preliminary investigation report on Mission, SD, p. 6.

Golden, S., 1987, Golden Graphics System, p. 1-3, 3-16, A-1, C-2. 
Hodson, R., 1990, Twin City testing, Preliminary investigation of soils, sediments and groundwater contamination, Mission, SD, p. 18-19.

Huq, S.Y., 1986, Initial report of groundwater contamination by hydrocarbons in the city of Mission, p. 1-7.

1989, Groundwater contamination in the city of Mission: South Dakota Water Congress, special issue, p. 1-15.

1990, Groundwater contamination by hydrocarbons in the city of Mission, SD: Hazardous Material Spills Conference, Houston, Texas, p. 7.
Huq, S.Y., and Mack, C.L., 1992, Annual report on water resource: Bureau of Indian Affairs, P.L. 93-638, p. 137.

1995, Radon in houses; its relation to hydrogeology on Rosebud Reservation, South Dakota: NAEP 20th Annual Conference Proceedings, p. 897-910.

Rogers, D., 1988, Groundwater quality for nine reservations in South Dakota, p. 117-125.

TCT, 1992, Quarterly groundwater and free product recovery system monitoring report, 3rd quarter 1992: Twin City Testing Corporation, City of Mission, S. Dak. 


\section{ILLUSTRATIONS}





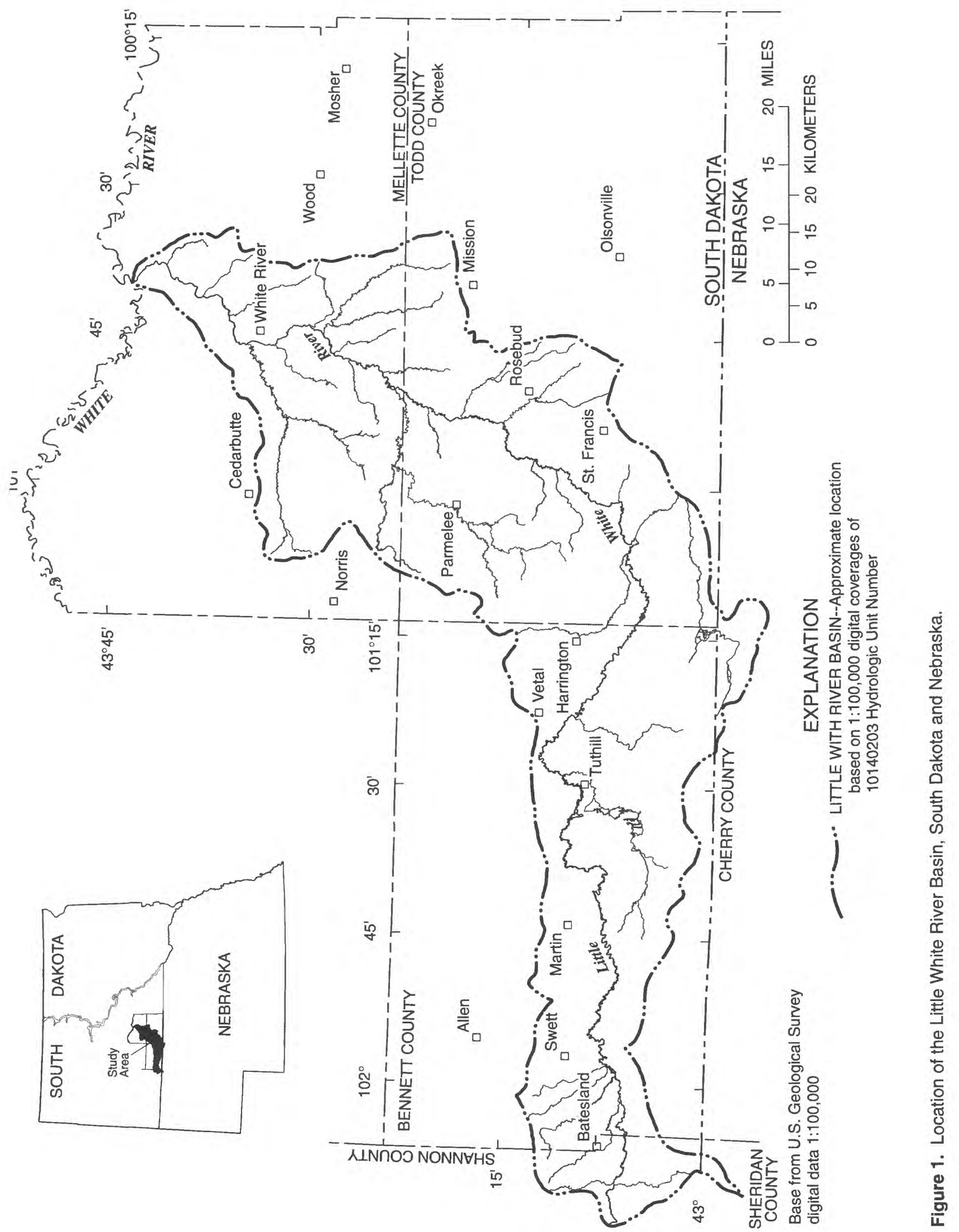

Figure $1 \quad 13$ 


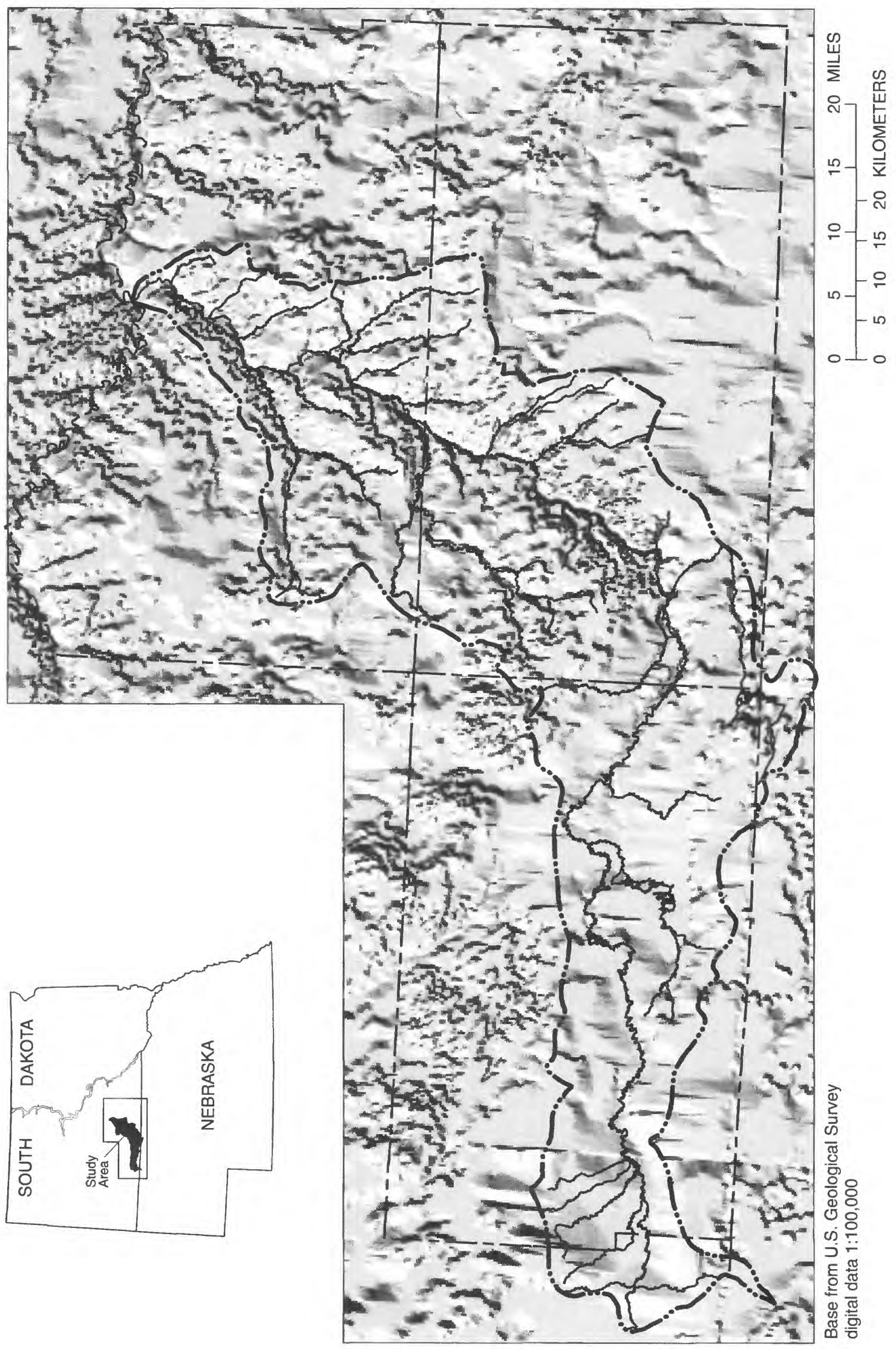

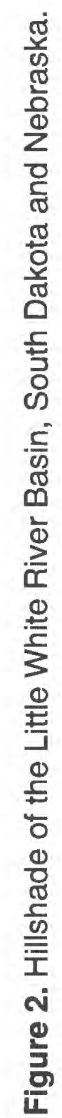




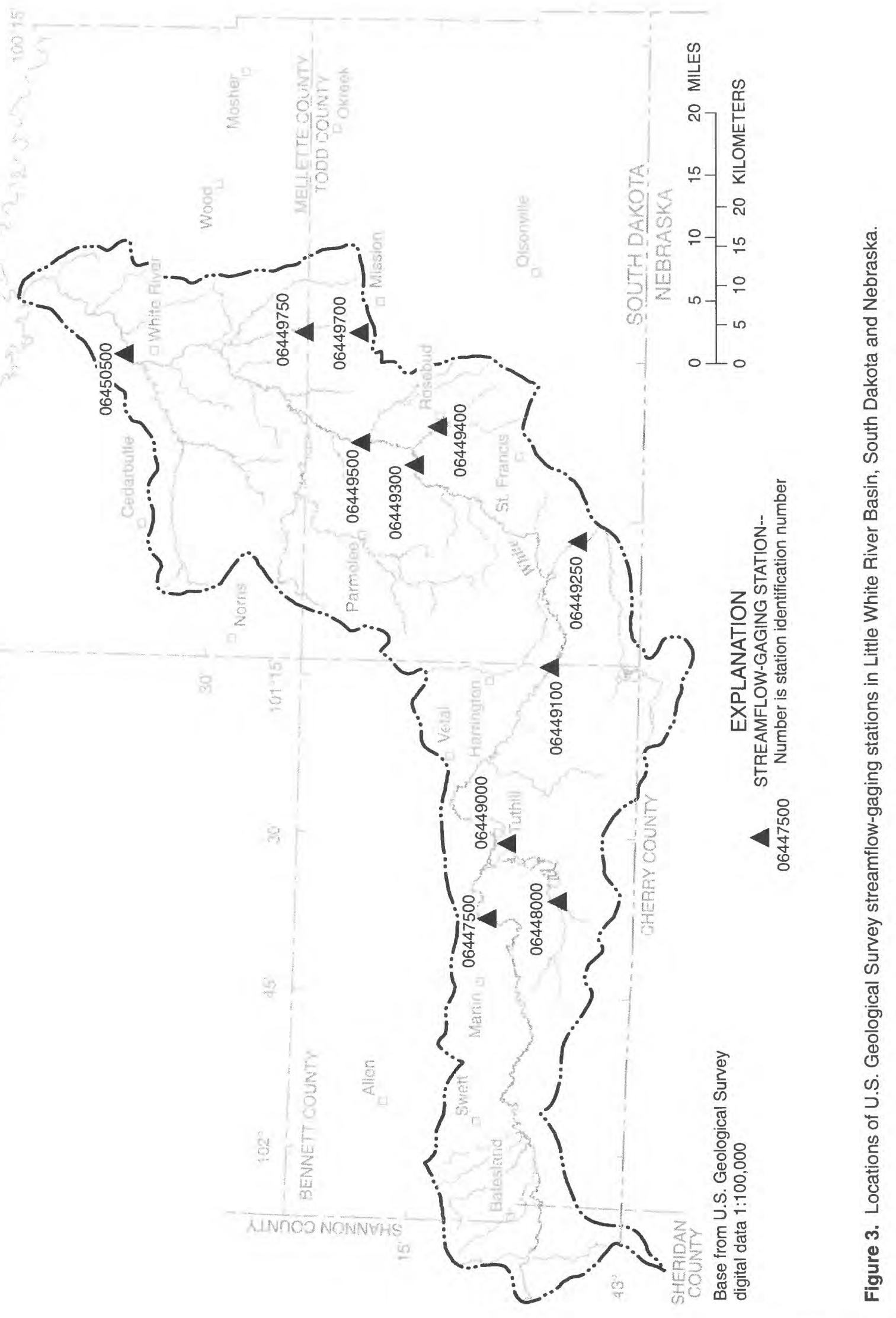

Figure $3 \quad 15$ 

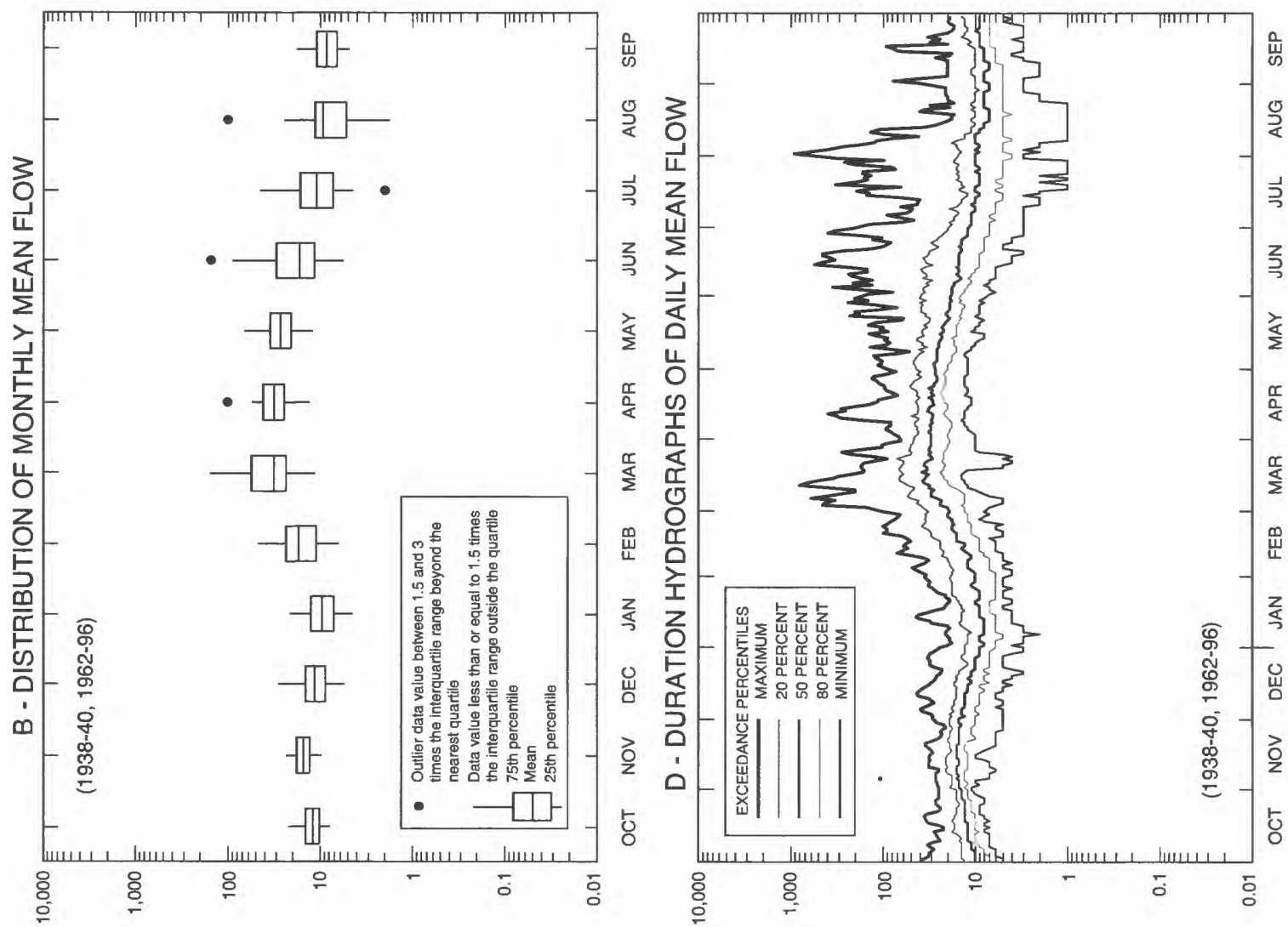

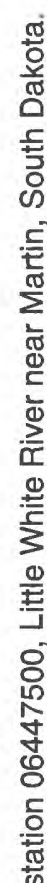
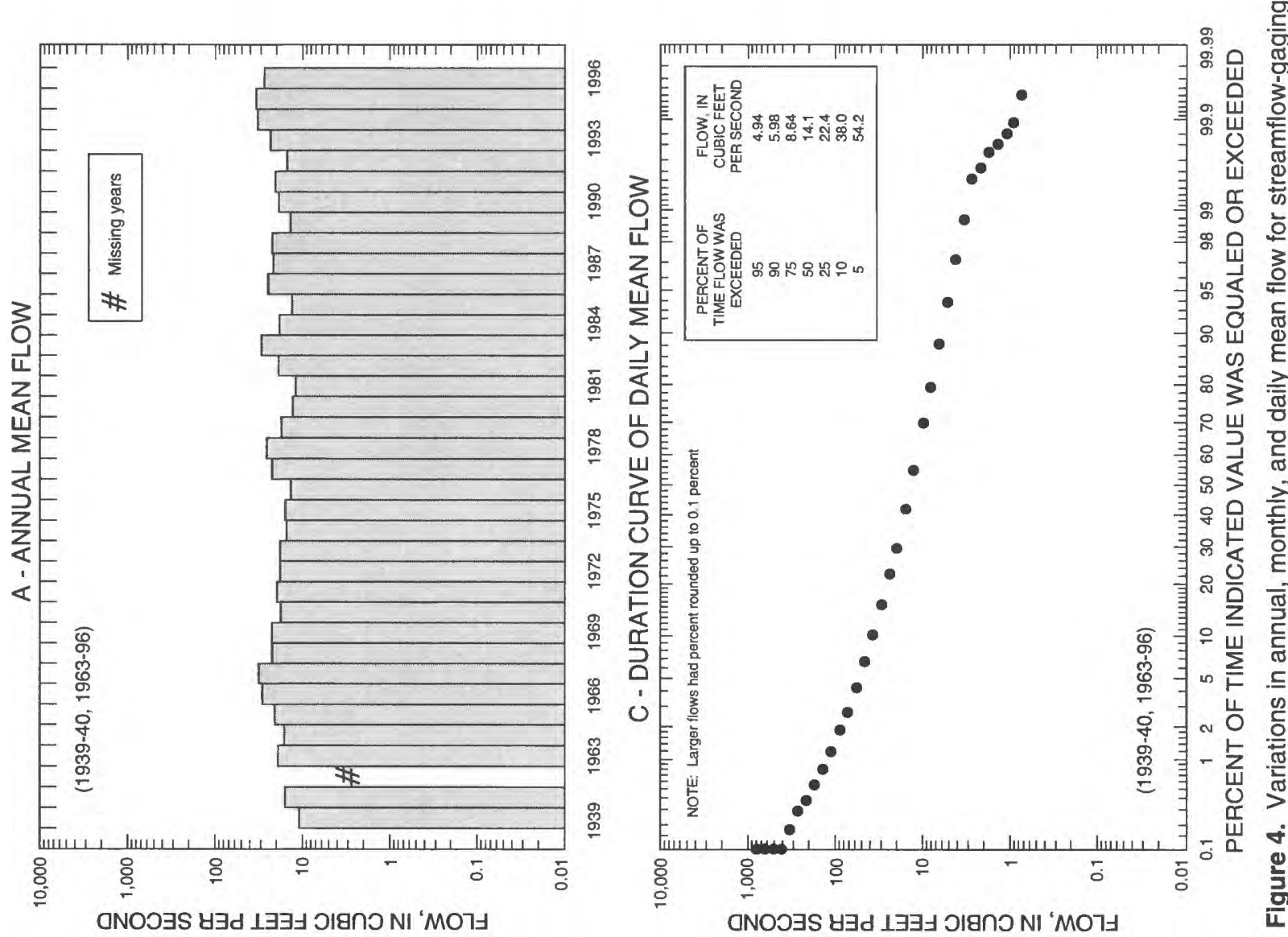

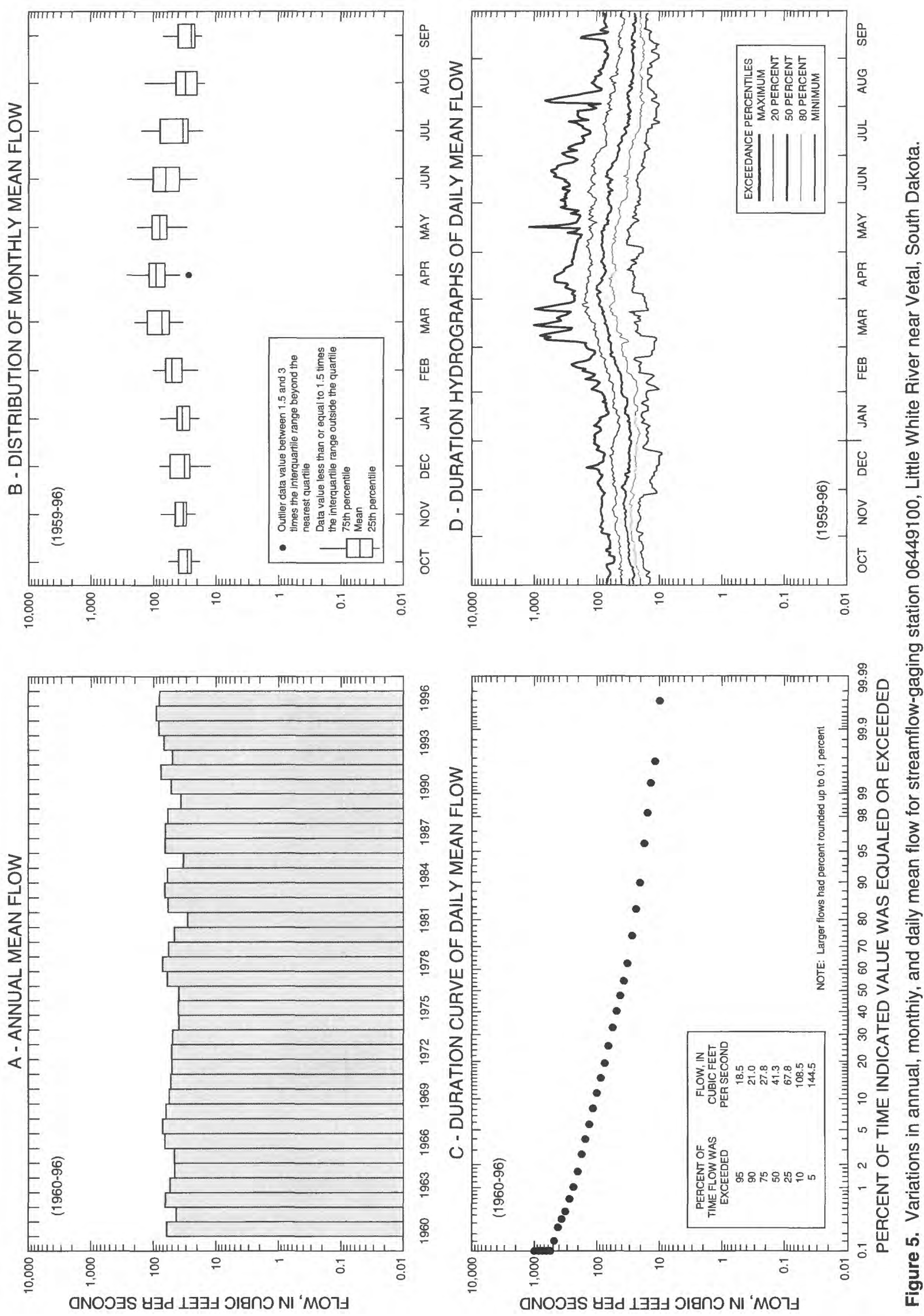

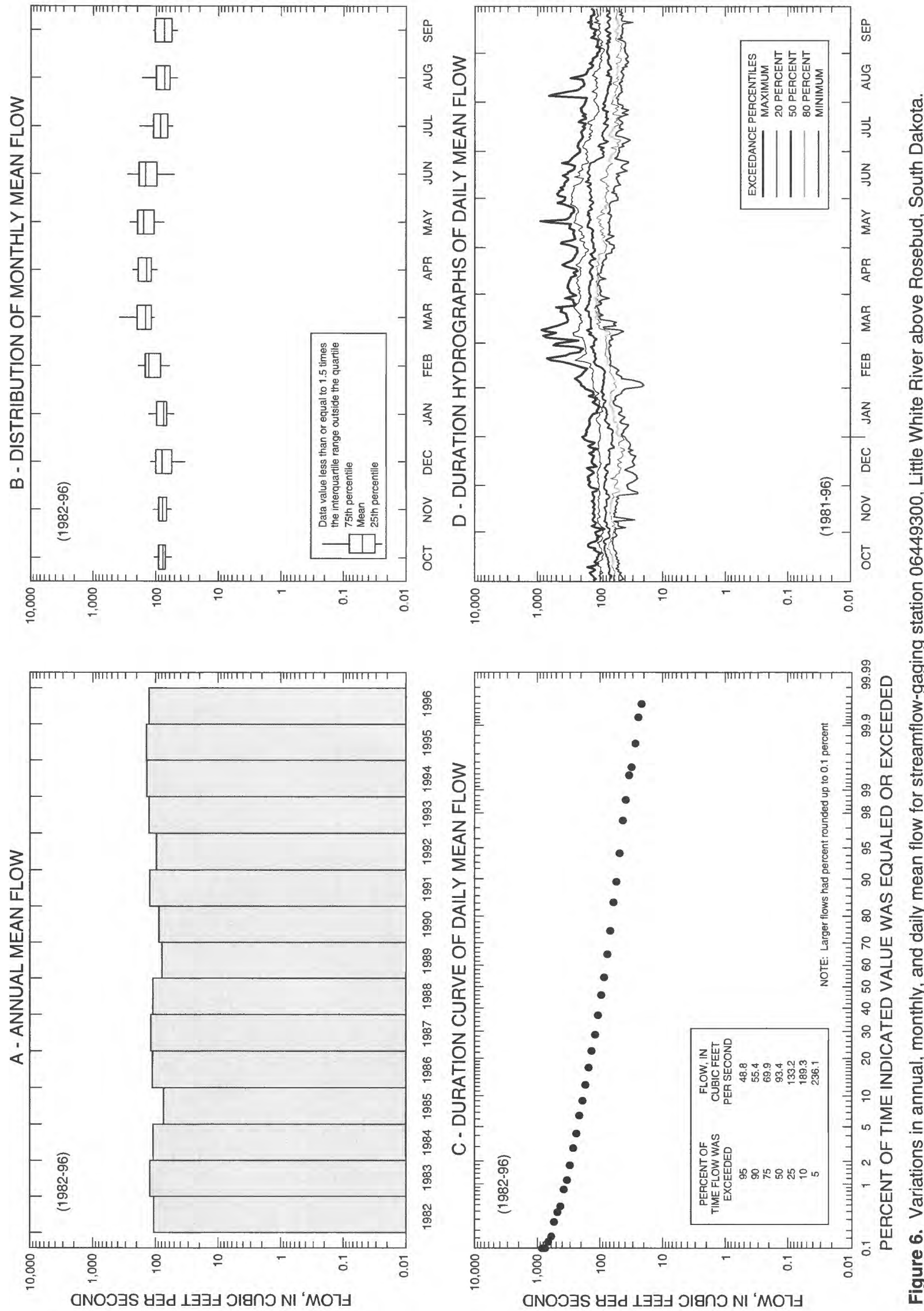

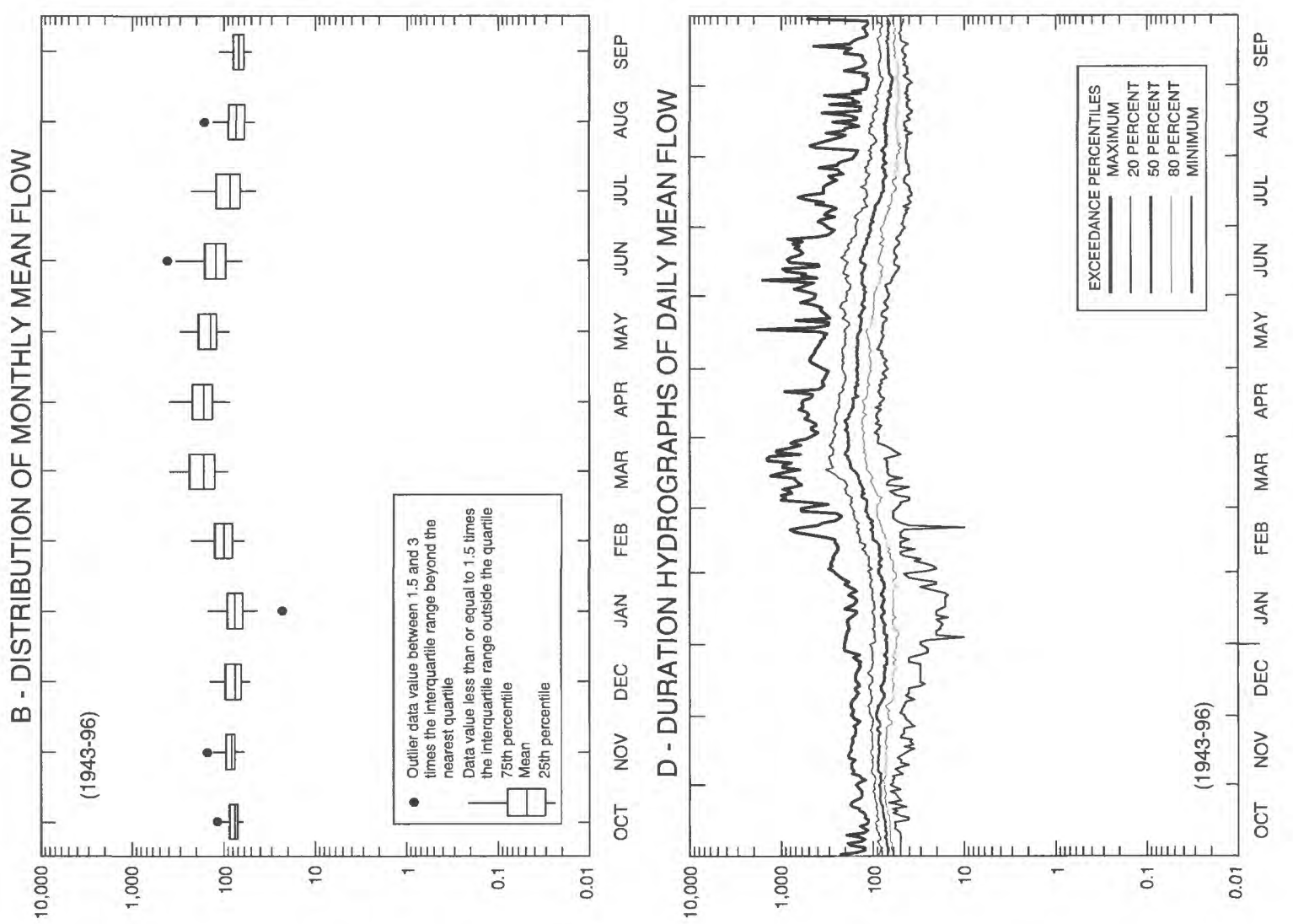

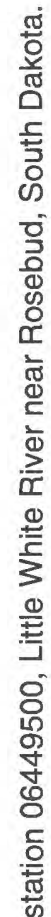
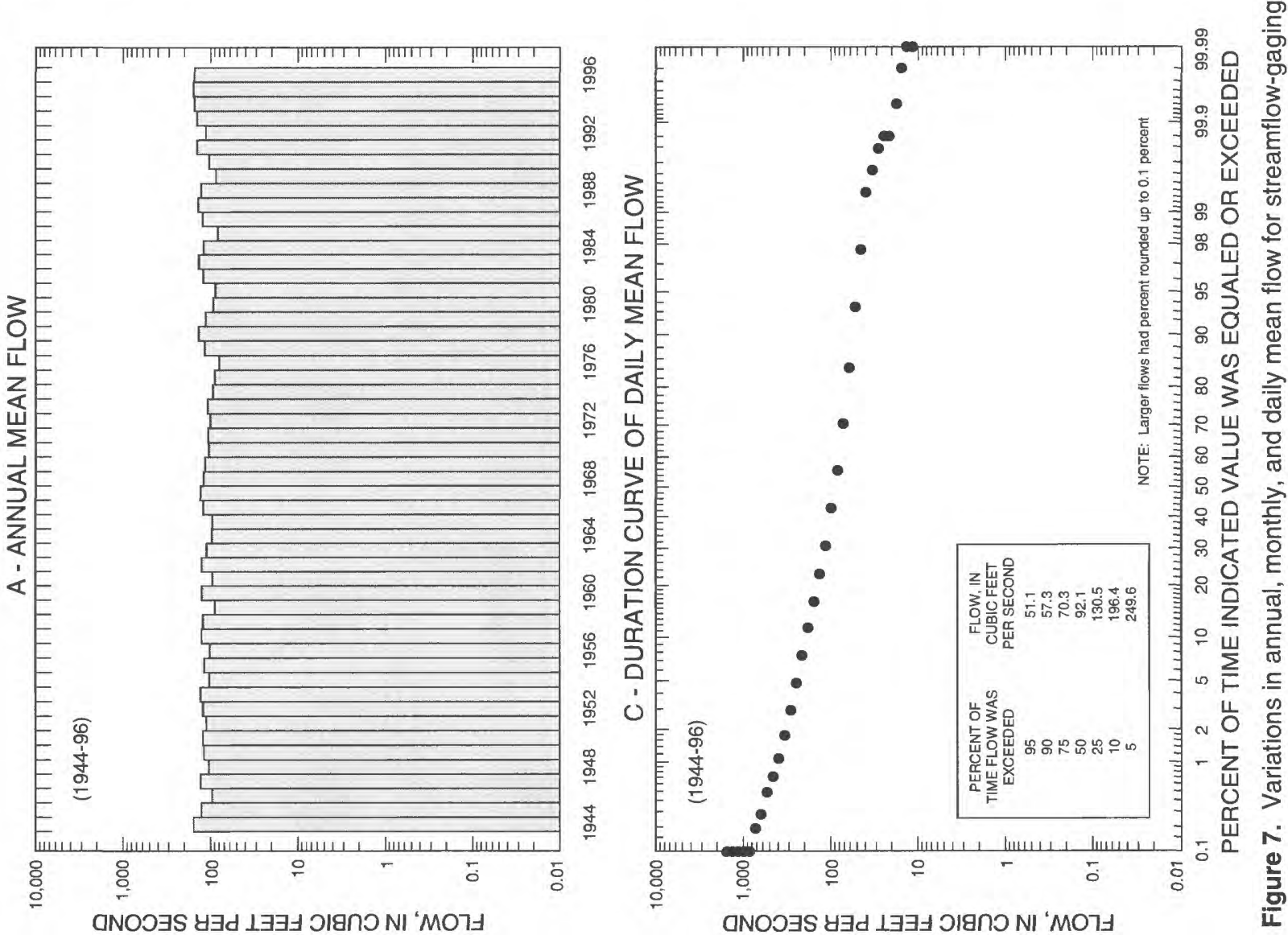

Figure 7 

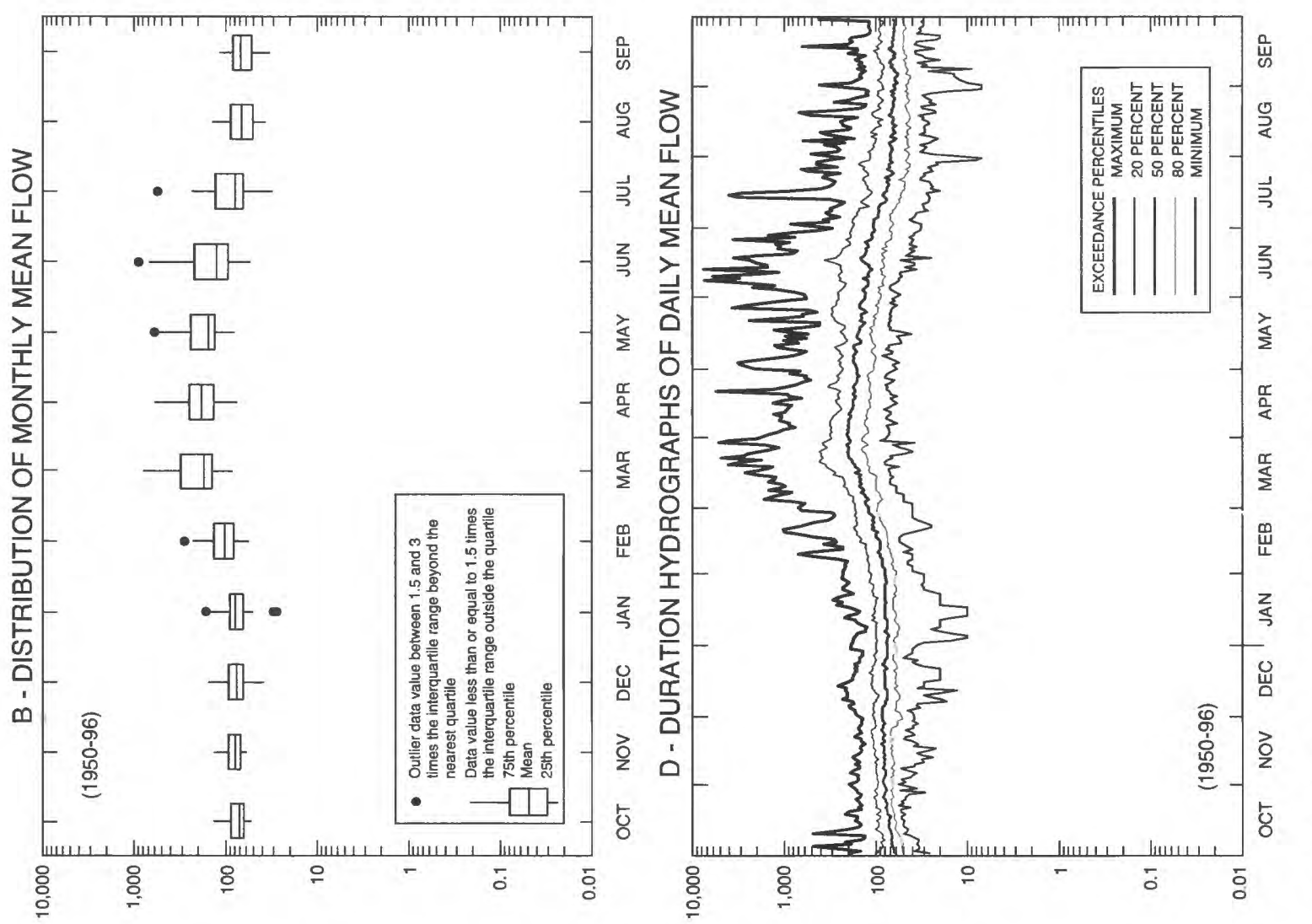

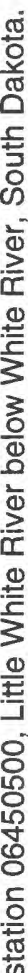
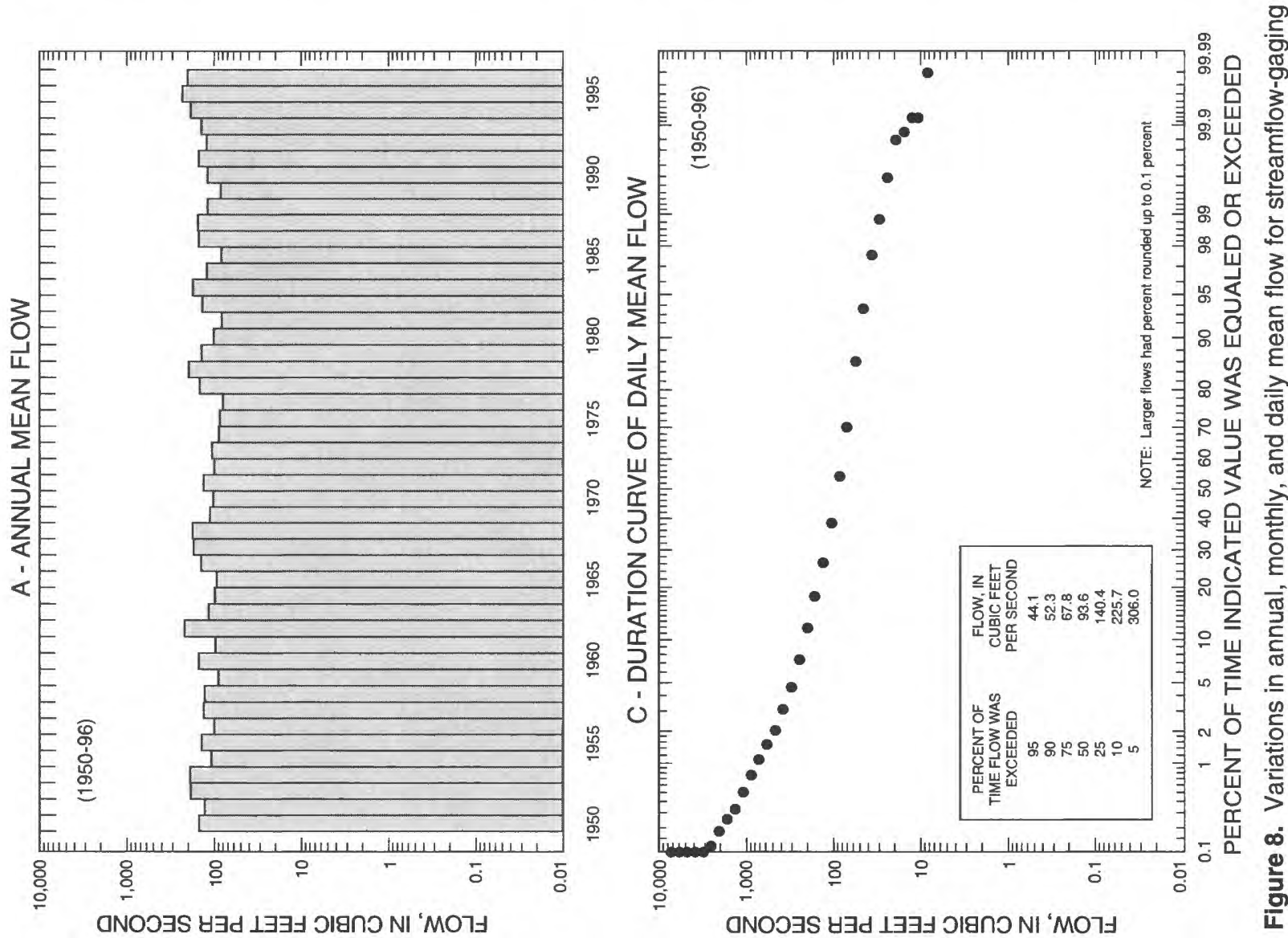

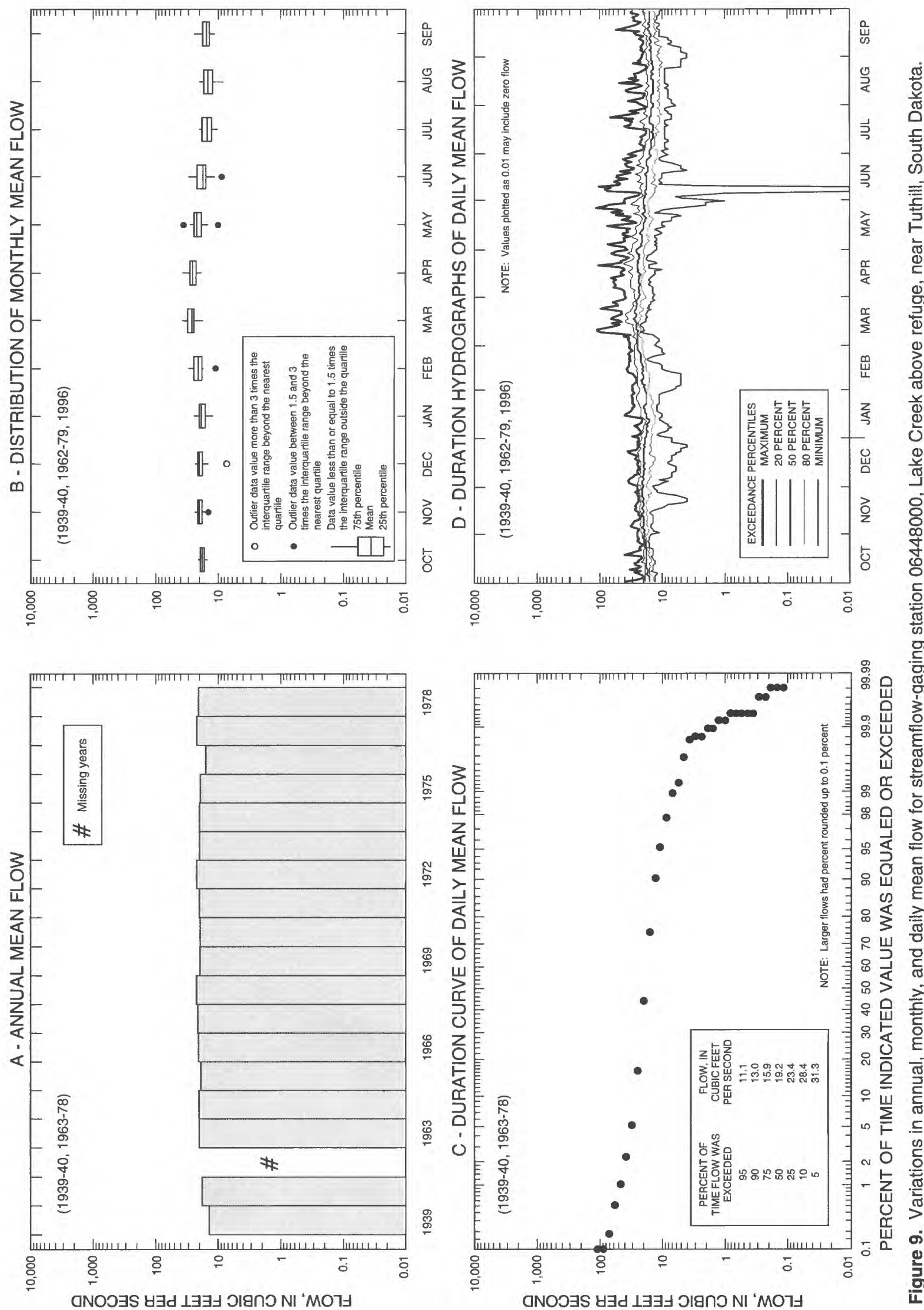

Figure 9 

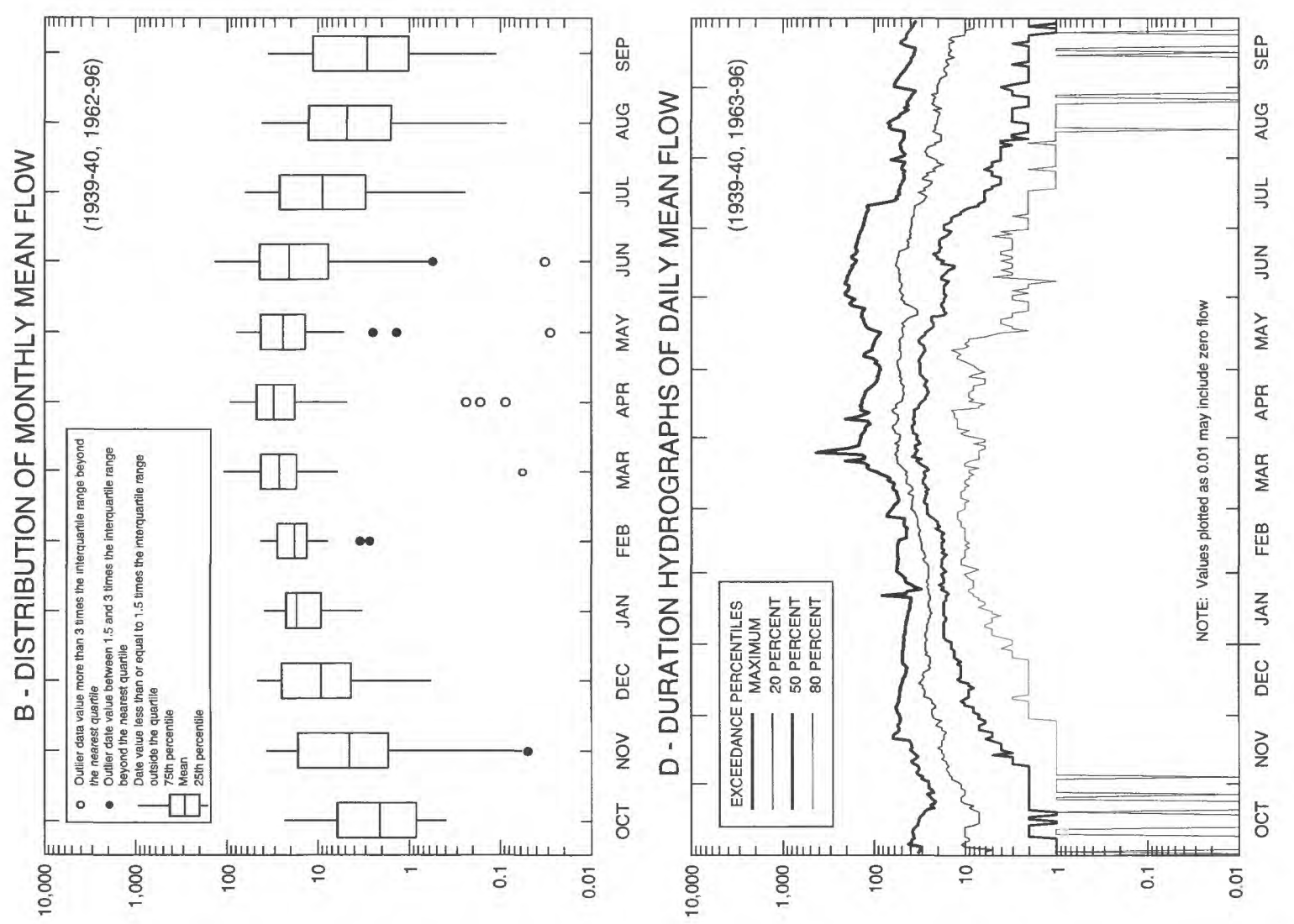

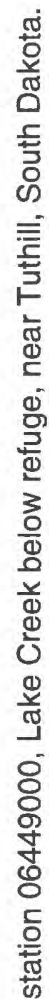
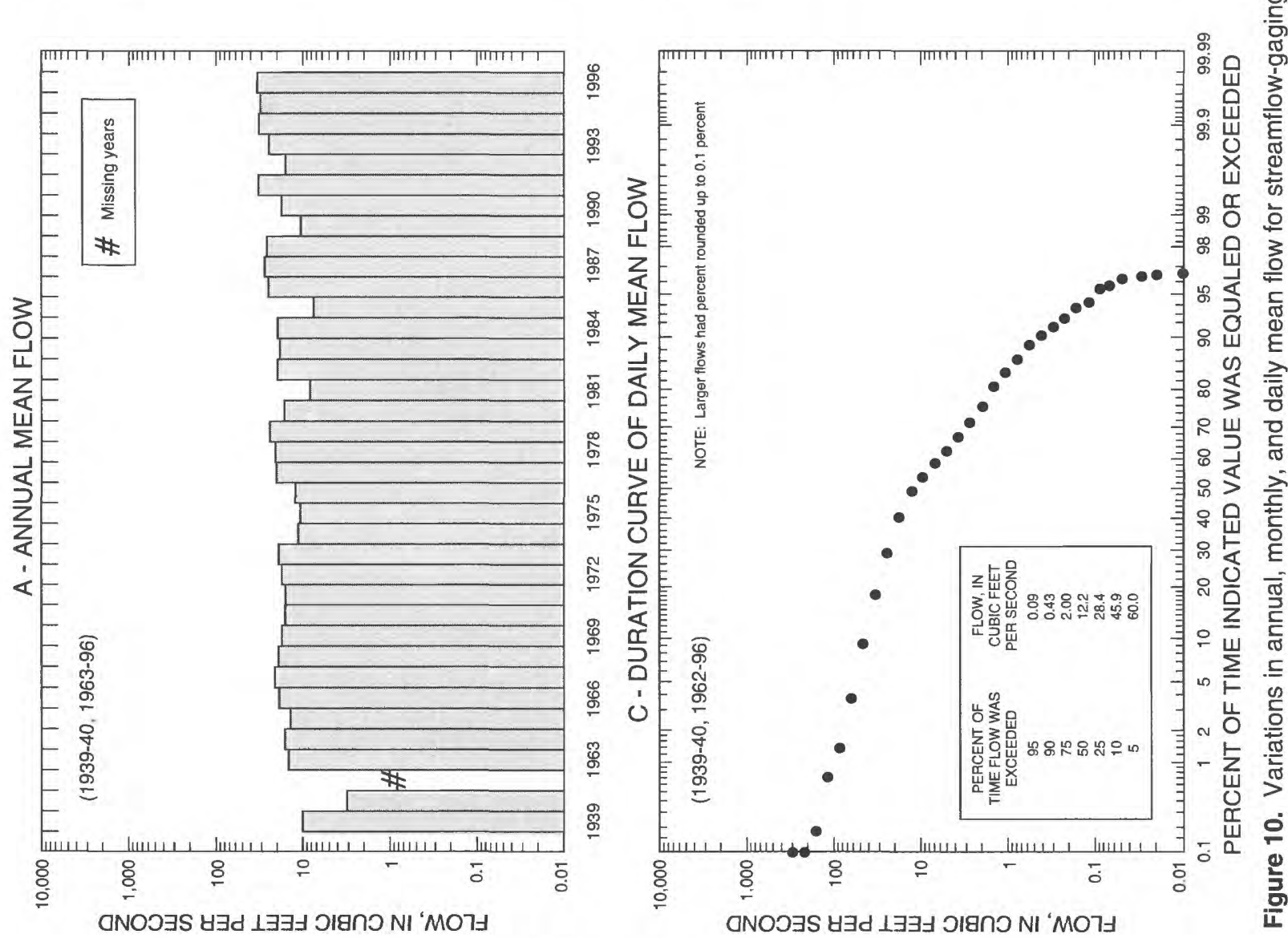

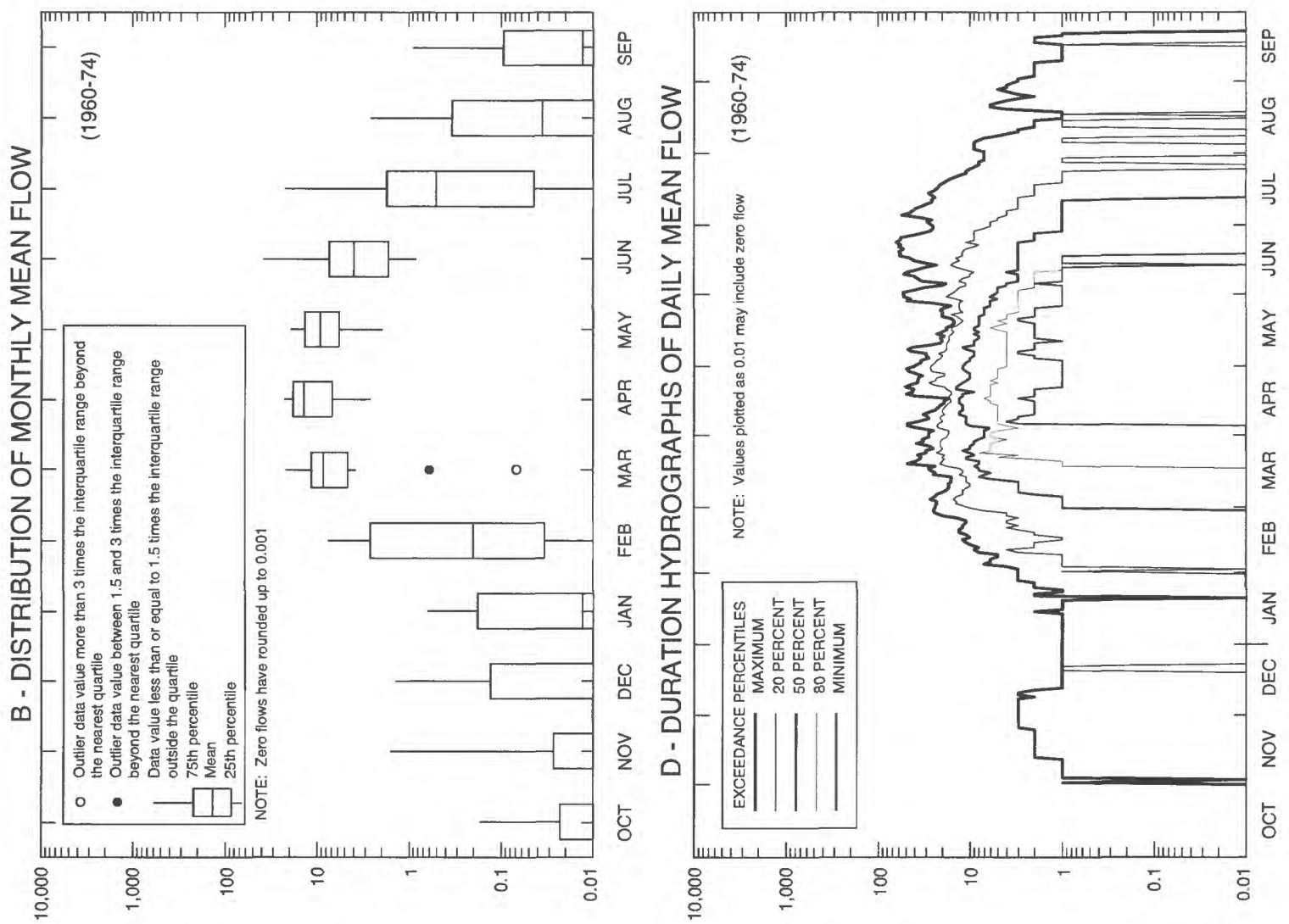

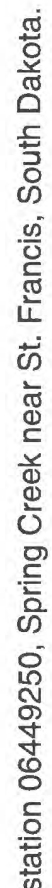
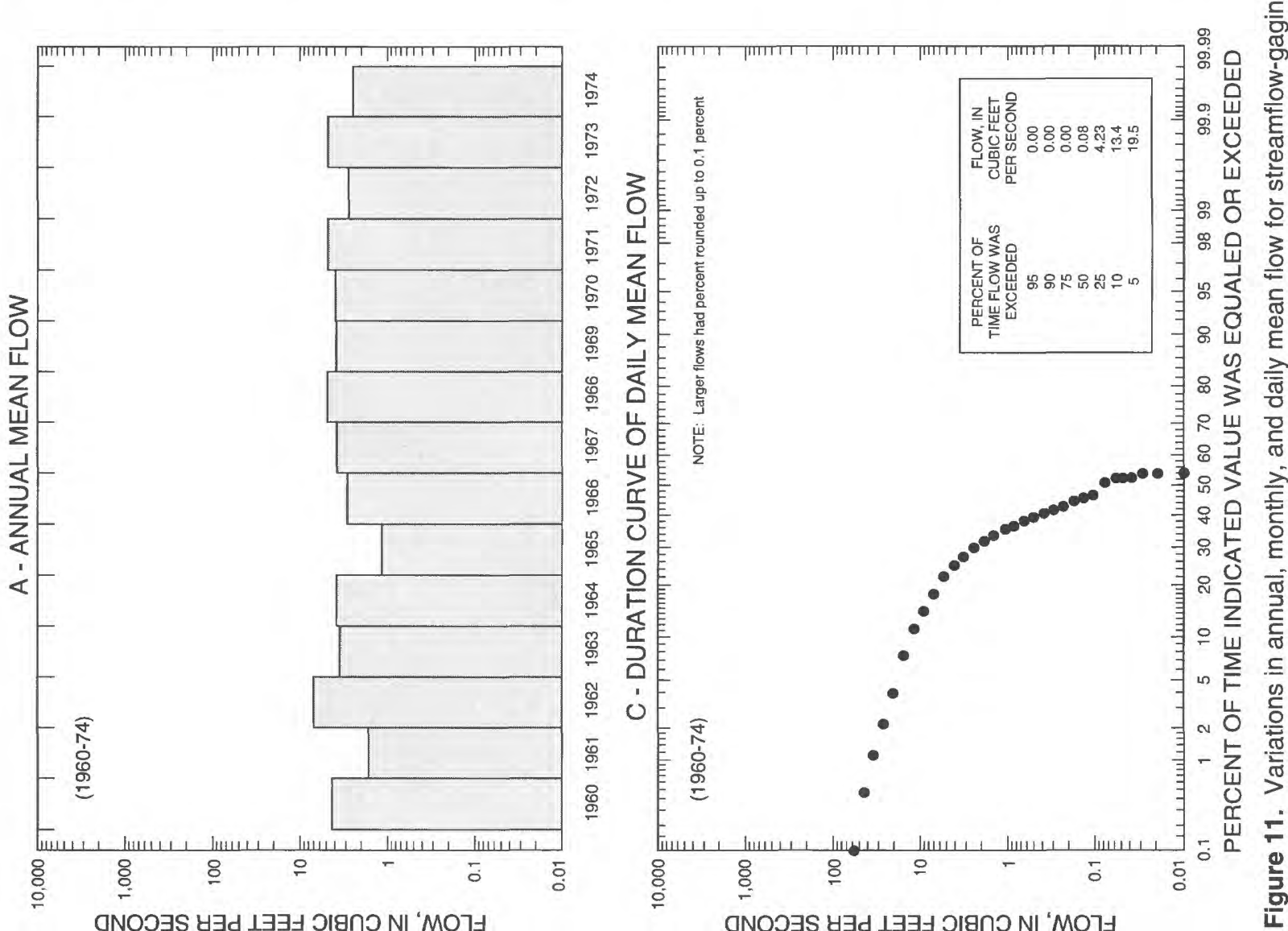

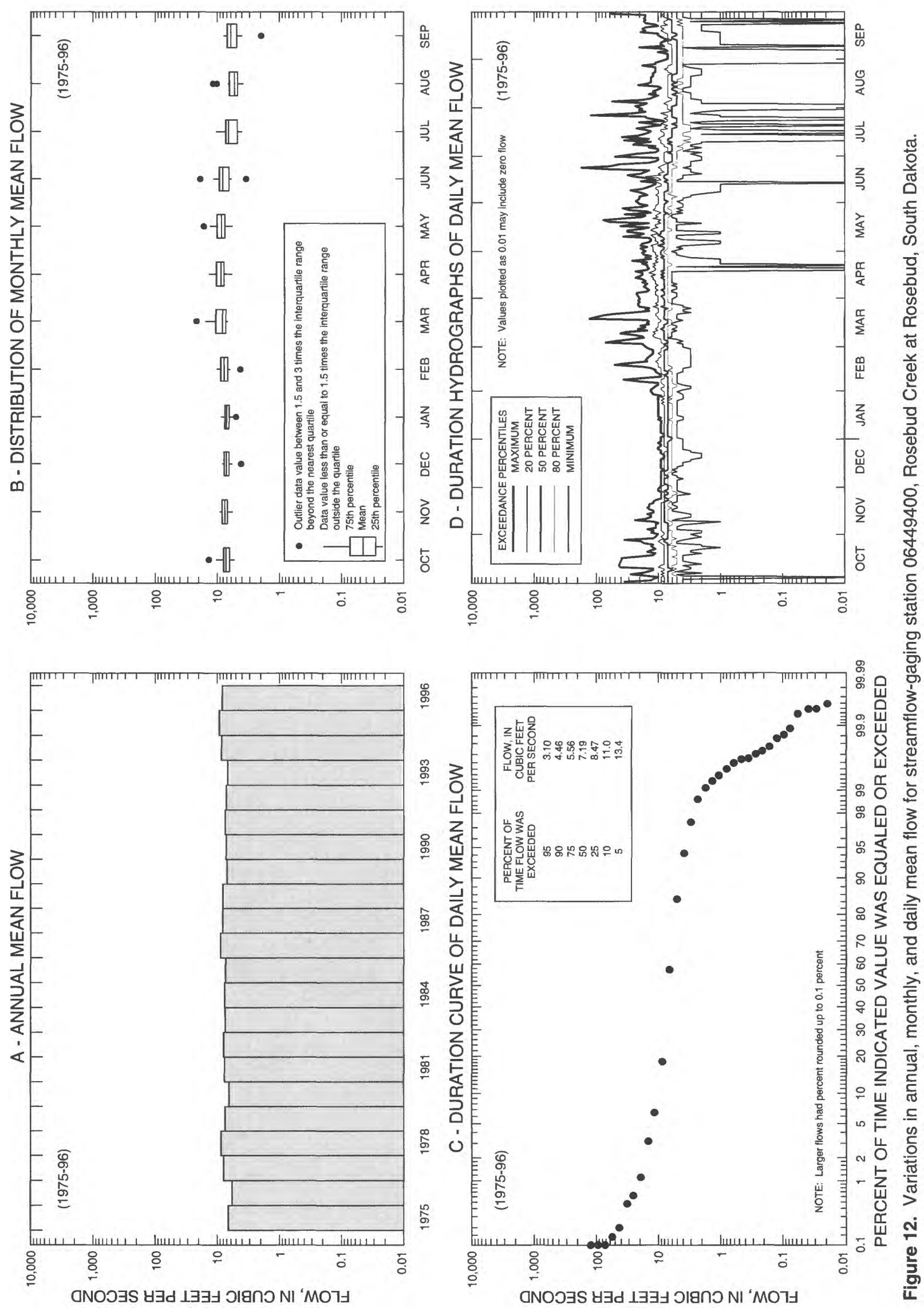


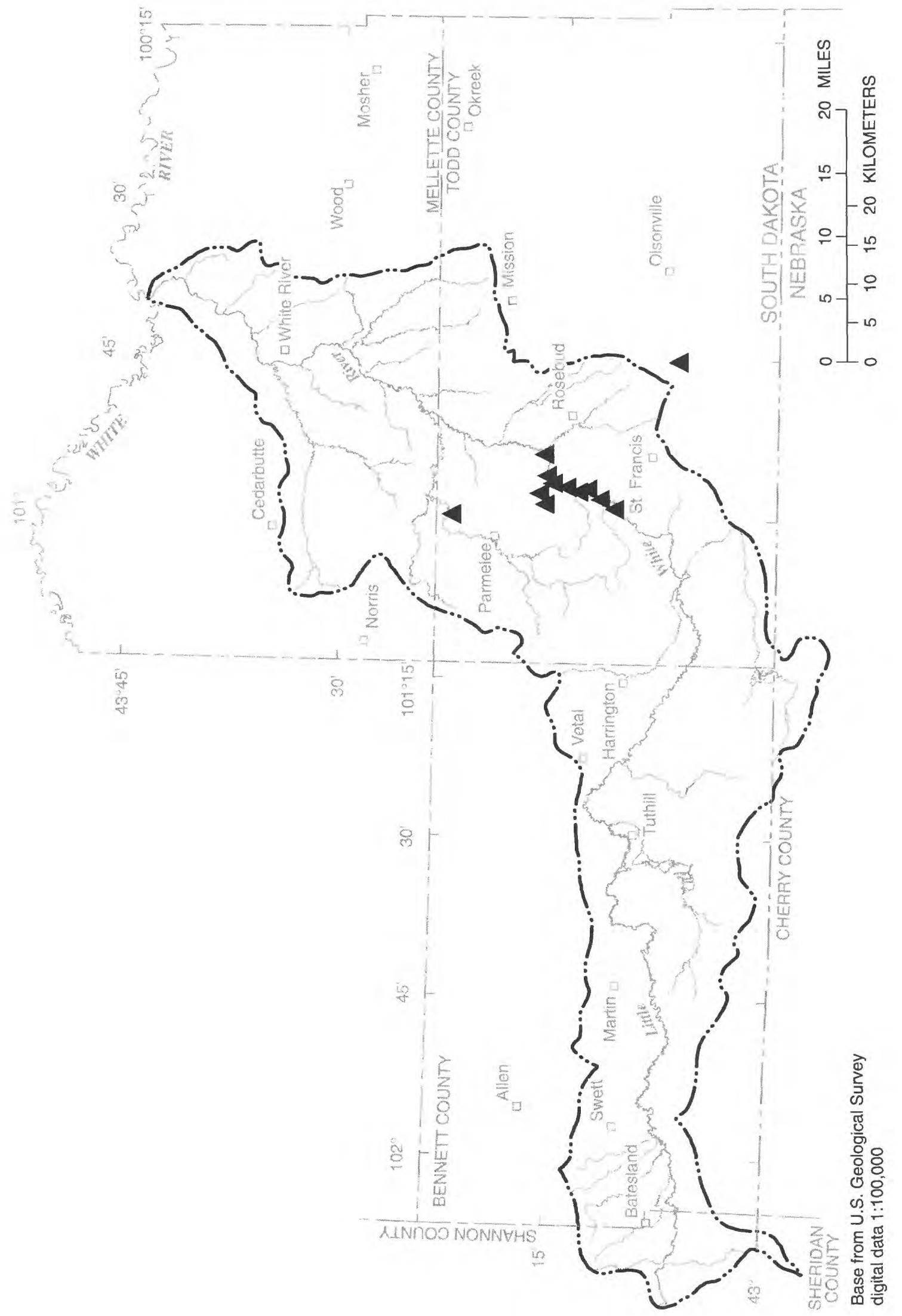

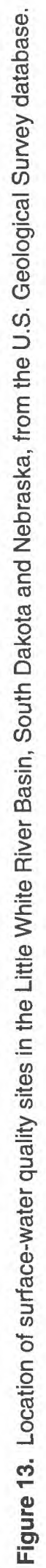




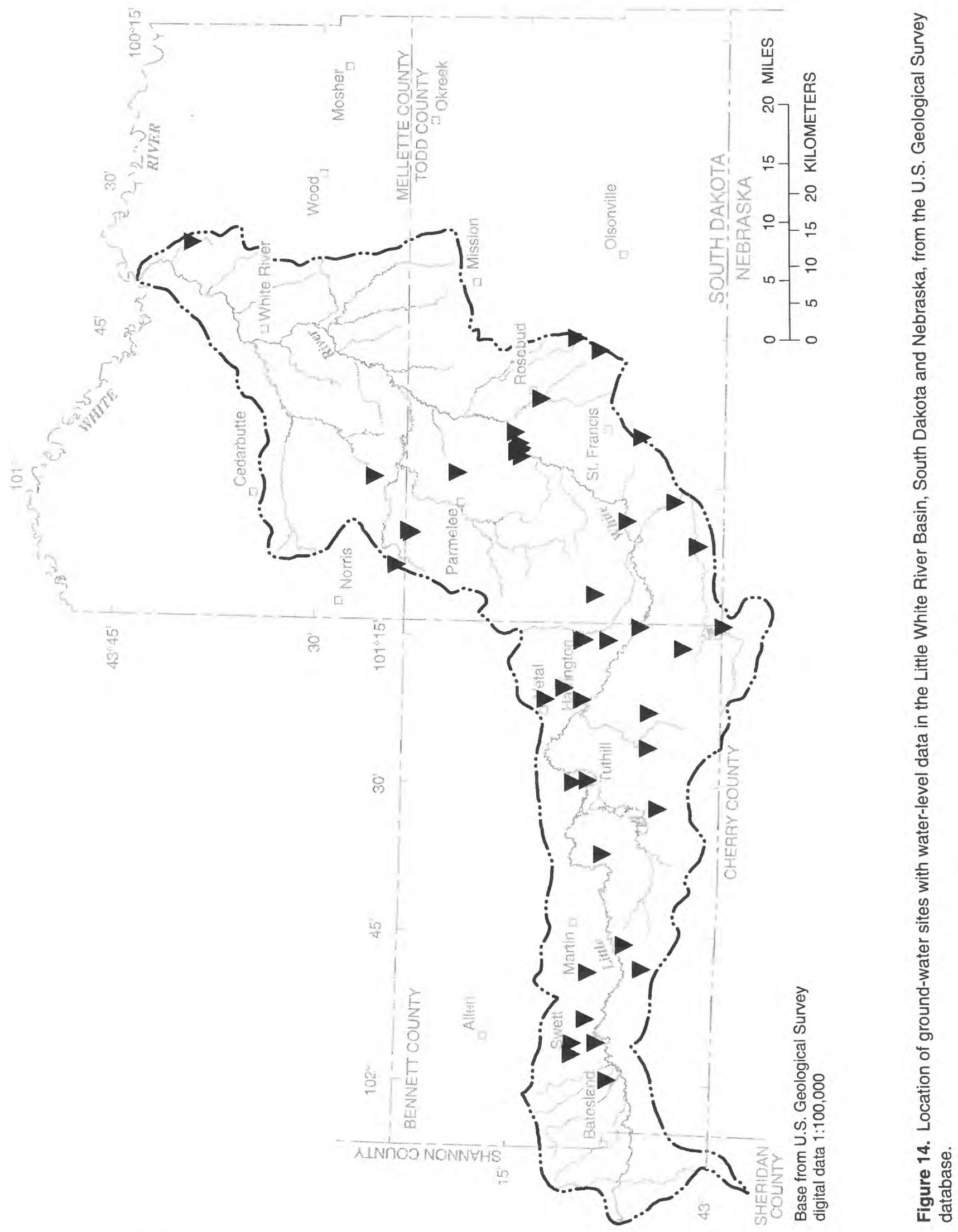




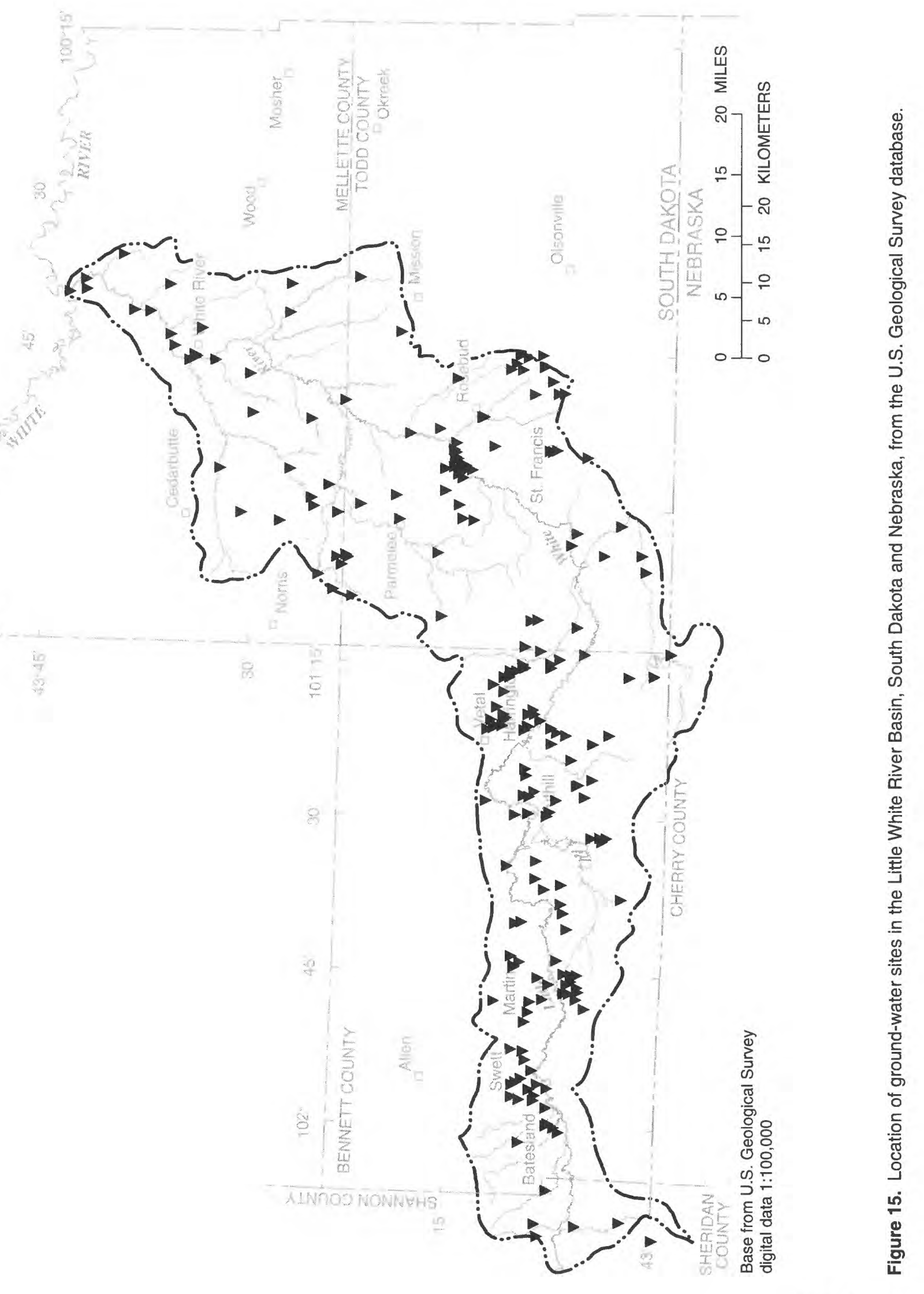

Figure 15 


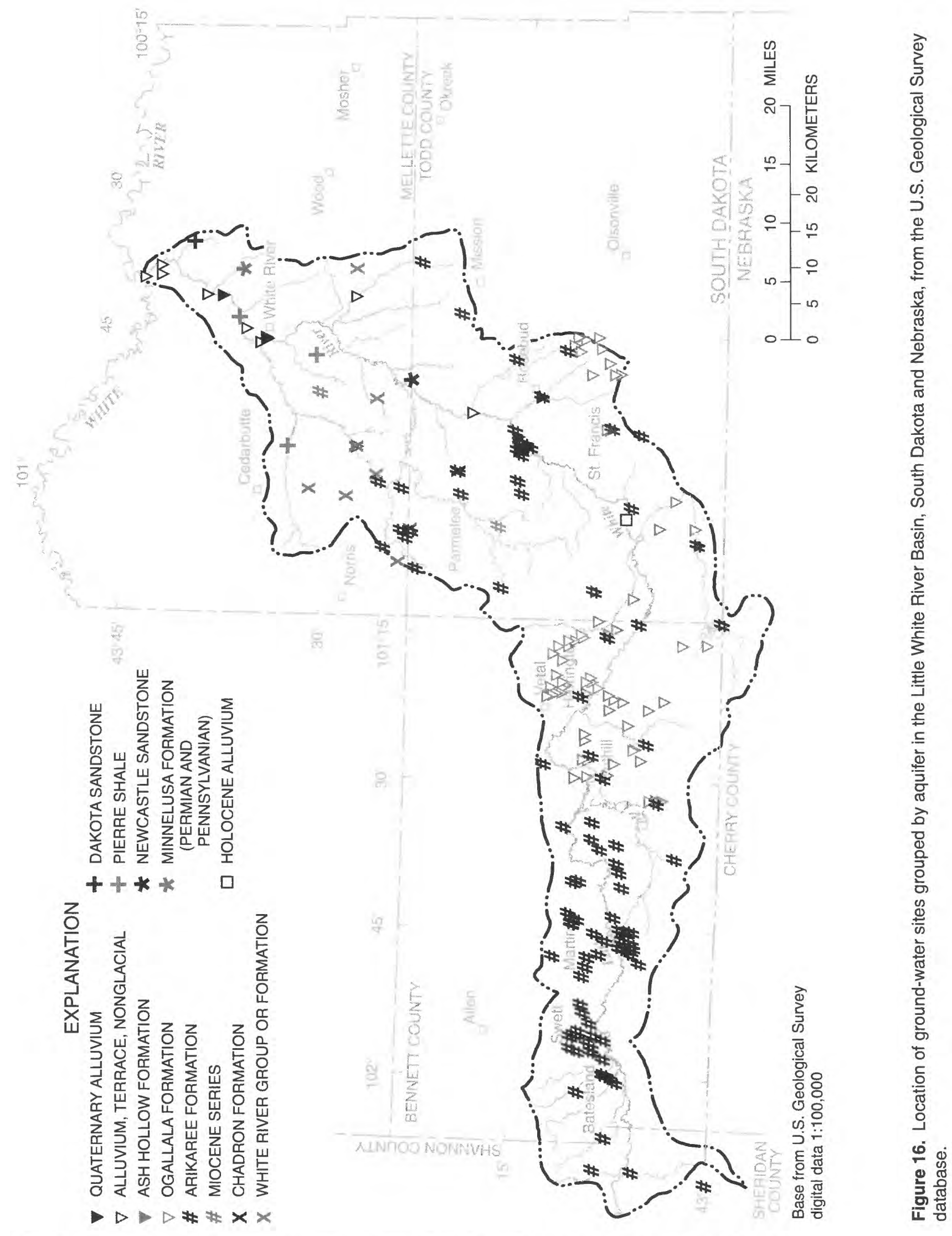




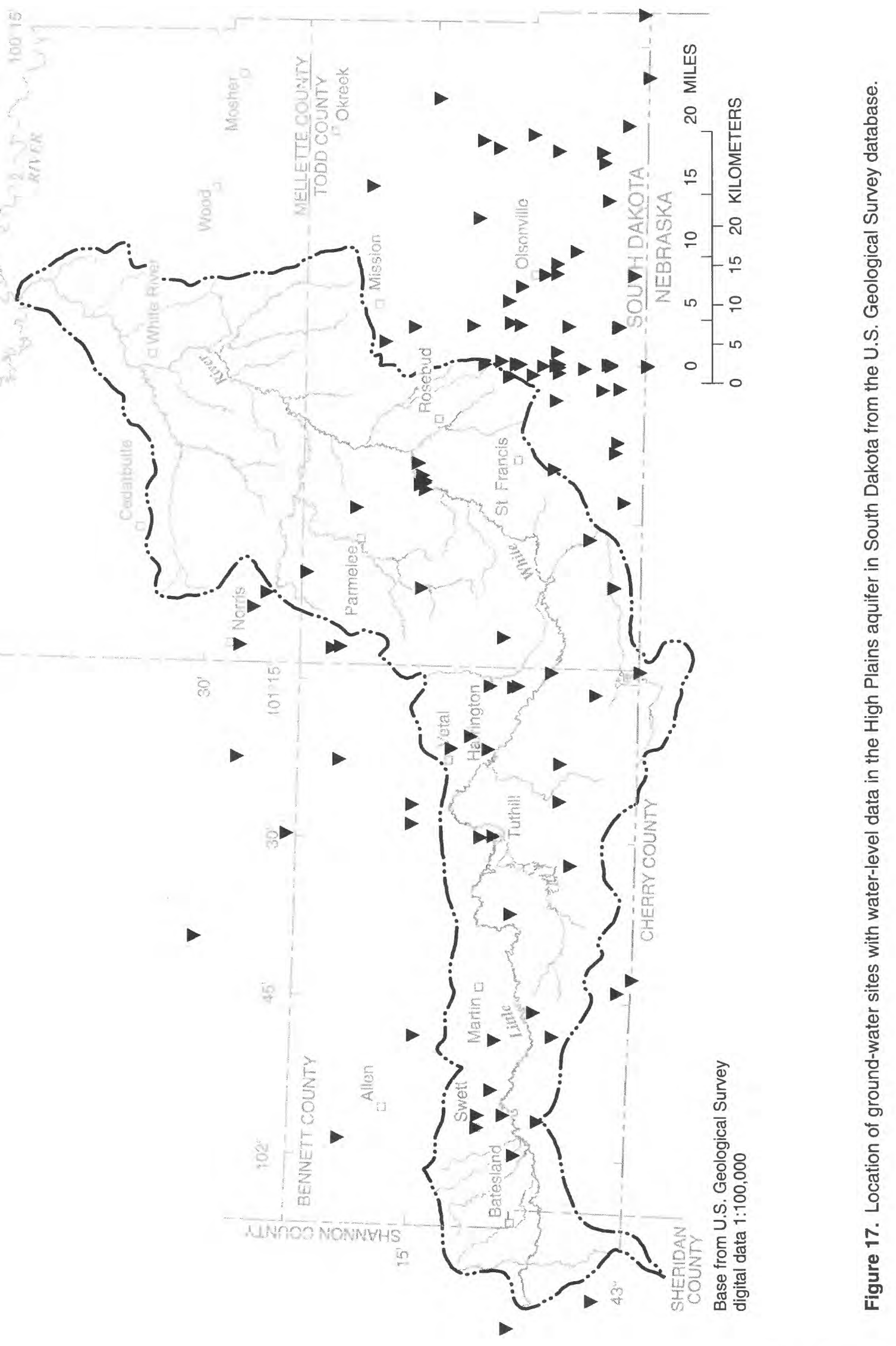




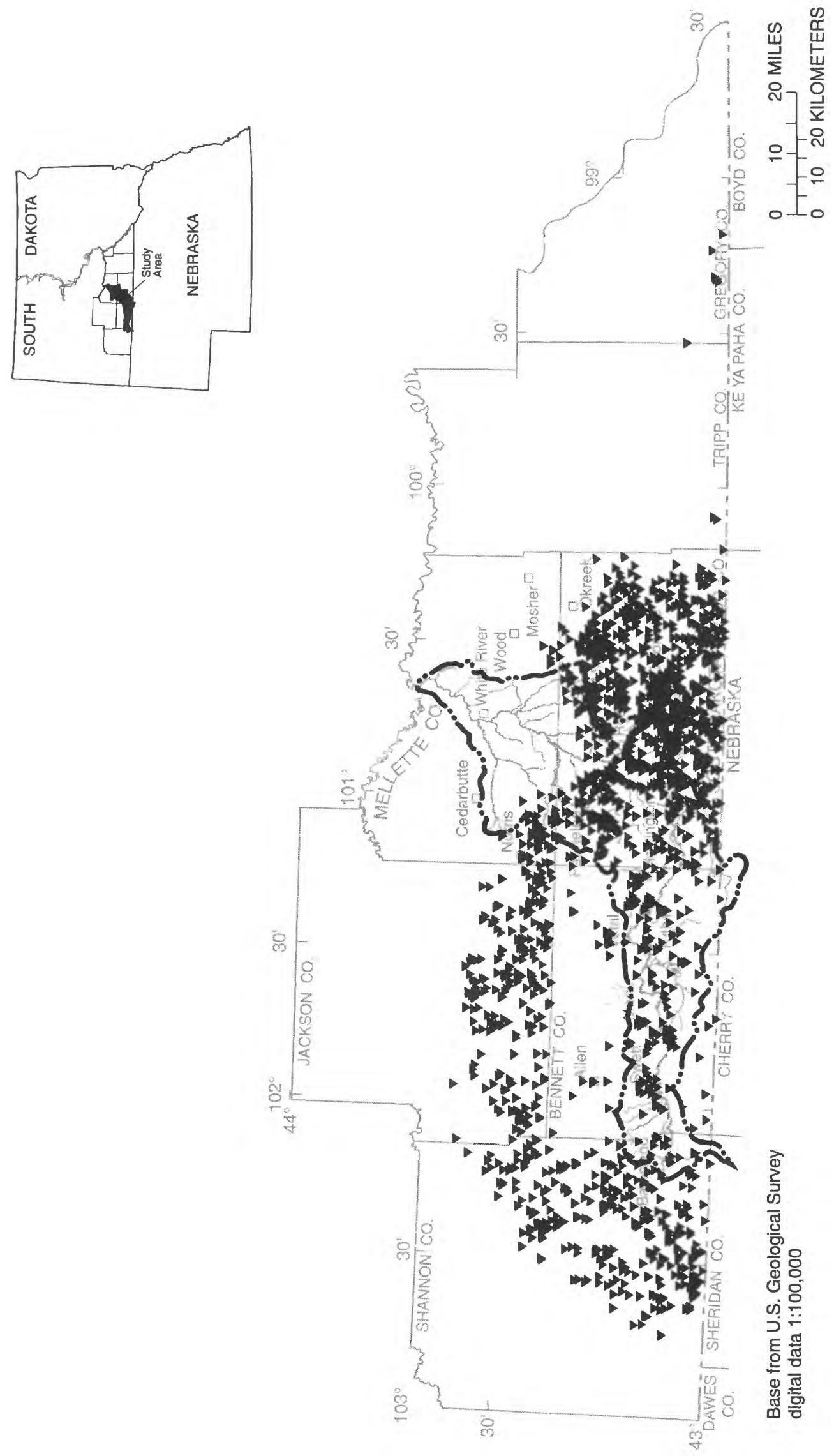

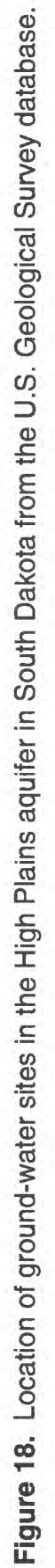




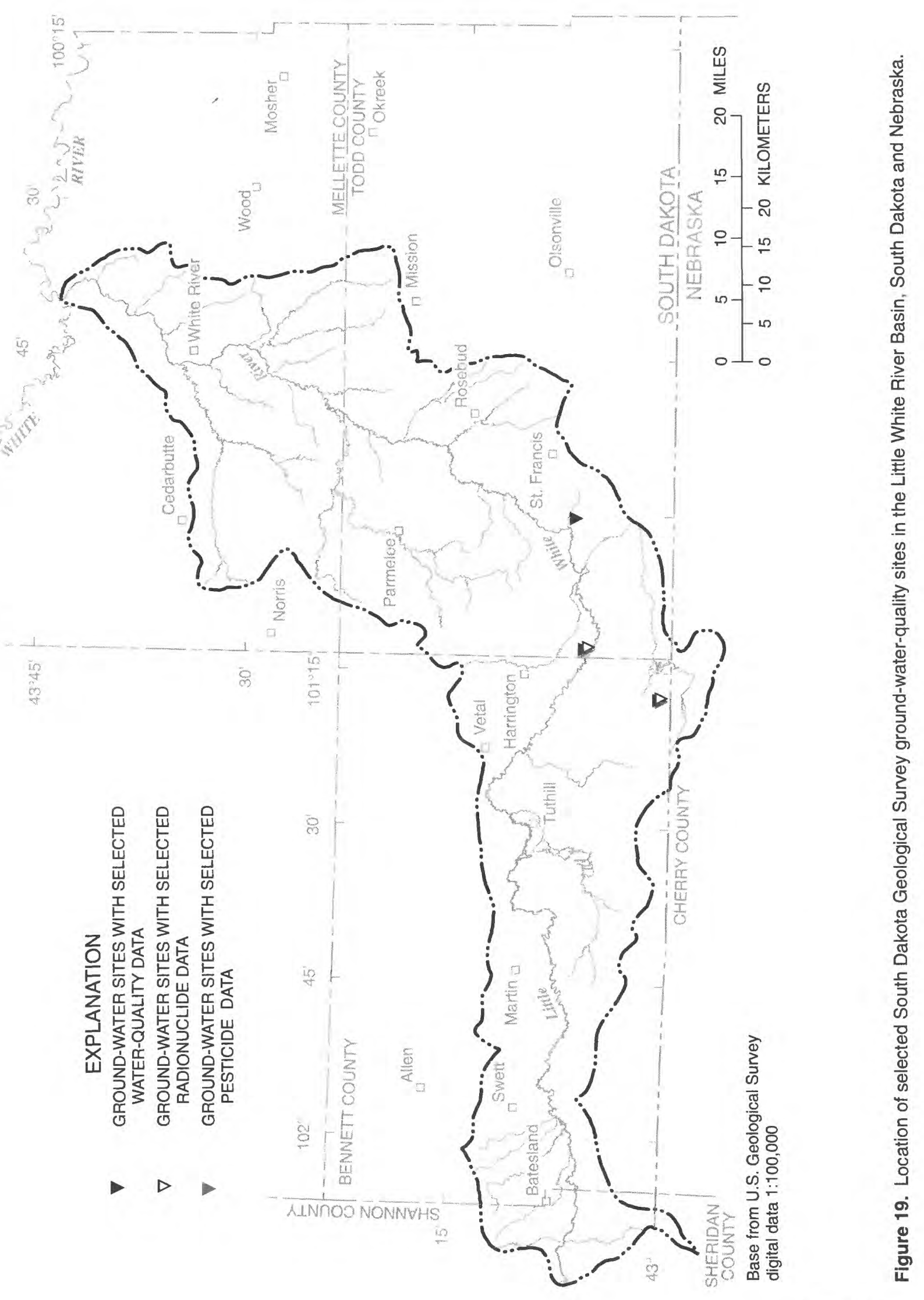

Figure $19 \quad 31$ 

TABLES 

Table 1. Monthly mean and annual flow, in cubic feet per second, for U.S. Geological Survey streamflow-gaging station 06447500, Little White River near Martin, South Dakota

$\left[{ }^{*}\right.$, no data]

\begin{tabular}{|c|c|c|c|c|c|c|c|c|c|c|c|c|c|}
\hline \multirow{2}{*}{$\begin{array}{c}\text { Water } \\
\text { year }\end{array}$} & \multicolumn{12}{|c|}{ Month } & \multirow{2}{*}{ Annua } \\
\hline & Oct & Nov & Dec & Jan & Feb & Mar & Apr & May & Jun & Jul & Aug & Sep & \\
\hline 1938 & $*$ & $*$ & $*$ & $*$ & $*$ & 31.0 & 25.6 & 30.4 & 12.6 & 9.40 & 3.42 & 5.50 & $*$ \\
\hline 1939 & 8.61 & 11.6 & 7.19 & 12.0 & 11.4 & 45.0 & 25.8 & 37.9 & 11.4 & 7.91 & 4.91 & 4.87 & 15.8 \\
\hline 1940 & 9.77 & 11.9 & 11.2 & 5.48 & 8.48 & 33.6 & 22.7 & 12.2 & 5.65 & 2.01 & 1.80 & 5.46 & 10.9 \\
\hline 1962 & $*$ & $*$ & $*$ & $*$ & $*$ & $*$ & $*$ & $*$ & $*$ & $*$ & 10.4 & 9.12 & $*$ \\
\hline 1963 & 12.1 & 17.9 & 16.0 & 7.42 & 20.0 & 34.1 & 27.7 & 18.5 & 47.5 & 11.5 & 7.51 & 10.7 & 19.2 \\
\hline 1964 & 11.5 & 14.2 & 7.97 & 7.16 & 10.8 & 24.0 & 41.4 & 31.6 & 22.9 & 10.4 & 5.38 & 6.74 & 16.2 \\
\hline 1965 & 10.5 & 9.73 & 8.26 & 9.13 & 12.3 & 18.7 & 34.6 & 30.2 & 55.0 & 39.5 & 9.83 & 13.5 & 20.9 \\
\hline 1966 & 18.2 & 18.3 & 16.6 & 10.3 & 11.2 & 157 & 38.7 & 21.7 & 15.5 & 9.30 & 12.1 & 15.2 & 28.9 \\
\hline 1967 & 14.4 & 15.1 & 14.8 & 15.4 & 22.2 & 30.1 & 17.9 & 34.3 & 157 & 38.6 & 10.5 & 10.2 & 31.6 \\
\hline 1968 & 12.2 & 15.6 & 9.71 & 12.8 & 16.6 & 35.5 & 45.8 & 23.5 & 63.6 & 14.2 & 9.99 & 9.14 & 22.3 \\
\hline 1969 & 12.9 & 18.4 & 12.8 & 11.0 & 11.2 & 57.8 & 41.1 & 25.8 & 16.0 & 44.5 & 9.98 & 7.55 & 22.5 \\
\hline 1970 & 13.1 & 18.3 & 16.3 & 12.2 & 17.1 & 23.1 & 50.2 & 27.6 & 16.0 & 7.40 & 4.24 & 5.72 & 17.6 \\
\hline 1971 & 9.54 & 18.1 & 12.8 & 7.26 & 19.4 & 44.9 & 42.8 & 36.2 & 23.2 & 8.12 & 4.51 & 8.32 & 19.6 \\
\hline 1972 & 13.7 & 15.1 & 11.5 & 6.89 & 17.4 & 32.3 & 28.7 & 46.1 & 16.9 & 10.9 & 8.93 & 7.78 & 18.0 \\
\hline 1973 & 11.5 & 16.3 & 7.55 & 10.5 & 26.0 & 55.4 & 30.3 & 25.5 & 13.6 & 6.90 & 4.57 & 8.88 & 18.0 \\
\hline 1974 & 14.6 & 17.9 & 13.6 & 14.3 & 18.4 & 24.2 & 35.5 & 16.7 & 10.7 & 5.19 & 5.66 & 6.61 & 15.2 \\
\hline 1975 & 10.3 & 12.8 & 9.65 & 7.50 & 10.6 & 15.7 & 55.9 & 27.7 & 18.0 & 7.14 & 7.70 & 4.95 & 15.6 \\
\hline 1976 & 9.16 & 10.7 & 8.69 & 7.60 & 27.3 & 23.4 & 24.7 & 20.7 & 7.28 & 4.70 & 6.94 & 13.5 & 13.6 \\
\hline 1977 & 9.55 & 9.97 & 9.39 & 4.90 & 12.2 & 11.4 & 104 & 28.8 & 31.7 & 11.9 & 24.6 & 11.5 & 22.4 \\
\hline 1978 & 20.6 & 20.6 & 11.7 & 8.34 & 8.24 & 97.5 & 47.9 & 36.8 & 21.7 & 13.3 & 10.9 & 8.62 & 25.7 \\
\hline 1979 & 12.3 & 13.5 & 8.61 & 6.79 & 9.67 & 38.1 & 33.3 & 27.0 & 20.0 & 19.5 & 11.3 & 7.74 & 17.4 \\
\hline 1980 & 7.86 & 9.80 & 10.3 & 6.78 & 8.26 & 24.5 & 39.2 & 17.6 & 11.4 & 4.85 & 4.77 & 8.58 & 12.8 \\
\hline 1981 & 14.4 & 14.1 & 12.8 & 11.5 & 14.0 & 15.5 & 13.0 & 15.1 & 6.09 & 7.31 & 13.4 & 6.90 & 12.0 \\
\hline 1982 & 11.5 & 14.9 & 13.5 & 4.51 & 21.7 & 30.7 & 24.8 & 50.5 & 27.6 & 11.7 & 6.37 & 8.44 & 18.8 \\
\hline 1983 & 15.5 & 16.6 & 12.2 & 14.3 & 23.2 & 26.3 & 28.3 & 55.9 & 14.9 & 25.2 & 102 & 18.0 & 29.6 \\
\hline 1984 & 14.4 & 17.2 & 13.9 & 18.2 & 27.9 & 36.4 & 32.2 & 22.2 & 11.9 & 10.3 & 10.2 & 6.39 & 18.4 \\
\hline 1985 & 11.8 & 17.7 & 10.5 & 7.33 & 7.30 & 25.0 & 24.1 & 12.4 & 8.16 & 15.9 & 7.08 & 10.4 & 13.2 \\
\hline 1986 & 13.5 & 10.7 & 5.59 & 12.5 & 23.7 & 97.1 & 45.6 & 25.5 & 24.7 & 12.0 & 12.1 & 15.1 & 24.9 \\
\hline 1987 & 22.5 & 20.9 & 17.4 & 14.1 & 17.4 & 64.9 & 48.7 & 23.5 & 10.9 & 5.91 & 5.73 & 8.09 & 21.7 \\
\hline 1988 & 12.1 & 14.9 & 11.5 & 4.98 & 28.4 & 73.0 & 31.0 & 54.7 & 16.5 & 9.47 & 4.81 & 5.72 & 22.3 \\
\hline 1989 & 10.4 & 14.2 & 14.6 & 8.12 & 6.26 & 42.9 & 24.4 & 18.8 & 8.32 & 4.39 & 4.26 & 7.58 & 13.7 \\
\hline 1990 & 9.63 & 13.4 & 8.06 & 11.3 & 13.8 & 22.3 & 18.2 & 30.5 & 58.9 & 12.1 & 19.4 & 6.55 & 18.7 \\
\hline 1991 & 10.0 & 13.1 & 10.2 & 5.28 & 22.8 & 28.3 & 25.7 & 31.4 & 40.9 & 30.0 & 19.1 & 9.22 & 20.5 \\
\hline 1992 & 11.0 & 18.5 & 11.6 & 11.4 & 18.1 & 27.1 & 20.6 & 13.0 & 13.1 & 11.4 & 12.0 & 10.9 & 14.9 \\
\hline 1993 & 11.4 & 15.7 & 8.57 & 8.37 & 30.3 & 69.6 & 38.6 & 29.9 & 27.7 & 17.2 & 10.3 & 13.1 & 23.3 \\
\hline 1994 & 20.8 & 23.8 & 28.8 & 13.9 & 35.1 & 124 & 31.9 & 27.3 & 28.7 & 37.6 & 10.1 & 9.24 & 32.7 \\
\hline 1995 & 16.8 & 16.5 & 20.6 & 21.6 & 28.6 & 22.5 & 53.6 & 66.4 & 89.5 & 40.7 & 20.4 & 12.4 & 34.1 \\
\hline 1996 & 20.8 & 22.9 & 16.4 & 18.0 & 48.0 & 57.5 & 37.9 & 44.6 & 32.3 & 11.1 & 9.11 & 13.9 & 27.6 \\
\hline
\end{tabular}


Table 2. Monthly mean and annual flow, in cubic feet per second, for U.S. Geological Survey streamflow-gaging station 06449100, Little White River near Vetal, South Dakota

[*, no data]

\begin{tabular}{|c|c|c|c|c|c|c|c|c|c|c|c|c|c|}
\hline \multirow{2}{*}{$\begin{array}{c}\text { Water } \\
\text { year }\end{array}$} & \multicolumn{12}{|c|}{ Month } & \multirow{2}{*}{ Annual } \\
\hline & Oct & Nov & Dec & Jan & Feb & Mar & Apr & May & Jun & Jul & Aug & Sep & \\
\hline 1959 & $*$ & $*$ & $*$ & $*$ & $*$ & $*$ & $*$ & $*$ & $*$ & $*$ & 21.6 & 18.5 & $*$ \\
\hline 1960 & 28.3 & 32.3 & 31.3 & 28.5 & 51.6 & 186 & 111 & 91.7 & 64.6 & 28.4 & 25.5 & 47.8 & 60.6 \\
\hline 1961 & 23.2 & 49.5 & 55.5 & 40.4 & 66.8 & 80.4 & 39.6 & 82.3 & 23.7 & 17.1 & 15.1 & 19.1 & 42.6 \\
\hline 1962 & 41.8 & 44.4 & 31.5 & 24.1 & 32.5 & 64.7 & 94.1 & 111 & 170 & 86.5 & 44.1 & 26.6 & 64.4 \\
\hline 1963 & 39.6 & 44.8 & 57.1 & 36.8 & 62.8 & 89.5 & 77.9 & 52.7 & 108 & 28.0 & 24.6 & 25.1 & 53.8 \\
\hline 1964 & 29.8 & 42.7 & 30.8 & 26.5 & 36.5 & 51.5 & 91.9 & 94.0 & 62.0 & 38.8 & 18.6 & 22.3 & 45.4 \\
\hline 1965 & 21.3 & 25.6 & 21.9 & 35.0 & 42.4 & 36.0 & 52.7 & 52.2 & 74.4 & 105 & 43.3 & 39.8 & 45.8 \\
\hline 1966 & 47.5 & 51.8 & 55.6 & 43.7 & 41.1 & 202 & 86.3 & 73.7 & 29.7 & 37.9 & 66.4 & 46.8 & 65.6 \\
\hline 1967 & 30.1 & 31.0 & 37.4 & 45.0 & 71.3 & 59.9 & 37.8 & 61.9 & 267 & 156 & 37.0 & 22.6 & 71.2 \\
\hline 1968 & 27.2 & 35.6 & 28.5 & 42.1 & 53.9 & 71.9 & 126 & 61.9 & 143 & 79.7 & 49.3 & 34.5 & 62.6 \\
\hline 1969 & 32.0 & 32.7 & 32.5 & 27.2 & 36.0 & 136 & 130 & 56.2 & 38.6 & 81.9 & 33.6 & 27.6 & 55.5 \\
\hline 1970 & 34.0 & 33.0 & 29.7 & 36.4 & 45.7 & 96.5 & 143 & 103 & 48.7 & 21.5 & 17.4 & 19.0 & 52.3 \\
\hline 1971 & 25.9 & 38.9 & 35.1 & 27.3 & 27.9 & 51.6 & 101 & 136 & 85.4 & 28.2 & 20.8 & 22.1 & 50.1 \\
\hline 1972 & 25.7 & 35.8 & 26.1 & 26.7 & 44.8 & 70.5 & 67.8 & 136 & 60.2 & 41.8 & 42.5 & 27.0 & 50.5 \\
\hline 1973 & 19.3 & 43.8 & 34.9 & 35.5 & 43.4 & 129 & 103 & 63.5 & 58.3 & 19.8 & 16.0 & 22.7 & 49.1 \\
\hline 1974 & 39.3 & 32.9 & 24.1 & 42.5 & 57.0 & 50.5 & 82.3 & 63.4 & 28.4 & 16.2 & 16.9 & 17.9 & 39.1 \\
\hline 1975 & 24.8 & 24.7 & 12.5 & 21.0 & 28.5 & 38.2 & 139 & 89.2 & 36.9 & 28.8 & 20.3 & 16.5 & 40.0 \\
\hline 1976 & 21.3 & 29.5 & 23.0 & 28.9 & 65.1 & 55.6 & 60.0 & 64.7 & 45.7 & 40.3 & 19.6 & 20.0 & 39.3 \\
\hline 1977 & 18.0 & 21.3 & 25.0 & 19.6 & 19.2 & 40.4 & 273 & 101 & 97.3 & 43.1 & 37.8 & 24.0 & 59.8 \\
\hline 1978 & 34.5 & 44.5 & 56.9 & 43.7 & 31.7 & 205 & 142 & 108 & 88.4 & 45.1 & 32.7 & 23.5 & 71.6 \\
\hline 1979 & 26.5 & 30.0 & 24.8 & 26.3 & 34.1 & 110 & 96.9 & 73.2 & 73.6 & 82.0 & 70.0 & 35.4 & 57.0 \\
\hline 1980 & 29.1 & 46.3 & 62.0 & 40.0 & 49.2 & 66.8 & 99.7 & 63.3 & 39.0 & 17.9 & 15.2 & 24.3 & 46.0 \\
\hline 1981 & 28.3 & 30.1 & 22.3 & 18.5 & 25.9 & 33.5 & 27.3 & 36.3 & 28.2 & 26.3 & 38.6 & 23.3 & 28.2 \\
\hline 1982 & 25.5 & 33.2 & 34.8 & 22.2 & 91.7 & 73.7 & 65.3 & 99.5 & 100 & 91.2 & 25.9 & 35.5 & 58.0 \\
\hline 1983 & 39.4 & 33.8 & 38.1 & 39.9 & 51.9 & 74.5 & 75.1 & 159 & 63.8 & 33.5 & 137 & 32.8 & 65.2 \\
\hline 1984 & 40.4 & 58.2 & 53.8 & 53.9 & 76.9 & 115 & 110 & 77.0 & 52.7 & 32.9 & 19.0 & 18.3 & 58.9 \\
\hline 1985 & 25.0 & 32.0 & 30.1 & 28.2 & 27.7 & 67.5 & 64.6 & 40.0 & 20.3 & 20.3 & 21.5 & 21.1 & 33.2 \\
\hline 1986 & 28.8 & 24.8 & 22.4 & 26.7 & 62.3 & 189 & 161 & 95.5 & 72.7 & 31.7 & 31.2 & 40.2 & 65.4 \\
\hline 1987 & 42.9 & 54.2 & 55.3 & 41.6 & 73.1 & 161 & 155 & 78.4 & 42.0 & 24.8 & 24.1 & 22.8 & 64.5 \\
\hline 1988 & 28.0 & 35.6 & 28.8 & 19.2 & 58.2 & 139 & 82.0 & 128 & 105 & 30.7 & 25.6 & 24.1 & 58.7 \\
\hline 1989 & 23.4 & 29.6 & 33.7 & 27.9 & 27.4 & 69.4 & 68.4 & 52.1 & 32.8 & 34.1 & 18.3 & 18.2 & 36.3 \\
\hline 1990 & 24.2 & 28.9 & 27.2 & 32.8 & 39.6 & 82.0 & 65.4 & 60.5 & 148 & 30.7 & 41.6 & 40.4 & 51.7 \\
\hline 1991 & 32.9 & 23.6 & 35.2 & 41.1 & 50.7 & 52.9 & 70.9 & 185 & 222 & 58.2 & 80.4 & 56.9 & 75.8 \\
\hline 1992 & 49.4 & 56.9 & 70.6 & 55.1 & 80.1 & 84.6 & 39.6 & 28.5 & 31.0 & 33.0 & 30.1 & 33.4 & 49.3 \\
\hline 1993 & 26.9 & 33.9 & 32.0 & 26.2 & 52.8 & 124 & 122 & 85.5 & 82.2 & 78.7 & 76.8 & 69.4 & 67.6 \\
\hline 1994 & 58.1 & 55.6 & 80.5 & 78.3 & 56.2 & 196 & 92.7 & 80.7 & 87.4 & 80.1 & 65.8 & 43.3 & 81.6 \\
\hline 1995 & 45.9 & 53.1 & 53.7 & 69.0 & 76.6 & 57.2 & 114 & 178 & 225 & 108 & 41.7 & 47.2 & 89.0 \\
\hline 1996 & 51.8 & 79.2 & 80.2 & 52.4 & 102 & 101 & 110 & 114 & 121 & 63.9 & 44.4 & 43.8 & 80.1 \\
\hline
\end{tabular}


Table 3. Monthly mean and annual flow, in cubic feet per second, for U.S. Geological Survey streamflow-gaging station 06449300, Little White River above Rosebud, South Dakota

[*, no data]

\begin{tabular}{|c|c|c|c|c|c|c|c|c|c|c|c|c|c|}
\hline \multirow{2}{*}{$\begin{array}{c}\text { Water } \\
\text { year }\end{array}$} & \multicolumn{12}{|c|}{ Month } & \multirow{2}{*}{ Annual } \\
\hline & Oct & Nov & Dec & Jan & Feb & Mar & Apr & May & Jun & Jul & Aug & Sep & \\
\hline 1982 & 77.6 & 78.8 & 67.4 & 51.1 & 161 & 131 & 94.9 & 167 & 165 & 143 & 69.9 & 77.6 & 107 \\
\hline 1983 & 93.8 & 82.1 & 81.6 & 86.9 & 98.0 & 135 & 142 & 268 & 148 & 105 & 161 & 68.2 & 123 \\
\hline 1984 & 76.0 & 111 & 91.8 & 93.0 & 145 & 199 & 190 & 124 & 114 & 67.7 & 54.5 & 45.2 & 109 \\
\hline 1985 & 69.6 & 70.8 & 56.1 & 67.8 & 69.3 & 119 & 127 & 89.6 & 50.8 & 54.0 & 58.2 & 56.3 & 74.0 \\
\hline 1986 & 74.2 & 56.0 & 56.3 & 73.0 & 143 & 203 & 218 & 157 & 120 & 68.4 & 60.2 & 110 & 111 \\
\hline 1987 & 84.4 & 90.9 & 101 & 97.3 & 131 & 252 & 239 & 136 & 97.4 & 64.5 & 62.5 & 56.4 & 118 \\
\hline 1988 & 66.1 & 89.7 & 80.1 & 57.7 & 88.1 & 210 & 143 & 181 & 187 & 85.8 & 64.9 & 64.0 & 110 \\
\hline 1989 & 70.8 & 75.9 & 64.6 & 62.5 & 60.3 & 140 & 125 & 108 & 69.2 & 70.7 & 45.4 & 51.9 & 78.8 \\
\hline 1990 & 55.5 & 62.9 & 51.1 & 74.9 & 74.6 & 106 & 114 & 126 & 171 & 64.1 & 78.6 & 78.8 & 88.1 \\
\hline 1991 & 81.7 & 68.2 & 59.9 & 71.4 & 118 & 107 & 129 & 238 & 281 & 90.5 & 123 & 104 & 123 \\
\hline 1992 & 90.9 & 101 & 103 & 95.0 & 147 & 160 & 102 & 72.7 & 69.0 & 66.0 & 73.8 & 74.5 & 96.1 \\
\hline 1993 & 71.2 & 58.5 & 34.9 & 75.5 & 147 & 158 & 198 & 160 & 159 & 186 & 168 & 110 & 127 \\
\hline 1994 & 108 & 76.5 & 113 & 130 & 196 & 388 & 119 & 104 & 140 & 105 & 100 & 66.1 & 137 \\
\hline 1995 & 78.9 & 83.8 & 80.7 & 98.1 & 135 & 108 & 193 & 263 & 291 & 155 & 79.5 & 106 & 139 \\
\hline 1996 & 102 & 116 & 123 & 99.2 & 85.8 & 147 & 169 & 199 & 192 & 110 & 91.5 & 90.0 & 127 \\
\hline
\end{tabular}


Table 4. Monthly mean and annual flow, in cubic feet per second, for U.S. Geological Survey streamflow-gaging station 06449500, Little White River near Rosebud, South Dakota

$[*$, no data $]$

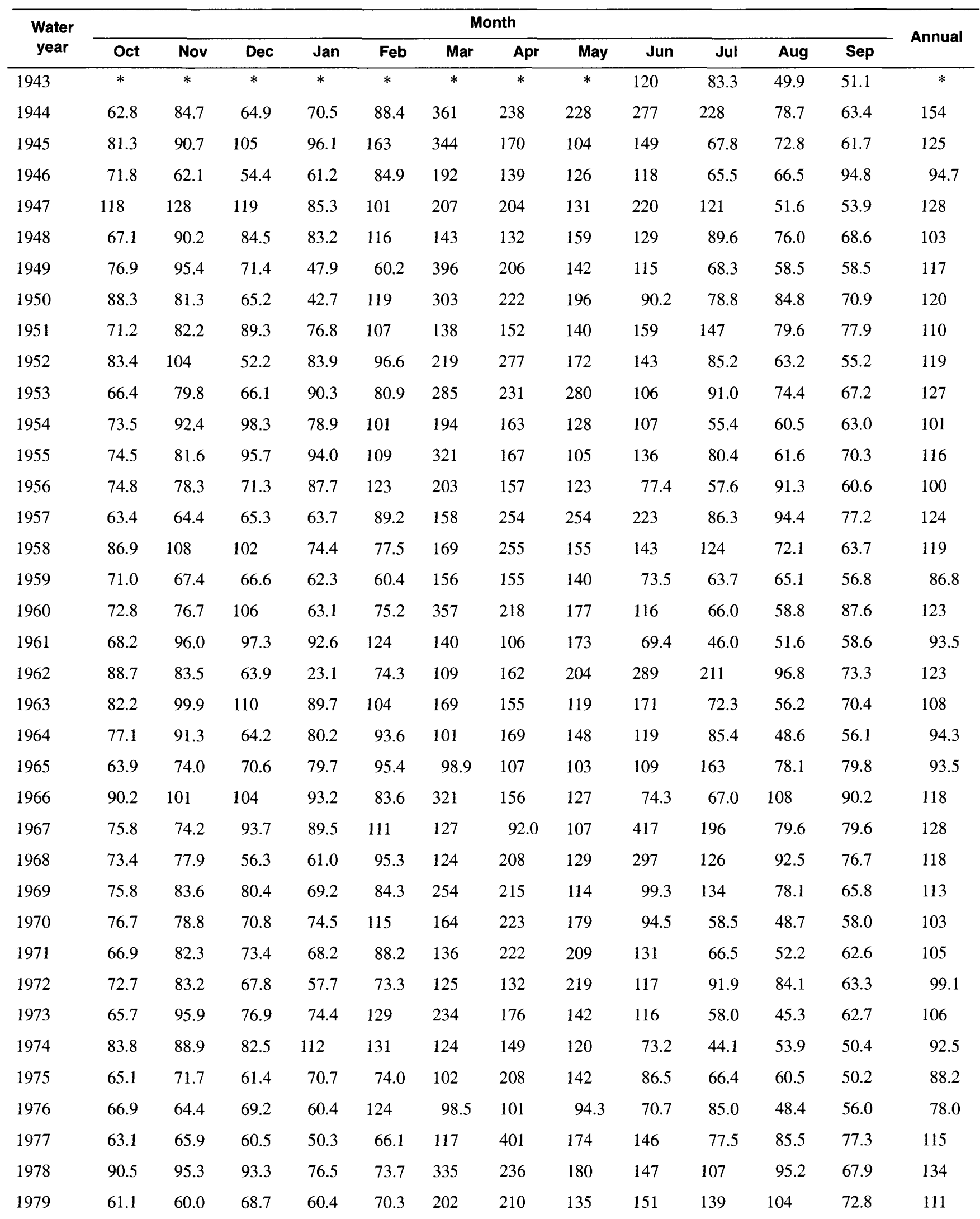


Table 4. Monthly mean and annual flow, in cubic feet per second, for U.S. Geological Survey streamflow-gaging station 06449500, Little White River near Rosebud, South Dakota-Continued

$[*$, no data $]$

\begin{tabular}{|c|c|c|c|c|c|c|c|c|c|c|c|c|c|}
\hline \multirow{2}{*}{$\begin{array}{l}\text { Water } \\
\text { year }\end{array}$} & \multicolumn{12}{|c|}{ Month } & \multirow{2}{*}{ Annual } \\
\hline & Oct & Nov & Dec & Jan & Feb & Mar & Apr & May & Jun & Jul & Aug & Sep & \\
\hline 1980 & 80.6 & 102 & 89.2 & 55.2 & 85.6 & 98.4 & 173 & 117 & 97.3 & 54.2 & 63.6 & 80.0 & 91.1 \\
\hline 1981 & 81.3 & 81.0 & 76.0 & 71.7 & 94.2 & 91.6 & 85.9 & 97.5 & 78.6 & 74.4 & 117 & 83.2 & 86.0 \\
\hline 1982 & 87.0 & 85.0 & 73.1 & 56.1 & 168 & 143 & 103 & 196 & 193 & 163 & 79.7 & 74.5 & 118 \\
\hline 1983 & 95.9 & 95.0 & 88.6 & 100 & 107 & 146 & 156 & 282 & 150 & 124 & 164 & 83.2 & 133 \\
\hline 1984 & 99.9 & 117 & 101 & 103 & 141 & 204 & 201 & 124 & 110 & 85.6 & 61.6 & 51.8 & 117 \\
\hline 1985 & 76.0 & 80.5 & 63.6 & 75.6 & 75.6 & 129 & 130 & 92.7 & 62.5 & 56.4 & 65.4 & 62.9 & 80.9 \\
\hline 1986 & 85.1 & 64.7 & 57.8 & 76.7 & 156 & 226 & 239 & 154 & 126 & 91.1 & 74.3 & 89.8 & 120 \\
\hline 1987 & 105 & 106 & 121 & 104 & 138 & 255 & 285 & 185 & 115 & 67.8 & 71.6 & 70.7 & 135 \\
\hline 1988 & 79.0 & 93.0 & 85.1 & 74.0 & 105 & 247 & 168 & 214 & 200 & 94.0 & 71.8 & 69.4 & 125 \\
\hline 1989 & 80.5 & 88.4 & 79.0 & 50.2 & 74.2 & 139 & 130 & 107 & 77.1 & 68.5 & 53.0 & 65.9 & 84.5 \\
\hline 1990 & 76.4 & 66.6 & 63.0 & 85.5 & 92.2 & 132 & 125 & 147 & 184 & 90.7 & 76.5 & 77.4 & 101 \\
\hline 1991 & 92.5 & 85.6 & 65.9 & 90.0 & 132 & 113 & 132 & 277 & 325 & 98.4 & 132 & 116 & 138 \\
\hline 1992 & 91.0 & 131 & 131 & 106 & 152 & 169 & 106 & 87.5 & 86.2 & 93.6 & 88.5 & 85.0 & 110 \\
\hline 1993 & 76.3 & 76.4 & 51.4 & 109 & 199 & 205 & 240 & 182 & 153 & 128 & 123 & 116 & 138 \\
\hline 1994 & 97.2 & 77.1 & 121 & 150 & 231 & 385 & 142 & 123 & 136 & 119 & 123 & 82.2 & 149 \\
\hline 1995 & 82.3 & 93.8 & 86.9 & 111 & 144 & 135 & 225 & 302 & 340 & 146 & 77.8 & 83.9 & 152 \\
\hline 1996 & 116 & 154 & 144 & 104 & 138 & 204 & 213 & 219 & 208 & 111 & 93.6 & 95.7 & 150 \\
\hline
\end{tabular}


Table 5. Monthly mean and annual flow, in cubic feet per second, for U.S. Geological Survey streamflow-gaging station 06450500, Little White River below White River, South Dakota

[*, no data]

\begin{tabular}{|c|c|c|c|c|c|c|c|c|c|c|c|c|c|}
\hline \multirow{2}{*}{$\begin{array}{c}\text { Water } \\
\text { year }\end{array}$} & \multicolumn{12}{|c|}{ Month } & \multirow{2}{*}{ Annual } \\
\hline & Oct & Nov & Dec & Jan & Feb & Mar & Apr & May & Jun & Jul & Aug & Sep & \\
\hline 1950 & 111 & 76.7 & 57.1 & 31.0 & 99.8 & 505 & 247 & 308 & 98.9 & 70.2 & 86.2 & 79.6 & 148 \\
\hline 1951 & 72.8 & 73.6 & 79.5 & 82.4 & 89.1 & 155 & 151 & 159 & 257 & 170 & 143 & 100 & 128 \\
\hline 1952 & 114 & 73.7 & 39.1 & 91.6 & 135 & 675 & 515 & 246 & 148 & 87.9 & 61.2 & 33.0 & 185 \\
\hline 1953 & 60.7 & 69.8 & 61.0 & 80.3 & 85.9 & 533 & 344 & 545 & 255 & 102 & 69.2 & 54.0 & 189 \\
\hline 1954 & 73.9 & 96.0 & 77.6 & 89.7 & 142 & 206 & 161 & 144 & 110 & 60.4 & 64.8 & 60.9 & 107 \\
\hline 1955 & 83.5 & 86.7 & 94.2 & 92.4 & 117 & 476 & 200 & 143 & 172 & 74.3 & 60.3 & 71.8 & 139 \\
\hline 1956 & 75.5 & 69.9 & 73.1 & 82.7 & 109 & 224 & 137 & 143 & 70.6 & 53.8 & 110 & 52.8 & 100 \\
\hline 1957 & 62.1 & 77.8 & 89.2 & 85.6 & 106 & 170 & 269 & 256 & 220 & 75.8 & 93.7 & 62.7 & 131 \\
\hline 1958 & 83.2 & 103 & 117 & 99.7 & 85.4 & 173 & 301 & 152 & 151 & 134 & 75.4 & 57.4 & 128 \\
\hline 1959 & 71.3 & 69.3 & 66.7 & 65.9 & 79.5 & 173 & 167 & 137 & 76.5 & 57.0 & 56.1 & 54.1 & 89.5 \\
\hline 1960 & 66.2 & 72.2 & 95.3 & 80.8 & 94.0 & 593 & 239 & 200 & 128 & 72.1 & 65.0 & 86.3 & 150 \\
\hline 1961 & 64.1 & 91.1 & 94.7 & 91.8 & 150 & 155 & 102 & 165 & 80.5 & 66.7 & 51.7 & 42.1 & 96.0 \\
\hline 1962 & 81.3 & 97.9 & 79.1 & 28.5 & 57.5 & 178 & 177 & 433 & 714 & 574 & 110 & 74.3 & 218 \\
\hline 1963 & 90.6 & 105 & 111 & 93.2 & 105 & 178 & 174 & 133 & 198 & 76.4 & 61.1 & 71.3 & 116 \\
\hline 1964 & 73.4 & 88.8 & 55.2 & 71.9 & 90.3 & 98.2 & 179 & 163 & 165 & 84.9 & 49.1 & 53.3 & 97.5 \\
\hline 1965 & 56.9 & 67.3 & 69.4 & 79.8 & 86.8 & 103 & 117 & 118 & 125 & 155 & 71.7 & 76.0 & 93.8 \\
\hline 1966 & 86.6 & 99.3 & 93.4 & 87.7 & 84.5 & 478 & 195 & 148 & 84.2 & 71.8 & 133 & 111 & 140 \\
\hline 1967 & 71.2 & 63.8 & 85.2 & 92.3 & 112 & 151 & 93.1 & 114 & 907 & 244 & 68.9 & 73.9 & 172 \\
\hline 1968 & 71.0 & 71.0 & 58.4 & 56.6 & 96.6 & 146 & 288 & 139 & 931 & 123 & 87.2 & 63.3 & 176 \\
\hline 1969 & 63.3 & 79.7 & 70.5 & 68.8 & 87.5 & 286 & 229 & 117 & 88.5 & 136 & 64.7 & 53.6 & 112 \\
\hline 1970 & 75.1 & 81.4 & 78.0 & 76.9 & 122 & 147 & 250 & 170 & 100 & 41.7 & 39.8 & 42.9 & 102 \\
\hline 1971 & 58.4 & 84.3 & 69.3 & 69.0 & 98.4 & 149 & 481 & 245 & 168 & 65.3 & 46.5 & 52.9 & 132 \\
\hline 1972 & 78.5 & 78.7 & 63.2 & 62.6 & 81.2 & 109 & 122 & 200 & 129 & 133 & 88.4 & 55.0 & 100 \\
\hline 1973 & 64.8 & 87.2 & 77.3 & 75.4 & 130 & 213 & 184 & 159 & 110 & 49.0 & 41.2 & 84.7 & 106 \\
\hline 1974 & 89.5 & 84.1 & 86.4 & 114 & 137 & 118 & 139 & 105 & 67.1 & 31.3 & 37.1 & 45.4 & 87.6 \\
\hline 1975 & 57.6 & 70.6 & 62.6 & 72.5 & 74.9 & 109 & 222 & 131 & 102 & 44.2 & 44.2 & 44.9 & 86.0 \\
\hline 1976 & 68.6 & 60.5 & 69.4 & 59.8 & 143 & 108 & 100 & 98.8 & 67.4 & 78.4 & 44.7 & 52.3 & 79.0 \\
\hline 1977 & 53.3 & 66.9 & 66.6 & 52.3 & 72.1 & 201 & 613 & 208 & 126 & 114 & 96.3 & 89.1 & 146 \\
\hline 1978 & 111 & 95.7 & 99.8 & 85.5 & 83.0 & 815 & 330 & 325 & 144 & 101 & 75.4 & 59.4 & 195 \\
\hline 1979 & 76.2 & 81.2 & 77.9 & 66.5 & 73.8 & 211 & 205 & 211 & 251 & 203 & 91.0 & 112 & 139 \\
\hline 1980 & 91.1 & 62.1 & 94.5 & 60.7 & 83.9 & 131 & 203 & 147 & 123 & 46.5 & 72.8 & 94.1 & 101 \\
\hline 1981 & 89.9 & 107 & 83.7 & 74.9 & 89.7 & 85.9 & 76.9 & 86.0 & 82.8 & 74.4 & 62.1 & 70.8 & 81.9 \\
\hline 1982 & 65.1 & 71.3 & 61.6 & 56.7 & 212 & 147 & 131 & 263 & 308 & 162 & 74.5 & 84.3 & 136 \\
\hline 1983 & 141 & 125 & 103 & 108 & 121 & 138 & 171 & 614 & 204 & 133 & 117 & 120 & 175 \\
\hline 1984 & 88.1 & 83.7 & 102 & 108 & 170 & 236 & 231 & 136 & 117 & 80.2 & 49.2 & 49.9 & 121 \\
\hline 1985 & 71.1 & 83.1 & 71.2 & 76.5 & 83.3 & 158 & 132 & 82.5 & 54.7 & 48.5 & 67.1 & 57.4 & 82.1 \\
\hline 1986 & 68.9 & 61.8 & 56.5 & 76.1 & 158 & 307 & 398 & 204 & 226 & 81.5 & 64.0 & 92.0 & 149 \\
\hline
\end{tabular}


Table 5. Monthly mean and annual flow, in cubic feet per second, for U.S. Geological Survey streamflow-gaging station 06450500, Little White River below White River, South Dakota - Continued

[*, no data]

\begin{tabular}{lcccccccccccccc}
\hline $\begin{array}{c}\text { Water } \\
\text { year }\end{array}$ & \multicolumn{1}{c}{ Oct } & Nov & Dec & Jan & Feb & Mar & Apr & May & Jun & Jul & Aug & Sep & Annual \\
\hline 1987 & 95.5 & 98.0 & 121 & 123 & 236 & 340 & 379 & 187 & 97.2 & 65.9 & 51.5 & 55.3 & 153 \\
1988 & 68.0 & 91.3 & 111 & 56.9 & 75.9 & 245 & 167 & 248 & 159 & 76.2 & 52.0 & 62.6 & 118 \\
1989 & 73.2 & 74.4 & 71.6 & 66.4 & 75.4 & 192 & 113 & 97.1 & 75.7 & 68.8 & 43.7 & 59.4 & 84.3 \\
1990 & 65.3 & 80.7 & 79.1 & 83.6 & 117 & 135 & 140 & 159 & 275 & 117 & 89.5 & 74.4 & 118 \\
1991 & 73.1 & 70.7 & 60.9 & 82.0 & 110 & 119 & 145 & 319 & 502 & 102 & 112 & 93.6 & 149 \\
1992 & 87.3 & 125 & 148 & 169 & 179 & 181 & 106 & 111 & 93.0 & 97.0 & 81.2 & 83.0 & 122 \\
1993 & 90.0 & 122 & 91.5 & 89.8 & 133 & 328 & 190 & 159 & 126 & 104 & 119 & 117 & 139 \\
1994 & 125 & 110 & 160 & 149 & 289 & 526 & 209 & 159 & 161 & 120 & 123 & 72.2 & 183 \\
1995 & 64.3 & 96.0 & 95.8 & 122 & 193 & 392 & 511 & 583 & 410 & 142 & 73.3 & 75.1 & 230 \\
1996 & 116 & 140 & 150 & 117 & 170 & 318 & 256 & 480 & 282 & 152 & 106 & 104 & 200 \\
\hline
\end{tabular}


Table 6. Monthly mean and annual flow, in cubic feet per second, for U.S. Geological Survey streamflow-gaging station 06448000, Lake Creek above refuge, near Tuthill, South Dakota.

$[*$, no data]

\begin{tabular}{|c|c|c|c|c|c|c|c|c|c|c|c|c|c|}
\hline \multirow{2}{*}{$\begin{array}{l}\text { Water } \\
\text { year }\end{array}$} & \multicolumn{12}{|c|}{ Month } & \multirow{2}{*}{ Annua } \\
\hline & Oct & Nov & Dec & Jan & Feb & Mar & Apr & May & Jun & Jul & Aug & Sep & \\
\hline 1938 & $*$ & * & $*$ & $*$ & * & 23.6 & 22.9 & 22.5 & 16.1 & 15.8 & 13.1 & 15.6 & $*$ \\
\hline 1939 & 15.8 & 14.5 & 7.39 & 15.0 & 11.1 & 26.5 & 18.9 & 9.99 & 8.83 & 10.9 & 13.3 & 13.7 & 13.8 \\
\hline 1940 & 17.9 & 20.8 & 23.4 & 13.4 & 21.2 & 26.9 & 25.2 & 14.6 & 11.3 & 10.3 & 15.7 & 15.3 & 18.0 \\
\hline 1962 & $*$ & $*$ & $*$ & * & $*$ & * & $*$ & $*$ & $*$ & $*$ & 17.0 & 17.3 & $*$ \\
\hline 1963 & 19.1 & 23.1 & 20.2 & 17.6 & 24.4 & 26.4 & 23.3 & 19.0 & 20.6 & 15.5 & 14.9 & 17.9 & 20.1 \\
\hline 1964 & 19.8 & 18.3 & 18.1 & 19.9 & 20.7 & 26.7 & 31.6 & 23.0 & 22.0 & 13.5 & 12.9 & 15.6 & 20.2 \\
\hline 1965 & 16.9 & 16.3 & 14.7 & 19.8 & 17.1 & 20.5 & 22.3 & 23.8 & 21.5 & 17.4 & 15.3 & 19.5 & 18.8 \\
\hline 1966 & 20.9 & 19.3 & 21.1 & 18.0 & 20.9 & 37.6 & 23.2 & 18.0 & 16.9 & 14.7 & 17.3 & 18.7 & 20.6 \\
\hline 1967 & 19.5 & 19.8 & 21.2 & 18.0 & 20.7 & 24.6 & 20.7 & 28.4 & 29.9 & 18.8 & 15.9 & 17.3 & 21.2 \\
\hline 1968 & 18.2 & 20.5 & 21.3 & 21.4 & 23.2 & 25.5 & 26.0 & 22.0 & 29.3 & 19.9 & 20.2 & 17.6 & 22.1 \\
\hline 1969 & 18.8 & 21.2 & 14.5 & 18.7 & 23.3 & 32.0 & 27.6 & 17.3 & 17.3 & 20.3 & 9.57 & 13.7 & 19.5 \\
\hline 1970 & 17.9 & 22.7 & 21.1 & 20.1 & 25.7 & 25.9 & 28.5 & 22.0 & 17.2 & 12.3 & 8.40 & 11.5 & 19.4 \\
\hline 1971 & 18.5 & 22.1 & 18.5 & 21.7 & 24.5 & 25.2 & 28.5 & 24.6 & 19.5 & 13.3 & 9.66 & 14.6 & 20.0 \\
\hline 1972 & 19.8 & 23.8 & 23.0 & 21.1 & 26.0 & 26.6 & 27.4 & 26.0 & 18.7 & 19.9 & 17.3 & 15.0 & 22.0 \\
\hline 1973 & 17.6 & 20.4 & 17.6 & 20.0 & 24.7 & 31.4 & 23.6 & 23.6 & 16.0 & 14.2 & 11.3 & 18.2 & 19.8 \\
\hline 1974 & 18.7 & 20.8 & 23.2 & 24.4 & 29.7 & 23.0 & 25.9 & 20.6 & 13.9 & 10.3 & 13.4 & 14.3 & 19.8 \\
\hline 1975 & 16.6 & 17.8 & 18.7 & 19.8 & 18.3 & 30.2 & 30.0 & 18.7 & 23.6 & 14.8 & 11.9 & 11.5 & 19.3 \\
\hline 1976 & 15.0 & 16.6 & 19.1 & 12.9 & 18.4 & 17.5 & 18.6 & 18.8 & 13.5 & 12.9 & 13.2 & 13.5 & 15.8 \\
\hline 1977 & 16.5 & 18.8 & 19.8 & 16.1 & 25.1 & 38.1 & 37.3 & 23.8 & 15.9 & 15.7 & 19.9 & 16.7 & 21.9 \\
\hline 1978 & 19.9 & 20.3 & 20.6 & 16.1 & 20.7 & 31.1 & 27.8 & 25.4 & 18.1 & 16.5 & 15.9 & 14.7 & 20.6 \\
\hline 1979 & 18.1 & 20.4 & 20.7 & 12.2 & 17.2 & $*$ & $*$ & $*$ & $*$ & $*$ & $*$ & $*$ & * \\
\hline 1996 & $*$ & $*$ & $*$ & $*$ & $*$ & $*$ & $*$ & 36.4 & 22.9 & 18.6 & 18.2 & 24.1 & $*$ \\
\hline
\end{tabular}


Table 7. Monthly mean and annual flow, in cubic feet per second, for U.S. Geological Survey streamflow-gaging station 06449000, Lake Creek below refuge, near Tuthill, South Dakota

[*, no data]

\begin{tabular}{|c|c|c|c|c|c|c|c|c|c|c|c|c|c|}
\hline \multirow{2}{*}{$\begin{array}{l}\text { Water } \\
\text { year }\end{array}$} & \multicolumn{12}{|c|}{ Month } & \multirow{2}{*}{ Annual } \\
\hline & Oct & Nov & Dec & Jan & Feb & Mar & Apr & May & Jun & Jul & Aug & Sep & \\
\hline 1939 & 23.8 & 17.6 & 17.2 & 20.9 & 14.9 & 24.9 & 0.17 & 0.029 & 0.033 & 0.24 & 0.084 & 0.11 & 10.0 \\
\hline 1940 & .000 & .000 & .000 & .000 & .000 & .058 & 15.8 & 9.73 & .56 & 3.58 & 4.88 & 2.40 & 3.09 \\
\hline 1962 & $*$ & $*$ & $*$ & $*$ & $*$ & $*$ & * & $*$ & $*$ & * & 10.4 & 3.39 & * \\
\hline 1963 & 3.59 & 18.8 & 22.7 & 9.00 & 18.0 & 39.2 & 29.5 & 13.7 & 11.3 & 7.64 & 1.16 & .30 & 14.5 \\
\hline 1964 & 6.71 & 14.3 & 9.00 & 19.2 & 30.1 & 12.8 & 30.2 & 36.6 & 14.9 & 14.9 & 2.06 & 1.16 & 15.9 \\
\hline 1965 & 1.07 & 3.99 & 18.9 & 20.6 & 21.7 & 7.76 & .090 & 5.15 & 19.7 & 36.4 & 19.9 & 10.5 & 13.8 \\
\hline 1966 & 16.7 & 20.9 & 26.8 & 17.6 & 18.0 & 22.0 & 17.6 & 23.5 & 2.11 & 9.13 & 34.6 & 15.1 & 18.7 \\
\hline 1967 & 2.50 & .69 & 9.03 & 18.3 & 28.7 & 18.6 & .24 & 2.56 & 105 & 63.4 & 3.52 & .91 & 21.0 \\
\hline 1968 & 1.05 & 5.22 & 10.0 & 17.4 & 18.6 & 7.52 & 43.9 & 19.6 & 47.2 & 40.9 & 9.11 & 9.36 & 19.1 \\
\hline 1969 & 4.82 & 3.36 & 5.31 & 14.8 & 21.5 & 49.2 & 53.2 & 13.2 & 8.83 & 27.8 & 5.85 & .29 & 17.3 \\
\hline 1970 & .94 & .62 & 1.85 & 17.2 & 14.8 & 39.8 & 53.9 & 39.4 & 14.8 & 1.54 & 1.49 & 2.93 & 15.8 \\
\hline 1971 & 2.15 & 6.84 & 7.82 & 5.35 & 3.49 & 20.6 & 35.1 & 69.9 & 30.7 & 2.59 & 1.49 & 1.81 & 15.7 \\
\hline 1972 & 2.30 & 2.10 & 3.54 & 11.9 & 12.3 & 17.5 & 27.0 & 72.7 & 19.6 & 13.8 & 18.6 & 7.20 & 17.4 \\
\hline 1973 & 2.09 & 9.21 & 17.1 & 19.7 & 20.9 & 49.0 & 48.5 & 21.7 & 32.4 & 2.37 & 1.04 & 3.11 & 18.9 \\
\hline 1974 & 1.11 & 5.68 & 8.95 & 28.5 & 18.8 & 11.5 & 28.4 & 23.8 & 4.35 & 2.17 & 1.27 & .79 & 11.2 \\
\hline 1975 & .82 & 1.26 & 1.17 & 3.65 & 13.2 & 13.2 & 57.0 & 26.0 & 4.04 & 4.18 & 1.51 & 1.71 & 10.6 \\
\hline 1976 & .61 & 2.07 & 2.07 & 8.10 & 22.8 & 22.7 & 19.1 & 17.7 & 23.5 & 23.2 & 2.41 & 1.16 & 12.1 \\
\hline 1977 & .57 & 2.31 & .58 & 3.56 & 2.79 & 24.3 & 96.1 & 55.1 & 46.4 & 6.69 & 4.04 & 1.61 & 20.3 \\
\hline 1978 & 2.91 & 5.50 & 28.4 & 23.6 & 7.88 & 35.5 & 50.3 & 46.9 & 25.6 & 13.0 & 5.06 & 2.71 & 20.7 \\
\hline 1979 & .73 & 1.17 & 8.37 & 12.2 & 17.3 & 44.5 & 47.0 & 31.7 & 29.9 & 46.4 & 36.6 & 12.6 & 24.1 \\
\hline 1980 & .58 & 18.6 & 32.7 & 12.0 & 17.9 & 28.0 & 48.0 & 25.5 & 7.13 & 2.86 & 2.57 & .43 & 16.3 \\
\hline 1981 & .40 & .051 & 1.01 & 10.6 & 39.7 & 5.98 & 4.85 & 7.38 & 7.72 & 8.72 & 13.5 & 1.29 & 8.23 \\
\hline 1982 & 2.52 & 2.33 & 8.07 & 19.6 & 26.3 & 26.3 & 32.4 & 23.3 & 63.4 & 29.8 & 1.91 & .32 & 19.6 \\
\hline 1983 & 2.15 & 1.90 & 17.3 & 23.2 & 24.5 & 30.6 & 17.5 & 58.2 & 27.8 & 3.01 & 6.03 & 8.88 & 18.4 \\
\hline 1984 & 11.9 & 37.2 & 31.3 & 17.1 & 15.3 & 39.2 & 41.6 & 26.9 & 8.21 & 3.47 & .98 & .71 & 19.5 \\
\hline 1985 & .67 & 2.71 & 5.56 & 17.3 & 11.1 & 18.0 & 18.0 & 9.46 & 1.76 & 1.86 & .50 & 3.03 & 7.48 \\
\hline 1986 & 1.04 & 1.72 & 14.0 & 9.04 & 37.6 & 50.3 & 89.9 & 44.4 & 27.8 & 9.28 & 4.43 & 13.2 & 25.0 \\
\hline 1987 & 11.0 & 21.6 & 26.0 & 24.4 & 30.4 & 109 & 72.3 & 20.3 & 8.67 & 3.12 & 2.08 & 1.89 & 27.5 \\
\hline 1988 & 1.93 & 1.73 & 4.11 & 26.5 & 37.2 & 58.3 & 37.0 & 61.1 & 60.6 & 5.13 & 12.0 & 6.56 & 26.0 \\
\hline 1989 & .43 & .058 & 3.13 & 7.69 & 9.80 & 30.0 & 27.6 & 17.2 & 12.0 & 16.1 & 1.69 & .54 & 10.5 \\
\hline 1990 & 1.41 & 1.03 & 5.55 & 3.24 & 14.5 & 44.5 & 28.5 & 15.1 & 61.9 & .96 & 7.50 & 27.5 & 17.5 \\
\hline 1991 & .93 & 5.62 & 25.9 & 18.9 & 11.4 & 7.29 & 25.3 & 79.3 & 139 & 9.33 & 30.7 & 35.0 & 32.4 \\
\hline 1992 & 19.6 & 12.3 & 26.2 & 28.6 & 30.9 & 26.7 & 9.17 & 1.41 & .60 & 6.11 & 7.51 & 18.6 & 15.6 \\
\hline 1993 & 3.32 & 8.64 & 9.76 & 10.6 & 28.4 & 52.0 & 36.0 & 36.0 & 22.6 & 21.2 & 28.9 & 35.8 & 24.4 \\
\hline 1994 & 17.5 & 17.8 & 38.8 & 39.0 & 25.2 & 52.9 & 39.1 & 32.6 & 37.7 & 31.5 & 42.8 & 6.67 & 31.9 \\
\hline 1995 & 9.60 & 19.9 & 23.7 & 26.5 & 43.9 & 32.9 & 30.0 & 52.7 & 72.2 & 36.9 & 5.58 & 15.1 & 30.6 \\
\hline 1996 & 15.8 & 35.0 & 47.3 & 26.3 & 30.1 & 38.5 & 44.3 & 38.7 & 48.7 & 30.5 & 23.9 & 18.7 & 33.1 \\
\hline
\end{tabular}


Table 8. Monthly mean and annual flow, in cubic feet per second, for U.S. Geological Survey streamflow-gaging station 06449250, Spring Creek near St. Francis, South Dakota

[*, no data]

\begin{tabular}{|c|c|c|c|c|c|c|c|c|c|c|c|c|c|}
\hline \multirow{2}{*}{$\begin{array}{l}\text { Water } \\
\text { year }\end{array}$} & \multicolumn{12}{|c|}{ Month } & \multirow{2}{*}{ Annual } \\
\hline & Oct & Nov & Dec & Jan & Feb & Mar & Apr & May & Jun & Jul & Aug & Sep & \\
\hline 1960 & 0.048 & 0.057 & 0.000 & 0.000 & 0.034 & 7.76 & 22.2 & 19.2 & 1.67 & 0.51 & 0.065 & 0.000 & 4.30 \\
\hline 1961 & .000 & .023 & .000 & .000 & .071 & 4.69 & 4.08 & 8.53 & 1.67 & .55 & .016 & .013 & 1.65 \\
\hline 1962 & .000 & .000 & .000 & .013 & .036 & .60 & 7.11 & 12.2 & 38.1 & 22.5 & 2.63 & .020 & 6.94 \\
\hline 1963 & .000 & .000 & .068 & .18 & 2.64 & 15.1 & 11.6 & 6.31 & 5.09 & .45 & .071 & .12 & 3.47 \\
\hline 1964 & .023 & .000 & .016 & .032 & .20 & 8.10 & 17.7 & 13.7 & 4.12 & 1.74 & .000 & .013 & 3.81 \\
\hline 1965 & .000 & .000 & .000 & .000 & .000 & .068 & 5.27 & 5.02 & 3.37 & .029 & .013 & .023 & 1.15 \\
\hline 1966 & .005 & .006 & .52 & .63 & .32 & 11.6 & 14.0 & 5.85 & .82 & .10 & .34 & .095 & 2.87 \\
\hline 1967 & .10 & .062 & .33 & .43 & 5.85 & 10.1 & 2.63 & 1.94 & 19.0 & 4.56 & .17 & .32 & 3.76 \\
\hline 1968 & .000 & .000 & .000 & .000 & 1.32 & 8.64 & 18.2 & 9.34 & 15.7 & 1.94 & 1.84 & .91 & 4.80 \\
\hline 1969 & .17 & 1.61 & 1.43 & .039 & .075 & 12.6 & 17.7 & 7.30 & 3.37 & 1.51 & .036 & .000 & 3.83 \\
\hline 1970 & .000 & .027 & .015 & .026 & .49 & 10.3 & 22.9 & 11.2 & 1.66 & .044 & .000 & .000 & 3.90 \\
\hline 1971 & .000 & .000 & .000 & .000 & .000 & 7.52 & 22.8 & 19.6 & 7.34 & .11 & .000 & .000 & 4.79 \\
\hline 1972 & .000 & .000 & .000 & .000 & .000 & 3.79 & 6.92 & 15.0 & 5.30 & .51 & 1.44 & .000 & 2.76 \\
\hline 1973 & .000 & .000 & .000 & .000 & 5.06 & 21.8 & 16.3 & 9.75 & 4.00 & .004 & .000 & .000 & 4.74 \\
\hline 1974 & .000 & .000 & .13 & .44 & 7.82 & 8.76 & 6.94 & 4.86 & .98 & .002 & .003 & .000 & 2.46 \\
\hline
\end{tabular}


Table 9. Monthly mean and annual flow, in cubic feet per second, for U.S. Geological Survey streamflow-gaging station 06449400, Rosebud Creek at Rosebud, South Dakota

$[*$, no data]

\begin{tabular}{|c|c|c|c|c|c|c|c|c|c|c|c|c|c|}
\hline \multirow{2}{*}{$\begin{array}{l}\text { Water } \\
\text { year }\end{array}$} & \multicolumn{12}{|c|}{ Month } & \multirow{2}{*}{ Annual } \\
\hline & Oct & Nov & Dec & Jan & Feb & Mar & Apr & May & Jun & Jul & Aug & Sep & \\
\hline 1975 & 6.79 & 7.09 & 7.07 & 6.90 & 6.18 & 7.35 & 10.2 & 7.89 & 6.25 & 4.47 & 4.68 & 4.31 & 6.60 \\
\hline 1976 & 5.42 & 5.64 & 5.97 & 6.44 & 7.08 & 6.73 & 6.52 & 5.64 & 3.47 & 7.57 & 3.79 & 4.14 & 5.70 \\
\hline 1977 & 5.30 & 8.68 & 6.25 & 7.15 & 7.61 & 8.46 & 12.0 & 9.92 & 8.33 & 6.94 & 6.64 & 7.79 & 7.91 \\
\hline 1978 & 9.36 & 7.64 & 7.21 & 6.45 & 6.69 & 21.6 & 10.7 & 9.27 & 3.43 & 4.64 & 10.2 & 6.03 & 8.63 \\
\hline 1979 & 6.81 & 7.58 & 7.83 & 8.88 & 8.54 & 9.73 & 8.59 & 7.40 & 10.9 & 7.02 & 4.13 & 1.96 & 7.44 \\
\hline 1980 & 6.23 & 6.74 & 6.77 & 7.27 & 8.43 & 7.38 & 8.34 & 7.55 & 6.00 & 3.96 & 4.73 & 3.96 & 6.44 \\
\hline 1981 & 10.2 & 9.12 & 7.59 & 5.62 & 8.34 & 6.90 & 5.69 & 7.47 & 6.68 & 7.67 & 11.6 & 4.17 & 7.60 \\
\hline 1982 & 13.7 & 7.04 & 5.58 & 5.40 & 9.64 & 8.68 & 8.13 & 9.98 & 9.03 & 5.89 & 4.05 & 7.76 & 7.89 \\
\hline 1983 & 8.62 & 8.09 & 7.14 & 5.84 & 6.18 & 7.68 & 8.18 & 12.9 & 8.18 & 5.51 & 5.16 & 5.48 & 7.43 \\
\hline 1984 & 6.80 & 8.17 & 7.12 & 6.96 & 7.51 & 9.75 & 9.46 & 8.01 & 7.13 & 7.12 & 6.44 & 5.88 & 7.53 \\
\hline 1985 & 7.23 & 8.32 & 7.09 & 6.72 & 8.67 & 11.0 & 9.78 & 6.82 & 6.55 & 4.32 & 5.11 & 6.06 & 7.29 \\
\hline 1986 & 6.40 & 7.60 & 8.36 & 8.09 & 10.1 & 15.3 & 13.5 & 8.53 & 8.27 & 6.59 & 5.99 & 7.38 & 8.84 \\
\hline 1987 & 7.47 & 8.49 & 7.86 & 6.73 & 7.96 & 10.6 & 10.6 & 9.22 & 10.0 & 6.96 & 5.65 & 6.40 & 8.16 \\
\hline 1988 & 7.23 & 7.49 & 7.48 & 8.14 & 7.23 & 8.00 & 8.32 & 16.4 & 8.38 & 6.85 & 4.86 & 5.98 & 8.04 \\
\hline 1989 & 7.27 & 7.74 & 7.52 & 7.52 & 6.90 & 9.03 & 7.72 & 7.83 & 5.94 & 4.78 & 4.45 & 5.91 & 6.89 \\
\hline 1990 & 6.22 & 6.72 & 5.99 & 6.88 & 6.80 & 7.16 & 7.22 & 8.12 & 8.95 & 8.34 & 6.38 & 6.78 & 7.13 \\
\hline 1991 & 7.21 & 6.98 & 6.54 & 7.51 & 9.05 & 7.11 & 8.98 & 9.68 & 10.0 & 5.96 & 4.81 & 5.06 & 7.39 \\
\hline 1992 & 7.30 & 8.33 & 7.79 & 7.47 & 6.63 & 6.98 & 7.82 & 6.23 & 7.45 & 6.61 & 5.96 & 6.42 & 7.08 \\
\hline 1993 & 5.49 & 6.27 & 4.13 & 5.05 & 4.24 & 10.5 & 9.73 & 9.86 & 8.40 & 4.49 & 5.54 & 6.51 & 6.70 \\
\hline 1994 & 7.63 & 8.88 & 7.76 & 7.17 & 9.96 & 10.6 & 7.55 & 8.43 & 11.4 & 10.4 & 6.32 & 6.41 & 8.53 \\
\hline 1995 & 7.13 & 6.85 & 6.93 & 7.25 & 8.48 & 7.94 & 11.5 & 13.0 & 19.1 & 8.63 & 6.85 & 7.22 & 9.23 \\
\hline 1996 & 10.3 & 8.66 & 7.91 & 7.80 & 8.98 & 7.75 & 8.85 & 12.4 & 7.68 & 6.40 & 5.14 & 7.43 & 8.27 \\
\hline
\end{tabular}




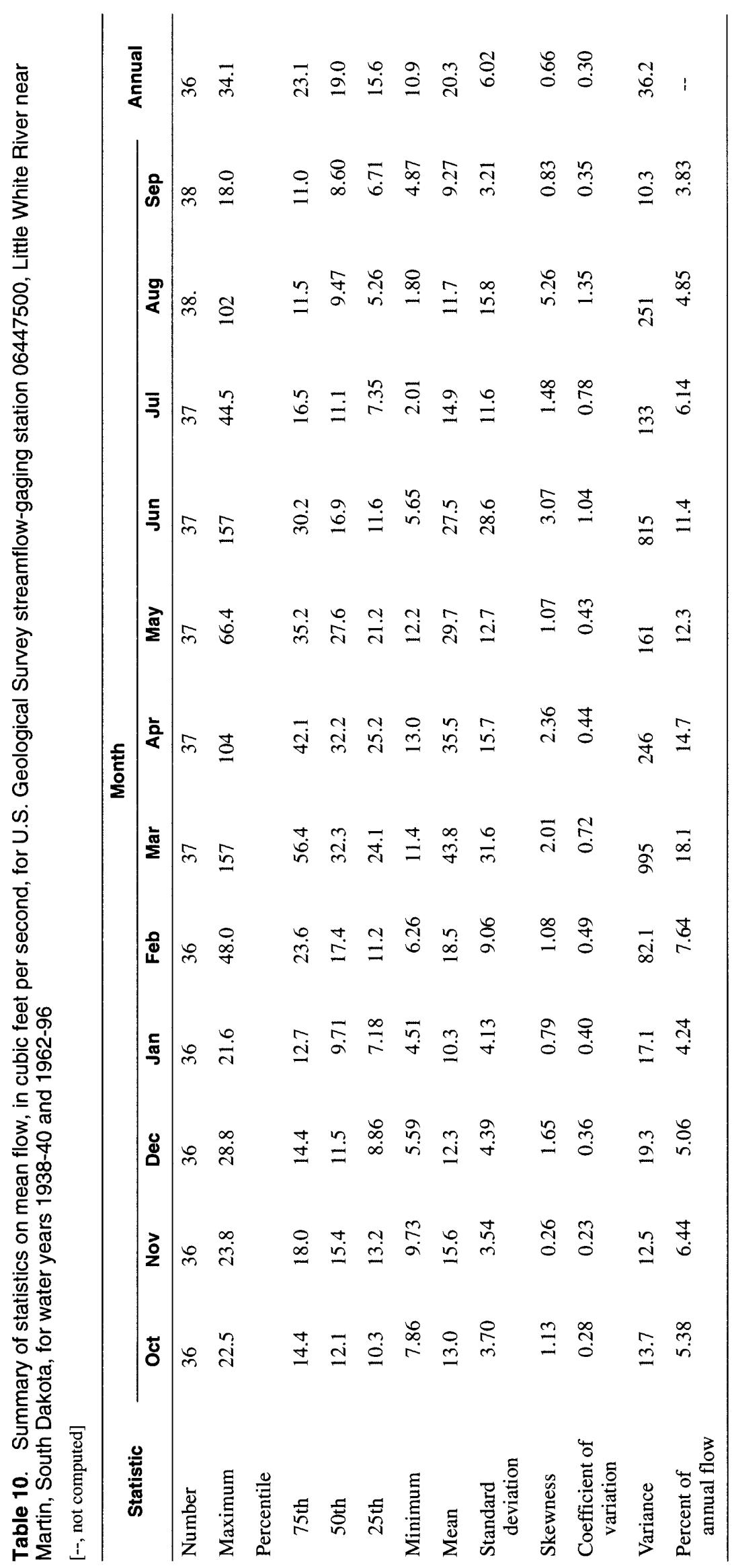




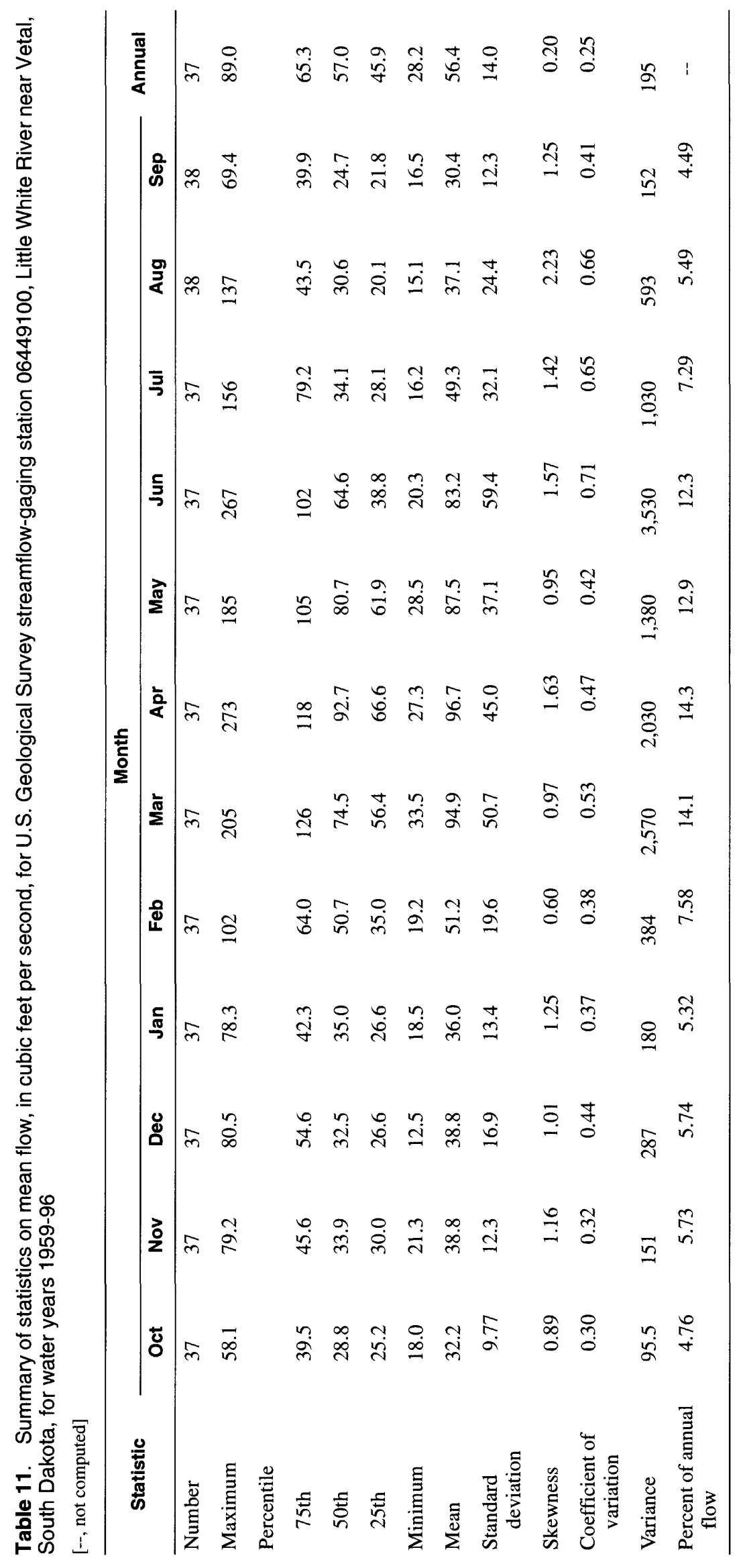




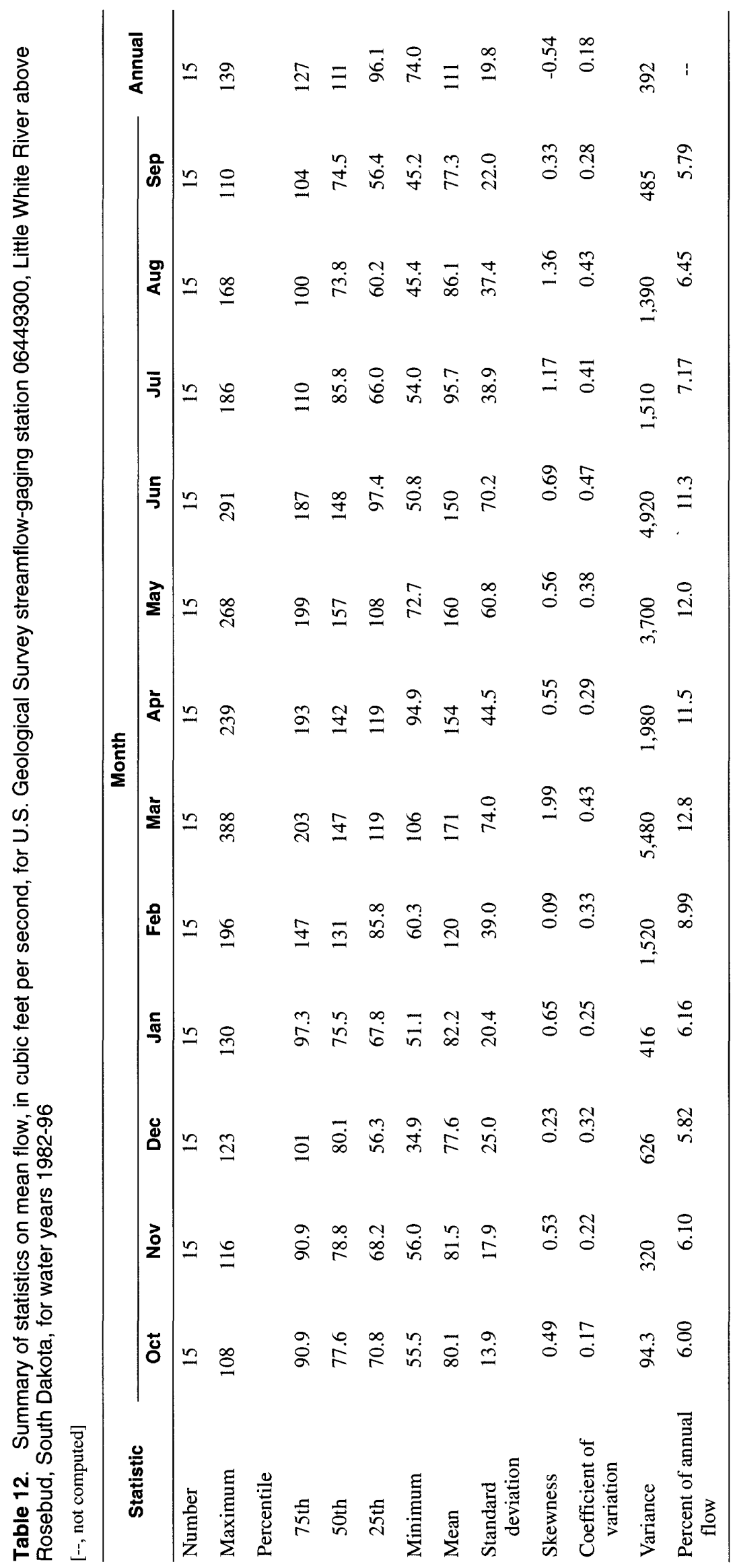




\begin{tabular}{|c|c|c|c|c|c|}
\hline 离 & 嵒 & $n$ & 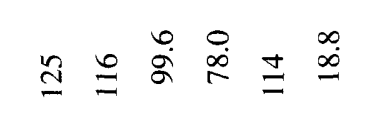 & $\frac{9}{0} \frac{5}{0}$ & 呄 \\
\hline 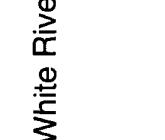 & ळ̆ & if & 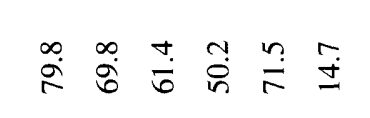 & $\stackrel{8}{-} \bar{g}$ & $\frac{n}{N}$ \\
\hline 5 & $\frac{9}{2}$ & If & 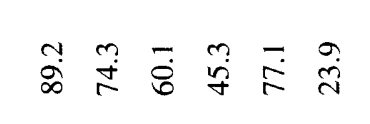 & $\bar{m}$ & $\overbrace{n}^{\infty}$ \\
\hline 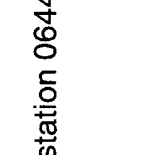 & $\overline{5}$ & 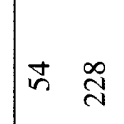 & 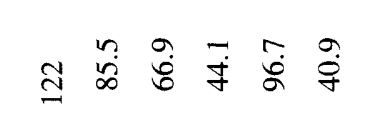 & $\stackrel{\infty}{=} \underset{0}{f}$ & 党 \\
\hline 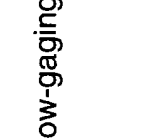 & 立 & 䎡孚 & త్ర & & \\
\hline$\frac{\Phi}{5}$ & 齐 & in : & 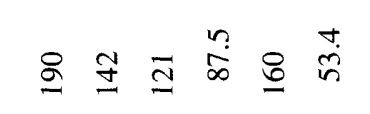 & $\stackrel{0}{\circ}:$ & \\
\hline 厄. & $\underline{\frac{a}{4}}$ & in $\bar{q}$ & 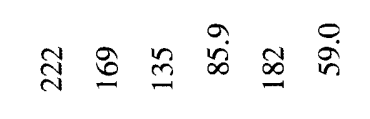 & : & \\
\hline כ̦ & $\overline{\mathbf{m}}$ & $\approx \circ$ & 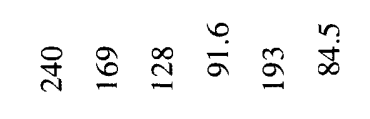 & Sิ & \\
\hline 位 & $\stackrel{8}{\mathbb{L}}$ & $\therefore \bar{i}$ & 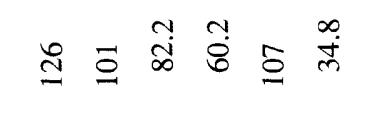 & 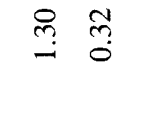 & \\
\hline$\frac{0}{0}$ & 雨 & in & 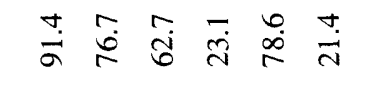 & 영 뎡 & 㤐 \\
\hline 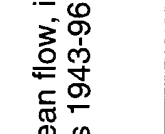 & 品 & 的表 & 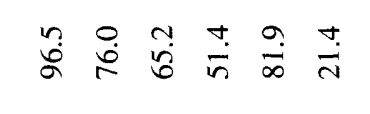 & & \\
\hline 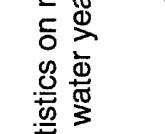 & $\frac{3}{2}$ & in & 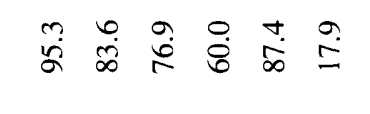 & & \\
\hline 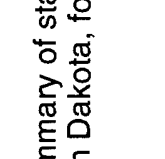 & $\ddot{\circ}$ & $n \stackrel{\infty}{=}$ & 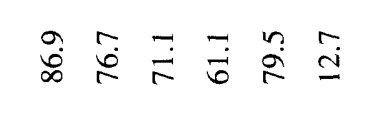 & & 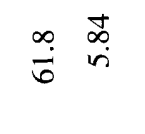 \\
\hline 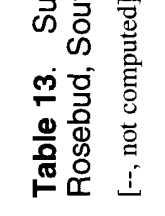 & $\frac{0}{\frac{0}{\bar{w}}}$ & 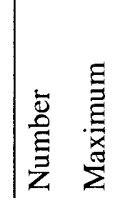 & 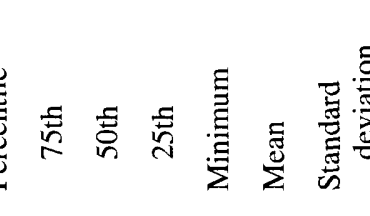 & $\frac{1}{2}$ & 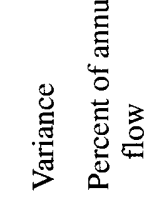 \\
\hline
\end{tabular}




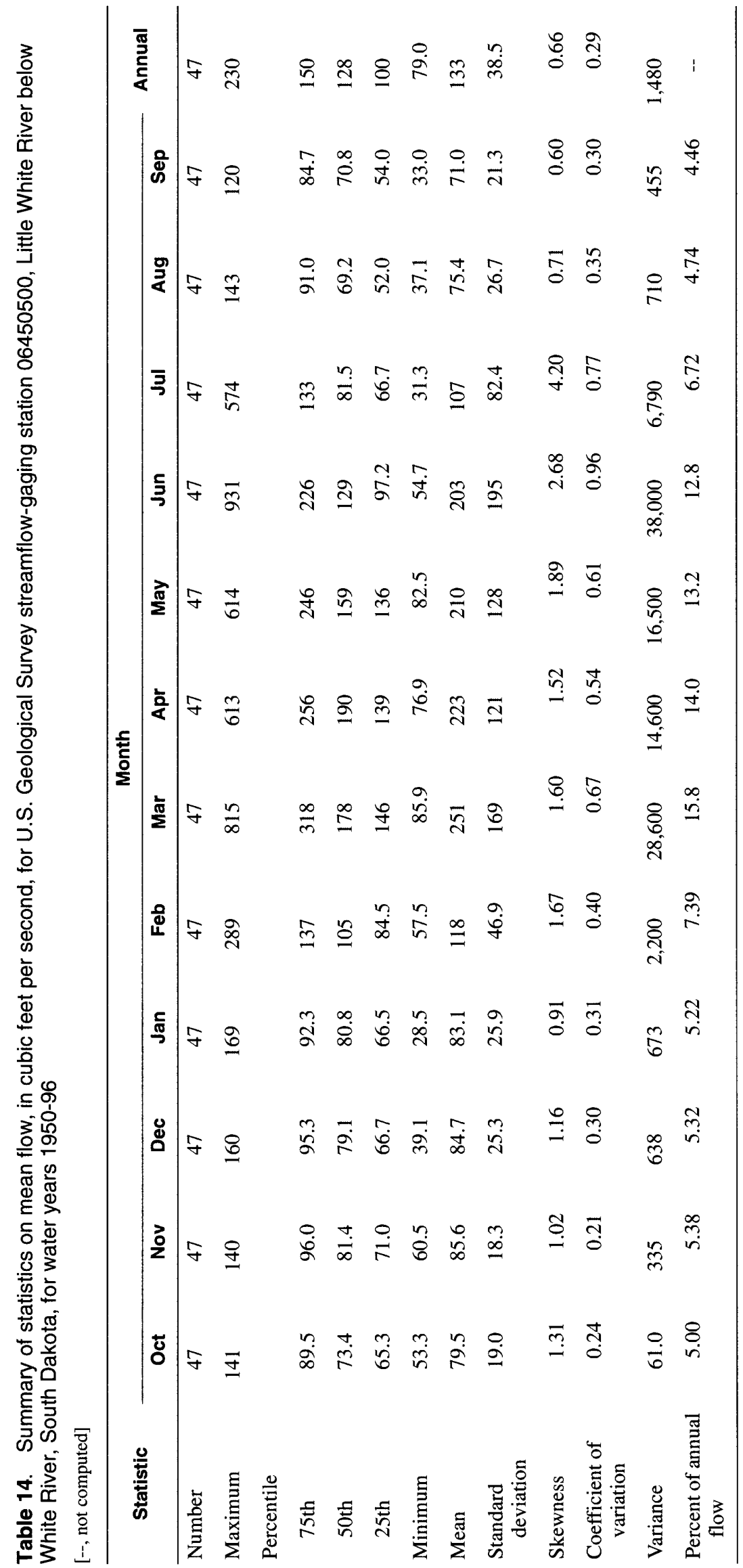




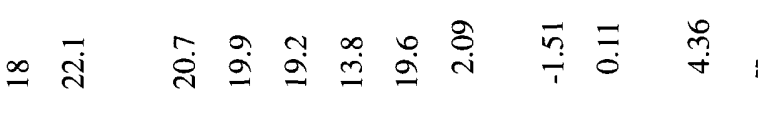

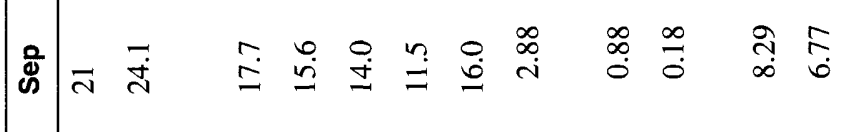

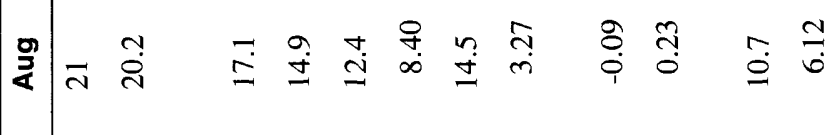

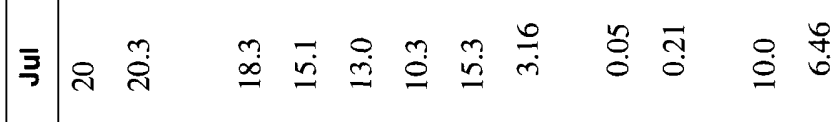

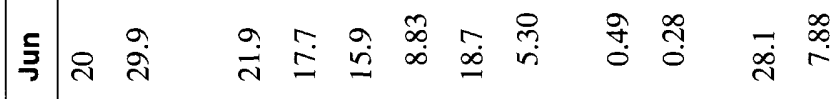

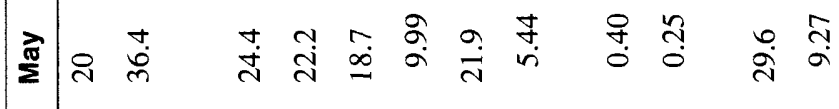

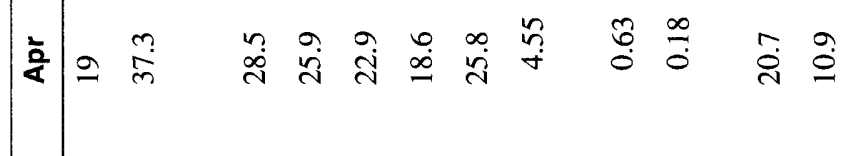

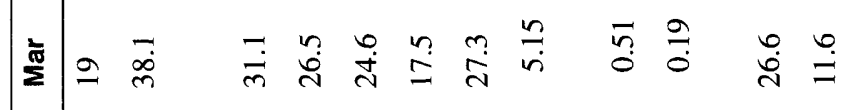

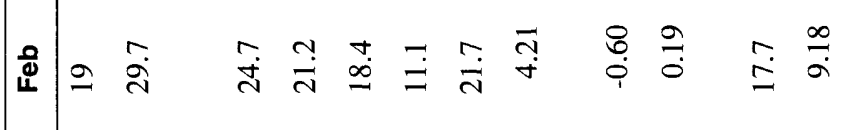

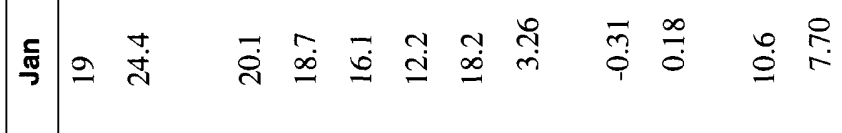

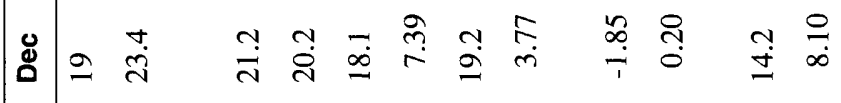

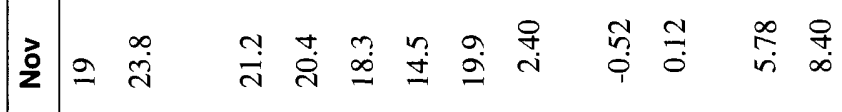

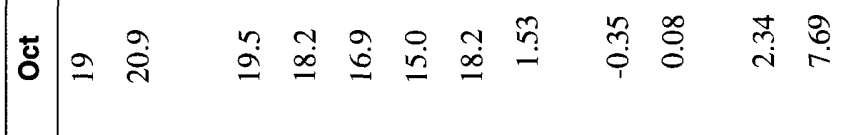

금

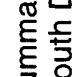

仓吅 $\overline{\mathrm{Q}}$

绝言言

迹䨔

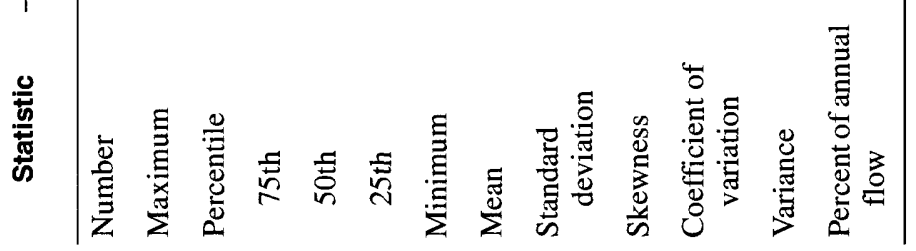




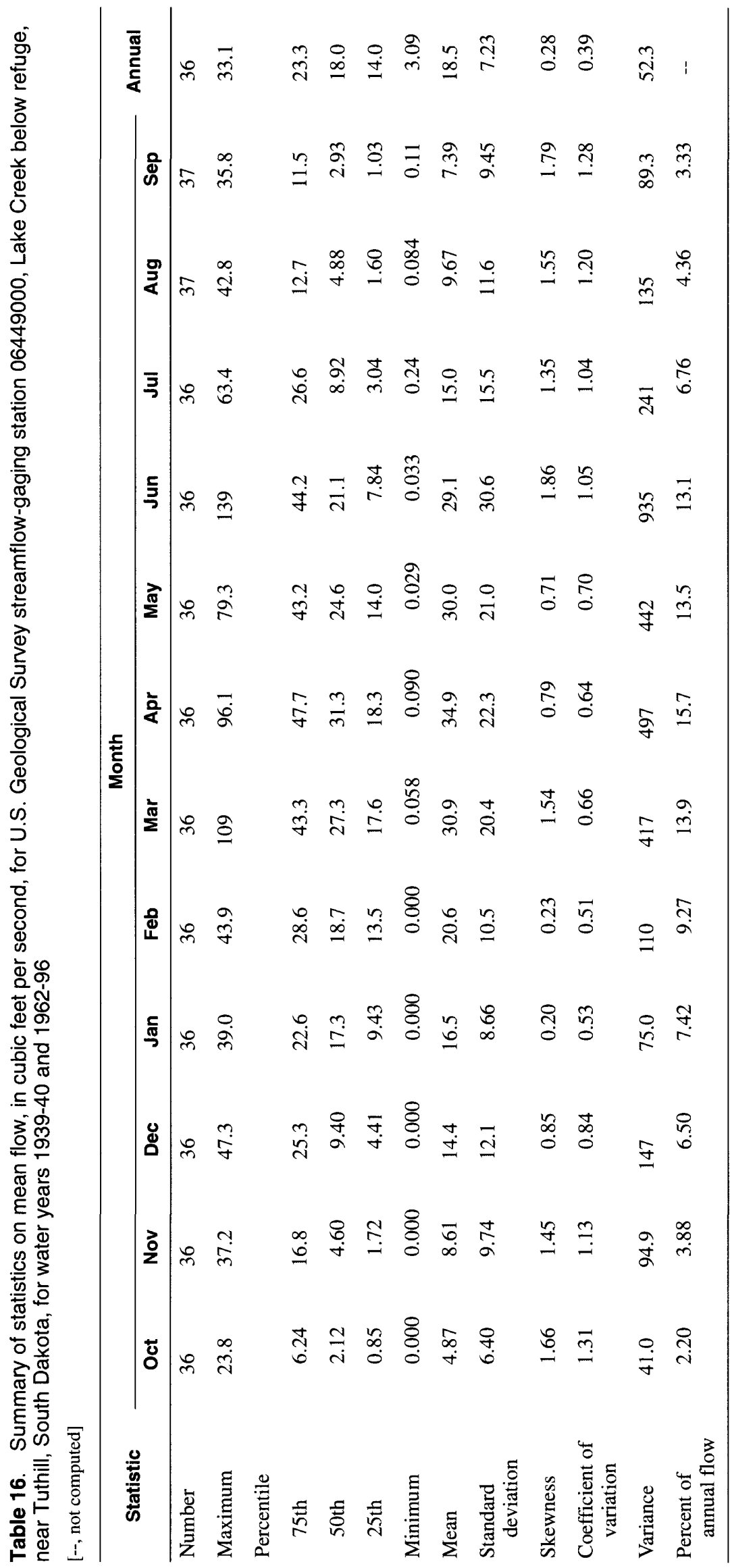




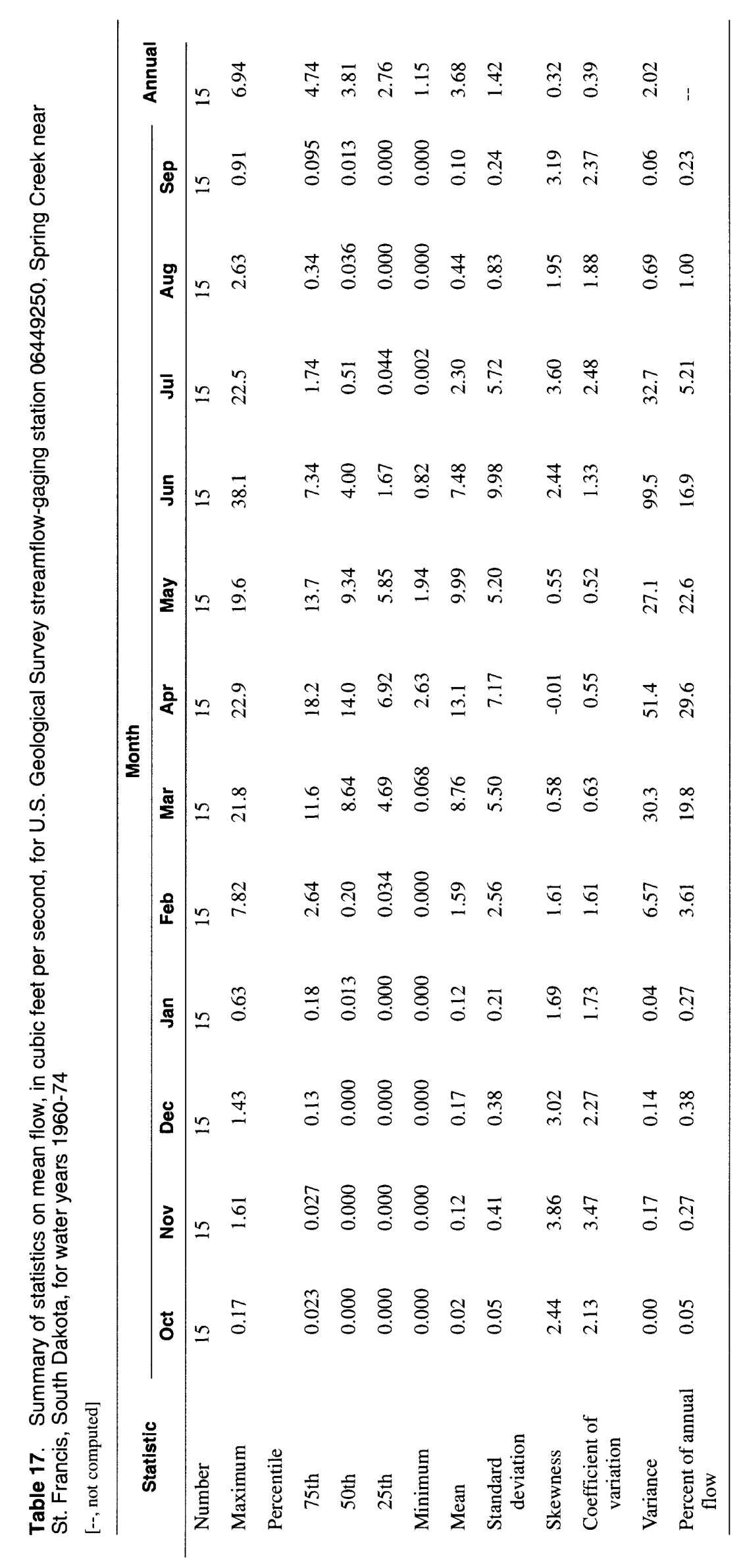




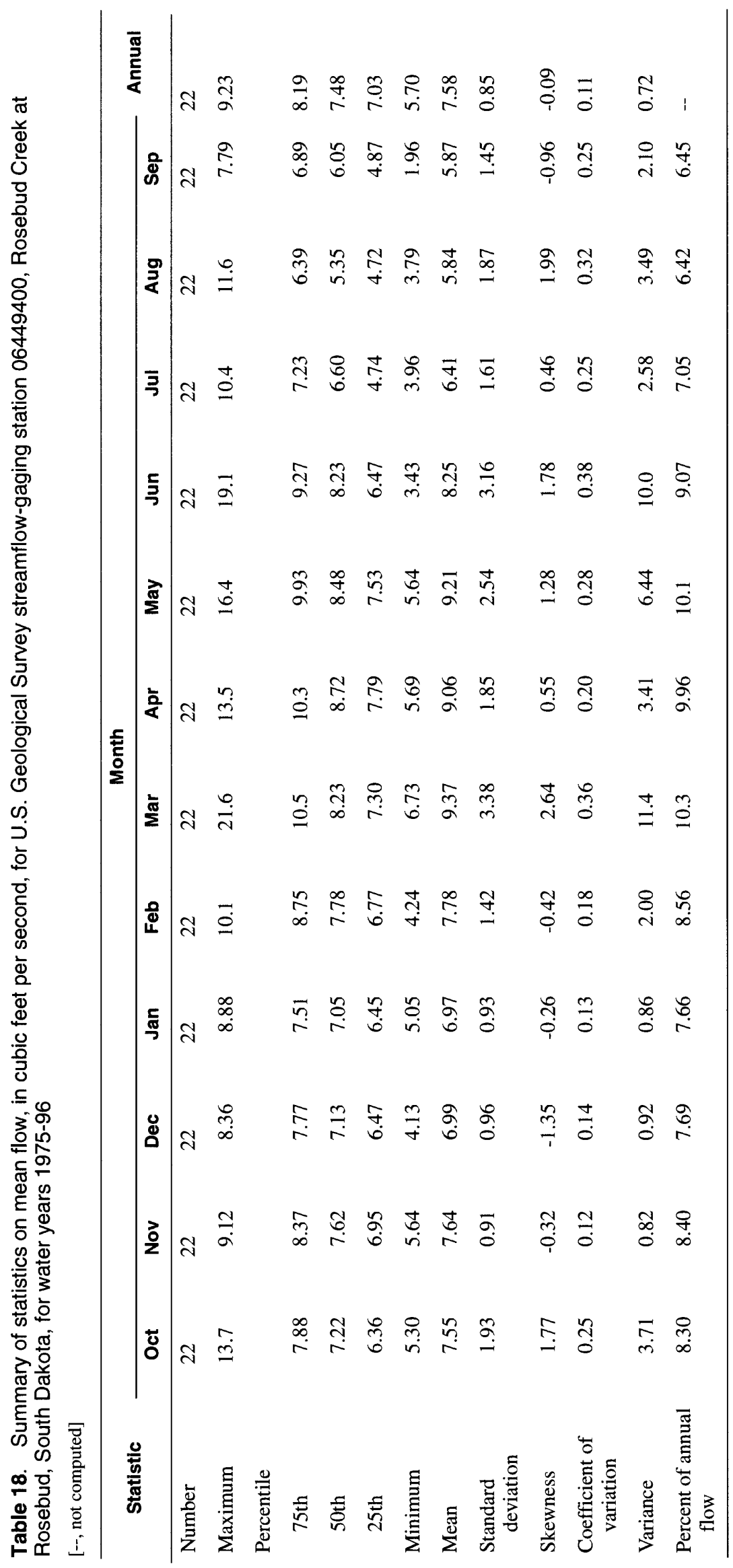

54 Summary of Water-Resources Data within the Little White River Basin, South Dakota and Nebraska 


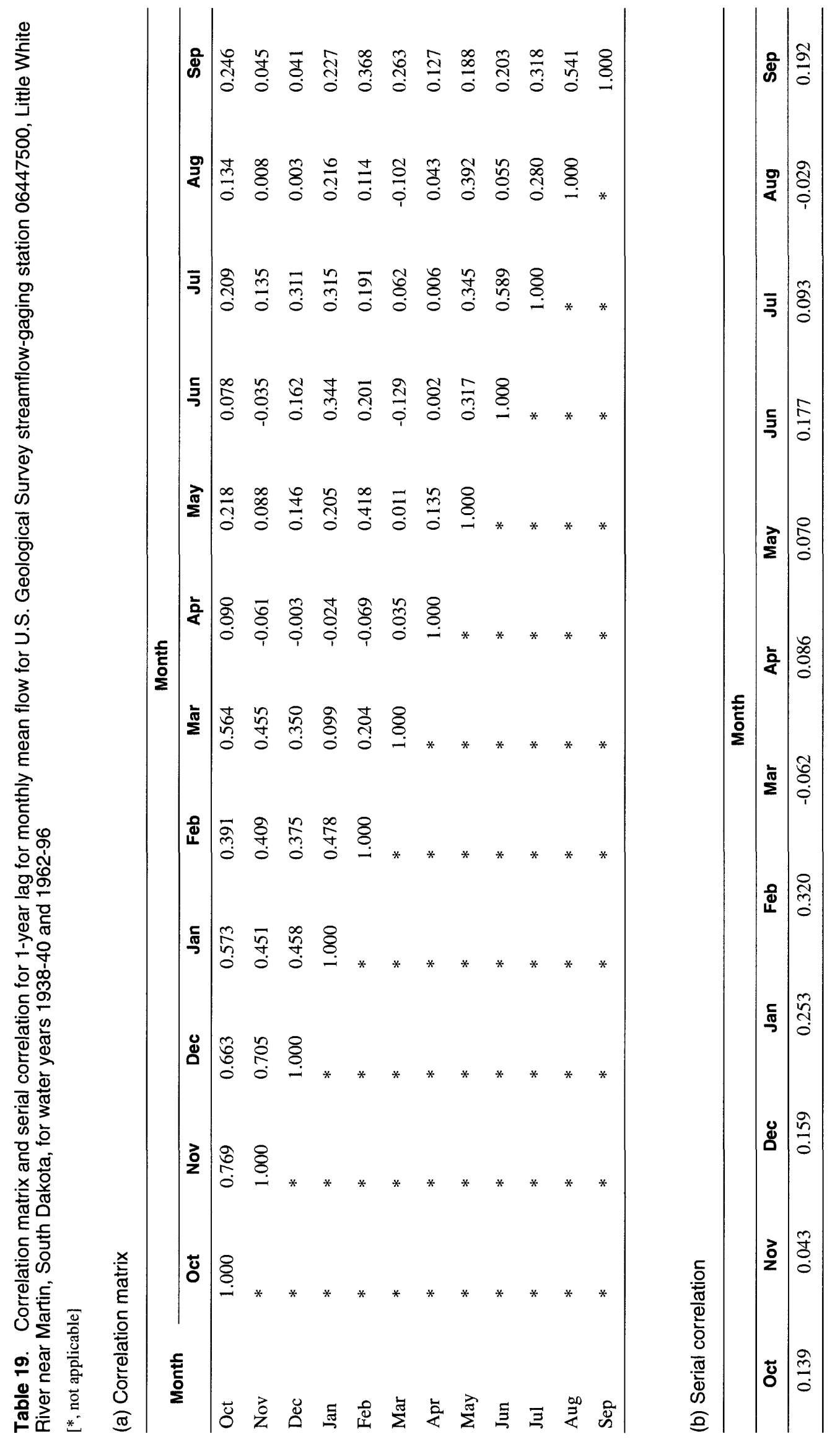




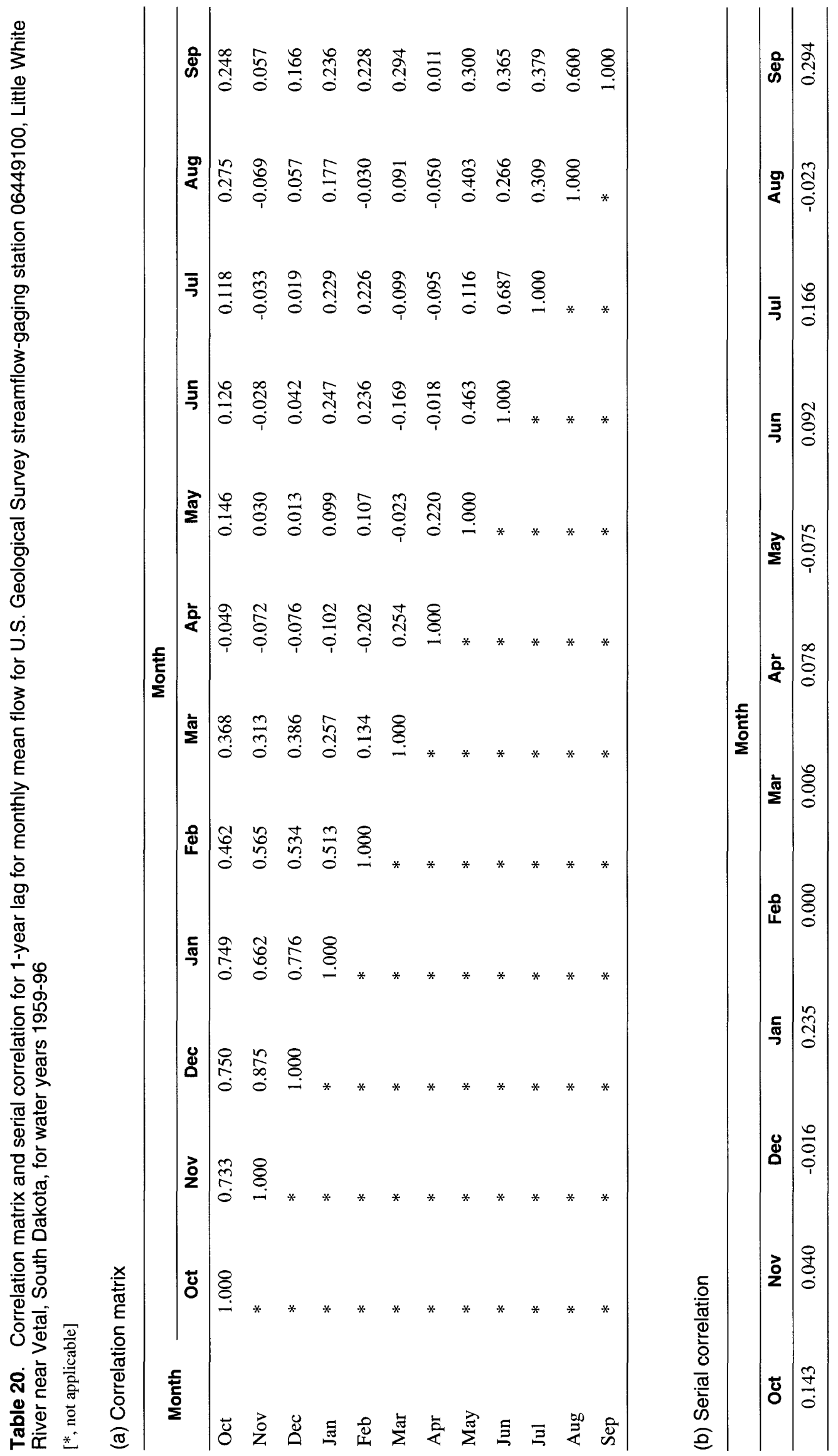

56 Summary of Water-Resources Data within the Little White River Basin, South Dakota and Nebraska 


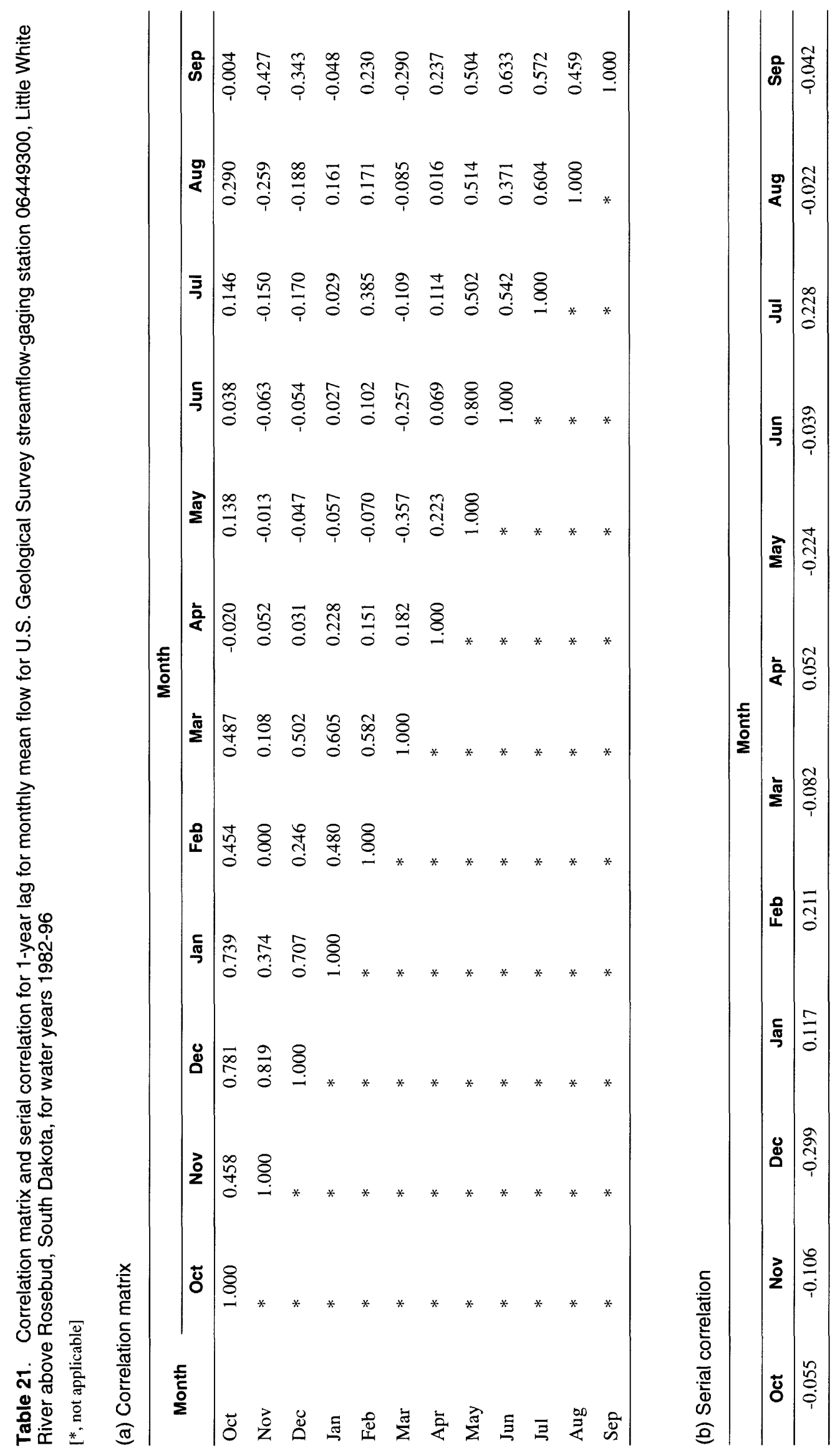



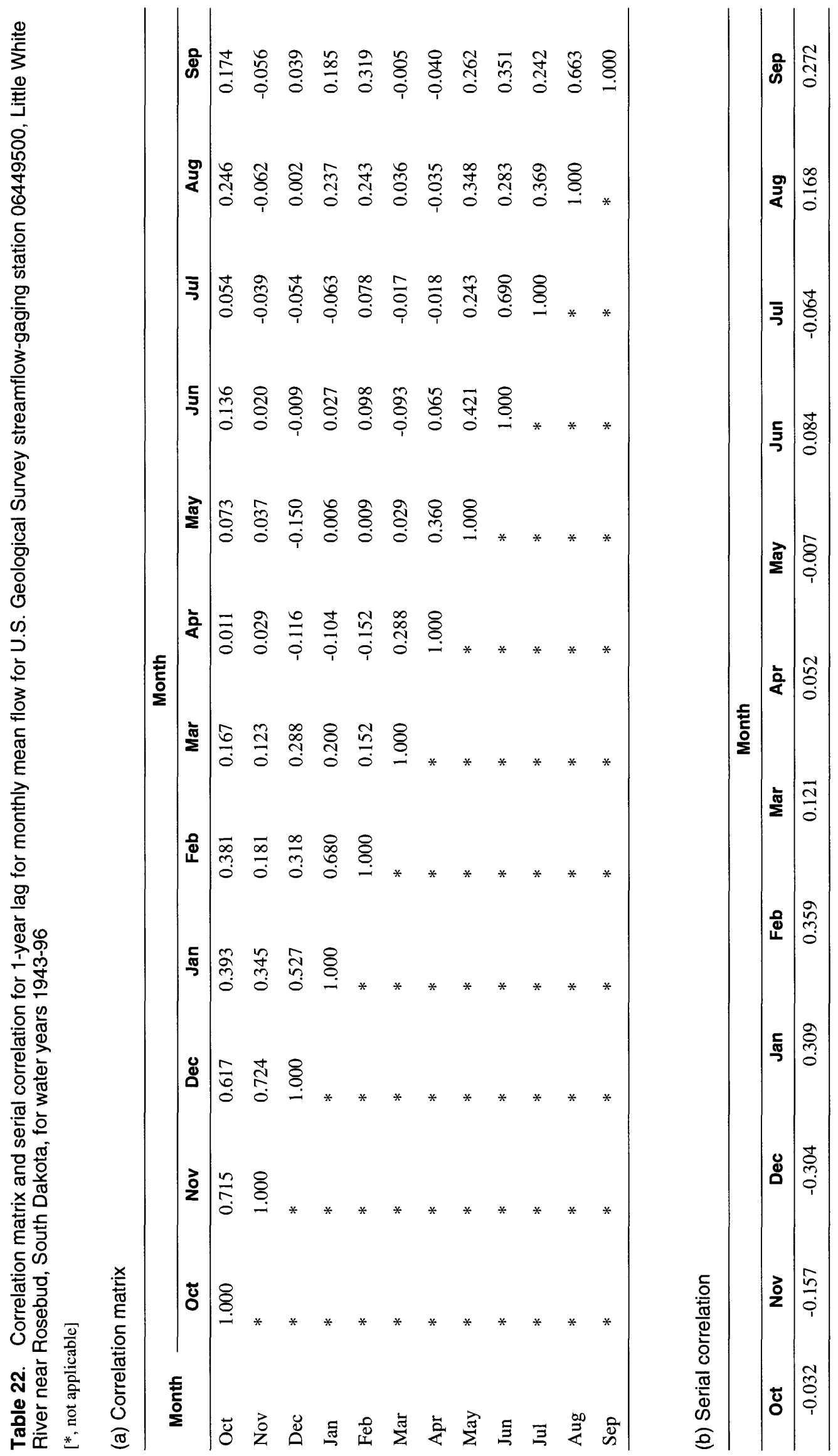


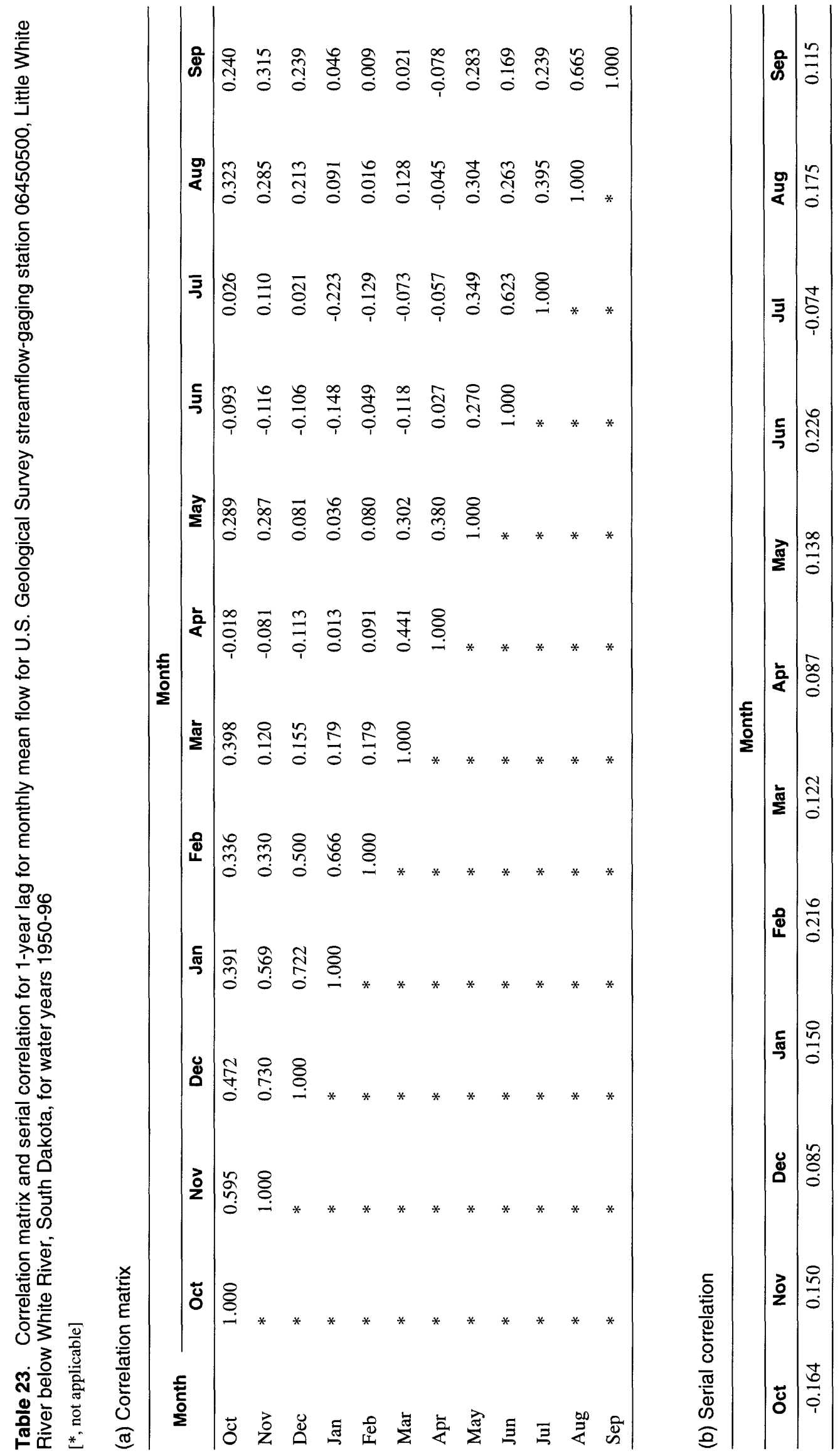




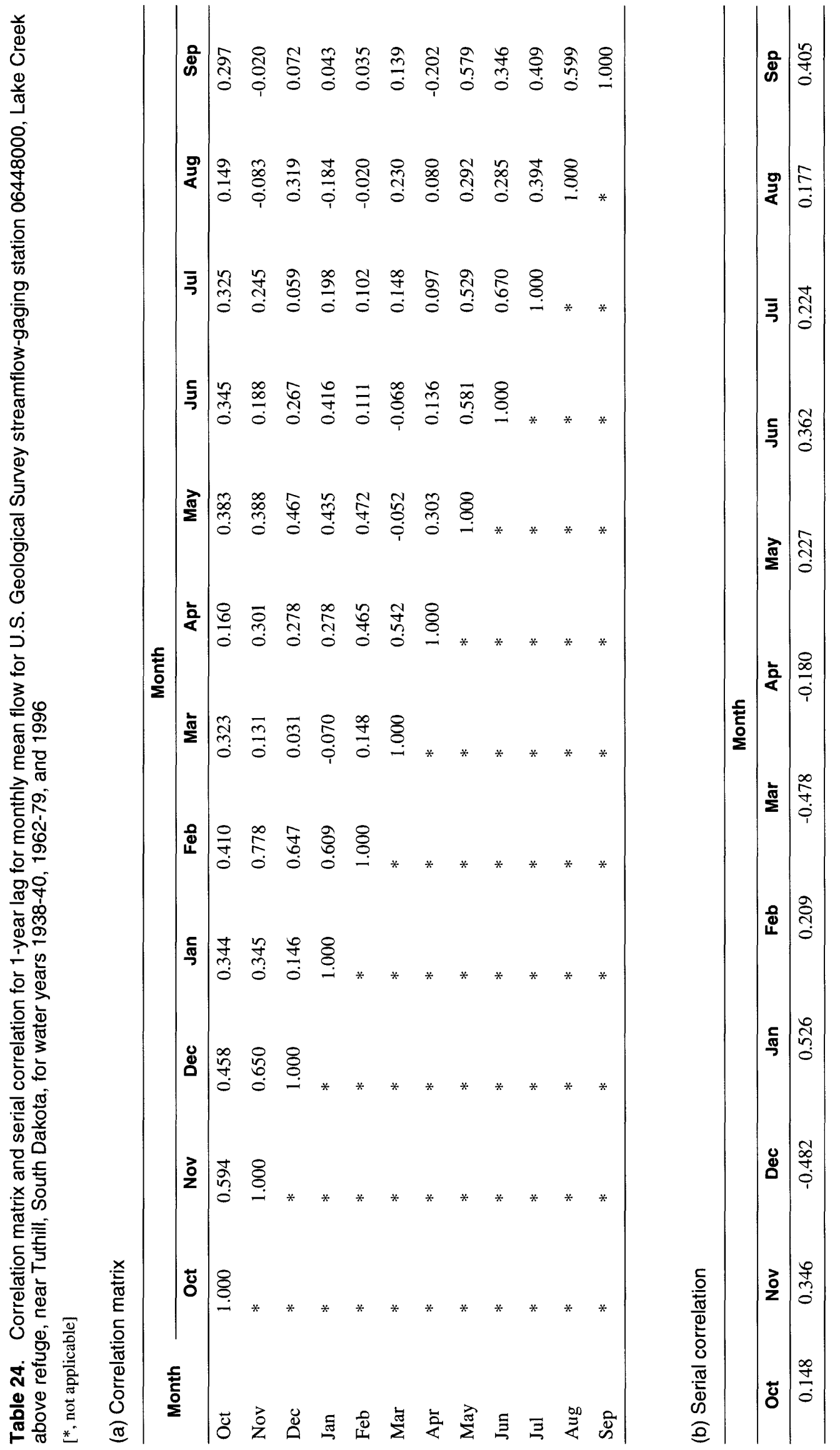




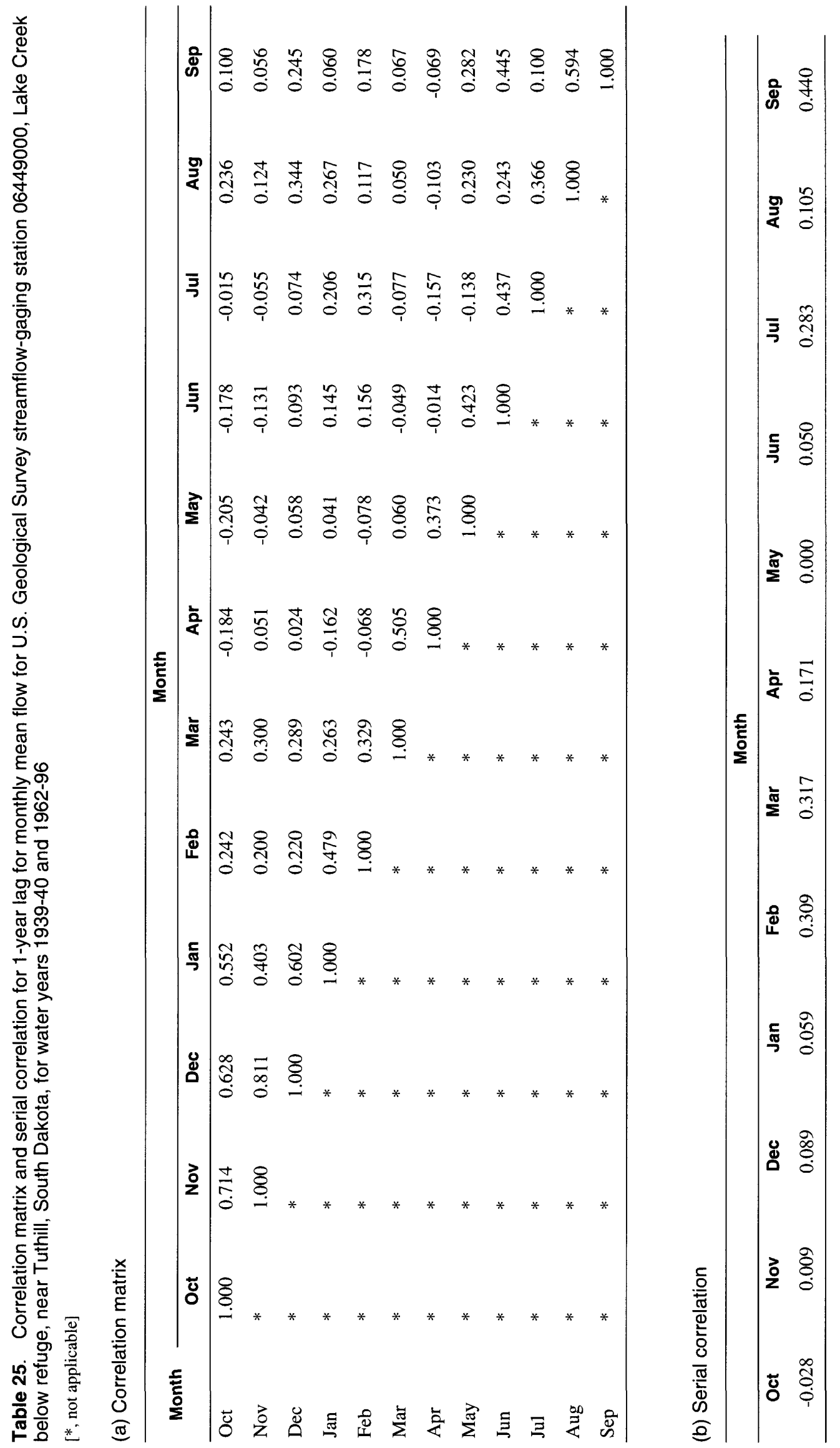




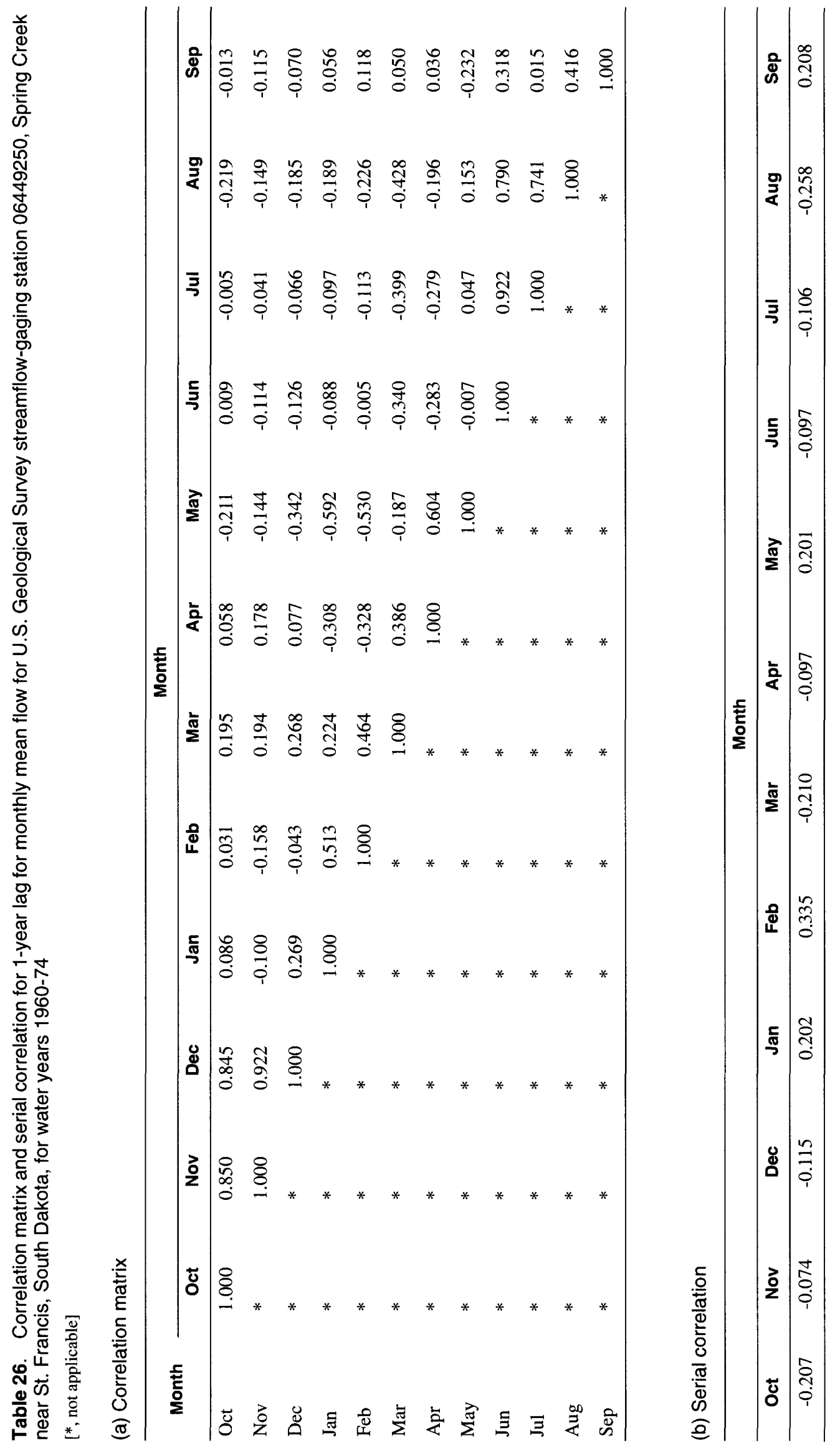

62 Summary of Water-Resources Data within the Little White River Basin, South Dakota and Nebraska 


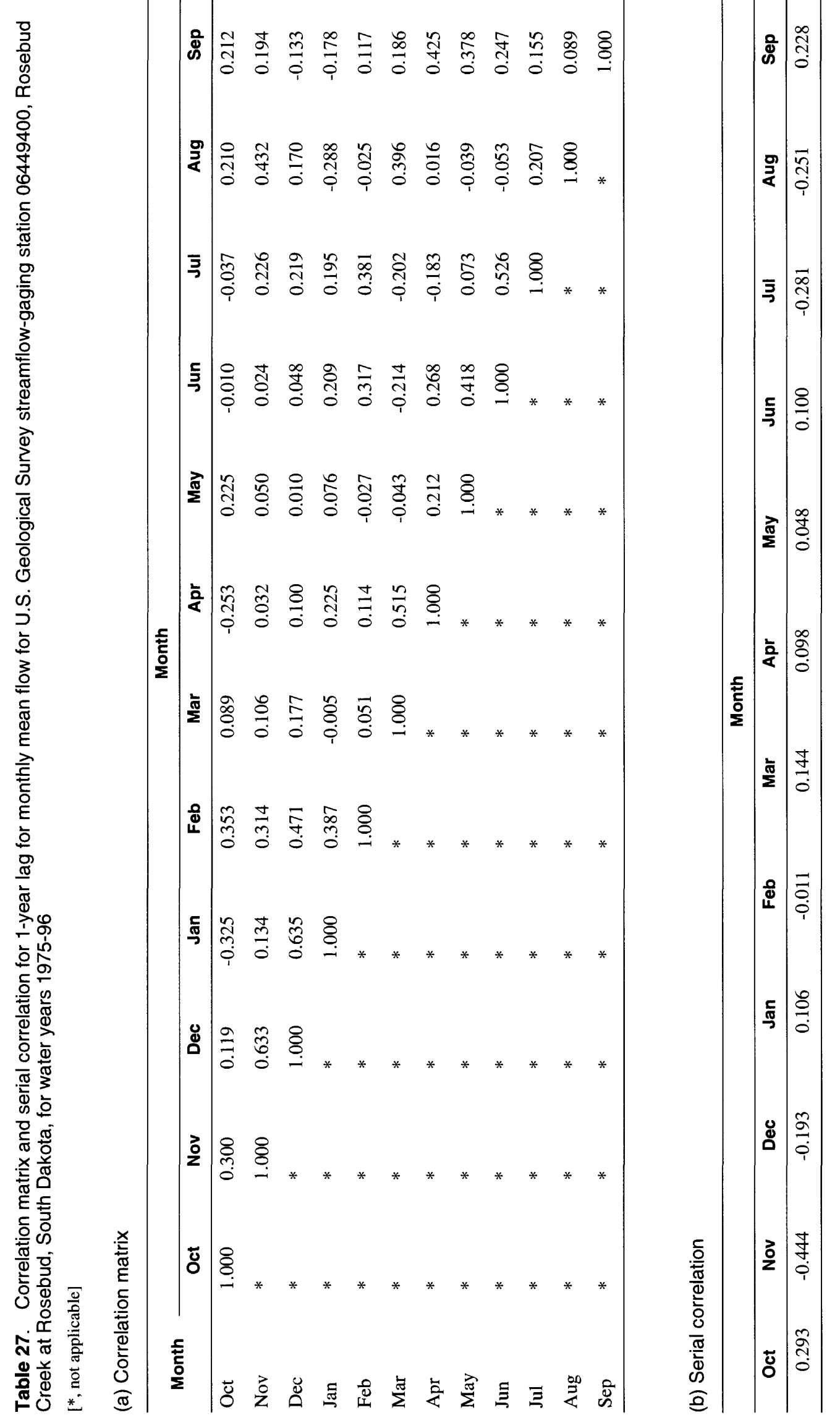


Table 28. Water-quality data for U.S. Geological Survey streamflow-gaging station 06447500 , Little White River near Martin, South Dakota

[ $\mu \mathrm{S} / \mathrm{cm}$; microsiemens per centimeter; --, no data]

\begin{tabular}{|c|c|c|c|c|c|}
\hline $\begin{array}{c}\text { Date of } \\
\text { sampling } \\
\text { (year/month/day) }\end{array}$ & $\begin{array}{l}\text { Sample } \\
\text { number }\end{array}$ & $\begin{array}{c}\text { Discharge, } \\
\text { instantaneous } \\
\text { (cubic feet } \\
\text { per second) }\end{array}$ & $\begin{array}{c}\text { Specific } \\
\text { conductance } \\
(\mu \mathrm{S} / \mathrm{cm} \text { at } 25 \\
\text { degrees Celsius) }\end{array}$ & $\begin{array}{c}\text { Temperature, } \\
\text { air } \\
\text { (degrees } \\
\text { Celsius) }\end{array}$ & $\begin{array}{c}\text { Water } \\
\text { temperature } \\
\text { (degrees } \\
\text { Celsius) }\end{array}$ \\
\hline 19751007 & 97600062 & 7 & - & 12 & 12 \\
\hline 19751105 & 97600063 & 13 & -- & 24 & 8.5 \\
\hline 19751203 & 97600064 & 7.1 & -- & 6 & 0 \\
\hline 19760105 & 97600065 & 3 & -- & 1 & 0 \\
\hline 19760127 & 97600066 & 8.5 & -- & -1 & 0 \\
\hline 19760224 & 97600067 & 39 & -- & 14 & 0 \\
\hline 19760323 & 97600068 & 27 & -- & 14 & 5 \\
\hline 19760420 & 97600069 & 51 & -- & 8 & 8 \\
\hline 19760517 & 97600070 & 15 & -- & 21.5 & 16.5 \\
\hline 19760615 & 97600071 & 5.9 & -- & 10.5 & 12 \\
\hline 19760713 & 97600072 & 3.5 & -- & 20 & 21.5 \\
\hline 19760809 & 97600073 & 7.2 & -- & 38 & 36 \\
\hline 19760908 & 97600074 & 2.8 & -- & 10 & 13 \\
\hline 19761004 & 97700085 & 6.8 & -- & 10 & 12 \\
\hline 19761102 & 97700086 & 11.7 & -- & 11.5 & 6 \\
\hline 19761130 & 97700087 & 6.9 & -- & 2 & 0 \\
\hline 19761228 & 97700088 & 10.7 & -- & 3 & 1 \\
\hline 19770125 & 97700089 & 5.3 & -- & -3 & 0 \\
\hline 19770222 & 97700090 & 41.8 & -- & 13 & .5 \\
\hline 19770314 & 97700091 & 13.5 & -- & 20 & 1 \\
\hline 19770324 & 97700092 & 4.0 & -- & 18 & 5 \\
\hline 19770412 & 97700093 & 246 & -- & 5 & 7.5 \\
\hline 19770413 & 97700094 & 286 & -- & 17 & 8 \\
\hline 19770419 & 97700095 & 70.1 & -- & 5 & 10 \\
\hline 19770516 & 97700096 & 19.5 & -- & 18 & 16.5 \\
\hline 19770614 & $97700097^{1}$ & 12.8 & -- & 18.5 & 21 \\
\hline 19770712 & 97700098 & 9.8 & -- & 19.5 & 20 \\
\hline 19770809 & 97700099 & 13.5 & -- & 26 & 23 \\
\hline 19770907 & 97700100 & 9.5 & -- & 23.5 & 21 \\
\hline 19771101 & 97800080 & 16 & 240 & 30 & 5 \\
\hline 19771129 & 97800081 & 17 & 305 & 5 & 0 \\
\hline 19771228 & 97800082 & 9.8 & 130 & -7 & 0 \\
\hline 19780127 & 97800083 & 7.9 & 280 & -5 & 0 \\
\hline 19780228 & 97800084 & 8.3 & 250 & -10 & 0 \\
\hline 19780315 & 97800085 & 192 & 190 & 1 & .5 \\
\hline 19780509 & 97800086 & 60 & 400 & 19.5 & 11.5 \\
\hline
\end{tabular}


Table 28. Water-quality data for U.S. Geological Survey streamflow-gaging station 06447500, Little White River near Martin, South Dakota-Continued

[ $\mu \mathrm{S} / \mathrm{cm}$; microsiemens per centimeter; --, no data]

\begin{tabular}{|c|c|c|c|c|c|}
\hline $\begin{array}{c}\text { Date of } \\
\text { sampling } \\
\text { (year/month/day) }\end{array}$ & $\begin{array}{l}\text { Sample } \\
\text { number }\end{array}$ & $\begin{array}{l}\text { Discharge, } \\
\text { instantaneous } \\
\text { (cubic feet } \\
\text { per second) }\end{array}$ & $\begin{array}{c}\text { Specific } \\
\text { conductance } \\
(\mu \mathrm{S} / \mathrm{cm} \text { at } 25 \\
\text { degrees Celsius) }\end{array}$ & $\begin{array}{c}\text { Temperature, } \\
\text { air } \\
\text { (degrees } \\
\text { Celsius) }\end{array}$ & $\begin{array}{c}\text { Water } \\
\text { temperature } \\
\text { (degrees } \\
\text { Celsius) }\end{array}$ \\
\hline 19780613 & 97800087 & 17 & 510 & 23 & 16 \\
\hline 19780711 & 97800088 & 12 & 310 & 22.5 & 19 \\
\hline 19781102 & 97900062 & 15 & 240 & 20 & 6 \\
\hline 19781127 & 97900063 & 12 & 240 & -5 & 0 \\
\hline 19781220 & 97900064 & 9.6 & 200 & 0 & 0 \\
\hline 19790417 & 97900068 & 40 & 360 & 27 & 16 \\
\hline 19790513 & 97900069 & 36 & 340 & 22 & 17 \\
\hline 19790612 & 97900070 & 12 & 300 & 27 & 25 \\
\hline 19790710 & 97900071 & 15 & 310 & 31 & 28 \\
\hline 19790807 & 97900072 & 12 & 375 & 30 & 27.5 \\
\hline 19790905 & 97900073 & 8 & 270 & 28 & 26 \\
\hline 19791001 & 98000065 & 6.4 & 280 & 20 & 17 \\
\hline 19800318 & 98000071 & 51.6 & 180 & 17 & 4.5 \\
\hline 19800414 & 98000072 & 40.1 & 455 & 16 & 10.5 \\
\hline 19800513 & 98000073 & 21.2 & 280 & 18.5 & 13.5 \\
\hline 19800609 & 98000074 & 13.3 & 240 & 23.5 & 22 \\
\hline 19800708 & 98000075 & 4.6 & 350 & 25 & 26.5 \\
\hline 19800804 & 98000076 & 3.1 & 230 & 23 & 21 \\
\hline 19800902 & 98000077 & 4.9 & 320 & 29 & 24 \\
\hline 19801002 & 98100067 & 14 & 250 & -- & -- \\
\hline 19801028 & 98100068 & 12 & 290 & 3 & 6.5 \\
\hline 19801124 & 98100069 & 14 & 200 & 9 & 2 \\
\hline 19810120 & 98100070 & 12 & 240 & -- & -- \\
\hline 19810318 & 98100071 & 17 & 200 & -- & -- \\
\hline 19810415 & 98100072 & 11 & 240 & 15 & 12 \\
\hline 19810513 & 98100073 & 18 & -- & 19 & 13 \\
\hline
\end{tabular}


Table 28. Water-quality data for U.S. Geological Survey streamflow-gaging station 06447500, Little White River near Martin, South Dakota-Continued

$[\mu \mathrm{S} / \mathrm{cm}$; microsiemens per centimeter; --, no data]

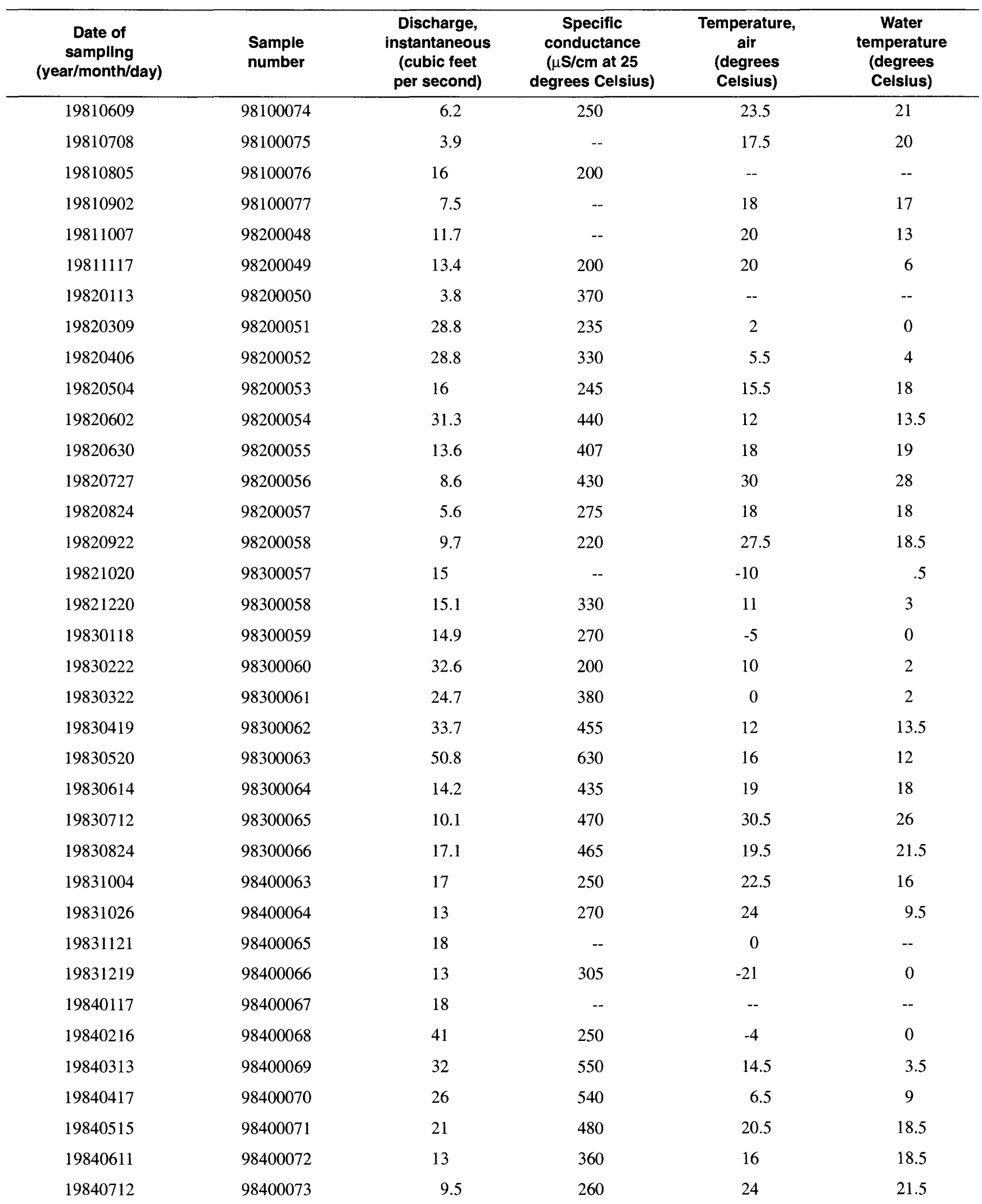


Table 28. Water-quality data for U.S. Geological Survey streamflow-gaging station 06447500 , Little White River near Martin, South Dakota-Continued

[ $\mu \mathrm{S} / \mathrm{cm}$; microsiemens per centimeter; --, no data]

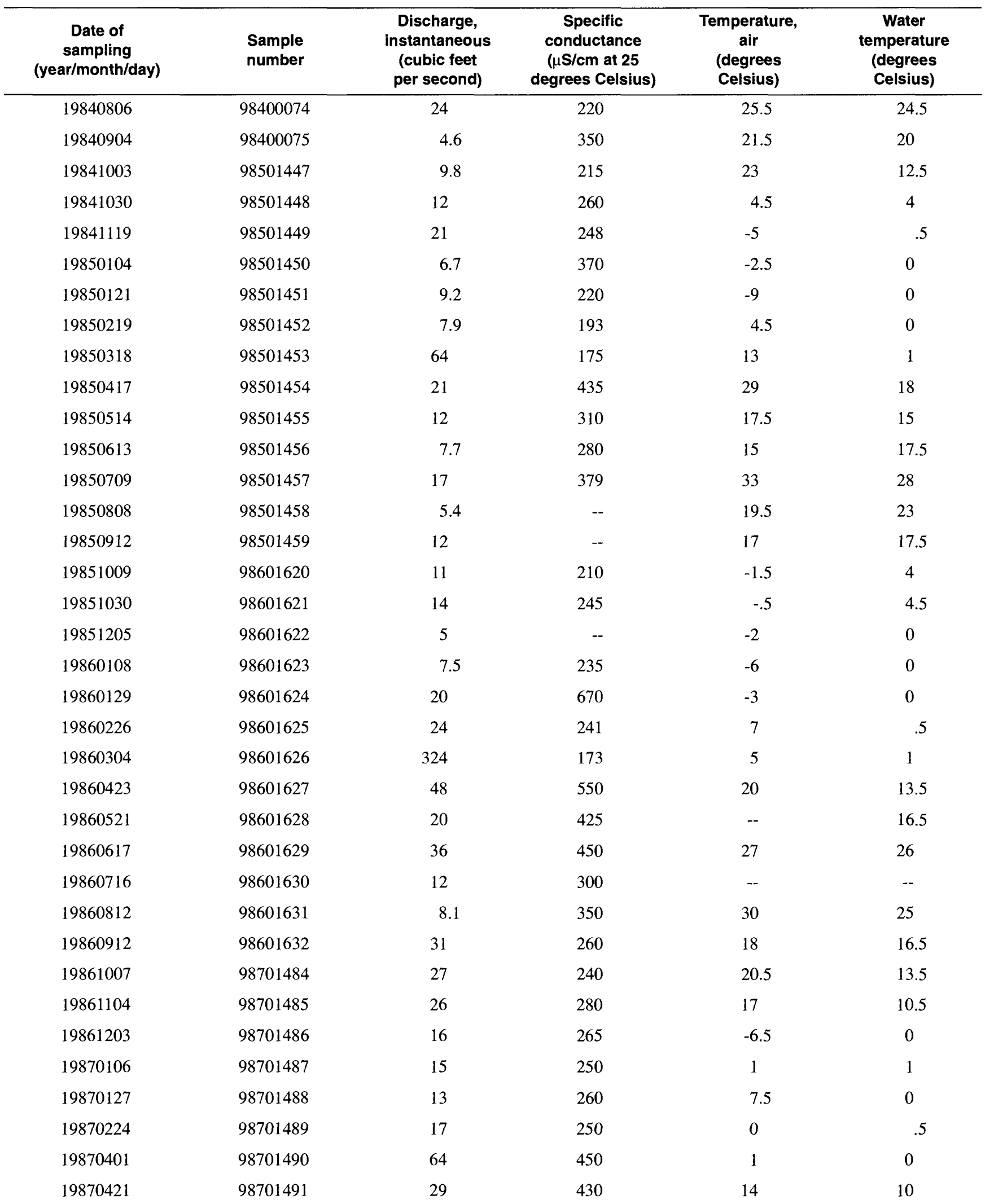


Table 28. Water-quality data for U.S. Geological Survey streamflow-gaging station 06447500, Little White River near Martin, South Dakota-Continued

$[\mu \mathrm{S} / \mathrm{cm}$; microsiemens per centimeter; --, no data]

\begin{tabular}{|c|c|c|c|c|c|}
\hline $\begin{array}{c}\text { Date of } \\
\text { sampling } \\
\text { (year/month/day) }\end{array}$ & $\begin{array}{l}\text { Sample } \\
\text { number }\end{array}$ & $\begin{array}{l}\text { Discharge, } \\
\text { instantaneous } \\
\text { (cubic feet } \\
\text { per second) }\end{array}$ & $\begin{array}{c}\text { Specific } \\
\text { conductance } \\
\text { ( } \mu \text { S/cm at } 25 \\
\text { degrees Celsius) }\end{array}$ & $\begin{array}{c}\text { Temperature, } \\
\text { air } \\
\text { (degrees } \\
\text { Celsius) }\end{array}$ & $\begin{array}{c}\text { Water } \\
\text { temperature } \\
\text { (degrees } \\
\text { Celsius) }\end{array}$ \\
\hline 19870519 & 98701492 & 16 & 450 & 21.5 & 22 \\
\hline 19870617 & 98701493 & 10 & 350 & 15 & 15 \\
\hline 19870715 & 98701494 & 6.3 & 315 & 16.5 & 19 \\
\hline 19870811 & 98701495 & 5.7 & 240 & 35 & 34 \\
\hline 19870909 & 98701496 & 6.4 & 212 & 14 & 15 \\
\hline 19871006 & 98800696 & 10 & -- & 16.5 & 13 \\
\hline 19871028 & 98800697 & 13 & 219 & 8.5 & 4 \\
\hline 19871202 & 98800698 & 14 & -- & 4 & 1 \\
\hline 19880113 & 98800699 & 4.5 & 245 & -6 & 0 \\
\hline 19880203 & 98800700 & 5.1 & -- & -1 & 0 \\
\hline 19880217 & 98800701 & - & - & -1 & 0 \\
\hline 19880405 & 98800702 & 37 & 372 & 17 & 11.5 \\
\hline 19880510 & 98800703 & 33 & 438 & 20 & 16.5 \\
\hline 19880613 & 98800704 & 16 & 450 & 19 & 20 \\
\hline 19880712 & 98800705 & 6.4 & 370 & -- & 24 \\
\hline 19880817 & 98800706 & 3.5 & 275 & 21.5 & 22.5 \\
\hline 19880928 & 98800707 & 7.5 & 225 & 8.5 & 11.5 \\
\hline 19881103 & 98901369 & 13.2 & 201 & 7 & 6 \\
\hline 19881214 & 98901370 & 18.7 & 240 & -1 & 0 \\
\hline 19890111 & 98901371 & 6.8 & 264 & 3 & 0 \\
\hline 19890214 & 98901372 & 6.4 & 234 & 4 & 0 \\
\hline 19890329 & 98901373 & 38.5 & 254 & 7 & 9.5 \\
\hline 19890426 & 98901374 & 17.9 & 305 & 10 & 7 \\
\hline 19890524 & 98901375 & 12.7 & 295 & -- & 19 \\
\hline 19890613 & 98901376 & 7.8 & 254 & 9.5 & 14 \\
\hline 19890724 & 98901377 & 3.6 & 222 & 30.5 & 25.5 \\
\hline 19890915 & 98901378 & 8.1 & 217 & 10 & 12.5 \\
\hline 19891018 & 99001173 & 9.6 & 218 & 1.5 & 5 \\
\hline 19891206 & 99001174 & 18.2 & 195 & 0 & 0 \\
\hline 19900118 & 99001175 & 14.7 & 193 & -- & 0 \\
\hline 19900227 & 99001176 & 36.8 & 177 & -1 & .5 \\
\hline 19900403 & 99001177 & 19.5 & 245 & 14 & 8 \\
\hline 19900430 & 99001178 & 28.8 & 287 & 7.5 & 8 \\
\hline 19900606 & 99001179 & 48.3 & 386 & 19.5 & 19.5 \\
\hline 19900710 & 99001180 & 12.9 & 325 & 15 & 19 \\
\hline 19900906 & 99001398 & 6.3 & 293 & 37 & 25 \\
\hline
\end{tabular}


Table 28. Water-quality data for U.S. Geological Survey streamflow-gaging station 06447500, Little White River near Martin, South Dakota-Continued

[ $\mu \mathrm{S} / \mathrm{cm}$; microsiemens per centimeter; --, no data]

\begin{tabular}{|c|c|c|c|c|c|}
\hline $\begin{array}{c}\text { Date of } \\
\text { sampling } \\
\text { (year/month/day) }\end{array}$ & $\begin{array}{l}\text { Sample } \\
\text { number }\end{array}$ & $\begin{array}{c}\text { Discharge, } \\
\text { instantaneous } \\
\text { (cubic feet } \\
\text { per second) }\end{array}$ & $\begin{array}{c}\text { Specific } \\
\text { conductance } \\
(\mu \mathrm{S} / \mathrm{cm} \text { at } 25 \\
\text { degrees Celsius) }\end{array}$ & $\begin{array}{c}\text { Temperature, } \\
\text { air } \\
\text { (degrees } \\
\text { Celsius) }\end{array}$ & $\begin{array}{c}\text { Water } \\
\text { temperature } \\
\text { (degrees } \\
\text { Celsius) }\end{array}$ \\
\hline 19901031 & 99101016 & 11.8 & 221 & 15 & 8 \\
\hline 19901205 & 99101017 & 18.6 & 249 & 2.5 & 1 \\
\hline 19910111 & 99101018 & 3.1 & 320 & -10 & 0 \\
\hline 19910226 & 99101019 & 36.3 & 209 & -3.5 & 0 \\
\hline 19910410 & 99101020 & 18.6 & 258 & 9 & 7 \\
\hline 19910508 & 99101021 & 28.7 & 362 & 9 & 10 \\
\hline 19910612 & 99101022 & 54.4 & 371 & 28.5 & 26 \\
\hline 19910717 & 99101023 & 8.7 & 339 & 25.5 & 24 \\
\hline 19910819 & 99101024 & 12.5 & 347 & 26.5 & 25 \\
\hline 19911002 & 99200991 & 10 & 252 & 12 & 14.5 \\
\hline 19911113 & 99200992 & 31.4 & 193 & 7 & 0 \\
\hline 19920108 & 99200993 & 11.2 & 228 & -7 & 0 \\
\hline 19920219 & 99200994 & 15.6 & 234 & -8 & 0 \\
\hline 19920330 & 99200995 & 19.7 & 331 & 3 & 10 \\
\hline 19920520 & 99200996 & 8.4 & 282 & 22 & 18 \\
\hline 19920609 & 99200997 & 11.9 & 252 & 26 & 22 \\
\hline 19920714 & 99200998 & 15.8 & 312 & 29 & 26.5 \\
\hline 19920825 & 99200999 & 14 & 203 & 16 & 17 \\
\hline 19921014 & 99301798 & 10.3 & 227 & 10 & 6 \\
\hline 19921123 & 99301799 & 16 & 230 & 0 & 0 \\
\hline 19921228 & 99301800 & 7.8 & 190 & 0 & 0 \\
\hline 19930208 & 99301801 & 32 & 209 & 4 & .5 \\
\hline 19930324 & 99301802 & 67.8 & 221 & 22 & .5 \\
\hline 19930505 & 99301803 & 22.9 & 433 & 22 & 15 \\
\hline 19930616 & 99301804 & 17.4 & 499 & -- & -- \\
\hline 19930707 & 99301805 & 13.6 & 460 & 29.5 & 19 \\
\hline 19930805 & 99301806 & 10.2 & 350 & 23 & 22.5 \\
\hline 19930909 & 99301807 & 14.8 & 254 & 18 & 15 \\
\hline 19931014 & 99400855 & 21.7 & 254 & 17 & 10.2 \\
\hline 19931129 & 99400856 & 18.1 & 420 & 6 & .5 \\
\hline 19931230 & 99400857 & 25 & 527 & 2 & 0 \\
\hline 19940126 & 99400858 & 13.1 & 440 & -1.5 & 0 \\
\hline 19940525 & 99400859 & 27.9 & 375 & 22 & 22.5 \\
\hline 19940629 & 99400860 & 10.9 & 350 & -- & 21 \\
\hline 19940810 & 99400861 & 10.4 & 310 & 24 & 23 \\
\hline 19940831 & 99400862 & 7.7 & 292 & 11.5 & 17 \\
\hline
\end{tabular}


Table 28. Water-quality data for U.S. Geological Survey streamflow-gaging station 06447500 , Little White River near Martin, South Dakota-Continued

$[\mu \mathrm{S} / \mathrm{cm}$; microsiemens per centimeter; --, no data]

\begin{tabular}{|c|c|c|c|c|c|}
\hline $\begin{array}{c}\text { Date of } \\
\text { sampling } \\
\text { (year/month/day) }\end{array}$ & $\begin{array}{l}\text { Sample } \\
\text { number }\end{array}$ & $\begin{array}{l}\text { Discharge, } \\
\text { instantaneous } \\
\text { (cubic feet } \\
\text { per second) }\end{array}$ & $\begin{array}{c}\text { Specific } \\
\text { conductance } \\
\text { ( } \mu \text { S/cm at } 25 \\
\text { degrees Celsius) }\end{array}$ & $\begin{array}{c}\text { Temperature, } \\
\text { air } \\
\text { (degrees } \\
\text { Celsius) }\end{array}$ & $\begin{array}{c}\text { Water } \\
\text { temperature } \\
\text { (degrees } \\
\text { Celsius) }\end{array}$ \\
\hline 19941012 & 99500710 & 14.7 & 261 & 15 & 12 \\
\hline 19941123 & 99500711 & 15.8 & 262 & 8 & .5 \\
\hline 19950105 & 99500712 & 10.4 & 314 & 4 & 0 \\
\hline 19950208 & 99500713 & 35 & 244 & 7 & 0 \\
\hline 19950314 & 99500714 & 37.2 & 212 & 20 & 10 \\
\hline 19950419 & 99500715 & 64.8 & 404 & 4 & 4.5 \\
\hline 19950614 & 99500716 & 217 & 499 & 28.5 & 22 \\
\hline 19950809 & 99500717 & 35 & 325 & 27 & 25.5 \\
\hline 19950907 & 99500718 & 9.4 & 324 & 17 & 19.5 \\
\hline 19951011 & 99600733 & 26.1 & 352 & 27 & 11 \\
\hline 19951205 & 99600734 & 24.6 & 309 & -3 & 0 \\
\hline 19960123 & 99600735 & 18.9 & 338 & 3 & 0 \\
\hline 19960312 & 99600736 & -- & -- & -- & -- \\
\hline 19960410 & 99600737 & 34.1 & 395 & 27 & 17 \\
\hline 19960520 & 99600738 & 22.3 & 474 & -- & 20 \\
\hline 19960612 & 99600739 & 27 & 550 & 25 & 25 \\
\hline 19960722 & 99600740 & 9.5 & 328 & 22 & 26.5 \\
\hline 19960805 & 99600741 & -- & - & -- & -- \\
\hline 19960910 & 99600742 & 9.9 & 246 & 22 & 18.5 \\
\hline
\end{tabular}

${ }^{1}$ Additional water-quality data for this sample includes: Sediment, bed material, fall diameter, distilled water, percent finer than 0.062 mm (11); sediment, bed material, fall diameter, distilled water, percent finer than $0.125 \mathrm{~mm}$ (24); sediment, bed material, fall diameter, distilled water, percent finer than $0.250 \mathrm{~mm}$ (61); sediment, bed material, fall diameter, distilled water, percent finer than $0.500 \mathrm{~mm}(78)$; sediment, bed material, fall diameter, distilled water, percent finer than $1.00 \mathrm{~mm}$ (78); sediment, bed material, sieve diameter, percent finer than $2.00 \mathrm{~mm}$ (79); sediment, bed material, sieve diameter, percent finer than $4.00 \mathrm{~mm}$ (83); sediment, bed material, sieve diameter, percent finer than $8.00 \mathrm{~mm}$ (88); sediment, bed material, sieve diameter, percent finer than $16.0 \mathrm{~mm}(98)$; and sediment, bed material, sieve diameter, percent finer than $32.0 \mathrm{~mm}$ (100). 
Table 29. Water-quality data for U.S. Geological Survey streamflow-gaging station 06448000 , Lake Creek above refuge, near Tuthill, South Dakota

$[\mu \mathrm{S} / \mathrm{cm}$, microsiemens per centimeter; --, no data]

\begin{tabular}{|c|c|c|c|c|c|}
\hline $\begin{array}{l}\text { Date of sampling } \\
\text { (year/month/day) }\end{array}$ & Sample number & $\begin{array}{c}\text { Discharge, } \\
\text { instantaneous } \\
\text { (cubic feet } \\
\text { per second) }\end{array}$ & $\begin{array}{c}\text { Specific } \\
\text { conductance } \\
\text { ( } \mu \text { S/cm at } 25 \\
\text { degrees Celsius) }\end{array}$ & $\begin{array}{l}\text { Temperature, air } \\
\text { (degrees } \\
\text { Celsius) }\end{array}$ & $\begin{array}{l}\text { Water temperature } \\
\text { (degrees Celsius) }\end{array}$ \\
\hline 19751006 & 97600075 & 13 & -- & 31.5 & 14.5 \\
\hline 19751105 & 97600076 & 17 & -- & 24 & 10.5 \\
\hline 19760106 & 97600078 & 8.1 & -- & -2 & 0 \\
\hline 19760126 & 97600079 & 14 & - & 12 & 0 \\
\hline 19760224 & 97600080 & 20 & -- & 6 & 6.5 \\
\hline 19760322 & 97600081 & 17 & -- & 14 & 12 \\
\hline 19760713 & 97600085 & 12 & -- & 24 & 20 \\
\hline 19760809 & 97600086 & 13 & -- & 38 & 36 \\
\hline 19760907 & 97600087 & 9.9 & -- & 13.5 & 16 \\
\hline 19761004 & 97700101 & 15.1 & -- & 10 & 12 \\
\hline 19761102 & 97700102 & 19.2 & -- & 15 & 7 \\
\hline 19761130 & 97700103 & 16.2 & -- & 2 & 0 \\
\hline 19761227 & 97700104 & 24 & -- & 8 & 1 \\
\hline 19770517 & 97700110 & 16.9 & -- & 12.5 & 12.5 \\
\hline 19770614 & 97700111 & 16.6 & -- & 20 & 20 \\
\hline 19770712 & 97700112 & 14.4 & -- & 23 & 18 \\
\hline 19770810 & 97700113 & 35.2 & -. & 11 & 17 \\
\hline 19770906 & 97700114 & 15.1 & -- & 29 & 23.5 \\
\hline 19771031 & 97800090 & 19 & 100 & 10 & 12 \\
\hline 19771129 & 97800091 & 24 & 110 & 10 & 20 \\
\hline 19771228 & 97800092 & 19 & 130 & -1 & 0 \\
\hline 19780126 & 97800093 & 14 & 130 & -6 & 0 \\
\hline 19780228 & 97800094 & 28 & 105 & -8 & 0 \\
\hline 19780315 & 97800095 & 30 & 120 & 1 & 2 \\
\hline 19780411 & 97800096 & 23 & 120 & 14 & 6.5 \\
\hline 19780510 & 97800097 & 24 & 130 & 14.5 & 9 \\
\hline 19780613 & 97800098 & 18 & 110 & 22 & 16 \\
\hline
\end{tabular}


Table 29. Water-quality data for U.S. Geological Survey streamflow-gaging station 06448000 , Lake Creek above refuge, near Tuthill, South Dakota-Continued

$[\mu \mathrm{S} / \mathrm{cm}$, microsiemens per centimeter; --, no data]

\begin{tabular}{cccccc}
\hline $\begin{array}{c}\text { Date of sampling } \\
\text { (year/month/day) }\end{array}$ & Sample number & $\begin{array}{c}\text { Discharge, } \\
\text { instantaneous } \\
\text { (cubic feet } \\
\text { per second) }\end{array}$ & $\begin{array}{c}\text { Specific } \\
\text { conductance } \\
(\mu \text { S/cm at 25 } \\
\text { degrees Celsius) }\end{array}$ & $\begin{array}{c}\text { Temperature, air } \\
\text { (degrees } \\
\text { Celsius) }\end{array}$ & $\begin{array}{c}\text { Water temperature } \\
\text { (degrees Celsius) }\end{array}$ \\
\hline 19780711 & 97800099 & 15 & 100 & 23 & 18.5 \\
19780829 & 97800100 & 16 & 110 & 13 & 13 \\
19781003 & 97900074 & 15 & 110 & 9 & 10 \\
19781102 & 97900075 & 21 & 120 & 18 & 6 \\
19781127 & 97900076 & 21 & 110 & -5 & 1 \\
19781219 & 97900077 & 24 & 120 & 2 & 0 \\
19790123 & 97900078 & 12 & 130 & -5 & 0 \\
19790220 & 97900079 & 22 & 230 & 16.5 & 3 \\
19960311 & 99600706 & 46 & 110 & 16 & 6.5 \\
19960312 & 99600707 & 33.7 & 117 & 31 & 15 \\
19960410 & 99600708 & 28.6 & 112 & - & 21 \\
19960520 & 99600709 & 22.8 & 117 & 26 & 26 \\
19960612 & 99600710 & 25.2 & 121 & 32 & 25.5 \\
19960722 & 99600711 & 18.2 & 101 & 33 & 23 \\
19960909 & 99600712 & 19.9 & 106 & & 6 \\
\hline
\end{tabular}


Table 30. Water-quality data for U.S. Geological Survey streamflow-gaging station 06449000 , Lake Creek below refuge, near Tuthill, South Dakota

[ $\mu \mathrm{S} / \mathrm{cm}$, microsiemens per centimeter; --, no data; E, estimated]

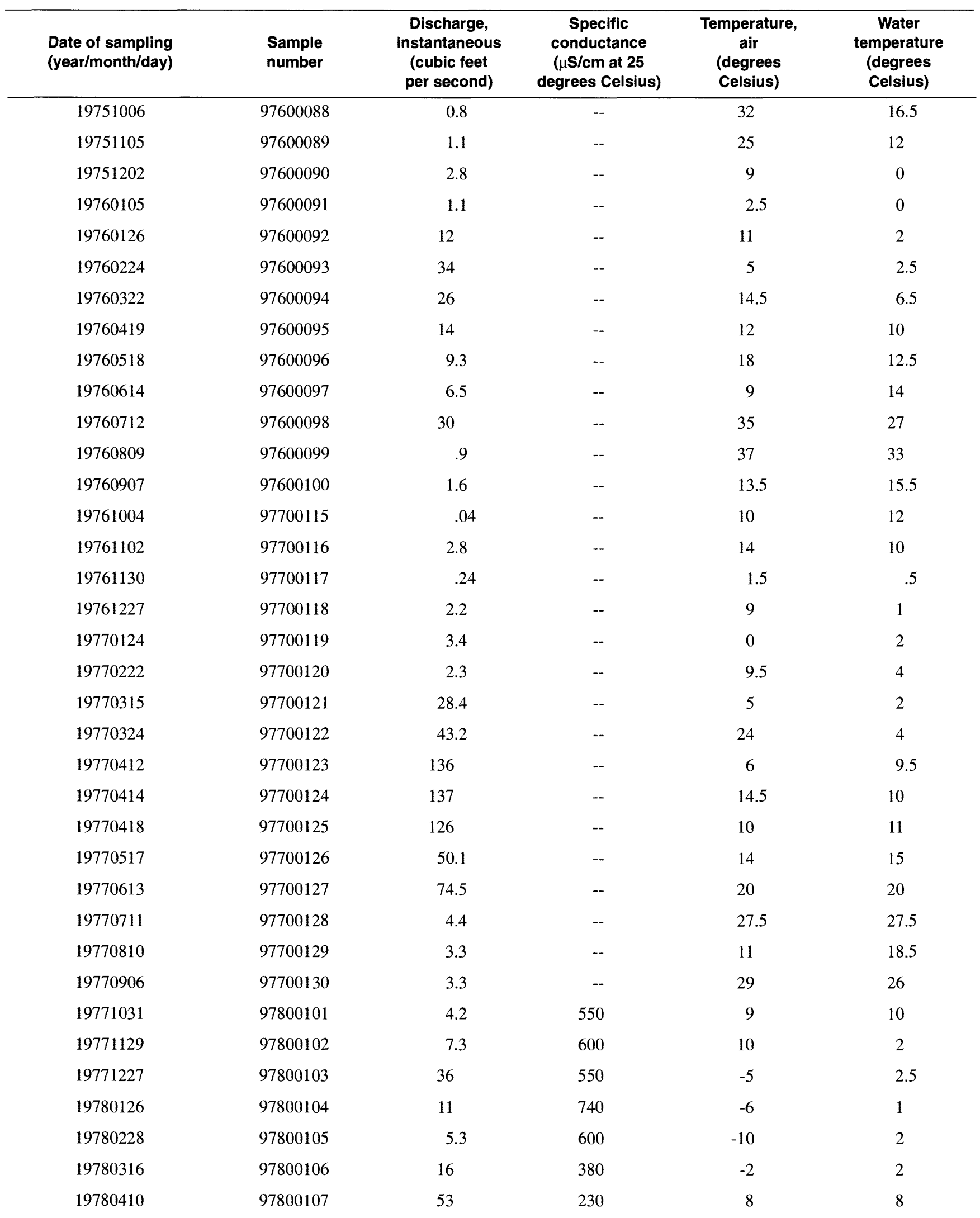


Table 30. Water-quality data for U.S. Geological Survey streamflow-gaging station 06449000 , Lake Creek below refuge, near Tuthill, South Dakota-Continued

$[\mu \mathrm{S} / \mathrm{cm}$, microsiemens per centimeter; --, no data; E, estimated]

\begin{tabular}{|c|c|c|c|c|c|}
\hline $\begin{array}{l}\text { Date of sampling } \\
\text { (year/month/day) }\end{array}$ & $\begin{array}{l}\text { Sample } \\
\text { number }\end{array}$ & $\begin{array}{l}\text { Discharge, } \\
\text { instantaneous } \\
\text { (cubic feet } \\
\text { per second) }\end{array}$ & $\begin{array}{c}\text { Specific } \\
\text { conductance } \\
(\mu \mathrm{S} / \mathrm{cm} \text { at } 25 \\
\text { degrees Celsius) }\end{array}$ & $\begin{array}{c}\text { Temperature, } \\
\text { air } \\
\text { (degrees } \\
\text { Celsius) }\end{array}$ & $\begin{array}{c}\text { Water } \\
\text { temperature } \\
\text { (degrees } \\
\text { Celsius) }\end{array}$ \\
\hline 19780510 & 97800108 & 59 & 300 & 15.5 & 13 \\
\hline 19780612 & 97800109 & 20 & 430 & 25 & 24 \\
\hline 19780710 & 97800110 & 14 & 440 & 25.5 & 23 \\
\hline 19780828 & 97800111 & 4.6 & 230 & 20 & 23 \\
\hline 19781003 & 97900080 & .8 & 540 & 13 & 12 \\
\hline 19781102 & 97900081 & .5 & 680 & 16 & 6 \\
\hline 19781127 & 97900082 & 1.6 & 760 & -5 & .5 \\
\hline 19781219 & 97900083 & 9.2 & 820 & 3 & 0 \\
\hline 19790123 & 97900084 & 11 & 820 & 0 & 1 \\
\hline 19790220 & 97900085 & 16 & 660 & -1 & 3 \\
\hline 19790320 & 97900086 & 56 & 420 & 6 & 0 \\
\hline 19790417 & 97900087 & 46 & 330 & 27 & 12 \\
\hline 19790515 & 97900088 & 33 & 370 & 21 & 15 \\
\hline 19790612 & 97900089 & 26 & 490 & 29 & 24 \\
\hline 19790710 & 97900090 & 45 & 420 & 32 & 24 \\
\hline 19790807 & 97900091 & 35 & 440 & 30 & 26 \\
\hline 19790906 & 97900092 & 21 & 410 & 18 & 20 \\
\hline 19791106 & 98000078 & 9.4 & 470 & -4 & 1 \\
\hline 19791126 & 98000079 & 52.1 & 590 & 4 & 1 \\
\hline 19791217 & 98000080 & 31.1 & 470 & 8 & 2 \\
\hline 19800122 & 98000081 & 9.8 & 440 & 0 & 0 \\
\hline 19800219 & 98000082 & 25.1 & 400 & 9 & 4 \\
\hline 19800318 & 98000083 & 35.1 & 220 & 14 & 4.5 \\
\hline 19800414 & 98000084 & 64.8 & 290 & 15.5 & 12 \\
\hline 19800513 & 98000085 & 25.2 & 320 & 16 & 13.5 \\
\hline 19800609 & 98000086 & 8.3 & 420 & 23.5 & 24.5 \\
\hline 19800708 & 98000087 & 9.1 & 500 & 25 & 24 \\
\hline 19800804 & 98000088 & 2.4 & 395 & 23.5 & 22.5 \\
\hline 19800902 & 98000089 & .4 & 620 & 27.5 & 23 \\
\hline 19801002 & 98100078 & 1.1 & 850 & 16.5 & 14 \\
\hline 19801028 & 98100079 & E. 01 & -- & -- & -- \\
\hline 19801124 & 98100080 & 0 & -- & -- & -- \\
\hline 19810120 & 98100081 & 2.4 & - & 5 & 0 \\
\hline 19810318 & 98100082 & 3.1 & 370 & -- & -- \\
\hline 19810415 & 98100083 & 5.7 & 430 & 17 & 13 \\
\hline 19810513 & 98100084 & 7.1 & 480 & 19 & 15 \\
\hline
\end{tabular}


Table 30. Water-quality data for U.S. Geological Survey streamflow-gaging station 06449000 , Lake Creek below refuge, near Tuthill, South Dakota-Continued

$[\mu \mathrm{S} / \mathrm{cm}$, microsiemens per centimeter; --, no data; E, estimated]

\begin{tabular}{|c|c|c|c|c|c|}
\hline $\begin{array}{l}\text { Date of sampling } \\
\text { (year/month/day) }\end{array}$ & $\begin{array}{l}\text { Sample } \\
\text { number }\end{array}$ & $\begin{array}{c}\text { Discharge, } \\
\text { instantaneous } \\
\text { (cubic feet } \\
\text { per second) }\end{array}$ & $\begin{array}{c}\text { Specific } \\
\text { conductance } \\
\text { ( } \mu \mathrm{S} / \mathrm{cm} \text { at } 25 \\
\text { degrees Celsius) }\end{array}$ & $\begin{array}{c}\text { Temperature, } \\
\text { air } \\
\text { (degrees } \\
\text { Celsius) }\end{array}$ & $\begin{array}{c}\text { Water } \\
\text { temperature } \\
\text { (degrees } \\
\text { Celsius) }\end{array}$ \\
\hline 19810609 & 98100085 & 1.4 & 510 & 19 & 21 \\
\hline 19810707 & 98100086 & 9.2 & -- & 31.5 & 27 \\
\hline 19810805 & 98100087 & 21 & 490 & -- & -- \\
\hline 19810901 & 98100088 & 2.4 & -- & 28 & 23.5 \\
\hline 19811007 & 98200059 & .1 & -- & 21.5 & 17 \\
\hline 19811117 & 98200060 & 2.0 & 550 & 20 & 9 \\
\hline 19820112 & 98200061 & 13.8 & 520 & -- & -- \\
\hline 19820308 & 98200062 & 25.6 & 295 & -2 & 2 \\
\hline 19820406 & 98200063 & 37.7 & 340 & 5.5 & 4.5 \\
\hline 19820504 & 98200064 & 23.7 & 355 & 16 & 16.5 \\
\hline 19820607 & 98200065 & 22.2 & 440 & 23 & 19.5 \\
\hline 19820629 & 98200066 & 71.4 & 431 & -- & -- \\
\hline 19820727 & 98200067 & 11.6 & 530 & 30.5 & 29 \\
\hline 19821019 & 98300067 & 6.6 & 580 & -3 & .5 \\
\hline 19821220 & 98300068 & 21.5 & -- & 10.5 & 2.5 \\
\hline 19830117 & 98300069 & 25.6 & 470 & -5 & 2 \\
\hline 19830222 & 98300070 & 18.3 & 360 & 13.5 & 9 \\
\hline 19830322 & 98300071 & 27.7 & 470 & 0 & 2.5 \\
\hline 19830520 & 98300072 & 101 & 390 & 21 & 13.5 \\
\hline 19830614 & 98300073 & 24.9 & 435 & 18 & 18.5 \\
\hline 19830712 & 98300074 & 1.2 & 430 & 30 & 28 \\
\hline 19830823 & 98300075 & 6.6 & 550 & 27 & 25 \\
\hline 19831004 & 98400076 & 15 & 470 & 24 & 18 \\
\hline 19831026 & 98400077 & 13 & -- & 24 & 11 \\
\hline 19831121 & 98400078 & 60 & 490 & 0 & 1 \\
\hline 19831219 & 98400079 & 30 & 610 & -17 & 0 \\
\hline 19840117 & 98400080 & 17 & -- & -18 & 0 \\
\hline 19840215 & 98400081 & 8.6 & 450 & 4 & 5 \\
\hline 19840313 & 98400082 & 42 & 500 & 14.5 & 2 \\
\hline 19840416 & 98400083 & 56 & 340 & 15 & 8.5 \\
\hline 19840515 & 98400084 & 32 & 540 & 19 & 19 \\
\hline 19840611 & 98400085 & 7.8 & 420 & 19.5 & 19 \\
\hline 19840712 & 98400086 & 5.5 & 460 & 25.5 & 24 \\
\hline 19840806 & 98400087 & 1.0 & 520 & 23.5 & 26 \\
\hline 19840904 & 98400088 & .2 & 580 & 22 & 25 \\
\hline 19840904 & 98401532 & .2 & 580 & 22 & 25 \\
\hline
\end{tabular}


Table 30. Water-quality data for U.S. Geological Survey streamflow-gaging station 06449000, Lake Creek below refuge, near Tuthill, South Dakota-Continued

[ $\mu \mathrm{S} / \mathrm{cm}$, microsiemens per centimeter; --, no data; E, estimated]

\begin{tabular}{|c|c|c|c|c|c|}
\hline $\begin{array}{l}\text { Date of sampling } \\
\text { (year/month/day) }\end{array}$ & $\begin{array}{l}\text { Sample } \\
\text { number }\end{array}$ & $\begin{array}{l}\text { Discharge, } \\
\text { instantaneous } \\
\text { (cubic feet } \\
\text { per second) }\end{array}$ & $\begin{array}{c}\text { Specific } \\
\text { conductance } \\
(\mu \mathrm{S} / \mathrm{cm} \text { at } 25 \\
\text { degrees Celsius) }\end{array}$ & $\begin{array}{c}\text { Temperature, } \\
\text { air } \\
\text { (degrees } \\
\text { Celsius) }\end{array}$ & $\begin{array}{c}\text { Water } \\
\text { temperature } \\
\text { (degrees } \\
\text { Celsius) }\end{array}$ \\
\hline 19841003 & 98501460 & 0.8 & 620 & 20.5 & 16.5 \\
\hline 19841030 & 98501461 & 1.6 & 730 & 6 & 4 \\
\hline 19841119 & 98501462 & .1 & 715 & -2.5 & 4.5 \\
\hline 19850103 & 98501463 & 27 & 650 & .5 & 1.5 \\
\hline 19850121 & 98501464 & 12 & 600 & -8 & .5 \\
\hline 19850219 & 98501465 & 8.9 & 630 & 4.5 & .5 \\
\hline 19850318 & 98501466 & 18 & 367 & 16 & 5 \\
\hline 19850417 & 98501467 & 28 & 395 & 29 & 18.5 \\
\hline 19850514 & 98501468 & 13 & 410 & 17 & 15 \\
\hline 19850612 & 98501469 & 0 & 510 & 21.5 & 22 \\
\hline 19850709 & 98501470 & 2.6 & 550 & 37 & 31 \\
\hline 19850808 & 98501471 & .3 & -- & 25 & $-\cdot$ \\
\hline 19850911 & 98501472 & 8.6 & 635 & 19 & 18 \\
\hline 19851008 & 98601593 & .4 & 260 & 5.5 & 8 \\
\hline 19851029 & 98601594 & 1.2 & 679 & 4.5 & 9 \\
\hline 19851205 & 98601595 & 1.8 & -- & -2 & .5 \\
\hline 19860108 & 98601596 & 7 & 750 & -5 & 1 \\
\hline 19860129 & 98601597 & 12 & 670 & -2 & 1 \\
\hline 19860225 & 98601598 & 44 & 424 & 13.5 & 2.5 \\
\hline 19860422 & 98601599 & -- & 350 & 27 & 14 \\
\hline 19860521 & 98601600 & 35 & 360 & 13 & 16 \\
\hline 19860617 & 98601601 & 40 & 390 & 32 & 25.5 \\
\hline 19860716 & 98601602 & 9.7 & 440 & 34.5 & 31 \\
\hline 19860812 & 98601603 & 4.1 & 450 & 28 & 26 \\
\hline 19860909 & 98601604 & 7.5 & 580 & 21.5 & 20.5 \\
\hline 19861007 & 98701497 & 16 & 475 & 21.5 & 15 \\
\hline 19861104 & 98701498 & 16 & 480 & 16 & 9 \\
\hline 19861202 & 98701499 & 29 & 470 & 1 & .5 \\
\hline 19870106 & 98701500 & 25 & 450 & 2 & 1.5 \\
\hline 19870127 & 98701501 & 24 & 450 & 8.5 & 4 \\
\hline 19870224 & 98701502 & 32 & 345 & .5 & .5 \\
\hline 19870331 & 98701503 & 140 & 340 & 7 & 2.5 \\
\hline 19870408 & 98701504 & 98 & 330 & 9 & 8 \\
\hline 19870421 & 98701505 & 43 & 360 & 13 & 10 \\
\hline 19870519 & 98701506 & 12 & 400 & 28 & 23 \\
\hline 19870617 & 98701507 & 16 & 430 & 15 & 14.5 \\
\hline
\end{tabular}


Table 30. Water-quality data for U.S. Geological Survey streamflow-gaging station 06449000 , Lake Creek below refuge, near Tuthill, South Dakota-Continued

[ $\mu \mathrm{S} / \mathrm{cm}$, microsiemens per centimeter; --, no data; E, estimated]

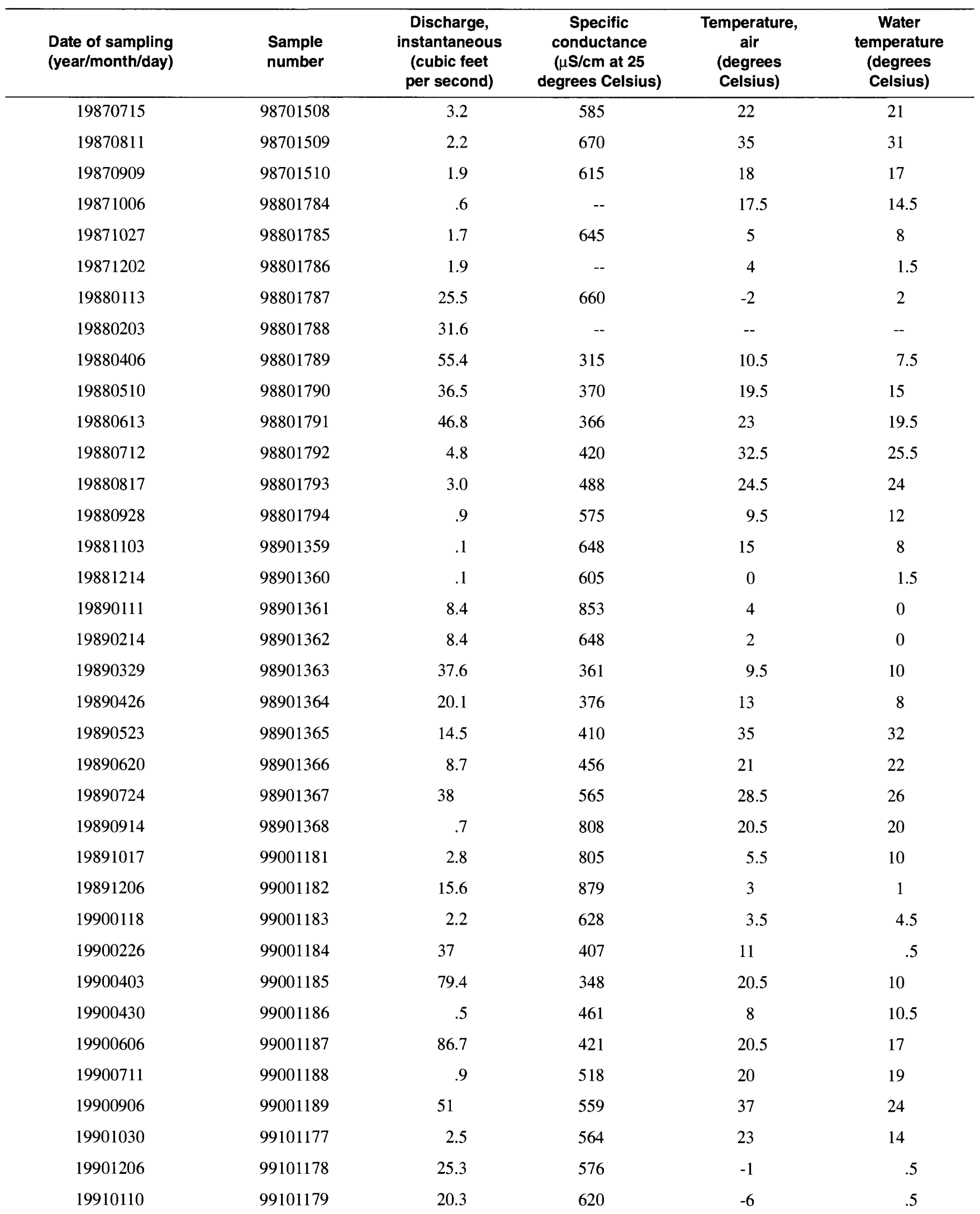


Table 30. Water-quality data for U.S. Geological Survey streamflow-gaging station 06449000, Lake Creek below refuge, near Tuthill, South Dakota-Continued

$[\mu \mathrm{S} / \mathrm{cm}$, microsiemens per centimeter; --, no data; E, estimated]

\begin{tabular}{|c|c|c|c|c|c|}
\hline $\begin{array}{l}\text { Date of sampling } \\
\text { (year/month/day) }\end{array}$ & $\begin{array}{l}\text { Sample } \\
\text { number }\end{array}$ & $\begin{array}{c}\text { Discharge, } \\
\text { instantaneous } \\
\text { (cubic feet } \\
\text { per second) }\end{array}$ & $\begin{array}{c}\text { Specific } \\
\text { conductance } \\
\text { ( } \mu \text { S/cm at } 25 \\
\text { degrees Celsius) }\end{array}$ & $\begin{array}{c}\text { Temperature, } \\
\text { air } \\
\text { (degrees } \\
\text { Celsius) }\end{array}$ & $\begin{array}{c}\text { Water } \\
\text { temperature } \\
\text { (degrees } \\
\text { Celsius) }\end{array}$ \\
\hline 19910226 & 99101180 & 8.4 & 221 & 0 & 1 \\
\hline 19910409 & 99101181 & 33.8 & 309 & 14.5 & 10 \\
\hline 19910507 & 99101182 & 18.3 & 380 & 11 & 10.5 \\
\hline 19910516 & 99101183 & 87.6 & 370 & 12 & 16 \\
\hline 19910521 & 99101184 & 125 & 382 & 19 & 18 \\
\hline 19910613 & 99101185 & 171 & 375 & 25 & 23.5 \\
\hline 19910716 & 99101186 & 5.6 & 479 & $33.5^{\circ}$ & 31 \\
\hline 19910819 & 99101187 & 53.8 & 458 & 27.5 & 24.5 \\
\hline 19911002 & 99200972 & 32.1 & 496 & 17.5 & 16 \\
\hline 19911113 & 99200973 & 11.9 & 397 & 14 & 3 \\
\hline 19920108 & 99200974 & 33 & 330 & -6 & 1.5 \\
\hline 19920219 & 99200975 & 34.3 & 272 & 0 & 1 \\
\hline 19920331 & 99200976 & 14.1 & 326 & 6 & 6 \\
\hline 19920520 & 99200977 & .8 & 505 & 26 & 18 \\
\hline 19920609 & 99200978 & .1 & 572 & 27 & -- \\
\hline 19920714 & 99200979 & 2.0 & 580 & 30 & 29 \\
\hline 19920825 & 99200980 & .9 & 550 & 16 & 16 \\
\hline 19921124 & 99301613 & 11.9 & 510 & 0 & 0 \\
\hline 19921229 & 99301614 & 6.2 & 630 & 18 & 11 \\
\hline 19930208 & 99301615 & 27.1 & 470 & 4 & .5 \\
\hline 19930324 & 99301616 & 84.2 & 260 & 21 & .5 \\
\hline 19930505 & 99301617 & 32.4 & 382 & 13 & 15 \\
\hline 19930623 & 99301618 & 54.3 & 418 & 26 & 22.5 \\
\hline 19930707 & 99301619 & 22.2 & 392 & 29 & 19 \\
\hline 19930805 & 99301620 & 21.2 & 410 & 23 & 22.5 \\
\hline 19930908 & 99301621 & 53.3 & 375 & 24 & 19 \\
\hline 19930909 & 99301622 & 41.1 & -- & -- & -- \\
\hline 19931014 & 99400840 & 16.2 & 385 & 16 & 11.5 \\
\hline 19931129 & 99400841 & 19.3 & 415 & 6 & .5 \\
\hline 19931230 & 99400842 & 48.3 & 390 & 5 & .5 \\
\hline 19940125 & 99400843 & 30.4 & 440 & -4.5 & 0 \\
\hline 19940525 & 99400844 & 32.4 & 312 & 19.5 & 19 \\
\hline 19940628 & 99400845 & 25.6 & 354 & 30 & 26 \\
\hline 19940810 & 99400846 & 45.7 & 345 & 22 & 25 \\
\hline 19940831 & 99400847 & 22.9 & 370 & 12 & 15 \\
\hline 19941012 & 99500675 & .1 & 503 & 13 & 11 \\
\hline
\end{tabular}


Table 30. Water-quality data for U.S. Geological Survey streamflow-gaging station 06449000 , Lake Creek below refuge, near Tuthill, South Dakota-Continued

$[\mu \mathrm{S} / \mathrm{cm}$, microsiemens per centimeter; --, no data; E, estimated]

\begin{tabular}{|c|c|c|c|c|c|}
\hline $\begin{array}{l}\text { Date of sampling } \\
\text { (year/month/day) }\end{array}$ & $\begin{array}{l}\text { Sample } \\
\text { number }\end{array}$ & $\begin{array}{c}\text { Discharge, } \\
\text { instantaneous } \\
\text { (cubic feet } \\
\text { per second) }\end{array}$ & $\begin{array}{c}\text { Specific } \\
\text { conductance } \\
(\mu \mathrm{S} / \mathrm{cm} \text { at } 25 \\
\text { degrees Celsius) }\end{array}$ & $\begin{array}{c}\text { Temperature, } \\
\text { air } \\
\text { (degrees } \\
\text { Celsius) }\end{array}$ & $\begin{array}{c}\text { Water } \\
\text { temperature } \\
\text { (degrees } \\
\text { Celsius) }\end{array}$ \\
\hline 19941123 & 99500676 & 16.9 & 324 & 8 & 2 \\
\hline 19950105 & 99500677 & 27.2 & 277 & -7.5 & 0 \\
\hline 19950208 & 99500678 & 46.5 & 217 & 7 & 0 \\
\hline 19950314 & 99500679 & 28 & 236 & 18 & 18.5 \\
\hline 19950419 & 99500680 & 40 & 382 & 6 & 7 \\
\hline 19950612 & 99500681 & 73 & 369 & 28 & 18.5 \\
\hline 19950809 & 99500682 & .1 & 472 & 28 & 28 \\
\hline 19950907 & 99500683 & 17.3 & 497 & 17 & 22.5 \\
\hline 19951011 & 99600713 & 12.5 & 377 & 20 & 11 \\
\hline 19951204 & 99600714 & 57 & 305 & 11 & 5 \\
\hline 19960123 & 99600715 & 25.5 & 331 & 2 & 1 \\
\hline 19960311 & 99600716 & 31.6 & 233 & 15 & 5 \\
\hline 19960417 & 99600717 & 47.2 & 259 & 26 & 12.5 \\
\hline 19960520 & 99600718 & 25.2 & 341 & -- & 21 \\
\hline 19960612 & 99600719 & 50.9 & 348 & 26 & 24.5 \\
\hline 19960722 & 99600720 & 29 & 411 & 29 & 28.5 \\
\hline 19960805 & 99600721 & -- & -- & -- & -- \\
\hline 19960909 & 99600722 & 16 & 443 & 32 & 26.5 \\
\hline
\end{tabular}




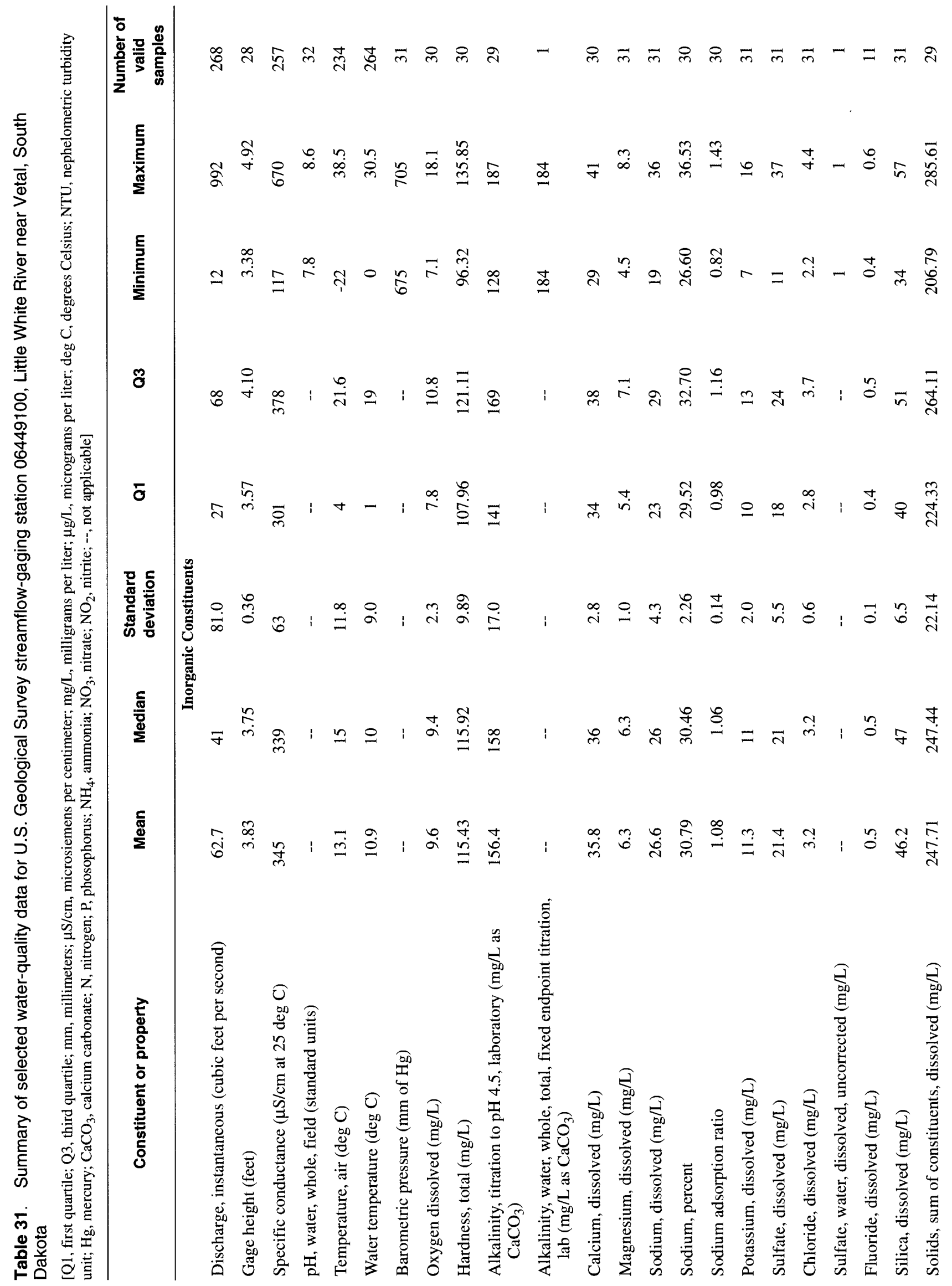




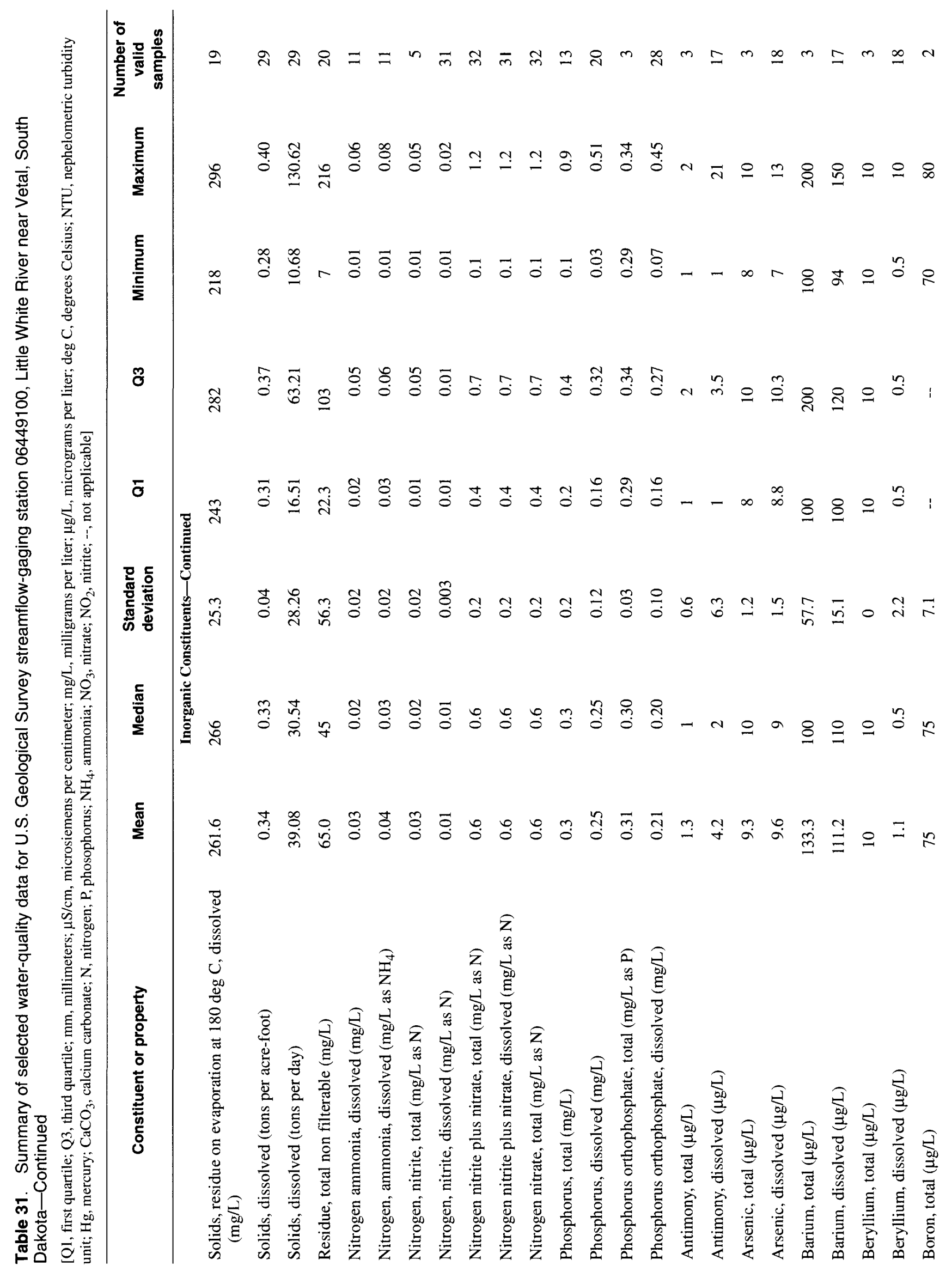




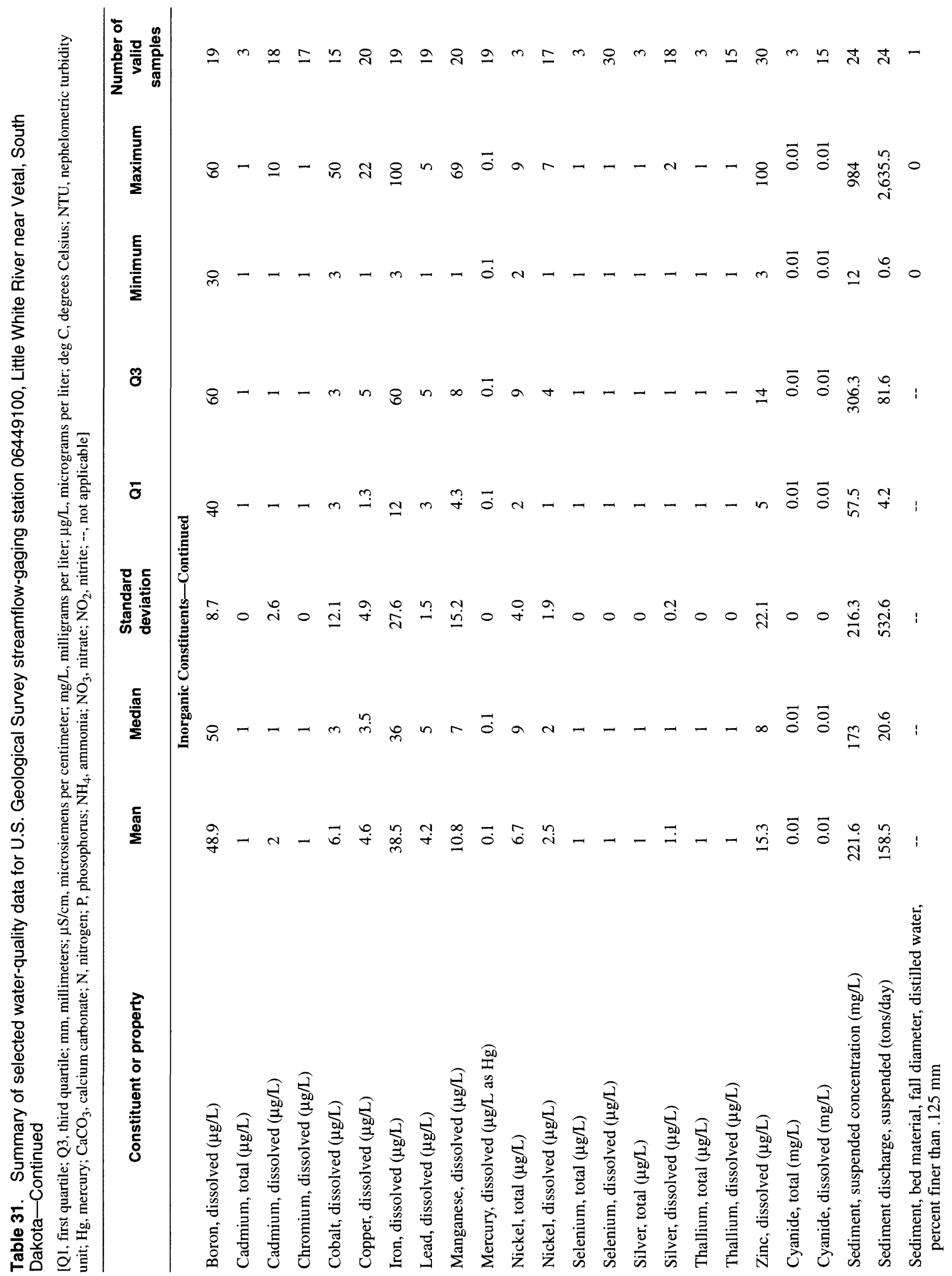




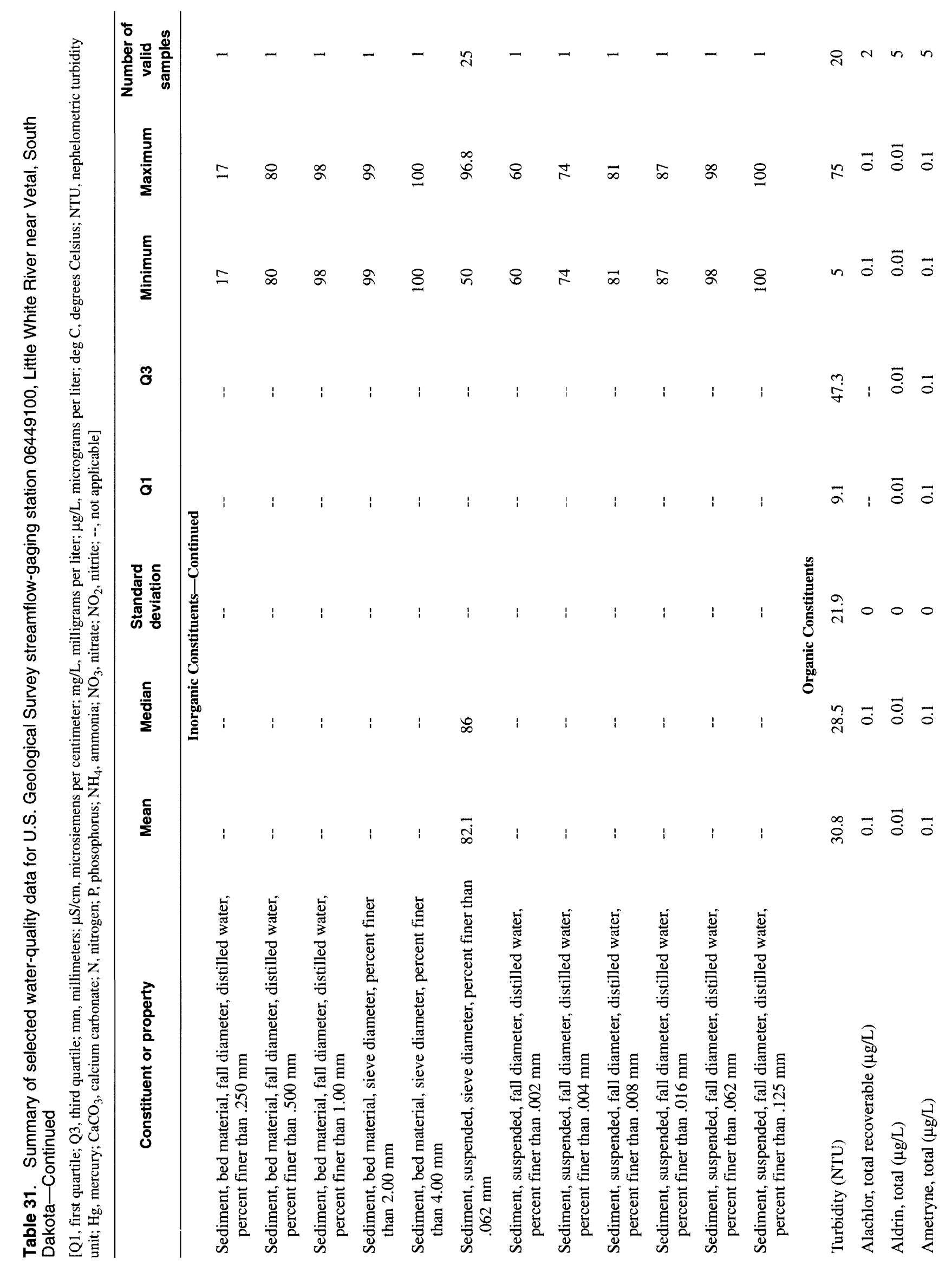




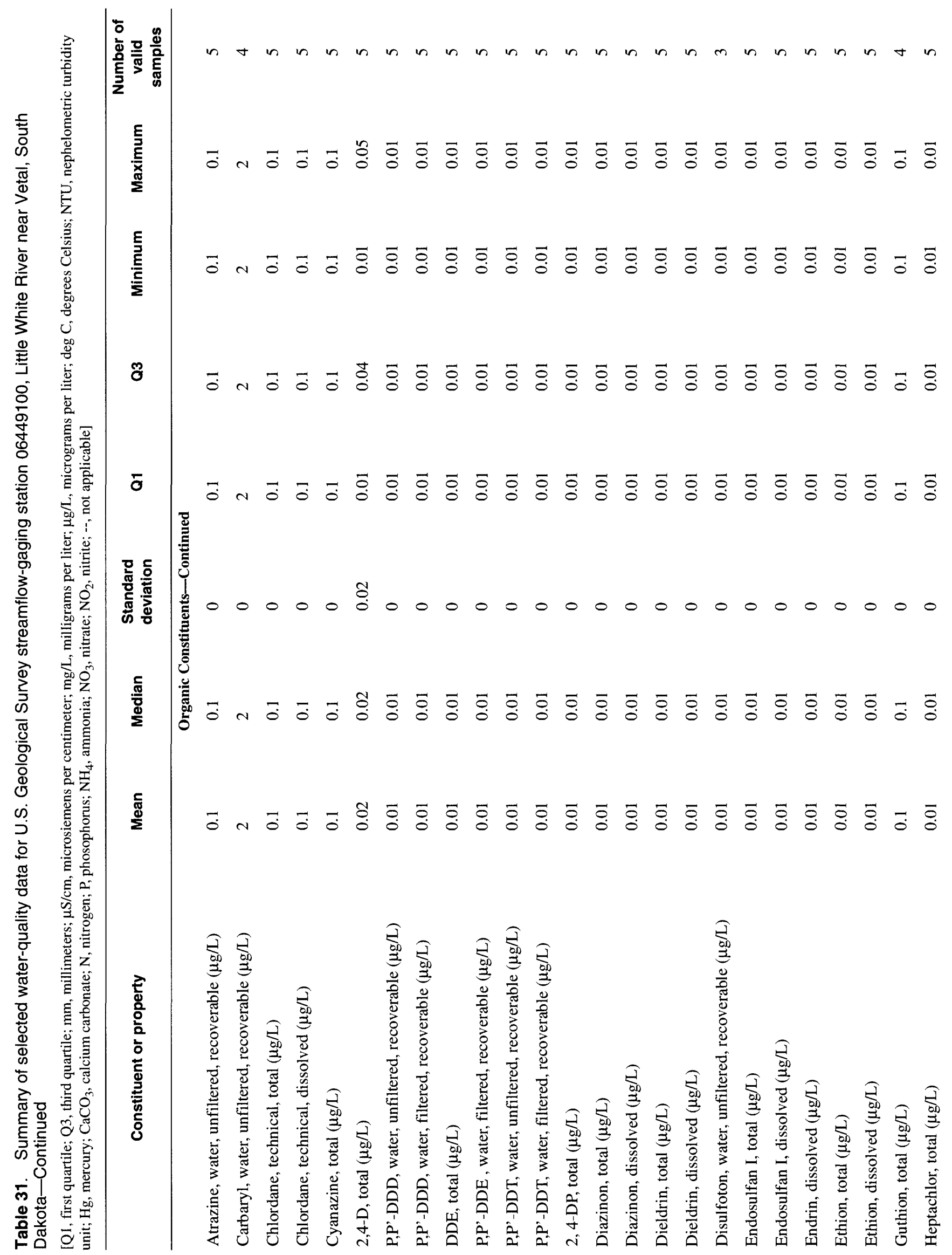




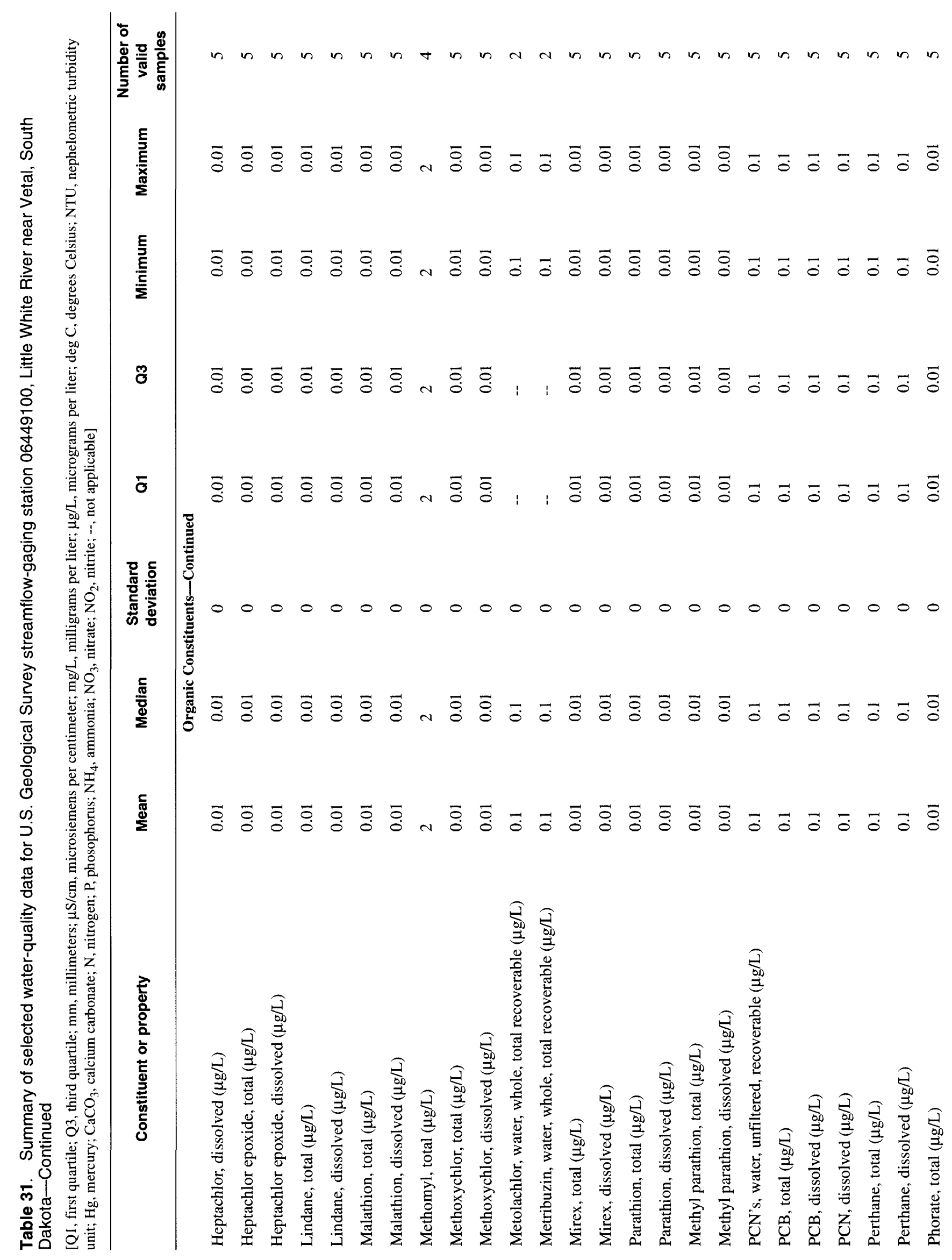




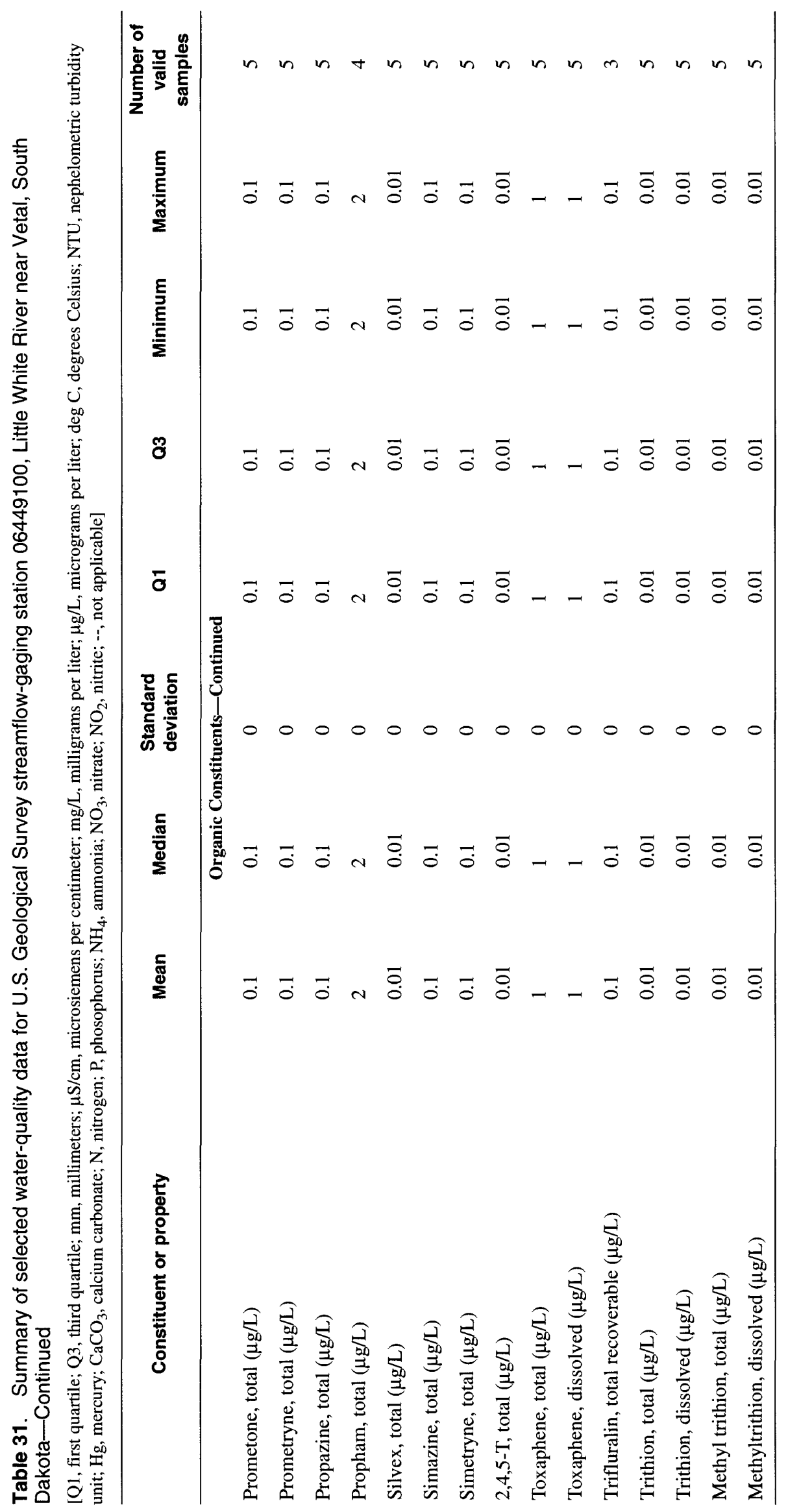




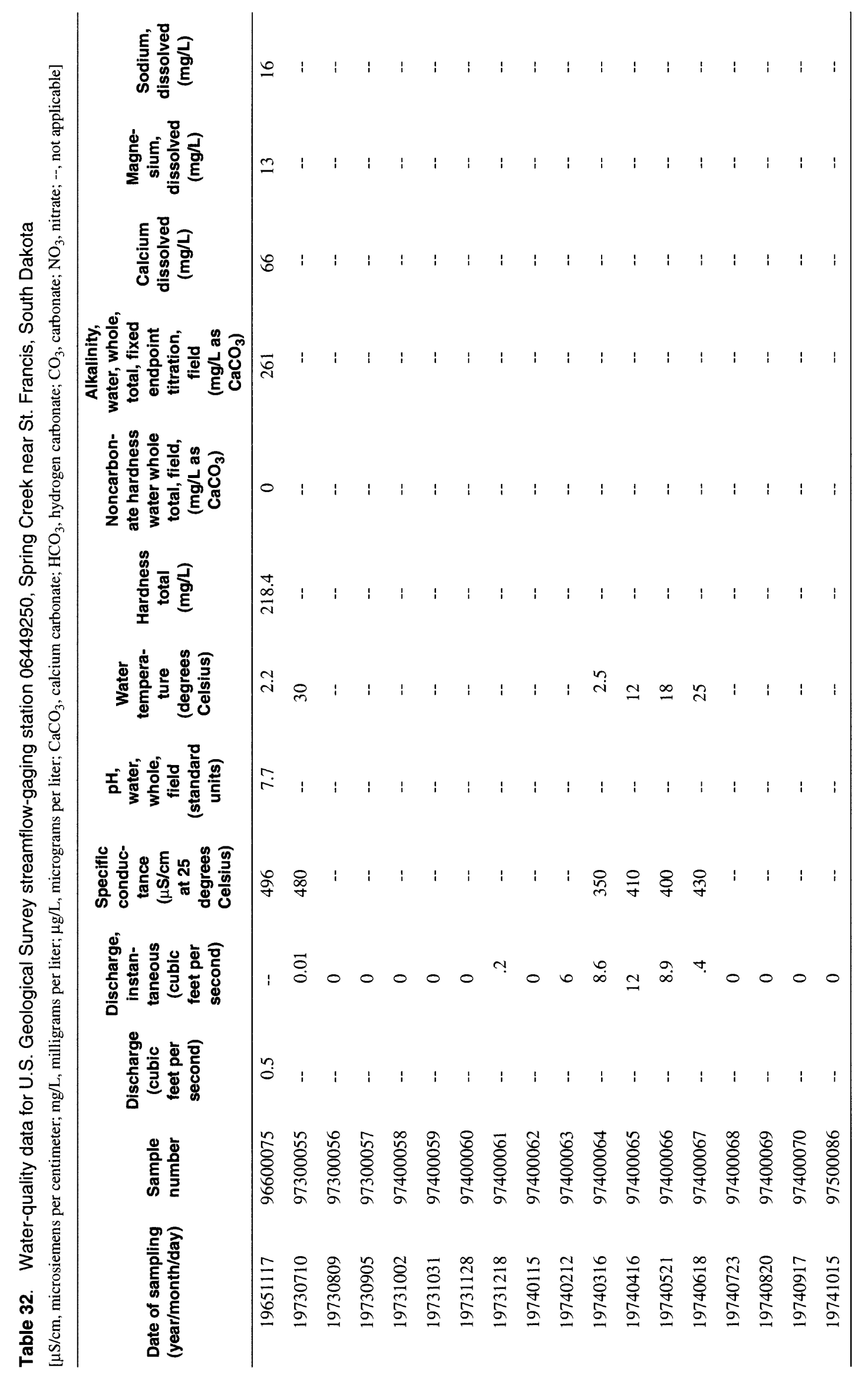




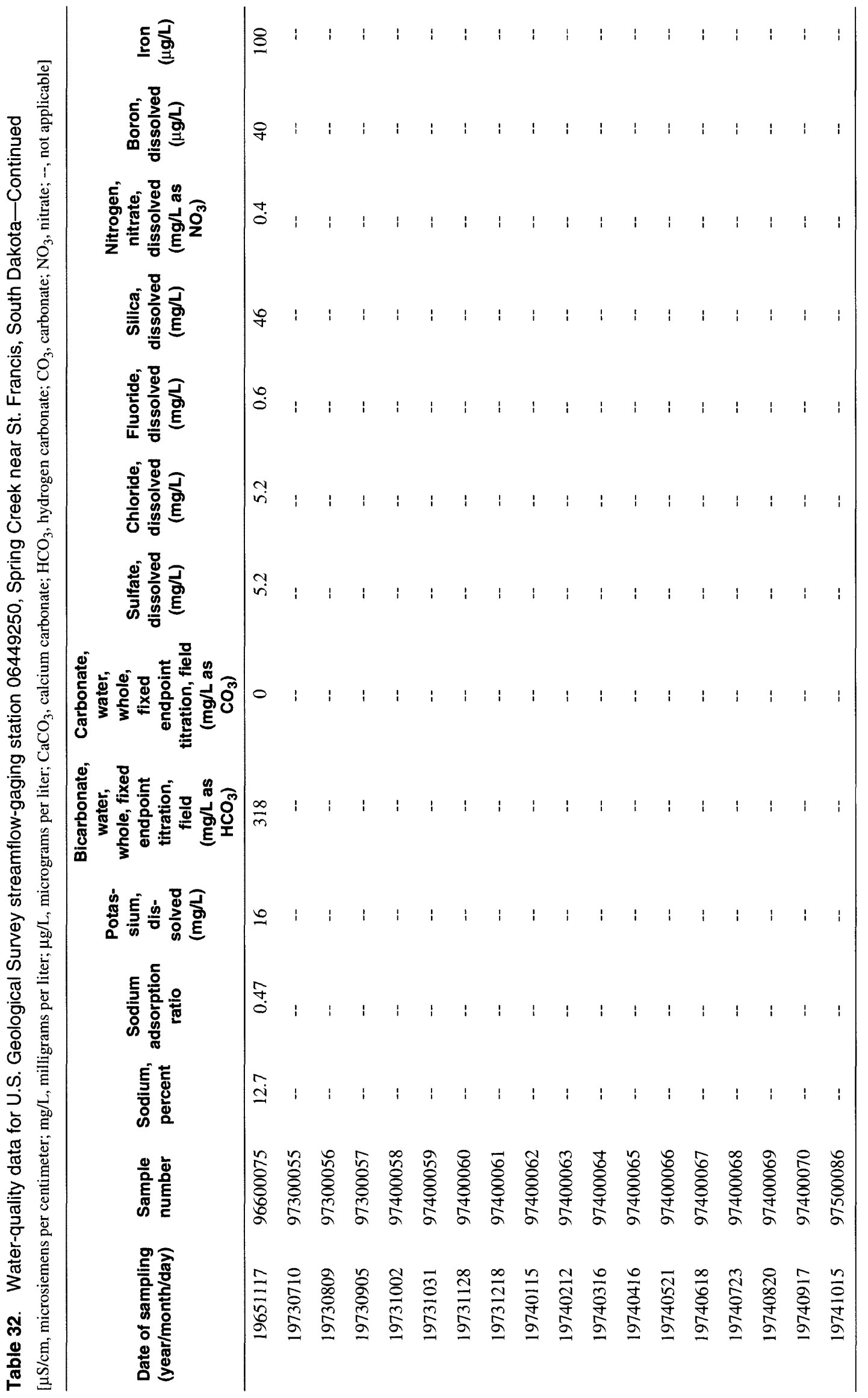




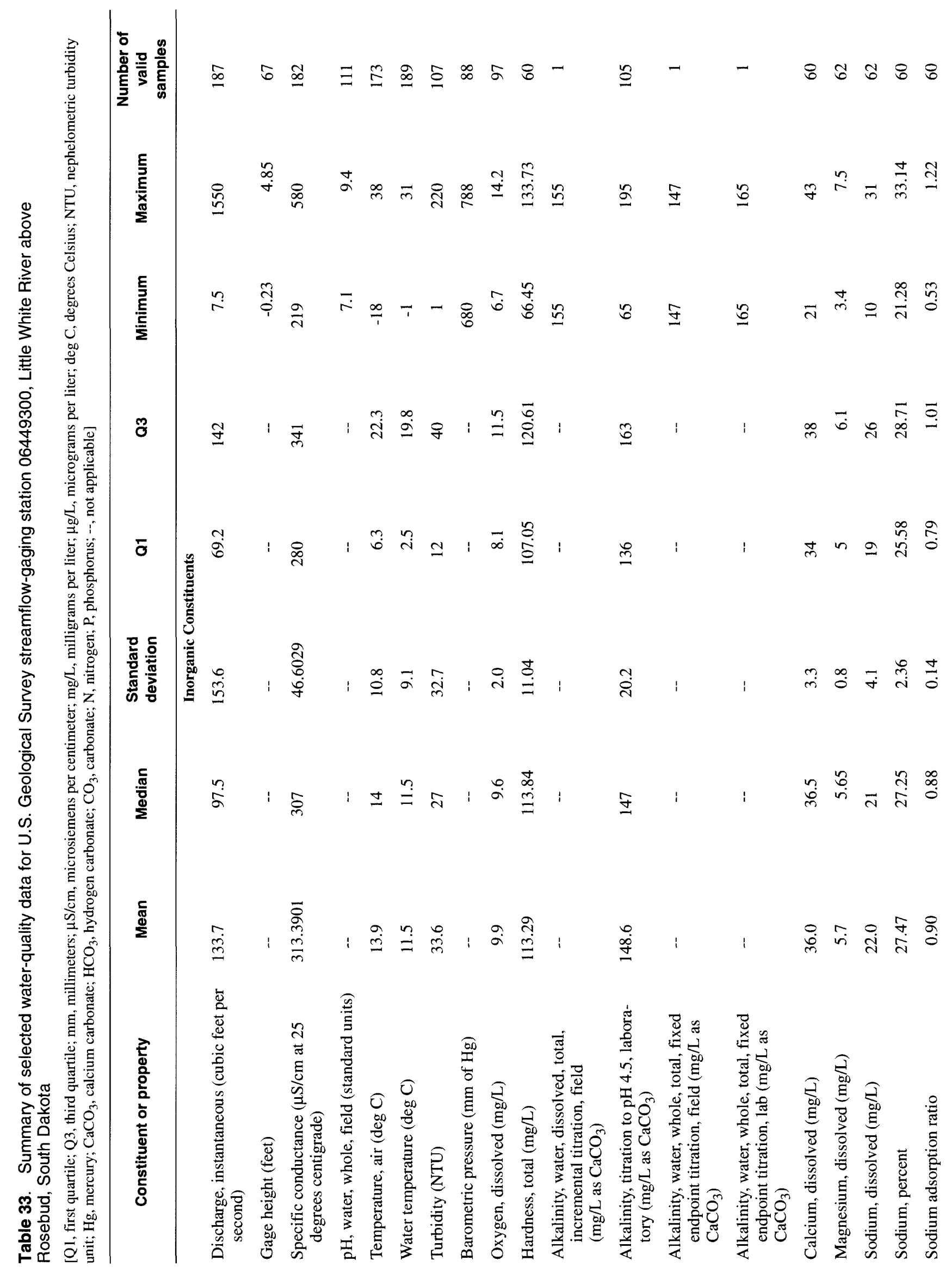




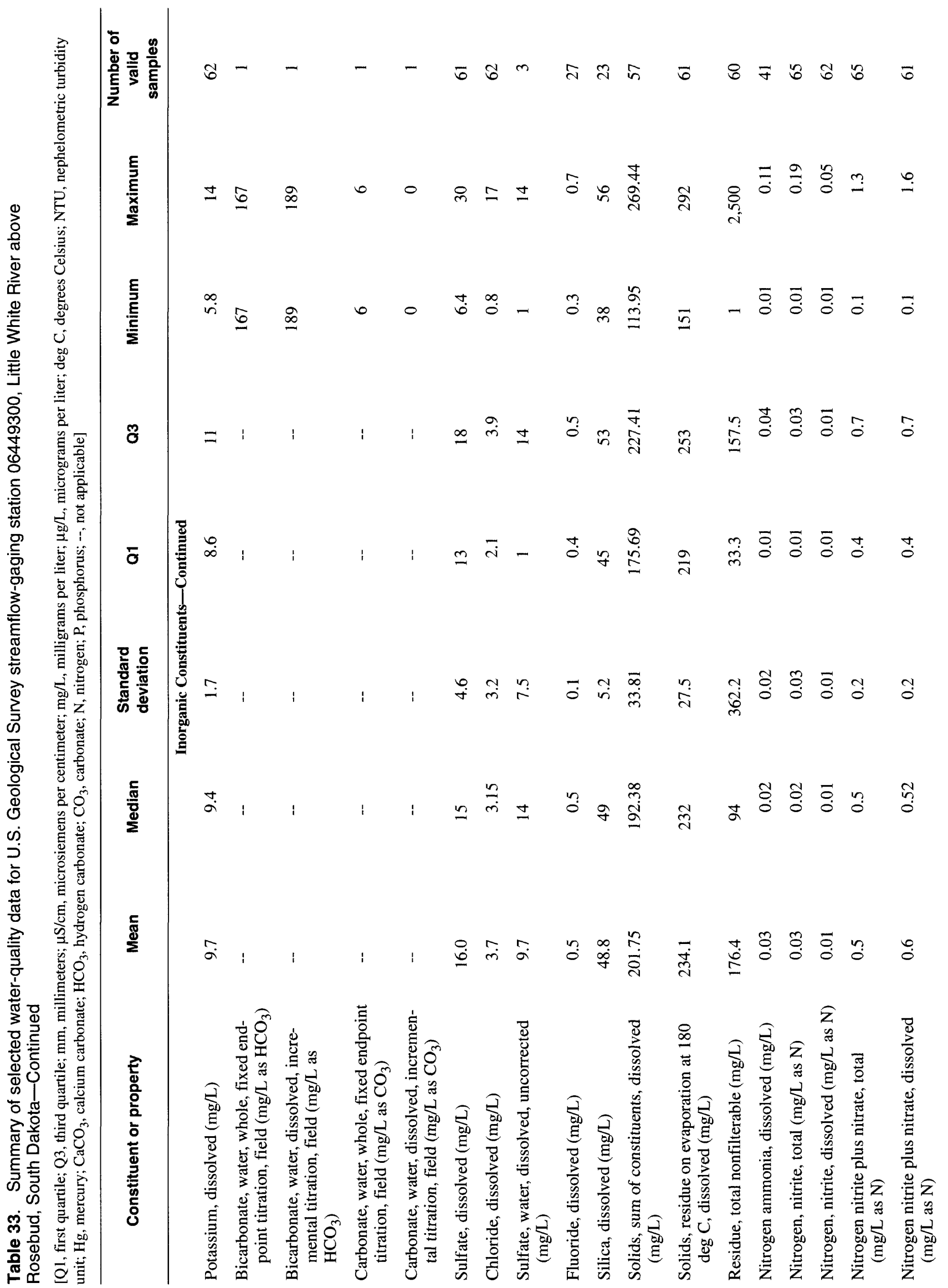




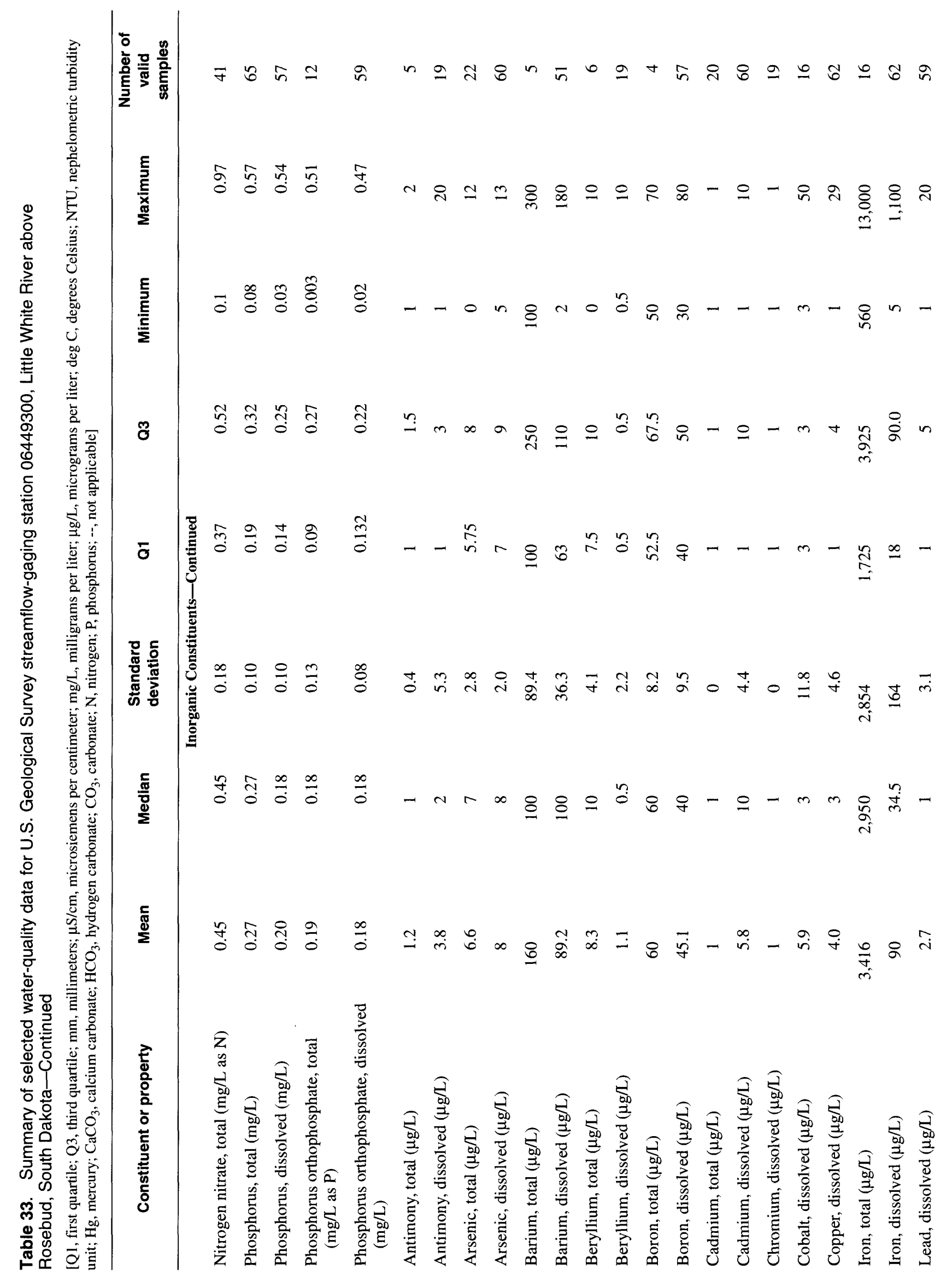




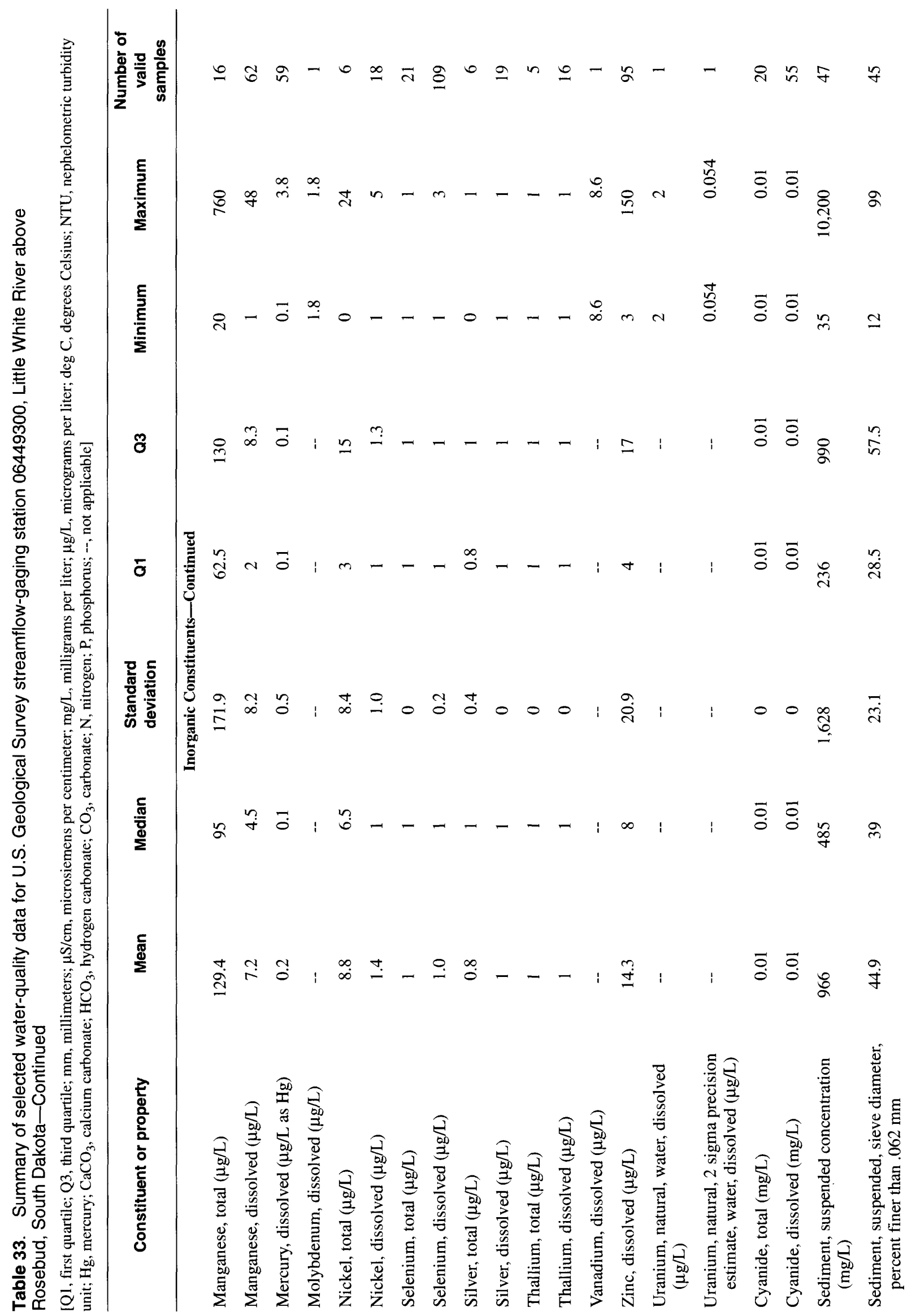




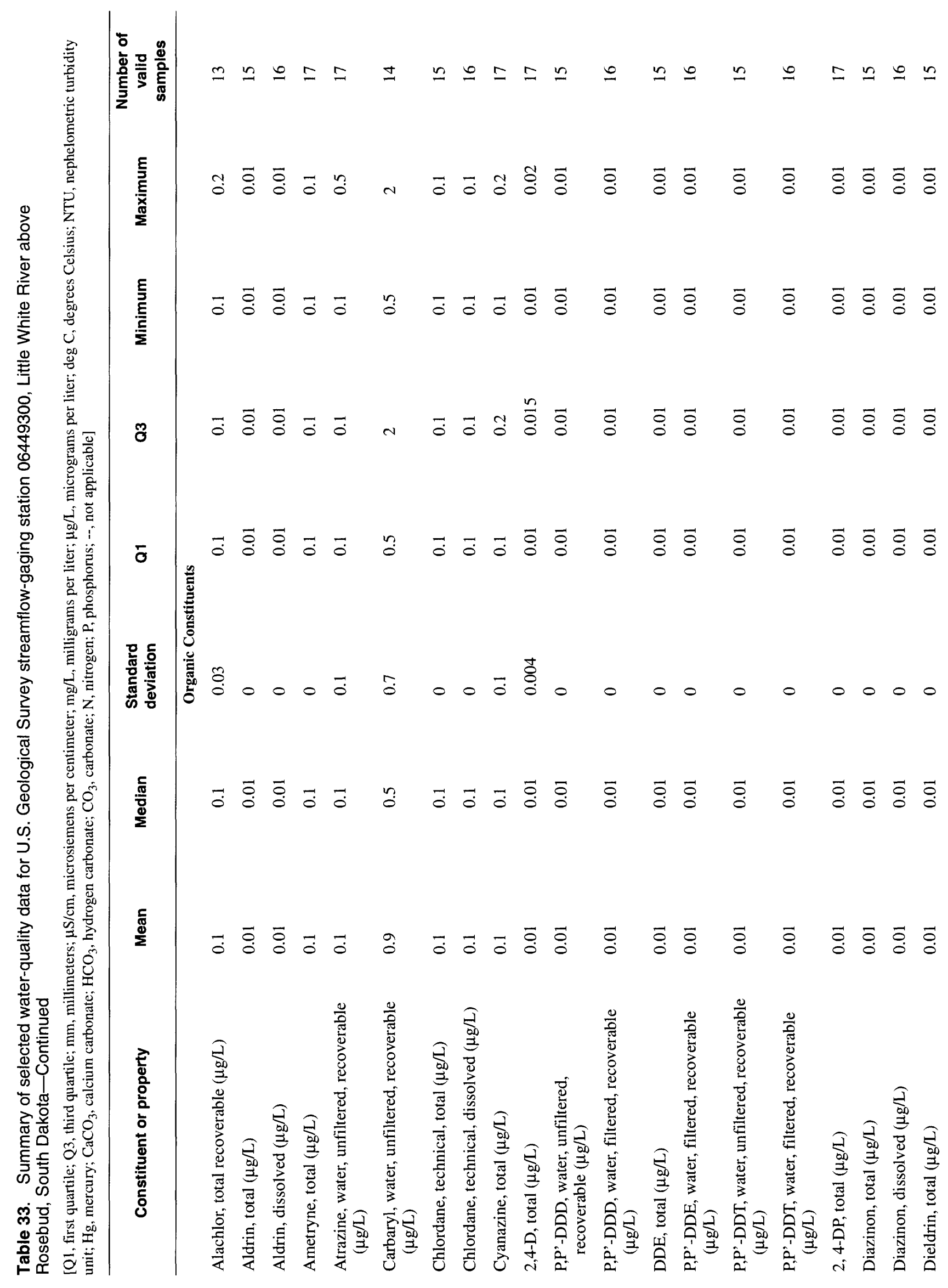




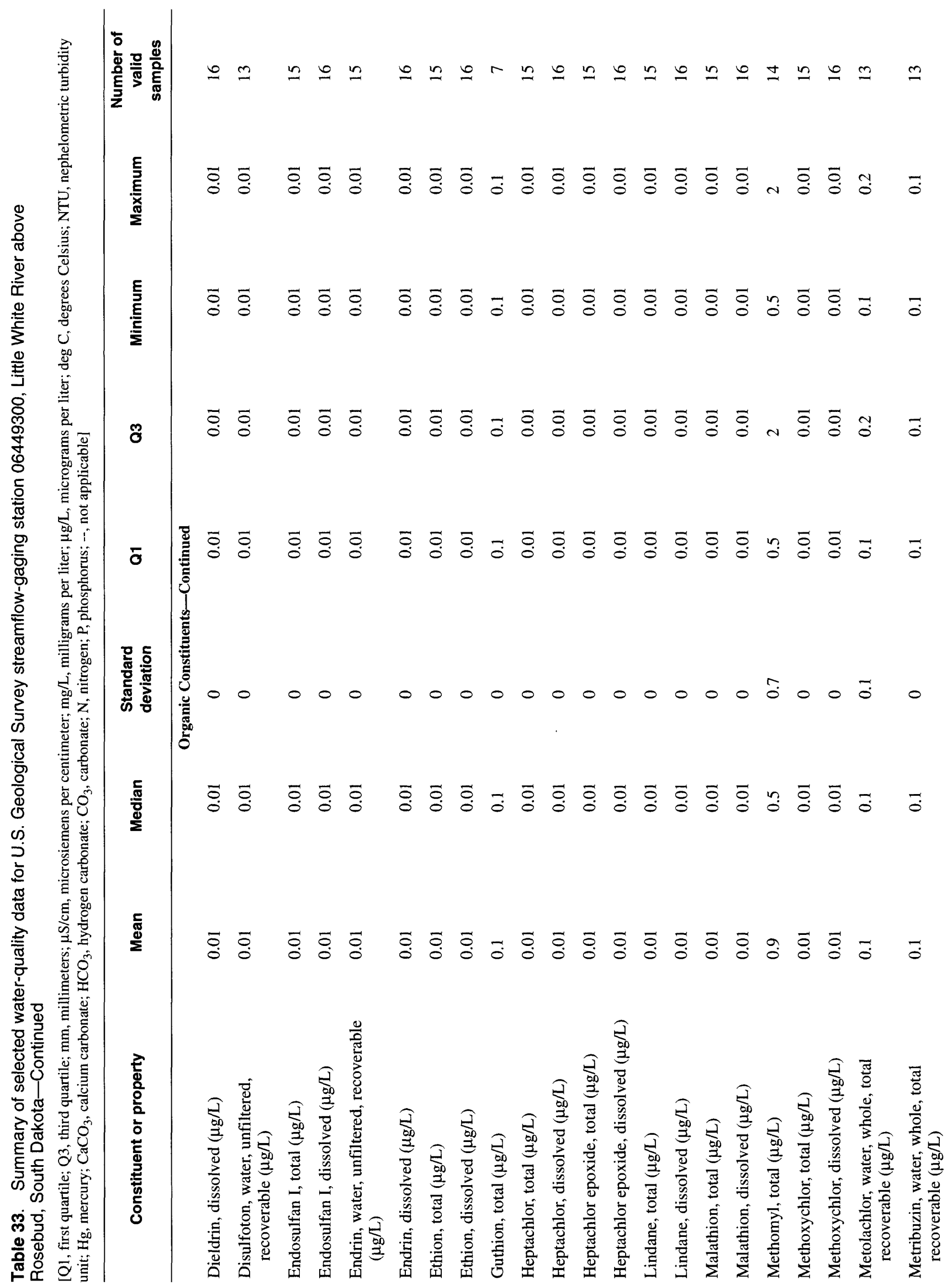




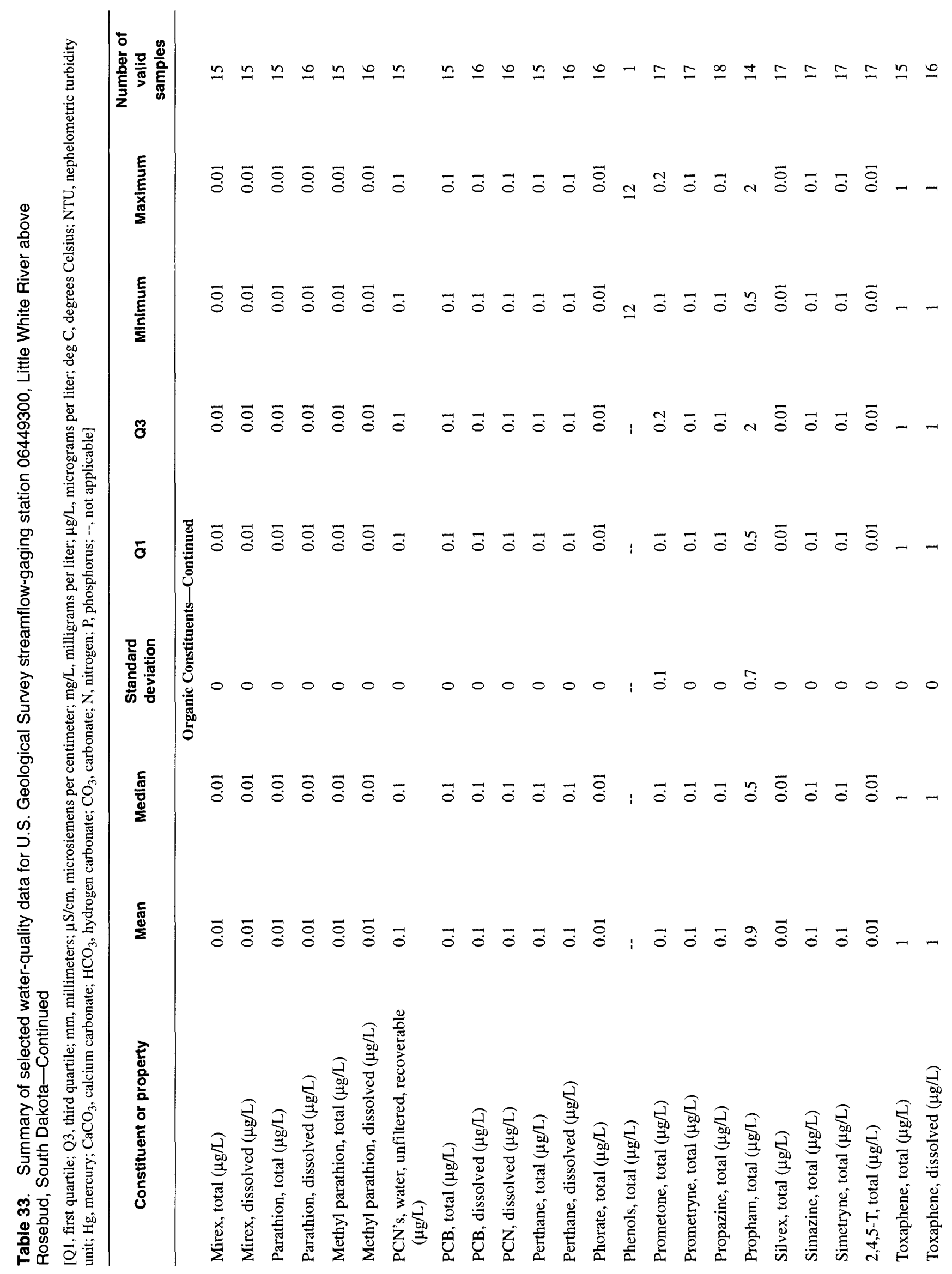




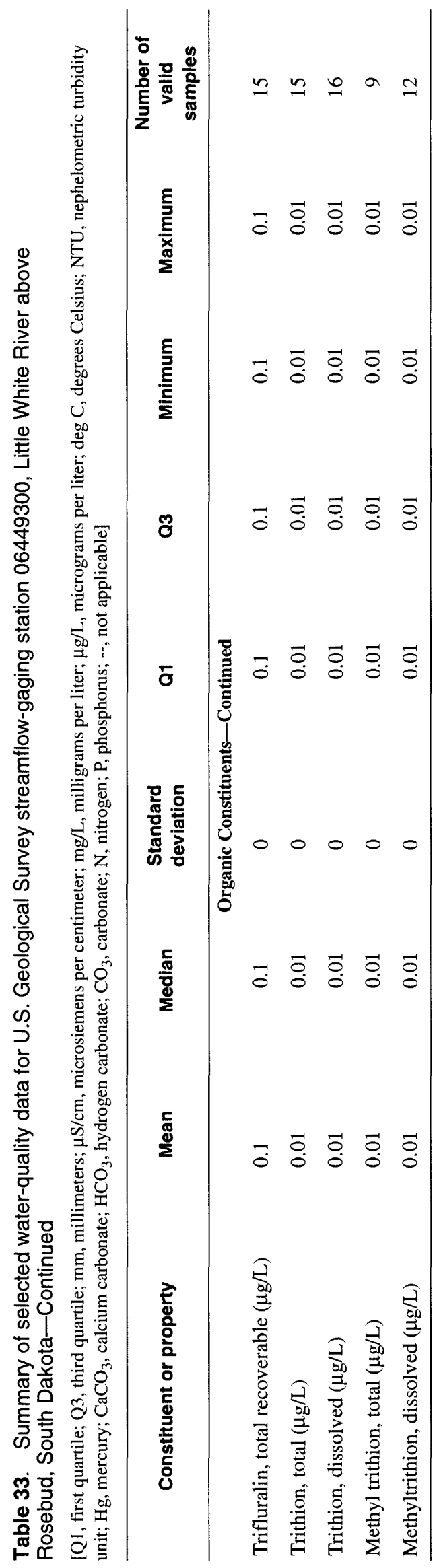


Table 34. Water-quality data for U.S. Geological Survey streamflow-gaging station 06449400 , Rosebud Creek at Rosebud, South Dakota

[ $\mu \mathrm{S} / \mathrm{cm}$, microsiemens per centimeters; --, no data]

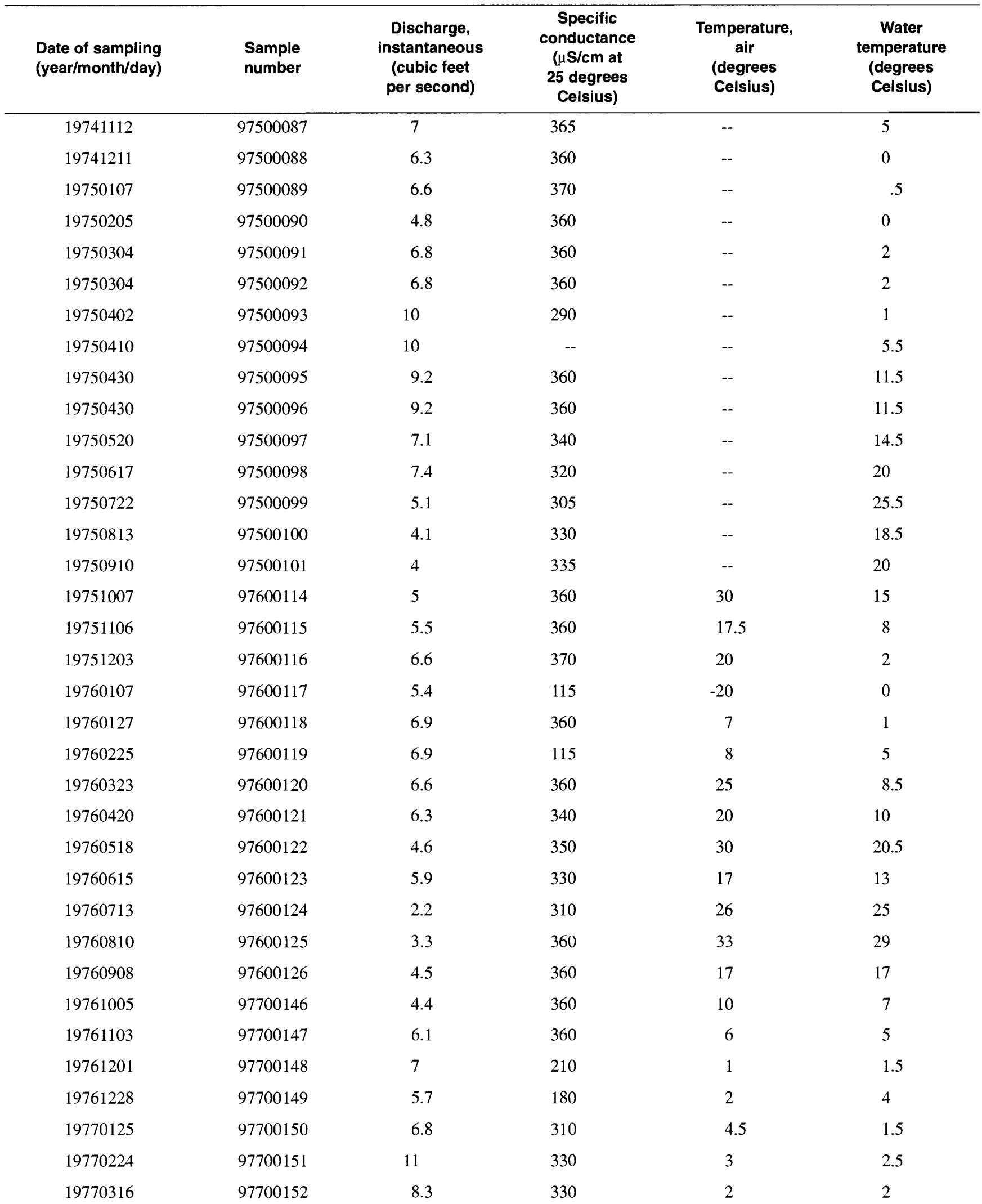


Table 34. Water-quality data for U.S. Geological Survey streamflow-gaging station 06449400 , Rosebud Creek at Rosebud, South Dakota-Continued

$[\mu \mathrm{S} / \mathrm{cm}$, microsiemens per centimeters; --, no data]

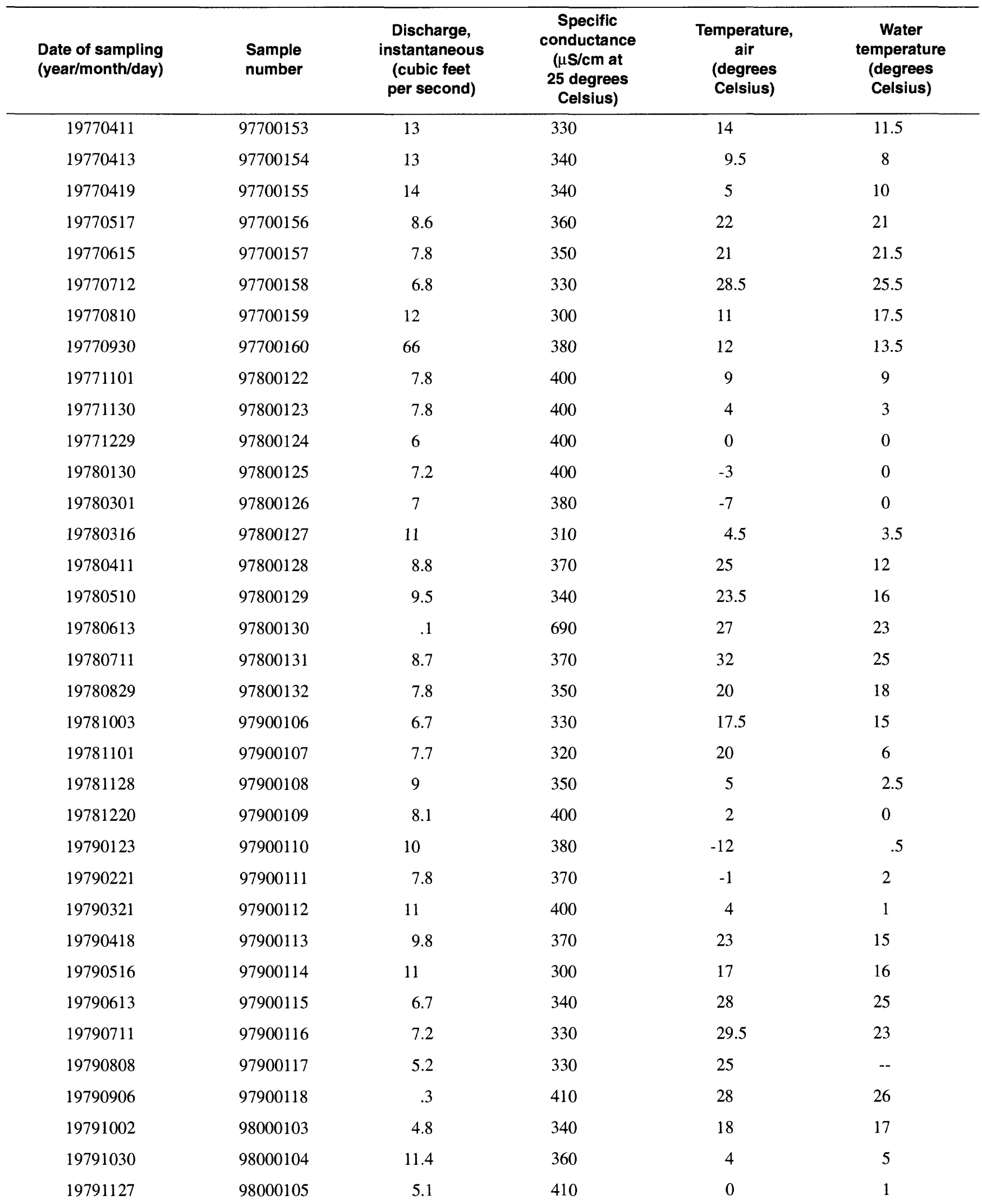


Table 34. Water-quality data for U.S. Geological Survey streamflow-gaging station 06449400 , Rosebud Creek at Rosebud, South Dakota-Continued

$[\mu \mathrm{S} / \mathrm{cm}$, microsiemens per centimeters; --, no data]

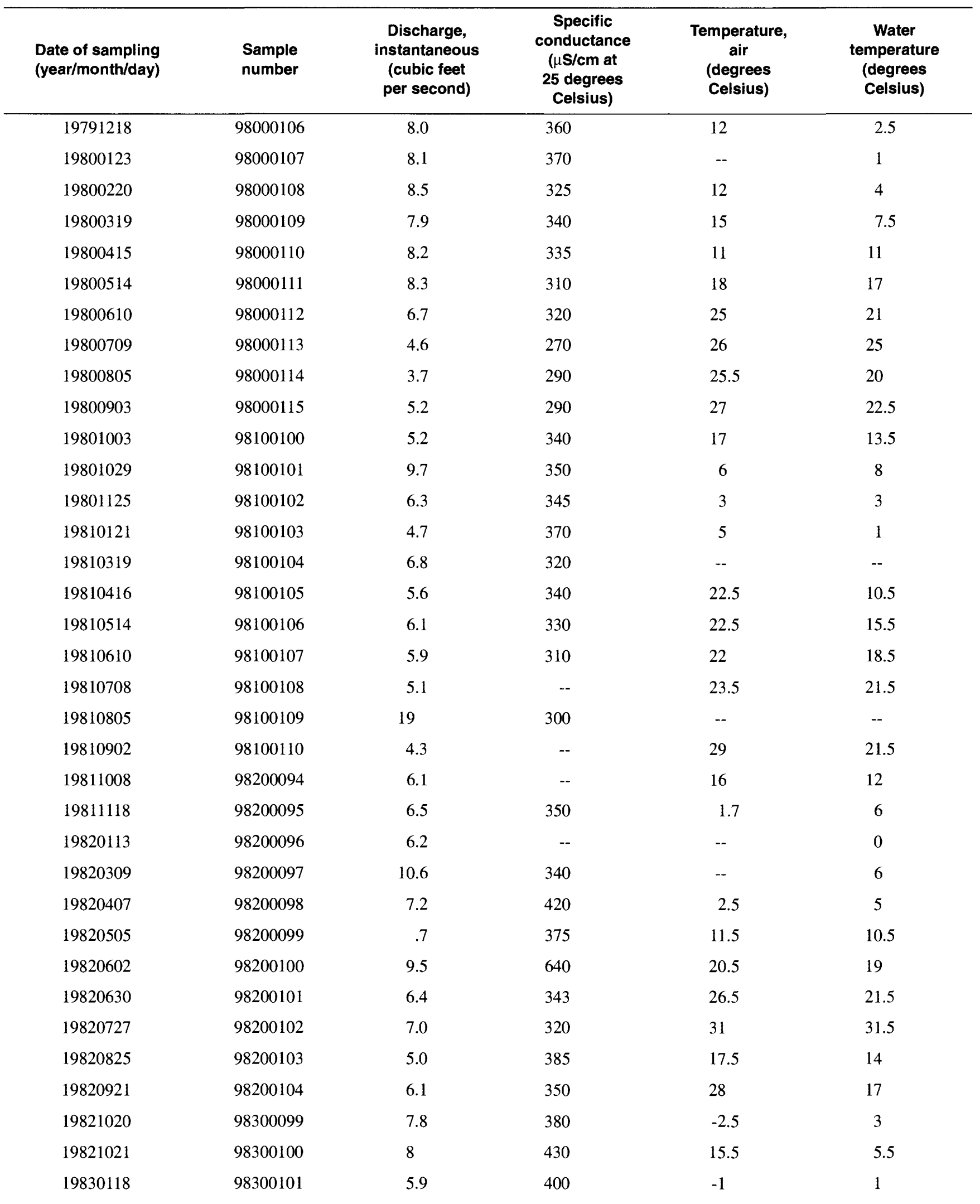


Table 34. Water-quality data for U.S. Geological Survey streamflow-gaging station 06449400 , Rosebud Creek at Rosebud, South Dakota-Continued

$[\mu \mathrm{S} / \mathrm{cm}$, microsiemens per centimeters; --, no data]

\begin{tabular}{|c|c|c|c|c|c|}
\hline $\begin{array}{l}\text { Date of sampling } \\
\text { (year/month/day) }\end{array}$ & $\begin{array}{l}\text { Sample } \\
\text { number }\end{array}$ & $\begin{array}{l}\text { Discharge, } \\
\text { instantaneous } \\
\text { (cubic feet } \\
\text { per second) }\end{array}$ & $\begin{array}{c}\text { Specific } \\
\text { conductance } \\
(\mu \mathrm{S} / \mathrm{cm} \text { at } \\
25 \text { degrees } \\
\text { Celsius) }\end{array}$ & $\begin{array}{c}\text { Temperature, } \\
\text { air } \\
\text { (degrees } \\
\text { Celsius) }\end{array}$ & $\begin{array}{c}\text { Water } \\
\text { temperature } \\
\text { (degrees } \\
\text { Celsius) }\end{array}$ \\
\hline 19830223 & 98300102 & 7 & 340 & 11 & 5 \\
\hline 19830323 & 98300103 & 7.4 & 370 & 0 & 3.5 \\
\hline 19830420 & 98300104 & 7.9 & 360 & 18 & 14 \\
\hline 19830518 & 98300105 & 14.1 & 420 & 9 & 10.5 \\
\hline 19830615 & 98300106 & 7.8 & 340 & 17 & 17 \\
\hline 19830713 & 98300107 & 4.9 & 480 & 24 & 24.5 \\
\hline 19830824 & 98300108 & 5.4 & 340 & 26 & 24.5 \\
\hline 19831005 & 98400113 & 6.6 & 280 & 15 & 13.5 \\
\hline 19831027 & 98400114 & 7.1 & 340 & 18 & 10 \\
\hline 19831222 & 98400115 & 6.5 & 400 & -25 & 0 \\
\hline 19840216 & 98400116 & 7.4 & 350 & 6 & 5 \\
\hline 19840314 & 98400117 & 9.9 & 440 & 13.5 & 8.5 \\
\hline 19840417 & 98400118 & 8.7 & 360 & 16 & 13.5 \\
\hline 19840516 & 98400119 & 8.2 & 340 & 27 & 19.5 \\
\hline 19840612 & 98400120 & 7.8 & 340 & 18 & 17 \\
\hline 19840712 & 98400121 & 8.4 & 320 & 32.5 & 27 \\
\hline 19840807 & 98400122 & 5.8 & 320 & 22 & 23 \\
\hline 19840905 & 98400123 & 5.6 & 330 & 17 & 17 \\
\hline 19841004 & 98501500 & 6.8 & 340 & 23 & 12 \\
\hline 19841031 & 98501501 & 7.3 & 410 & 8 & 5 \\
\hline 19841120 & 98501502 & 9.1 & 340 & 6 & 2 \\
\hline 19850104 & 98501503 & 6.2 & 340 & .5 & 0 \\
\hline 19850122 & 98501504 & 7.4 & 380 & -1.5 & 0 \\
\hline 19850220 & 98501505 & 16 & 210 & 3.5 & 0 \\
\hline 19850319 & 98501506 & 8.4 & 325 & 15 & 7 \\
\hline 19850418 & 98501507 & 7.4 & 360 & 30 & 26 \\
\hline 19850515 & 98501508 & 6.5 & 350 & 12 & 14 \\
\hline 19850613 & 98501509 & 5.9 & 330 & 21 & 18 \\
\hline 19850710 & 98501510 & 3.8 & 318 & 31 & 24 \\
\hline 19850808 & 98501511 & 4.3 & 302 & 31.5 & 25 \\
\hline 19850912 & 98501512 & 7.4 & 345 & 17.5 & 18 \\
\hline 19851009 & 98601637 & 5.9 & 350 & 3 & 5 \\
\hline 19851030 & 98601638 & 6.6 & 347 & 13 & 8.5 \\
\hline 19851205 & 98601639 & 7.9 & -- & 1 & 0 \\
\hline 19860108 & 98601640 & 7.1 & 345 & 3.5 & .5 \\
\hline
\end{tabular}


Table 34. Water-quality data for U.S. Geological Survey streamflow-gaging station 06449400, Rosebud Creek at Rosebud, South Dakota-Continued

$[\mu \mathrm{S} / \mathrm{cm}$, microsiemens per centimeters; --, no data]

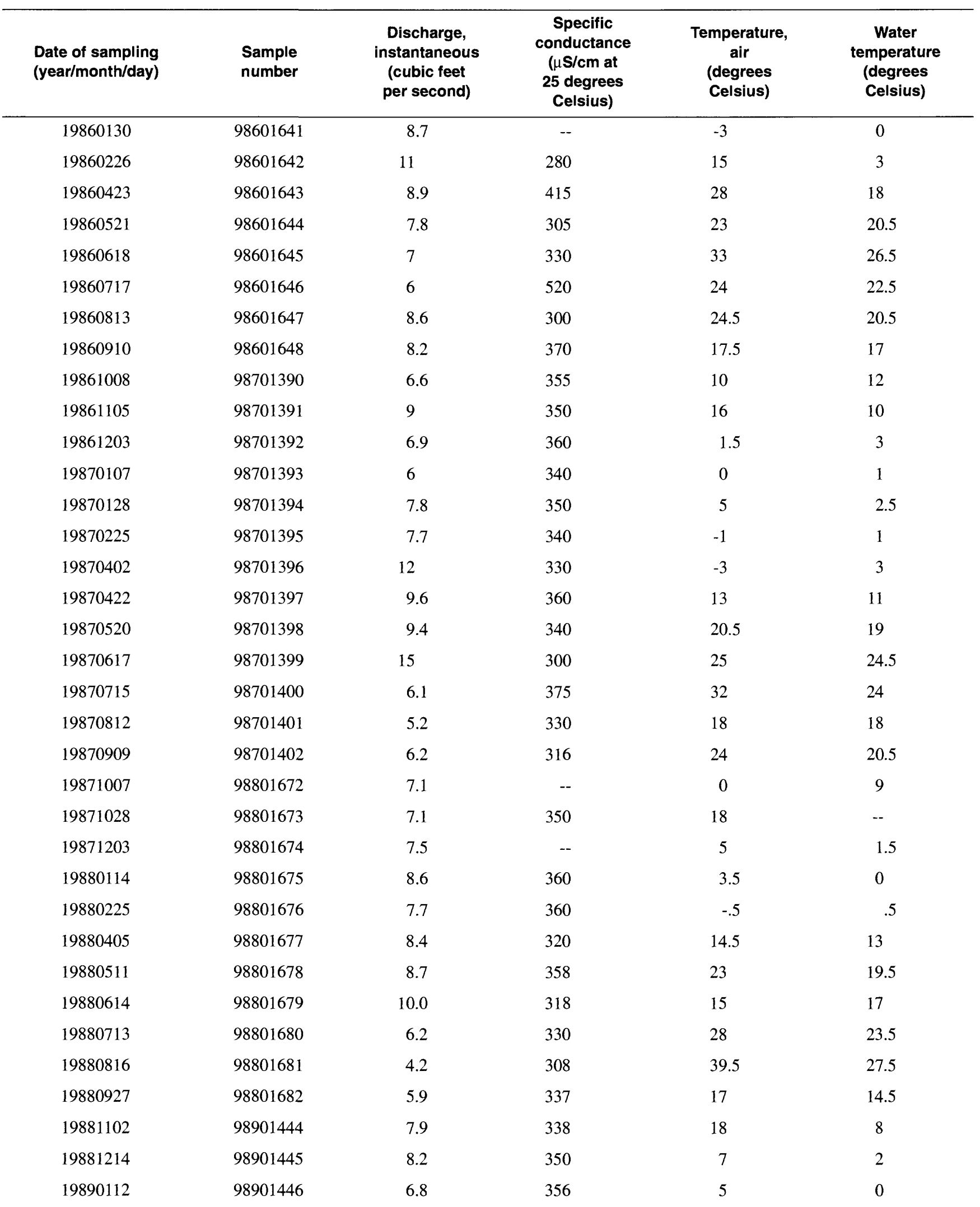


Table 34. Water-quality data for U.S. Geological Survey streamflow-gaging station 06449400, Rosebud Creek at Rosebud, South Dakota-Continued

$[\mu \mathrm{S} / \mathrm{cm}$, microsiemens per centimeters; --, no data]

\begin{tabular}{|c|c|c|c|c|c|}
\hline $\begin{array}{l}\text { Date of sampling } \\
\text { (year/month/day) }\end{array}$ & $\begin{array}{l}\text { Sample } \\
\text { number }\end{array}$ & $\begin{array}{l}\text { Discharge, } \\
\text { instantaneous } \\
\text { (cubic feet } \\
\text { per second) }\end{array}$ & $\begin{array}{c}\text { Specific } \\
\text { conductance } \\
(\mu \mathrm{S} / \mathrm{cm} \text { at } \\
25 \text { degrees } \\
\text { Celsius })\end{array}$ & $\begin{array}{c}\text { Temperature, } \\
\text { air } \\
\text { (degrees } \\
\text { Celsius) }\end{array}$ & $\begin{array}{c}\text { Water } \\
\text { temperature } \\
\text { (degrees } \\
\text { Celsius) }\end{array}$ \\
\hline 19890216 & 98901447 & 7.4 & 338 & 11 & 0 \\
\hline 19890330 & 98901448 & 7.8 & 337 & 2 & 6.5 \\
\hline 19890427 & 98901449 & 8.5 & 339 & 2.5 & 12 \\
\hline 19890524 & 98901450 & 6.9 & 342 & 18 & 21.5 \\
\hline 19890621 & 98901451 & 4.8 & 312 & 16 & 19.5 \\
\hline 19890725 & 98901452 & 4.8 & 299 & 32 & 23.5 \\
\hline 19890914 & 98901453 & 5.9 & 337 & 23 & 16 \\
\hline 19891018 & 99001145 & 6.3 & 354 & 4.5 & 8 \\
\hline 19891205 & 99001146 & 7.4 & 355 & 8 & 5 \\
\hline 19900122 & 99001147 & 6.9 & 355 & 9.5 & 3.5 \\
\hline 19900227 & 99001148 & 7.4 & 334 & 3 & 6 \\
\hline 19900404 & 99001149 & 7 & 322 & 9.5 & 10 \\
\hline 19900501 & 99001150 & 7.0 & 348 & 14.5 & 12 \\
\hline 19900612 & 99001151 & 6.8 & 343 & 20.5 & 20 \\
\hline 19900711 & 99001152 & 15.7 & 284 & 24 & 19 \\
\hline 19900905 & 99001397 & 4.8 & 324 & 34.5 & 26 \\
\hline 19901010 & 99101294 & 6.5 & 353 & 18.5 & 10.5 \\
\hline 19901205 & 99101295 & 6.5 & 364 & 9 & 3.5 \\
\hline 19910109 & 99101296 & 7.1 & 364 & -- & 0 \\
\hline 19910225 & 99101297 & 9.6 & 355 & 0 & 3 \\
\hline 19910410 & 99101298 & 7.3 & 332 & 17 & 11 \\
\hline 19910508 & 99101299 & 7.9 & 321 & 21 & 15 \\
\hline 19910611 & 99101300 & 7.7 & 344 & 28.5 & 25 \\
\hline 19910716 & 99101301 & 4.8 & 355 & 31.5 & 23 \\
\hline 19910821 & 99101302 & 4.6 & 324 & 29.5 & 24.5 \\
\hline 19911002 & 99201066 & 5.9 & 351 & 16 & 17 \\
\hline 19911114 & 99201067 & 8.2 & 357 & 3 & 4 \\
\hline 19920109 & 99201068 & 7.6 & 356 & -6 & 0 \\
\hline 19920220 & 99201069 & 7.2 & 352 & 0 & 2 \\
\hline 19920331 & 99201070 & 6.7 & 340 & 9 & 9 \\
\hline 19920513 & 99201071 & 5.9 & 323 & 10 & 15 \\
\hline 19920608 & 99201072 & 7.9 & 355 & 18 & 17 \\
\hline 19920709 & 99201073 & 6.5 & 331 & 22 & 22.5 \\
\hline 19920818 & 99201074 & 5.8 & 330 & 25.5 & 23 \\
\hline 19921005 & 99301715 & 9 & 385 & 15 & 15 \\
\hline
\end{tabular}


Table 34. Water-quality data for U.S. Geological Survey streamflow-gaging station 06449400, Rosebud Creek at Rosebud, South Dakota-Continued

$[\mu \mathrm{S} / \mathrm{cm}$, microsiemens per centimeters; --, no data]

\begin{tabular}{|c|c|c|c|c|c|}
\hline $\begin{array}{l}\text { Date of sampling } \\
\text { (year/month/day) }\end{array}$ & $\begin{array}{l}\text { Sample } \\
\text { number }\end{array}$ & $\begin{array}{c}\text { Discharge, } \\
\text { instantaneous } \\
\text { (cubic feet } \\
\text { per second) }\end{array}$ & $\begin{array}{c}\text { Specific } \\
\text { conductance } \\
(\mu \mathrm{S} / \mathrm{cm} \text { at } \\
25 \text { degrees } \\
\text { Celsius })\end{array}$ & $\begin{array}{c}\text { Temperature, } \\
\text { air } \\
\text { (degrees } \\
\text { Celsius) }\end{array}$ & $\begin{array}{c}\text { Water } \\
\text { temperature } \\
\text { (degrees } \\
\text { Celsius) }\end{array}$ \\
\hline 19921119 & 99301716 & 9.2 & 370 & 5 & 4 \\
\hline 19930106 & 99301717 & 6.0 & 373 & -5 & .5 \\
\hline 19930303 & 99301718 & 11.7 & 380 & 2 & 0 \\
\hline 19930406 & 99301719 & 8.4 & 373 & 6 & 5.5 \\
\hline 19930513 & 99301720 & 7.6 & 387 & 21.5 & 16 \\
\hline 19930617 & 99301721 & 30.2 & 249 & 25 & 19 \\
\hline 19930630 & 99301722 & 1.3 & -- & 24 & 21 \\
\hline 19930811 & 99301723 & 5.5 & 346 & 29 & 21 \\
\hline 19930908 & 99301724 & 5.6 & 341 & 25.5 & 19.5 \\
\hline 19931019 & 99400222 & 7.0 & 350 & 11 & 9 \\
\hline 19931201 & 99401670 & 7.3 & 356 & 5.5 & 2 \\
\hline 19931229 & 99401671 & 6.2 & 409 & -3 & 2 \\
\hline 19940216 & 99401672 & 11.8 & 341 & 5 & 3 \\
\hline 19940601 & 99401673 & 8.0 & 321 & 17.5 & 20 \\
\hline 19940628 & 99401674 & 5.5 & 314 & 22.5 & 23.5 \\
\hline 19940810 & 99401675 & 5.8 & 320 & 20 & -- \\
\hline 19941011 & 99502444 & 6.9 & 335 & 24 & 14 \\
\hline 19941114 & 99502445 & 6.5 & 349 & 4.5 & 6 \\
\hline 19950110 & 99502446 & 8.1 & 358 & -4 & 0 \\
\hline 19950221 & 99502447 & 8.3 & 333 & 19.5 & 6.5 \\
\hline 19950413 & 99502448 & 12.6 & 311 & 11.5 & 7.5 \\
\hline 19950414 & 99502449 & 11.4 & 321 & 15.5 & 10.5 \\
\hline 19950424 & 99502450 & 12.1 & 341 & 13.5 & 10.5 \\
\hline 19950511 & 99502451 & 11.2 & 364 & 19.5 & 14.5 \\
\hline 19950626 & 99502452 & 12.1 & 348 & 24 & 19 \\
\hline 19950817 & 99502453 & 6.4 & 330 & 32.5 & 25 \\
\hline 19951017 & 99602046 & 8.1 & 359 & 16.5 & 11.5 \\
\hline 19951204 & 99602047 & 7.8 & 360 & 11.5 & 4 \\
\hline 19960206 & 99602048 & 7.4 & 363 & 7.5 & 0 \\
\hline 19960416 & 99602049 & 8.9 & 359 & 15 & 9 \\
\hline 19960520 & 99602050 & 8.5 & 354 & 19 & 19 \\
\hline 19960718 & 99602052 & 6.5 & 320 & 28 & 26.5 \\
\hline 19960826 & 99602053 & 5.8 & 319 & 20.5 & 21.5 \\
\hline
\end{tabular}




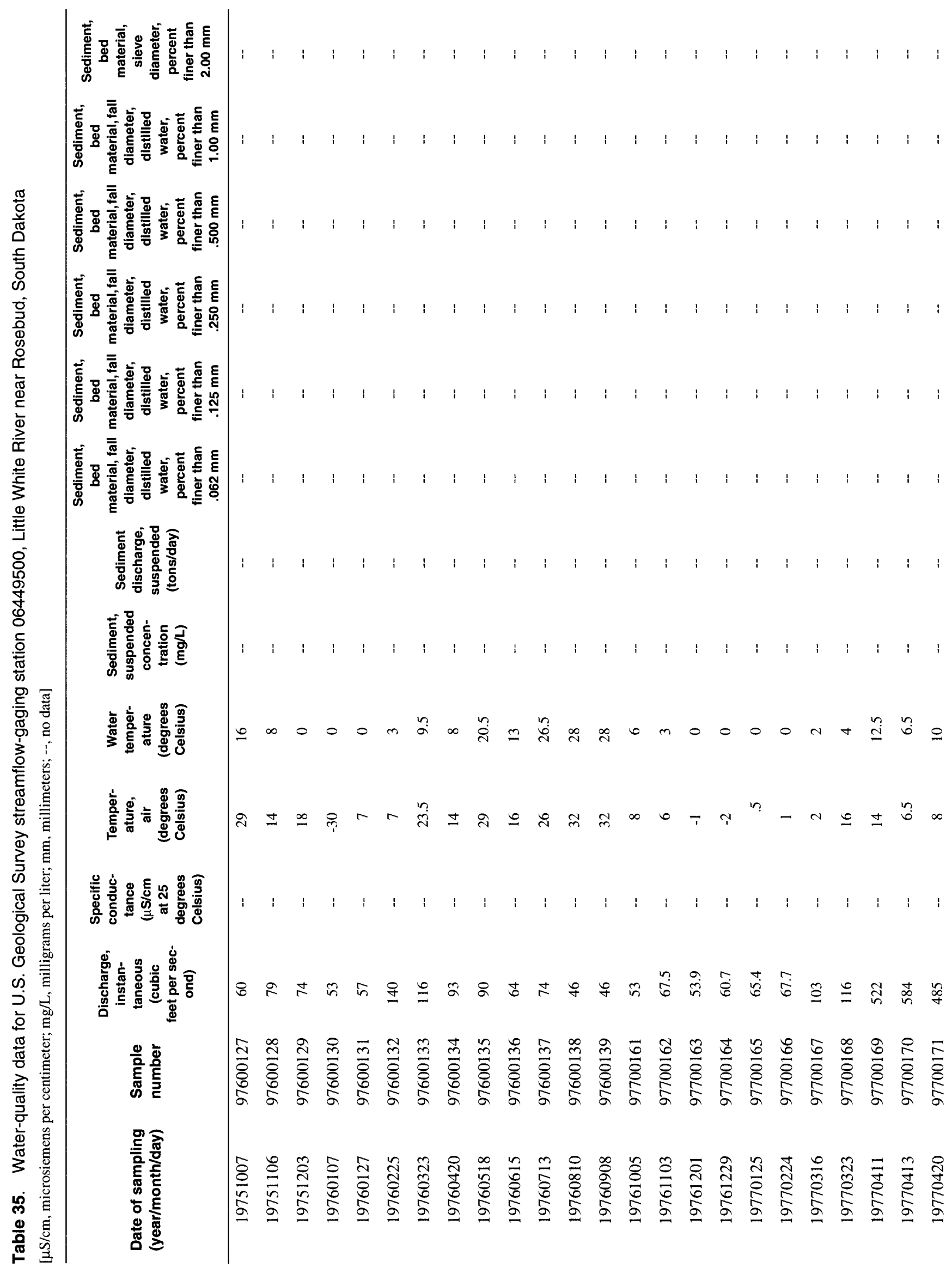




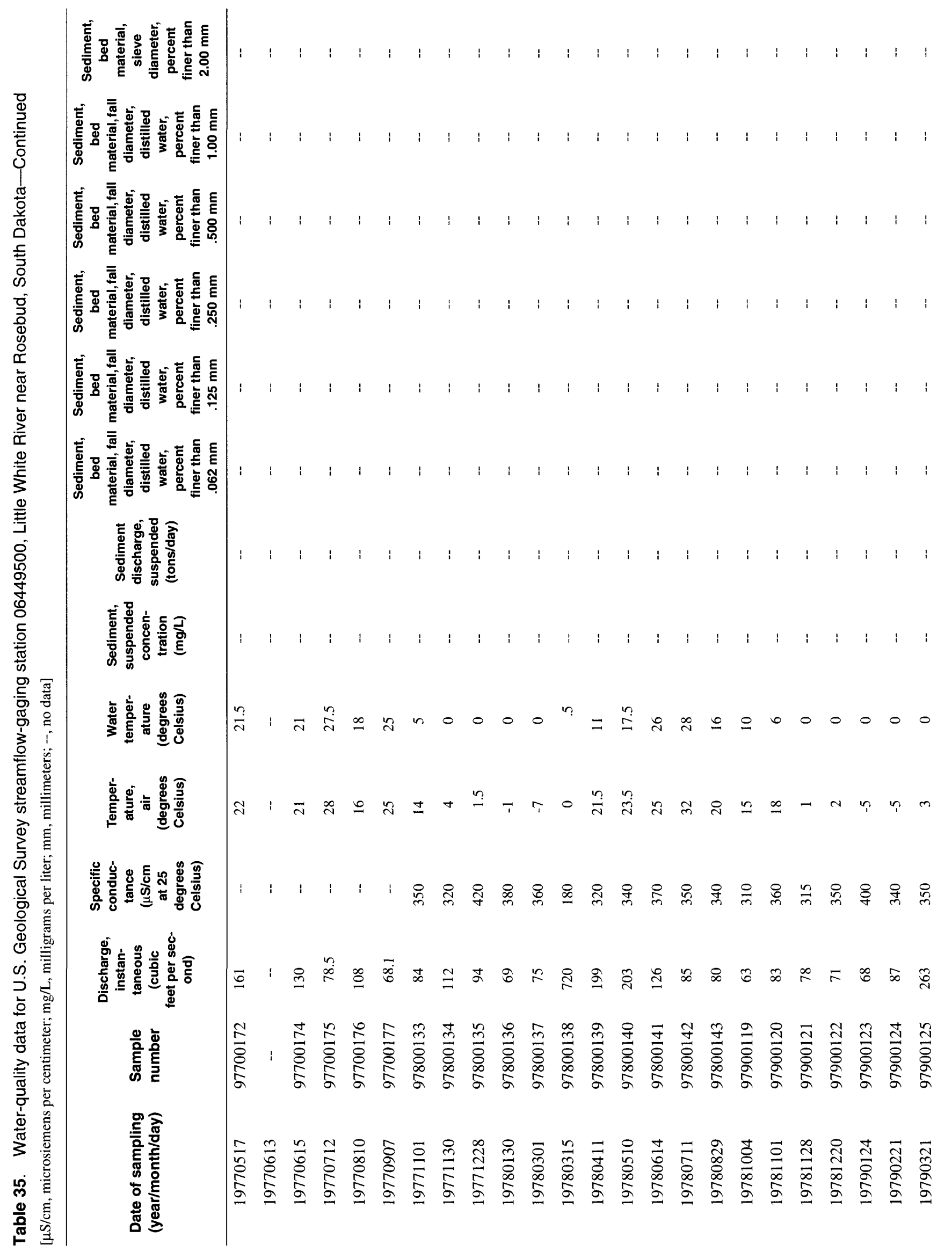




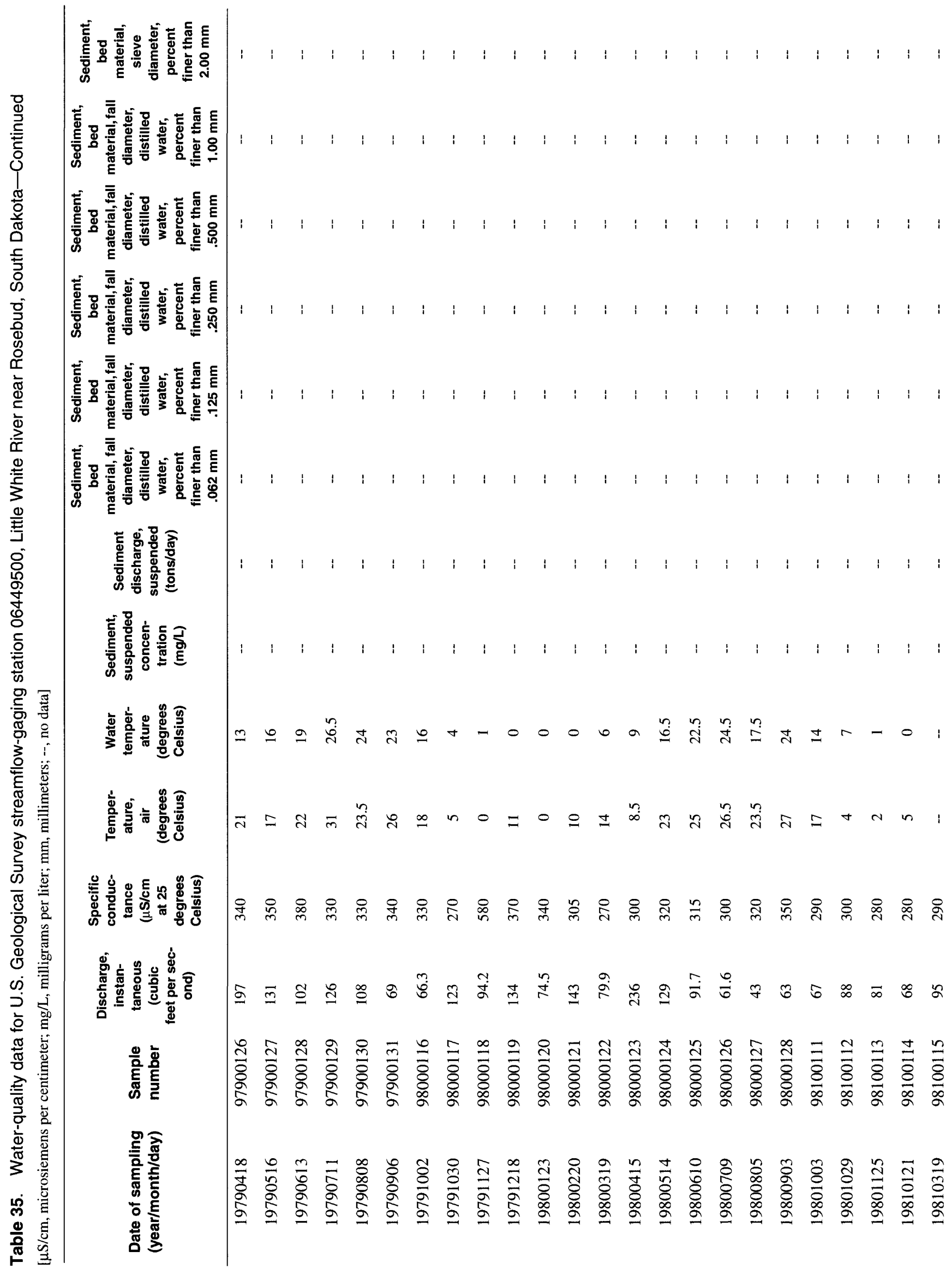




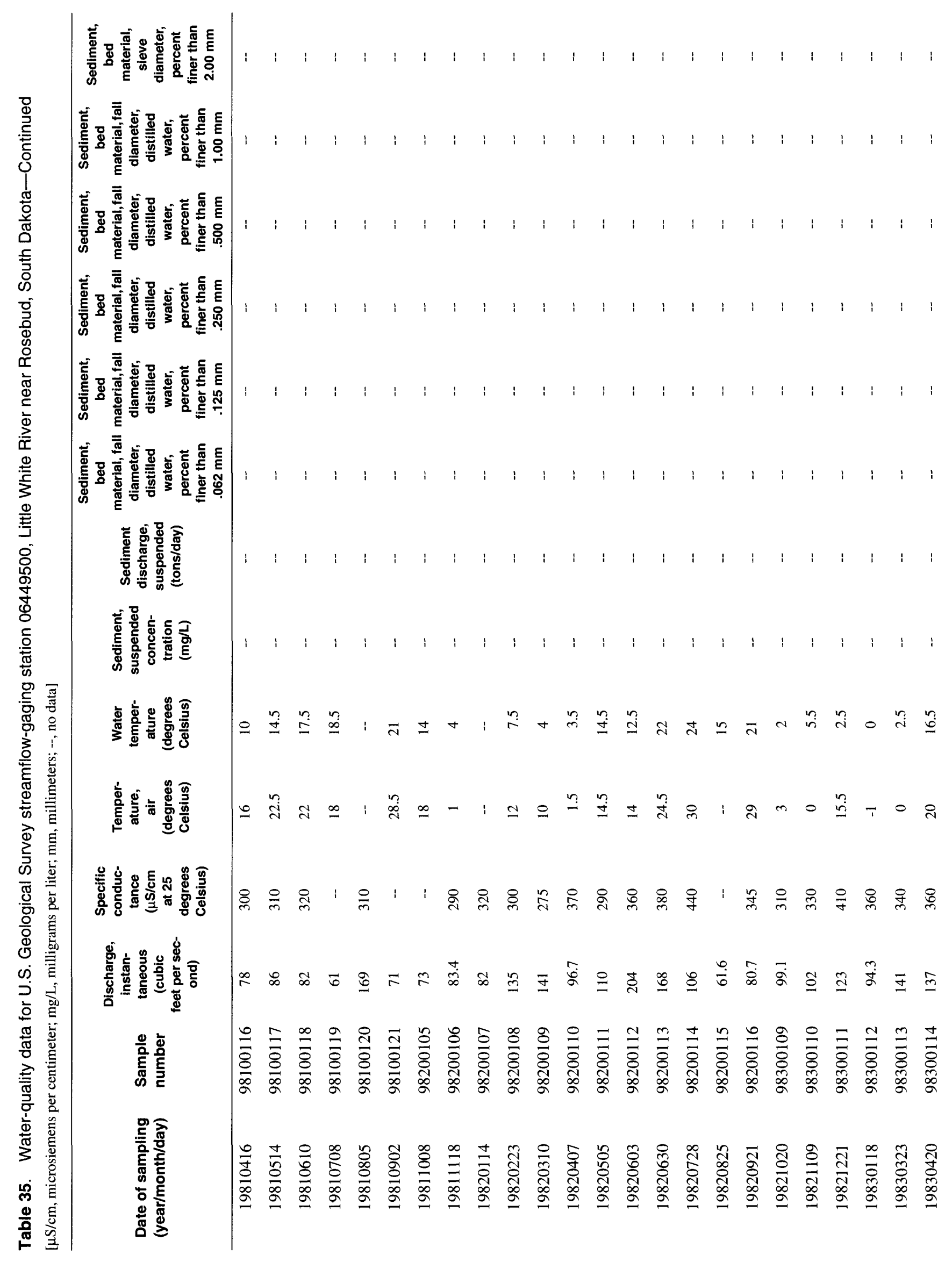




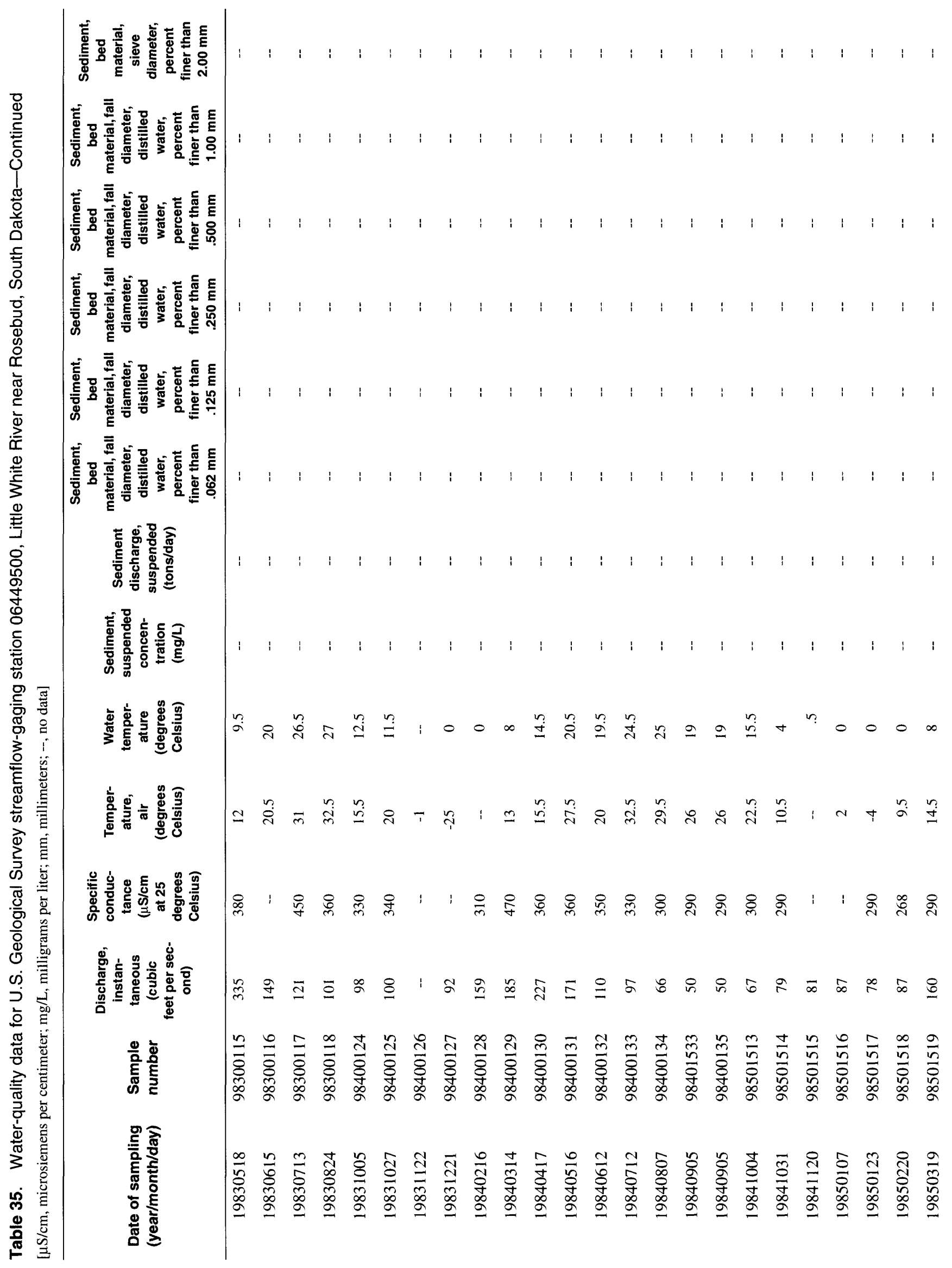


要

은

龸 哥

(⿻)

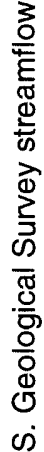

के है

흘

$\frac{\pi}{\pi}$

क्ष

줄

굴

离

.

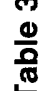

MIn! Inlmin! Fin

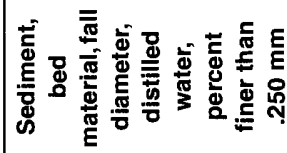

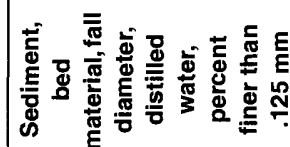

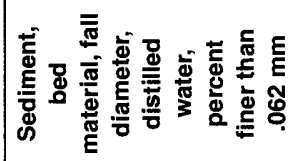

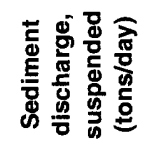

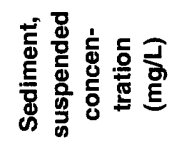

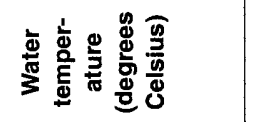

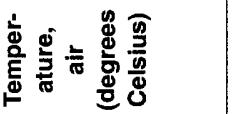

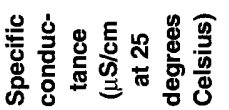

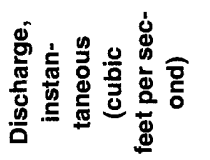
亘高

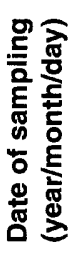

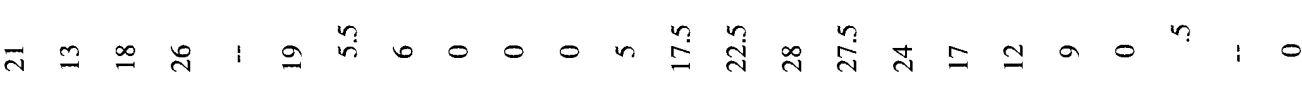

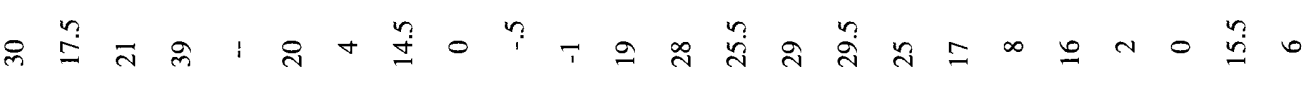

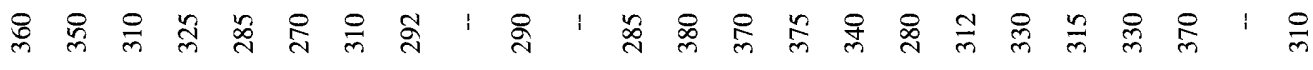

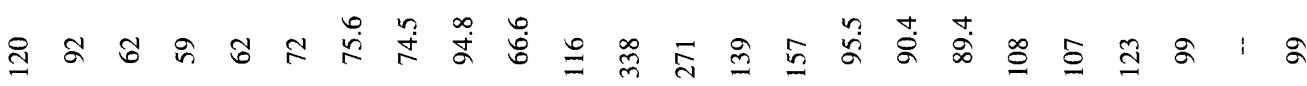

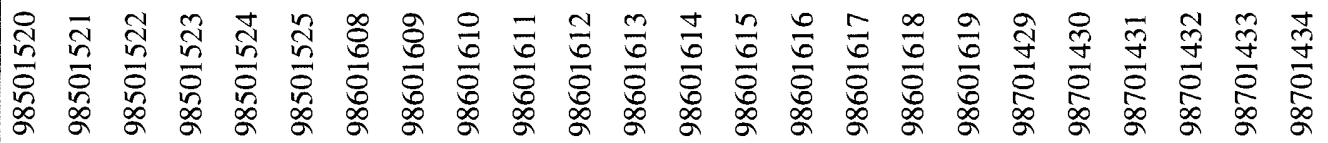

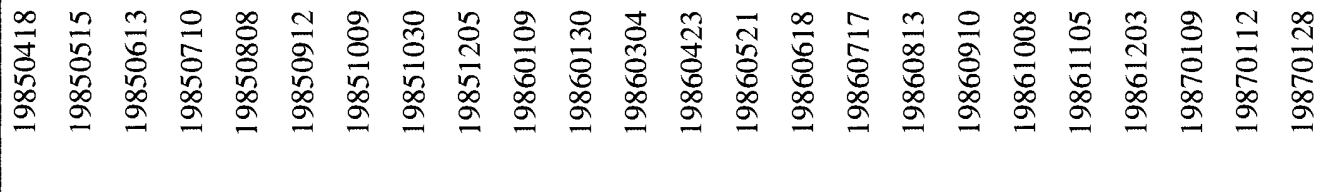




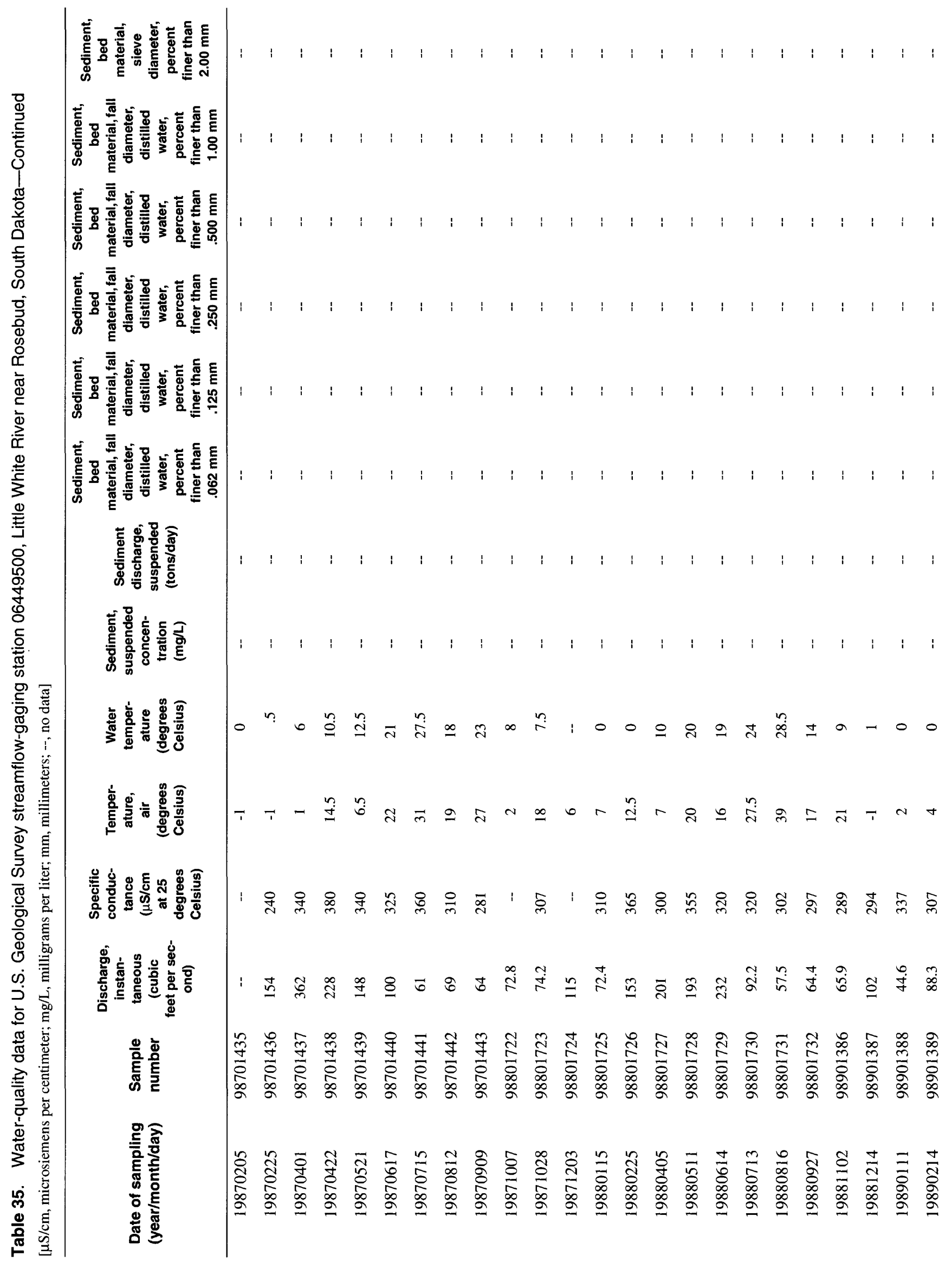




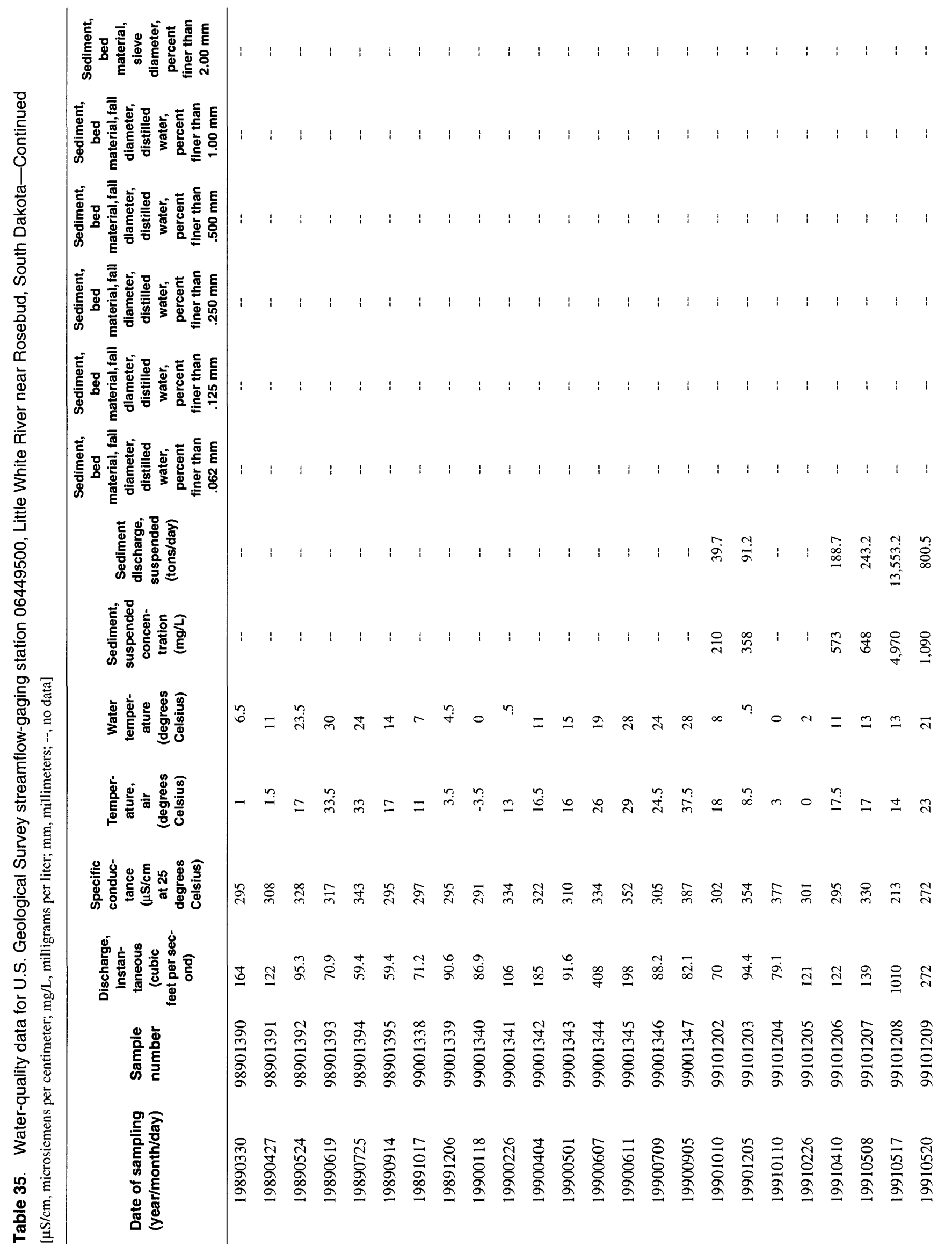




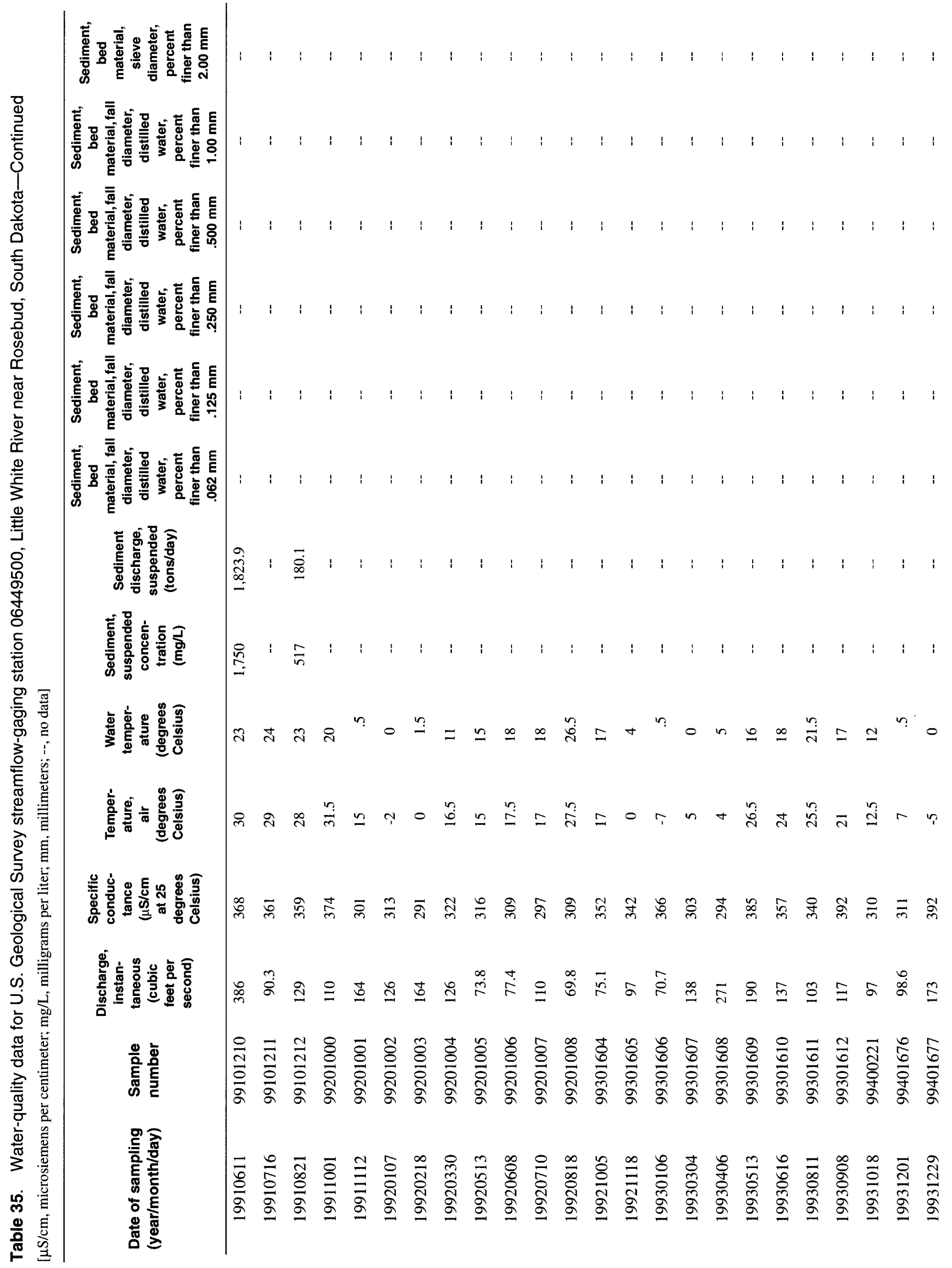




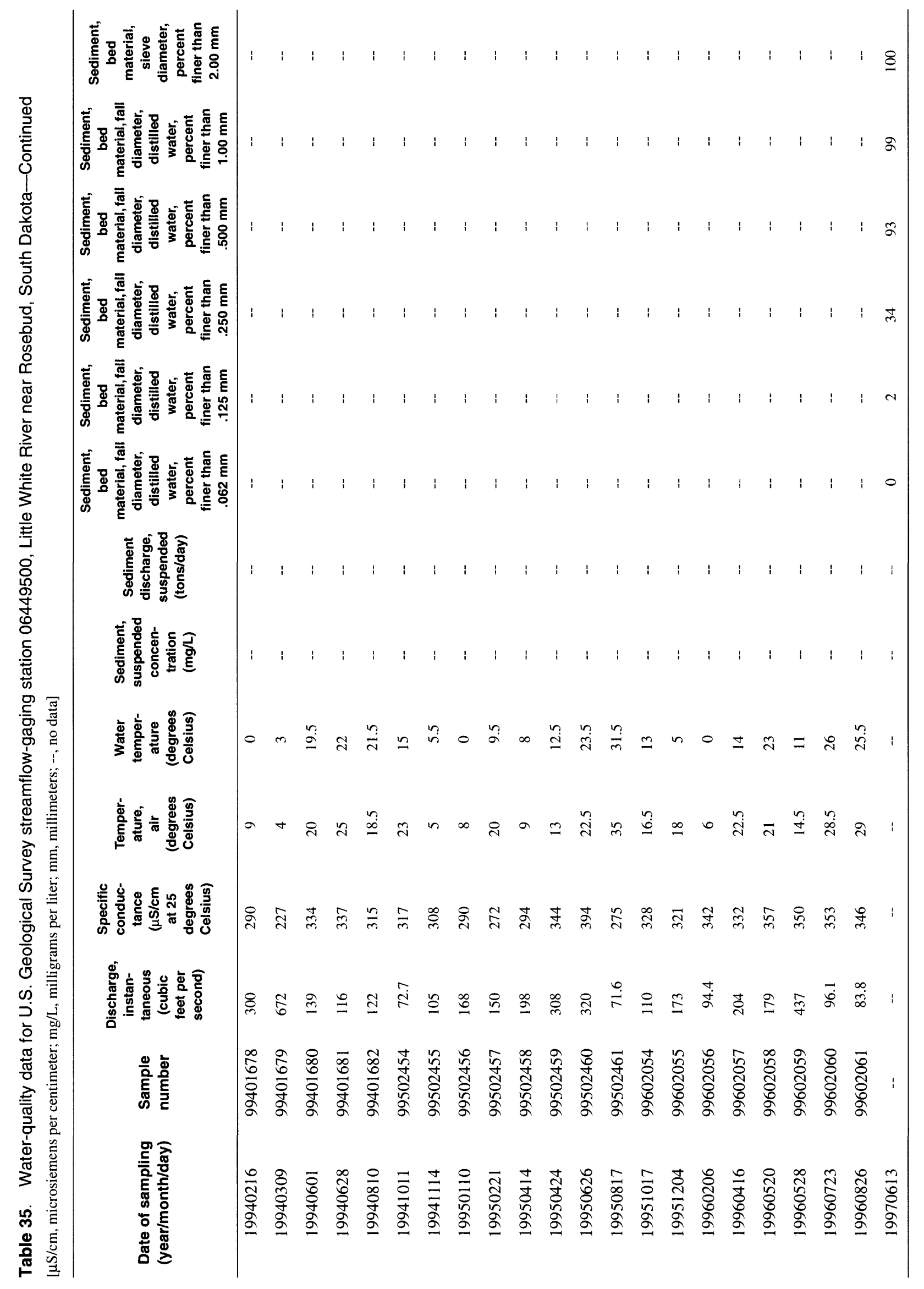




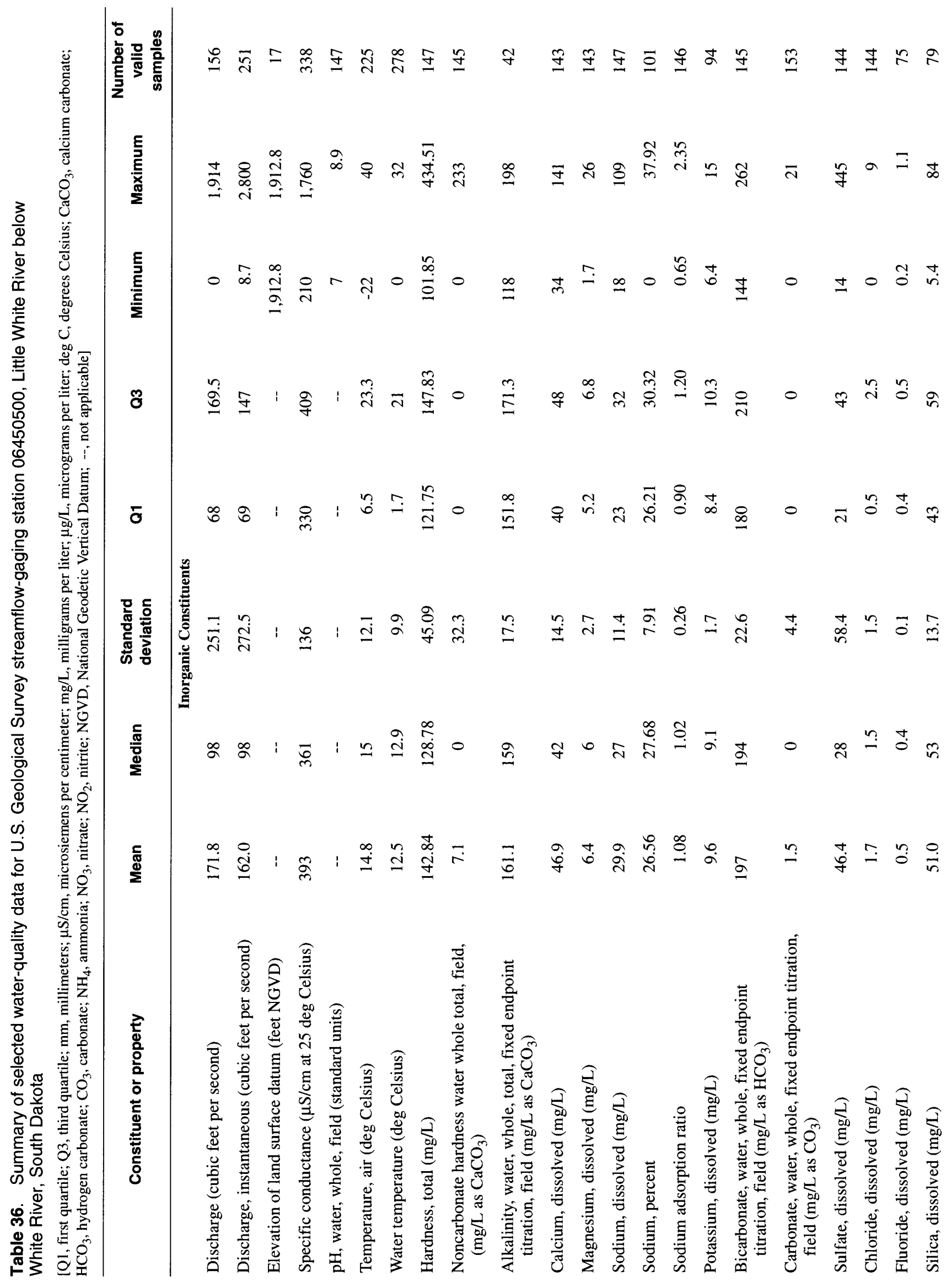




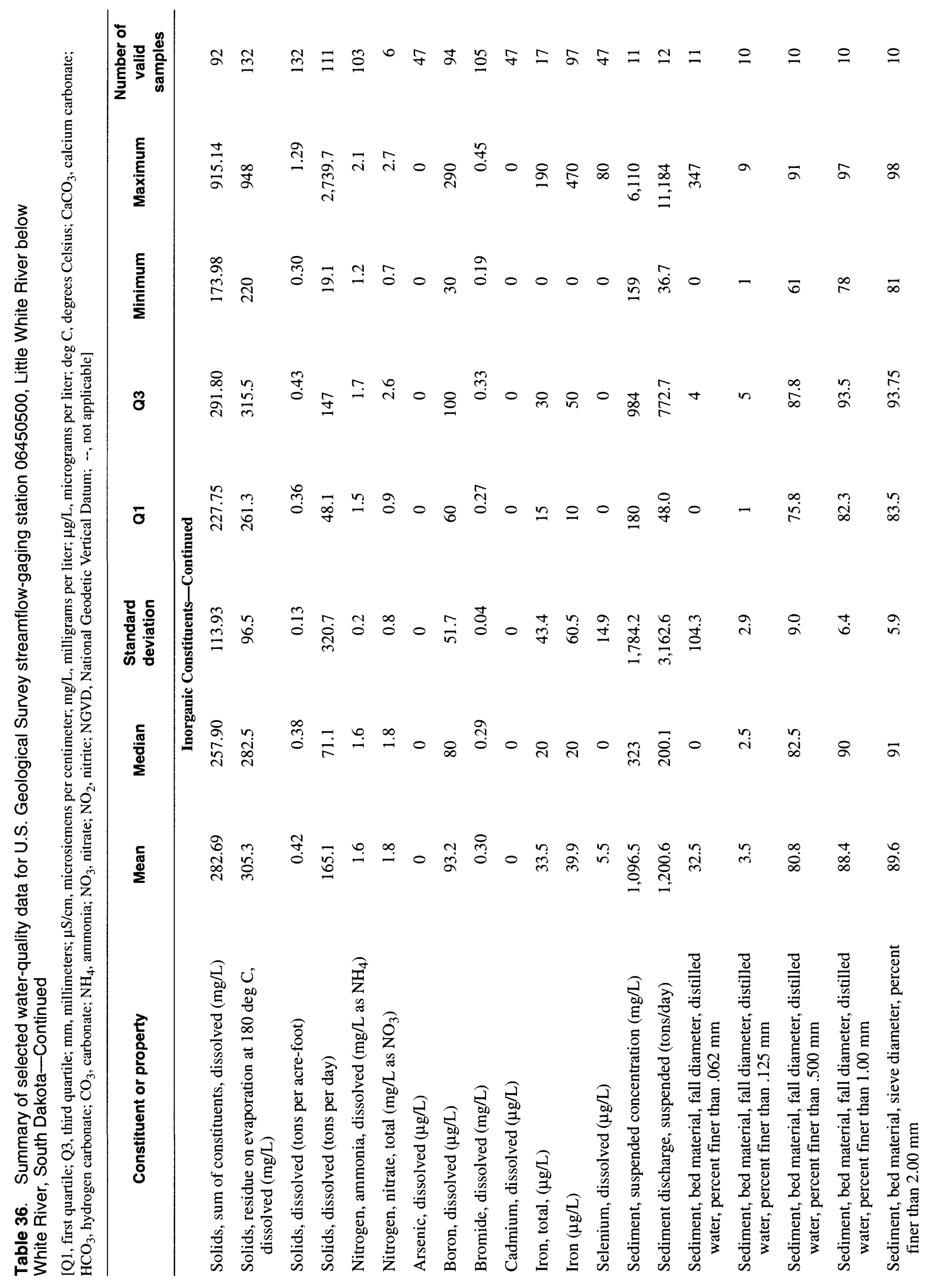




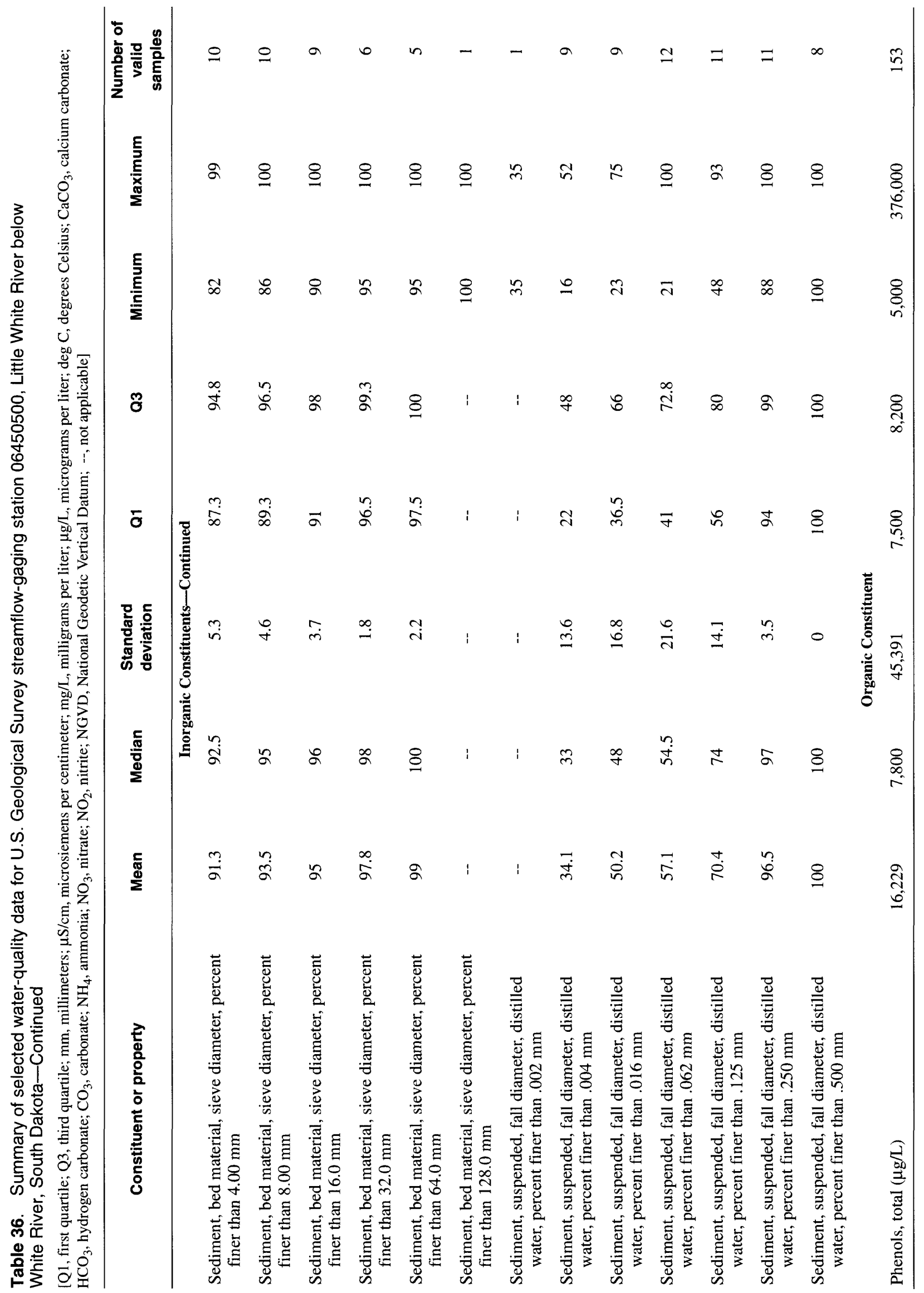




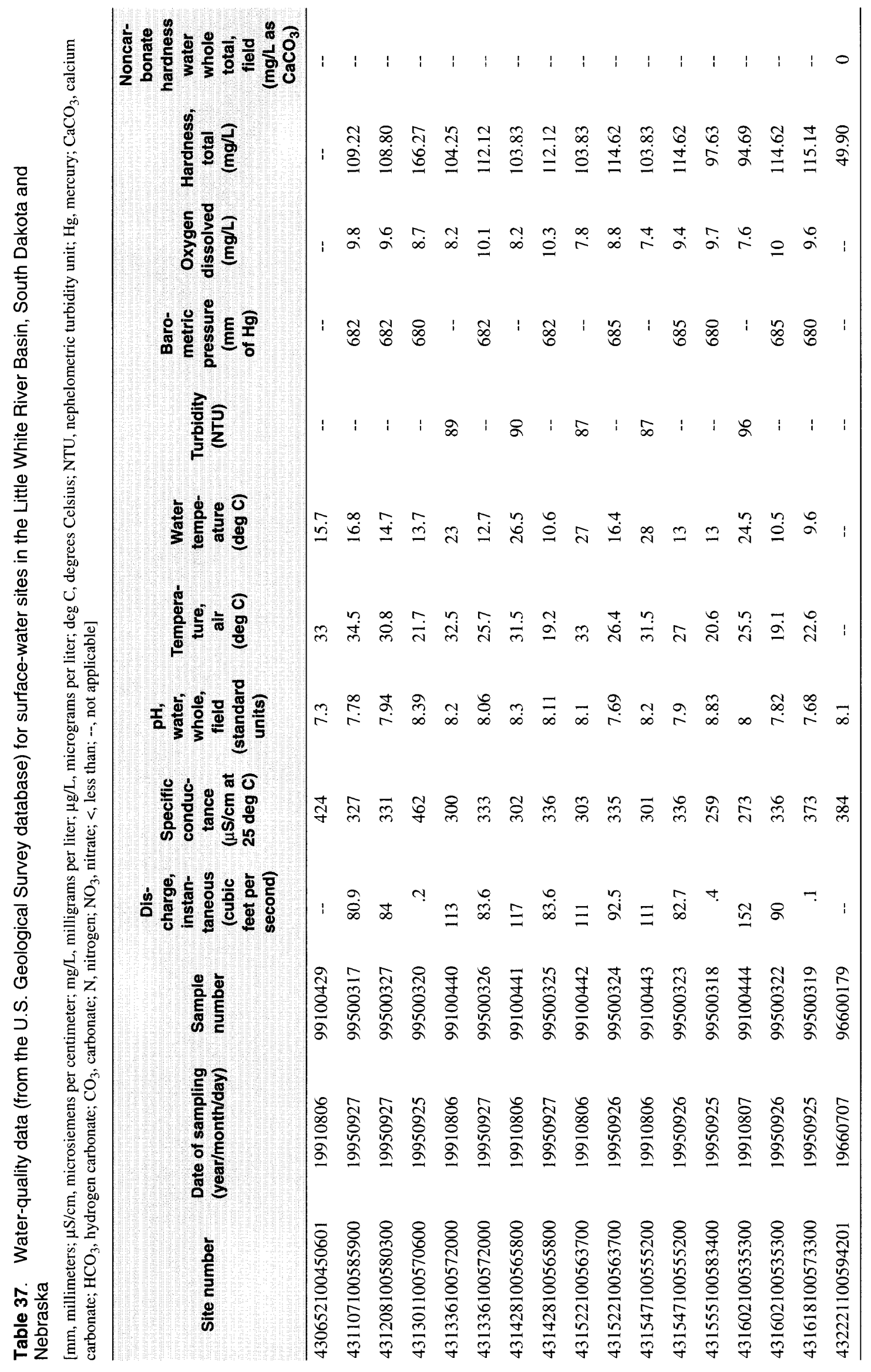




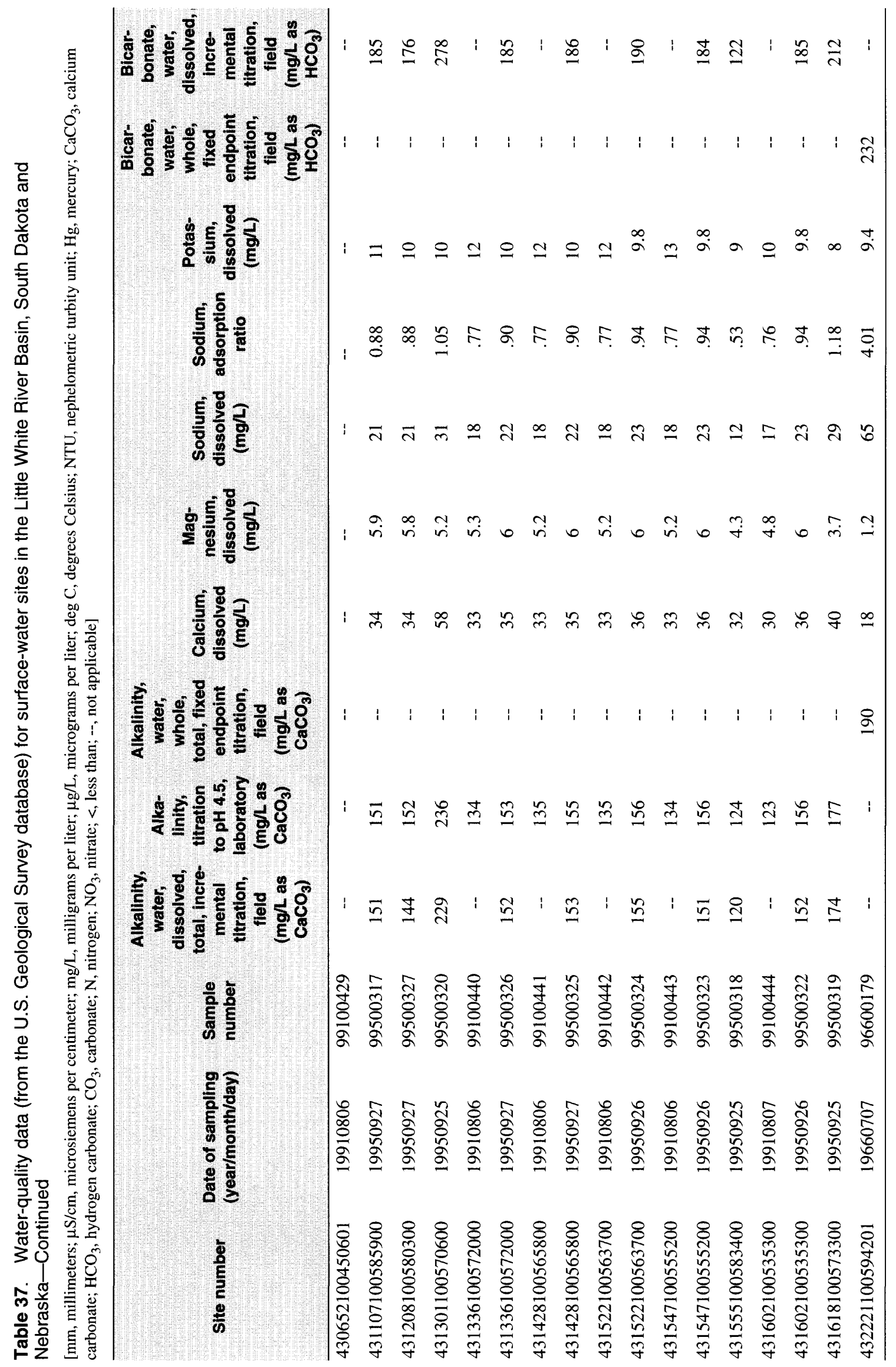




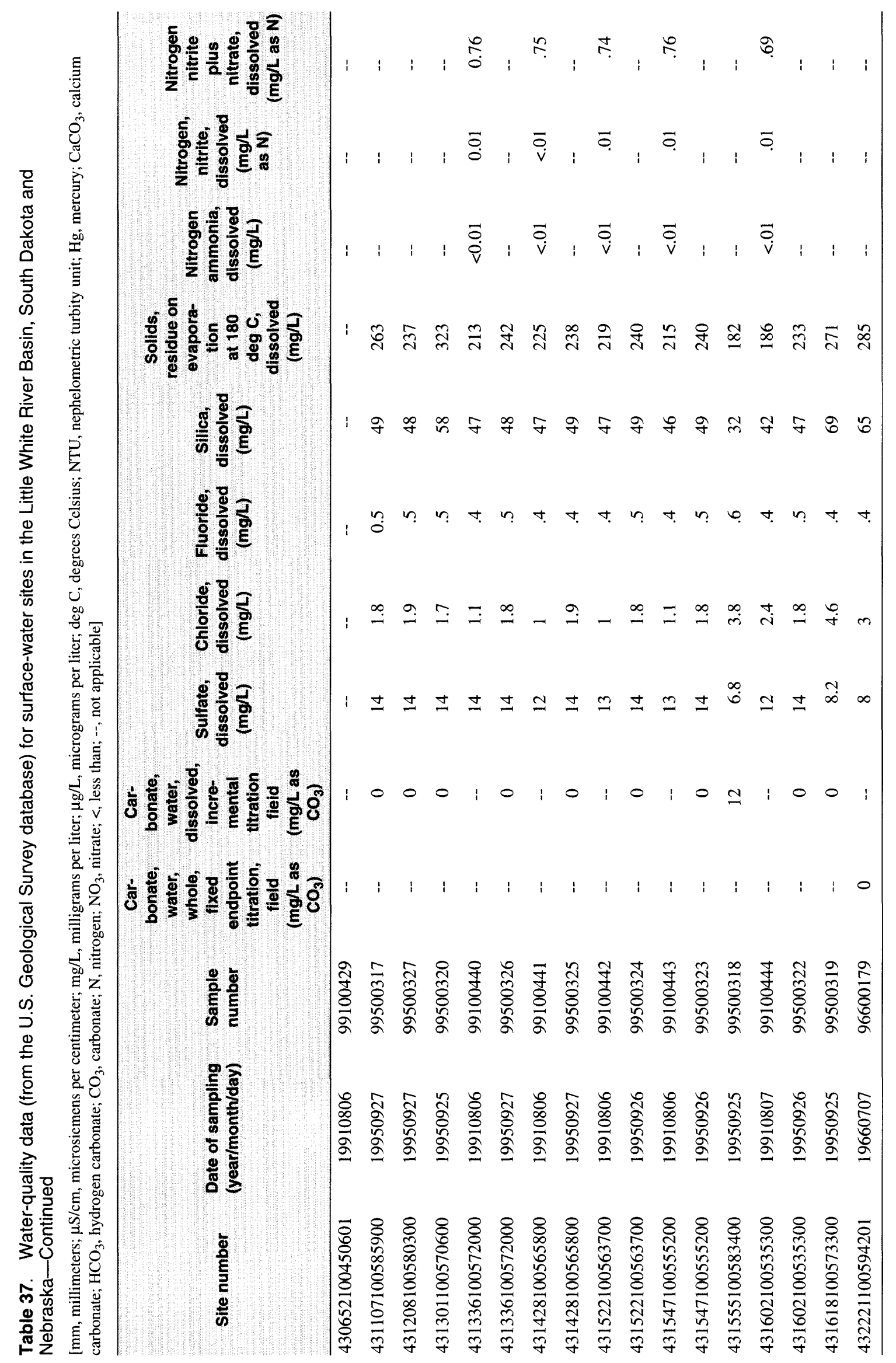




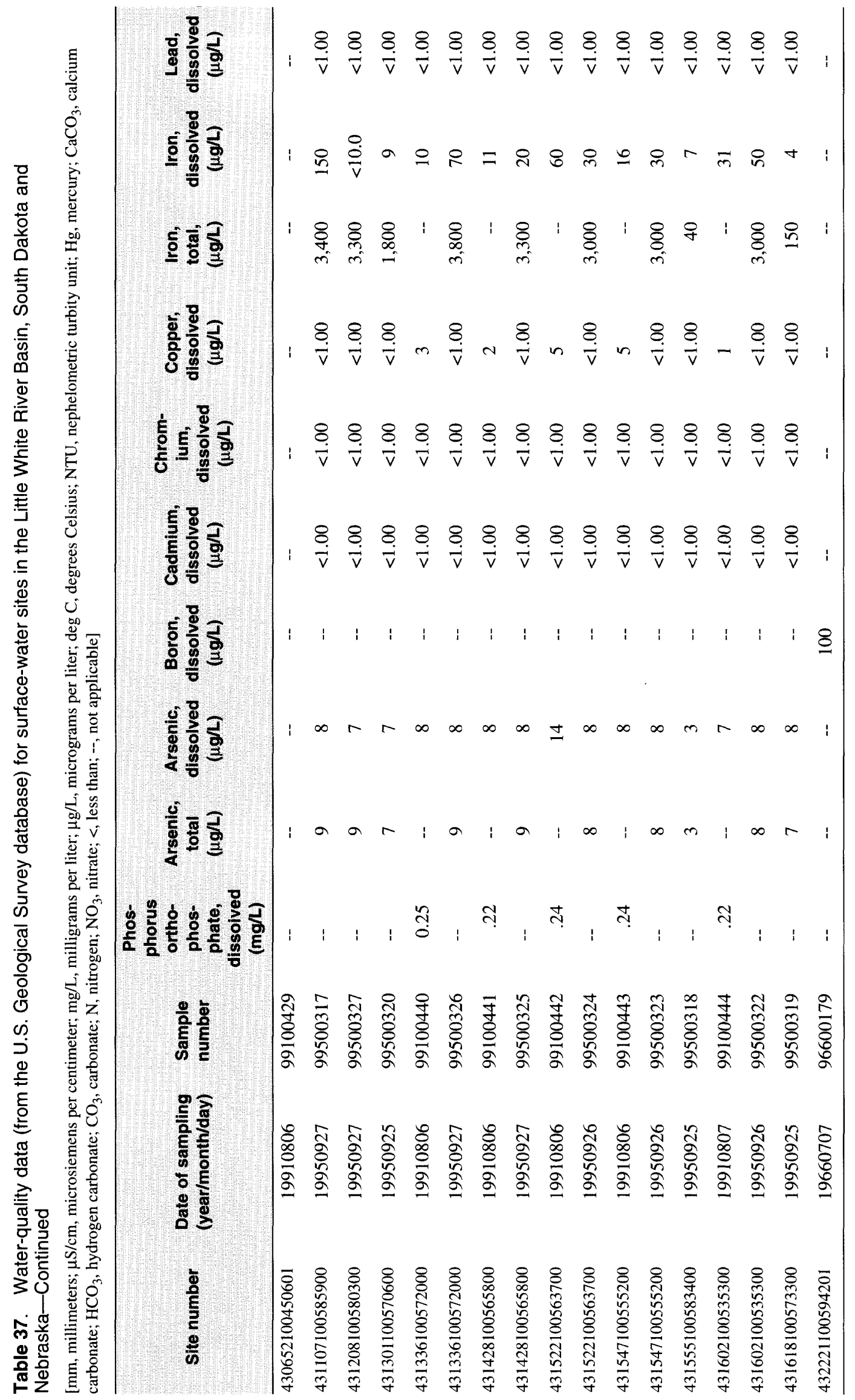




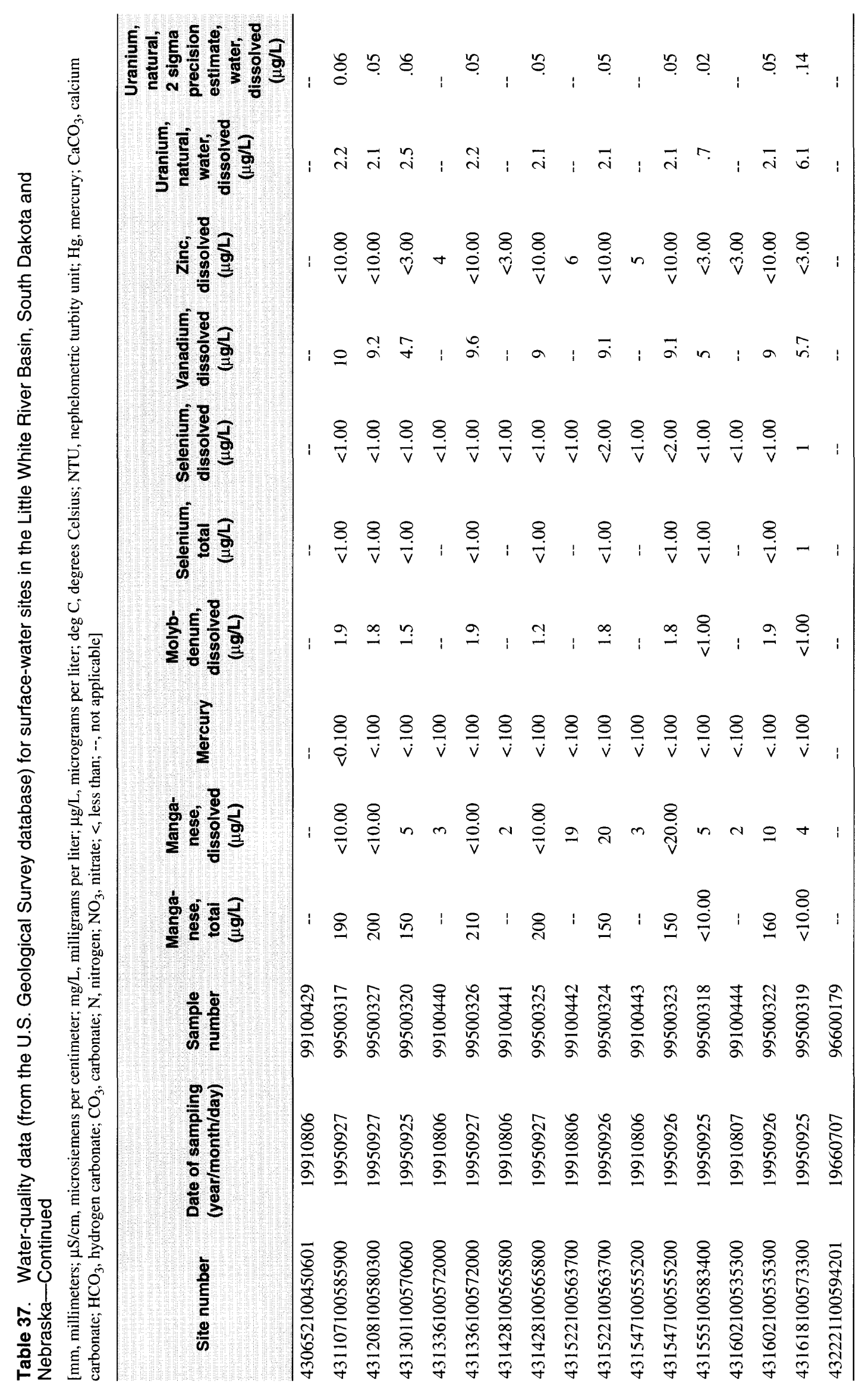


Table 38. Summary of daily ground-water level data (from the U.S. Geological Survey database), in feet below land surface, for one site in the Little White River Basin, South Dakota and Nebraska

\begin{tabular}{|c|c|c|c|c|c|c|}
\hline \multirow[b]{2}{*}{ Site number } & \multirow[b]{2}{*}{ Month } & \multicolumn{4}{|c|}{ Ground-water level (in feet below land surface) } & \multirow{2}{*}{$\begin{array}{c}\text { Number of } \\
\text { valid } \\
\text { samples }\end{array}$} \\
\hline & & Mean & $\begin{array}{l}\text { Standard } \\
\text { deviation }\end{array}$ & Minimum & Maximum & \\
\hline \multirow[t]{12}{*}{432306099284801} & 1 & 171.27 & 1.20 & 169.26 & 172.97 & 155 \\
\hline & 2 & 171.30 & 1.15 & 169.38 & 172.95 & 141 \\
\hline & 3 & 171.24 & 1.16 & 169.25 & 173.00 & 155 \\
\hline & 4 & 171.30 & 1.14 & 169.45 & 172.99 & 148 \\
\hline & 5 & 171.19 & 1.11 & 169.31 & 172.84 & 155 \\
\hline & 6 & 171.04 & 1.20 & 169.15 & 172.76 & 133 \\
\hline & 7 & 171.09 & 1.07 & 169.43 & 172.64 & 123 \\
\hline & 8 & 171.27 & 1.18 & 169.58 & 173.05 & 110 \\
\hline & 9 & 171.04 & 1.17 & 169.37 & 173.03 & 144 \\
\hline & 10 & 170.53 & .84 & 169.29 & 171.69 & 123 \\
\hline & 11 & 170.76 & .93 & 169.30 & 171.99 & 143 \\
\hline & 12 & 171.05 & 1.11 & 169.27 & 172.80 & 155 \\
\hline
\end{tabular}


Table 39. Summary of periodic ground-water level data (from the U.S. Geological Survey database), in feet below land surface, for sites in the Little White River Basin, South Dakota and Nebraska

[Negative numbers mean water surface above land surface]

\begin{tabular}{|c|c|c|c|c|c|c|}
\hline Site number & Month & Mean & $\begin{array}{l}\text { Standard } \\
\text { deviation }\end{array}$ & Minimum & Maximum & $\begin{array}{l}\text { Number of } \\
\text { valid } \\
\text { samples }\end{array}$ \\
\hline \multirow[t]{4}{*}{425956101134501} & 1 & -- & -- & 6.59 & 6.59 & 1 \\
\hline & 9 & 8.12 & 1.25 & 7.24 & 9.00 & 2 \\
\hline & 10 & -- & -- & 8.92 & 8.92 & 1 \\
\hline & 11 & -- & -- & 7.13 & 7.13 & 1 \\
\hline \multirow[t]{2}{*}{425956101134502} & 9 & -- & -- & 7.75 & 7.75 & 1 \\
\hline & 10 & -- & -- & 9.52 & 9.52 & 1 \\
\hline \multirow[t]{4}{*}{425956101134503} & 1 & -- & -- & 7.10 & 7.10 & 1 \\
\hline & 9 & 7.91 & .08 & 7.85 & 7.96 & 2 \\
\hline & 10 & -- & -- & 9.46 & 9.46 & 1 \\
\hline & 11 & -- & -- & 7.56 & 7.56 & 1 \\
\hline \multirow[t]{2}{*}{430152101054803} & 8 & -- & -- & 7.30 & 7.30 & 1 \\
\hline & 10 & -- & -- & 9.29 & 9.29 & 1 \\
\hline \multirow[t]{2}{*}{430153101054902} & 8 & -- & -- & 11.80 & 11.80 & 1 \\
\hline & 10 & -- & -- & 9.92 & 9.92 & 1 \\
\hline \multirow[t]{10}{*}{430256101160001} & 3 & 5.50 & .66 & 5.00 & 6.60 & 5 \\
\hline & 4 & 5.59 & .85 & 4.40 & 7.20 & 13 \\
\hline & 5 & 5.41 & .37 & 4.80 & 5.80 & 9 \\
\hline & 6 & 5.80 & .67 & 4.70 & 7.60 & 14 \\
\hline & 7 & 5.99 & .45 & 5.40 & 6.70 & 9 \\
\hline & 8 & 6.17 & .55 & 5.60 & 7.90 & 15 \\
\hline & 9 & 6.41 & .65 & 6.00 & 8.00 & 8 \\
\hline & 10 & 6.05 & .55 & 5.08 & 6.70 & 8 \\
\hline & 11 & 6.43 & .71 & 5.80 & 7.80 & 6 \\
\hline & 12 & 6.17 & 1.10 & 5.30 & 7.40 & 3 \\
\hline \multirow[t]{11}{*}{430340101012301} & 2 & 7.05 & 2.76 & 5.10 & 9.00 & 2 \\
\hline & 3 & 6.72 & .68 & 5.90 & 7.80 & 6 \\
\hline & 4 & 6.42 & .93 & 5.00 & 7.90 & 13 \\
\hline & 5 & 6.42 & 1.13 & 4.70 & 8.60 & 13 \\
\hline & 6 & 6.60 & 1.29 & 3.20 & 8.70 & 15 \\
\hline & 7 & 7.31 & 1.32 & 4.80 & 8.90 & 13 \\
\hline & 8 & 7.66 & .88 & 6.10 & 9.40 & 18 \\
\hline & 9 & 8.66 & .66 & 8.10 & 9.80 & 8 \\
\hline & 10 & 8.05 & .58 & 6.90 & 8.70 & 7 \\
\hline & 11 & 8.27 & .61 & 7.70 & 9.20 & 6 \\
\hline & 12 & 7.50 & .71 & 7.00 & 8.00 & 2 \\
\hline
\end{tabular}


Table 39. Summary of periodic ground-water level data (from the U.S. Geological Survey database), in feet below land surface, for sites in the Little White River Basin, South Dakota and Nebraska-Continued

[Negative numbers mean water surface above land surface]

\begin{tabular}{|c|c|c|c|c|c|c|}
\hline Site number & Month & Mean & $\begin{array}{l}\text { Standard } \\
\text { deviation }\end{array}$ & Minimum & Maximum & $\begin{array}{c}\text { Number of } \\
\text { valid } \\
\text { samples }\end{array}$ \\
\hline \multirow[t]{11}{*}{430429101320401} & 2 & -- & -- & 5.00 & 5.00 & 1 \\
\hline & 3 & 6.69 & 2.42 & 4.00 & 10.90 & 8 \\
\hline & 4 & 5.18 & 1.93 & 2.30 & 10.90 & 14 \\
\hline & 5 & 5.79 & 2.08 & 3.60 & 11.40 & 12 \\
\hline & 6 & 6.83 & 2.27 & 2.40 & 11.70 & 20 \\
\hline & 7 & 7.79 & 1.78 & 4.80 & 10.90 & 17 \\
\hline & 8 & 8.94 & 1.71 & 4.40 & 11.20 & 21 \\
\hline & 9 & 9.32 & 2.37 & 6.10 & 12.80 & 10 \\
\hline & 10 & 9.17 & 2.35 & 6.30 & 13.70 & 9 \\
\hline & 11 & 8.64 & 2.59 & 4.90 & 13.50 & 8 \\
\hline & 12 & 8.78 & 3.97 & 3.10 & 13.00 & 6 \\
\hline \multirow[t]{10}{*}{430520101260401} & 3 & 31.37 & .94 & 30.20 & 32.60 & 7 \\
\hline & 4 & 31.31 & 1.05 & 29.30 & 33.00 & 13 \\
\hline & 5 & 31.18 & .74 & 30.30 & 32.30 & 10 \\
\hline & 6 & 31.33 & .74 & 30.20 & 32.70 & 17 \\
\hline & 7 & 31.49 & .87 & 30.00 & 32.70 & 14 \\
\hline & 8 & 31.19 & .84 & 29.80 & 32.70 & 17 \\
\hline & 9 & 31.33 & .87 & 30.40 & 32.60 & 8 \\
\hline & 10 & 31.21 & .56 & 30.50 & 32.20 & 8 \\
\hline & 11 & 31.55 & .82 & 30.60 & 32.70 & 6 \\
\hline & 12 & 31.50 & .74 & 31.00 & 32.60 & 4 \\
\hline \multirow[t]{10}{*}{430521101223401} & 3 & 13.90 & 1.01 & 12.70 & 15.30 & 8 \\
\hline & 4 & 13.72 & 1.00 & 12.50 & 15.30 & 14 \\
\hline & 5 & 13.69 & .89 & 12.60 & 15.20 & 13 \\
\hline & 6 & 13.60 & .82 & 12.40 & 15.30 & 20 \\
\hline & 7 & 13.85 & 1.02 & 12.30 & 15.50 & 17 \\
\hline & 8 & 13.85 & .92 & 12.30 & 15.50 & 21 \\
\hline & 9 & 14.01 & 1.07 & 12.60 & 15.60 & 10 \\
\hline & 10 & 13.96 & .85 & 12.70 & 15.60 & 10 \\
\hline & 11 & 14.29 & .90 & 13.20 & 15.70 & 8 \\
\hline & 12 & 16.38 & 4.81 & 13.50 & 26.10 & 6 \\
\hline
\end{tabular}


Table 39. Summary of periodic ground-water level data (from the U.S. Geological Survey database), in feet below land surface, for sites in the Little White River Basin, South Dakota and Nebraska-Continued

[Negative numbers mean water surface above land surface]

\begin{tabular}{|c|c|c|c|c|c|c|}
\hline Site number & Month & Mean & $\begin{array}{l}\text { Standard } \\
\text { deviation }\end{array}$ & Minimum & Maximum & $\begin{array}{c}\text { Number of } \\
\text { valid } \\
\text { samples }\end{array}$ \\
\hline \multirow{7}{*}{430524101481601} & 3 & 6.51 & 0.31 & 6.00 & 7.00 & 7 \\
\hline & 4 & 6.61 & .56 & 5.90 & 7.70 & 13 \\
\hline & 6 & 7.25 & .77 & 5.90 & 8.60 & 16 \\
\hline & 7 & 7.96 & .86 & 6.50 & 9.40 & 14 \\
\hline & 8 & 8.28 & .67 & 7.20 & 9.30 & 17 \\
\hline & 9 & 8.33 & .37 & 7.90 & 9.00 & 7 \\
\hline & 12 & 7.30 & .54 & 6.60 & 7.90 & 4 \\
\hline \multirow[t]{3}{*}{430605101135701} & 1 & -- & -- & -6.59 & -6.59 & 1 \\
\hline & 8 & -- & -- & -13.94 & -13.94 & 1 \\
\hline & 10 & -- & -- & -18.03 & -18.03 & 1 \\
\hline \multirow[t]{2}{*}{430605101135702} & 8 & -- & -- & -14.60 & -14.60 & 1 \\
\hline & 10 & -6.40 & 11.88 & -14.80 & 2.00 & 2 \\
\hline 430613100544901 & 10 & 59.14 & -- & 59.14 & 59.14 & 1 \\
\hline \multirow[t]{6}{*}{430642101455801} & 2 & 15.00 & -- & 15.00 & 15.00 & 1 \\
\hline & 8 & 15.92 & 1.92 & 13.20 & 20.70 & 23 \\
\hline & 9 & 15.87 & 1.02 & 14.00 & 18.00 & 11 \\
\hline & 10 & 15.36 & 1.25 & 13.40 & 17.20 & 11 \\
\hline & 11 & 15.92 & 1.04 & 14.50 & 17.80 & 9 \\
\hline & 12 & 14.83 & .99 & 13.40 & 15.90 & 6 \\
\hline 430715101031901 & 10 & -- & -- & 11.28 & 11.28 & 1 \\
\hline
\end{tabular}


Table 39. Summary of periodic ground-water level data (from the U.S. Geological Survey database), in feet below land surface, for sites in the Little White River Basin, South Dakota and Nebraska-Continued

[Negative numbers mean water surface above land surface]

\begin{tabular}{|c|c|c|c|c|c|c|}
\hline Site number & Month & Mean & $\begin{array}{l}\text { Standard } \\
\text { deviation }\end{array}$ & Minimum & Maximum & $\begin{array}{c}\text { Number of } \\
\text { valid } \\
\text { samples }\end{array}$ \\
\hline \multirow[t]{10}{*}{430744101593201} & 3 & 0.00 & 1.28 & -2.00 & 2.10 & 9 \\
\hline & 4 & -.21 & 1.40 & -2.20 & 2.20 & 13 \\
\hline & 5 & -.34 & 1.59 & -2.20 & 3.40 & 13 \\
\hline & 6 & 3.69 & 7.56 & -2.20 & 23.50 & 20 \\
\hline & 7 & 9.15 & 9.13 & -1.60 & 27.30 & 15 \\
\hline & 8 & 19.96 & 12.03 & .70 & 36.70 & 21 \\
\hline & 9 & 18.99 & 12.68 & 5.10 & 45.20 & 10 \\
\hline & 10 & 8.09 & 4.52 & 1.30 & 15.60 & 8 \\
\hline & 11 & 7.76 & 6.03 & 1.10 & 17.70 & 8 \\
\hline & 12 & 2.08 & 2.55 & -.40 & 5.60 & 5 \\
\hline \multirow[t]{11}{*}{430825101151801} & 2 & -- & -- & 57.90 & 57.90 & 1 \\
\hline & 3 & 58.28 & .28 & 58.00 & 58.70 & 8 \\
\hline & 4 & 58.16 & .39 & 57.60 & 58.90 & 13 \\
\hline & 5 & 58.20 & .40 & 57.50 & 58.80 & 10 \\
\hline & 6 & 58.22 & .44 & 57.40 & 59.00 & 16 \\
\hline & 7 & 58.32 & .29 & 57.80 & 58.80 & 13 \\
\hline & 8 & 58.22 & .29 & 57.80 & 58.60 & 16 \\
\hline & 9 & 58.28 & .36 & 57.80 & 58.80 & 9 \\
\hline & 10 & 58.30 & .51 & 57.40 & 58.90 & 8 \\
\hline & 11 & 58.42 & .36 & 58.00 & 59.00 & 6 \\
\hline & 12 & 58.43 & .33 & 58.10 & 58.80 & 4 \\
\hline \multirow[t]{11}{*}{430832101364701} & 2 & -- & -- & 127.70 & 127.70 & 1 \\
\hline & 3 & 126.78 & .69 & 125.90 & 127.70 & 9 \\
\hline & 4 & 126.57 & .77 & 125.40 & 127.90 & 13 \\
\hline & 5 & 126.89 & .85 & 125.80 & 128.20 & 14 \\
\hline & 6 & 126.78 & .77 & 125.60 & 128.10 & 21 \\
\hline & 7 & 126.90 & .69 & 125.60 & 127.80 & 16 \\
\hline & 8 & 126.77 & .68 & 125.60 & 127.70 & 23 \\
\hline & 9 & 126.76 & .62 & 125.90 & 127.40 & 11 \\
\hline & 10 & 126.69 & .57 & 125.70 & 127.30 & 11 \\
\hline & 11 & 127.02 & .76 & 126.10 & 127.90 & 9 \\
\hline & 12 & 126.92 & .31 & 126.50 & 127.30 & 6 \\
\hline
\end{tabular}


Table 39. Summary of periodic ground-water level data (from the U.S. Geological Survey database), in feet below land surface, for sites in the Little White River Basin, South Dakota and Nebraska-Continued

[Negative numbers mean water surface above land surface]

\begin{tabular}{|c|c|c|c|c|c|c|}
\hline Slte number & Month & Mean & $\begin{array}{l}\text { Standard } \\
\text { devlatlon }\end{array}$ & MInimum & Maximum & $\begin{array}{l}\text { Number of } \\
\text { valld } \\
\text { samples }\end{array}$ \\
\hline \multirow[t]{10}{*}{430835101554601} & 3 & 17.07 & 2.85 & 10.50 & 19.60 & 9 \\
\hline & 4 & 17.25 & 2.26 & 12.40 & 20.50 & 14 \\
\hline & 5 & 17.44 & 2.07 & 13.60 & 20.70 & 14 \\
\hline & 6 & 17.48 & 2.10 & 13.90 & 21.20 & 21 \\
\hline & 7 & 17.68 & 1.97 & 14.00 & 21.10 & 15 \\
\hline & 8 & 17.73 & 2.49 & 14.30 & 22.30 & 23 \\
\hline & 9 & 18.31 & 1.74 & 15.90 & 20.70 & 11 \\
\hline & 10 & 17.36 & 1.55 & 15.20 & 19.80 & 11 \\
\hline & 11 & 18.43 & 1.66 & 16.20 & 20.80 & 9 \\
\hline & 12 & 17.95 & 1.85 & 15.70 & 20.30 & 6 \\
\hline \multirow[t]{11}{*}{430923101484301} & 2 & -- &.- & 39.10 & 39.10 & 1 \\
\hline & 3 & 37.80 & 1.89 & 35.30 & 40.00 & 8 \\
\hline & 4 & 36.54 & 2.37 & 32.80 & 40.20 & 14 \\
\hline & 5 & 36.99 & 2.33 & 33.90 & 40.70 & 11 \\
\hline & 6 & 37.04 & 2.06 & 33.70 & 39.90 & 20 \\
\hline & 7 & 36.93 & 3.67 & 25.30 & 39.70 & 16 \\
\hline & 8 & 36.99 & 2.11 & 33.50 & 39.60 & 21 \\
\hline & 9 & 37.32 & 1.88 & 35.20 & 39.50 & 10 \\
\hline & 10 & 36.93 & 1.66 & 35.10 & 39.40 & 10 \\
\hline & 11 & 37.09 & 1.71 & 35.50 & 39.40 & 7 \\
\hline & 12 & 38.04 & 1.81 & 35.70 & 39.70 & 5 \\
\hline \multirow[t]{12}{*}{430924100460601} & 1 & -- & -- & 74.00 & 74.00 & 1 \\
\hline & 2 & 78.15 & 3.75 & 75.50 & 80.80 & 2 \\
\hline & 3 & 77.56 & 2.51 & 74.50 & 81.00 & 9 \\
\hline & 4 & 78.03 & 2.13 & 75.10 & 81.40 & 15 \\
\hline & 5 & 77.24 & 2.51 & 73.70 & 81.20 & 18 \\
\hline & 6 & 77.06 & 2.41 & 74.10 & 81.20 & 21 \\
\hline & 7 & 77.10 & 2.34 & 74.40 & 81.40 & 17 \\
\hline & 8 & 77.26 & 2.54 & 74.10 & 81.90 & 25 \\
\hline & 9 & 77.52 & 2.78 & 73.90 & 81.40 & 13 \\
\hline & 10 & 77.98 & 3.08 & 74.40 & 84.39 & 12 \\
\hline & 11 & 77.27 & 2.34 & 74.60 & 81.00 & 9 \\
\hline & 12 & 76.15 & 1.45 & 74.40 & 78.00 & 6 \\
\hline
\end{tabular}


Table 39. Summary of periodic ground-water level data (from the U.S. Geological Survey database), in feet below land surface, for sites in the Little White River Basin, South Dakota and Nebraska-Continued

[Negative numbers mean water surface above land surface]

\begin{tabular}{|c|c|c|c|c|c|c|}
\hline Site number & Month & Mean & $\begin{array}{l}\text { Standard } \\
\text { deviation }\end{array}$ & Minimum & Maximum & $\begin{array}{c}\text { Number of } \\
\text { valid } \\
\text { samples }\end{array}$ \\
\hline \multirow[t]{11}{*}{430928101532401} & 2 & 27.30 & -- & 27.30 & 27.30 & 1 \\
\hline & 3 & 25.36 & 2.02 & 22.60 & 27.70 & 8 \\
\hline & 4 & 24.04 & 2.33 & 20.30 & 27.70 & 14 \\
\hline & 5 & 24.69 & 2.24 & 21.40 & 27.70 & 14 \\
\hline & 6 & 24.72 & 2.28 & 20.60 & 27.90 & 21 \\
\hline & 7 & 24.85 & 2.23 & 20.70 & 27.60 & 13 \\
\hline & 8 & 25.14 & 2.16 & 21.60 & 28.10 & 23 \\
\hline & 9 & 25.44 & 2.25 & 22.50 & 28.20 & 11 \\
\hline & 10 & 24.56 & 1.76 & 22.30 & 27.50 & 10 \\
\hline & 11 & 25.48 & 2.05 & 22.90 & 28.00 & 9 \\
\hline & 12 & 25.70 & 1.71 & 23.60 & 27.60 & 6 \\
\hline \multirow[t]{4}{*}{430929101104202} & 1 & -- & -- & 78.25 & 78.25 & 1 \\
\hline & 8 & -- & -- & 77.98 & 77.98 & 1 \\
\hline & 10 & 78.36 & 2.38 & 76.68 & 80.04 & 2 \\
\hline & 11 & 78.42 & -- & 78.42 & 78.42 & 1 \\
\hline \multirow[t]{3}{*}{430929101104203} & 8 & 77.38 & -- & 77.38 & 77.38 & 1 \\
\hline & 10 & 78.18 & 1.10 & 77.40 & 78.95 & 2 \\
\hline & 11 & -- & -- & 77.86 & 77.86 & 1 \\
\hline \multirow[t]{12}{*}{430949101292701} & 1 & -- & -- & 21.30 & 21.30 & 1 \\
\hline & 2 & -- & -- & 21.50 & 21.50 & 1 \\
\hline & 3 & 20.38 & .92 & 18.20 & 21.40 & 10 \\
\hline & 4 & 20.71 & .59 & 19.70 & 21.90 & 14 \\
\hline & 5 & 20.52 & .49 & 19.90 & 21.70 & 15 \\
\hline & 6 & 20.81 & .57 & 19.30 & 21.90 & 23 \\
\hline & 7 & 21.14 & .51 & 20.30 & 22.20 & 19 \\
\hline & 8 & 21.08 & .58 & 19.90 & 22.20 & 23 \\
\hline & 9 & 21.37 & .48 & 20.50 & 22.40 & 13 \\
\hline & 10 & 21.26 & .42 & 20.70 & 22.00 & 11 \\
\hline & 11 & 21.62 & .36 & 21.00 & 22.10 & 11 \\
\hline & 12 & 21.28 & .33 & 20.80 & 21.80 & 6 \\
\hline
\end{tabular}


Table 39. Summary of periodic ground-water level data (from the U.S. Geological Survey database), in feet below land surface, for sites in the Little White River Basin, South Dakota and Nebraska-Continued

[Negative numbers mean water surface above land surface]

\begin{tabular}{|c|c|c|c|c|c|c|}
\hline Site number & Month & Mean & $\begin{array}{l}\text { Standard } \\
\text { deviation }\end{array}$ & Minimum & Maximum & $\begin{array}{c}\text { Number of } \\
\text { valid } \\
\text { samples }\end{array}$ \\
\hline \multirow[t]{12}{*}{431018101152001} & 1 & -- & -- & 67.00 & 67.00 & 1 \\
\hline & 2 & -- & -- & 67.60 & 67.60 & 1 \\
\hline & 3 & 67.60 & 0.95 & 66.10 & 68.70 & 9 \\
\hline & 4 & 67.54 & .87 & 65.90 & 68.90 & 14 \\
\hline & 5 & 67.22 & .92 & 65.90 & 68.70 & 14 \\
\hline & 6 & 67.42 & .84 & 65.80 & 68.70 & 21 \\
\hline & 7 & 67.79 & .71 & 66.70 & 68.80 & 19 \\
\hline & 8 & 67.97 & .87 & 66.50 & 69.50 & 23 \\
\hline & 9 & 68.35 & .94 & 66.60 & 69.50 & 13 \\
\hline & 10 & 68.42 & 1.00 & 66.70 & 69.90 & 12 \\
\hline & 11 & 68.16 & 1.02 & 66.50 & 69.60 & 10 \\
\hline & 12 & 67.68 & .64 & 66.70 & 68.30 & 6 \\
\hline \multirow[t]{11}{*}{431018101212001} & 2 & -- & -- & 29.20 & 29.20 & 1 \\
\hline & 3 & 27.38 & 2.34 & 23.30 & 29.90 & 10 \\
\hline & 4 & 27.21 & 2.14 & 23.20 & 30.30 & 14 \\
\hline & 5 & 27.39 & 1.91 & 24.50 & 29.70 & 12 \\
\hline & 6 & 27.57 & 1.69 & 24.10 & 30.20 & 21 \\
\hline & 7 & 27.94 & 1.86 & 23.70 & 30.10 & 17 \\
\hline & 8 & 27.45 & 1.88 & 23.50 & 30.00 & 21 \\
\hline & 9 & 27.44 & 1.79 & 25.30 & 30.00 & 11 \\
\hline & 10 & 27.69 & 1.64 & 25.10 & 30.00 & 9 \\
\hline & 11 & 27.79 & 1.39 & 25.40 & 29.70 & 8 \\
\hline & 12 & 28.22 & 1.12 & 26.40 & 29.80 & 6 \\
\hline \multirow[t]{11}{*}{431022101555001} & 2 & -- & - & 25.60 & 25.60 & 1 \\
\hline & 3 & 21.95 & 2.78 & 17.80 & 25.30 & 6 \\
\hline & 4 & 21.34 & 2.67 & 17.60 & 25.70 & 12 \\
\hline & 5 & 21.10 & 2.17 & 18.00 & 25.70 & 11 \\
\hline & 6 & 21.74 & 2.38 & 17.30 & 26.30 & 17 \\
\hline & 7 & 21.83 & 2.15 & 17.60 & 24.90 & 11 \\
\hline & 8 & 21.59 & 2.39 & 18.00 & 26.20 & 17 \\
\hline & 9 & 21.99 & 2.38 & 19.80 & 26.20 & 8 \\
\hline & 10 & 21.82 & 1.62 & 19.80 & 25.30 & 9 \\
\hline & 11 & 22.18 & 2.13 & 20.40 & 26.30 & 6 \\
\hline & 12 & 22.77 & 2.63 & 21.20 & 25.80 & 3 \\
\hline
\end{tabular}


Table 39. Summary of periodic ground-water level data (from the U.S. Geological Survey database), in feet below land surface, for sites in the Little White River Basin, South Dakota and Nebraska-Continued

[Negative numbers mean water surface above land surface]

\begin{tabular}{|c|c|c|c|c|c|c|}
\hline Site number & Month & Mean & $\begin{array}{l}\text { Standard } \\
\text { deviation }\end{array}$ & Minimum & Maximum & $\begin{array}{l}\text { Number of } \\
\text { valid } \\
\text { samples }\end{array}$ \\
\hline \multirow[t]{10}{*}{431022101570101} & 3 & 24.40 & 5.19 & 18.00 & 30.90 & 10 \\
\hline & 4 & 23.90 & 5.39 & 14.20 & 32.20 & 13 \\
\hline & 5 & 23.45 & 4.61 & 14.50 & 29.30 & 14 \\
\hline & 6 & 24.21 & 5.30 & 14.60 & 33.90 & 20 \\
\hline & 7 & 23.37 & 4.79 & 16.70 & 33.80 & 15 \\
\hline & 8 & 24.23 & 5.71 & 15.80 & 34.30 & 21 \\
\hline & 9 & 24.48 & 6.60 & 18.30 & 34.10 & 10 \\
\hline & 10 & 23.04 & 5.68 & 15.50 & 34.10 & 9 \\
\hline & 11 & 24.25 & 4.42 & 19.40 & 32.90 & 8 \\
\hline & 12 & 22.25 & 6.16 & 15.60 & 33.40 & 6 \\
\hline \multirow[t]{12}{*}{431045101293701} & 1 & 16.20 & 1.41 & 15.20 & 17.20 & 2 \\
\hline & 2 & -- & -- & 17.30 & 17.30 & 1 \\
\hline & 3 & 15.65 & 1.70 & 12.40 & 20.00 & 13 \\
\hline & 4 & 16.11 & 1.29 & 13.80 & 18.80 & 12 \\
\hline & 5 & 16.20 & .69 & 15.40 & 16.90 & 5 \\
\hline & 6 & 16.11 & .98 & 14.00 & 17.10 & 12 \\
\hline & 7 & 16.29 & 1.22 & 13.90 & 18.20 & 21 \\
\hline & 8 & 16.75 & .67 & 15.40 & 17.90 & 12 \\
\hline & 9 & 16.93 & .57 & 15.80 & 17.60 & 7 \\
\hline & 10 & 16.34 & 1.42 & 13.60 & 17.70 & 7 \\
\hline & 11 & 17.19 & .98 & 15.50 & 18.60 & 14 \\
\hline & 12 & 16.41 & .79 & 15.20 & 17.60 & 9 \\
\hline \multirow[t]{12}{*}{431109100445901} & 1 & -- & -- & 95.10 & 95.10 & 1 \\
\hline & 2 & 98.65 & 2.48 & 96.90 & 100.40 & 2 \\
\hline & 3 & 98.23 & 1.77 & 96.00 & 100.30 & 8 \\
\hline & 4 & 98.54 & 1.41 & 96.10 & 100.50 & 14 \\
\hline & 5 & 97.95 & 1.74 & 95.30 & 100.30 & 18 \\
\hline & 6 & 98.03 & 1.55 & 95.40 & 100.50 & 20 \\
\hline & 7 & 97.90 & 1.50 & 95.50 & 100.30 & 19 \\
\hline & 8 & 97.79 & 1.67 & 95.30 & 100.40 & 24 \\
\hline & 9 & 98.07 & 1.74 & 95.60 & 100.40 & 13 \\
\hline & 10 & 98.40 & 2.25 & 95.50 & 103.40 & 12 \\
\hline & 11 & 97.79 & 1.73 & 95.60 & 100.20 & 9 \\
\hline & 12 & 96.93 & 1.51 & 95.10 & 98.70 & 7 \\
\hline
\end{tabular}


Table 39. Summary of periodic ground-water level data (from the U.S. Geological Survey database), in feet below land surface, for sites in the Little White River Basin, South Dakota and Nebraska-Continued

[Negative numbers mean water surface above land surface]

\begin{tabular}{|c|c|c|c|c|c|c|}
\hline Site number & Month & Mean & $\begin{array}{l}\text { Standard } \\
\text { deviation }\end{array}$ & Minimum & Maximum & $\begin{array}{l}\text { Number of } \\
\text { valid } \\
\text { samples }\end{array}$ \\
\hline \multirow[t]{12}{*}{431136101200901} & 1 & -- & -- & 22.40 & 22.40 & 1 \\
\hline & 2 & -- & -- & 24.00 & 24.00 & 1 \\
\hline & 3 & 21.86 & 1.05 & 19.80 & 23.40 & 9 \\
\hline & 4 & 21.54 & 1.49 & 19.50 & 23.90 & 14 \\
\hline & 5 & 21.15 & 1.50 & 19.30 & 24.30 & 14 \\
\hline & 6 & 22.15 & 1.74 & 19.30 & 26.10 & 22 \\
\hline & 7 & 23.68 & 2.12 & 21.10 & 28.80 & 19 \\
\hline & 8 & 24.50 & 2.24 & 21.40 & 30.60 & 23 \\
\hline & 9 & 24.56 & 1.58 & 21.90 & 26.50 & 13 \\
\hline & 10 & 23.50 & 1.85 & 21.40 & 27.50 & 11 \\
\hline & 11 & 23.44 & 1.60 & 20.80 & 25.30 & 11 \\
\hline & 12 & 22.30 & 1.25 & 21.00 & 24.30 & 6 \\
\hline \multirow[t]{12}{*}{431255101212001} & 1 & -- & -- & 68.30 & 68.30 & 1 \\
\hline & 2 & -- & -- & 68.70 & 68.70 & 1 \\
\hline & 3 & 68.09 & .69 & 66.90 & 69.20 & 8 \\
\hline & 4 & 67.92 & .82 & 66.60 & 69.20 & 13 \\
\hline & 5 & 68.00 & .60 & 67.00 & 68.70 & 12 \\
\hline & 6 & 68.23 & .55 & 67.30 & 69.30 & 17 \\
\hline & 7 & 69.01 & .72 & 67.40 & 70.30 & 15 \\
\hline & 8 & 69.51 & .83 & 67.80 & 71.00 & 19 \\
\hline & 9 & 69.77 & .86 & 68.50 & 71.00 & 10 \\
\hline & 10 & 69.21 & .71 & 68.20 & 70.50 & 8 \\
\hline & 11 & 69.15 & .78 & 68.10 & 70.00 & 8 \\
\hline & 12 & 68.45 & .79 & 67.60 & 69.50 & 4 \\
\hline 431348100510401 & 11 & -- & -- & 16.50 & 16.50 & 1 \\
\hline 431505100560901 & 9 & -- & -- & 134.94 & 134.94 & 1 \\
\hline \multirow[t]{2}{*}{431505100565801} & 9 & -- & -- & 16.25 & 16.25 & 1 \\
\hline & 10 & -. & -- & 18.67 & 18.67 & 1 \\
\hline \multirow[t]{2}{*}{431508100562101} & 9 & -- & -- & 100.94 & 100.94 & 1 \\
\hline & 10 & -- & -- & 101.99 & 101.99 & 1 \\
\hline 431515100553901 & 10 & -- & -- & 145.54 & 145.54 & 1 \\
\hline \multirow[t]{2}{*}{431517100562301} & 9 & 103.11 & .01 & 103.10 & 103.11 & 2 \\
\hline & 10 & -- & -- & 104.14 & 104.14 & 1 \\
\hline \multirow[t]{2}{*}{431526100563202} & 9 & -- & -- & 10.96 & 10.96 & 1 \\
\hline & 10 & -- & -- & 13.15 & 13.15 & 1 \\
\hline
\end{tabular}


Table 39. Summary of periodic ground-water level data (from the U.S. Geological Survey database), in feet below land surface, for sites in the Little White River Basin, South Dakota and Nebraska-Continued

[Negative numbers mean water surface above land surface]

\begin{tabular}{|c|c|c|c|c|c|c|}
\hline Site number & Month & Mean & $\begin{array}{l}\text { Standard } \\
\text { deviatlon }\end{array}$ & Minimum & Maximum & $\begin{array}{l}\text { Number of } \\
\text { valid } \\
\text { samples }\end{array}$ \\
\hline \multirow[t]{2}{*}{431526100563203} & 9 & 11.40 & 0.01 & 11.39 & 11.40 & 2 \\
\hline & 10 & -- & -- & 13.44 & 13.44 & 1 \\
\hline 431536100543101 & 9 & -- & -- & 18.87 & 18.87 & 1 \\
\hline 431949100584601 & 9 & -- & -- & 87.10 & 87.10 & 1 \\
\hline 431949100584602 & 9 & -- & -- & 62.00 & 62.00 & 1 \\
\hline \multirow[t]{11}{*}{432310101045501} & 2 & 32.20 & 4.67 & 28.90 & 35.50 & 2 \\
\hline & 3 & 33.47 & 2.20 & 31.30 & 36.10 & 6 \\
\hline & 4 & 32.40 & 1.98 & 28.80 & 35.80 & 12 \\
\hline & 5 & 32.72 & 1.65 & 31.20 & 35.80 & 13 \\
\hline & 6 & 32.95 & 1.95 & 30.90 & 36.50 & 15 \\
\hline & 7 & 33.52 & 2.11 & 30.50 & 36.40 & 13 \\
\hline & 8 & 32.91 & 1.96 & 30.20 & 36.30 & 18 \\
\hline & 9 & 32.98 & 2.08 & 31.00 & 36.30 & 9 \\
\hline & 10 & 32.42 & 2.54 & 27.60 & 36.20 & 9 \\
\hline & 11 & 32.93 & 1.97 & 31.30 & 36.30 & 6 \\
\hline & 12 & 33.78 & 1.98 & 31.50 & 36.00 & 4 \\
\hline \multirow[t]{2}{*}{432320101044101} & 9 & -- & -- & 74.32 & 74.32 & 1 \\
\hline & 10 & -- & -- & 76.27 & 76.27 & 1 \\
\hline \multirow[t]{11}{*}{432411101080701} & 2 & 20.95 & 1.20 & 20.10 & 21.80 & 2 \\
\hline & 3 & 21.31 & 2.36 & 15.40 & 23.20 & 9 \\
\hline & 4 & 21.08 & 2.12 & 15.00 & 23.60 & 13 \\
\hline & 5 & 21.28 & 1.93 & 15.20 & 22.80 & 14 \\
\hline & 6 & 21.04 & 2.06 & 15.60 & 23.10 & 19 \\
\hline & 7 & 21.77 & .95 & 20.40 & 23.20 & 16 \\
\hline & 8 & 21.56 & .94 & 20.10 & 23.20 & 21 \\
\hline & 9 & 21.91 & 1.05 & 20.20 & 22.90 & 11 \\
\hline & 10 & 21.50 & .91 & 20.50 & 22.80 & 9 \\
\hline & 11 & 20.78 & 2.95 & 13.90 & 23.10 & 8 \\
\hline & 12 & 21.25 & 1.10 & 20.60 & 22.90 & 4 \\
\hline \multirow[t]{5}{*}{432555100591302} & 1 & -- & -- & 59.93 & 59.93 & 1 \\
\hline & 7 & -- & -- & 58.60 & 58.60 & 1 \\
\hline & 9 & -- & -- & 61.35 & 61.35 & 1 \\
\hline & 10 & -- & -- & 59.78 & 59.780 & 1 \\
\hline & 11 & -- & -- & 59.97 & 59.97 & 1 \\
\hline 433939100355001 & 9 & -- & -- & 150.00 & 150.00 & 1 \\
\hline
\end{tabular}


Table 40. Selected descriptive data (from the U.S. Geological Survey database) for ground-water sites in the Little White River Basin, South Dakota and Nebraska

[County code: 7 = Bennett County, 95 = Mellette County, 113 = Shannon County, 121 = Todd County, $123=$ Tripp County; 110 ALVM, Quaternary alluvium; 110AVTN, Alluvium, terrace, non-glacial; 111 ALVM, Holocene Alluvium; 121ASHL, Ash Hollow Formation; 121OGLL, Ogallala Formation; 122ARKR, Arikaree Formation; 122MOCN, Miocene Series; 123CRDN, Chadron Formation; 123WRVR, White River Group or Formation; 211DKOT, Dakota Sandstone or Formation; 211PIRR, Pierre Shale; 217NCSL, Newcastle Sandstone; 310 MNLS, Minnelusa Formation. Datum, National Geodetic Vertical Datum; --, no data]

\begin{tabular}{|c|c|c|c|c|c|}
\hline Site number & Local identifier & County code & $\begin{array}{l}\text { Depth of well, total } \\
\text { (feet) }\end{array}$ & $\begin{array}{l}\text { Altitude of land } \\
\text { surface, datum } \\
\text { (feet) }\end{array}$ & Geologic unit \\
\hline 425956101134501 & $35 \mathrm{~N} 33 \mathrm{~W} 20 \mathrm{ABCC}$ & 7 & 580 & 3,039 & 122ARKR \\
\hline 425956101134502 & $35 \mathrm{~N} 33 \mathrm{~W} 20 \mathrm{ABCC} 2$ & 7 & 64 & 3,040 & 121OGLL \\
\hline 425956101134503 & $35 \mathrm{~N} 33 \mathrm{~W} 20 \mathrm{ABCC} 3$ & 7 & 165 & 3,040 & 121OGLL \\
\hline 430000102103001 & $35 \mathrm{~N} 41 \mathrm{~W} 4 \mathrm{BCC}$ & 113 & 120 & 3,470 & 122ARKR \\
\hline 430104101155501 & $35 \mathrm{~N} 34 \mathrm{~W} 12 \mathrm{DCC}$ & 7 & 250 & -- & 121OGLL \\
\hline 430152101054803 & $35 \mathrm{~N} 32 \mathrm{~W} 9 \mathrm{BABB} 3$ & 121 & 67 & 2,935 & 121ASHL \\
\hline 430153101054902 & $35 \mathrm{~N} 32 \mathrm{~W}$ 9BABB5 & 121 & 452 & 2,938 & 122ARKR \\
\hline 430212101041201 & $35 \mathrm{~N} 32 \mathrm{~W}$ 3DAB & 121 & 110 & 2,910 & 1210GLL \\
\hline 430223102084901 & WAKPAMN WELL & 113 & -- & 3,480 & -- \\
\hline 430256101160001 & $36 \mathrm{~N} 34 \mathrm{~W} 36 \mathrm{DBCC}$ & 7 & 205 & 2,930 & 121OGLL \\
\hline 430300101373501 & $36 \mathrm{~N} 37 \mathrm{~W} 36 \mathrm{CCA}$ & 7 & 250 & -- & 122ARKR \\
\hline 430340101012301 & 36N32W25DDDD & 121 & 125 & 2,841 & 121OGLL \\
\hline 430413101214101 & $36 \mathrm{~N} 34 \mathrm{~W} 30 \mathrm{AAC}$ & 7 & 230 & -- & 121OGLL \\
\hline 430414101314401 & $36 \mathrm{~N} 36 \mathrm{~W} 26 \mathrm{BBD}$ & 7 & 340 & -- & 121OGLL \\
\hline 430429101320401 & 36N36W22DDDD2 & 7 & 263 & 3,068 & 122ARKR \\
\hline 430438101314301 & $36 \mathrm{~N} 36 \mathrm{~W} 23 \mathrm{CCA}$ & 7 & 350 & -- & 121OGLL \\
\hline 430451101041601 & $36 \mathrm{~N} 32 \mathrm{~W} 22 \mathrm{DBB}$ & 121 & 83 & 2,855 & 121OGLL \\
\hline 430504101314401 & $36 \mathrm{~N} 36 \mathrm{~W} 23 \mathrm{BBD}$ & 7 & 320 & -- & 121OGLL \\
\hline 430520101260401 & $36 \mathrm{~N} 35 \mathrm{~W} 15 \mathrm{CCCC}$ & 7 & 165 & 3,090 & 122ARKR \\
\hline 430521101223401 & $36 \mathrm{~N} 34 \mathrm{~W} 18 \mathrm{CCCC} 2$ & 7 & 78.2 & 3,071 & 121OGLL \\
\hline 430524101481601 & $36 \mathrm{~N} 38 \mathrm{~W} 16 \mathrm{CCCC}$ & 7 & 279 & 3,230 & 122ARKR \\
\hline 430530102092301 & $36 \mathrm{~N} 41 \mathrm{~W} 29 \mathrm{AAA}$ & 113 & 120 & 3,530 & 122ARKR \\
\hline 430550101274501 & $36 \mathrm{~N} 35 \mathrm{~W} 17 \mathrm{ACC}$ & 7 & 210 & -- & 121OGLL \\
\hline 430553101463901 & $36 \mathrm{~N} 38 \mathrm{~W} 15 \mathrm{BDD}$ & 7 & 260 & 3,247 & 122ARKR \\
\hline 430600101450201 & $36 \mathrm{~N} 38 \mathrm{~W} 14 \mathrm{AAC}$ & 7 & 150 & 3,231 & 122ARKR \\
\hline 430600101461301 & $36 \mathrm{~N} 38 \mathrm{~W} 15 \mathrm{AAC}$ & 7 & 360 & 3,280 & 122ARKR \\
\hline 430600101472401 & $36 \mathrm{~N} 38 \mathrm{~W} 16 \mathrm{AAC}$ & 7 & 375 & 3,211 & 122ARKR \\
\hline 430605101135701 & $36 \mathrm{~N} 33 \mathrm{~W} 17 \mathrm{BACA}$ & 7 & 216 & 2,807 & 122ARKR \\
\hline
\end{tabular}


Table 40. Selected descriptive data (from the U.S. Geological Survey database) for ground-water sites in the Little White River Basin, South Dakota and Nebraska-Continued

[County code: 7 = Bennett County, 95 = Mellette County, 113 = Shannon County, 121 = Todd County, 123 = Tripp County; 110ALVM, Quaternary alluvium; 110AVTN, Alluvium, terrace, non-glacial; 111ALVM, Holocene Alluvium; 121ASHL, Ash Hollow Formation; 121OGLL, Ogallala Formation; 122ARKR, Arikaree Formation; 122MOCN, Miocene Series; 123CRDN, Chadron Formation; 123WRVR, White River Group or Formation; $211 D K O T$, Dakota Sandstone or Formation; 211PIRR, Pierre Shale; 217NCSL, Newcastle Sandstone; 310 MNLS, Minnelusa Formation. Datum, National Geodetic Vertical Datum; --, no data]

\begin{tabular}{|c|c|c|c|c|c|}
\hline Site number & Local identifier & County code & $\begin{array}{l}\text { Depth of well, total } \\
\text { (feet) }\end{array}$ & $\begin{array}{l}\text { Altitude of land } \\
\text { surface, datum } \\
\text { (feet) }\end{array}$ & Geologic unit \\
\hline 430605101135702 & 36N33W17BACA2 & 7 & 290 & 2,808 & 122ARKR \\
\hline 430613100544901 & $36 \mathrm{~N} 31 \mathrm{~W} 12 \mathrm{DCCD}$ & 121 & 194 & 2,899 & 122ARKR \\
\hline 430617101263501 & $36 \mathrm{~N} 35 \mathrm{~W}$ 9DCC & 7 & 350 & -- & 121OGLL \\
\hline 430625101264101 & $36 \mathrm{~N} 35 \mathrm{~W} 9 \mathrm{DBCC}$ & 7 & 350 & -- & 121OGLL \\
\hline 430625101450101 & 36N38W11DDB & 7 & 475 & 3,241 & 122ARKR \\
\hline 430625101453801 & 36N38W11CCA & 7 & 350 & 3,215 & 122ARKR \\
\hline 430630101465001 & $36 \mathrm{~N} 38 \mathrm{~W} 10 \mathrm{CC}$ & 7 & 500 & 3,221 & 122ARKR \\
\hline 430640101112001 & $36 \mathrm{~N} 33 \mathrm{~W} 10 \mathrm{ABCA}$ & 121 & 65 & 2,881 & 121OGLL \\
\hline 430642101455801 & 36N38W10ADDD & 7 & 443 & 3,199 & 122ARKR \\
\hline 430650101021001 & $36 \mathrm{~N} 32 \mathrm{~W} 1 \mathrm{DCDC}$ & 121 & 60 & 2,623 & 122ARKR \\
\hline 430651101241001 & 36N35W11AAC & 7 & 270 & -- & 1210GLL \\
\hline 430651101403501 & 36N37W 9AAC & 7 & 525 & -- & 122ARKR \\
\hline 430653101464801 & 36N38W10BBD & 7 & 350 & 3,182 & 122ARKR \\
\hline 430656101445501 & 36N38W11AAC & 7 & 480 & 3,228 & 122ARKR \\
\hline 430656102002101 & 36N40W10AAC & 7 & 50 & 3,284 & 122ARKR \\
\hline 430704101390601 & $36 \mathrm{~N} 37 \mathrm{~W} 11 \mathrm{BBBB} 2$ & 7 & 320 & 3,159 & 122ARKR \\
\hline 430715101031901 & $36 \mathrm{~N} 32 \mathrm{~W} 2 \mathrm{CDBD}$ & 121 & 44 & 2,655 & 111ALVM \\
\hline 430716101214801 & $36 \mathrm{~N} 34 \mathrm{~W} 6 \mathrm{DBDC}$ & 7 & 220 & -- & 1210GLL \\
\hline 430716101595401 & $36 \mathrm{~N} 40 \mathrm{~W} 2 \mathrm{CCA}$ & 7 & 60 & 3,290 & 122ARKR \\
\hline 430717101381201 & $36 \mathrm{~N} 37 \mathrm{~W} 2 \mathrm{DDB}$ & 7 & 500 & -- & 122ARKR \\
\hline 430720101361901 & $36 \mathrm{~N} 36 \mathrm{~W} 6 \mathrm{CDD}$ & 7 & 480 & -- & 122ARKR \\
\hline 430727101433601 & $36 \mathrm{~N} 38 \mathrm{~W}$ 1DAA & 7 & 450 & 3,198 & 122ARKR \\
\hline 430738102055901 & $36 \mathrm{~N} 41 \mathrm{~W} 1 \mathrm{BBCB}$ & 113 & 250 & 3,418 & 122ARKR \\
\hline 430744101593201 & $36 \mathrm{~N} 40 \mathrm{~W} 2 \mathrm{ABBB}$ & 7 & 398 & 3,300 & 122ARKR \\
\hline 430750101594301 & 37N40W36DDBC & 7 & 400 & 3,264 & 122ARKR \\
\hline 430751101142801 & 37N33W32DCAA & 7 & 370 & 2,940 & 1210GLL \\
\hline 430751101280601 & 37N35W33CCA & 7 & 320 & 3,018 & 121OGLL \\
\hline 430752101560701 & 37N39W33DDB & 7 & 50 & 3,274 & 122ARKR \\
\hline
\end{tabular}


Table 40. Selected descriptive data (from the U.S. Geological Survey database) for ground-water sites in the Little White River Basin, South Dakota and Nebraska-Continued

[County code: 7 = Bennett County, 95 = Mellette County, 113 = Shannon County, 121 = Todd County, 123 = Tripp County; 110ALVM, Quaternary alluvium; 110AVTN, Alluvium, terrace, non-glacial; 111ALVM, Holocene Alluvium; 121ASHL, Ash Hollow Formation; 121OGLL, Ogallala Formation; 122ARKR, Arikaree Formation; 122MOCN, Miocene Series; 123CRDN, Chadron Formation; 123WRVR, White River Group or Formation; 211DKOT, Dakota Sandstone or Formation; 211PIRR, Pierre Shale; 217NCSL, Newcastle Sandstone; 310 MNLS, Minnelusa Formation. Datum, National Geodetic Vertical Datum; --, no data]

\begin{tabular}{|c|c|c|c|c|c|}
\hline Site number & Local identifier & County code & $\begin{array}{c}\text { Depth of well, total } \\
\text { (feet) }\end{array}$ & $\begin{array}{c}\text { Altitude of land } \\
\text { surface, datum } \\
\text { (feet) }\end{array}$ & Geologic unit \\
\hline 430752101580001 & $37 \mathrm{~N} 39 \mathrm{~W} 32 \mathrm{CCA}$ & 7 & 40 & 3,297 & 122ARKR \\
\hline 430757100484001 & 37N30W36D & 121 & -- & 2,980 & 121OGLL \\
\hline 430757101213301 & 37N34W32DACB & 7 & 350 & 3,001 & 121OGLL \\
\hline 430759101460001 & $37 \mathrm{~N} 38 \mathrm{~W} 36 \mathrm{CCA}$ & 7 & 520 & 3,202 & 122ARKR \\
\hline 430811102102701 & $37 N 45 W 32(2)$ & 113 & -- & 3,177 & -- \\
\hline 430818101291701 & $37 N 35 W 32 B$ & 7 & 350 & 2,998 & 121OGLL \\
\hline 430819101223701 & $37 \mathrm{~N} 34 \mathrm{~W} 31 \mathrm{AADC}$ & 7 & 350 & 2,988 & 121OGLL \\
\hline 430822101210701 & 37N34W33BBDB & 7 & 164 & 2,988 & $1210 G L L$ \\
\hline 430823100484101 & 37 N30W36A & 121 & -- & 2,940 & 121OGLL \\
\hline 430824101472701 & 37N38W34AADC & 7 & 50 & 3,231 & 122ARKR \\
\hline 430824102091401 & $37 \mathrm{~N} 41 \mathrm{~W} 34 \mathrm{~A}$ & 113 & 250 & 3,428 & 122ARKR \\
\hline 430825100541001 & $37 \mathrm{~N} 30 \mathrm{~W} 32 \mathrm{BB}$ & 121 & 295 & 2,987 & 122ARKR \\
\hline 430825101151801 & $37 \mathrm{~N} 33 \mathrm{~W} 32 \mathrm{BBBB} 3$ & 7 & 143 & 2,960 & 122ARKR \\
\hline 430828101145601 & $37 N 33 W 32 B A B A$ & 7 & 350 & 2,943 & 121OGLL \\
\hline 430829101294101 & 37N35W31AAAA & 7 & 302 & 2,999 & 122ARKR \\
\hline 430832101364701 & $37 N 36 \mathrm{~W} 31 \mathrm{~B}$ & 7 & 425 & 3,189 & 122ARKR \\
\hline 430835101554601 & 37 N39W34CCCC & 7 & 382 & 3,284 & 122ARKR \\
\hline 430842100541801 & $37 N 30 W 29 \mathrm{CCBD}$ & 121 & 274 & 2,980 & 121OGLL \\
\hline 430844101565001 & $37 \mathrm{~N} 39 \mathrm{~W} 28 \mathrm{CCA}$ & 7 & 130 & 3,303 & 122ARKR \\
\hline 430846101572001 & $37 N 39 W 29 D D B$ & 7 & 400 & 3,310 & 122ARKR \\
\hline 430847101452001 & 37N38W25DDB & 7 & 500 & 3,244 & 122ARKR \\
\hline 430848100473101 & $37 N 29 W 30 D$ & 121 & -- & 2,905 & 121OGLL \\
\hline 430855100541501 & $37 \mathrm{~N} 30 \mathrm{~W} 29 \mathrm{CB}$ & 121 & 265 & 2,980 & 121OGLL \\
\hline 430859101542401 & $37 N 39 W 26 \mathrm{CBAA}$ & 7 & 300 & 3,313 & 122ARKR \\
\hline 430907101561701 & 37N39W28ACC & 7 & 500 & 3,307 & 122ARKR \\
\hline 430910101202401 & $37 \mathrm{~N} 34 \mathrm{~W} 28 \mathrm{AAC}$ & 7 & 340 & 2,962 & 121OGLL \\
\hline 430910101340301 & $37 N 36 W 27 B B C$ & 7 & 220 & 3,100 & 122ARKR \\
\hline 430910101354901 & 37N36W29AAC & 7 & 250 & 3,165 & 122ARKR \\
\hline
\end{tabular}


Table 40. Selected descriptive data (from the U.S. Geological Survey database) for ground-water sites in the Little White River Basin, South Dakota and Nebraska-Continued

[County code: 7 = Bennett County, 95 = Mellette County, $113=$ Shannon County, 121 = Todd County, $123=$ Tripp County; 110ALVM, Quaternary alluvium; 110AVTN, Alluvium, terrace, non-glacial; 111ALVM, Holocene Alluvium; 121 ASHL, Ash Hollow Formation; 121OGLL, Ogallala Formation; 122ARKR, Arikaree Formation; 122MOCN, Miocene Series; 123CRDN, Chadron Formation; 123WRVR, White River Group or Formation; 211DKOT, Dakota Sandstone or Formation; 211PIRR, Pierre Shale; 217NCSL, Newcastle Sandstone; 310 MNLS, Minnelusa Formation. Datum, National Geodetic Vertical Datum; --, no data]

\begin{tabular}{|c|c|c|c|c|c|}
\hline Site number & Local identifier & County code & $\begin{array}{l}\text { Depth of well, total } \\
\text { (feet) }\end{array}$ & $\begin{array}{l}\text { Altitude of land } \\
\text { surface, datum } \\
\text { (feet) }\end{array}$ & Geologic unit \\
\hline 430914101134001 & 37N33W28BAAA & 7 & 266 & 2,961 & 121OGLL \\
\hline 430917101474301 & 37N38W27AAC & 7 & 370 & 3,264 & 122ARKR \\
\hline 430923101484301 & 37N38W28AAAA & 7 & 398 & 3,268 & 122ARKR \\
\hline 430924100460601 & 37N29W29AAAA & 121 & 204 & 2,868 & 121OGLL \\
\hline 430926101271801 & 37N35W21DDDD2 & 7 & -- & 2,975 & 122ARKR \\
\hline 430928101532401 & 37N39W25BBBB & 7 & 382 & 3,287 & 122ARKR \\
\hline 430929101104202 & 37N33W26AAAA2 & 121 & 395 & 2,953 & 122ARKR \\
\hline 430929101104203 & 37N33W26AAAA3 & 121 & 236 & 2,953 & 121OGLL \\
\hline 430937100445801 & 37N29W21DDAD & 121 & -- & 2,880 & 121OGLL \\
\hline 430937101194901 & $37 \mathrm{~N} 34 \mathrm{~W} 22 \mathrm{CCA}$ & 7 & 350 & 2,949 & 121OGLL \\
\hline 430938101494101 & $37 \mathrm{~N} 38 \mathrm{~W} 21 \mathrm{CCA}$ & 7 & 450 & 3,247 & 122ARKR \\
\hline 430938101523101 & 37N39W24DDB & 7 & 130 & 3,297 & 122ARKR \\
\hline 430943101192301 & 37 N34W22DBB & 7 & 370 & 2,965 & 1210GLL \\
\hline 430943101551401 & $37 \mathrm{~N} 39 \mathrm{~W} 22 \mathrm{DBCC}$ & 7 & 160 & 3,287 & $122 \mathrm{ARKR}$ \\
\hline 430946102012401 & 37N40W23CAA & 7 & 400 & 1,030 & 122ARKR \\
\hline 430947101274801 & $37 N 35 W 21 D B B$ & 7 & 200 & 2,992 & 121OGLL \\
\hline 430948101571601 & 37N39W20DABC & 7 & 460 & 3,343 & 122ARKR \\
\hline 430949101292701 & 37N35W20CBBA & 7 & 128 & 2,995 & 121OGLL \\
\hline 430958101104701 & 37N33W23ADCA & 121 & -- & 3,008 & -- \\
\hline 431002101194901 & $37 \mathrm{~N} 34 \mathrm{~W} 22 \mathrm{BBD}$ & 7 & 370 & 2,969 & 121OGLL \\
\hline 431002101195001 & 37N34W22BBD2 & 7 & 350 & 2,969 & 121OGLL \\
\hline 431002101205901 & $37 \mathrm{~N} 34 \mathrm{~W} 21 \mathrm{BBD}$ & 7 & 350 & 2,956 & 1210GLL \\
\hline 431002101210001 & 37N34W21BBD2 & 7 & 350 & 2,956 & 121OGLL \\
\hline 431002101254501 & 37N35W23BBD & 7 & 260 & 2,969 & 1210GLL \\
\hline 431003101395901 & $37 \mathrm{~N} 37 \mathrm{~W} 23 \mathrm{~B}$ & 7 & 420 & 3,231 & 122ARKR \\
\hline 431005100484401 & $37 \mathrm{~N} 30 \mathrm{~W} 24 \mathrm{~A}$ & 121 & -- & 2,900 & 121OGLL \\
\hline 431005101553501 & $37 \mathrm{~N} 39 \mathrm{~W} 22 \mathrm{BBD}$ & 7 & 480 & 3,330 & $122 \mathrm{ARKR}$ \\
\hline 431007101250601 & 37N35W23A & 7 & 470 & 2,978 & 121OGLL \\
\hline
\end{tabular}


Table 40. Selected descriptive data (from the U.S. Geological Survey database) for ground-water sites in the Little White River Basin, South Dakota and Nebraska-Continued

[County code: 7 = Bennett County, 95 = Mellette County, $113=$ Shannon County, 121 = Todd County, $123=$ Tripp County; 110ALVM, Quaternary alluvium; 110AVTN, Alluvium, terrace, non-glacial; 111ALVM, Holocene Alluvium; 121 ASHL, Ash Hollow Formation; 121OGLL, Ogallala Formation; 122ARKR, Arikaree Formation; 122MOCN, Miocene Series; 123CRDN, Chadron Formation; 123WRVR, White River Group or Formation; 211DKOT, Dakota Sandstone or Formation; 211PIRR, Pierre Shale; 217NCSL, Newcastle Sandstone; 310 MNLS, Minnelusa Formation. Datum, National Geodetic Vertical Datum; --, no data]

\begin{tabular}{|c|c|c|c|c|c|}
\hline Site number & Local identifier & County code & $\begin{array}{c}\text { Depth of well, total } \\
\text { (feet) }\end{array}$ & $\begin{array}{c}\text { Altitude of land } \\
\text { surface, datum } \\
\text { (feet) }\end{array}$ & Geologic unit \\
\hline 431007101435101 & $37 N 37 \mathrm{~W} 20 \mathrm{BBCC}$ & 7 & -- & 3,264 & 122ARKR \\
\hline 431009101274101 & $37 N 35 W 21 \mathrm{ABB}$ & 7 & 390 & 3,002 & 1210GLL \\
\hline 431018101152001 & 37N33W17CCCC & 7 & 181 & 2,998 & 121OGLL \\
\hline 431018101212001 & 37N34W17DDDD & 7 & 43.7 & 2,956 & 122ARKR \\
\hline 431022101131701 & $37 N 33 W 16 D$ & 121 & -- & 2,989 & -- \\
\hline 431022101555001 & 37N39W16DDDD & 7 & 565 & 3,350 & 122ARKR \\
\hline 431022101570101 & 37N39W17DDDD & 7 & 305 & 3,337 & 122ARKR \\
\hline 431029101150401 & 37N33W 17CBDD & 7 & 300 & 2,992 & 1210GLL \\
\hline 431029101400902 & $37 \mathrm{~N} 37 \mathrm{~W} 17 \mathrm{CB} 2$ & 7 & 500 & 3,287 & 122ARKR \\
\hline 431029101441601 & $37 \mathrm{~N} 37 \mathrm{~W} 18 \mathrm{DCAB}$ & 7 & -- & 3,290 & 122ARKR \\
\hline 431031101435201 & $37 \mathrm{~N} 37 \mathrm{~W} 17 \mathrm{CCBB}$ & 7 & -- & 3,297 & 122ARKR \\
\hline 431031101442201 & $37 N 37 W 18 D C B A$ & 7 & -- & 3,287 & 122ARKR \\
\hline 431031101522201 & $37 N 39 W 13$ & 113 & -- & 3,340 & -- \\
\hline 431034100451201 & $37 \mathrm{~N} 29 \mathrm{~W} 16 \mathrm{D}$ & 121 & -- & 2,858 & 121OGLL \\
\hline 431044101431801 & 37N37W17CAAA & 7 & -- & 3,208 & 122ARKR \\
\hline 431045101293701 & 37N35W18DAAA & 7 & 27.1 & 2,995 & 1210GLL \\
\hline 431054101154001 & 37N33W18AAC & 7 & 225 & 3,005 & 121OGLL \\
\hline 431058100462401 & $37 \mathrm{~N} 29 \mathrm{~W} 17 \mathrm{~A}$ & 121 & -- & 2,850 & 121OGLL \\
\hline 431109100445901 & 37N29W16AAAA & 121 & 184 & 2,852 & 121OGLL \\
\hline 431117101343501 & 37N36W 9DDB & 7 & 400 & 3,083 & 122ARKR \\
\hline 431121101154001 & 37N33W 7DDB & 7 & 390 & 3,001 & 121OGLL \\
\hline 431124101161501 & $37 N 33 W 7 C$ & 7 & 340 & 3,034 & 121OGLL \\
\hline 431125100454801 & $37 N 29 W 9 C$ & 121 & -- & 2,825 & 1210GLL \\
\hline 431136101200901 & 37N34W 9AAAA & 7 & 93.6 & 2,978 & 1210GLL \\
\hline 431147101161501 & 37N33W 7BBD & 7 & 360 & 3,054 & 121OGLL \\
\hline 431147101202601 & 37N34W 9AAC & 7 & 260 & 2,985 & 121OGLL \\
\hline 431148101174001 & $37 \mathrm{~N} 34 \mathrm{~W} 12 \mathrm{BBC}$ & 7 & 320 & 3,047 & 1210GLL \\
\hline 431148101195101 & $37 \mathrm{~N} 34 \mathrm{~W} 10 \mathrm{~B}$ & 7 & 255 & 2,992 & 1210GLL \\
\hline
\end{tabular}


Table 40. Selected descriptive data (from the U.S. Geological Survey database) for ground-water sites in the Little White River Basin, South Dakota and Nebraska-Continued

[County code: 7 = Bennett County, 95 = Mellette County, $113=$ Shannon County, $121=$ Todd County, $123=$ Tripp County; 110ALVM, Quaternary alluvium; 110AVTN, Alluvium, terrace, non-glacial; 111ALVM, Holocene Alluvium; 121ASHL, Ash Hollow Formation; 121OGLL, Ogallala Formation; 122ARKR, Arikaree Formation; 122MOCN, Miocene Series; 123CRDN, Chadron Formation; 123WRVR, White River Group or Formation; 211DKOT, Dakota Sandstone or Formation; 211PIRR, Pierre Shale; 217NCSL, Newcastle Sandstone; 310 MNLS, Minnelusa Formation. Datum, National Geodetic Vertical Datum; --, no data]

\begin{tabular}{|c|c|c|c|c|c|}
\hline Site number & Local identifier & County code & $\begin{array}{l}\text { Depth of well, total } \\
\text { (feet) }\end{array}$ & $\begin{array}{l}\text { Altitude of land } \\
\text { surface, datum } \\
\text { (feet) }\end{array}$ & Geologic unit \\
\hline 431149100462301 & $37 \mathrm{~N} 29 \mathrm{~W} 8 \mathrm{AADC}$ & 121 & -- & 2,810 & 122ARKR \\
\hline 431151101205701 & 37N34W 9BACB & 7 & 375 & 2,986 & 121OGLL \\
\hline 431151101474101 & 37N38W10AAC & 7 & 420 & 3,362 & 122ARKR \\
\hline 431214101210001 & $37 \mathrm{~N} 34 \mathrm{~W} 4 \mathrm{CCA}$ & 7 & 250 & 2,988 & 1210GLL \\
\hline 431215101191001 & $37 \mathrm{~N} 34 \mathrm{~W}$ 3DDB & 7 & 230 & 3,038 & 1210GLL \\
\hline 431230101211001 & 37N34W 4BCCA & 7 & 300 & 3,001 & 121OGLL \\
\hline 431233101170101 & $37 \mathrm{~N} 34 \mathrm{~W} 1 \mathrm{ACBD}$ & 7 & 300 & 3,051 & $1210 G L L$ \\
\hline 431239101202401 & $37 \mathrm{~N} 34 \mathrm{~W} 4 \mathrm{AAC}$ & 7 & 350 & 3,018 & 121OGLL \\
\hline 431253100535201 & 37N30W31DAB & 121 & -- & 2,720 & -- \\
\hline 431255101212001 & 38N34W32DDDD & 7 & 172 & 3,031 & $1210 G L L$ \\
\hline 431257101281501 & $38 \mathrm{~N} 35 \mathrm{~W} 33 \mathrm{CC}$ & 7 & 96 & 2,969 & 122ARKR \\
\hline 431340100510202 & 38N30W34AA2 & 121 & -- & 2,645 & $122 \mathrm{ARKR}$ \\
\hline 431348100510401 & 38N30W27DDDC & 121 & 58 & 2,582 & 110ALVM \\
\hline 431415101010601 & $38 \mathrm{~N} 31 \mathrm{~W} 29 \mathrm{BCCBR}$ & 121 & -- & 2,920 & -- \\
\hline 431427100561201 & $38 \mathrm{~N} 31 \mathrm{~W} 25 \mathrm{BBAC}$ & 121 & 360 & 2,722 & 122ARKR \\
\hline 431455100554801 & 38N31W24DADB & 121 & 300 & 2,680 & 122ARKR \\
\hline 431501101005701 & 38N31W20CBAA & 121 & 140 & 2,837 & 122ARKR \\
\hline 431505100560901 & $38 \mathrm{~N} 31 \mathrm{~W} 24 \mathrm{ACCA}$ & 121 & 2,272 & 71.97 & 122ARKR \\
\hline 431505100565801 & 38N31W23ACCBR & 121 & 732 & 59.49 & 122ARKR \\
\hline 431508100562101 & 38N31W24BDADR & 121 & 2,622 & 04.68 & 122ARKR \\
\hline 431515100553901 & 38N30W19BCBAR & 121 & 2,742 & 59.35 & 122ARKR \\
\hline 431515100562701 & 38N31W24BDAC & 121 & -- & 2,600 & -- \\
\hline 431517100562301 & 38N31W24BDAC2R & 121 & 2,422 & 73.99 & -- \\
\hline 431520100593601 & 38N31W16DBAA & 121 & 128 & 2,710 & 122ARKR \\
\hline 431525100563801 & $38 \mathrm{~N} 31 \mathrm{~W} 23 \mathrm{ABAD} 2$ & 121 & -- & 2,446 & -- \\
\hline 431526100563201 & 38N31W23AAB2 & 121 & 33 & 2,445 & -- \\
\hline 431526100563202 & 38N31W23AAAB2R & 121 & 602 & 42.71 & 122ARKR \\
\hline 431526100563203 & 38N31W23AAAB3R & 121 & 332 & 42.55 & 110ALVM \\
\hline
\end{tabular}


Table 40. Selected descriptive data (from the U.S. Geological Survey database) for ground-water sites in the Little White River Basin, South Dakota and Nebraska-Continued

[County code: 7 = Bennett County, 95 = Mellette County, 113 = Shannon County, $121=$ Todd County, $123=$ Tripp County; 110 ALVM, Quaternary alluvium; 110AVTN, Alluvium, terrace, non-glacial; 111 ALVM, Holocene Alluvium; 121ASHL, Ash Hollow Formation; 121OGLL, Ogallala Formation; 122ARKR, Arikaree Formation; 122MOCN, Miocene Series; 123CRDN, Chadron Formation; 123WRVR, White River Group or Formation; 211DKOT, Dakota Sandstone or Formation; 211PIRR, Pierre Shale; 217NCSL, Newcastle Sandstone; 310 MNLS, Minnelusa Formation. Datum, National Geodetic Vertical Datum; --, no data]

\begin{tabular}{|c|c|c|c|c|c|}
\hline Site number & Local identifier & County code & $\begin{array}{l}\text { Depth of well, total } \\
\text { (feet) }\end{array}$ & $\begin{array}{l}\text { Altitude of land } \\
\text { surface, datum } \\
\text { (feet) }\end{array}$ & Geologic unit \\
\hline 431530100550801 & 38N30W18DBCD & 121 & 28 & 411.5 & 110ALVM \\
\hline 431536100472201 & $38 \mathrm{~N} 29 \mathrm{~W} 17 \mathrm{BCDA}$ & 121 & 100 & 2,687 & 122ARKR \\
\hline 431536100533601 & $38 \mathrm{~N} 30 \mathrm{~W} 17 \mathrm{DCD}$ & 121 & 35 & 2,550 & -- \\
\hline 431536100543101 & 38N30W18DDAD & 121 & 602 & 19.62 & 122ARKR \\
\hline 431540100545801 & 38N30W18DCBD & 121 & 27 & 2,425 & -- \\
\hline 431545100541501 & 38N30W17CBDD & 121 & -- & 2,408 & -- \\
\hline 431547100555601 & 38N31W13DABC & 121 & -- & 2,425 & -- \\
\hline 431620100560601 & 38N30W18BAAAR & 121 & -- & 2,562 & -- \\
\hline 431620100581301 & 38N31W10DCCCR & 121 & -- & 2,772 & -- \\
\hline 431625101103001 & $38 \mathrm{~N} 33 \mathrm{~W} 12 \mathrm{CCDC}$ & 121 & 150 & 2,943 & 122ARKR \\
\hline 431645101042001 & $38 \mathrm{~N} 32 \mathrm{~W} 11 \mathrm{CBD}$ & 121 & 123 & 2,785 & $122 \mathrm{MOCN}$ \\
\hline 431648100521601 & 38N30W 9ABD & 121 & -- & 2,565 & -- \\
\hline 431855100524501 & 39N30W28CDBD & 121 & 40 & 2,330 & 110AVTN \\
\hline 431933101010501 & $39 \mathrm{~N} 31 \mathrm{~W} 29 \mathrm{BCB}$ & 121 & 240 & 2,640 & 122ARKR \\
\hline 431943100425001 & 39N29W26AABA & 121 & 60 & 2,542 & 122ARKR \\
\hline 431949100584601 & 39N31W27BBBB & 121 & 565 & 2,622 & $123 \mathrm{CRDN}$ \\
\hline 431949100584602 & 39N31W27BBBB2 & 121 & 95 & 2,622 & 122ARKR \\
\hline 432221100594201 & 39N31W 9BBA & 121 & -- & 2,480 & -- \\
\hline 432244100373601 & 39N28W 3DBAC2 & 121 & 35 & 2,482 & 122ARKR \\
\hline 432250101084501 & $39 \mathrm{~N} 32 \mathrm{~W} 6 \mathrm{AC}$ & 121 & 150 & 2,667 & 122ARKR \\
\hline 432310101045501 & 39N32W 3AAAA & 121 & 125 & 2,610 & 122ARKR \\
\hline 432320101044101 & $39 \mathrm{~N} 32 \mathrm{~W} 3 \mathrm{AAAA} 2$ & 121 & 526 & 2,607 & 123WRVR \\
\hline 432336100494001 & 99N76W15CDA & 123 & 1,320 & 1,953 & $217 \mathrm{NCSL}$ \\
\hline 432338101053901 & 40N32W33DA & 95 & 180 & 2,609 & 122ARKR \\
\hline 432358101045501 & $40 \mathrm{~N} 32 \mathrm{~W} 34 \mathrm{AC}$ & 95 & 270 & 2,610 & 122ARKR \\
\hline 432359101003501 & $40 \mathrm{~N} 31 \mathrm{~W} 32 \mathrm{AC}$ & 95 & 220 & 2,540 & 122ARKR \\
\hline 432411101080701 & $40 \mathrm{~N} 32 \mathrm{~W} 32 \mathrm{BBBB}$ & 95 & 265 & 2,640 & 123WRVR \\
\hline 432440100575501 & $40 \mathrm{~N} 31 \mathrm{~W} 28 \mathrm{CAA}$ & 95 & -- & 2,457 & -- \\
\hline
\end{tabular}


Table 40. Selected descriptive data (from the U.S. Geological Survey database) for ground-water sites in the Little White River Basin, South Dakota and Nebraska-Continued

[County code: 7 = Bennett County, 95 = Mellette County, $113=$ Shannon County, 121 = Todd County, $123=$ Tripp County; 110ALVM, Quaternary alluvium; 110AVTN, Alluvium, terrace, non-glacial; 111ALVM, Holocene Alluvium; 121 ASHL, Ash Hollow Formation; 121OGLL, Ogallala Formation; 122ARKR, Arikaree Formation; 122MOCN, Miocene Series; 123CRDN, Chadron Formation; 123WRVR, White River Group or Formation; 211DKOT, Dakota Sandstone or Formation; 211PIRR, Pierre Shale; 217NCSL, Newcastle Sandstone; 310 MNLS, Minnelusa Formation. Datum, National Geodetic Vertical Datum; --, no data]

\begin{tabular}{|c|c|c|c|c|c|}
\hline Site number & Local identifier & County code & $\begin{array}{l}\text { Depth of well, total } \\
\text { (feet) }\end{array}$ & $\begin{array}{l}\text { Altitude of land } \\
\text { surface, datum } \\
\text { (feet) }\end{array}$ & Geologic unit \\
\hline 432517101064001 & $40 \mathrm{~N} 32 \mathrm{~W} 21 \mathrm{CAACC}$ & 95 & 240 & 2,600 & 122ARKR \\
\hline 432542101000101 & $40 \mathrm{~N} 31 \mathrm{~W} 21 \mathrm{BC}$ & 95 & 200 & 2,550 & 122ARKR \\
\hline 432555100591302 & 40N31W21AAAA2 & 95 & 81.2 & 2,587 & 123WRVR \\
\hline 432558100513303 & $40 \mathrm{~N} 30 \mathrm{~W} 22 \mathrm{BAB}$ & 95 & 60 & 2,360 & 123WRVR \\
\hline 432728100562702 & 40N31W12BDB2 & 95 & 89 & 2,345 & 123WRVR \\
\hline 432728100562703 & $40 \mathrm{~N} 31 \mathrm{~W} 12 \mathrm{BDB} 3$ & 95 & 40 & 2,345 & 110AVTN \\
\hline 432737100382201 & $40 \mathrm{~N} 28 \mathrm{~W} 9 \mathrm{ABAC}$ & 95 & 16 & 2,400 & 123WRVR \\
\hline 432741100411201 & $40 \mathrm{~N} 28 \mathrm{~W}$ 7BCAA & 95 & 45 & 2,200 & 110AVTN \\
\hline 432808101012902 & $40 \mathrm{~N} 31 \mathrm{~W} 5 \mathrm{CBB} 2$ & 95 & 105 & 2,610 & 123WRVR \\
\hline 433013100510501 & 38N30W34AABA & 121 & 200 & 2,600 & $122 \mathrm{MOCN}$ \\
\hline 433027100471601 & 41N29W20DDD2 & 95 & -- & 2,346 & 211PIRR \\
\hline 433055101004902 & 41N31W21BDB2 & 95 & 40 & 2,345 & 123WRVR \\
\hline 433229100563101 & 41N30W 7DDBD & 95 & 32 & 2,188 & 211PIRR \\
\hline 433256100460001 & $41 \mathrm{~N} 29 \mathrm{~W} 10 \mathrm{BAB}$ & 95 & -- & -- & -- \\
\hline 433355100425301 & $42 \mathrm{~N} 29 \mathrm{~W} 36 \mathrm{DCD}$ & 95 & -- & -- & -- \\
\hline 433416100454101 & 42N29W34CAAC2 & 95 & 18 & 1,959 & 110ALVM \\
\hline 433425100453001 & $42 \mathrm{~N} 29 \mathrm{~W} 34 \mathrm{CAAC} 2$ & 95 & 13 & 1,959 & 110AVTN \\
\hline 433427100454801 & $42 \mathrm{~N} 29 \mathrm{~W} 34 \mathrm{BDBD}$ & 95 & 15 & 1,958 & 110ALVM \\
\hline 433447100460401 & $42 \mathrm{~N} 29 \mathrm{~W} 27 \mathrm{CDC}$ & 95 & 48 & 1,952 & 110AVTN \\
\hline 433552100444001 & $42 \mathrm{~N} 29 \mathrm{~W} 23 \mathrm{CACC}$ & 95 & 48 & 1,929 & 110AVTN \\
\hline 433613100433201 & $42 \mathrm{~N} 29 \mathrm{~W} 24 \mathrm{BCAD} 2$ & 95 & 47 & 1,912 & 211PIRR \\
\hline 433617100384101 & $42 \mathrm{~N} 28 \mathrm{~W} 22 \mathrm{~B}$ & 95 & 2,360 & 2,101 & 310MNLS \\
\hline 433738100411901 & $42 \mathrm{~N} 28 \mathrm{~W} 8 \mathrm{CBC}$ & 95 & 50 & 1,875 & 110ALVM \\
\hline 433847100411401 & $42 \mathrm{~N} 28 \mathrm{~W} 5 \mathrm{BCD}$ & 95 & 14 & 1,865 & 110AVTN \\
\hline 433939100355001 & $43 \mathrm{~N} 28 \mathrm{~W} 36 \mathrm{AC}$ & 95 & 1,990 & 2,112 & 211DKOT \\
\hline 434213100391601 & 43N28W15ACCD & 95 & -- & 1,798 & $110 \mathrm{AVTN}$ \\
\hline 434216100381601 & 43N28W15ACBD & 95 & -- & 1,795 & 110AVTN \\
\hline 434325100393301 & $43 \mathrm{~N} 28 \mathrm{~W} 9 \mathrm{ABBB}$ & 95 & -- & 1,790 & 110AVTN \\
\hline
\end{tabular}




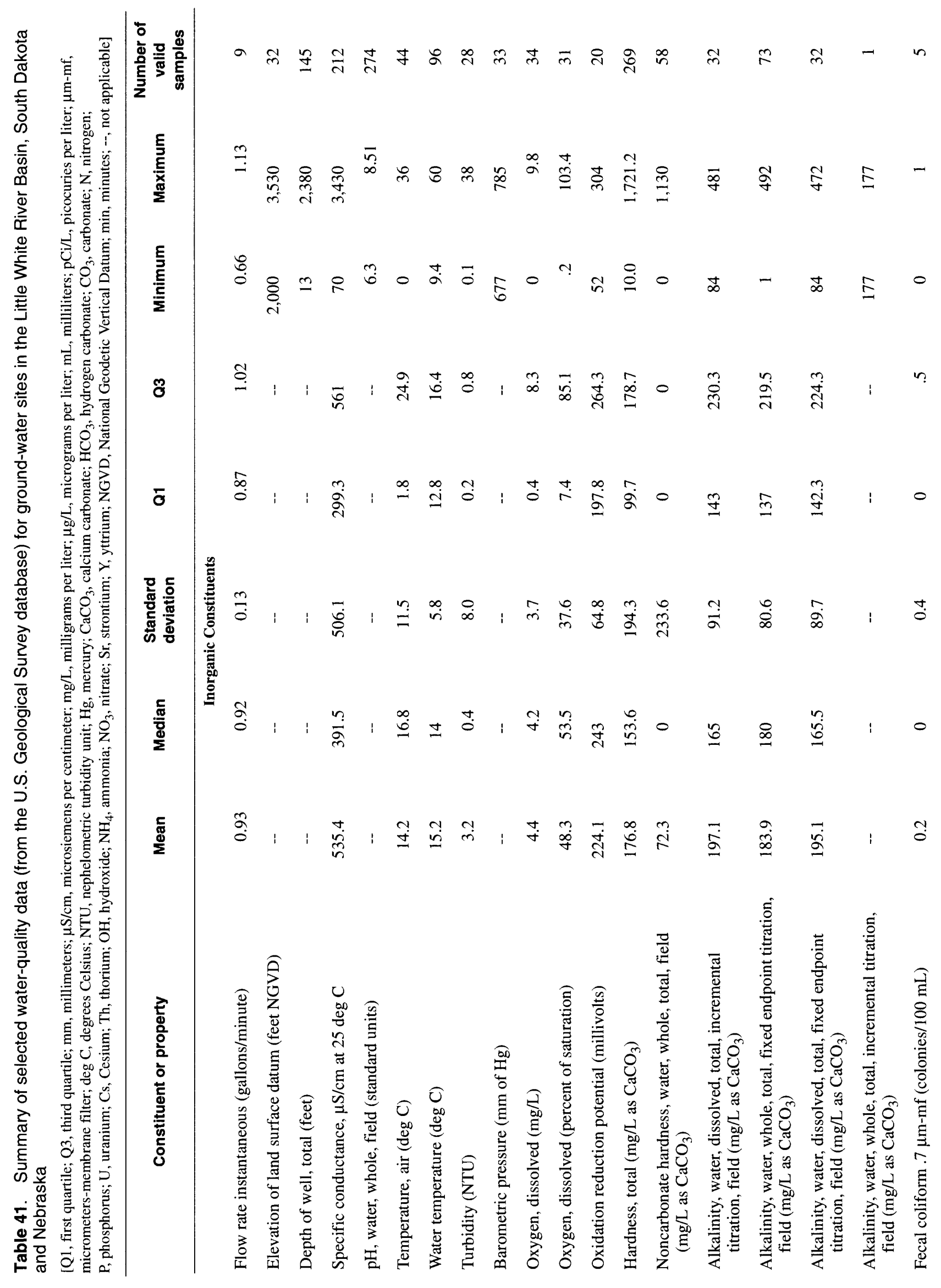




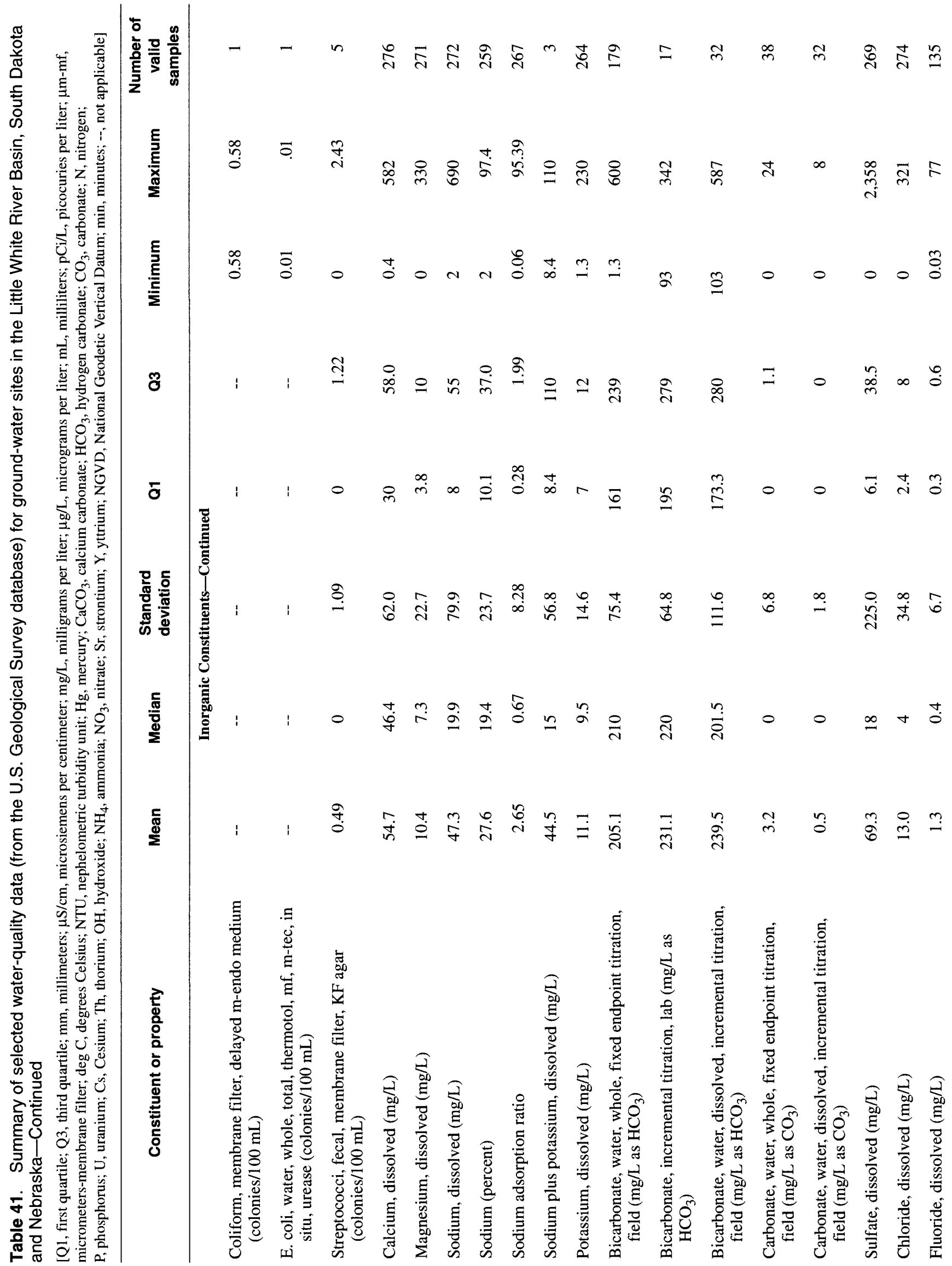




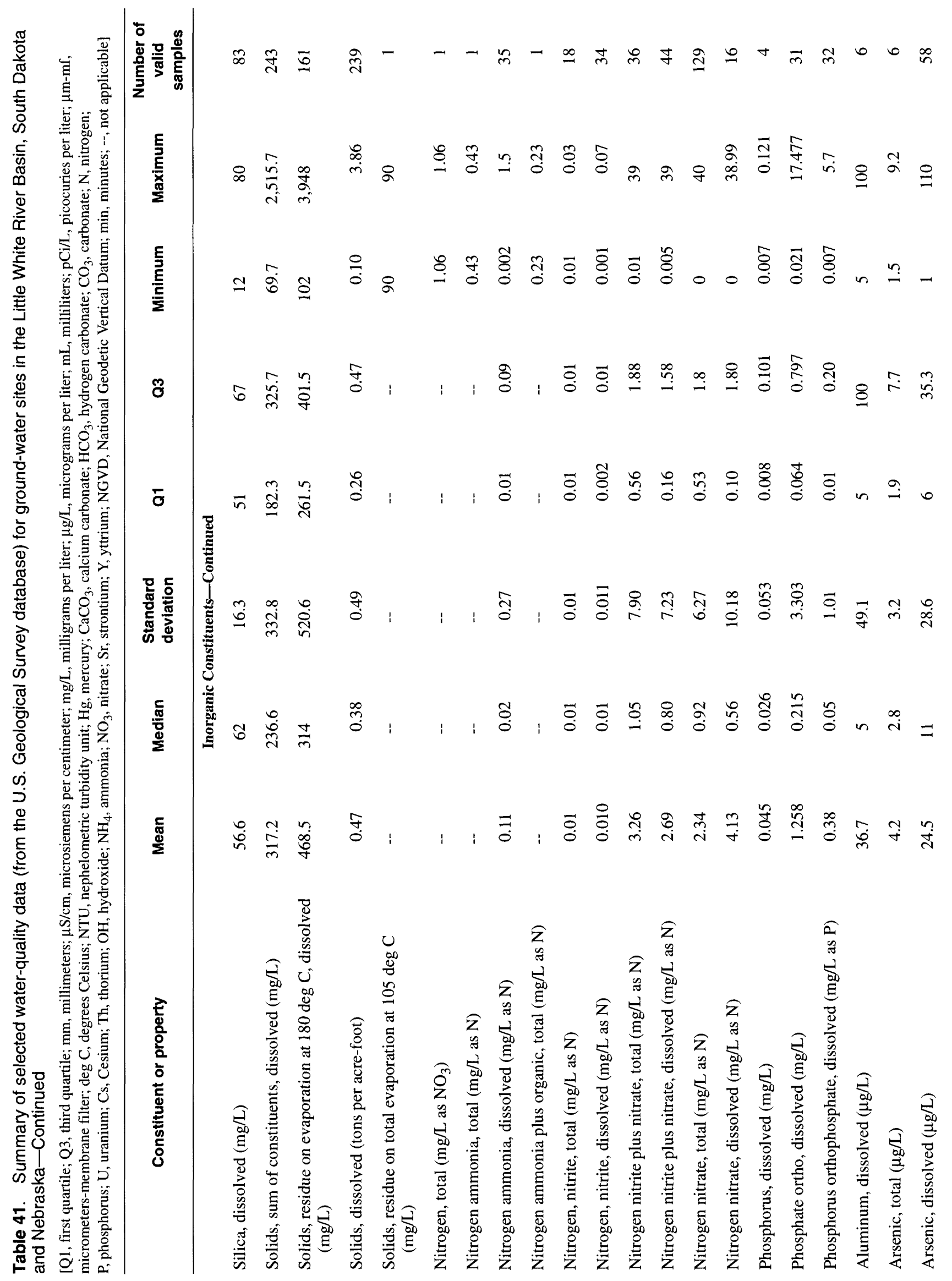




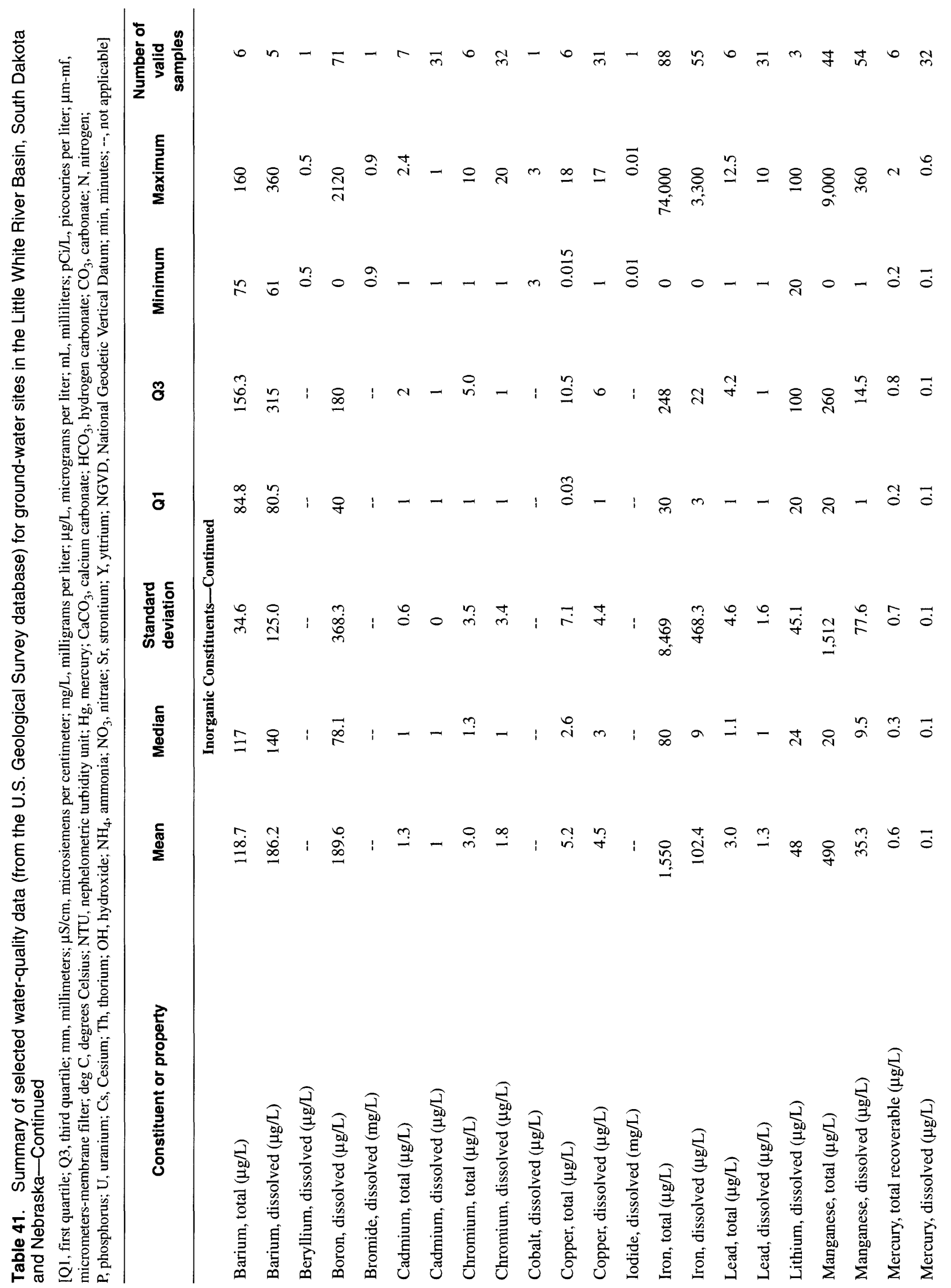




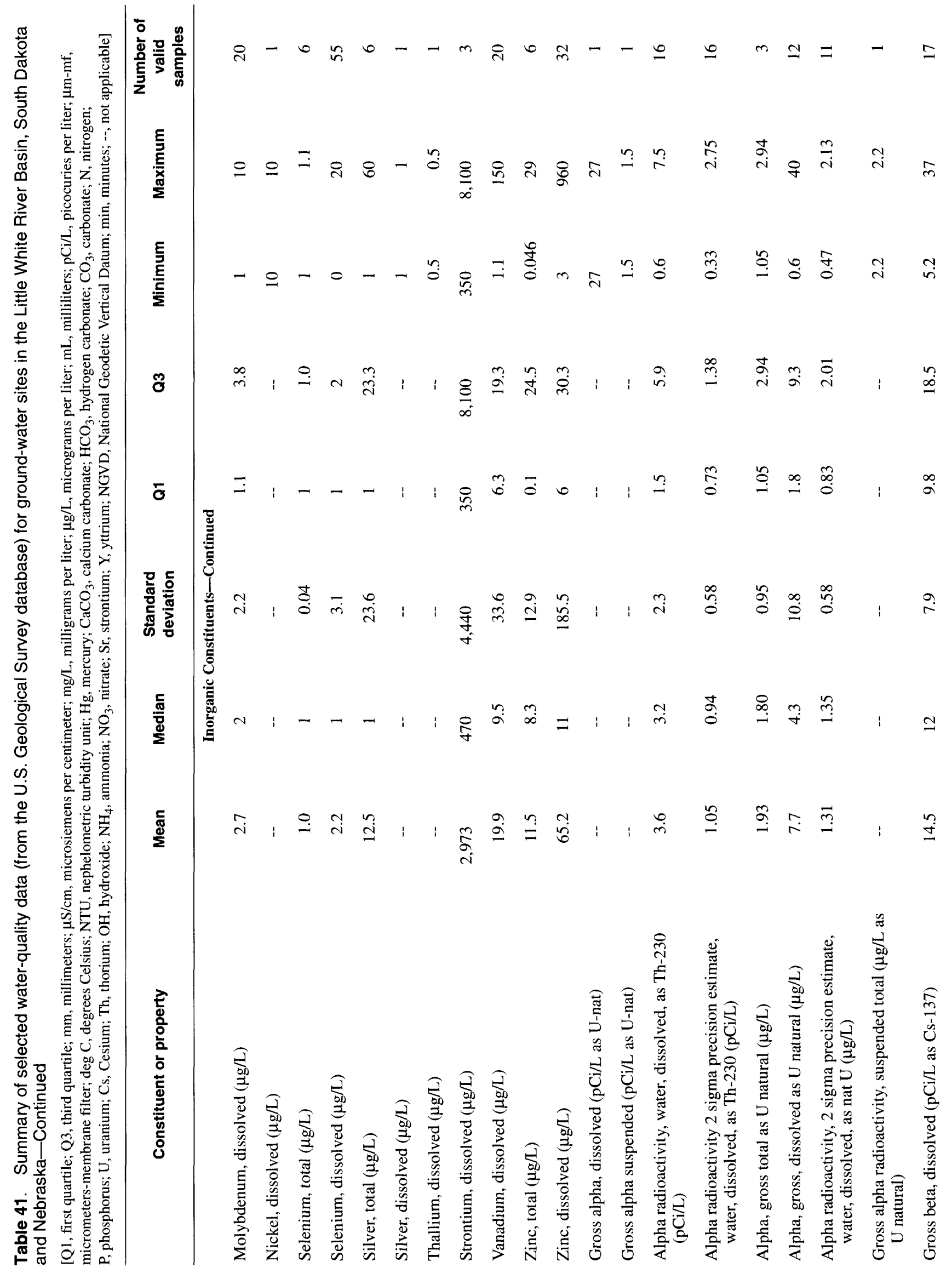




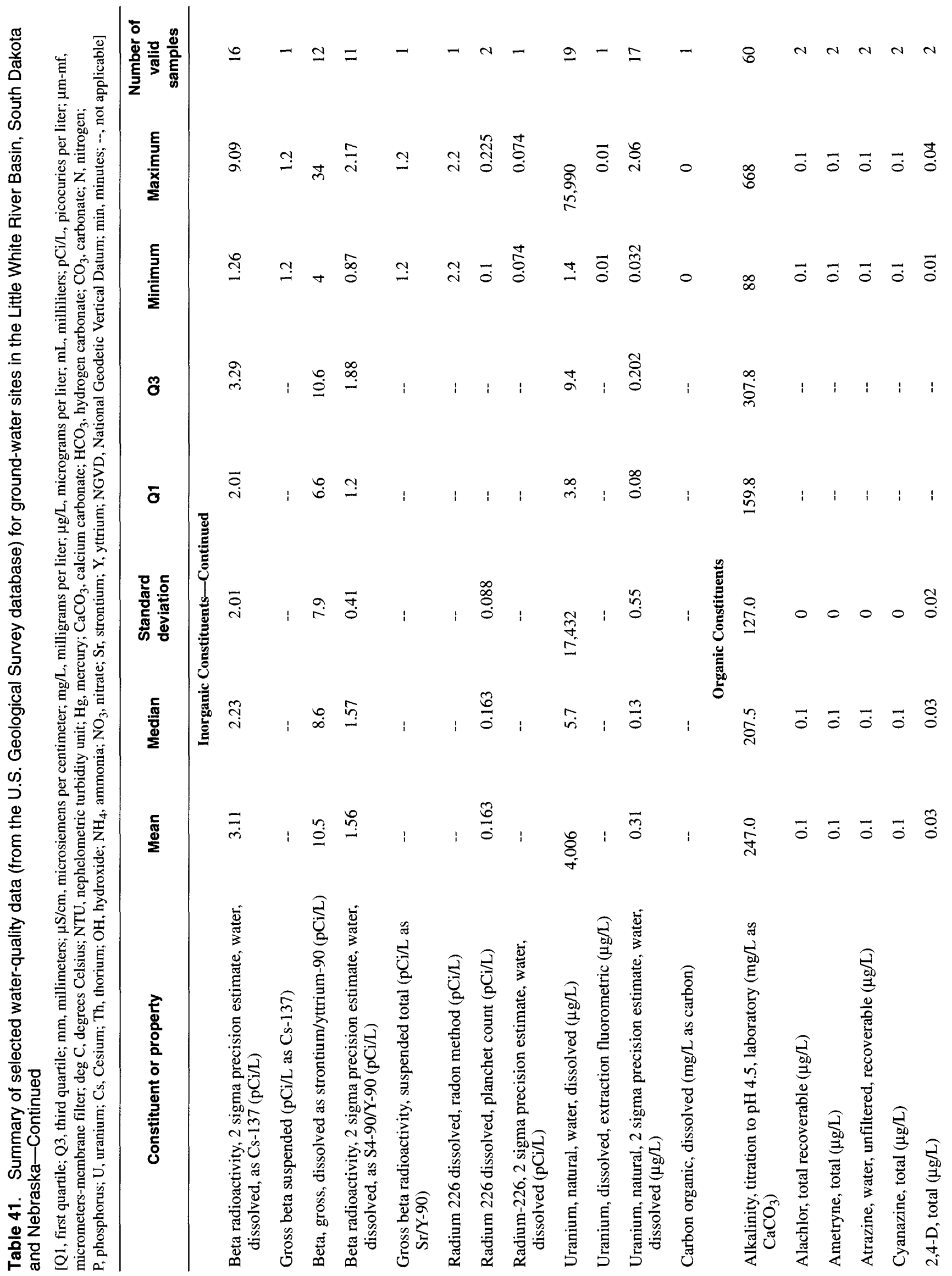




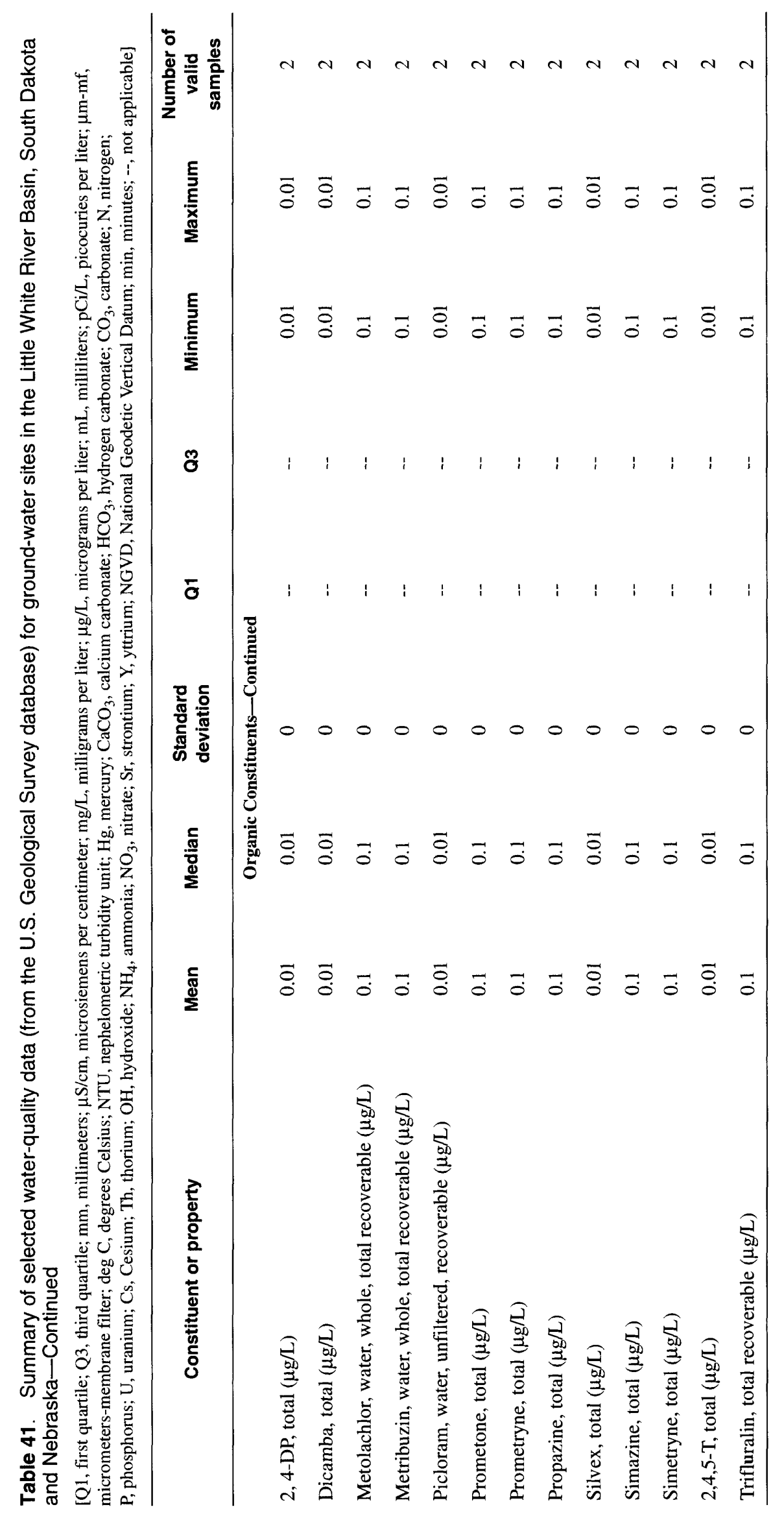




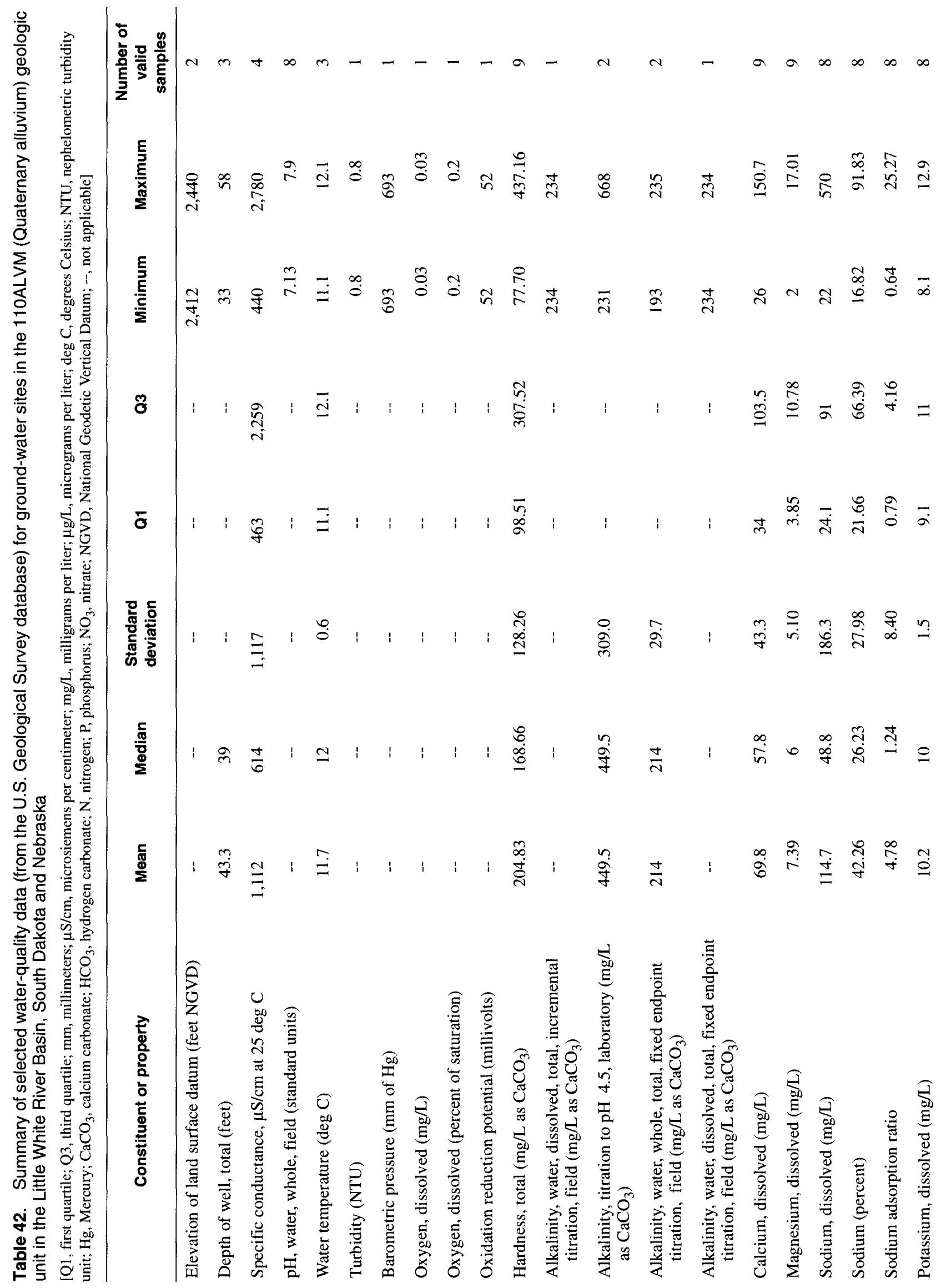




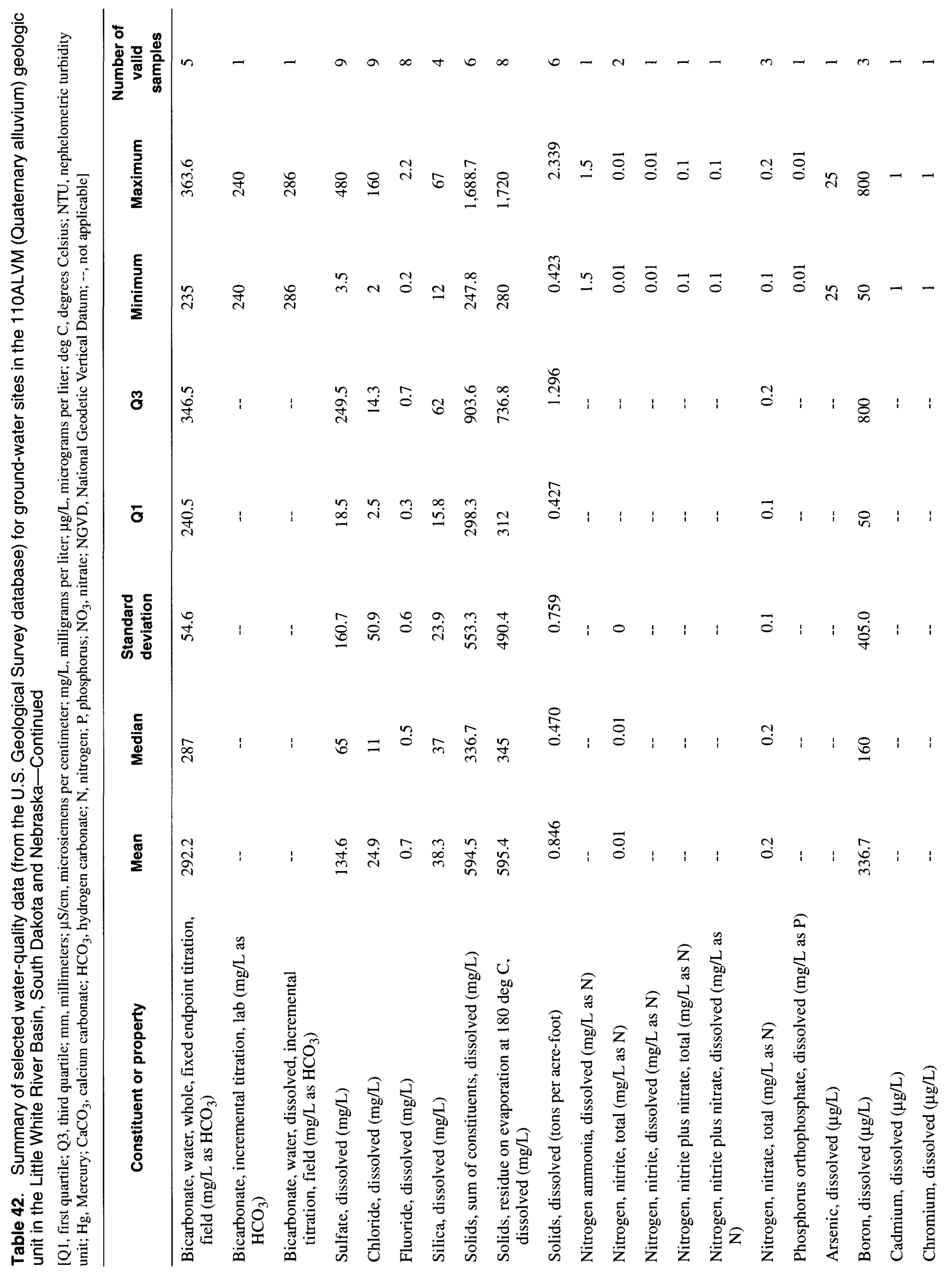




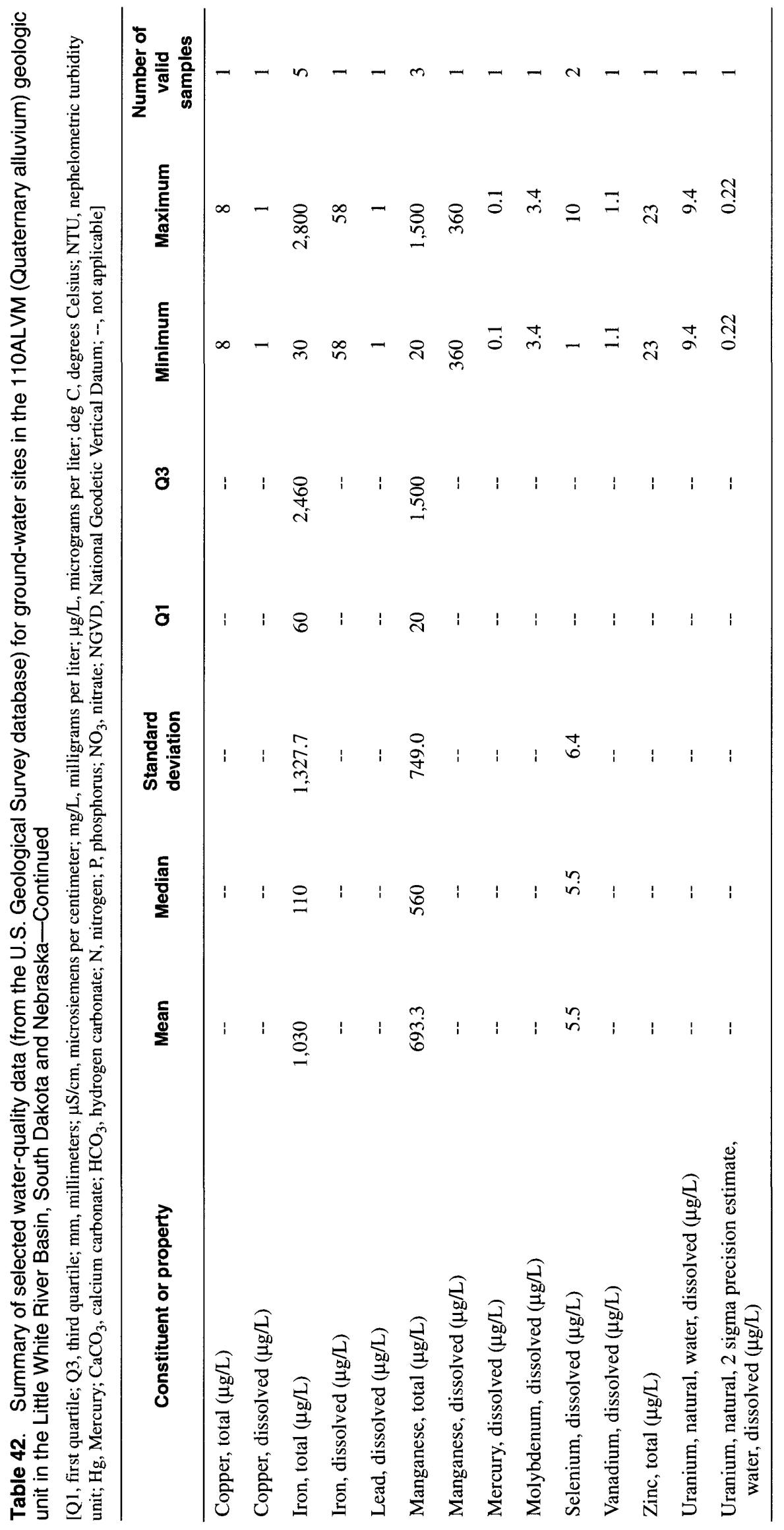




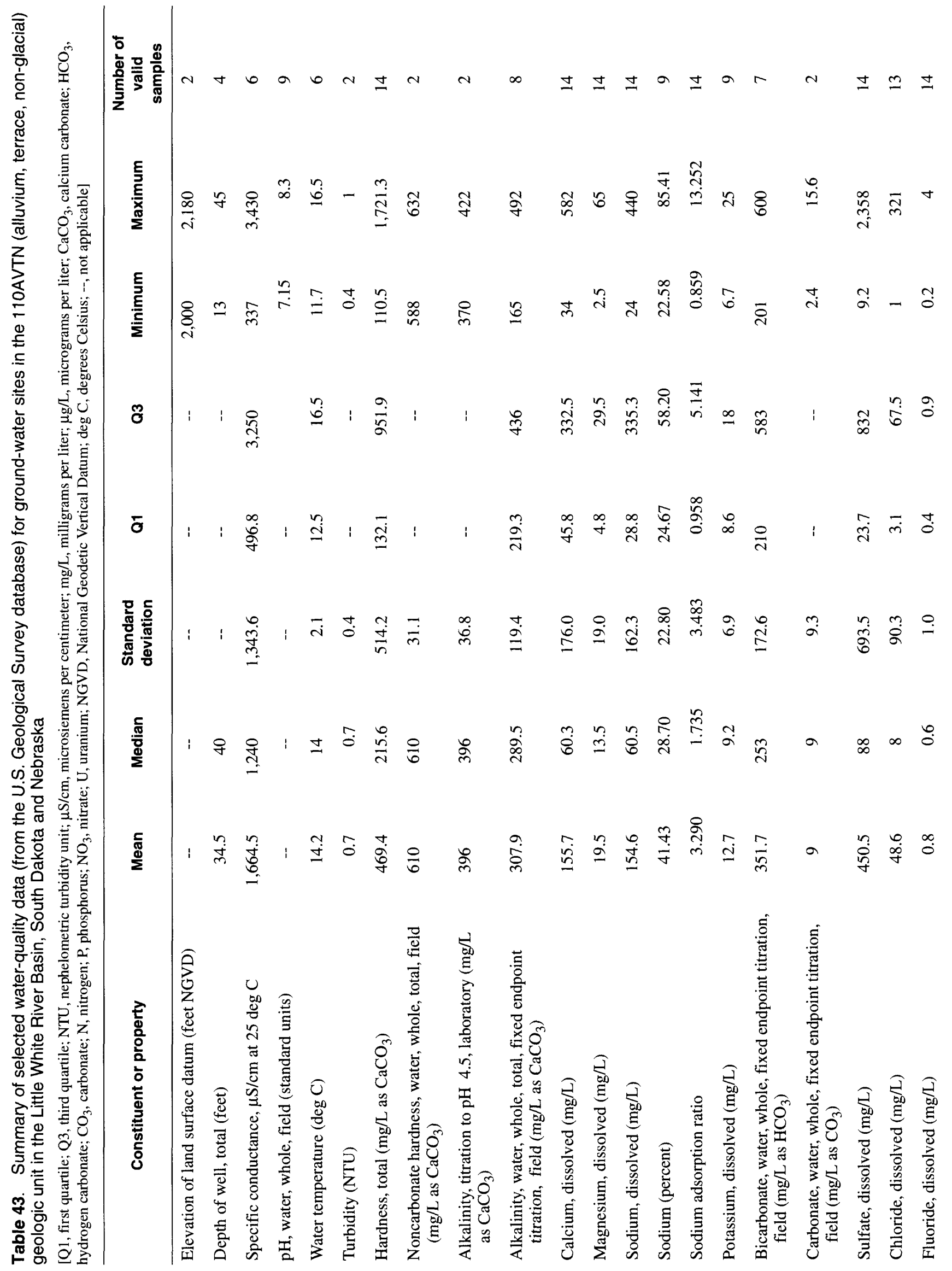




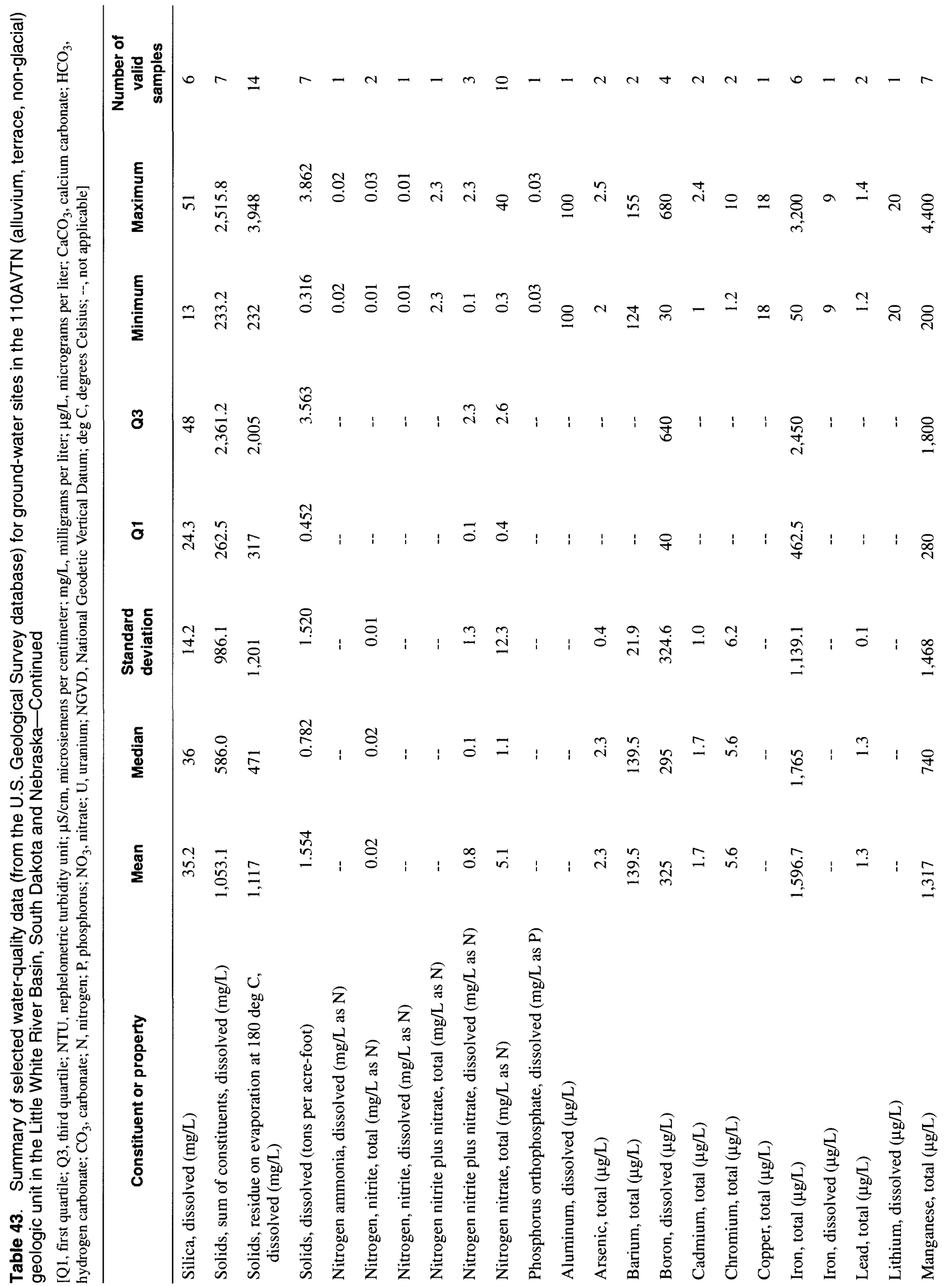




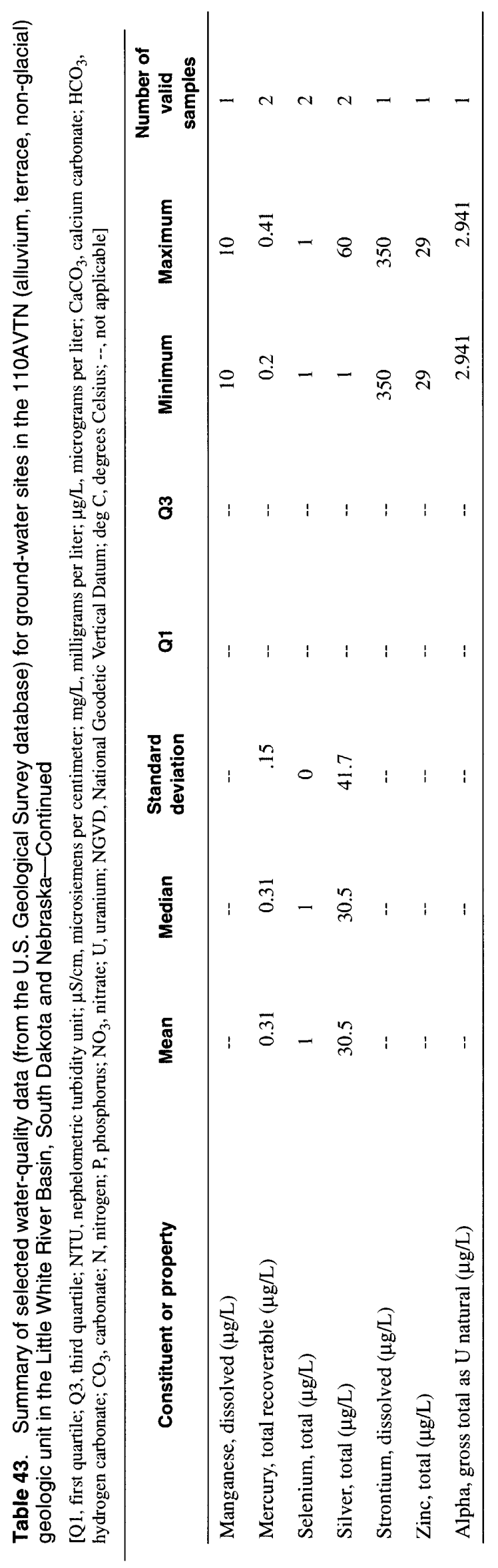

Table 43

153 


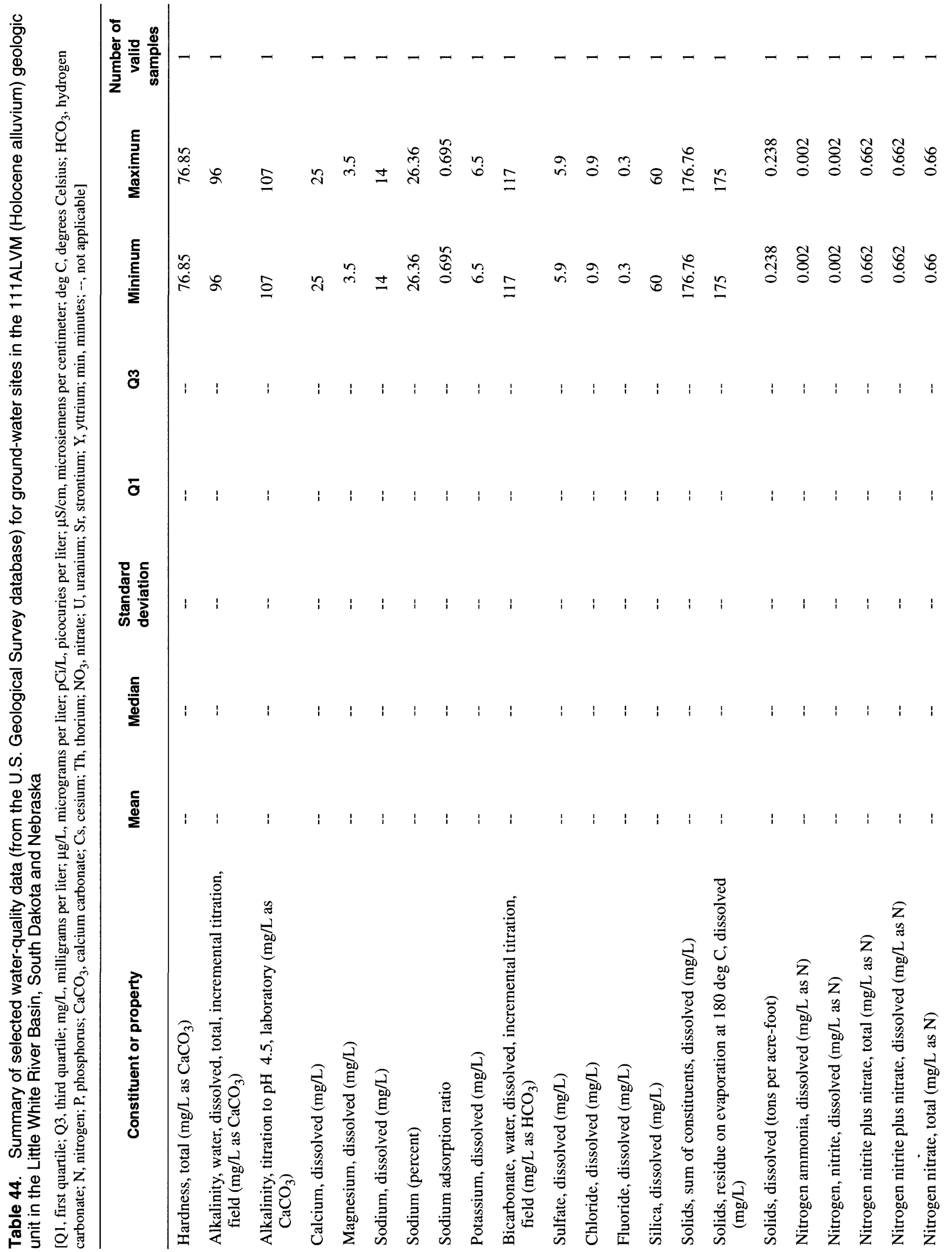




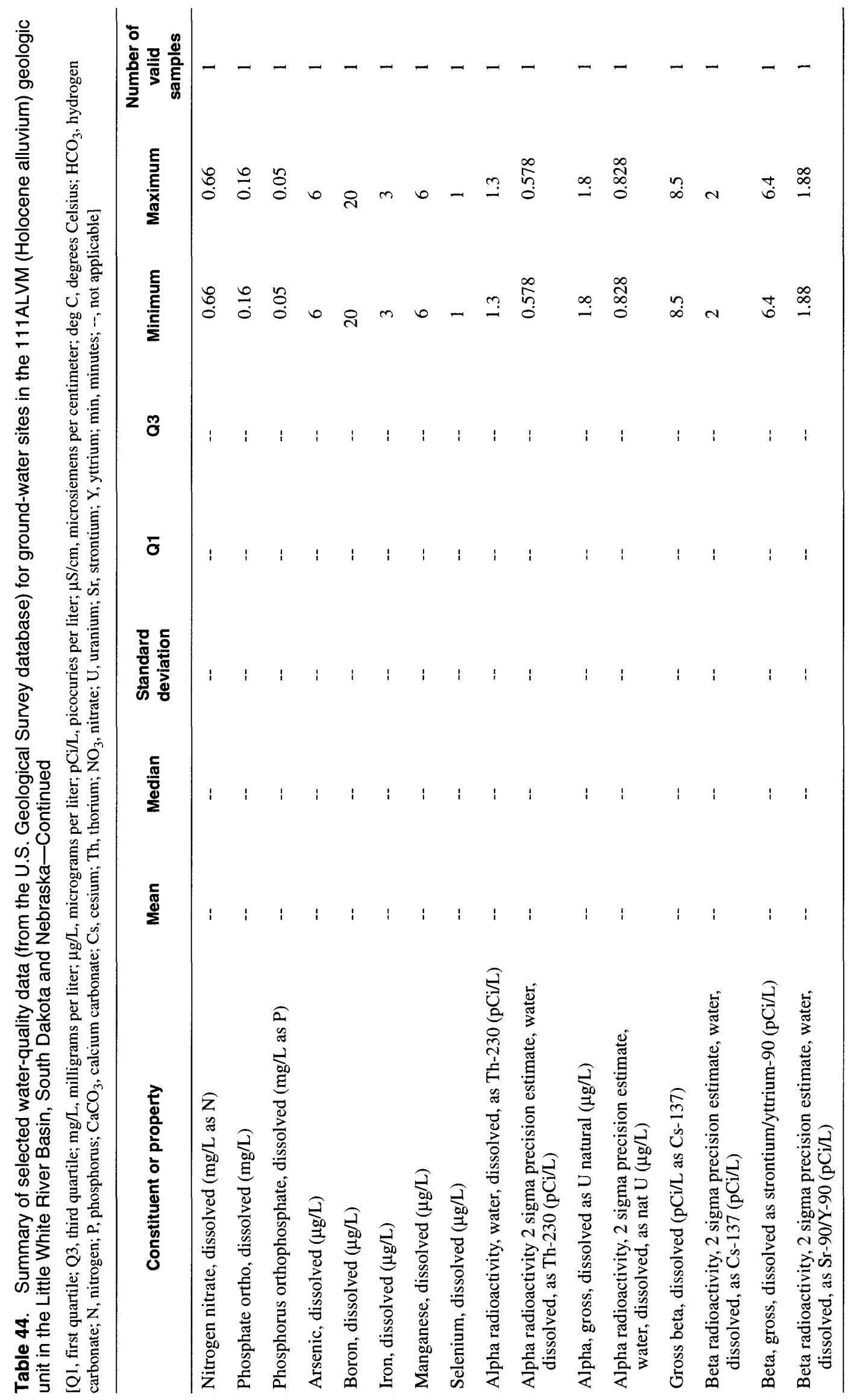




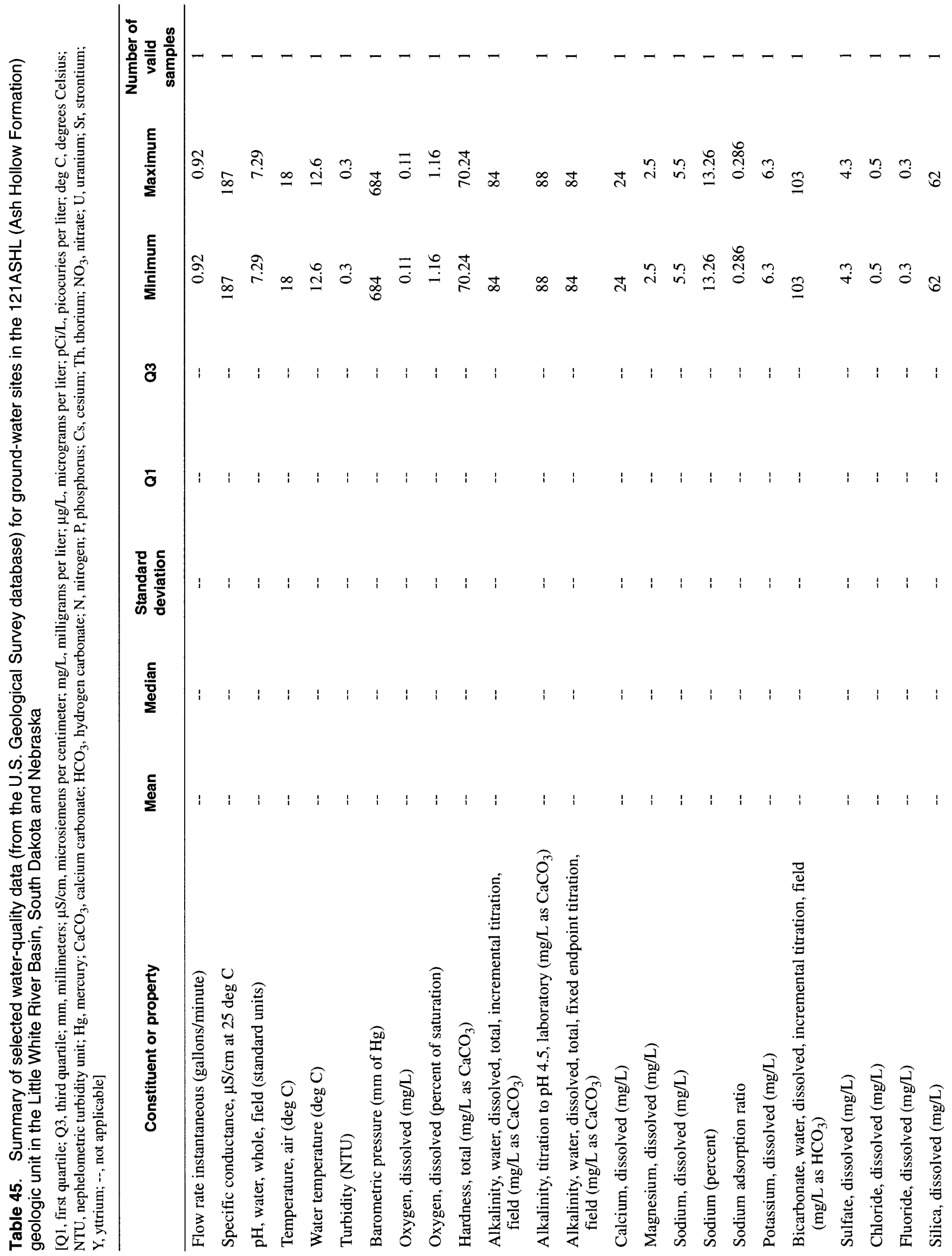




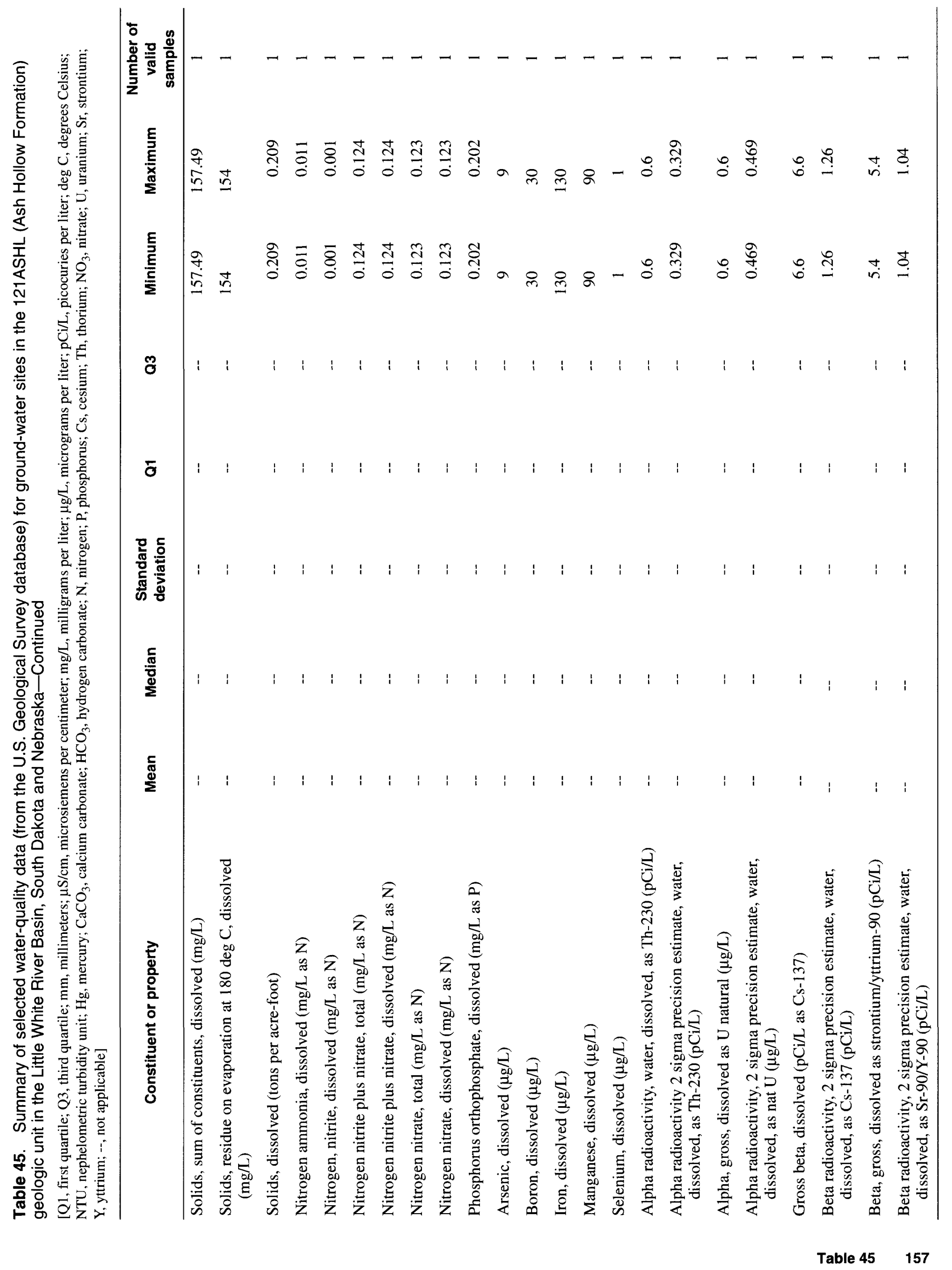




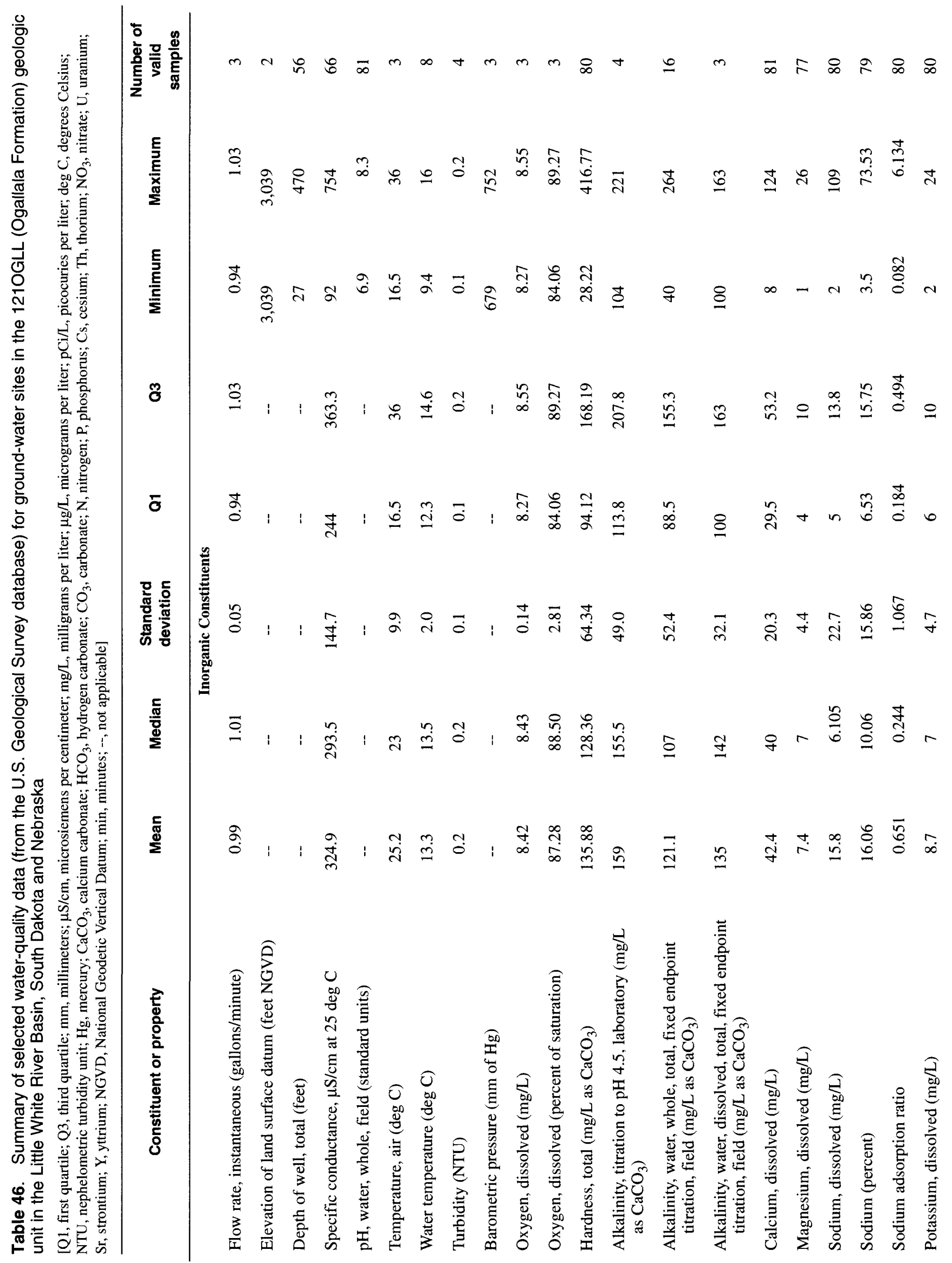




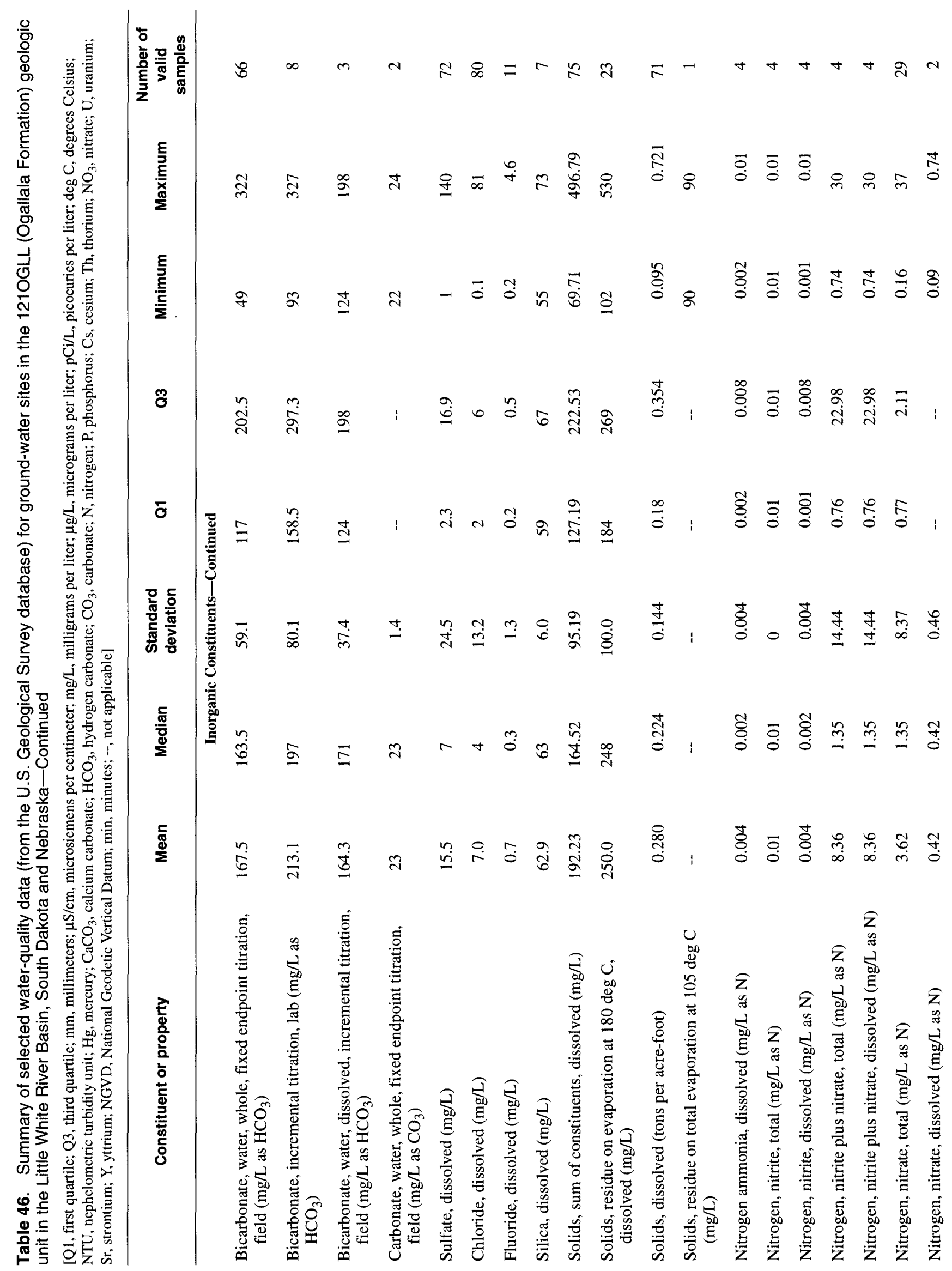




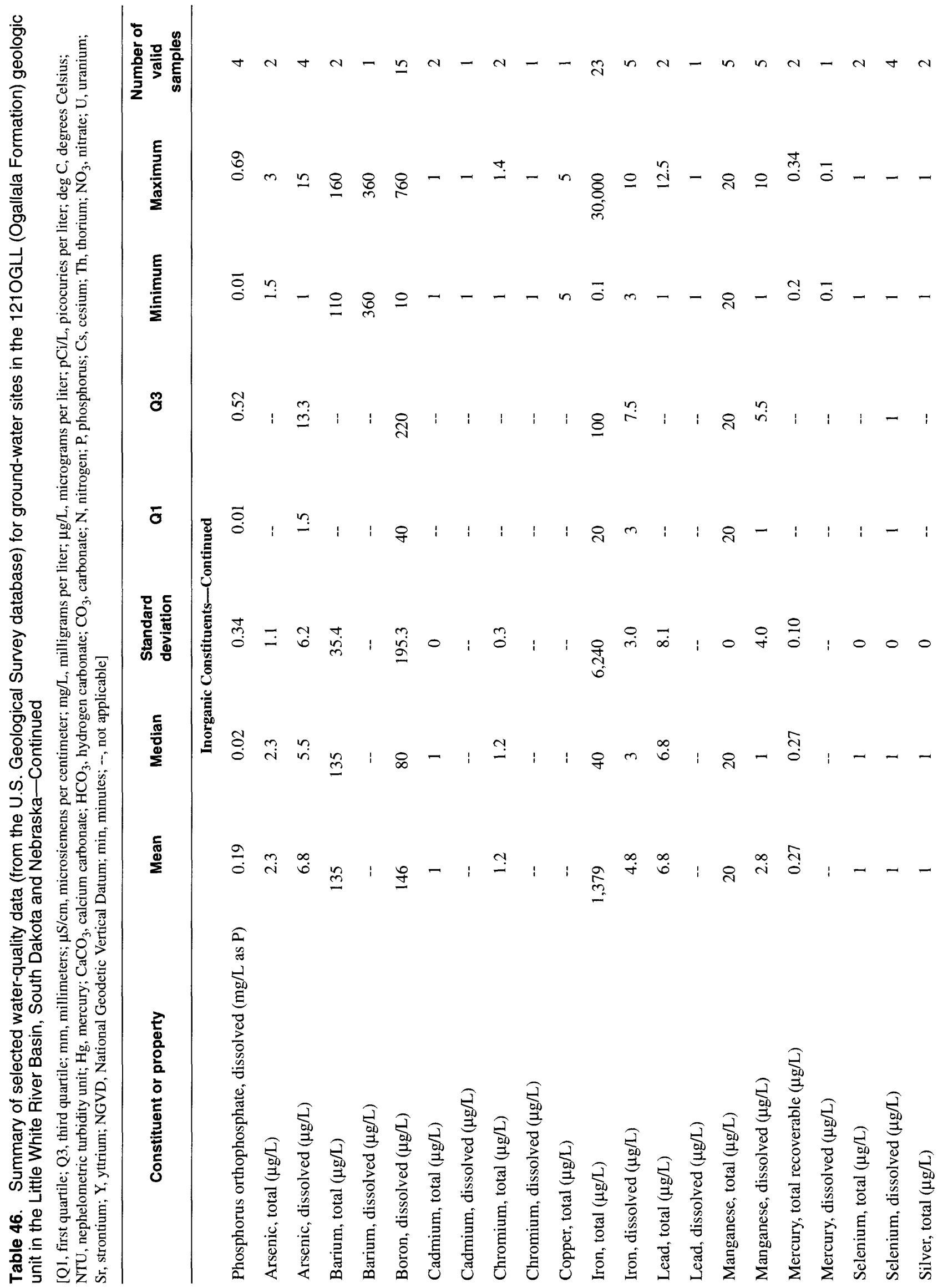




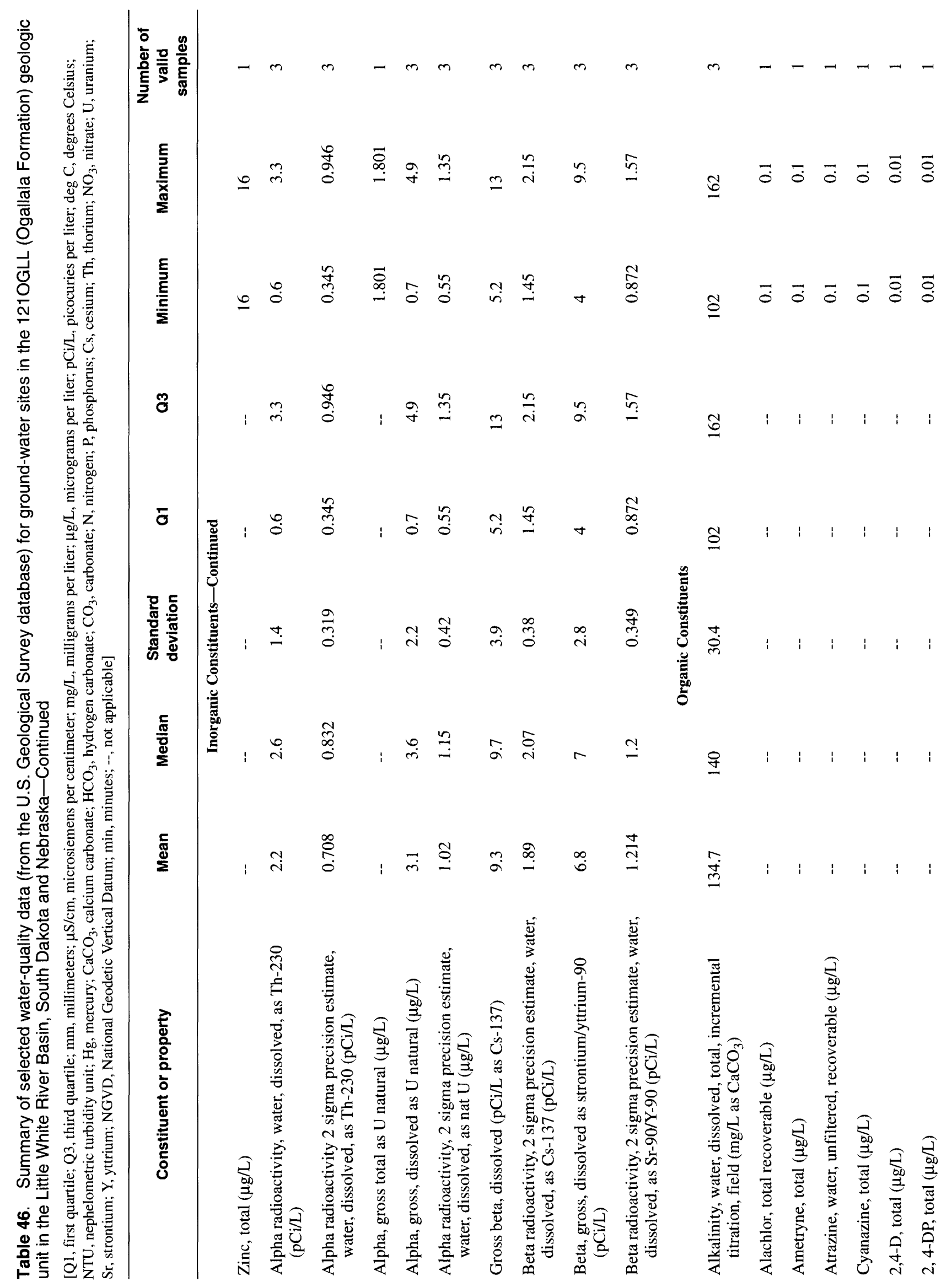




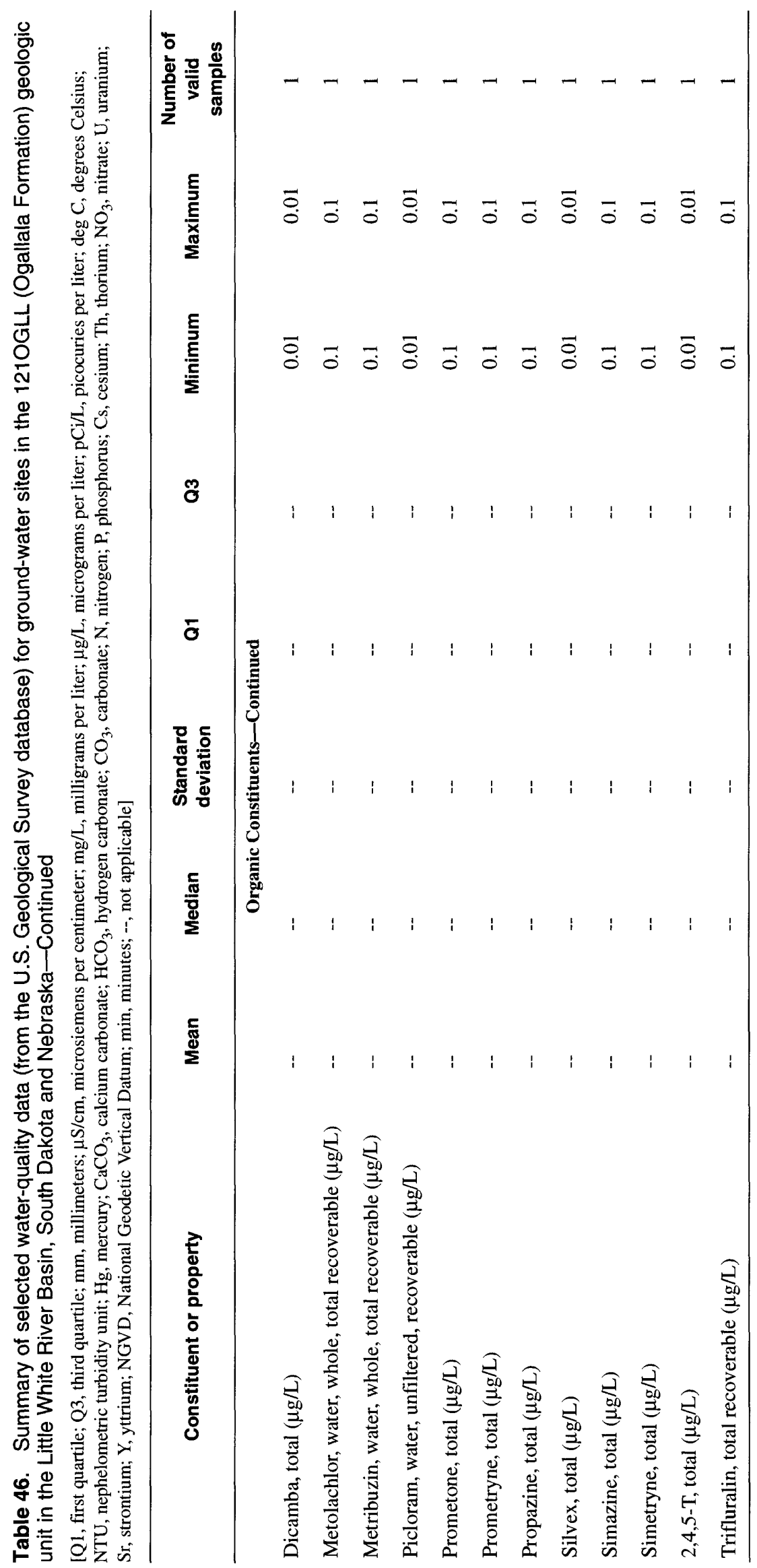




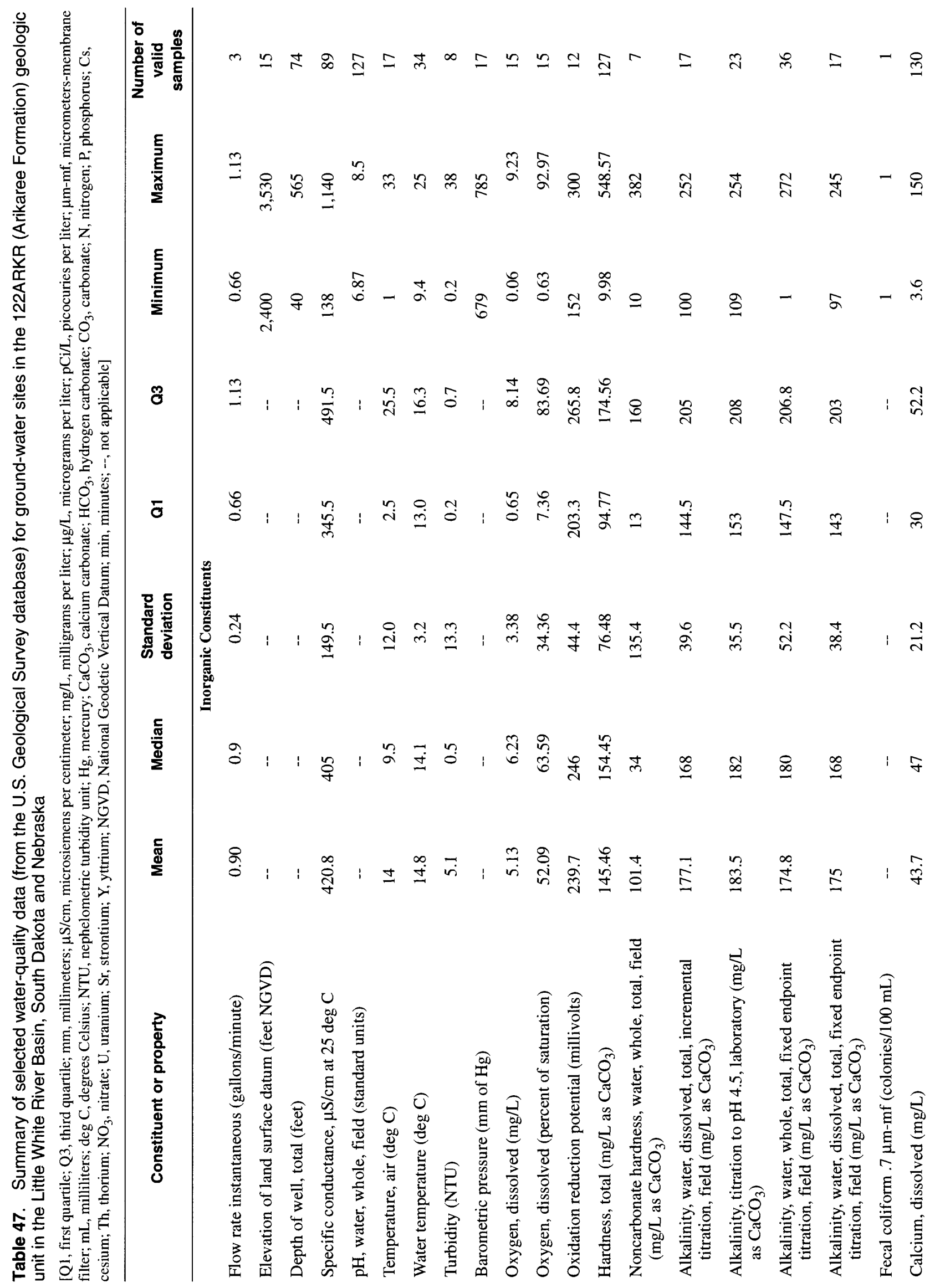




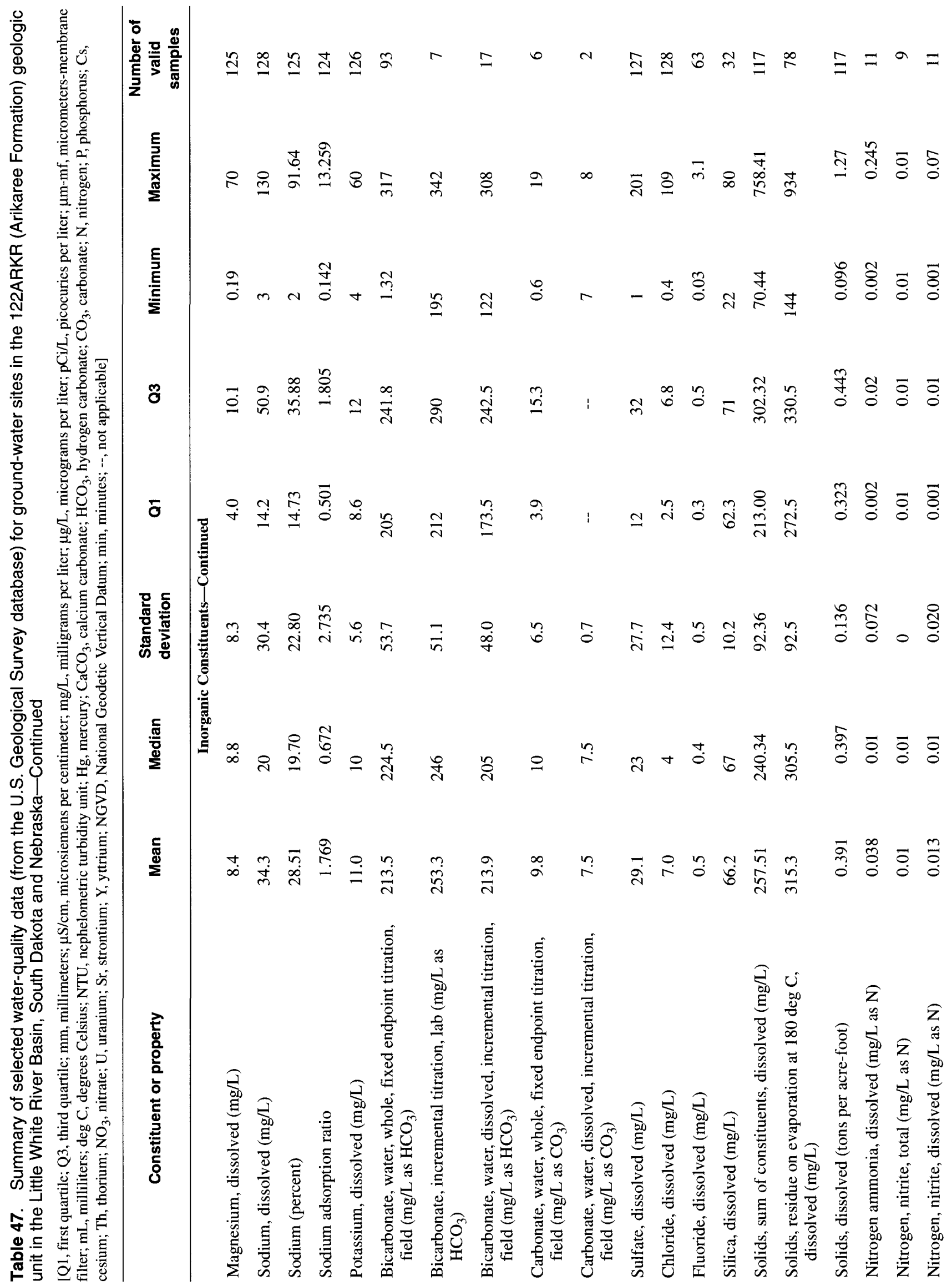




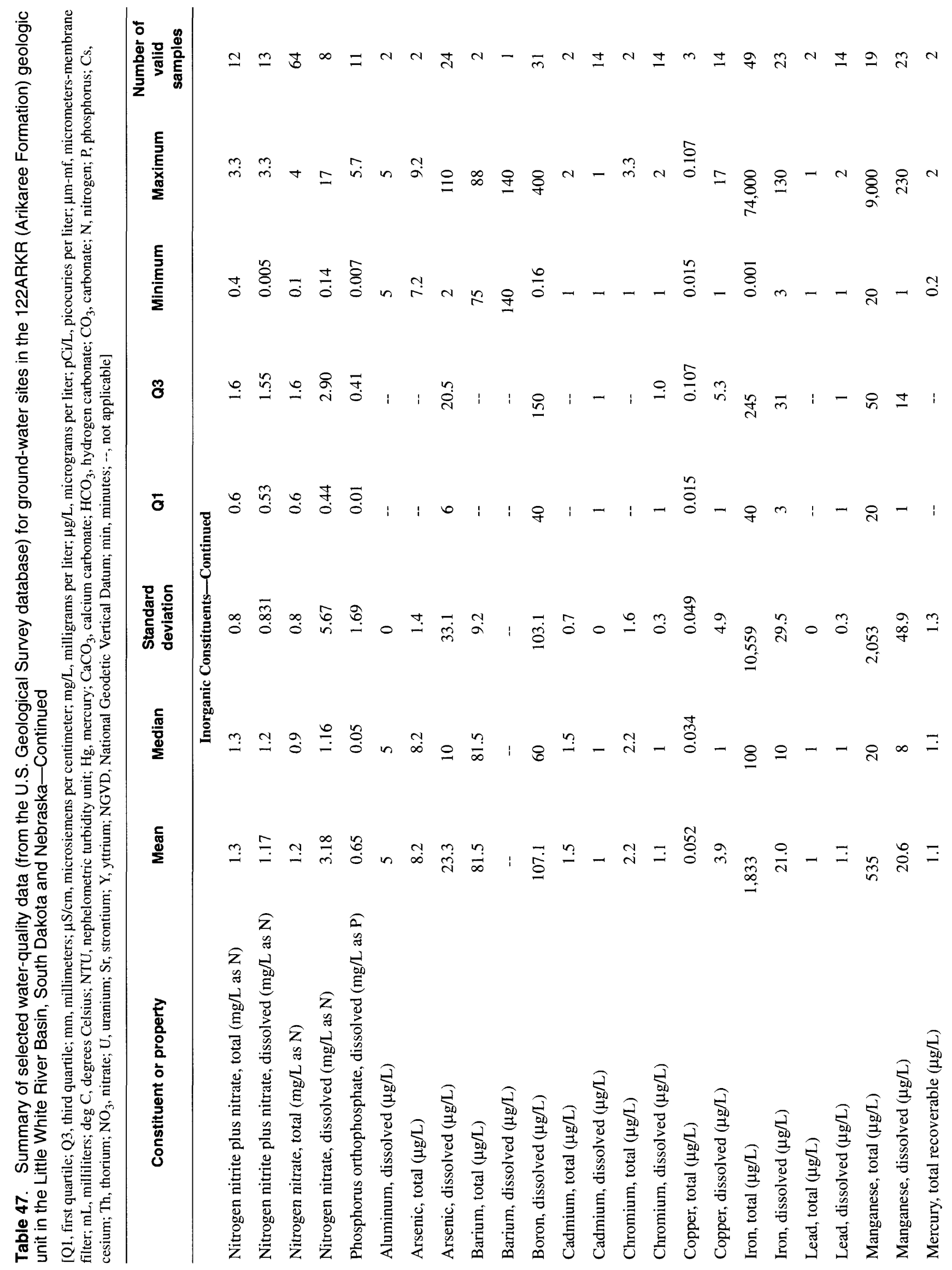




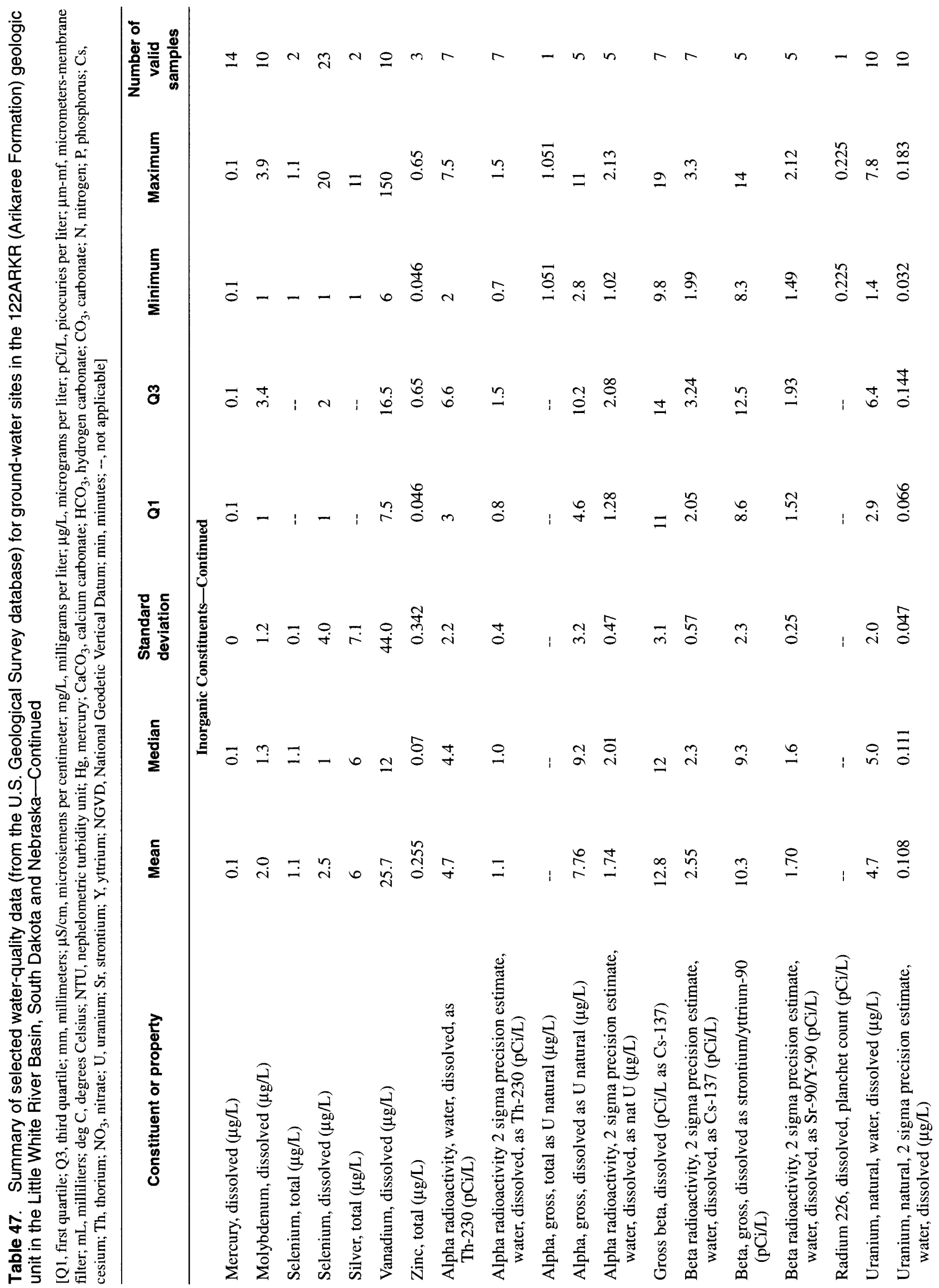




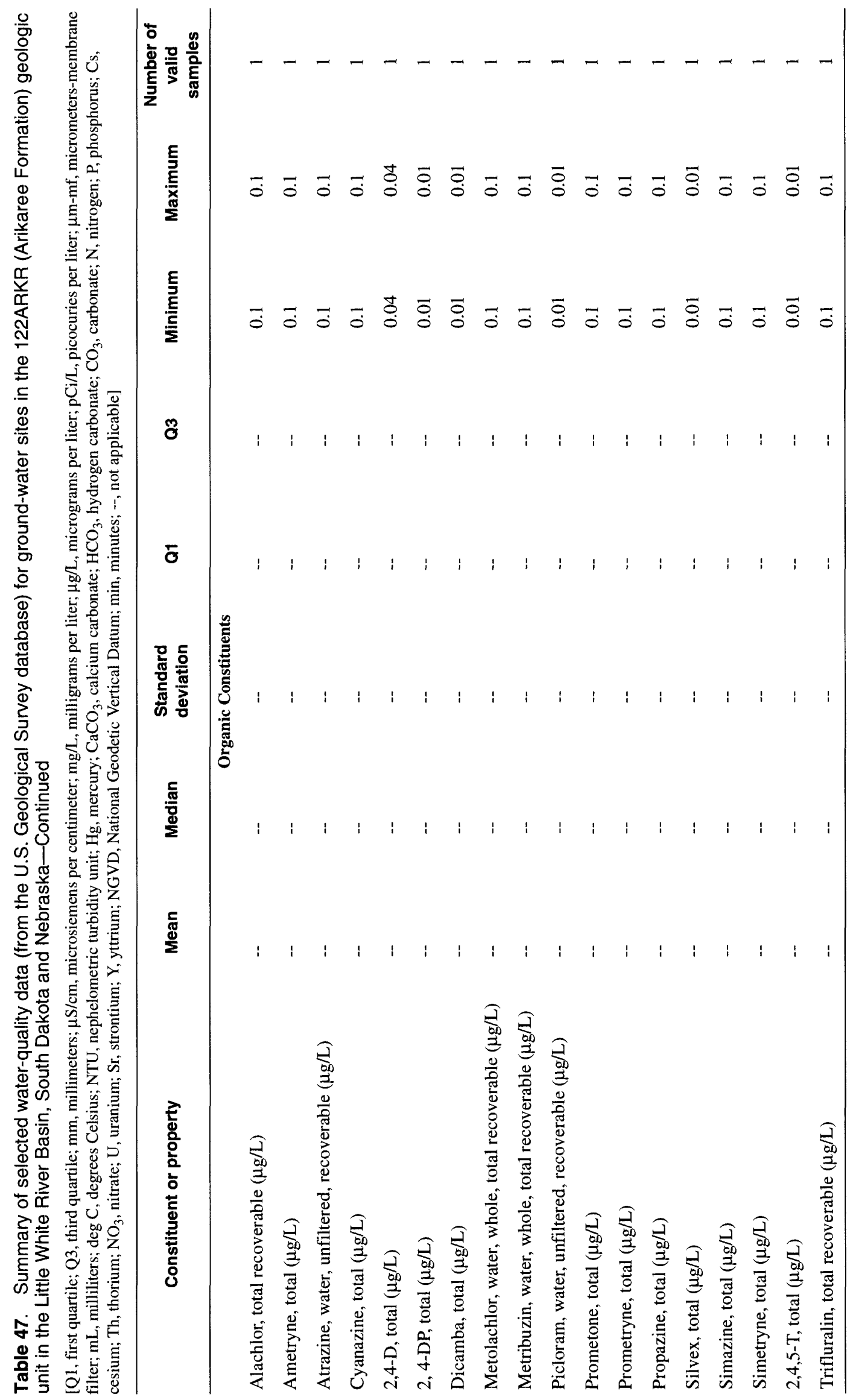




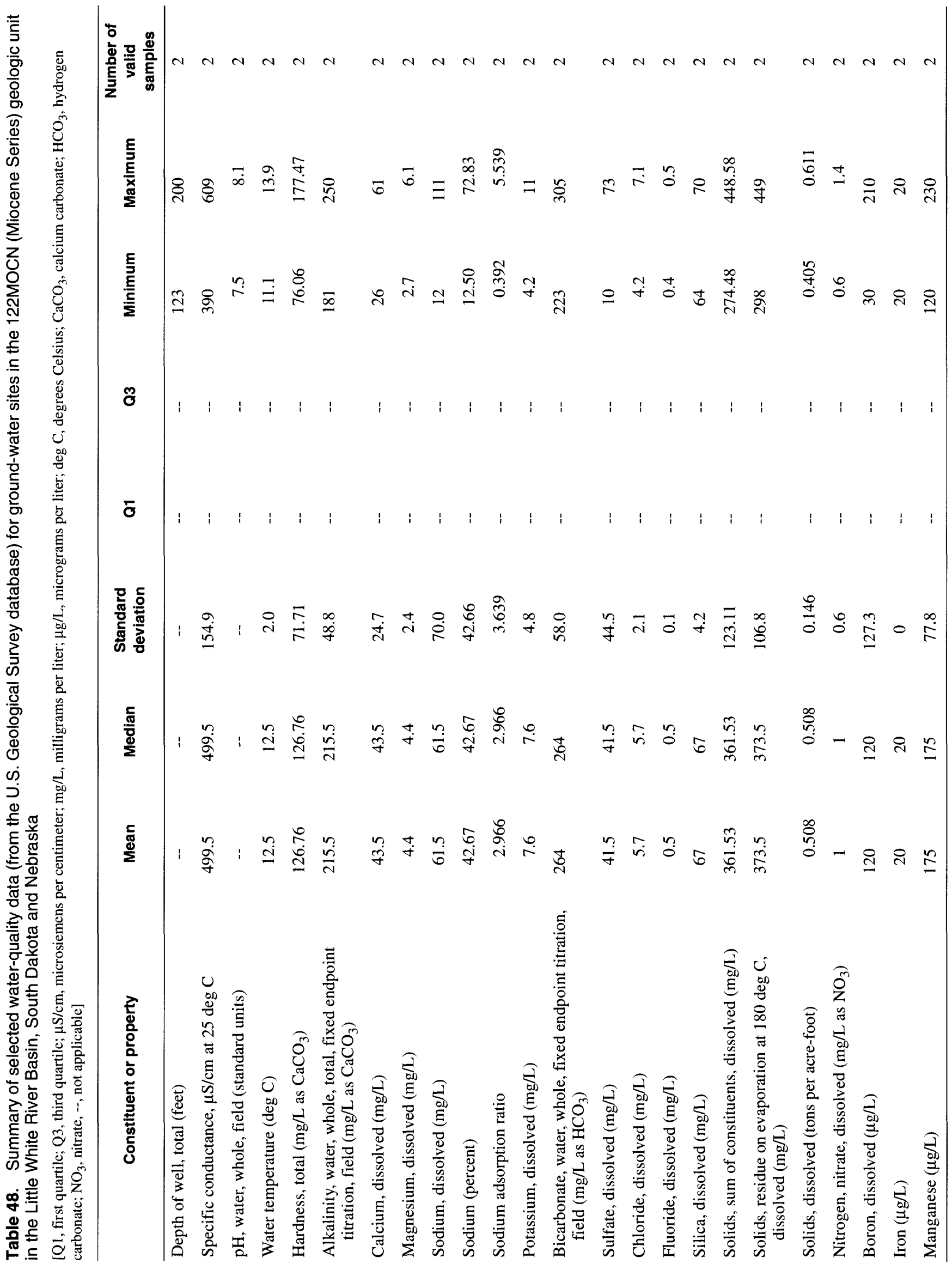




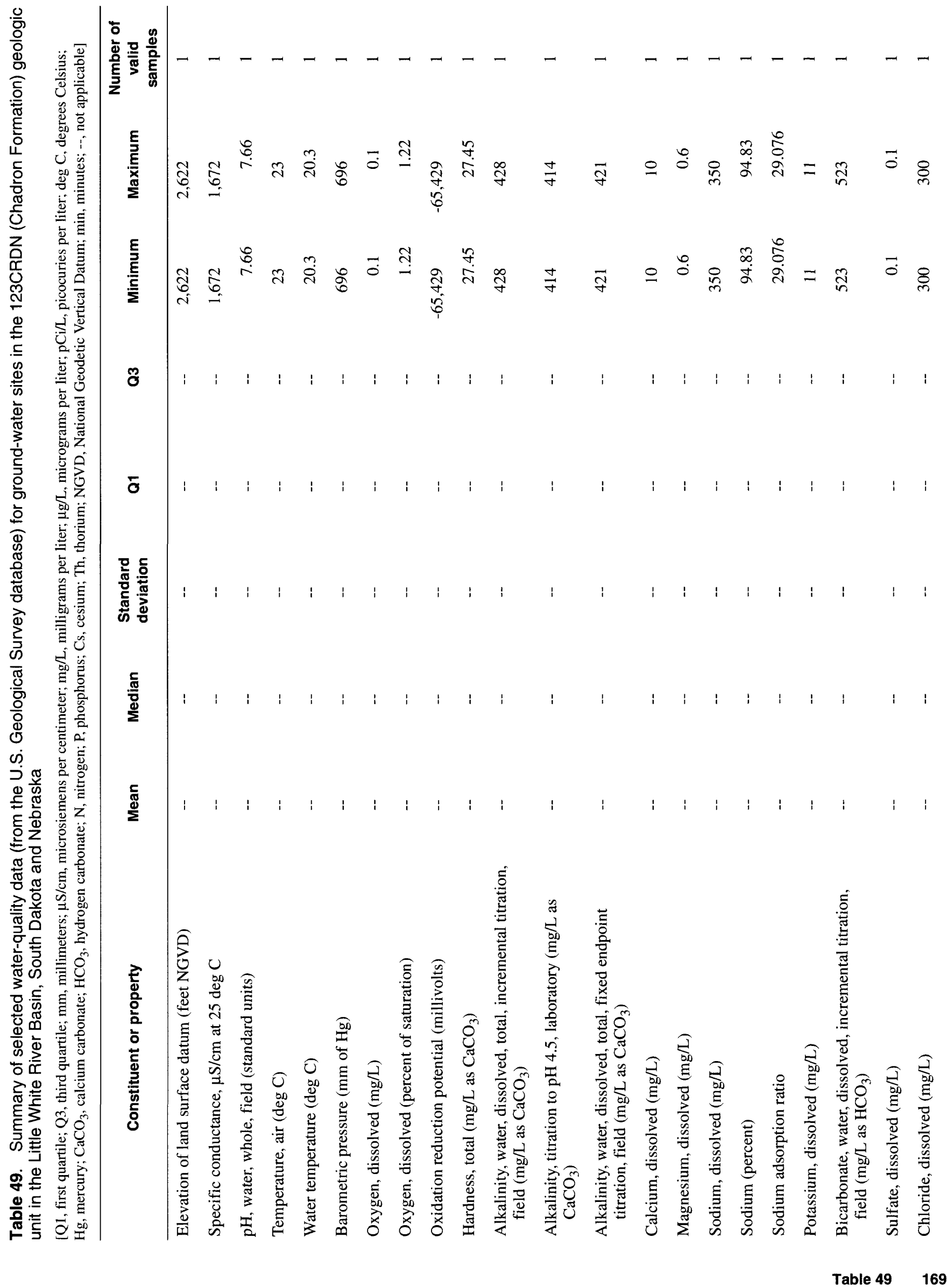




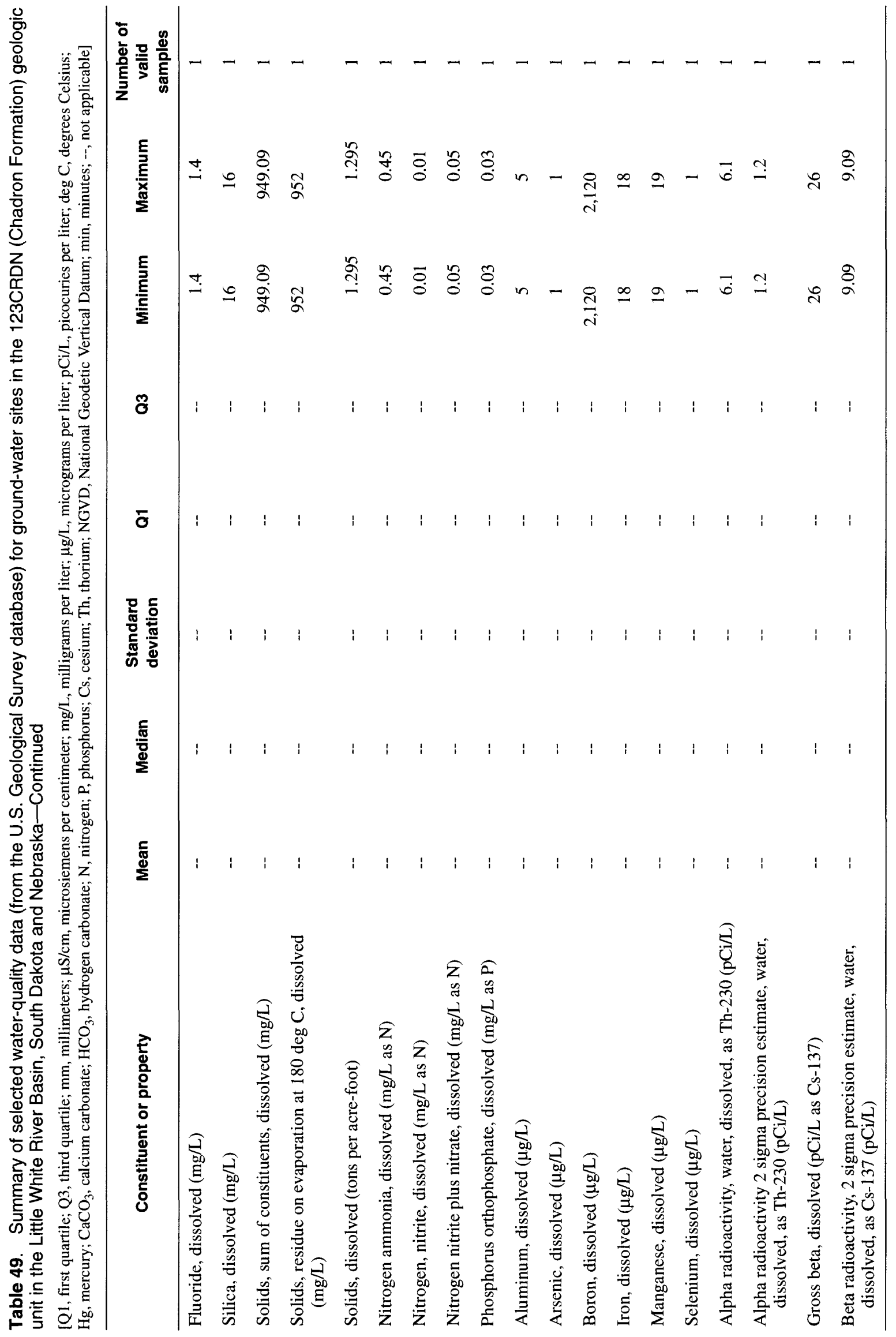




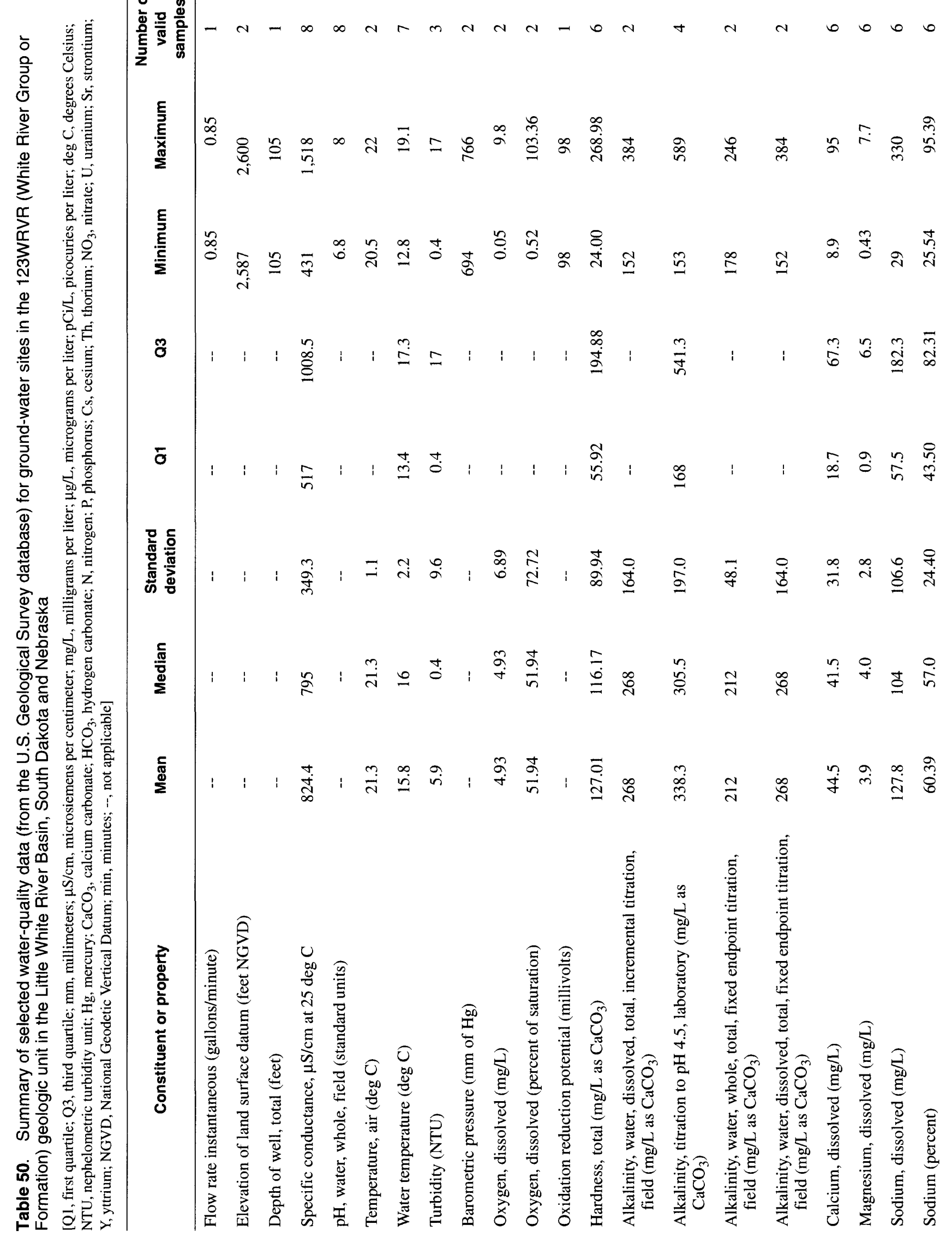




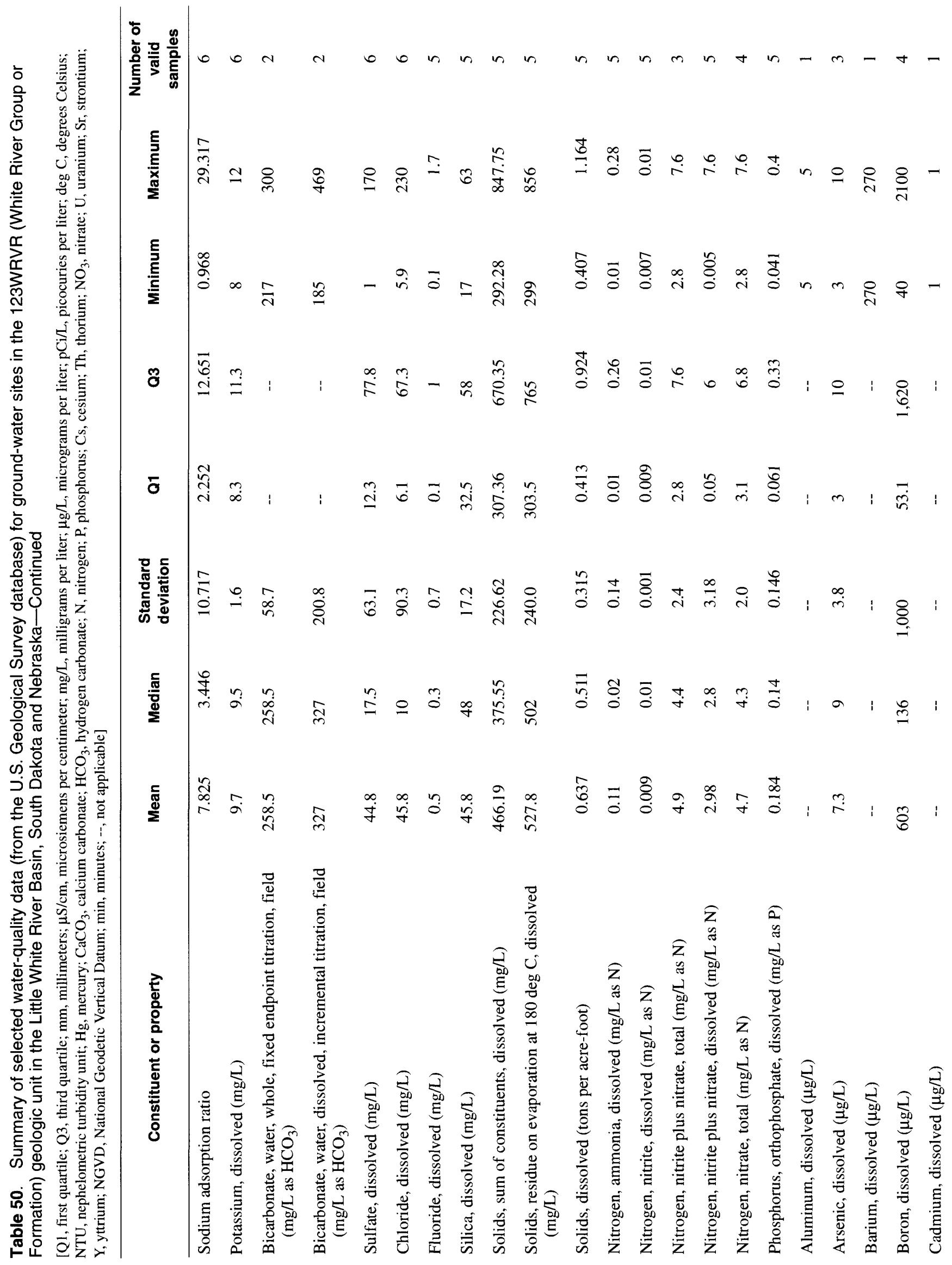




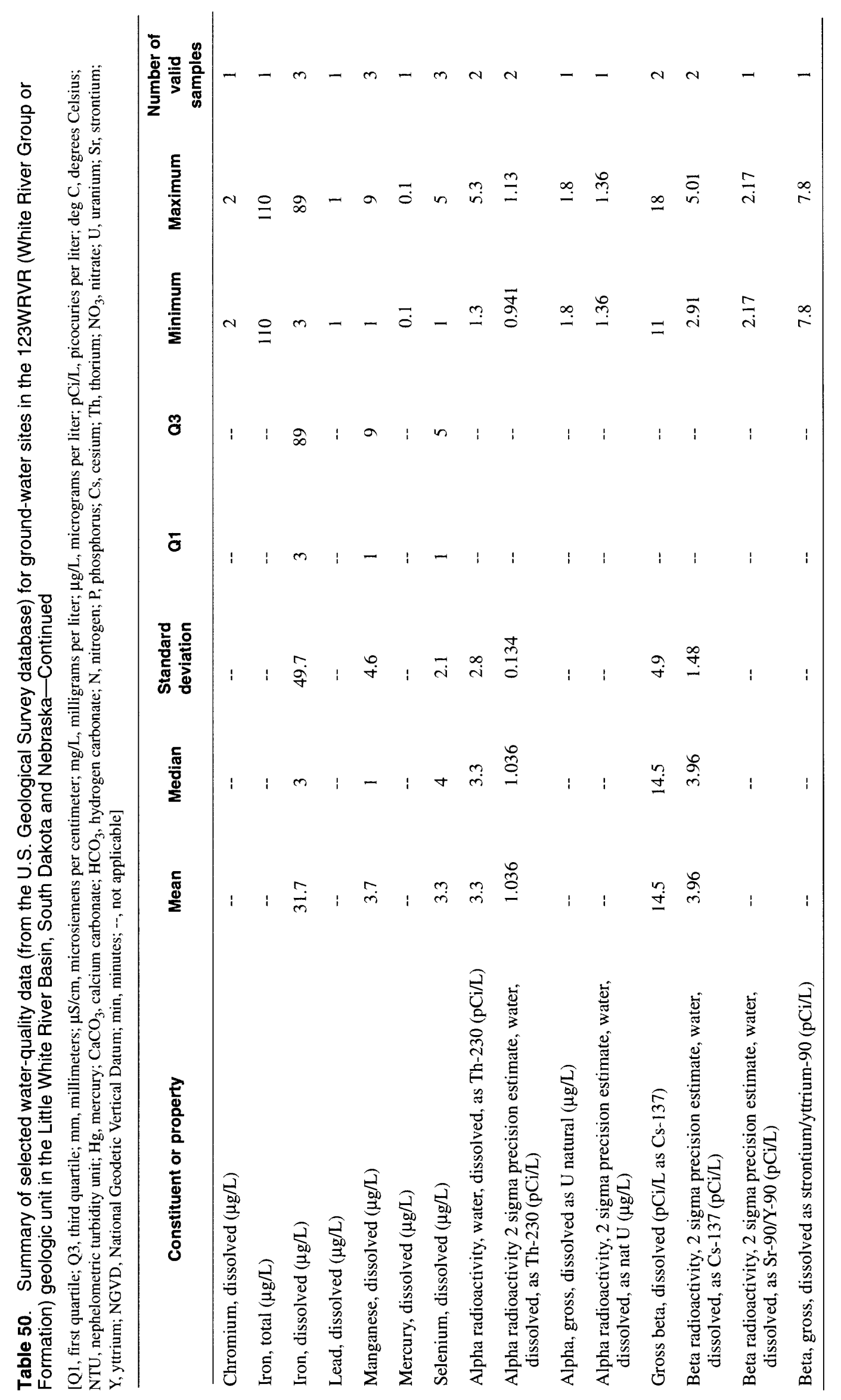




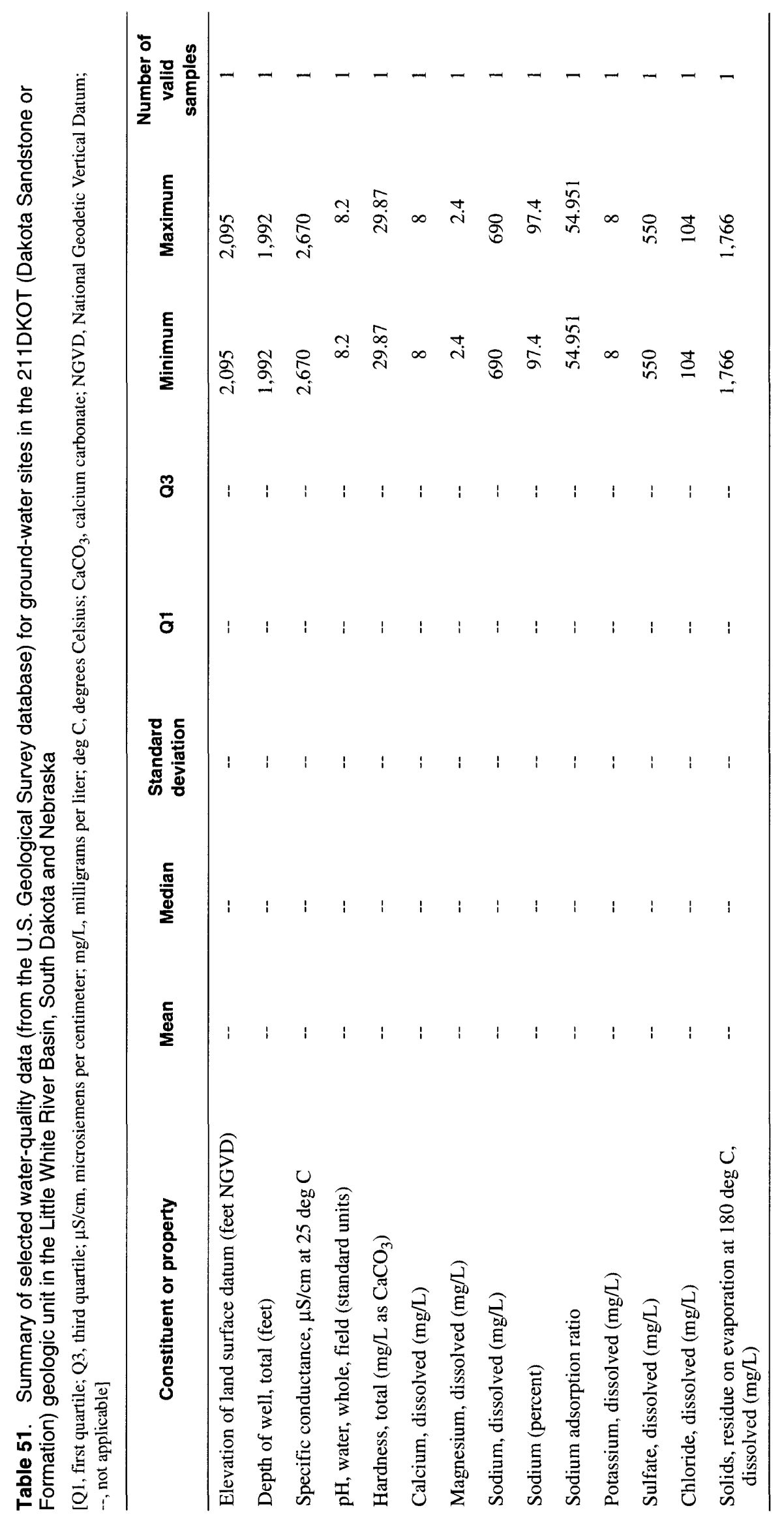

174 Summary of Water-Resources Data within the Little White River Basin, South Dakota and Nebraska 


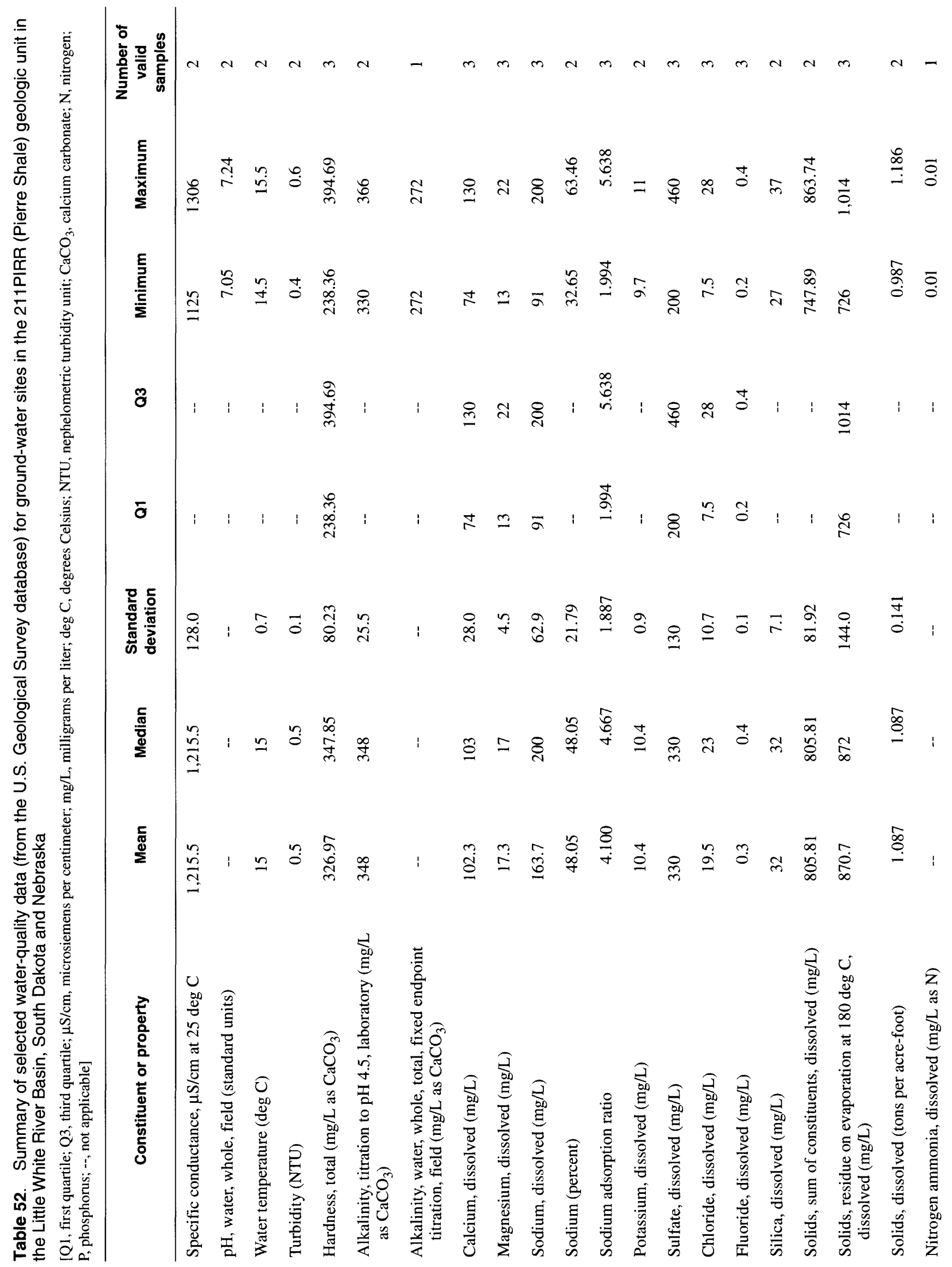




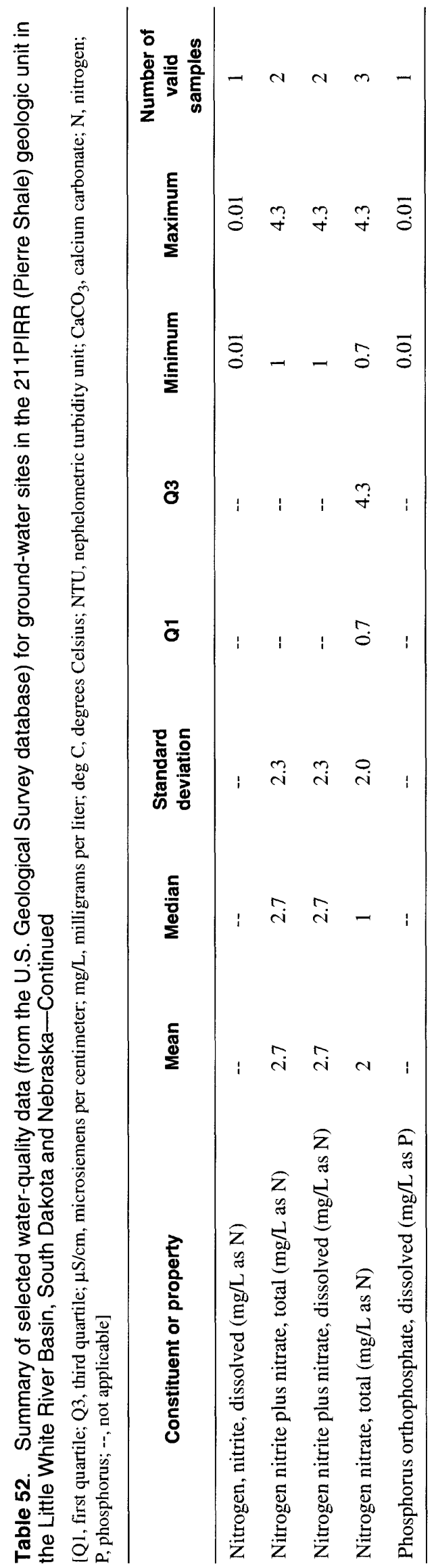




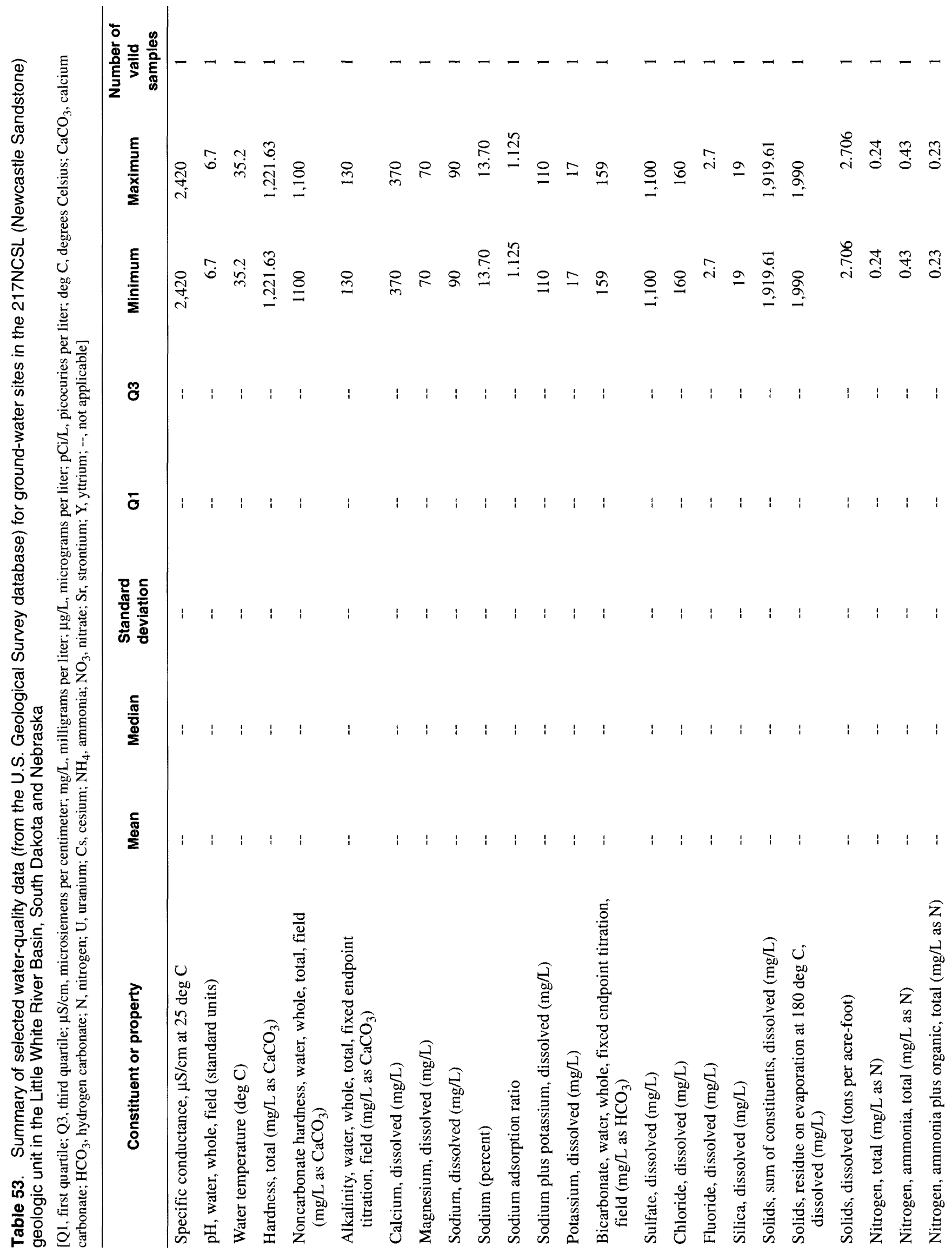




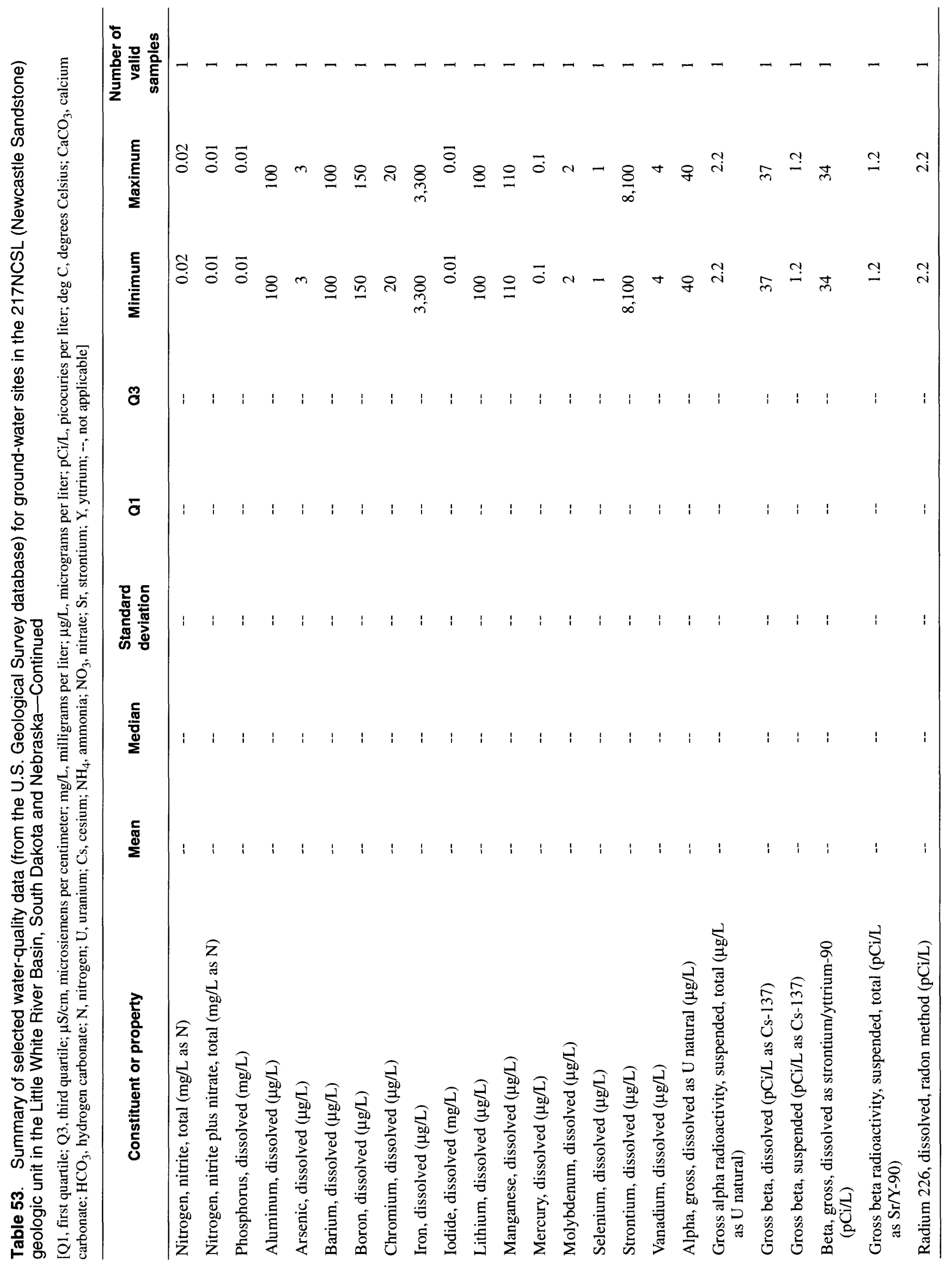




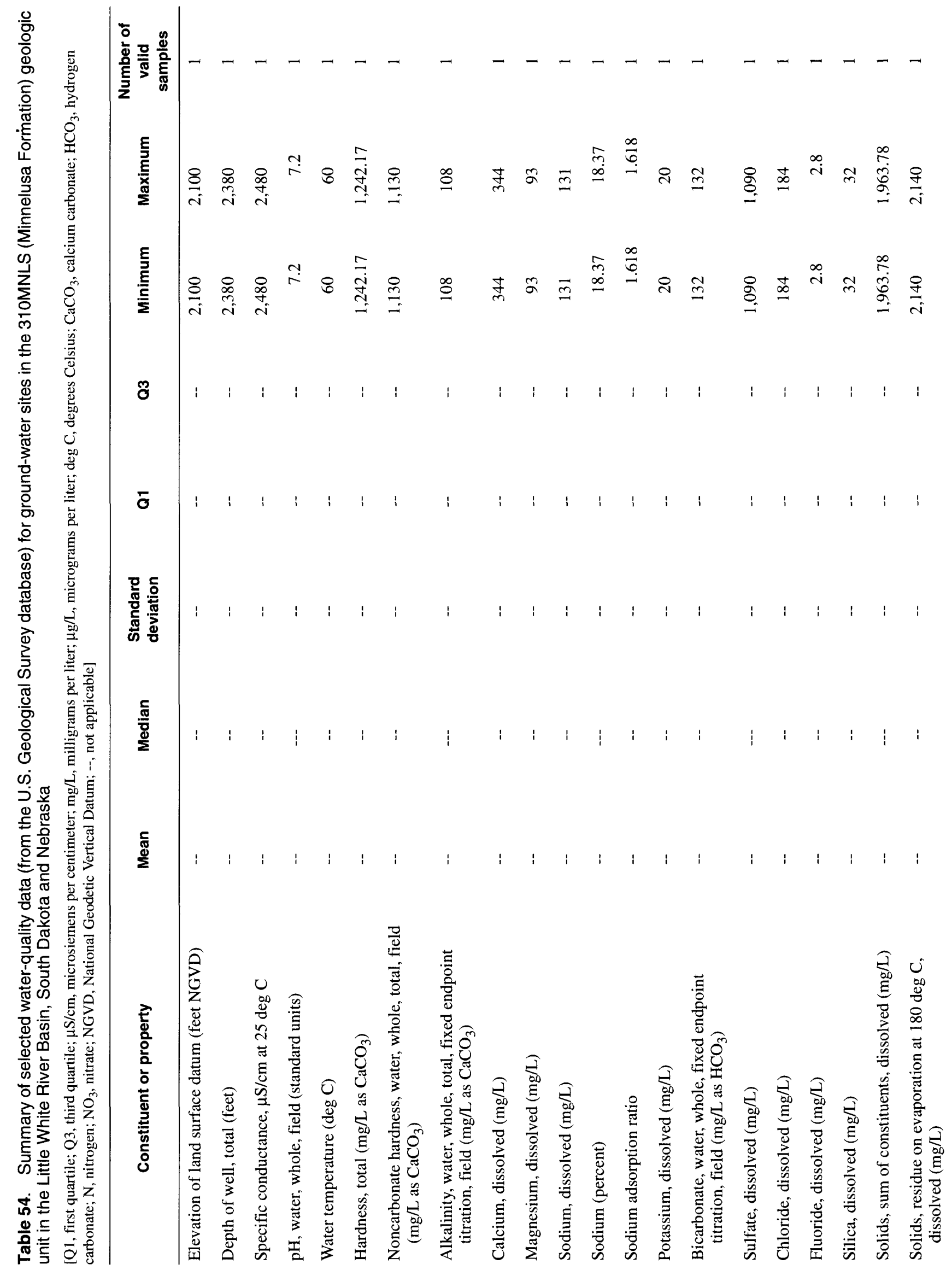




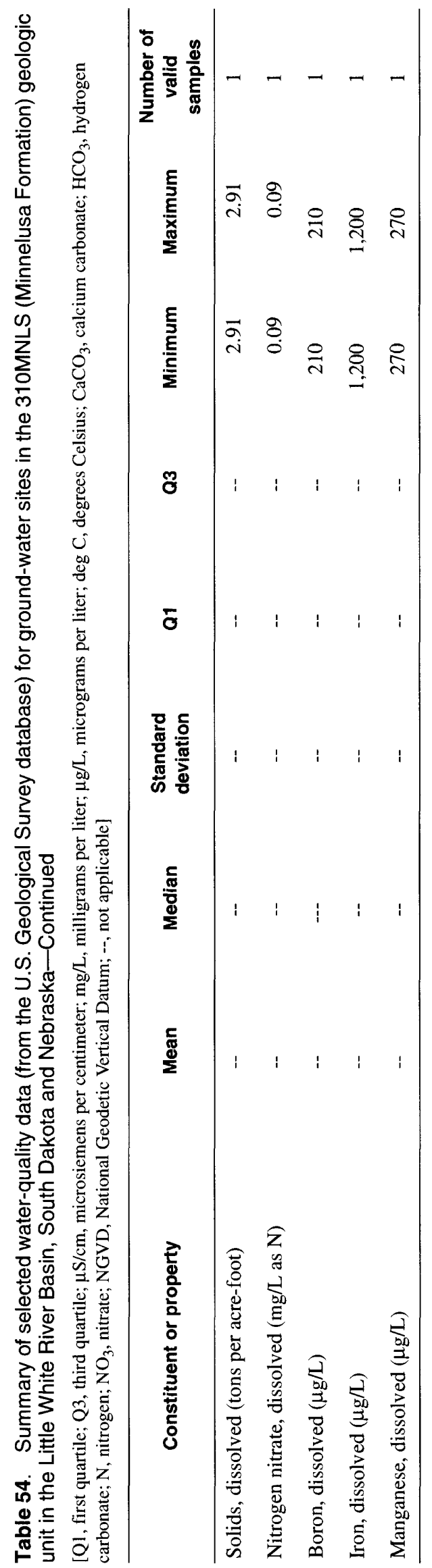

180 Summary of Water-Resources Data within the Little White River Basin, South Dakota and Nebraska 
Table 55. Summary of daily ground-water-level data (from the U.S. Geological Survey database), in feet below land surface, for two sites in the High Plains aquifer in South Dakota

\begin{tabular}{|c|c|c|c|c|c|c|}
\hline Site number & Month & Mean & $\begin{array}{l}\text { Standard } \\
\text { deviation }\end{array}$ & Minimum & Maximum & $\begin{array}{l}\text { Number of } \\
\text { valid } \\
\text { samples }\end{array}$ \\
\hline \multirow[t]{12}{*}{430027102311801} & January & 42.92 & 0.07 & 42.78 & 43.08 & 155 \\
\hline & February & 42.92 & .07 & 42.79 & 43.05 & 141 \\
\hline & March & 42.91 & .07 & 42.74 & 43.09 & 155 \\
\hline & April & 42.91 & .08 & 42.76 & 43.06 & 150 \\
\hline & May & 42.91 & .08 & 42.75 & 43.07 & 155 \\
\hline & June & 42.92 & .08 & 42.75 & 43.05 & 172 \\
\hline & July & 42.92 & .08 & 42.73 & 43.04 & 186 \\
\hline & August & 42.91 & .08 & 42.71 & 43.07 & 186 \\
\hline & September & 42.91 & .09 & 42.71 & 43.09 & 180 \\
\hline & October & 42.93 & .07 & 42.80 & 43.08 & 155 \\
\hline & November & 42.93 & .06 & 42.80 & 43.05 & 150 \\
\hline & December & 42.93 & .07 & 42.79 & 43.13 & 155 \\
\hline \multirow[t]{12}{*}{430027102311806} & January & 36.90 & .48 & 35.96 & 38.03 & 216 \\
\hline & February & 36.92 & .47 & 36.05 & 37.86 & 192 \\
\hline & March & 36.90 & .59 & 35.97 & 38.50 & 202 \\
\hline & April & 36.77 & .46 & 35.94 & 37.94 & 189 \\
\hline & May & 36.85 & .58 & 35.96 & 38.47 & 203 \\
\hline & June & 36.85 & .43 & 36.01 & 37.68 & 232 \\
\hline & July & 36.97 & .32 & 36.38 & 37.70 & 237 \\
\hline & August & 37.03 & .30 & 36.59 & 37.71 & 217 \\
\hline & September & 37.07 & .31 & 36.55 & 37.69 & 210 \\
\hline & October & 36.99 & .38 & 36.25 & 37.80 & 246 \\
\hline & November & 37.02 & .61 & 36.18 & 39.14 & 239 \\
\hline & December & 36.91 & .63 & 35.95 & 38.99 & 236 \\
\hline
\end{tabular}


Table 56. Summary of periodic ground-water-level data (from the U.S. Geological Survey database), in feet below land surface, for sites in the High Plains aquifer in South Dakota

[Negative values means water levels above land surface. --, not applicable]

\begin{tabular}{|c|c|c|c|c|c|c|}
\hline Site number & Month & Mean & $\begin{array}{l}\text { Standard } \\
\text { deviation }\end{array}$ & Minimum & Maximum & $\begin{array}{l}\text { Number of } \\
\text { valid } \\
\text { samples }\end{array}$ \\
\hline \multirow[t]{4}{*}{425956101134501} & January & -- & -- & 6.59 & 6.59 & 1 \\
\hline & September & 8.12 & 1.25 & 7.24 & 9.00 & 2 \\
\hline & October & -- & -- & 8.92 & 8.92 & 1 \\
\hline & November & -- & -- & 7.13 & 7.13 & 1 \\
\hline \multirow[t]{2}{*}{425956101134502} & September & -- & -- & 7.75 & 7.75 & 1 \\
\hline & October & -- & -- & 9.52 & 9.52 & 1 \\
\hline \multirow[t]{4}{*}{425956101134503} & January & -- & -- & 7.10 & 7.10 & 1 \\
\hline & September & 7.91 & .08 & 7.85 & 7.96 & 2 \\
\hline & October & -- & -- & 9.46 & 9.46 & 1 \\
\hline & November & -- & -- & 7.56 & 7.56 & 1 \\
\hline \multirow[t]{11}{*}{425956101424101} & January & -- & -- & 6.50 & 6.50 & 1 \\
\hline & March & 6.20 & .65 & 5.20 & 7.10 & 7 \\
\hline & April & 5.61 & .87 & 3.50 & 6.70 & 14 \\
\hline & May & 5.63 & .60 & 4.70 & 6.60 & 14 \\
\hline & June & 5.70 & .60 & 4.20 & 6.70 & 21 \\
\hline & July & 5.80 & .66 & 4.10 & 6.80 & 15 \\
\hline & August & 5.69 & .63 & 4.10 & 6.60 & 20 \\
\hline & September & 6.12 & .50 & 5.40 & 6.80 & 9 \\
\hline & October & 5.81 & .41 & 5.20 & 6.60 & 12 \\
\hline & November & 6.15 & .54 & 5.30 & 6.80 & 8 \\
\hline & December & 5.87 & .76 & 5.10 & 6.80 & 6 \\
\hline \multirow[t]{2}{*}{425957100445302} & May & -- & -- & 81.18 & 81.18 & 1 \\
\hline & October & -- & -- & 83.05 & 83.05 & 1 \\
\hline \multirow[t]{12}{*}{425957100445601} & January & -- & -- & 85.60 & 85.60 & 1 \\
\hline & February & 86.00 & 1.27 & 85.10 & 86.90 & 2 \\
\hline & March & 85.65 & .47 & 85.00 & 86.70 & 10 \\
\hline & April & 85.76 & .79 & 84.70 & 87.30 & 18 \\
\hline & May & 85.86 & .94 & 84.60 & 87.50 & 16 \\
\hline & June & 85.83 & .91 & 84.80 & 87.40 & 16 \\
\hline & July & 85.96 & .78 & 84.80 & 87.60 & 22 \\
\hline & August & 86.00 & .97 & 84.60 & 87.70 & 23 \\
\hline & September & 86.00 & 1.04 & 84.70 & 87.50 & 11 \\
\hline & October & 85.89 & .91 & 84.70 & 87.80 & 12 \\
\hline & November & 86.03 & .80 & 84.70 & 87.40 & 17 \\
\hline & December & 86.47 & .89 & 85.10 & 87.70 & 7 \\
\hline 430002100174801 & October & -- & -- & 5.07 & 5.07 & 1 \\
\hline
\end{tabular}


Table 56. Summary of periodic ground-water-level data (from the U.S. Geological Survey database), in feet below land surface, for sites in the High Plains aquifer in South Dakota-Continued

[Negative values means water levels above land surface. --, not applicable]

\begin{tabular}{|c|c|c|c|c|c|c|}
\hline Site number & Month & Mean & $\begin{array}{l}\text { Standard } \\
\text { deviation }\end{array}$ & Minimum & Maximum & $\begin{array}{l}\text { Number of } \\
\text { valid } \\
\text { samples }\end{array}$ \\
\hline \multirow[t]{2}{*}{430003100174802} & June & -- & -- & 3.22 & 3.22 & 1 \\
\hline & October & -- & -- & 4.22 & 4.22 & 1 \\
\hline \multirow[t]{2}{*}{430023100115602} & August & -- & -- & 8.00 & 8.00 & 1 \\
\hline & October & -- & -- & 10.01 & 10.01 & 1 \\
\hline \multirow[t]{10}{*}{430027102311801} & January & -- & -- & 43.10 & 43.10 & 1 \\
\hline & February & -- & -- & 42.95 & 42.95 & 1 \\
\hline & March & -- & -- & 42.95 & 42.95 & 1 \\
\hline & April & -- & -- & 42.83 & 42.83 & 1 \\
\hline & May & 42.88 & 0.04 & 42.85 & 42.93 & 3 \\
\hline & June & 43.22 & .16 & 43.08 & 43.41 & 4 \\
\hline & July & 43.02 & .08 & 42.94 & 43.09 & 3 \\
\hline & September & 43.40 & .39 & 43.00 & 43.77 & 3 \\
\hline & October & 43.66 & .38 & 43.30 & 44.30 & 9 \\
\hline & December & -- & -- & 42.05 & 42.05 & 1 \\
\hline \multirow[t]{12}{*}{430027102311806} & January & 37.07 & .51 & 36.67 & 37.65 & 3 \\
\hline & February & 37.02 & .37 & 36.76 & 37.28 & 2 \\
\hline & March & 36.92 & .24 & 36.75 & 37.09 & 2 \\
\hline & April & -- & -- & 37.07 & 37.07 & 1 \\
\hline & May & 36.85 & .08 & 36.81 & 36.94 & 3 \\
\hline & June & 37.19 & .12 & 37.09 & 37.35 & 4 \\
\hline & July & 37.03 & .10 & 36.95 & 37.15 & 3 \\
\hline & August & -- & -- & 36.94 & 36.94 & 1 \\
\hline & September & -- & -- & 36.93 & 36.93 & 1 \\
\hline & October & 38.57 & 1.00 & 36.92 & 39.54 & 8 \\
\hline & November & -- & -- & 36.96 & 36.96 & 1 \\
\hline & December & -- & -- & 38.33 & 38.33 & 1 \\
\hline \multirow[t]{4}{*}{430055100362702} & January & -- & -- & 12.09 & 12.09 & 1 \\
\hline & August & -- & -- & 11.53 & 11.53 & 1 \\
\hline & October & 11.30 & .26 & 11.12 & 11.48 & 2 \\
\hline & November & -- & -- & 12.18 & 12.18 & 1 \\
\hline
\end{tabular}


Table 56. Summary of periodic ground-water-level data (from the U.S. Geological Survey database), in feet below land surface, for sites in the High Plains aquifer in South Dakota-Continued

[Negative values means water levels above land surface. --, not applicable]

\begin{tabular}{|c|c|c|c|c|c|c|}
\hline Site number & Month & Mean & $\begin{array}{l}\text { Standard } \\
\text { deviation }\end{array}$ & Minimum & Maximum & $\begin{array}{c}\text { Number of } \\
\text { valid } \\
\text { samples }\end{array}$ \\
\hline \multirow[t]{10}{*}{430055101440002} & March & 24.18 & 0.96 & 23.00 & 25.10 & 4 \\
\hline & April & 23.42 & .90 & 22.40 & 24.80 & 6 \\
\hline & May & 23.69 & 1.30 & 22.00 & 25.30 & 8 \\
\hline & June & 23.78 & 1.14 & 22.40 & 25.60 & 11 \\
\hline & July & 24.51 & 1.32 & 22.80 & 26.30 & 8 \\
\hline & August & 24.39 & .97 & 22.80 & 26.00 & 10 \\
\hline & September & 25.60 & 1.25 & 24.20 & 26.80 & 4 \\
\hline & October & 24.50 & .92 & 23.30 & 25.40 & 4 \\
\hline & November & 25.13 & .60 & 24.70 & 26.00 & 4 \\
\hline & December & 24.95 & 1.06 & 24.20 & 25.70 & 2 \\
\hline \multirow[t]{2}{*}{430120100574901} & September & 5.09 & .98 & 4.40 & 5.78 & 2 \\
\hline & October & -- & -- & 5.76 & 5.76 & 1 \\
\hline 430126100221901 & October & -- & -- & 48.25 & 48.25 & 1 \\
\hline \multirow[t]{2}{*}{430126100222001} & May & -- & -- & 45.60 & 45.60 & 1 \\
\hline & October & -- & -- & 45.80 & 45.80 & 1 \\
\hline \multirow[t]{11}{*}{430129099201201} & February & 36.25 & 4.60 & 33.00 & 39.50 & 2 \\
\hline & March & 36.45 & 3.38 & 32.60 & 41.40 & 8 \\
\hline & April & 36.56 & 3.30 & 31.70 & 41.50 & 11 \\
\hline & May & 35.90 & 2.91 & 31.50 & 40.90 & 14 \\
\hline & June & 37.00 & 3.28 & 31.50 & 41.30 & 17 \\
\hline & July & 37.51 & 2.83 & 32.90 & 41.60 & 12 \\
\hline & August & 36.88 & 3.41 & 31.40 & 41.80 & 21 \\
\hline & September & 37.04 & 3.00 & 32.50 & 41.70 & 9 \\
\hline & October & 35.68 & 3.29 & 31.40 & 41.40 & 8 \\
\hline & November & 35.46 & 3.62 & 32.00 & 41.10 & 5 \\
\hline & December & 35.95 & 4.39 & 31.00 & 41.10 & 4 \\
\hline \multirow[t]{12}{*}{430148100471001} & January & -- & -- & 65.10 & 65.10 & 1 \\
\hline & February & 65.75 & .21 & 65.60 & 65.90 & 2 \\
\hline & March & 65.62 & .45 & 64.80 & 66.50 & 12 \\
\hline & April & 65.66 & .45 & 64.70 & 66.40 & 18 \\
\hline & May & 65.75 & .51 & 64.90 & 66.60 & 17 \\
\hline & June & 65.68 & .50 & 64.80 & 66.40 & 16 \\
\hline & July & 65.73 & .41 & 64.90 & 66.40 & 23 \\
\hline & August & 65.73 & .43 & 64.80 & 66.60 & 24 \\
\hline & September & 65.69 & .39 & 65.00 & 66.40 & 10 \\
\hline & October & 65.84 & .79 & 65.00 & 67.75 & 13 \\
\hline & November & 66.09 & .78 & 65.10 & 68.00 & 18 \\
\hline & December & 65.94 & .73 & 65.10 & 67.10 & 8 \\
\hline
\end{tabular}


Table 56. Summary of periodic ground-water-level data (from the U.S. Geological Survey database), in feet below land surface, for sites in the High Plains aquifer in South Dakota-Continued

[Negative values means water levels above land surface. --, not applicable]

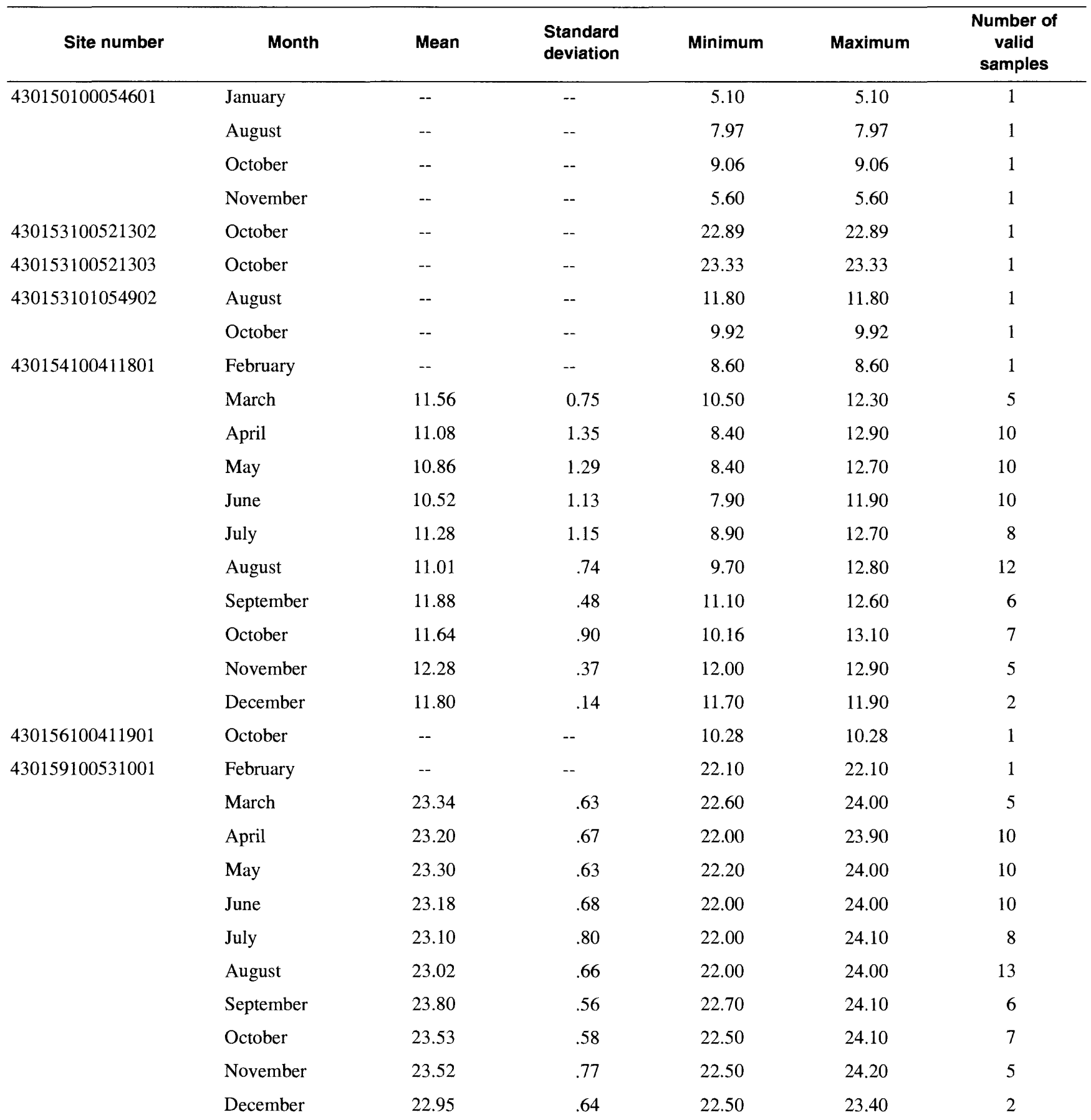


Table 56. Summary of periodic ground-water-level data (from the U.S. Geological Survey database), in feet below land surface, for sites in the High Plains aquifer in South Dakota-Continued

[Negative values means water levels above land surface. --, not applicable]

\begin{tabular}{|c|c|c|c|c|c|c|}
\hline Site number & Month & Mean & $\begin{array}{l}\text { Standard } \\
\text { deviation }\end{array}$ & Minimum & Maximum & $\begin{array}{l}\text { Number of } \\
\text { valid } \\
\text { samples }\end{array}$ \\
\hline \multirow[t]{10}{*}{430200102130001} & March & 29.89 & 0.80 & 28.60 & 30.70 & 7 \\
\hline & April & 29.91 & .70 & 28.60 & 30.70 & 12 \\
\hline & May & 30.03 & .58 & 28.70 & 30.80 & 12 \\
\hline & June & 29.93 & .55 & 28.70 & 30.70 & 15 \\
\hline & July & 29.91 & .61 & 29.00 & 30.80 & 10 \\
\hline & August & 29.97 & .57 & 29.00 & 30.60 & 15 \\
\hline & September & 30.42 & .70 & 29.30 & 31.00 & 6 \\
\hline & October & 30.14 & .52 & 29.30 & 30.70 & 8 \\
\hline & November & 30.34 & .56 & 29.30 & 31.10 & 7 \\
\hline & December & -- & -- & 29.90 & 29.90 & 1 \\
\hline 430224100445701 & July & -- & -- & 21.23 & 21.23 & 1 \\
\hline \multirow[t]{2}{*}{430225100445002} & July & -- & -- & 17.65 & 17.65 & 1 \\
\hline & October & -- & -- & 18.00 & 18.00 & 1 \\
\hline \multirow[t]{10}{*}{430226100445201} & February & -- & -- & 16.30 & 16.30 & 1 \\
\hline & March & -- & -- & 18.40 & 18.40 & 1 \\
\hline & April & 17.90 & 1.23 & 16.50 & 18.80 & 3 \\
\hline & May & 18.27 & .51 & 17.70 & 18.70 & 3 \\
\hline & June & 18.20 & .74 & 17.00 & 18.80 & 5 \\
\hline & July & 17.90 & 1.14 & 16.60 & 18.70 & 3 \\
\hline & August & 17.98 & 1.02 & 16.50 & 18.80 & 4 \\
\hline & September & 18.65 & .21 & 18.50 & 18.80 & 2 \\
\hline & October & 18.53 & .24 & 18.36 & 18.70 & 2 \\
\hline & November & -- & -- & 18.90 & 18.90 & 1 \\
\hline \multirow[t]{2}{*}{430226100445203} & July & -- & -- & 17.21 & 17.21 & 1 \\
\hline & October & -- & -- & 19.37 & 19.37 & 1 \\
\hline 430237100445001 & July & -- & -- & 18.16 & 18.16 & 1 \\
\hline \multirow[t]{2}{*}{430245100292701} & May & -- & -- & 12.99 & 12.99 & 1 \\
\hline & October & -- & - & 11.96 & 11.96 & 1 \\
\hline \multirow[t]{4}{*}{430245100292801} & January & -- & -- & 11.69 & 11.69 & 1 \\
\hline & August & -- & -- & 11.58 & 11.58 & 1 \\
\hline & October & 11.55 & .10 & 11.48 & 11.62 & 2 \\
\hline & November & -- & -- & 11.60 & 11.60 & 1 \\
\hline
\end{tabular}


Table 56. Summary of periodic ground-water-level data (from the U.S. Geological Survey database), in feet below land surface, for sites in the High Plains aquifer in South Dakota-Continued

[Negative values means water levels above land surface. --, not applicable]

\begin{tabular}{|c|c|c|c|c|c|c|}
\hline Site number & Month & Mean & $\begin{array}{l}\text { Standard } \\
\text { deviation }\end{array}$ & Minimum & Maximum & $\begin{array}{l}\text { Number of } \\
\text { valid } \\
\text { samples }\end{array}$ \\
\hline \multirow[t]{10}{*}{430256101160001} & March & 5.50 & 0.66 & 5.00 & 6.60 & 5 \\
\hline & April & 5.59 & .85 & 4.40 & 7.20 & 13 \\
\hline & May & 5.41 & .37 & 4.80 & 5.80 & 9 \\
\hline & June & 5.80 & .67 & 4.70 & 7.60 & 14 \\
\hline & July & 5.99 & .45 & 5.40 & 6.70 & 9 \\
\hline & August & 6.17 & .55 & 5.60 & 7.90 & 15 \\
\hline & September & 6.41 & .65 & 6.00 & 8.00 & 8 \\
\hline & October & 6.05 & .55 & 5.08 & 6.70 & 8 \\
\hline & November & 6.43 & .71 & 5.80 & 7.80 & 6 \\
\hline & December & 6.17 & 1.10 & 5.30 & 7.40 & 3 \\
\hline \multirow[t]{11}{*}{430258100471401} & February & -- & -- & 48.80 & 48.80 & 1 \\
\hline & March & 49.24 & .15 & 49.00 & 49.40 & 5 \\
\hline & April & 48.88 & .48 & 48.10 & 49.60 & 10 \\
\hline & May & 48.92 & .51 & 48.20 & 49.60 & 10 \\
\hline & June & 48.90 & .49 & 48.20 & 49.60 & 10 \\
\hline & July & 48.99 & .41 & 48.20 & 49.50 & 8 \\
\hline & August & 48.88 & .45 & 48.10 & 49.50 & 14 \\
\hline & September & 48.97 & .47 & 48.20 & 49.40 & 6 \\
\hline & October & 49.09 & .62 & 48.30 & 50.22 & 7 \\
\hline & November & 48.86 & .44 & 48.30 & 49.30 & 5 \\
\hline & December & 48.55 & .35 & 48.30 & 48.80 & 2 \\
\hline 430302100255101 & July & -- & -- & 59.00 & 59.00 & 1 \\
\hline \multirow[t]{11}{*}{430310100245501} & February & 25.65 & 4.60 & 22.40 & 28.90 & 2 \\
\hline & March & 26.13 & .85 & 25.50 & 27.80 & 6 \\
\hline & April & 25.56 & 1.63 & 22.30 & 28.90 & 12 \\
\hline & May & 25.49 & 1.37 & 23.90 & 29.20 & 13 \\
\hline & June & 25.65 & 1.59 & 22.60 & 29.40 & 15 \\
\hline & July & 26.43 & 1.92 & 22.70 & 29.60 & 13 \\
\hline & August & 26.08 & 1.64 & 23.00 & 29.60 & 17 \\
\hline & September & 26.77 & 1.35 & 25.70 & 29.70 & 9 \\
\hline & October & 26.11 & 1.24 & 23.72 & 28.40 & 9 \\
\hline & November & 26.67 & 1.48 & 25.60 & 29.60 & 6 \\
\hline & December & 25.60 & .36 & 25.30 & 26.00 & 3 \\
\hline
\end{tabular}


Table 56. Summary of periodic ground-water-level data (from the U.S. Geological Survey database), in feet below land surface, for sites in the High Plains aquifer in South Dakota-Continued

[Negative values means water levels above land surface. --, not applicable]

\begin{tabular}{|c|c|c|c|c|c|c|}
\hline Site number & Month & Mean & $\begin{array}{l}\text { Standard } \\
\text { deviation }\end{array}$ & Minimum & Maximum & $\begin{array}{l}\text { Number of } \\
\text { valid } \\
\text { samples }\end{array}$ \\
\hline \multirow[t]{11}{*}{430340101012301} & February & 7.05 & 2.76 & 5.10 & 9.00 & 2 \\
\hline & March & 6.72 & .68 & 5.90 & 7.80 & 6 \\
\hline & April & 6.42 & .93 & 5.00 & 7.90 & 13 \\
\hline & May & 6.42 & 1.13 & 4.70 & 8.60 & 13 \\
\hline & June & 6.60 & 1.29 & 3.20 & 8.70 & 15 \\
\hline & July & 7.31 & 1.32 & 4.80 & 8.90 & 13 \\
\hline & August & 7.66 & .88 & 6.10 & 9.40 & 18 \\
\hline & September & 8.66 & .66 & 8.10 & 9.80 & 8 \\
\hline & October & 8.05 & .58 & 6.90 & 8.70 & 7 \\
\hline & November & 8.27 & .61 & 7.70 & 9.20 & 6 \\
\hline & December & 7.50 & .71 & 7.00 & 8.00 & 2 \\
\hline 430415100451401 & August & -- & -- & 21.27 & 21.27 & 1 \\
\hline \multirow[t]{11}{*}{430429101320401} & February & -- & -- & 5.00 & 5.00 & 1 \\
\hline & March & 6.69 & 2.42 & 4.00 & 10.90 & 8 \\
\hline & April & 5.18 & 1.93 & 2.30 & 10.90 & 14 \\
\hline & May & 5.79 & 2.08 & 3.60 & 11.40 & 12 \\
\hline & June & 6.83 & 2.27 & 2.40 & 11.70 & 20 \\
\hline & July & 7.79 & 1.78 & 4.80 & 10.90 & 17 \\
\hline & August & 8.94 & 1.71 & 4.40 & 11.20 & 21 \\
\hline & September & 9.32 & 2.37 & 6.10 & 12.80 & 10 \\
\hline & October & 9.17 & 2.35 & 6.30 & 13.70 & 9 \\
\hline & November & 8.64 & 2.59 & 4.90 & 13.50 & 8 \\
\hline & December & 8.78 & 3.97 & 3.10 & 13.00 & 6 \\
\hline 430454100341801 & May & -- & -- & 18.00 & 18.00 & 1 \\
\hline \multirow[t]{10}{*}{430520101260401} & March & 31.37 & .94 & 30.20 & 32.60 & 7 \\
\hline & April & 31.31 & 1.05 & 29.30 & 33.00 & 13 \\
\hline & May & 31.18 & .74 & 30.30 & 32.30 & 10 \\
\hline & June & 31.33 & .74 & 30.20 & 32.70 & 17 \\
\hline & July & 31.49 & .87 & 30.00 & 32.70 & 14 \\
\hline & August & 31.19 & .84 & 29.80 & 32.70 & 17 \\
\hline & September & 31.33 & .87 & 30.40 & 32.60 & 8 \\
\hline & October & 31.21 & .56 & 30.50 & 32.20 & 8 \\
\hline & November & 31.55 & .82 & 30.60 & 32.70 & 6 \\
\hline & December & 31.50 & .74 & 31.00 & 32.60 & 4 \\
\hline
\end{tabular}


Table 56. Summary of periodic ground-water-level data (from the U.S. Geological Survey database), in feet below land surface, for sites in the High Plains aquifer in South Dakota-Continued

[Negative values means water levels above land surface. --, not applicable]

\begin{tabular}{|c|c|c|c|c|c|c|}
\hline Site number & Month & Mean & $\begin{array}{l}\text { Standard } \\
\text { deviation }\end{array}$ & Minimum & Maximum & $\begin{array}{l}\text { Number of } \\
\text { valid } \\
\text { samples }\end{array}$ \\
\hline \multirow[t]{10}{*}{430521101223401} & March & 13.90 & 1.01 & 12.70 & 15.30 & 8 \\
\hline & April & 13.72 & 1.00 & 12.50 & 15.30 & 14 \\
\hline & May & 13.69 & .89 & 12.60 & 15.20 & 13 \\
\hline & June & 13.60 & .82 & 12.40 & 15.30 & 20 \\
\hline & July & 13.85 & 1.02 & 12.30 & 15.50 & 17 \\
\hline & August & 13.85 & .92 & 12.30 & 15.50 & 21 \\
\hline & September & 14.01 & 1.07 & 12.60 & 15.60 & 10 \\
\hline & October & 13.96 & .85 & 12.70 & 15.60 & 10 \\
\hline & November & 14.29 & .90 & 13.20 & 15.70 & 8 \\
\hline & December & 16.38 & 4.81 & 13.50 & 26.10 & 6 \\
\hline \multirow[t]{2}{*}{430522100411902} & June & -- & -- & 74.86 & 74.86 & 1 \\
\hline & October & -- & -- & 72.95 & 72.95 & 1 \\
\hline \multirow[t]{11}{*}{430524101481601} & February & -- & -- & 7.90 & 7.90 & 1 \\
\hline & March & 6.51 & .31 & 6.00 & 7.00 & 7 \\
\hline & April & 6.61 & .56 & 5.90 & 7.70 & 13 \\
\hline & May & 6.64 & .79 & 5.30 & 7.90 & 10 \\
\hline & June & 7.25 & .77 & 5.90 & 8.60 & 16 \\
\hline & July & 7.96 & .86 & 6.50 & 9.40 & 14 \\
\hline & August & 8.28 & .67 & 7.20 & 9.30 & 17 \\
\hline & September & 8.33 & .37 & 7.90 & 9.00 & 7 \\
\hline & October & 8.20 & .72 & 7.30 & 9.00 & 8 \\
\hline & November & 8.27 & .34 & 7.90 & 8.80 & 6 \\
\hline & December & 7.30 & .54 & 6.60 & 7.90 & 4 \\
\hline 430600100450801 & July & -- & -- & 93.74 & 93.74 & 1 \\
\hline 430600100454101 & December & -- & -- & 73.00 & 73.00 & 1 \\
\hline 430604100445201 & July & -- & -- & 68.48 & 68.48 & 1 \\
\hline \multirow[t]{2}{*}{430604100445302} & July & -- & -- & 68.74 & 68.74 & 1 \\
\hline & October & -- & -- & 72.33 & 72.33 & 1 \\
\hline \multirow[t]{10}{*}{430604100445401} & February & -- & -- & 69.70 & 69.70 & 1 \\
\hline & March & 69.60 & .28 & 69.40 & 69.80 & 2 \\
\hline & April & 69.87 & .21 & 69.70 & 70.10 & 3 \\
\hline & May & 69.53 & .45 & 69.10 & 70.00 & 3 \\
\hline & June & 69.54 & .39 & 69.10 & 70.00 & 5 \\
\hline & July & 69.63 & .45 & 69.20 & 70.10 & 3 \\
\hline & August & 69.83 & .17 & 69.60 & 70.00 & 4 \\
\hline & September & 69.37 & .21 & 69.20 & 69.60 & 3 \\
\hline & October & 70.53 & 1.74 & 69.30 & 71.76 & 2 \\
\hline & November & -- & -- & 69.40 & 69.40 & 1 \\
\hline
\end{tabular}


Table 56. Summary of periodic ground-water-level data (from the U.S. Geological Survey database), in feet below land surface, for sites in the High Plains aquifer in South Dakota-Continued

[Negative values means water levels above land surface. --, not applicable]

\begin{tabular}{|c|c|c|c|c|c|c|}
\hline Site number & Month & Mean & $\begin{array}{l}\text { Standard } \\
\text { deviation }\end{array}$ & Minimum & Maximum & $\begin{array}{l}\text { Number of } \\
\text { valid } \\
\text { samples }\end{array}$ \\
\hline \multirow[t]{2}{*}{430604100445403} & July & -- & -- & 68.17 & 68.17 & 1 \\
\hline & October & -- & -- & 71.76 & 71.76 & 1 \\
\hline \multirow[t]{3}{*}{430605101135701} & January & -- & -- & -6.59 & -6.59 & 1 \\
\hline & August & -- & -- & -13.94 & -13.94 & 1 \\
\hline & October & -- & -- & -18.03 & -18.03 & 1 \\
\hline \multirow[t]{2}{*}{430605101135702} & August & -- & -- & -14.60 & -14.60 & 1 \\
\hline & October & -6.40 & 11.88 & -14.80 & 2.00 & 2 \\
\hline \multirow[t]{12}{*}{430609100434201} & January & -- & -- & 38.00 & 38.00 & 1 \\
\hline & February & 41.35 & 2.90 & 39.30 & 43.40 & 2 \\
\hline & March & 41.27 & 2.40 & 38.70 & 46.10 & 9 \\
\hline & April & 41.57 & 1.90 & 38.70 & 46.10 & 15 \\
\hline & May & 41.13 & 2.19 & 38.10 & 46.00 & 17 \\
\hline & June & 40.85 & 2.09 & 38.00 & 46.00 & 21 \\
\hline & July & 40.84 & 1.75 & 38.40 & 44.10 & 17 \\
\hline & August & 41.09 & 2.20 & 38.10 & 46.20 & 25 \\
\hline & September & 40.88 & 1.99 & 38.00 & 43.80 & 13 \\
\hline & October & 41.23 & 2.28 & 38.10 & 46.06 & 12 \\
\hline & November & 40.59 & 1.75 & 38.30 & 43.30 & 9 \\
\hline & December & 39.92 & 1.38 & 38.10 & 41.50 & 6 \\
\hline \multirow[t]{12}{*}{430610100481701} & January & -- & - & 72.70 & 72.70 & 1 \\
\hline & February & - & -- & 73.20 & 73.20 & 1 \\
\hline & March & 72.66 & .70 & 71.70 & 73.60 & 8 \\
\hline & April & 72.57 & .70 & 71.50 & 73.70 & 15 \\
\hline & May & 72.49 & .73 & 71.50 & 73.60 & 17 \\
\hline & June & 72.47 & .66 & 71.40 & 73.60 & 20 \\
\hline & July & 72.54 & .58 & 71.70 & 73.60 & 17 \\
\hline & August & 72.46 & .64 & 71.40 & 73.70 & 25 \\
\hline & September & 72.58 & .61 & 71.80 & 73.60 & 12 \\
\hline & October & 72.90 & 1.18 & 71.70 & 76.30 & 12 \\
\hline & November & 72.66 & .48 & 71.90 & 73.50 & 9 \\
\hline & December & 72.20 & .28 & 72.00 & 72.60 & 5 \\
\hline 430613100352901 & May & -- & -- & 19.00 & 19.00 & 1 \\
\hline 430613100445901 & August & -- & -- & 59.25 & 59.25 & 1 \\
\hline 430613100544901 & October & -- & -- & 59.14 & 59.14 & 1 \\
\hline
\end{tabular}


Table 56. Summary of periodic ground-water-level data (from the U.S. Geological Survey database), in feet below land surface, for sites in the High Plains aquifer in South Dakota-Continued

[Negative values means water levels above land surface. --, not applicable]

\begin{tabular}{|c|c|c|c|c|c|c|}
\hline Site number & Month & Mean & $\begin{array}{l}\text { Standard } \\
\text { deviation }\end{array}$ & Minimum & Maximum & $\begin{array}{c}\text { Number of } \\
\text { valid } \\
\text { samples }\end{array}$ \\
\hline \multirow[t]{11}{*}{430613101561701} & February & 137.50 & 0.71 & 137.00 & 138.00 & 2 \\
\hline & March & 138.03 & 1.37 & 136.30 & 139.40 & 6 \\
\hline & April & 138.03 & 1.57 & 136.20 & 141.40 & 11 \\
\hline & May & 138.27 & 1.15 & 136.10 & 139.80 & 12 \\
\hline & June & 138.93 & 1.84 & 136.20 & 141.60 & 15 \\
\hline & July & 138.33 & 1.51 & 136.10 & 140.10 & 11 \\
\hline & August & 139.40 & 1.38 & 136.40 & 142.20 & 16 \\
\hline & September & 139.16 & 1.22 & 136.40 & 140.20 & 8 \\
\hline & October & 139.72 & 1.74 & 136.40 & 142.20 & 8 \\
\hline & November & 138.94 & .96 & 137.50 & 139.80 & 5 \\
\hline & December & -- & -- & 140.40 & 140.40 & 1 \\
\hline 430614100244802 & September & -- & -- & 12.58 & 12.58 & 1 \\
\hline 430614100362502 & October & -- & -- & 26.87 & 26.87 & 1 \\
\hline 430614100362503 & October & -- & -- & 26.60 & 26.60 & 1 \\
\hline \multirow[t]{11}{*}{430642101455801} & February & -- & -- & 15.00 & 15.00 & 1 \\
\hline & March & 14.29 & .64 & 13.50 & 15.20 & 9 \\
\hline & April & 14.59 & 1.32 & 12.60 & 16.90 & 14 \\
\hline & May & 14.13 & 1.12 & 12.70 & 16.80 & 14 \\
\hline & June & 14.91 & 1.47 & 12.70 & 18.40 & 21 \\
\hline & July & 15.49 & 1.71 & 13.80 & 20.00 & 17 \\
\hline & August & 15.92 & 1.92 & 13.20 & 20.70 & 23 \\
\hline & September & 15.87 & 1.02 & 14.00 & 18.00 & 11 \\
\hline & October & 15.36 & 1.25 & 13.40 & 17.20 & 11 \\
\hline & November & 15.92 & 1.04 & 14.50 & 17.80 & 9 \\
\hline & December & 14.83 & .99 & 13.40 & 15.90 & 6 \\
\hline \multirow[t]{12}{*}{430701100363001} & January & -- & -- & 75.10 & 75.10 & 1 \\
\hline & February & 72.70 & 1.41 & 71.70 & 73.70 & 2 \\
\hline & March & 73.07 & .77 & 72.10 & 74.30 & 9 \\
\hline & April & 72.62 & 1.09 & 71.20 & 74.50 & 14 \\
\hline & May & 72.79 & 1.09 & 71.20 & 74.90 & 17 \\
\hline & June & 72.86 & 1.09 & 71.20 & 74.90 & 20 \\
\hline & July & 73.15 & 1.03 & 71.30 & 75.10 & 19 \\
\hline & August & 73.03 & 1.10 & 71.30 & 75.30 & 25 \\
\hline & September & 73.13 & 1.06 & 71.40 & 75.10 & 13 \\
\hline & October & 73.19 & 1.15 & 71.50 & 74.90 & 12 \\
\hline & November & 73.21 & 1.05 & 71.90 & 74.70 & 8 \\
\hline & December & 73.30 & 1.18 & 71.70 & 75.00 & 6 \\
\hline
\end{tabular}


Table 56. Summary of periodic ground-water-level data (from the U.S. Geological Survey database), in feet below land surface, for sites in the High Plains aquifer in South Dakota-Continued

[Negative values means water levels above land surface. --, not applicable]

\begin{tabular}{|c|c|c|c|c|c|c|}
\hline Site number & Month & Mean & $\begin{array}{l}\text { Standard } \\
\text { deviation }\end{array}$ & Minimum & Maximum & $\begin{array}{c}\text { Number of } \\
\text { valid } \\
\text { samples }\end{array}$ \\
\hline \multirow[t]{2}{*}{430705100450201} & August &.- & -- & 67.01 & 67.01 & 1 \\
\hline & October & -- & -- & 66.63 & 66.63 & 1 \\
\hline \multirow[t]{10}{*}{430744101593201} & March & 0 & 1.28 & -2.00 & 2.10 & 9 \\
\hline & April & -.21 & 1.40 & -2.20 & 2.20 & 13 \\
\hline & May & -.34 & 1.59 & -2.20 & 3.40 & 13 \\
\hline & June & 3.69 & 7.56 & -2.20 & 23.50 & 20 \\
\hline & July & 9.15 & 9.13 & -1.60 & 27.30 & 15 \\
\hline & August & 19.96 & 12.03 & .70 & 36.70 & 21 \\
\hline & September & 18.99 & 12.68 & 5.10 & 45.20 & 10 \\
\hline & October & 8.09 & 4.52 & 1.30 & 15.60 & 8 \\
\hline & November & 7.76 & 6.03 & 1.10 & 17.70 & 8 \\
\hline & December & 2.08 & 2.55 & -.40 & 5.60 & 5 \\
\hline \multirow[t]{12}{*}{430748100455601} & January & -- & - & 87.90 & 87.90 & 1 \\
\hline & February & -- & -- & 89.90 & 89.90 & 1 \\
\hline & March & 91.23 & 1.99 & 88.90 & 94.10 & 7 \\
\hline & April & 91.39 & 1.70 & 89.00 & 94.10 & 14 \\
\hline & May & 90.96 & 2.23 & 88.00 & 94.20 & 16 \\
\hline & June & 90.84 & 2.08 & 87.60 & 94.30 & 21 \\
\hline & July & 91.09 & 2.02 & 88.50 & 94.30 & 16 \\
\hline & August & 91.63 & 1.95 & 88.70 & 95.20 & 24 \\
\hline & September & 91.99 & 2.16 & 89.000 & 95.00 & 13 \\
\hline & October & 92.39 & 2.73 & 89.00 & 98.66 & 12 \\
\hline & November & 91.41 & 1.96 & 88.70 & 94.40 & 9 \\
\hline & December & 90.50 & 1.56 & 88.80 & 92.10 & 4 \\
\hline \multirow[t]{10}{*}{430748102154801} & March & 101.89 & 2.02 & 99.20 & 104.40 & 7 \\
\hline & April & 101.55 & 1.93 & 99.00 & 104.60 & 13 \\
\hline & May & 101.84 & 2.05 & 99.40 & 104.90 & 8 \\
\hline & June & 101.49 & 1.71 & 99.60 & 104.60 & 13 \\
\hline & July & 101.66 & 1.79 & 99.40 & 104.30 & 9 \\
\hline & August & 101.85 & 1.85 & 99.50 & 104.70 & 15 \\
\hline & September & 100.87 & 1.23 & 100.00 & 103.30 & 6 \\
\hline & October & 101.70 & 1.31 & 100.00 & 103.90 & 8 \\
\hline & November & 102.10 & 1.71 & 100.40 & 104.50 & 5 \\
\hline & December & 102.67 & 1.36 & 101.40 & 104.10 & 3 \\
\hline 430756100231601 & June & -- & -- & 16.00 & 16.00 & 1 \\
\hline
\end{tabular}


Table 56. Summary of periodic ground-water-level data (from the U.S. Geological Survey database), in feet below land surface, for sites in the High Plains aquifer in South Dakota-Continued

[Negative values means water levels above land surface. --, not applicable]

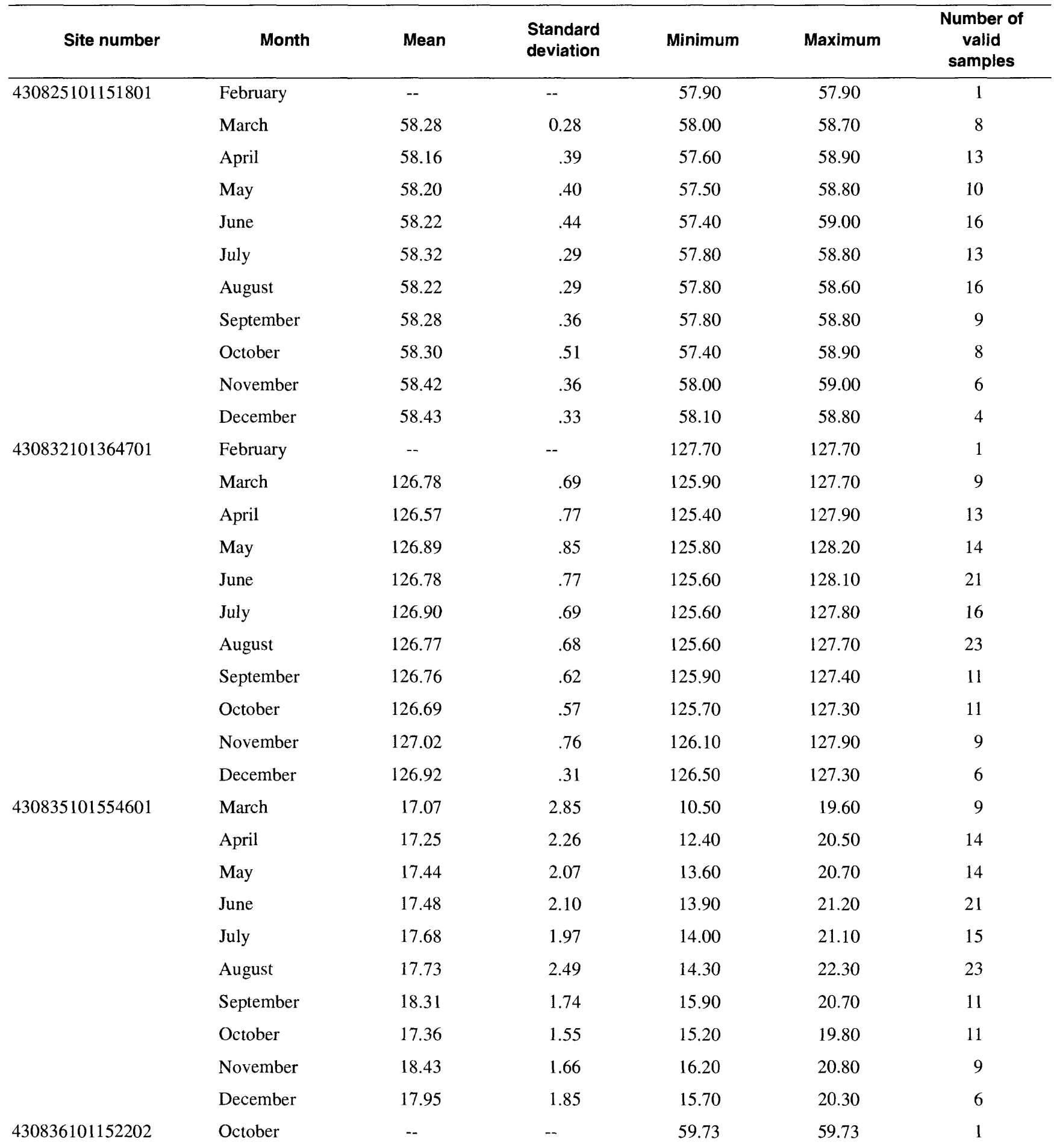


Table 56. Summary of periodic ground-water-level data (from the U.S. Geological Survey database), in feet below land surface, for sites in the High Plains aquifer in South Dakota-Continued

[Negative values means water levels above land surface. --, not applicable]

\begin{tabular}{|c|c|c|c|c|c|c|}
\hline Site number & Month & Mean & $\begin{array}{l}\text { Standard } \\
\text { deviation }\end{array}$ & Minimum & Maximum & $\begin{array}{c}\text { Number of } \\
\text { valid } \\
\text { samples }\end{array}$ \\
\hline \multirow[t]{12}{*}{430839100373801} & January & -- & -- & 78.00 & 78.00 & 1 \\
\hline & February & 78.35 & 0.64 & 77.90 & 78.80 & 2 \\
\hline & March & 78.34 & .73 & 77.30 & 79.20 & 9 \\
\hline & April & 78.11 & .79 & 76.80 & 79.20 & 15 \\
\hline & May & 77.88 & .88 & 76.20 & 79.20 & 17 \\
\hline & June & 78.22 & 1.00 & 77.10 & 81.40 & 21 \\
\hline & July & 78.63 & .78 & 77.20 & 79.90 & 17 \\
\hline & August & 78.74 & .75 & 77.50 & 80.10 & 25 \\
\hline & September & 78.79 & .80 & 77.30 & 79.80 & 13 \\
\hline & October & 78.91 & 1.57 & 77.50 & 83.36 & 12 \\
\hline & November & 78.27 & .72 & 77.20 & 79.50 & 9 \\
\hline & December & 77.80 & .46 & 77.40 & 78.40 & 4 \\
\hline \multirow[t]{12}{*}{430840100445601} & January & -- & -- & 62.60 & 62.60 & 1 \\
\hline & February & 67.35 & 2.90 & 65.30 & 69.40 & 2 \\
\hline & March & 66.90 & 3.13 & 62.90 & 71.50 & 9 \\
\hline & April & 67.49 & 2.63 & 62.70 & 71.20 & 14 \\
\hline & May & 66.99 & 2.93 & 62.50 & 71.10 & 15 \\
\hline & June & 65.81 & 3.11 & 61.30 & 70.90 & 21 \\
\hline & July & 65.92 & 2.89 & 61.80 & 70.80 & 18 \\
\hline & August & 66.52 & 3.04 & 61.70 & 71.40 & 25 \\
\hline & September & 67.72 & 3.41 & 62.50 & 72.00 & 11 \\
\hline & October & 68.31 & 3.61 & 63.00 & 73.38 & 10 \\
\hline & November & 67.29 & 3.20 & 63.20 & 71.50 & 9 \\
\hline & December & 66.24 & 2.15 & 63.30 & 68.70 & 5 \\
\hline \multirow[t]{12}{*}{430842100411301} & January & -- & -- & 24.80 & 24.80 & 1 \\
\hline & February & 24.60 & .57 & 24.20 & 25.00 & 2 \\
\hline & March & 24.66 & .82 & 23.70 & 25.90 & 9 \\
\hline & April & 24.47 & .90 & 23.10 & 25.90 & 15 \\
\hline & May & 24.20 & 1.16 & 22.30 & 26.50 & 17 \\
\hline & June & 24.38 & .97 & 22.50 & 26.60 & 21 \\
\hline & July & 25.16 & .96 & 23.50 & 26.60 & 17 \\
\hline & August & 25.55 & .94 & 23.70 & 27.00 & 25 \\
\hline & September & 25.86 & 1.04 & 24.00 & 27.30 & 13 \\
\hline & October & 25.99 & 1.13 & 24.40 & 28.35 & 10 \\
\hline & November & 25.46 & 1.15 & 23.50 & 27.00 & 9 \\
\hline & December & 24.75 & .56 & 24.10 & 25.50 & 6 \\
\hline 430857100445101 & July & - & -- & 70.96 & 70.96 & 1 \\
\hline
\end{tabular}


Table 56. Summary of periodic ground-water-level data (from the U.S. Geological Survey database), in feet below land surface, for sites in the High Plains aquifer in South Dakota-Continued

[Negative values means water levels above land surface. --, not applicable]

\begin{tabular}{|c|c|c|c|c|c|c|}
\hline Site number & Month & Mean & $\begin{array}{l}\text { Standard } \\
\text { deviation }\end{array}$ & Minimum & Maximum & $\begin{array}{l}\text { Number of } \\
\text { valid } \\
\text { samples }\end{array}$ \\
\hline \multirow[t]{2}{*}{430858100445302} & July & -- & -- & 70.92 & 70.92 & 1 \\
\hline & October & -- & -- & 75.10 & 75.10 & 1 \\
\hline 430858100445503 & July & -- & -- & 71.01 & 71.01 & 1 \\
\hline 430922100410302 & October & -- & -- & 71.89 & 71.89 & 1 \\
\hline \multirow[t]{11}{*}{430923101484301} & February & -- & -- & 39.10 & 39.10 & 1 \\
\hline & March & 37.80 & 1.89 & 35.30 & 40.00 & 8 \\
\hline & April & 36.54 & 2.37 & 32.80 & 40.20 & 14 \\
\hline & May & 36.99 & 2.33 & 33.90 & 40.70 & 11 \\
\hline & June & 37.04 & 2.06 & 33.70 & 39.90 & 20 \\
\hline & July & 36.93 & 3.67 & 25.30 & 39.70 & 16 \\
\hline & August & 36.99 & 2.11 & 33.50 & 39.60 & 21 \\
\hline & September & 37.32 & 1.88 & 35.20 & 39.50 & 10 \\
\hline & October & 36.93 & 1.66 & 35.10 & 39.40 & 10 \\
\hline & November & 37.09 & 1.71 & 35.50 & 39.40 & 7 \\
\hline & December & 38.04 & 1.81 & 35.70 & 39.70 & 5 \\
\hline \multirow[t]{12}{*}{430924100460601} & January & -- & -- & 74.00 & 74.00 & 1 \\
\hline & February & 78.15 & 3.75 & 75.50 & 80.80 & 2 \\
\hline & March & 77.56 & 2.51 & 74.50 & 81.00 & 9 \\
\hline & April & 78.03 & 2.13 & 75.10 & 81.40 & 15 \\
\hline & May & 77.24 & 2.51 & 73.70 & 81.20 & 18 \\
\hline & June & 77.06 & 2.41 & 74.10 & 81.20 & 21 \\
\hline & July & 77.10 & 2.34 & 74.40 & 81.40 & 17 \\
\hline & August & 77.26 & 2.54 & 74.10 & 81.90 & 25 \\
\hline & September & 77.52 & 2.78 & 73.90 & 81.40 & 13 \\
\hline & October & 77.98 & 3.08 & 74.40 & 84.39 & 12 \\
\hline & November & 77.27 & 2.34 & 74.60 & 81.00 & 9 \\
\hline & December & 76.15 & 1.45 & 74.40 & 78.00 & 6 \\
\hline \multirow[t]{11}{*}{430928101532401} & February & -- & -- & 27.30 & 27.30 & 1 \\
\hline & March & 25.36 & 2.02 & 22.60 & 27.70 & 8 \\
\hline & April & 24.04 & 2.33 & 20.30 & 27.70 & 14 \\
\hline & May & 24.69 & 2.24 & 21.40 & 27.70 & 14 \\
\hline & June & 24.72 & 2.28 & 20.60 & 27.90 & 21 \\
\hline & July & 24.85 & 2.23 & 20.70 & 27.60 & 13 \\
\hline & August & 25.14 & 2.16 & 21.60 & 28.10 & 23 \\
\hline & September & 25.44 & 2.25 & 22.50 & 28.20 & 11 \\
\hline & October & 24.56 & 1.76 & 22.30 & 27.50 & 10 \\
\hline & November & 25.48 & 2.05 & 22.90 & 28.00 & 9 \\
\hline & December & 25.70 & 1.71 & 23.60 & 27.60 & 6 \\
\hline
\end{tabular}


Table 56. Summary of periodic ground-water-level data (from the U.S. Geological Survey database), in feet below land surface, for sites in the High Plains aquifer in South Dakota-Continued

[Negative values means water levels above land surface. --, not applicable]

\begin{tabular}{|c|c|c|c|c|c|c|}
\hline Site number & Month & Mean & $\begin{array}{l}\text { Standard } \\
\text { deviation }\end{array}$ & Minimum & Maximum & $\begin{array}{c}\text { Number of } \\
\text { valid } \\
\text { samples }\end{array}$ \\
\hline \multirow[t]{4}{*}{430929101104202} & January & -- & -- & 78.25 & 78.25 & 1 \\
\hline & August & -- & -- & 77.98 & 77.98 & 1 \\
\hline & October & 78.36 & 2.38 & 76.68 & 80.04 & 2 \\
\hline & November & -- & -- & 78.42 & 78.42 & 1 \\
\hline \multirow[t]{3}{*}{430929101104203} & August & -- & - & 77.38 & 77.38 & 1 \\
\hline & October & 78.18 & 1.10 & 77.40 & 78.95 & 2 \\
\hline & November & -- & -- & 77.86 & 77.86 & 1 \\
\hline \multirow[t]{12}{*}{430932100390001} & January & -- & - & 47.10 & 47.10 & 1 \\
\hline & February & 46.30 & .14 & 46.20 & 46.40 & 2 \\
\hline & March & 46.15 & 1.35 & 45.10 & 48.60 & 8 \\
\hline & April & 45.39 & 1.44 & 43.50 & 48.00 & 15 \\
\hline & May & 45.30 & 1.46 & 43.50 & 48.10 & 16 \\
\hline & June & 45.28 & 1.21 & 43.60 & 48.20 & 21 \\
\hline & July & 45.94 & 1.31 & 43.90 & 48.10 & 17 \\
\hline & August & 46.37 & 1.31 & 44.40 & 48.80 & 25 \\
\hline & September & 46.60 & 1.07 & 44.90 & 48.70 & 12 \\
\hline & October & 46.28 & 1.29 & 44.90 & 48.60 & 11 \\
\hline & November & 46.07 & 1.00 & 44.80 & 47.40 & 9 \\
\hline & December & 45.73 & 1.12 & 44.70 & 47.30 & 4 \\
\hline \multirow[t]{12}{*}{430949101292701} & January & -- & -- & 21.30 & 21.30 & 1 \\
\hline & February & -- & - & 21.50 & 21.50 & 1 \\
\hline & March & 20.38 & .92 & 18.20 & 21.40 & 10 \\
\hline & April & 20.71 & .59 & 19.70 & 21.90 & 14 \\
\hline & May & 20.52 & .49 & 19.90 & 21.70 & 15 \\
\hline & June & 20.81 & .57 & 19.30 & 21.90 & 23 \\
\hline & July & 21.14 & .51 & 20.30 & 22.20 & 19 \\
\hline & August & 21.08 & .58 & 19.90 & 22.20 & 23 \\
\hline & September & 21.37 & .48 & 20.50 & 22.40 & 13 \\
\hline & October & 21.26 & .42 & 20.70 & 22.00 & 11 \\
\hline & November & 21.62 & .36 & 21.00 & 22.10 & 11 \\
\hline & December & 21.28 & .33 & 20.80 & 21.80 & 6 \\
\hline
\end{tabular}


Table 56. Summary of periodic ground-water-level data (from the U.S. Geological Survey database), in feet below land surface, for sites in the High Plains aquifer in South Dakota-Continued

[Negative values means water levels above land surface. --, not applicable]

\begin{tabular}{|c|c|c|c|c|c|c|}
\hline Site number & Month & Mean & $\begin{array}{l}\text { Standard } \\
\text { deviation }\end{array}$ & Minimum & Maximum & $\begin{array}{l}\text { Number of } \\
\text { valid } \\
\text { samples }\end{array}$ \\
\hline \multirow[t]{11}{*}{430959100444001} & February & 100.75 & 4.88 & 97.30 & 104.20 & 2 \\
\hline & March & 100.42 & 2.49 & 98.20 & 103.60 & 5 \\
\hline & April & 100.48 & 2.34 & 97.80 & 103.70 & 12 \\
\hline & May & 100.15 & 2.37 & 96.80 & 103.30 & 12 \\
\hline & June & 100.12 & 2.48 & 96.90 & 103.60 & 13 \\
\hline & July & 100.32 & 2.45 & 97.30 & 104.30 & 13 \\
\hline & August & 101.80 & 2.31 & 98.50 & 105.90 & 15 \\
\hline & September & 102.09 & 1.92 & 99.60 & 105.20 & 9 \\
\hline & October & 102.42 & 2.19 & 99.90 & 105.64 & 8 \\
\hline & November & 101.33 & 2.10 & 99.20 & 103.80 & 6 \\
\hline & December & 99.43 & .32 & 99.20 & 99.80 & 3 \\
\hline \multirow[t]{12}{*}{431018101152001} & January & -- & -- & 67.00 & 67.00 & 1 \\
\hline & February & -- & -- & 67.60 & 67.60 & 1 \\
\hline & March & 67.60 & .95 & 66.10 & 68.70 & 9 \\
\hline & April & 67.54 & .87 & 65.90 & 68.90 & 14 \\
\hline & May & 67.22 & .92 & 65.90 & 68.70 & 14 \\
\hline & June & 67.42 & .84 & 65.80 & 68.70 & 21 \\
\hline & July & 67.79 & .71 & 66.70 & 68.80 & 19 \\
\hline & August & 67.97 & .87 & 66.50 & 69.50 & 23 \\
\hline & September & 68.35 & .94 & 66.60 & 69.50 & 13 \\
\hline & October & 68.42 & 1.00 & 66.70 & 69.90 & 12 \\
\hline & November & 68.16 & 1.02 & 66.50 & 69.60 & 10 \\
\hline & December & 67.68 & .64 & 66.70 & 68.30 & 6 \\
\hline \multirow[t]{11}{*}{431018101212001} & February & -- & -- & 29.20 & 29.20 & 1 \\
\hline & March & 27.38 & 2.34 & 23.30 & 29.90 & 10 \\
\hline & April & 27.21 & 2.14 & 23.20 & 30.30 & 14 \\
\hline & May & 27.39 & 1.91 & 24.50 & 29.70 & 12 \\
\hline & June & 27.57 & 1.69 & 24.10 & 30.20 & 21 \\
\hline & July & 27.94 & 1.86 & 23.70 & 30.10 & 17 \\
\hline & August & 27.45 & 1.88 & 23.50 & 30.00 & 21 \\
\hline & September & 27.44 & 1.79 & 25.30 & 30.00 & 11 \\
\hline & October & 27.69 & 1.64 & 25.10 & 30.00 & 9 \\
\hline & November & 27.79 & 1.39 & 25.40 & 29.70 & 8 \\
\hline & December & 28.22 & 1.12 & 26.40 & 29.80 & 6 \\
\hline
\end{tabular}


Table 56. Summary of periodic ground-water-level data (from the U.S. Geological Survey database), in feet below land surface, for sites in the High Plains aquifer in South Dakota-Continued

[Negative values means water levels above land surface. --, not applicable]

\begin{tabular}{|c|c|c|c|c|c|c|}
\hline Site number & Month & Mean & $\begin{array}{l}\text { Standard } \\
\text { deviation }\end{array}$ & Minimum & Maximum & $\begin{array}{c}\text { Number of } \\
\text { valid } \\
\text { samples }\end{array}$ \\
\hline \multirow[t]{11}{*}{431020100243501} & February & 8.05 & 6.15 & 3.70 & 12.40 & 2 \\
\hline & March & 8.39 & 2.37 & 6.70 & 13.10 & 7 \\
\hline & April & 7.12 & 3.02 & 3.20 & 13.40 & 13 \\
\hline & May & 6.72 & 2.47 & 3.40 & 12.50 & 14 \\
\hline & June & 7.36 & 2.86 & 2.80 & 12.70 & 16 \\
\hline & July & 7.91 & 2.68 & 4.20 & 12.90 & 14 \\
\hline & August & 7.51 & 2.38 & 4.70 & 13.00 & 18 \\
\hline & September & 8.11 & 2.70 & 3.75 & 13.10 & 11 \\
\hline & October & 7.58 & 1.49 & 5.80 & 10.70 & 8 \\
\hline & November & 8.07 & 2.60 & 6.20 & 13.30 & 6 \\
\hline & December & 6.50 & .82 & 5.80 & 7.40 & 3 \\
\hline \multirow[t]{11}{*}{431022101555001} & February & -- & -- & 25.60 & 25.60 & 1 \\
\hline & March & 21.95 & 2.78 & 17.80 & 25.30 & 6 \\
\hline & April & 21.34 & 2.67 & 17.60 & 25.70 & 12 \\
\hline & May & 21.10 & 2.17 & 18.00 & 25.70 & 11 \\
\hline & June & 21.74 & 2.38 & 17.30 & 26.30 & 17 \\
\hline & July & 21.83 & 2.15 & 17.60 & 24.90 & 11 \\
\hline & August & 21.59 & 2.39 & 18.00 & 26.20 & 17 \\
\hline & September & 21.99 & 2.38 & 19.80 & 26.20 & 8 \\
\hline & October & 21.82 & 1.62 & 19.80 & 25.30 & 9 \\
\hline & November & 22.18 & 2.13 & 20.40 & 26.30 & 6 \\
\hline & December & 22.77 & 2.63 & 21.20 & 25.80 & 3 \\
\hline \multirow[t]{10}{*}{431022101570101} & March & 24.40 & 5.19 & 18.00 & 30.90 & 10 \\
\hline & April & 23.90 & 5.39 & 14.20 & 32.20 & 13 \\
\hline & May & 23.45 & 4.61 & 14.50 & 29.30 & 14 \\
\hline & June & 24.21 & 5.30 & 14.60 & 33.90 & 20 \\
\hline & July & 23.37 & 4.79 & 16.70 & 33.80 & 15 \\
\hline & August & 24.23 & 5.71 & 15.80 & 34.30 & 21 \\
\hline & September & 24.48 & 6.60 & 18.30 & 34.10 & 10 \\
\hline & October & 23.04 & 5.68 & 15.50 & 34.10 & 9 \\
\hline & November & 24.25 & 4.42 & 19.40 & 32.90 & 8 \\
\hline & December & 22.25 & 6.16 & 15.60 & 33.40 & 6 \\
\hline
\end{tabular}


Table 56. Summary of periodic ground-water-level data (from the U.S. Geological Survey database), in feet below land surface, for sites in the High Plains aquifer in South Dakota-Continued

[Negative values means water levels above land surface. --, not applicable]

\begin{tabular}{|c|c|c|c|c|c|c|}
\hline Site number & Month & Mean & $\begin{array}{l}\text { Standard } \\
\text { deviation }\end{array}$ & Minimum & Maximum & $\begin{array}{c}\text { Number of } \\
\text { valid } \\
\text { samples }\end{array}$ \\
\hline \multirow[t]{12}{*}{431045101293701} & January & 16.20 & 1.41 & 15.20 & 17.20 & 2 \\
\hline & February & -- & -- & 17.30 & 17.30 & 1 \\
\hline & March & 15.65 & 1.70 & 12.40 & 20.00 & 13 \\
\hline & April & 16.11 & 1.29 & 13.80 & 18.80 & 12 \\
\hline & May & 16.20 & .69 & 15.40 & 16.90 & 5 \\
\hline & June & 16.11 & .98 & 14.00 & 17.10 & 12 \\
\hline & July & 16.29 & 1.22 & 13.90 & 18.20 & 21 \\
\hline & August & 16.75 & .67 & 15.40 & 17.90 & 12 \\
\hline & September & 16.93 & .57 & 15.80 & 17.60 & 7 \\
\hline & October & 16.34 & 1.42 & 13.60 & 17.70 & 7 \\
\hline & November & 17.19 & .98 & 15.50 & 18.60 & 14 \\
\hline & December & 16.41 & .79 & 15.20 & 17.60 & 9 \\
\hline \multirow[t]{12}{*}{431109100445901} & January & -- & -- & 95.10 & 95.10 & 1 \\
\hline & February & 98.65 & 2.48 & 96.90 & 100.40 & 2 \\
\hline & March & 98.23 & 1.77 & 96.00 & 100.30 & 8 \\
\hline & April & 98.54 & 1.41 & 96.10 & 100.50 & 14 \\
\hline & May & 97.95 & 1.74 & 95.30 & 100.30 & 18 \\
\hline & June & 98.03 & 1.55 & 95.40 & 100.50 & 20 \\
\hline & July & 97.90 & 1.50 & 95.50 & 100.30 & 19 \\
\hline & August & 97.79 & 1.67 & 95.30 & 100.40 & 24 \\
\hline & September & 98.07 & 1.74 & 95.60 & 100.40 & 13 \\
\hline & October & 98.40 & 2.25 & 95.50 & 103.40 & 12 \\
\hline & November & 97.79 & 1.73 & 95.60 & 100.20 & 9 \\
\hline & December & 96.93 & 1.51 & 95.10 & 98.70 & 7 \\
\hline 431127100235201 & June & -- & -- & 26.00 & 26.00 & 1 \\
\hline \multirow[t]{12}{*}{431136101200901} & January & -- & -- & 22.40 & 22.40 & 1 \\
\hline & February & -- & -- & 24.00 & 24.00 & 1 \\
\hline & March & 21.86 & 1.05 & 19.80 & 23.40 & 9 \\
\hline & April & 21.54 & 1.49 & 19.50 & 23.90 & 14 \\
\hline & May & 21.15 & 1.50 & 19.30 & 24.30 & 14 \\
\hline & June & 22.15 & 1.74 & 19.30 & 26.10 & 22 \\
\hline & July & 23.68 & 2.12 & 21.10 & 28.80 & 19 \\
\hline & August & 24.50 & 2.24 & 21.40 & 30.60 & 23 \\
\hline & September & 24.56 & 1.58 & 21.90 & 26.50 & 13 \\
\hline & October & 23.50 & 1.85 & 21.40 & 27.50 & 11 \\
\hline & November & 23.44 & 1.60 & 20.80 & 25.30 & 11 \\
\hline & December & 22.30 & 1.25 & 21.00 & 24.30 & 6 \\
\hline 431139100311501 & August & -- & -- & 12.00 & 12.00 & 1 \\
\hline
\end{tabular}


Table 56. Summary of periodic ground-water-level data (from the U.S. Geological Survey database), in feet below land surface, for sites in the High Plains aquifer in South Dakota-Continued

[Negative values means water levels above land surface. --, not applicable]

\begin{tabular}{|c|c|c|c|c|c|c|}
\hline Site number & Month & Mean & $\begin{array}{l}\text { Standard } \\
\text { deviation }\end{array}$ & Minimum & Maximum & $\begin{array}{l}\text { Number of } \\
\text { valid } \\
\text { samples }\end{array}$ \\
\hline 431159100412102 & October & - & -- & 80.52 & 80.52 & 1 \\
\hline 431159100412103 & October & -- & -- & 80.24 & 80.24 & 1 \\
\hline \multirow[t]{12}{*}{431255101212001} & January & -- & -- & 68.30 & 68.30 & 1 \\
\hline & February & -- & -- & 68.70 & 68.70 & 1 \\
\hline & March & 68.09 & 0.69 & 66.90 & 69.20 & 8 \\
\hline & April & 67.92 & .82 & 66.60 & 69.20 & 13 \\
\hline & May & 68.00 & .60 & 67.00 & 68.70 & 12 \\
\hline & June & 68.23 & .55 & 67.30 & 69.30 & 17 \\
\hline & July & 69.01 & .72 & 67.40 & 70.30 & 15 \\
\hline & August & 69.51 & .83 & 67.80 & 71.00 & 19 \\
\hline & September & 69.77 & .86 & 68.50 & 71.00 & 10 \\
\hline & October & 69.21 & .71 & 68.20 & 70.50 & 8 \\
\hline & November & 69.15 & .78 & 68.10 & 70.00 & 8 \\
\hline & December & 68.45 & .79 & 67.60 & 69.50 & 4 \\
\hline \multirow[t]{11}{*}{431430100195901} & February & -- & -- & 22.30 & 22.30 & 1 \\
\hline & March & 26.34 & 1.47 & 24.80 & 29.10 & 7 \\
\hline & April & 25.89 & 1.89 & 22.30 & 29.30 & 14 \\
\hline & May & 25.28 & 1.26 & 23.40 & 27.30 & 12 \\
\hline & June & 25.52 & 2.02 & 21.60 & 29.60 & 15 \\
\hline & July & 26.15 & 2.33 & 21.70 & 29.90 & 13 \\
\hline & August & 25.79 & 1.97 & 22.10 & 29.70 & 18 \\
\hline & September & 26.16 & 1.97 & 22.30 & 29.90 & 10 \\
\hline & October & 25.96 & 1.13 & 23.90 & 27.30 & 8 \\
\hline & November & 26.63 & 1.82 & 24.50 & 29.80 & 6 \\
\hline & December & 24.90 & .79 & 24.00 & 25.50 & 3 \\
\hline \multirow[t]{10}{*}{431458101483001} & March & 187.80 & .62 & 187.00 & 188.70 & 5 \\
\hline & April & 187.79 & .29 & 187.40 & 188.40 & 13 \\
\hline & May & 187.98 & .46 & 187.60 & 188.90 & 9 \\
\hline & June & 187.92 & .50 & 186.50 & 188.80 & 17 \\
\hline & July & 187.84 & .47 & 187.00 & 188.60 & 10 \\
\hline & August & 187.94 & .47 & 187.30 & 189.30 & 18 \\
\hline & September & 188.16 & .77 & 187.50 & 189.80 & 8 \\
\hline & October & 187.91 & .28 & 187.60 & 188.30 & 7 \\
\hline & November & 188.30 & .35 & 187.90 & 188.90 & 6 \\
\hline & December & 188.25 & .35 & 187.90 & 188.60 & 4 \\
\hline 431505100560901 & September & -- & -- & 134.94 & 134.94 & 1 \\
\hline \multirow[t]{2}{*}{431505100565801} & September & -- & -- & 16.25 & 16.25 & 1 \\
\hline & October & -- & -- & 18.67 & 18.67 & 1 \\
\hline
\end{tabular}


Table 56. Summary of periodic ground-water-level data (from the U.S. Geological Survey database), in feet below land surface, for sites in the High Plains aquifer in South Dakota-Continued

[Negative values means water levels above land surface. --, not applicable]

\begin{tabular}{|c|c|c|c|c|c|c|}
\hline Site number & Month & Mean & $\begin{array}{l}\text { Standard } \\
\text { deviation }\end{array}$ & Minimum & Maximum & $\begin{array}{l}\text { Number of } \\
\text { valid } \\
\text { samples }\end{array}$ \\
\hline \multirow[t]{2}{*}{431508100562101} & September & -- & -- & 100.94 & 100.94 & 1 \\
\hline & October & -- & -- & 101.99 & 101.99 & 1 \\
\hline \multirow[t]{11}{*}{431514101061101} & February & -- & -- & 30.80 & 30.80 & 1 \\
\hline & March & 29.30 & 1.08 & 28.40 & 30.50 & 3 \\
\hline & April & 29.36 & .96 & 28.10 & 30.80 & 8 \\
\hline & May & 28.35 & 1.40 & 26.90 & 30.60 & 6 \\
\hline & June & 28.82 & 1.41 & 26.80 & 30.80 & 11 \\
\hline & July & 29.26 & 1.46 & 27.20 & 31.10 & 11 \\
\hline & August & 29.19 & 1.45 & 27.30 & 31.20 & 13 \\
\hline & September & 30.26 & 1.02 & 28.80 & 31.40 & 5 \\
\hline & October & 28.95 & 1.29 & 27.80 & 30.70 & 4 \\
\hline & November & 30.16 & .97 & 28.80 & 31.30 & 5 \\
\hline & December & 29.03 & 1.02 & 28.20 & 30.40 & 4 \\
\hline 431515100553901 & October & -- & -- & 145.54 & 145.54 & 1 \\
\hline \multirow[t]{2}{*}{431517100562301} & September & 103.11 & .01 & 103.10 & 103.11 & 2 \\
\hline & October & -- & -- & 104.14 & 104.14 & 1 \\
\hline \multirow[t]{2}{*}{431526100563202} & September & -- & -- & 10.96 & 10.96 & 1 \\
\hline & October & -- & -- & 13.15 & 13.15 & 1 \\
\hline \multirow[t]{11}{*}{431530101264101} & February & -- & -- & 19.80 & 19.80 & 1 \\
\hline & March & 16.25 & 2.28 & 11.90 & 19.40 & 10 \\
\hline & April & 13.58 & 6.05 & -.50 & 19.50 & 15 \\
\hline & May & 13.70 & 5.34 & .60 & 18.90 & 16 \\
\hline & June & 15.07 & 4.61 & 2.20 & 19.80 & 23 \\
\hline & July & 17.00 & 2.78 & 11.10 & 21.50 & 16 \\
\hline & August & 16.87 & 2.91 & 11.60 & 22.10 & 21 \\
\hline & September & 17.54 & 2.59 & 14.80 & 22.00 & 12 \\
\hline & October & 16.98 & 1.61 & 14.90 & 19.40 & 10 \\
\hline & November & 17.28 & 2.11 & 14.50 & 20.50 & 9 \\
\hline & December & 17.15 & 1.72 & 15.40 & 20.10 & 6 \\
\hline
\end{tabular}


Table 56. Summary of periodic ground-water-level data (from the U.S. Geological Survey database), in feet below land surface, for sites in the High Plains aquifer in South Dakota-Continued

[Negative values means water levels above land surface. --, not applicable]

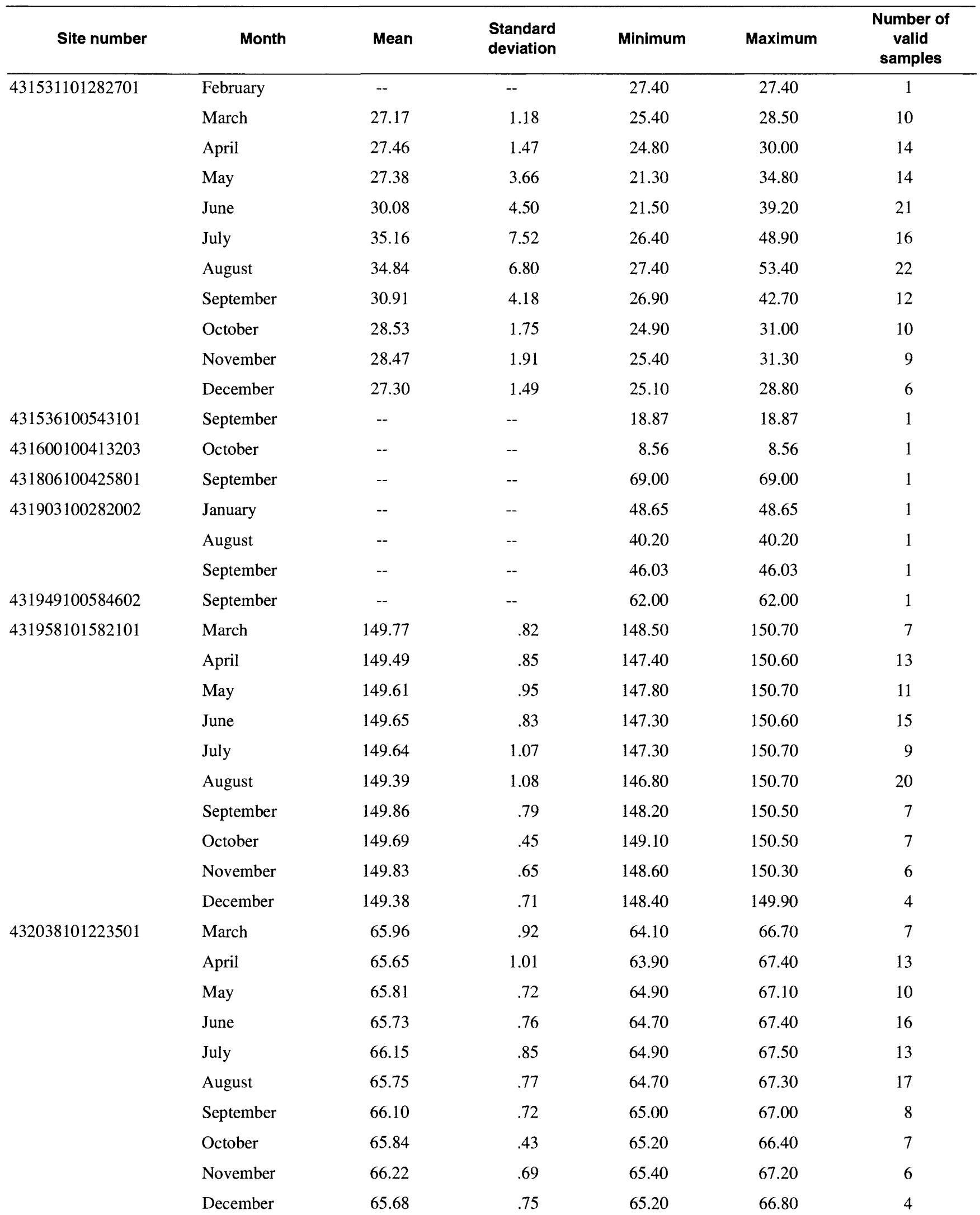


Table 56. Summary of periodic ground-water-level data (from the U.S. Geological Survey database), in feet below land surface, for sites in the High Plains aquifer in South Dakota-Continued

[Negative values means water levels above land surface. --, not applicable]

\begin{tabular}{|c|c|c|c|c|c|c|}
\hline Site number & Month & Mean & $\begin{array}{l}\text { Standard } \\
\text { deviation }\end{array}$ & Minimum & Maximum & $\begin{array}{l}\text { Number of } \\
\text { valid } \\
\text { samples }\end{array}$ \\
\hline \multirow[t]{10}{*}{432044101115201} & March & 37.90 & 0.58 & 37.20 & 38.80 & 5 \\
\hline & April & 37.10 & 1.25 & 34.30 & 39.20 & 12 \\
\hline & May & 37.06 & 1.06 & 35.30 & 38.70 & 12 \\
\hline & June & 37.00 & 1.26 & 33.70 & 39.00 & 15 \\
\hline & July & 37.50 & 1.47 & 33.90 & 39.40 & 12 \\
\hline & August & 37.48 & 1.16 & 34.40 & 39.50 & 18 \\
\hline & September & 37.77 & 1.47 & 34.40 & 39.60 & 9 \\
\hline & October & 37.67 & .66 & 36.70 & 38.40 & 7 \\
\hline & November & 38.37 & .91 & 36.90 & 39.70 & 6 \\
\hline & December & 37.80 & 1.21 & 36.70 & 39.10 & 3 \\
\hline \multirow[t]{10}{*}{432120101120001} & March & 34.84 & 2.00 & 31.60 & 37.30 & 8 \\
\hline & April & 35.13 & 2.09 & 30.80 & 39.10 & 13 \\
\hline & May & 35.36 & 1.95 & 32.70 & 38.80 & 10 \\
\hline & June & 35.82 & 2.35 & 29.00 & 38.10 & 13 \\
\hline & July & 36.46 & 2.50 & 31.70 & 39.20 & 11 \\
\hline & August & 37.14 & 1.89 & 32.60 & 40.10 & 16 \\
\hline & September & 38.68 & 1.59 & 36.50 & 40.40 & 6 \\
\hline & October & 37.87 & 2.10 & 34.50 & 40.90 & 9 \\
\hline & November & 38.48 & 1.33 & 36.30 & 39.40 & 5 \\
\hline & December & 36.70 & 2.08 & 34.30 & 37.90 & 3 \\
\hline \multirow[t]{11}{*}{432310101045501} & February & 32.20 & 4.67 & 28.90 & 35.50 & 2 \\
\hline & March & 33.47 & 2.20 & 31.30 & 36.10 & 6 \\
\hline & April & 32.40 & 1.98 & 28.80 & 35.80 & 12 \\
\hline & May & 32.72 & 1.65 & 31.20 & 35.80 & 13 \\
\hline & June & 32.95 & 1.95 & 30.90 & 36.50 & 15 \\
\hline & July & 33.52 & 2.11 & 30.50 & 36.40 & 13 \\
\hline & August & 32.91 & 1.96 & 30.20 & 36.30 & 18 \\
\hline & September & 32.98 & 2.08 & 31.00 & 36.30 & 9 \\
\hline & October & 32.42 & 2.54 & 27.60 & 36.20 & 9 \\
\hline & November & 32.93 & 1.97 & 31.30 & 36.30 & 6 \\
\hline & December & 33.78 & 1.98 & 31.50 & 36.00 & 4 \\
\hline
\end{tabular}


Table 56. Summary of periodic ground-water-level data (from the U.S. Geological Survey database), in feet below land surface, for sites in the High Plains aquifer in South Dakota-Continued

[Negative values means water levels above land surface. --, not applicable]

\begin{tabular}{|c|c|c|c|c|c|c|}
\hline Site number & Month & Mean & $\begin{array}{l}\text { Standard } \\
\text { deviation }\end{array}$ & Minimum & Maximum & $\begin{array}{l}\text { Number of } \\
\text { valid } \\
\text { samples }\end{array}$ \\
\hline \multirow[t]{10}{*}{432410101294002} & March & 53.48 & 1.42 & 50.30 & 54.60 & 8 \\
\hline & April & 52.34 & 1.77 & 49.80 & 54.50 & 7 \\
\hline & May & 53.40 & .79 & 52.30 & 54.60 & 8 \\
\hline & June & 53.40 & .69 & 52.20 & 54.50 & 10 \\
\hline & July & 53.63 & .60 & 52.70 & 54.60 & 7 \\
\hline & August & 53.20 & .61 & 52.10 & 53.90 & 12 \\
\hline & September & 53.95 & .77 & 53.00 & 54.90 & 6 \\
\hline & October & 53.58 & .51 & 53.10 & 54.30 & 4 \\
\hline & November & 53.93 & .74 & 53.10 & 54.80 & 4 \\
\hline & December & 53.47 & .47 & 53.10 & 54.00 & 3 \\
\hline \multirow[t]{11}{*}{432554101065601} & February & 13.75 & 7.00 & 8.80 & 18.70 & 2 \\
\hline & March & 15.82 & 2.81 & 12.50 & 19.90 & 9 \\
\hline & April & 14.98 & 4.68 & 7.80 & 22.40 & 14 \\
\hline & May & 13.47 & 5.40 & 6.10 & 21.30 & 14 \\
\hline & June & 13.36 & 4.16 & 5.20 & 20.40 & 19 \\
\hline & July & 13.73 & 4.90 & 6.40 & 21.60 & 16 \\
\hline & August & 15.71 & 4.03 & 7.60 & 22.10 & 21 \\
\hline & September & 18.91 & 3.72 & 9.80 & 23.50 & 11 \\
\hline & October & 17.34 & 3.95 & 11.62 & 22.20 & 10 \\
\hline & November & 20.36 & 3.47 & 14.20 & 24.00 & 8 \\
\hline & December & 16.94 & 4.33 & 12.30 & 22.90 & 5 \\
\hline 432649101081802 & October & -- & -- & 12.98 & 12.98 & 1 \\
\hline \multirow[t]{10}{*}{432740101223501} & March & 30.36 & 1.38 & 27.60 & 31.70 & 7 \\
\hline & April & 30.11 & 1.58 & 27.40 & 32.00 & 8 \\
\hline & May & 30.20 & 1.09 & 28.10 & 31.60 & 8 \\
\hline & June & 30.31 & 1.15 & 27.90 & 31.80 & 10 \\
\hline & July & 30.44 & 1.12 & 28.10 & 31.80 & 8 \\
\hline & August & 30.25 & 1.10 & 28.20 & 32.00 & 11 \\
\hline & September & 30.70 & 1.20 & 28.50 & 32.10 & 7 \\
\hline & October & 30.56 & 1.08 & 28.90 & 31.90 & 5 \\
\hline & November & 30.50 & 1.51 & 28.60 & 32.20 & 4 \\
\hline & December & 30.43 & 1.56 & 29.00 & 32.10 & 3 \\
\hline 432742101115802 & October & -- & -- & 25.40 & 25.40 & 1 \\
\hline
\end{tabular}


Table 56. Summary of periodic ground-water-level data (from the U.S. Geological Survey database), in feet below land surface, for sites in the High Plains aquifer in South Dakota-Continued

[Negative values means water levels above land surface. --, not applicable]

\begin{tabular}{lllcccc}
\hline Site number & Month & Mean & $\begin{array}{c}\text { Standard } \\
\text { deviation }\end{array}$ & Minimum & Maximum & $\begin{array}{c}\text { Number of } \\
\text { valid } \\
\text { samples }\end{array}$ \\
\hline 433020101394201 & March & 72.22 & 0.72 & 71.40 & 73.30 & 6 \\
& April & 71.60 & .47 & 71.20 & 72.50 & 6 \\
& May & 72.30 & .82 & 71.30 & 73.10 & 5 \\
& June & 72.17 & .73 & 71.30 & 73.10 & 6 \\
& July & 72.56 & .91 & 71.40 & 73.50 & 5 \\
& August & 72.24 & .60 & 71.50 & 73.00 & 8 \\
& September & 73.20 & .27 & 72.90 & 73.40 & 3 \\
& October & 72.63 & .83 & 71.70 & 73.30 & 3 \\
\\
November & 73.05 & .21 & 72.90 & 73.20 & 2 \\
\hline
\end{tabular}


Table 57. Selected descriptive data (from the U.S. Geological Survey database) for ground-water sites in the High Plains aquifer in South Dakota

[County code: 7 = Bennett County, $53=$ Gregory County, $71=$ Jackson County, $95=$ Mellette County, $113=$ Shannon County, and $121=$ Todd County; Datum, National Geodetic Vertical Datum; 1210GLL, Ogallala Formation; 122ARKR, Arikaree Formation; --, no data]

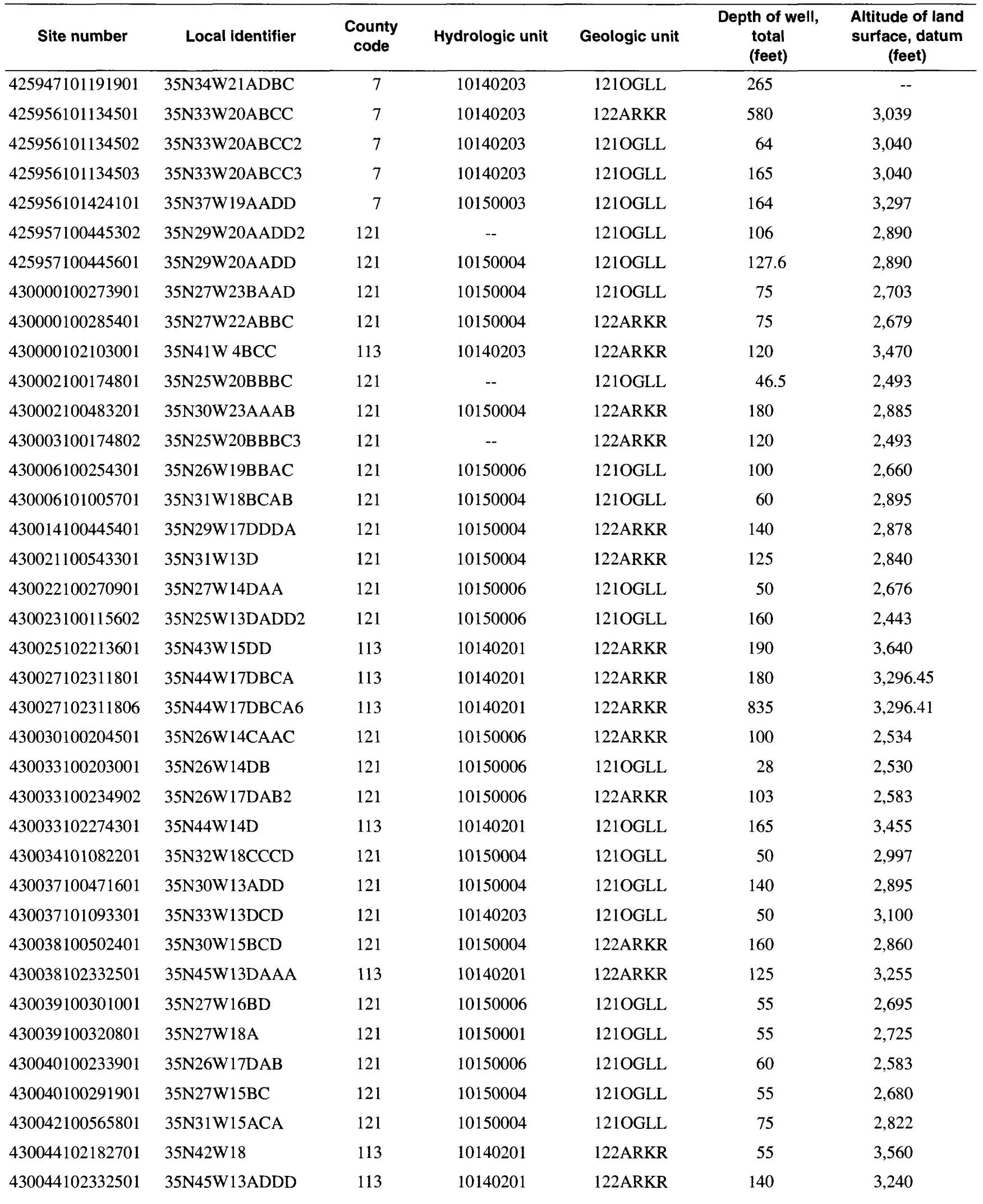


Table 57. Selected descriptive data (from the U.S. Geological Survey database) for ground-water sites in the High Plains aquifer in South Dakota - Continued

[County code: 7 = Bennett County, 53 = Gregory County, $71=$ Jackson County, $95=$ Mellette County, $113=$ Shannon County, and $121=$ Todd County; Datum, National Geodetic Vertical Datum; 1210GLL, Ogallala Formation; 122ARKR, Arikaree Formation; --, no data]

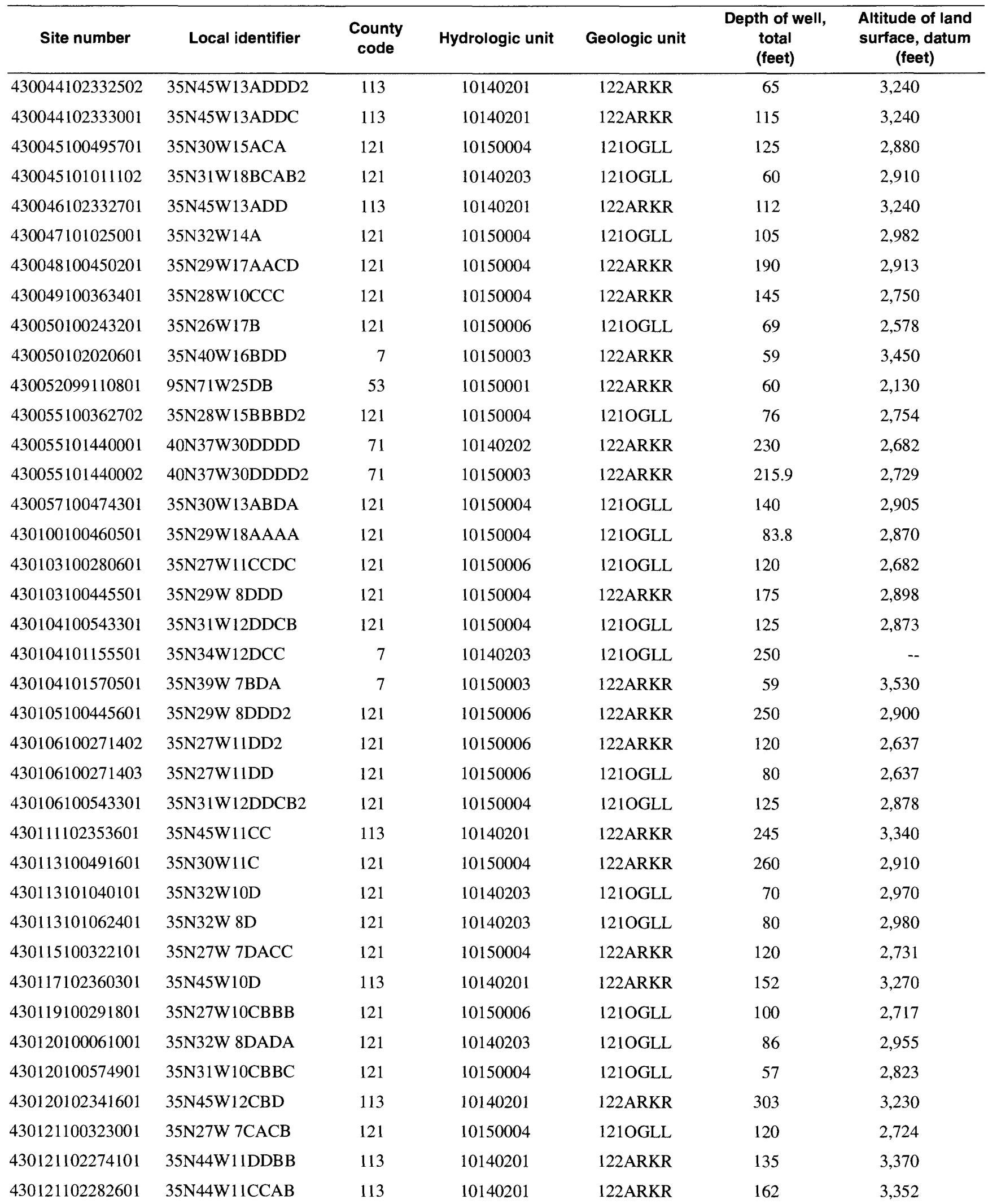


Table 57. Selected descriptive data (from the U.S. Geological Survey database) for ground-water sites in the High Plains aquifer in South Dakota-Continued

[County code: 7 = Bennett County, $53=$ Gregory County, $71=$ Jackson County, $95=$ Mellette County, $113=$ Shannon County, and $121=$ Todd County; Datum, National Geodetic Vertical Datum; 121OGLL, Ogallala Formation; 122ARKR, Arikaree Formation; --, no data]

\begin{tabular}{|c|c|c|c|c|c|c|}
\hline Site number & Local identifier & $\begin{array}{l}\text { County } \\
\text { code }\end{array}$ & Hydrologic unit & Geologic unit & $\begin{array}{c}\text { Depth of well, } \\
\text { total } \\
\text { (feet) }\end{array}$ & $\begin{array}{l}\text { Altitude of land } \\
\text { surface, datum } \\
\text { (feet) }\end{array}$ \\
\hline 430122100344501 & $35 \mathrm{~N} 28 \mathrm{~W} 11 \mathrm{DBBB}$ & 121 & 10150006 & 121OGLL & 94.1 & 2,728 \\
\hline 430123102321501 & $35 \mathrm{~N} 44 \mathrm{~W} 7 \mathrm{CAB}$ & 113 & 10140201 & 122ARKR & 284 & 3,300 \\
\hline 430124100415701 & $35 \mathrm{~N} 29 \mathrm{~W} 11 \mathrm{CAA}$ & 121 & 10150004 & 121OGLL & 42 & 2,777 \\
\hline 430124102342501 & $35 \mathrm{~N} 45 \mathrm{~W} 12 \mathrm{CB}$ & 113 & 10140201 & 122ARKR & 350 & 3,230 \\
\hline 430125102343201 & $35 \mathrm{~N} 45 \mathrm{~W} 12 \mathrm{CBBC}$ & 113 & 10140201 & 122ARKR & -- & 3,130 \\
\hline 430126100221901 & $35 \mathrm{~N} 26 \mathrm{~W} 10 \mathrm{CBBA} 3$ & 121 & -- & 122ARKR & 492 & 2,617 \\
\hline 430126100222001 & $35 \mathrm{~N} 26 \mathrm{~W} 10 \mathrm{CBBA} 2$ & 121 & -- & 121OGLL & 82 & 2,618 \\
\hline 430127100230601 & 35N26W 9BDAD & 121 & 10150006 & 122ARKR & 100 & 2,585 \\
\hline 430127100481701 & $35 \mathrm{~N} 30 \mathrm{~W} 12 \mathrm{BCCD}$ & 121 & 10150004 & 121OGLL & 150 & 2,915 \\
\hline 430127102333601 & $35 \mathrm{~N} 45 \mathrm{~W} 12 \mathrm{DAB}$ & 113 & 10140201 & 122ARKR & 120 & 3,230 \\
\hline 430129099201201 & 95N72W22DDDD & 53 & 10150006 & 122ARKR & 104 & 2,217 \\
\hline 430129101075401 & $35 \mathrm{~N} 32 \mathrm{~W} 7 \mathrm{BDD}$ & 121 & 10140203 & 121OGLL & 50 & 2,950 \\
\hline 430131102261601 & $35 \mathrm{~N} 44 \mathrm{~W} 12 \mathrm{DADA}$ & 113 & 10140201 & 122ARKR & 155 & 3,382 \\
\hline 430135100302401 & $35 \mathrm{~N} 27 \mathrm{~W} 9 \mathrm{BADB}$ & 121 & 10150004 & 122ARKR & 135 & 2,701 \\
\hline 430136102355401 & 35N45W10AD & 113 & 10140201 & 122ARKR & 127 & 3,250 \\
\hline 430138100470601 & 35N29W 7BCBA & 121 & 10150004 & 121OGLL & -- & 2,909 \\
\hline 430138102363201 & $35 \mathrm{~N} 45 \mathrm{~W} 10 \mathrm{BDBD}$ & 113 & 10140201 & 122ARKR & 170 & 3,340 \\
\hline 430139100264401 & $35 \mathrm{~N} 27 \mathrm{~W} 12 \mathrm{~B}$ & 121 & 10150006 & 1210GLL & 85 & 2,636 \\
\hline 430139100474801 & $35 \mathrm{~N} 30 \mathrm{~W} 12 \mathrm{ACBB}$ & 121 & 10150004 & 1210GLL & 160 & 2,935 \\
\hline 430140102273701 & 35N44W11ADCD & 113 & 10140201 & 122ARKR & 125 & 3,357 \\
\hline 430141100243201 & $35 \mathrm{~N} 26 \mathrm{~W} 8 \mathrm{~B}$ & 121 & 10150006 & 122ARKR & 105 & 2,627 \\
\hline 430142100580301 & $35 \mathrm{~N} 31 \mathrm{~W} 9 \mathrm{AACD}$ & 121 & 10150004 & 122ARKR & 120 & 2,840 \\
\hline 430142102093601 & 35N41W 8ADAA & 113 & 10140201 & 122ARKR & 120 & 3,550 \\
\hline 430142102341601 & $35 \mathrm{~N} 45 \mathrm{~W} 12 \mathrm{~B}$ & 113 & 10140201 & 122ARKR & 112 & 3,195 \\
\hline 430142102341602 & $35 \mathrm{~N} 45 \mathrm{~W} 12 \mathrm{~B} 2$ & 113 & 10140201 & 122ARKR & 92 & 3,195 \\
\hline 430144099194301 & $95 N 72 W 23 C A 2$ & 53 & 10150001 & 121OGLL & 100 & 2,220 \\
\hline 430146102322701 & $35 \mathrm{~N} 44 \mathrm{~W} 7 \mathrm{ADBB}$ & 113 & 10140201 & 122ARKR & -- & 3,249 \\
\hline 430146102325801 & $35 \mathrm{~N} 44 \mathrm{~W} 7 \mathrm{BDBA}$ & 113 & 10140201 & 122ARKR & -- & 3,265 \\
\hline 430147102343601 & $35 \mathrm{~N} 45 \mathrm{~W} 11 \mathrm{AADD}$ & 113 & 10140201 & 122ARKR & 129 & 3,215 \\
\hline 430148100471001 & $35 \mathrm{~N} 29 \mathrm{~W} 7 \mathrm{BBBB}$ & 121 & 10150004 & 121OGLL & 83.1 & 2,903 \\
\hline 430149102355401 & $35 \mathrm{~N} 45 \mathrm{~W} 10 \mathrm{AA}$ & 113 & 10140201 & 122ARKR & 127 & 3,280 \\
\hline 430150100054601 & $35 N 32 W$ 9BABB & 121 & 10140203 & 122ARKR & 678 & 2,935 \\
\hline 430150100523801 & $35 \mathrm{~N} 30 \mathrm{~W} 8 \mathrm{BAA}$ & 121 & 10150004 & 121OGLL & 95 & 2,840 \\
\hline 430150102303501 & 35N44W 9BBAD & 113 & 10140201 & 122ARKR & 245 & 3,280 \\
\hline 430151100173901 & $35 \mathrm{~N} 25 \mathrm{~W} 8 \mathrm{BB}$ & 121 & 10150006 & 1210GLL & 90 & 2,513 \\
\hline 430151100415401 & $35 \mathrm{~N} 29 \mathrm{~W} 11 \mathrm{ABBB}$ & 121 & 10150004 & 1210GLL & 65 & 2,800 \\
\hline 430151100415402 & $35 \mathrm{~N} 29 \mathrm{~W} 11 \mathrm{ABBB} 2$ & 121 & 10150004 & 1210GLL & 110 & 2,800 \\
\hline 430152102335401 & $35 \mathrm{~N} 45 \mathrm{~W} 12 \mathrm{ABB}$ & 113 & 10140201 & 122ARKR & 220 & 3,262 \\
\hline
\end{tabular}


Table 57. Selected descriptive data (from the U.S. Geological Survey database) for ground-water sites in the High Plains aquifer in South Dakota-Continued

[County code: 7 = Bennett County, $53=$ Gregory County, $71=$ Jackson County, $95=$ Mellette County, $113=$ Shannon County, and $121=$ Todd County; Datum, National Geodetic Vertical Datum; 1210GLL, Ogallala Formation; 122ARKR, Arikaree Formation; --, no data]

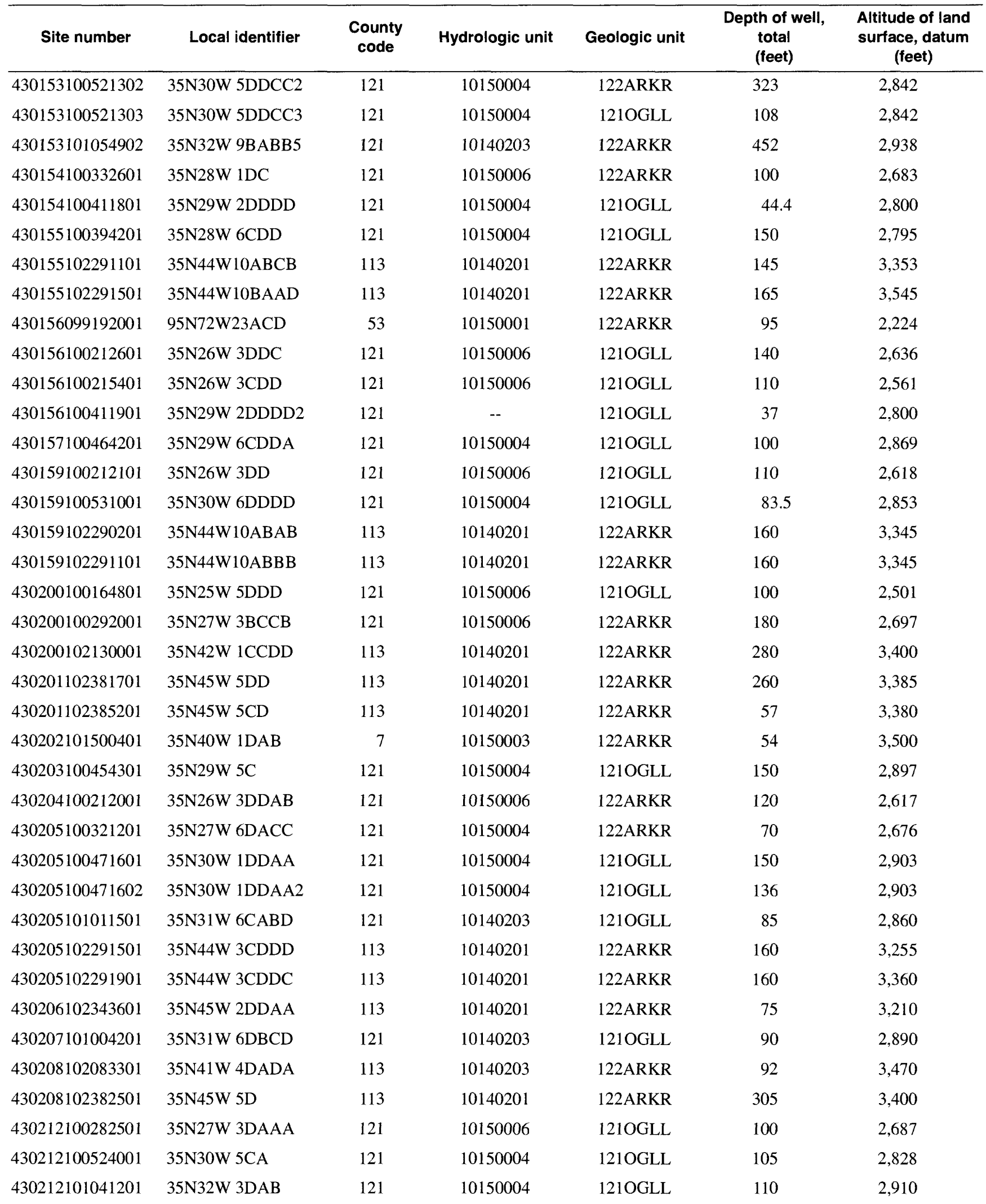


Table 57. Selected descriptive data (from the U.S. Geological Survey database) for ground-water sites in the High Plains aquifer in South Dakota-Continued

[County code: 7 = Bennett County, 53 = Gregory County, 71 = Jackson County, $95=$ Mellette County, $113=$ Shannon County, and $121=$ Todd County; Datum, National Geodetic Vertical Datum; 1210GLL, Ogallala Formation; 122ARKR, Arikaree Formation; --, no data]

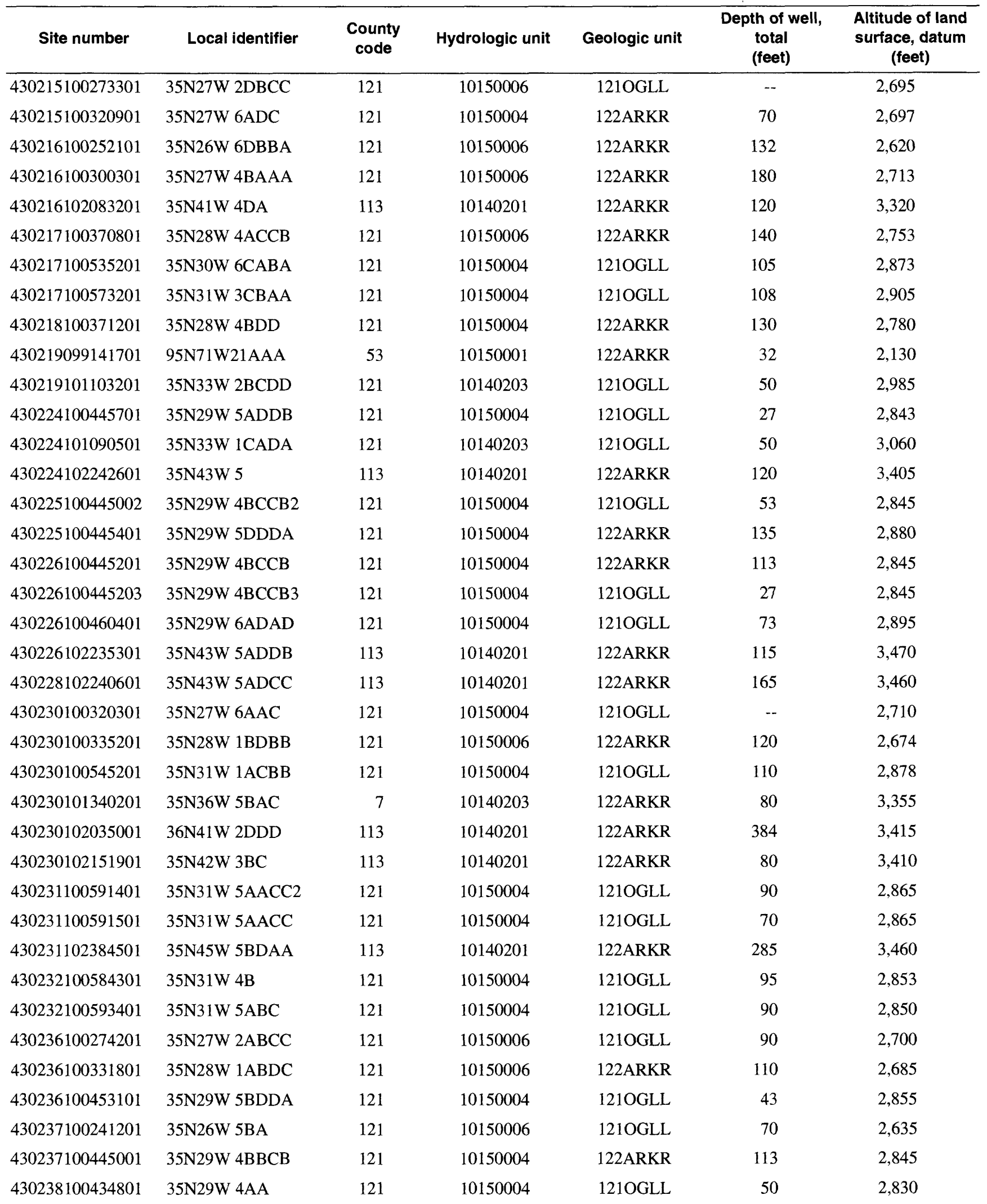


Table 57. Selected descriptive data (from the U.S. Geological Survey database) for ground-water sites in the High Plains aquifer in South Dakota-Continued

[County code: 7 = Bennett County, $53=$ Gregory County, $71=$ Jackson County, $95=$ Mellette County, $113=$ Shannon County, and $121=$ Todd County; Datum, National Geodetic Vertical Datum; 121OGLL, Ogallala Formation; 122ARKR, Arikaree Formation; --, no data]

\begin{tabular}{|c|c|c|c|c|c|c|}
\hline Site number & Local identifier & $\begin{array}{l}\text { County } \\
\text { code }\end{array}$ & Hydrologic unit & Geologic unit & $\begin{array}{l}\text { Depth of well, } \\
\text { total } \\
\text { (feet) }\end{array}$ & $\begin{array}{l}\text { Altitude of land } \\
\text { surface, datum } \\
\text { (feet) }\end{array}$ \\
\hline 430239100164801 & $35 \mathrm{~N} 25 \mathrm{~W} 5 \mathrm{AA}$ & 121 & 10150006 & 122ARKR & 100 & 2,513 \\
\hline 430239100174301 & $35 \mathrm{~N} 25 \mathrm{~W} 5 \mathrm{BBAA}$ & 121 & 10150006 & 1210GLL & 72 & 2,518 \\
\hline 430240102115301 & $35 \mathrm{~N} 41 \mathrm{~W} 6 \mathrm{BBCC}$ & 113 & 10140201 & 122ARKR & 140 & 3,550 \\
\hline 430242100184801 & $35 \mathrm{~N} 25 \mathrm{~W} 6 \mathrm{BBA}$ & 121 & 10150006 & 122ARKR & 100 & 2,525 \\
\hline 430242102210001 & $35 \mathrm{~N} 43 \mathrm{~W} 2 \mathrm{BA}$ & 113 & 10140201 & 122ARKR & 110 & 3,450 \\
\hline 430243100333301 & $35 \mathrm{~N} 28 \mathrm{~W} 1 \mathrm{ABB}$ & 121 & 10150006 & 122ARKR & 105 & 2,695 \\
\hline 430243100371701 & $36 \mathrm{~N} 28 \mathrm{~W} 33 \mathrm{BDDD}$ & 121 & 10150006 & $1210 \mathrm{OLL}$ & 74.8 & 2,753 \\
\hline 430243102125601 & $35 \mathrm{~N} 42 \mathrm{~W} 1 \mathrm{BB}$ & 113 & 10140201 & 122ARKR & 60 & 3,430 \\
\hline 430244101284101 & $36 \mathrm{~N} 35 \mathrm{~W} 31 \mathrm{D}$ & 7 & 10140203 & 122ARKR & 60 & 3,200 \\
\hline 430245100272401 & $36 \mathrm{~N} 27 \mathrm{~W} 35 \mathrm{DCD}$ & 121 & 10150006 & 122ARKR & 144 & 2,673 \\
\hline 430245100292701 & $35 \mathrm{~N} 27 \mathrm{~W} 3 \mathrm{BBBB} 4$ & 121 & -- & 122ARKR & 202 & 2,671 \\
\hline 430245100292801 & $35 \mathrm{~N} 27 \mathrm{~W} 3 \mathrm{BBBB}$ & 121 & 10150006 & 121OGLL & 47 & 2,671 \\
\hline 430246100222302 & $36 \mathrm{~N} 26 \mathrm{~W} 34 \mathrm{CCC} 2$ & 121 & 10150006 & 1210GLL & 110 & 2,634 \\
\hline 430247100433801 & $36 \mathrm{~N} 29 \mathrm{~W} 34 \mathrm{CCCC}$ & 121 & 10150004 & 1210GLL & 40 & 2,831 \\
\hline 430250100532701 & 36N30W31DCDA & 121 & 10150004 & 1210GLL & 50 & 2,868 \\
\hline 430250100584701 & 36N31W33CCDA & 121 & 10140203 & 121OGLL & 146 & 2,877 \\
\hline 430252100431301 & $36 \mathrm{~N} 29 \mathrm{~W} 34 \mathrm{CD}$ & 121 & 10150004 & 1210GLL & 100 & 2,804 \\
\hline 430252100531701 & 36N30W31DDBD & 121 & 10150004 & 121OGLL & 40 & 2.951 \\
\hline 430254100453001 & $36 \mathrm{~N} 29 \mathrm{~W} 32 \mathrm{CDAD}$ & 121 & 10150004 & 121OGLL & 60 & 2,851 \\
\hline 430254100515001 & $36 \mathrm{~N} 30 \mathrm{~W} 33 \mathrm{CCBD}$ & 121 & 10150004 & 1210GLL & 50 & 2,879 \\
\hline 430255100390101 & $36 \mathrm{~N} 28 \mathrm{~W} 31 \mathrm{DDA}$ & 121 & 10150006 & 1210GLL & 180 & 2,830 \\
\hline 430255101032301 & $36 \mathrm{~N} 32 \mathrm{~W} 35 \mathrm{CDBB}$ & 121 & 10140203 & 1210GLL & 85 & 2,897 \\
\hline 430255102191201 & $36 N 43 W 36 D D$ & 113 & 10140201 & 122ARKR & 80 & 3,400 \\
\hline 430256101160001 & $36 \mathrm{~N} 34 \mathrm{~W} 36 \mathrm{DBCC}$ & 7 & 10140203 & 1210GLL & 205 & 2,930 \\
\hline 430256102305101 & $36 \mathrm{~N} 44 \mathrm{~W} 33 \mathrm{CC}$ & 113 & 10140201 & 122ARKR & 380 & 3,320 \\
\hline 430257101003001 & 36N31W31D & 121 & 10140203 & 121OGLL & 63 & 2,843 \\
\hline 430257102340501 & 36N45W36CDAB & 113 & 10140201 & 122ARKR & 148 & 3,230 \\
\hline 430258100294701 & 36N27W33DAAC & 121 & 10150006 & 122ARKR & 140 & 2,673 \\
\hline 430258100445701 & $36 \mathrm{~N} 29 \mathrm{~W} 32 \mathrm{DDAB}$ & 121 & 10150004 & 121OGLL & 60 & 2,837 \\
\hline 430258100471401 & 36N30W36DDDA & 121 & 10150004 & 121OGLL & 123 & 2,885 \\
\hline 430258100491601 & 36N30W35CCAA & 121 & 10150004 & 121OGLL & 179 & 2,903 \\
\hline 430259100521001 & 36N30W32DACC & 121 & 10150004 & 121OGLL & 115 & 2,974 \\
\hline 430259102323501 & 36N44W31DCBB & 113 & 10140201 & 122ARKR & 38 & 3,265 \\
\hline 430300101012701 & 36N32W36DADC & 121 & 10140203 & 1210GLL & 146 & 2,858 \\
\hline 430300101373501 & $36 \mathrm{~N} 37 \mathrm{~W} 36 \mathrm{CCA}$ & 7 & 10140203 & 122ARKR & 250 & -- \\
\hline 430301100365801 & 36N28W33DBD & 121 & 10150006 & 122ARKR & 160 & 2,750 \\
\hline 430301100492101 & $36 \mathrm{~N} 30 \mathrm{~W} 35 \mathrm{CBCC}$ & 121 & 10150004 & 122ARKR & 179 & 2,917 \\
\hline 430301100555201 & $36 \mathrm{~N} 31 \mathrm{~W} 35 \mathrm{DBDC}$ & 121 & 10140203 & 121OGLL & 140 & 2,904 \\
\hline
\end{tabular}


Table 57. Selected descriptive data (from the U.S. Geological Survey database) for ground-water sites in the High Plains aquifer in South Dakota-Continued

[County code: 7 = Bennett County, $53=$ Gregory County, 71 = Jackson County, $95=$ Mellette County, $113=$ Shannon County, and $121=$ Todd County; Datum, National Geodetic Vertical Datum; 1210GLL, Ogallala Formation; 122ARKR, Arikaree Formation; --, no data]

\begin{tabular}{|c|c|c|c|c|c|c|}
\hline Site number & Local identifier & $\begin{array}{l}\text { County } \\
\text { code }\end{array}$ & Hydrologic unit & Geologic unit & $\begin{array}{c}\text { Depth of well, } \\
\text { total } \\
\text { (feet) }\end{array}$ & $\begin{array}{l}\text { Altitude of land } \\
\text { surface, datum } \\
\text { (feet) }\end{array}$ \\
\hline 430301102121101 & $36 \mathrm{~N} 42 \mathrm{~W} 36 \mathrm{D}$ & 113 & 10140201 & 122ARKR & -- & 3,500 \\
\hline 430302100255101 & 36N26W33DBDB & 121 & 10150006 & 121OGLL & 130 & 2,630 \\
\hline 430302100412001 & 36N29W35DAD & 121 & 10150004 & 121OGLL & 45 & 2,834 \\
\hline 430305100455401 & $36 \mathrm{~N} 29 \mathrm{~W} 32 \mathrm{CB}$ & 121 & 10150004 & 121OGLL & 60 & 2,835 \\
\hline 430305100531601 & 36N30W31DA & 121 & 10150004 & 121OGLL & 80 & 2,841 \\
\hline 430308100222801 & $36 \mathrm{~N} 26 \mathrm{~W} 33 \mathrm{DAA}$ & 121 & 10150006 & 121OGLL & 110 & 2,637 \\
\hline 430308102183601 & $36 \mathrm{~N} 42 \mathrm{~W} 31 \mathrm{CA}$ & 113 & 10140201 & 122ARKR & 20 & 3,375 \\
\hline 430309100290001 & $36 \mathrm{~N} 27 \mathrm{~W} 34 \mathrm{CAA}$ & 121 & 10150006 & 122ARKR & 110 & 2,673 \\
\hline 430310100245501 & 36N26W31 ADDD & 121 & 10150006 & 121OGLL & 125 & 2,620 \\
\hline 430310101125201 & $36 \mathrm{~N} 33 \mathrm{~W} 33 \mathrm{CABB}$ & 121 & 10140203 & 1210GLL & 99 & 3,015 \\
\hline 430313100491501 & $36 \mathrm{~N} 30 \mathrm{~W} 35 \mathrm{BDCC}$ & 121 & 10150004 & 121OGLL & 246 & 2,913 \\
\hline 430314100392301 & $36 \mathrm{~N} 28 \mathrm{~W} 31 \mathrm{ACDC}$ & 121 & 10150006 & 121OGLL & 95 & 2,839 \\
\hline 430315100184301 & $36 \mathrm{~N} 25 \mathrm{~W} 31 \mathrm{BDCB}$ & 121 & 10150006 & 121OGLL & 70 & 2,552 \\
\hline 430315100282501 & $36 \mathrm{~N} 27 \mathrm{~W} 34 \mathrm{ADDB}$ & 121 & 10150006 & 122ARKR & 100 & 2,670 \\
\hline 430315100303001 & $36 \mathrm{~N} 27 \mathrm{~W} 33 \mathrm{BCCC}$ & 121 & 10150006 & 122ARKR & 120 & 2,708 \\
\hline 430315102280101 & $36 \mathrm{~N} 44 \mathrm{~W} 35$ & 113 & 10140201 & 122ARKR & -- & 3,294 \\
\hline 430321100313401 & $36 \mathrm{~N} 27 \mathrm{~W} 32 \mathrm{BAAA}$ & 121 & 10150006 & 122ARKR & 140 & 2,706 \\
\hline 430322100280301 & $36 \mathrm{~N} 27 \mathrm{~W} 35 \mathrm{BBCD}$ & 121 & 10150006 & 122ARKR & 100 & 2,654 \\
\hline 430323100430001 & $36 \mathrm{~N} 29 \mathrm{~W} 34 \mathrm{ACBB}$ & 121 & 10150004 & 121OGLL & 100 & 2,827 \\
\hline 430324100161901 & $36 \mathrm{~N} 25 \mathrm{~W} 33 \mathrm{~B}$ & 121 & 10150006 & 122ARKR & 142 & 2,605 \\
\hline 430324100383901 & $36 \mathrm{~N} 28 \mathrm{~W} 32 \mathrm{~B}$ & 121 & 10150006 & 121OGLL & 151 & 2,810 \\
\hline 430324100491501 & $36 \mathrm{~N} 30 \mathrm{~W} 35 \mathrm{~B}$ & 121 & 10150004 & 121OGLL & -- & 2,890 \\
\hline 430324100571301 & 36 N31W34 & 121 & 10140203 & 121OGLL & 190 & 2,945 \\
\hline 430325100180701 & $36 \mathrm{~N} 25 \mathrm{~W} 31 \mathrm{~A}$ & 121 & 10150006 & 122ARKR & 150 & 2,577 \\
\hline 430325100540001 & $36 \mathrm{~N} 30 \mathrm{~W} 31 \mathrm{BACC}$ & 121 & 10150004 & 121OGLL & 128 & 2,885 \\
\hline 430326100185001 & $36 \mathrm{~N} 25 \mathrm{~W} 31 \mathrm{ABC}$ & 121 & 10150006 & 122ARKR & 100 & 2,597 \\
\hline 430326100431101 & $36 \mathrm{~N} 29 \mathrm{~W} 35 \mathrm{~B}$ & 121 & 10150004 & 121OGLL & 98 & 2,835 \\
\hline 430326100520801 & $36 \mathrm{~N} 30 \mathrm{~W} 32 \mathrm{AACC}$ & 121 & 10150004 & 121OGLL & 180 & 2,940 \\
\hline 430326102240801 & $36 \mathrm{~N} 43 \mathrm{~W} 32 \mathrm{~A}$ & 113 & 10140201 & 122ARKR & - & 3,409 \\
\hline 430327100512301 & 36N30W33BADD & 121 & 10150004 & 121OGLL & 180 & 2,945 \\
\hline 430329101123101 & $36 \mathrm{~N} 33 \mathrm{~W} 33 \mathrm{ABCB}$ & 121 & 10140203 & 121OGLL & 126 & 2,959 \\
\hline 430331100153301 & $36 \mathrm{~N} 25 \mathrm{~W} 33 \mathrm{AA}$ & 121 & 10150006 & 121OGLL & 70 & 2,585 \\
\hline 430331100455101 & $36 \mathrm{~N} 29 \mathrm{~W} 32 \mathrm{BB}$ & 121 & 10150004 & 1210GLL & 60 & 2,850 \\
\hline 430331100512901 & $36 \mathrm{~N} 30 \mathrm{~W} 33 \mathrm{BAA}$ & 121 & 10150004 & 121OGLL & 170 & 2,935 \\
\hline 430331101141201 & $36 \mathrm{~N} 33 \mathrm{~W} 32 \mathrm{BB}$ & 7 & 10140203 & 121OGLL & 150 & 2,999 \\
\hline 430332102200601 & $36 \mathrm{~N} 43 \mathrm{~W} 36 \mathrm{BB}$ & 113 & 10140201 & 122ARKR & 100 & 3,380 \\
\hline 430332102202401 & 36N43W35AA & 113 & 10140201 & 122ARKR & 125 & 3,390 \\
\hline 430332102202402 & $36 \mathrm{~N} 43 \mathrm{~W} 35 \mathrm{AA} 2$ & 113 & 10140201 & $122 \mathrm{ARKR}$ & 75 & 3,390 \\
\hline
\end{tabular}


Table 57. Selected descriptive data (from the U.S. Geological Survey database) for ground-water sites in the High Plains aquifer in South Dakota-Continued

[County code: $7=$ Bennett County, $53=$ Gregory County, $71=$ Jackson County, $95=$ Mellette County, $113=$ Shannon County, and $121=$ Todd County; Datum, National Geodetic Vertical Datum; 1210GLL, Ogallala Formation; 122ARKR, Arikaree Formation; --, no data]

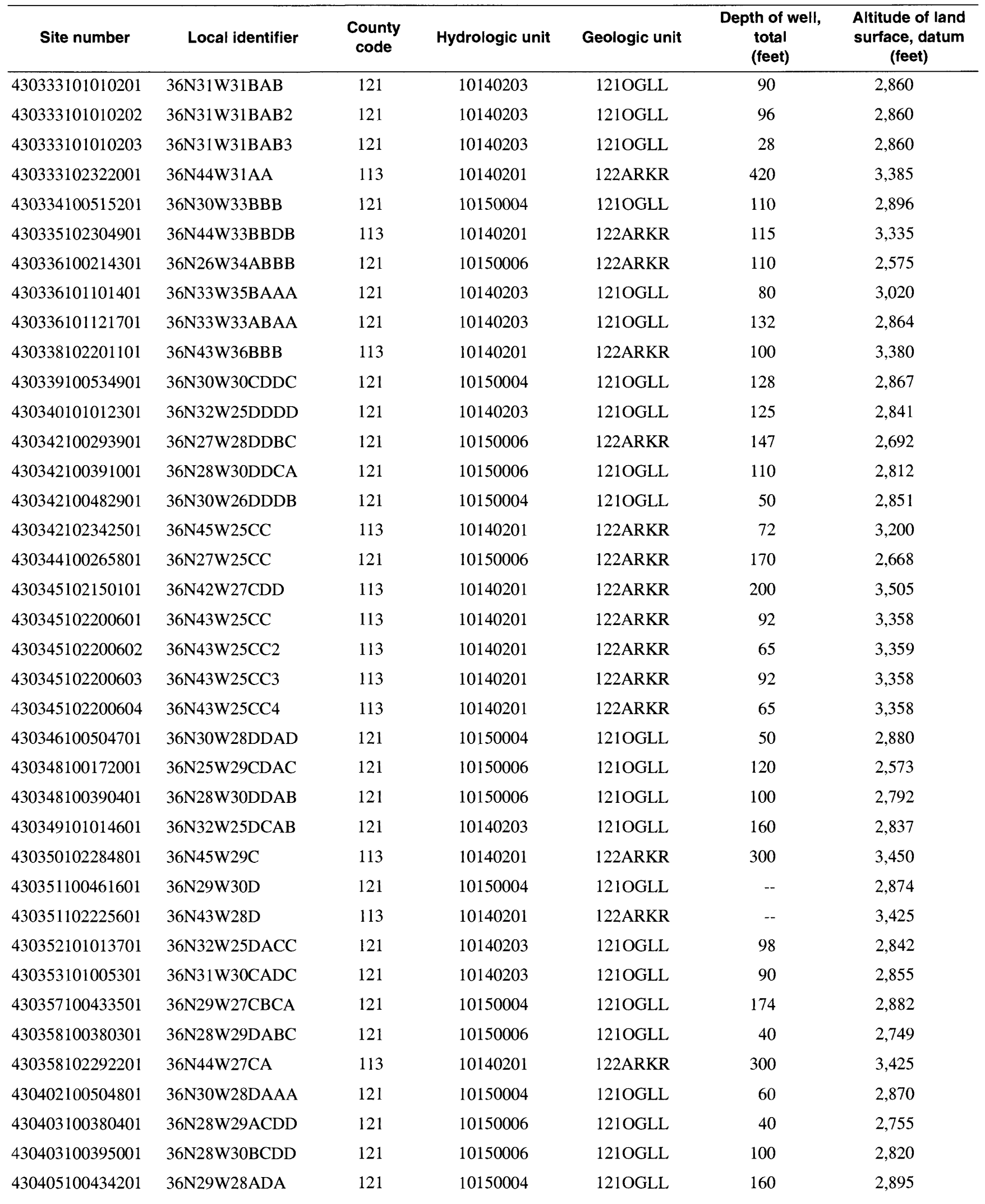


Table 57. Selected descriptive data (from the U.S. Geological Survey database) for ground-water sites in the High Plains aquifer in South Dakota-Continued

[County code: 7 = Bennett County, 53 = Gregory County, 71 = Jackson County, $95=$ Mellette County, $113=$ Shannon County, and $121=$ Todd County; Datum, National Geodetic Vertical Datum; 1210GLL, Ogallala Formation; 122ARKR, Arikaree Formation; --, no data]

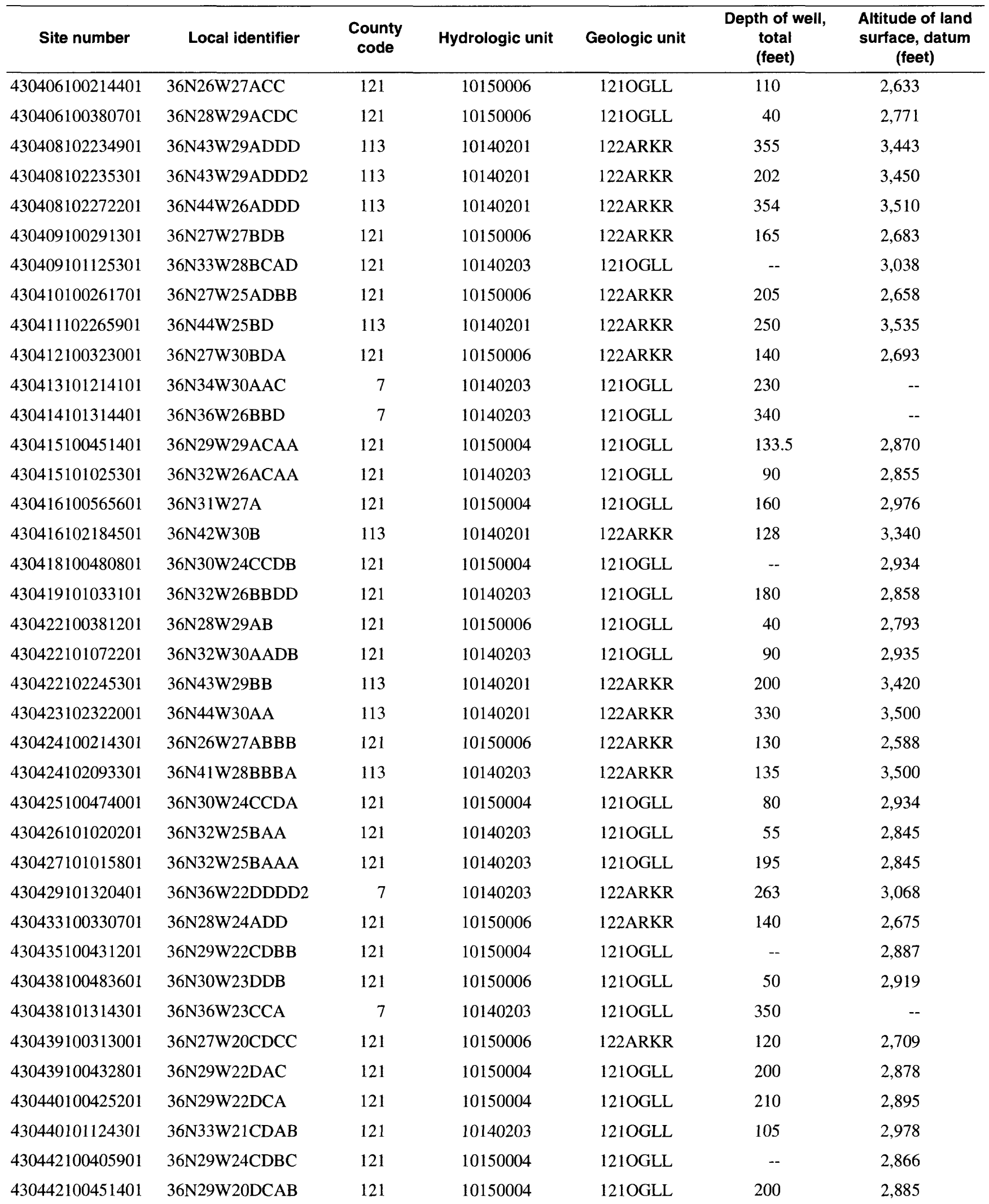


Table 57. Selected descriptive data (from the U.S. Geological Survey database) for ground-water sites in the High Plains aquifer in South Dakota - Continued

[County code: 7 = Bennett County, 53 = Gregory County, $71=$ Jackson County, 95 = Mellette County, $113=$ Shannon County, and $121=$ Todd County; Datum, National Geodetic Vertical Datum; 121OGLL, Ogallala Formation; 122ARKR, Arikaree Formation; --, no data]

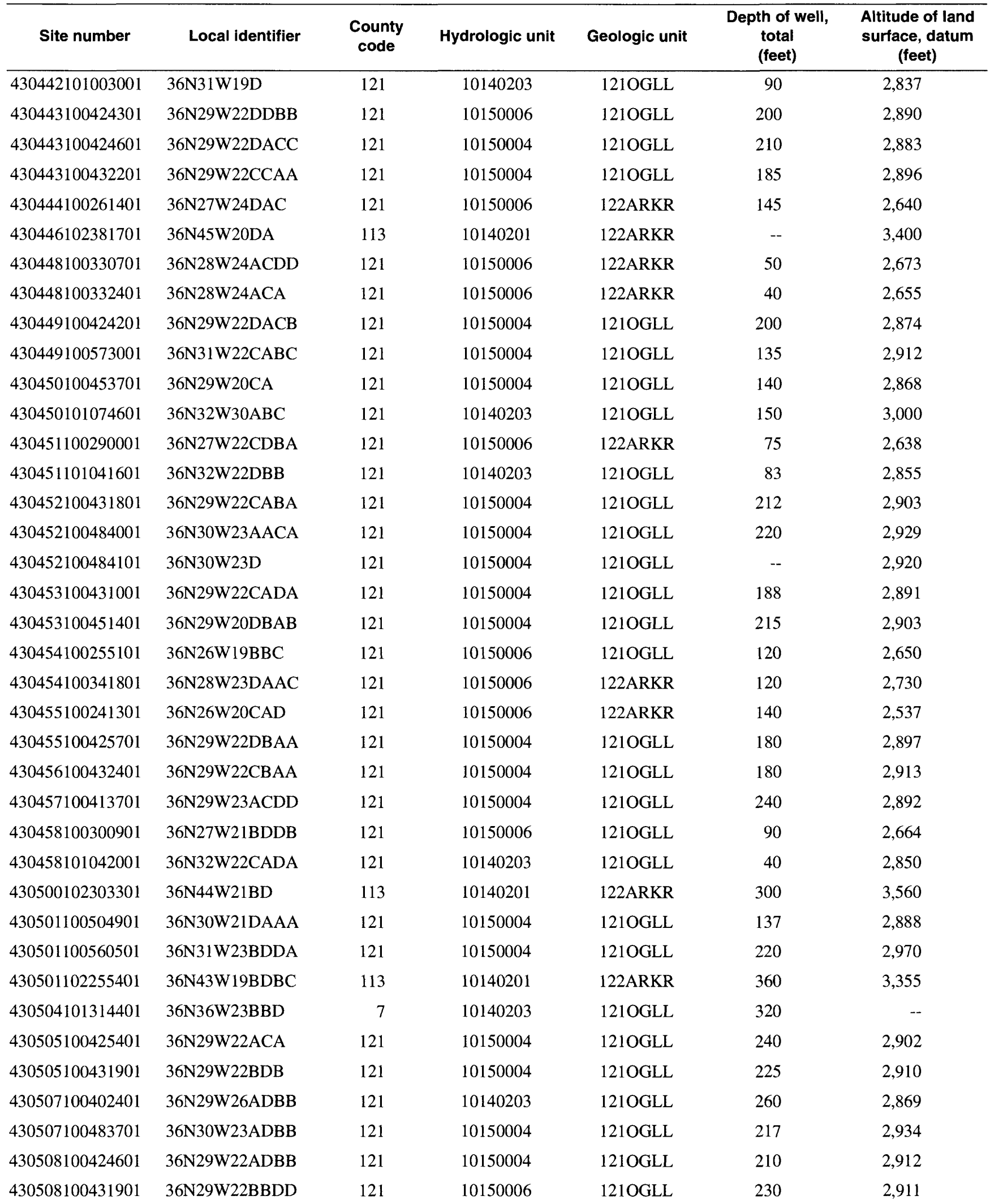


Table 57. Selected descriptive data (from the U.S. Geological Survey database) for ground-water sites in the High Plains aquifer in South Dakota-Continued

[County code: $7=$ Bennett County, $53=$ Gregory County, $71=$ Jackson County, $95=$ Mellette County, $113=$ Shannon County, and $121=$ Todd County; Datum, National Geodetic Vertical Datum; 121OGLL, Ogallala Formation; 122ARKR, Arikaree Formation; --, no data]

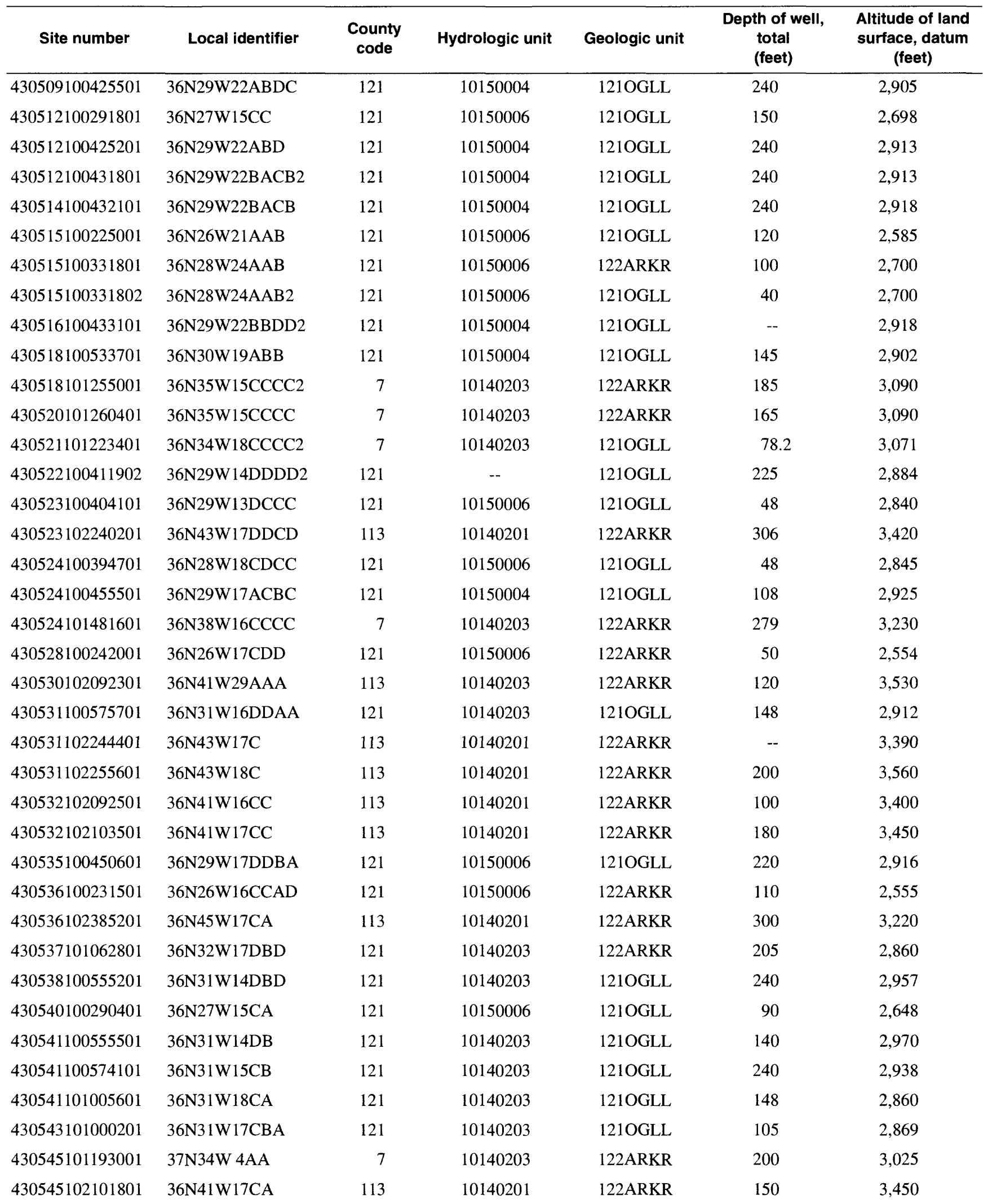


Table 57. Selected descriptive data (from the U.S. Geological Survey database) for ground-water sites in the High Plains aquifer in South Dakota-Continued

[County code: 7 = Bennett County, $53=$ Gregory County, 71 = Jackson County, $95=$ Mellette County, $113=$ Shannon County, and $121=$ Todd County; Datum, National Geodetic Vertical Datum; 1210GLL, Ogallala Formation; 122ARKR, Arikaree Formation; --, no data]

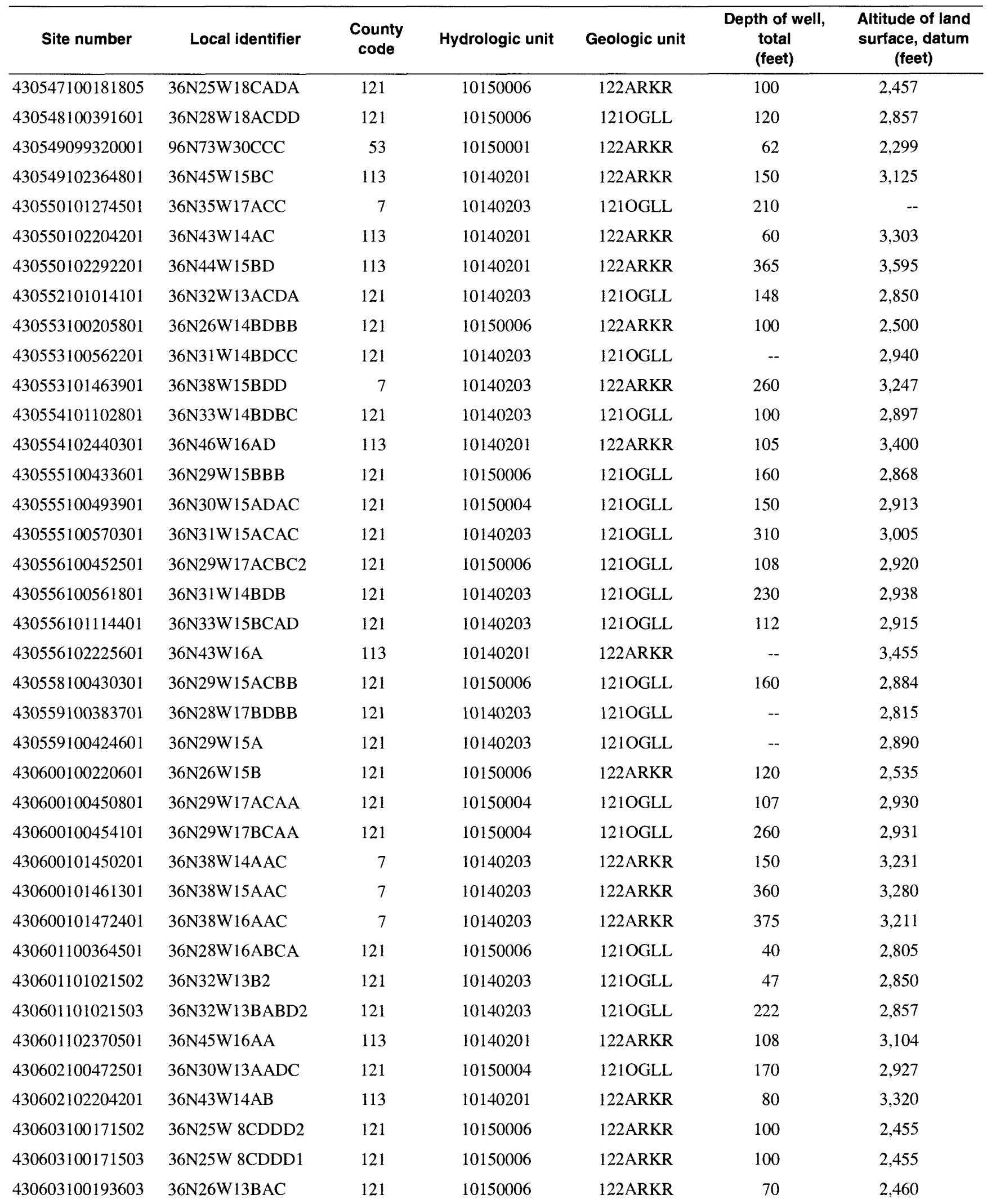


Table 57. Selected descriptive data (from the U.S. Geological Survey database) for ground-water sites in the High Plains aquifer in South Dakota-Continued

[County code: 7 = Bennett County, $53=$ Gregory County, $71=$ Jackson County, $95=$ Mellette County, $113=$ Shannon County, and $121=$ Todd County; Datum, National Geodetic Vertical Datum; 1210GLL, Ogallala Formation; 122ARKR, Arikaree Formation; --, no data]

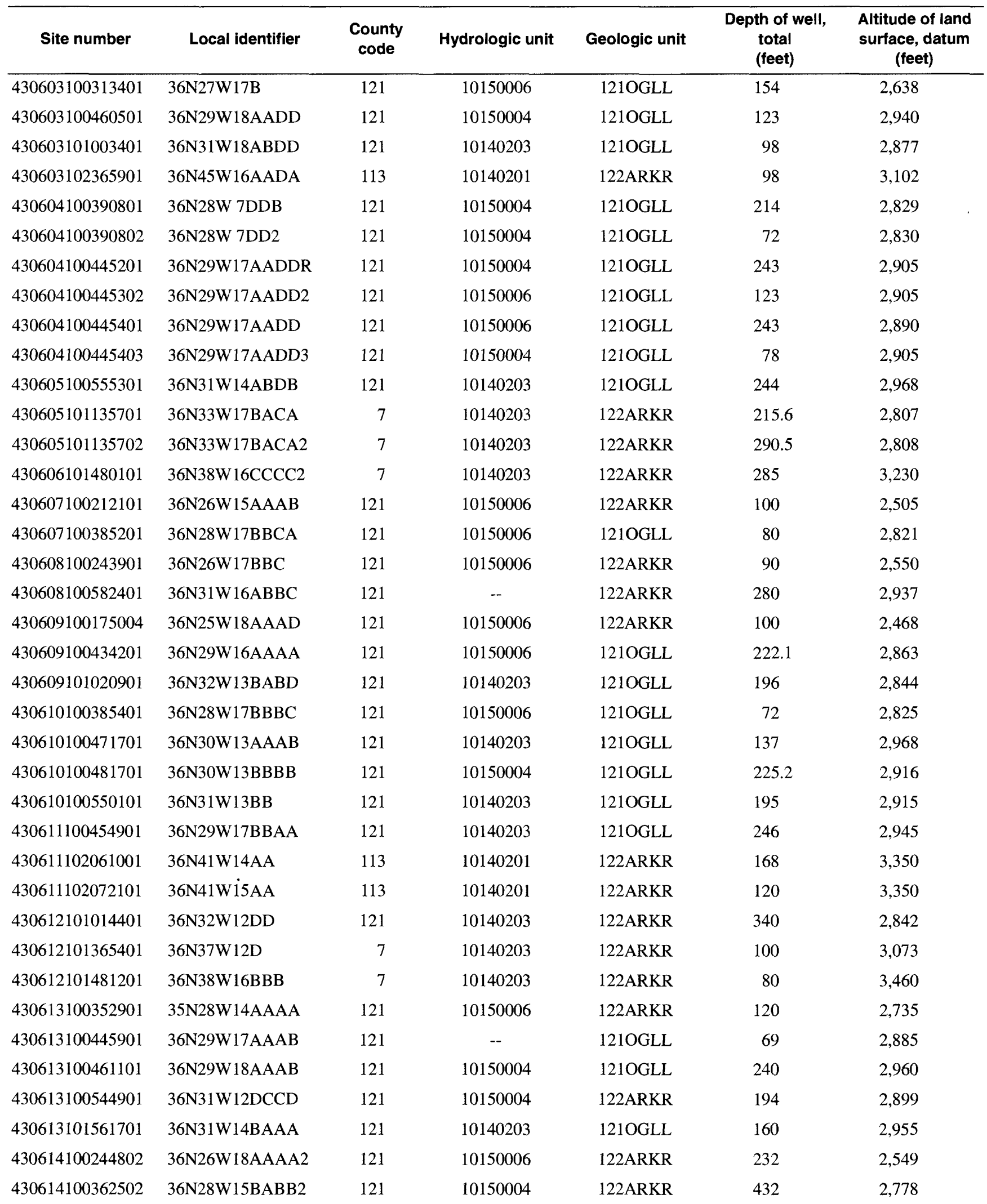


Table 57. Selected descriptive data (from the U.S. Geological Survey database) for ground-water sites in the High Plains aquifer in South Dakota-Continued

[County code: 7 = Bennett County, $53=$ Gregory County, $71=$ Jackson County, $95=$ Mellette County, $113=$ Shannon County, and $121=$ Todd County; Datum, National Geodetic Vertical Datum; 121OGLL, Ogallala Formation; 122ARKR, Arikaree Formation; --, no data]

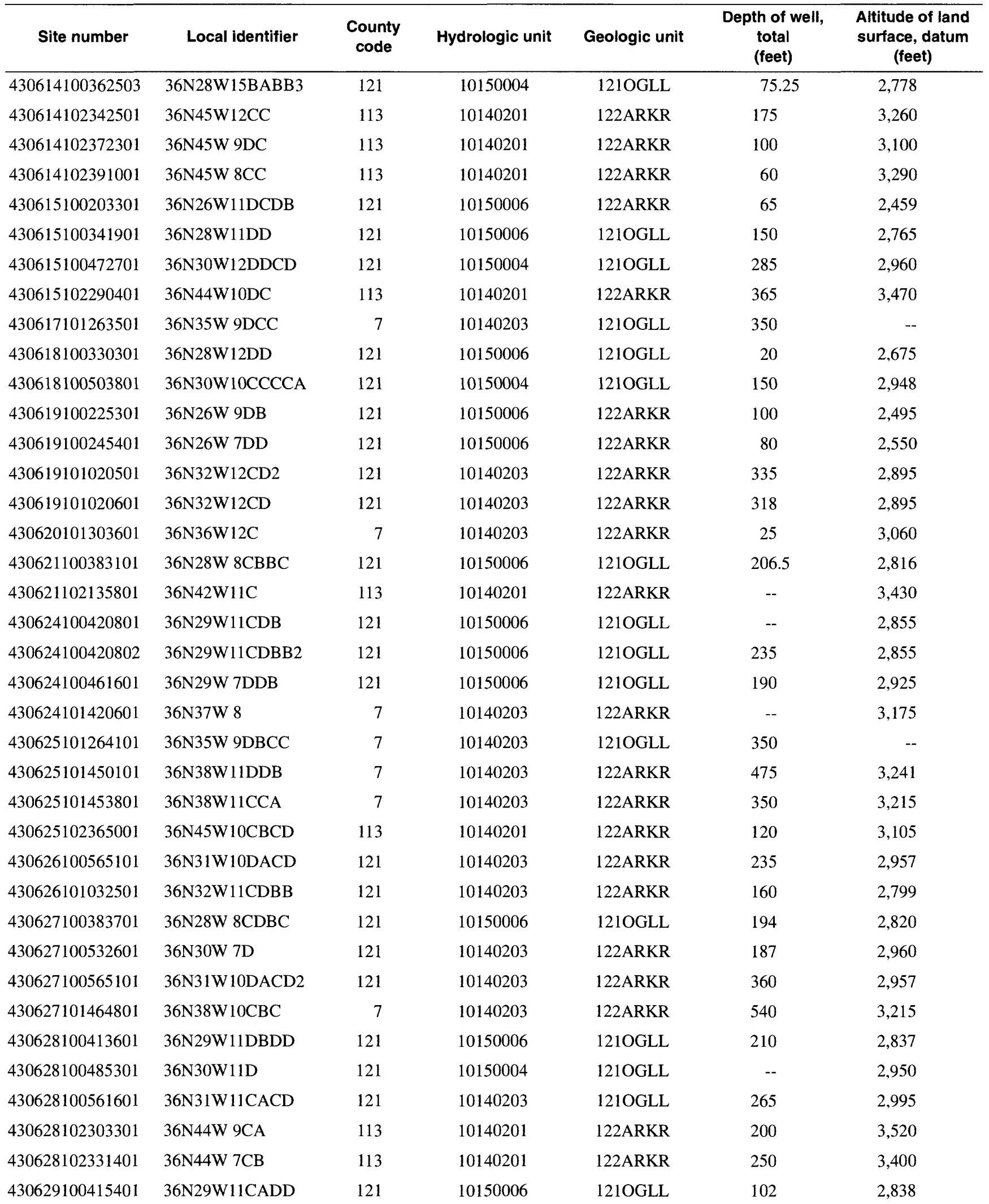


Table 57. Selected descriptive data (from the U.S. Geological Survey database) for ground-water sites in the High Plains aquifer in South Dakota-Continued

[County code: $7=$ Bennett County, $53=$ Gregory County, $71=$ Jackson County, $95=$ Mellette County, $113=$ Shannon County, and $121=$ Todd County; Datum, National Geodetic Vertical Datum; 1210GLL, Ogallala Formation; 122ARKR, Arikaree Formation; --, no data]

\begin{tabular}{|c|c|c|c|c|c|c|}
\hline Site number & Local identifier & $\begin{array}{l}\text { County } \\
\text { code }\end{array}$ & Hydrologic unit & Geologic unit & $\begin{array}{c}\text { Depth of well, } \\
\text { total } \\
\text { (feet) }\end{array}$ & $\begin{array}{l}\text { Altitude of land } \\
\text { surface, datum } \\
\text { (feet) }\end{array}$ \\
\hline 430629100434401 & $36 \mathrm{~N} 29 \mathrm{~W}$ 9DAD & 121 & 10150006 & 121OGLL & 209 & 2,885 \\
\hline 430630100565401 & 36N31W10DACD3 & 121 & 10140203 & 122ARKR & 305 & 2,958 \\
\hline 430630101465001 & $36 \mathrm{~N} 38 \mathrm{~W} 10 \mathrm{CC}$ & 7 & 10140203 & 122ARKR & 500 & 3,221 \\
\hline 430632100594601 & $36 \mathrm{~N} 31 \mathrm{~W} 8 \mathrm{CA}$ & 121 & 10140203 & 1210GLL & 180 & 2,965 \\
\hline 430632101340801 & $36 \mathrm{~N} 36 \mathrm{~W} 9 \mathrm{CAB}$ & 7 & 10140203 & 121OGLL & 140 & 3,037 \\
\hline 430633100290201 & 36N27W10DBBB & 121 & 10150006 & 1210GLL & 150 & 2,688 \\
\hline 430633102220301 & $36 \mathrm{~N} 43 \mathrm{~W} 10$ & 113 & 10140201 & 122ARKR & -- & 3,373 \\
\hline 430635100473001 & 36N30W12DBAD & 121 & 10150004 & 121OGLL & 285 & 2,950 \\
\hline 430635100510501 & 36N30W 9DBAD & 121 & 10150004 & 121OGLL & 150 & 2,957 \\
\hline 430639100383001 & $36 \mathrm{~N} 28 \mathrm{~W} 8 \mathrm{CAAB}$ & 121 & 10150006 & 121OGLL & 80 & 2,820 \\
\hline 430639100384901 & $36 \mathrm{~N} 28 \mathrm{~W} 29 \mathrm{BC}$ & 121 & 10150006 & 121OGLL & 40 & 2,807 \\
\hline 430640100222401 & $36 \mathrm{~N} 26 \mathrm{~W} 10 \mathrm{BCC}$ & 121 & 10150006 & 122ARKR & 165 & 2,528 \\
\hline 430640101112001 & $36 \mathrm{~N} 33 \mathrm{~W} 10 \mathrm{ABCA}$ & 121 & 10140203 & 121OGLL & 65 & 2,881 \\
\hline 430641100373601 & $36 \mathrm{~N} 28 \mathrm{~W} 9 \mathrm{BCDC}$ & 121 & 10150006 & 121OGLL & 140 & 2,833 \\
\hline 430642101455801 & 36N38W10ADDD & 7 & 10140203 & 122ARKR & 443 & 3,199 \\
\hline 430642102071501 & 36N41W10DAAA & 113 & 10140201 & 122ARKR & 135 & 3,400 \\
\hline 430649100364801 & $36 \mathrm{~N} 28 \mathrm{~W}$ 9ADDA & 121 & 10150006 & 121OGLL & 70 & 2,805 \\
\hline 430650100365301 & $36 \mathrm{~N} 28 \mathrm{~W}$ 9ACAA & 121 & 10150006 & 1210GLL & -- & 2,815 \\
\hline 430650100375101 & $36 \mathrm{~N} 28 \mathrm{~W}$ 8ADAA & 121 & 10150006 & 1210GLL & 140 & 2,838 \\
\hline 430650100450501 & $36 \mathrm{~N} 29 \mathrm{~W} 8 \mathrm{ADBB}$ & 121 & 10150004 & 121OGLL & 220 & 2,878 \\
\hline 430650101021001 & $36 \mathrm{~N} 32 \mathrm{~W} 1 \mathrm{DCDC}$ & 121 & 10140203 & 122ARKR & 60 & 2,623 \\
\hline 430651101095501 & $36 \mathrm{~N} 33 \mathrm{~W} 11 \mathrm{~A}$ & 121 & 10140203 & 122ARKR & 105 & 2,878 \\
\hline 430651101241001 & $36 \mathrm{~N} 35 \mathrm{~W} 11 \mathrm{AAC}$ & 7 & 10140203 & 121OGLL & 270 & -- \\
\hline 430651101403501 & 36N37W 9AAC & 7 & 10140203 & 122ARKR & 525 & -- \\
\hline 430652100450601 & $36 \mathrm{~N} 29 \mathrm{~W} 8 \mathrm{~A}$ & 121 & 10150006 & 121OGLL & 190 & 2,880 \\
\hline 430652100484001 & 36N30W11ADBB & 121 & 10150004 & 121OGLL & 300 & 2,949 \\
\hline 430652100495101 & $36 \mathrm{~N} 30 \mathrm{~W} 10 \mathrm{~A}$ & 121 & 10150004 & 121OGLL & -- & 2,960 \\
\hline 430652102125601 & $36 \mathrm{~N} 42 \mathrm{~W} 12 \mathrm{BB}$ & 113 & 10140201 & 122ARKR & 230 & 3,500 \\
\hline 430652102174201 & $36 \mathrm{~N} 42 \mathrm{~W} 8 \mathrm{BB}$ & 113 & 10140201 & 122ARKR & 100 & 3,370 \\
\hline 430652102363001 & $36 \mathrm{~N} 45 \mathrm{~W} 10 \mathrm{BA}$ & 113 & 10140201 & 122ARKR & 350 & 3,260 \\
\hline 430653100421201 & $36 \mathrm{~N} 29 \mathrm{~W} 11 \mathrm{BBDD}$ & 121 & 10150006 & 121OGLL & 245 & 2,844 \\
\hline 430653100491201 & $36 \mathrm{~N} 30 \mathrm{~W} 11 \mathrm{~B}$ & 121 & 10150004 & 121OGLL & -- & 2,960 \\
\hline 430653100513701 & $36 \mathrm{~N} 30 \mathrm{~W} 9 \mathrm{~B}$ & 121 & 10150004 & 121OGLL & -- & 2,973 \\
\hline 430653100562101 & $36 \mathrm{~N} 31 \mathrm{~W} 11 \mathrm{~B}$ & 121 & 10140203 & 122ARKR & 265 & 2,938 \\
\hline 430653100565705 & $36 \mathrm{~N} 31 \mathrm{~W} 10 \mathrm{~A} 5$ & 121 & 10140203 & 121OGLL & 115 & 2,990 \\
\hline 430653100565706 & $36 \mathrm{~N} 31 \mathrm{~W} 10 \mathrm{~A} 6$ & 121 & 10140203 & 122ARKR & 390 & 2,990 \\
\hline 430653100571003 & 36N31W10BDDA & 121 & 10140203 & 1210GLL & 269 & 2,926 \\
\hline 430653101464801 & $36 \mathrm{~N} 38 \mathrm{~W} 10 \mathrm{BBD}$ & 7 & 10140203 & 122ARKR & 350 & 3,182 \\
\hline
\end{tabular}


Table 57. Selected descriptive data (from the U.S. Geological Survey database) for ground-water sites in the High Plains aquifer in South Dakota-Continued

[County code: 7 = Bennett County, $53=$ Gregory County, $71=$ Jackson County. $95=$ Mellette County, $113=$ Shannon County, and $121=$ Todd County; Datum, National Geodetic Vertical Datum; 1210GLL, Ogallala Formation; 122ARKR, Arikaree Formation; --, no data]

\begin{tabular}{|c|c|c|c|c|c|c|}
\hline Site number & Local identifier & $\begin{array}{c}\text { County } \\
\text { code }\end{array}$ & Hydrologic unit & Geologic unit & $\begin{array}{c}\text { Depth of well, } \\
\text { total } \\
\text { (feet) }\end{array}$ & $\begin{array}{l}\text { Altitude of land } \\
\text { surface, datum } \\
\text { (feet) }\end{array}$ \\
\hline 430656100181806 & $36 \mathrm{~N} 25 \mathrm{~W} 7 \mathrm{ADCA}$ & 121 & 10150006 & 122ARKR & 30 & 2,522 \\
\hline 430656101445501 & 36N38W11AAC & 7 & 10140203 & 122ARKR & 480 & 3,228 \\
\hline 430656102002101 & $36 \mathrm{~N} 40 \mathrm{~W} 10 \mathrm{AAC}$ & 7 & 10140203 & 122ARKR & 50 & 3,284 \\
\hline 430659100434901 & 36N29W 9AA & 121 & 10150006 & 121OGLL & 200 & 2,845 \\
\hline 430659100434902 & $36 \mathrm{~N} 29 \mathrm{~W} 9 \mathrm{AA} 2$ & 121 & 10150006 & 1210GLL & 140 & 2,845 \\
\hline 430700100225701 & 36N26W 9AB & 121 & 10150006 & 122ARKR & 120 & 2,505 \\
\hline 430700100344501 & $36 \mathrm{~N} 28 \mathrm{~W} 11 \mathrm{ABB}$ & 121 & 10150006 & $121 \mathrm{OGLL}$ & 140 & 2,807 \\
\hline 430701100363001 & $36 \mathrm{~N} 28 \mathrm{~W} 10 \mathrm{BBBB}$ & 121 & 10150006 & 1210GLL & 182.5 & 2,823 \\
\hline 430701100494301 & 36N30W10AAAC & 121 & 10150004 & 121OGLL & 235 & 2,962 \\
\hline 430702100330501 & $36 \mathrm{~N} 28 \mathrm{~W} 12 \mathrm{AABA}$ & 121 & 10150006 & 122ARKR & 215 & 2,806 \\
\hline 430702100451701 & $36 \mathrm{~N} 29 \mathrm{~W} 8 \mathrm{ABAC}$ & 121 & 10150006 & 1210GLL & 150 & 2,874 \\
\hline 430703100451801 & $36 \mathrm{~N} 29 \mathrm{~W} 8 \mathrm{ABAB}$ & 121 & 10150006 & 121OGLL & 140 & 2,874 \\
\hline 430704100145901 & $36 \mathrm{~N} 25 \mathrm{~W} 10 \mathrm{BABB}$ & 121 & 10150006 & 121OGLL & 30 & 2,442 \\
\hline 430704101390601 & 36N37W11BBBB2 & 7 & 10140203 & 122ARKR & 320 & 3,159 \\
\hline 430704102385201 & $36 \mathrm{~N} 45 \mathrm{~W} 5 \mathrm{CD}$ & 113 & 10140201 & 122ARKR & 135 & 3,070 \\
\hline 430705100304701 & $36 \mathrm{~N} 27 \mathrm{~W} 5 \mathrm{DDD}$ & 121 & 10150006 & 121OGLL & 120 & 2,600 \\
\hline 430705100450201 & $36 \mathrm{~N} 29 \mathrm{~W} 8 \mathrm{AABA}$ & 121 & -- & 1210GLL & 77 & 2,865 \\
\hline 430705102200601 & 36N43W 1CC & 113 & 10140201 & 122ARKR & 100 & 3,345 \\
\hline 430705102273401 & $36 \mathrm{~N} 44 \mathrm{~W} 2 \mathrm{DD}$ & 113 & 10140201 & 122ARKR & 200 & 3,460 \\
\hline 430707100561201 & $36 \mathrm{~N} 31 \mathrm{~W} 2 \mathrm{CDDC}$ & 121 & 10140203 & 122ARKR & 249 & 2,923 \\
\hline 430707101115901 & $36 \mathrm{~N} 33 \mathrm{~W} 3 \mathrm{CCCC}$ & 121 & 10140203 & 121OGLL & 45 & 2,899 \\
\hline 430709100530401 & $36 \mathrm{~N} 30 \mathrm{~W} 5 \mathrm{C}$ & 121 & 10150004 & 122ARKR & 274 & 2,985 \\
\hline 430709100574701 & $36 \mathrm{~N} 31 \mathrm{~W} 3 \mathrm{CCC}$ & 121 & 10140203 & 1210GLL & 265 & 3,008 \\
\hline 430712100230601 & $36 \mathrm{~N} 26 \mathrm{~W} 4 \mathrm{DC}$ & 121 & 10150006 & 122ARKR & 160 & 2,524 \\
\hline 430712100371901 & $36 \mathrm{~N} 28 \mathrm{~W} 4 \mathrm{CD}$ & 121 & 10150006 & 121OGLL & 173 & 2,812 \\
\hline 430712100421301 & $36 \mathrm{~N} 29 \mathrm{~W} 2 \mathrm{CDCC}$ & 121 & 10150006 & 121OGLL & 200 & 2,850 \\
\hline 430712101042801 & $36 \mathrm{~N} 32 \mathrm{~W} 3 \mathrm{CD}$ & 121 & 10140203 & 122ARKR & 118 & 2,686 \\
\hline 430713100431701 & $36 \mathrm{~N} 29 \mathrm{~W} 3 \mathrm{CDBB}$ & 121 & 10150004 & 121OGLL & 225 & 2,848 \\
\hline 430714100445001 & $36 \mathrm{~N} 29 \mathrm{~W} 4 \mathrm{CCBC}$ & 121 & 10150006 & 121OGLL & 150 & 2,853 \\
\hline 430714100563401 & $36 \mathrm{~N} 31 \mathrm{~W} 2 \mathrm{CCB}$ & 121 & 10140203 & 121OGLL & 225 & 2,948 \\
\hline 430715100342901 & $36 \mathrm{~N} 28 \mathrm{~W} 2 \mathrm{DDBC}$ & 121 & 10150006 & 121OGLL & -- & 2,795 \\
\hline 430716101214801 & $36 \mathrm{~N} 34 \mathrm{~W} 6 \mathrm{DBDC}$ & 7 & 10140203 & 121OGLL & 220 & -- \\
\hline 430716101595401 & $36 \mathrm{~N} 40 \mathrm{~W} 2 \mathrm{CCA}$ & 7 & 10140203 & 122ARKR & 60 & 3,290 \\
\hline 430717100361501 & $36 \mathrm{~N} 28 \mathrm{~W} 3 \mathrm{CDBB}$ & 121 & 10150006 & 121OGLL & -- & 2,812 \\
\hline 430717101381201 & $36 \mathrm{~N} 37 \mathrm{~W} 2 \mathrm{DDB}$ & 7 & 10140203 & 122ARKR & 500 & -- \\
\hline 430718100354801 & $36 \mathrm{~N} 28 \mathrm{~W} 3 \mathrm{DBB}$ & 121 & 10150006 & 121OGLL & 160 & 2,787 \\
\hline 430718100393501 & $38 \mathrm{~N} 28 \mathrm{~W} 8 \mathrm{ABBA}$ & 121 & 10150006 & 122ARKR & 120 & 2,620 \\
\hline 430718100413601 & 36 N29W 2D2 & 121 & 10150006 & 121OGLL & -- & 2,845 \\
\hline
\end{tabular}


Table 57. Selected descriptive data (from the U.S. Geological Survey database) for ground-water sites in the High Plains aquifer in South Dakota-Continued

[County code: 7 = Bennett County, 53 = Gregory County, $71=$ Jackson County, 95 = Mellette County, $113=$ Shannon County, and $121=$ Todd County; Datum, National Geodetic Vertical Datum; 1210GLL, Ogallala Formation; 122ARKR, Arikaree Formation; --, no data]

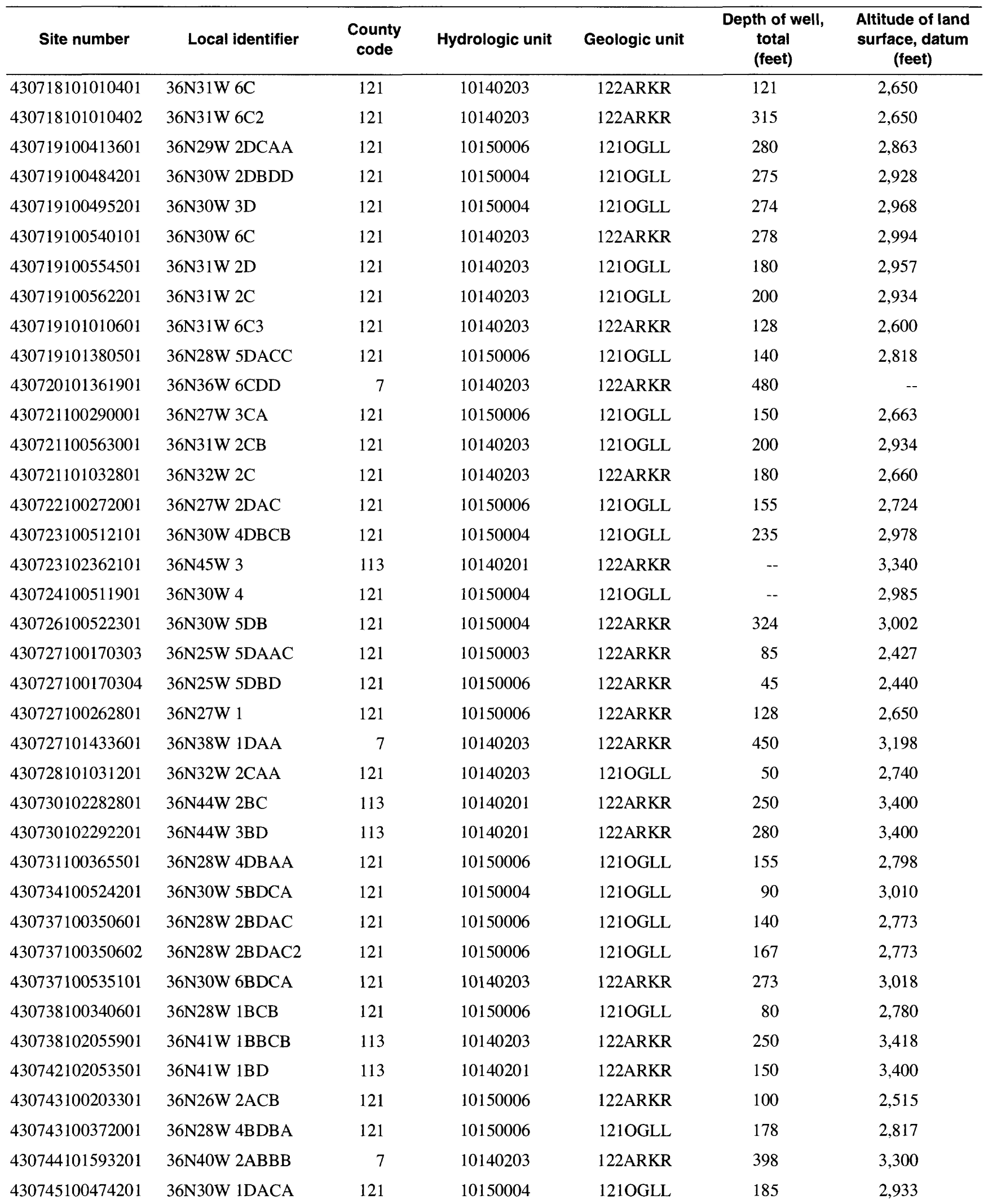


Table 57. Selected descriptive data (from the U.S. Geological Survey database) for ground-water sites in the High Plains aquifer in South Dakota-Continued

[County code: 7 = Bennett County, 53 = Gregory County, $71=$ Jackson County, 95 = Mellette County, $113=$ Shannon County, and $121=$ Todd County; Datum, National Geodetic Vertical Datum; 121OGLL, Ogallala Formation; 122ARKR, Arikaree Formation; --, no data]

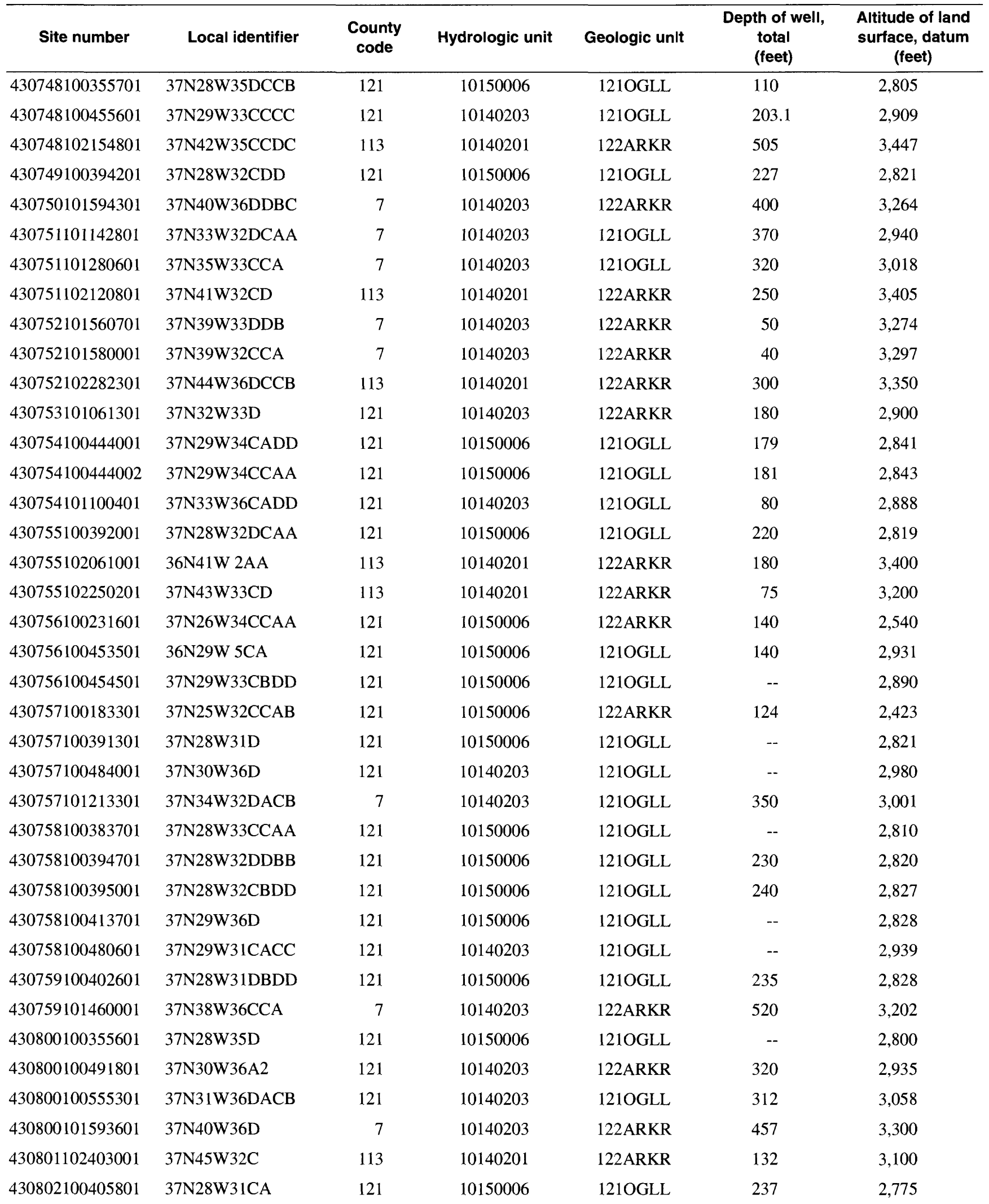


Table 57. Selected descriptive data (from the U.S. Geological Survey database) for ground-water sites in the High Plains aquifer in South Dakota-Continued

[County code: 7 = Bennett County, $53=$ Gregory County, $71=$ Jackson County, $95=$ Mellette County, $113=$ Shannon County, and $121=$ Todd County; Datum, National Geodetic Vertical Datum; 1210GLL, Ogallala Formation; 122ARKR, Arikaree Formation; --, no data]

\begin{tabular}{|c|c|c|c|c|c|c|}
\hline Site number & Local identifier & $\begin{array}{c}\text { County } \\
\text { code }\end{array}$ & Hydrologic unit & Geologic unit & $\begin{array}{c}\text { Depth of well, } \\
\text { total } \\
\text { (feet) }\end{array}$ & $\begin{array}{l}\text { Altitude of land } \\
\text { surface, datum } \\
\text { (feet) }\end{array}$ \\
\hline 430802101085101 & $37 \mathrm{~N} 32 \mathrm{~W} 31 \mathrm{DBBC}$ & 121 & 10140203 & 1210GLL & 80 & 2,860 \\
\hline 430802101543402 & 37N30W31DA2 & 121 & 10140203 & 121OGLL & 240 & -- \\
\hline 430803100212801 & 37N26W35DBDA & 121 & 10150006 & 122ARKR & 140 & 2,554 \\
\hline 430803100455501 & $37 \mathrm{~N} 29 \mathrm{~W} 33 \mathrm{CDCB}$ & 121 & 10150006 & 121OGLL & 300 & 2,880 \\
\hline 430804102072201 & $37 \mathrm{~N} 41 \mathrm{~W} 36 \mathrm{CA}$ & 113 & 10140201 & 122ARKR & 150 & 3,451 \\
\hline 430805102280801 & 37N44W36D & 113 & 10140201 & 122ARKR & -- & 3,300 \\
\hline 430805102325101 & 37N44W32D & 113 & 10140201 & 122ARKR & -- & 3,550 \\
\hline 430806100401001 & 37N28W31DAAD & 121 & 10150006 & 121OGLL & 120 & 2,805 \\
\hline 430806102402801 & $37 \mathrm{~N} 45 \mathrm{~W} 32 \mathrm{CACB}$ & 113 & 10140201 & 122ARKR & 132 & 3,090 \\
\hline 430807100450501 & 37N29W33DAB & 121 & 10150006 & 1210GLL & -- & 2,858 \\
\hline 430807100591001 & 37N31W33DAA & 121 & 10140203 & 122ARKR & 445 & 3,037 \\
\hline 430808102242701 & 37N43W33DA & 113 & 10140201 & 122ARKR & 90 & 3,315 \\
\hline 430809100372401 & $37 \mathrm{~N} 28 \mathrm{~W} 34 \mathrm{BDA} 2$ & 121 & 10150006 & 1210GLL & 171.1 & 2,800 \\
\hline 430809100372402 & $37 \mathrm{~N} 28 \mathrm{~W} 34 \mathrm{BBDC}$ & 121 & 10150006 & 122ARKR & 200 & 2,812 \\
\hline 430809102402801 & $37 \mathrm{~N} 45 \mathrm{~W} 32 \mathrm{CABC}$ & 113 & 10140201 & 122ARKR & 115 & 3,040 \\
\hline 430811101574401 & 37N39W32BD & 7 & 10140203 & 122ARKR & 573 & 3,303 \\
\hline 430811102214201 & 37N43W36CBAA & 113 & 10140201 & 122ARKR & 115 & 3,218 \\
\hline 430813102153301 & $37 N 42 W 35$ & 113 & 10140201 & 122ARKR & -- & 3,480 \\
\hline 430814100594101 & $37 \mathrm{~N} 31 \mathrm{~W} 33 \mathrm{BDCB}$ & 121 & -- & 122ARKR & 440 & 3,020 \\
\hline 430814102245301 & $37 N 43 W 33$ & 113 & 10140201 & 122ARKR & 162 & 3,210 \\
\hline 430815100404001 & $37 \mathrm{~N} 28 \mathrm{~W} 31 \mathrm{ACA}$ & 121 & 10150006 & 1210GLL & 170 & 2,795 \\
\hline 430817100263501 & 37N26W31BDD & 121 & 10150006 & 122ARKR & 145 & 2,722 \\
\hline 430817100312001 & 37N27W33BD & 121 & 10150006 & 122ARKR & 120 & 2,620 \\
\hline 430818101291701 & 37N35W32B & 7 & 10140203 & 1210GLL & 350 & 2,998 \\
\hline 430819101064801 & $37 N 32 W 33 B$ & 121 & 10140203 & 122ARKR & 180 & 2,870 \\
\hline 430819101223701 & $37 \mathrm{~N} 34 \mathrm{~W} 31 \mathrm{AADC}$ & 7 & 10140203 & 121OGLL & 350 & 2,988 \\
\hline 430820100371401 & 37N28W34ABDA & 121 & 10150006 & 121OGLL & 171.1 & 2,783 \\
\hline 430820102402101 & $37 N 45 W 32 B D$ & 113 & 10140201 & 122ARKR & 98 & 3,075 \\
\hline 430821100373401 & $37 \mathrm{~N} 28 \mathrm{~W} 34 \mathrm{BCAB}$ & 121 & 10150006 & 122ARKR & 200 & 2,808 \\
\hline 430821100435501 & $37 \mathrm{~N} 29 \mathrm{~W} 34 \mathrm{ADBA}$ & 121 & 10150006 & 121OGLL & -- & 2,801 \\
\hline 430821102211201 & $37 N 43 W 36 A C$ & 113 & 10140201 & 122ARKR & 110 & 3,260 \\
\hline 430821102214801 & $37 \mathrm{~N} 43 \mathrm{~W} 36 \mathrm{BC}$ & 113 & 10140201 & 122ARKR & 115 & 3,245 \\
\hline 430821102242701 & $37 N 43 W 33 A D$ & 113 & 10140201 & 122ARKR & 100 & 3,300 \\
\hline 430822101210701 & $37 \mathrm{~N} 34 \mathrm{~W} 33 \mathrm{BBDB}$ & 7 & 10140203 & 121OGLL & 164 & 2,988 \\
\hline 430823100441201 & $37 \mathrm{~N} 29 \mathrm{~W} 34 \mathrm{ACA}$ & 121 & 10150006 & 121OGLL & 150 & 2,830 \\
\hline 430823100451001 & $37 \mathrm{~N} 29 \mathrm{~W} 33 \mathrm{ADBB}$ & 121 & 10150006 & 1210GLL & 187 & 2,858 \\
\hline 430823100454801 & $37 \mathrm{~N} 29 \mathrm{~W} 33 \mathrm{BBDD}$ & 121 & 10150006 & 121OGLL & -- & 2,870 \\
\hline 430823100484101 & $37 \mathrm{~N} 30 \mathrm{~W} 36 \mathrm{~A}$ & 121 & 10140203 & 1210GLL & -- & 2,940 \\
\hline
\end{tabular}


Table 57. Selected descriptive data (from the U.S. Geological Survey database) for ground-water sites in the High Plains aquifer in South Dakota-Continued

[County code: 7 = Bennett County, $53=$ Gregory County, $71=$ Jackson County, $95=$ Mellette County, $113=$ Shannon County, and $121=$ Todd County; Datum, National Geodetic Vertical Datum; 12IOGLL, Ogallala Formation; 122ARKR, Arikaree Formation; --, no data]

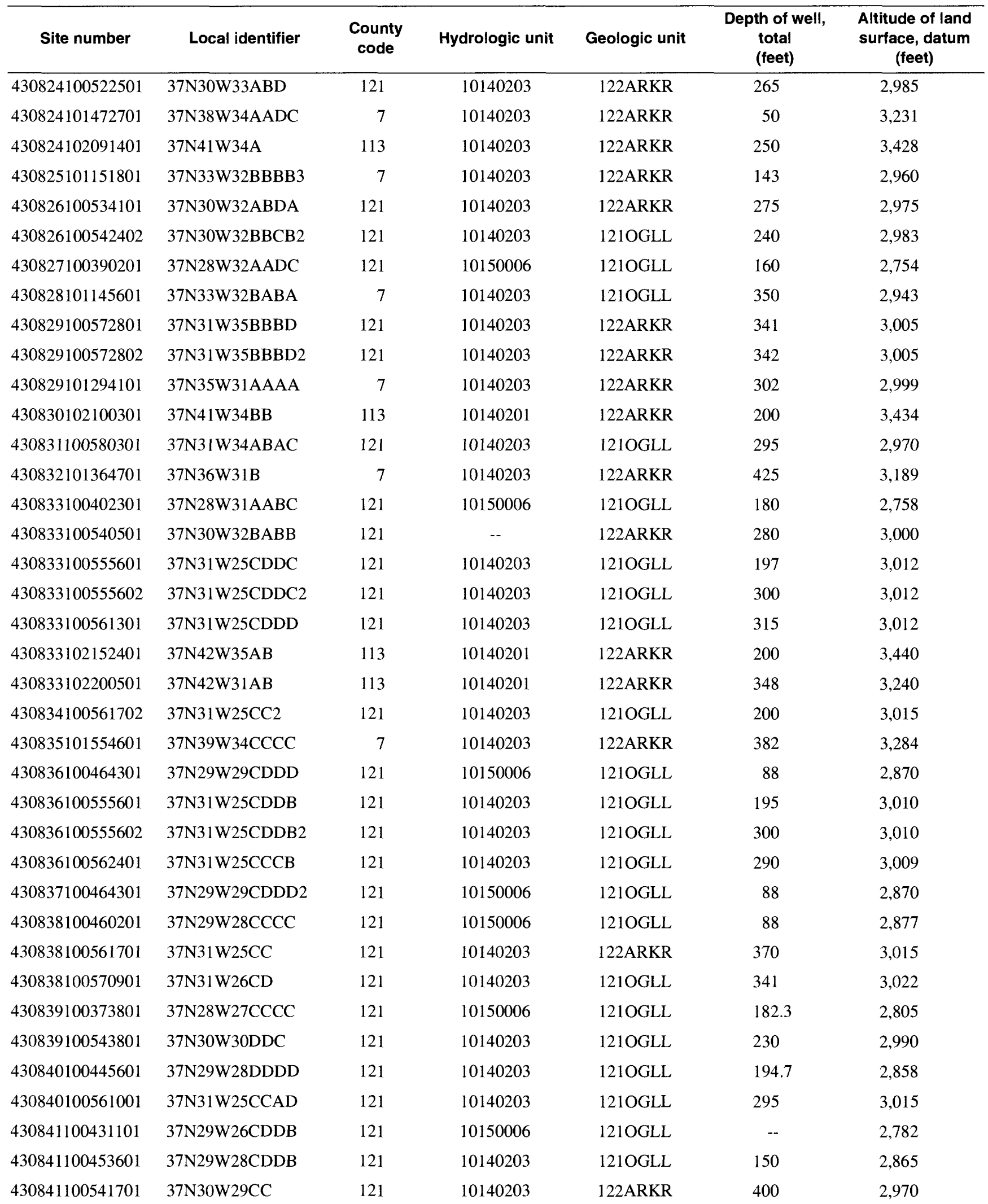


Table 57. Selected descriptive data (from the U.S. Geological Survey database) for ground-water sites in the High Plains aquifer in South Dakota-Continued

[County code: 7 = Bennett County, $53=$ Gregory County, $71=$ Jackson County, $95=$ Mellette County, $113=$ Shannon County, and $121=$ Todd County; Datum, National Geodetic Vertical Datum; 1210GLL, Ogallala Formation; 122ARKR, Arikaree Formation; --, no data]

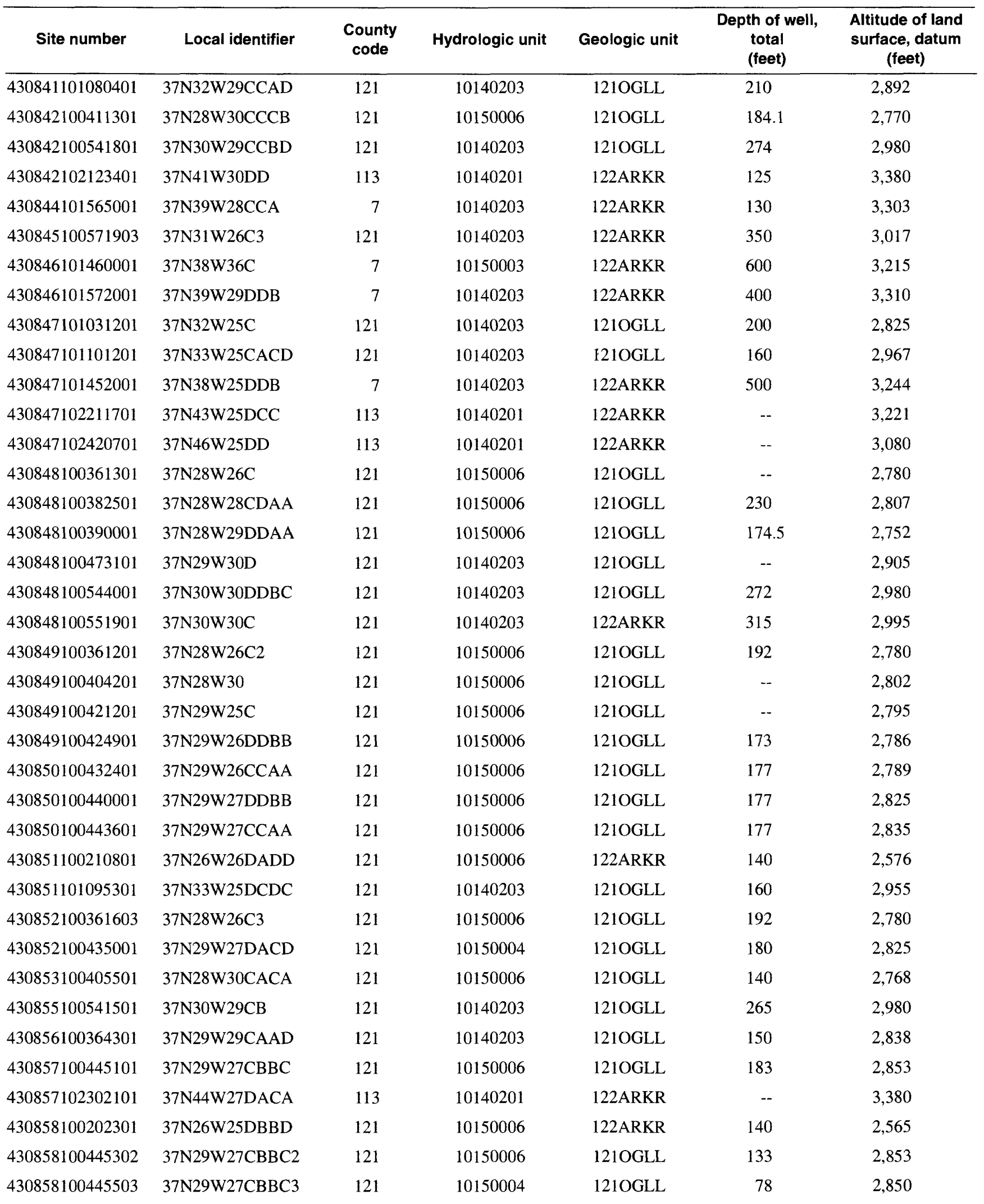


Table 57. Selected descriptive data (from the U.S. Geological Survey database) for ground-water sites in the High Plains aquifer in South Dakota-Continued

[County code: 7 = Bennett County, 53 = Gregory County, $71=$ Jackson County, $95=$ Mellette County, $113=$ Shannon County, and $121=$ Todd County; Datum, National Geodetic Vertical Datum; 1210GLL, Ogallala Formation; 122ARKR, Arikaree Formation; --, no data]

\begin{tabular}{|c|c|c|c|c|c|c|}
\hline Site number & Local identifier & $\begin{array}{l}\text { County } \\
\text { code }\end{array}$ & Hydrologic unit & Geologic unit & $\begin{array}{c}\text { Depth of well, } \\
\text { total } \\
\text { (feet) }\end{array}$ & $\begin{array}{l}\text { Altitude of land } \\
\text { surface, datum } \\
\text { (feet) }\end{array}$ \\
\hline 430859101542401 & $37 \mathrm{~N} 39 \mathrm{~W} 26 \mathrm{CBAA}$ & 7 & 10140203 & 122ARKR & 300 & 3,313 \\
\hline 430900100330201 & 37N27W30DAA & 121 & 10150006 & 121OGLL & 155 & 2,733 \\
\hline 430900100541101 & 37 N30W29C & 121 & 10140203 & 122ARKR & 400 & 2,980 \\
\hline 430900101342901 & 37N36W28DA & 7 & 10140203 & 122ARKR & 100 & 3,040 \\
\hline 430901100513001 & $37 \mathrm{~N} 30 \mathrm{~W} 27 \mathrm{CAAA}$ & 121 & 10140203 & 122ARKR & 220 & 2,880 \\
\hline 430905100395201 & 37N28W29BCDD & 121 & 10150006 & 121OGLL & 120 & 2,755 \\
\hline 430905102201301 & $37 \mathrm{~N} 42 \mathrm{~W} 30$ & 113 & 10140201 & 122ARKR & -- & 3,325 \\
\hline 430907100401001 & 37N28W30ADDA & 121 & 10150006 & 121OGLL & 80 & 2,757 \\
\hline 430907101073801 & 37N32W29ACB & 121 & 10140203 & 121OGLL & 165 & 2,910 \\
\hline 430907101561701 & $37 \mathrm{~N} 39 \mathrm{~W} 28 \mathrm{ACC}$ & 7 & 10140203 & 122ARKR & 500 & 3,307 \\
\hline 430908100175801 & 37N25W29ACDD & 121 & 10150006 & 122ARKR & 60 & 2,374 \\
\hline 430908100175802 & 37N25W29ACDD2 & 121 & 10150006 & 122ARKR & 30 & 2,374 \\
\hline 430909100333501 & 37N27W30BDDA & 121 & 10150006 & 121OGLL & 150 & 2,722 \\
\hline 430909100444501 & $37 \mathrm{~N} 29 \mathrm{~W} 27 \mathrm{BC}$ & 121 & 10150006 & 121OGLL & 140 & 2,797 \\
\hline 430909100452701 & $37 \mathrm{~N} 29 \mathrm{~W} 28 \mathrm{ACBC}$ & 121 & 10150006 & 121OGLL & 150 & 2,880 \\
\hline 430910100454701 & $37 \mathrm{~N} 29 \mathrm{~W} 28 \mathrm{BDBC}$ & 121 & 10150006 & 121OGLL & 177 & 2,840 \\
\hline 430910100490201 & $37 \mathrm{~N} 30 \mathrm{~W} 25 \mathrm{ACBC}$ & 121 & 10140203 & 121OGLL & 165 & 2,899 \\
\hline 430910101202401 & $37 \mathrm{~N} 34 \mathrm{~W} 28 \mathrm{AAC}$ & 7 & 10140203 & 121OGLL & 340 & 2,962 \\
\hline 430910101340301 & $37 \mathrm{~N} 36 \mathrm{~W} 27 \mathrm{BBC}$ & 7 & 10140203 & 122ARKR & 220 & 3,100 \\
\hline 430910101354901 & 37N36W29AAC & 7 & 10140203 & 122ARKR & 250 & 3,165 \\
\hline 430910102143001 & $37 \mathrm{~N} 42 \mathrm{~W} 25 \mathrm{BCC}$ & 113 & 10140201 & 122ARKR & 170 & 3,350 \\
\hline 430911100571901 & $37 N 31 W 26 B$ & 121 & 10140203 & 122ARKR & 344 & 3,025 \\
\hline 430912100415501 & $37 N 29 W 24$ & 121 & 10150006 & 121OGLL & 150 & 2,827 \\
\hline 430912100542301 & $37 \mathrm{~N} 30 \mathrm{~W} 29 \mathrm{BCBB}$ & 121 & 10140203 & 121OGLL & 216 & 2,997 \\
\hline 430913102220501 & 37N43W26AD & 113 & 10140201 & 122ARKR & 170 & 3,240 \\
\hline 430913102364301 & $37 N 45 W 26 B D$ & 113 & 10140201 & 122ARKR & 432 & 3,390 \\
\hline 430914100455201 & $37 \mathrm{~N} 29 \mathrm{~W} 28 \mathrm{BCAB}$ & 121 & 10140203 & 121OGLL & 110 & 2,848 \\
\hline 430914100501601 & $37 \mathrm{~N} 30 \mathrm{~W} 26 \mathrm{~B}$ & 121 & 10140203 & 1210GLL & 150 & 2,923 \\
\hline 430914101134001 & 37N33W28BAAA & 7 & 10140203 & 121OGLL & 266 & 2,961 \\
\hline 430915100383601 & $37 \mathrm{~N} 28 \mathrm{~W} 28 \mathrm{~B}$ & 121 & 10150006 & 121OGLL & -- & 2,755 \\
\hline 430915100421401 & $37 \mathrm{~N} 29 \mathrm{~W} 25 \mathrm{BBDD}$ & 121 & 10150006 & 121OGLL & 220 & 2,799 \\
\hline 430915100432301 & $37 \mathrm{~N} 29 \mathrm{~W} 26 \mathrm{~B}$ & 121 & 10150006 & 121OGLL & -- & 2,803 \\
\hline 430916100424901 & $37 \mathrm{~N} 29 \mathrm{~W} 26 \mathrm{AACC}$ & 121 & 10150006 & 121OGLL & 189 & 2,808 \\
\hline 430916100432501 & $37 \mathrm{~N} 29 \mathrm{~W} 26 \mathrm{BBDD}$ & 121 & 10150006 & 121OGLL & 161 & 2,799 \\
\hline 430916100443601 & 37N29W27BBDD & 121 & 10150006 & 121OGLL & 208 & 2,868 \\
\hline 430917101474301 & $37 N 38 W 27 A A C$ & 7 & 10140203 & 122ARKR & 370 & 3,264 \\
\hline 430918100391501 & $37 \mathrm{~N} 28 \mathrm{~W} 29 \mathrm{AACC}$ & 121 & 10150006 & 121OGLL & 180 & 2,733 \\
\hline 430920100213001 & $37 \mathrm{~N} 26 \mathrm{~W} 26 \mathrm{ABD}$ & 121 & 10150006 & 122ARKR & 100 & 2,565 \\
\hline
\end{tabular}


Table 57. Selected descriptive data (from the U.S. Geological Survey database) for ground-water sites in the High Plains aquifer in South Dakota-Continued

[County code: 7 = Bennett County, $53=$ Gregory County, $71=$ Jackson County, $95=$ Mellette County, $113=$ Shannon County, and $121=$ Todd County; Datum, National Geodetic Vertical Datum; 1210GLL, Ogallala Formation; 122ARKR, Arikaree Formation; --, no data]

\begin{tabular}{|c|c|c|c|c|c|c|}
\hline Site number & Local identifier & $\begin{array}{l}\text { County } \\
\text { code }\end{array}$ & Hydrologic unit & Geologic unit & $\begin{array}{c}\text { Depth of well, } \\
\text { total } \\
\text { (feet) }\end{array}$ & $\begin{array}{c}\text { Altitude of land } \\
\text { surface, datum } \\
\text { (feet) }\end{array}$ \\
\hline 430920100444801 & 37N29W27BBCA & 121 & 10150006 & 121OGLL & 190 & 2,877 \\
\hline 430920100581201 & $37 \mathrm{~N} 31 \mathrm{~W} 22 \mathrm{ABCD} 2$ & 121 & 10140203 & 122ARKR & 430 & 2,943 \\
\hline 430922100410302 & 37 N28W30BBAA2 & 121 & -- & 122ARKR & 320.5 & 2818 \\
\hline 430924100460601 & 37N29W29AAAA & 121 & 10150006 & 121OGLL & 204.3 & 2,868 \\
\hline 430925100251701 & 37N26W20CAAA3 & 121 & 10150006 & 122ARKR & -- & 2,559 \\
\hline 430925102144901 & $37 \mathrm{~N} 42 \mathrm{~W} 25 \mathrm{BB}$ & 113 & 10140201 & 122ARKR & 160 & 3,380 \\
\hline 430926100290301 & $37 \mathrm{~N} 27 \mathrm{~W} 26 \mathrm{BAB}$ & 121 & 10150006 & 122ARKR & 250 & 2,564 \\
\hline 430929101104202 & 37N33W26AAAA2 & 121 & 10140203 & 122ARKR & 395 & 2,953 \\
\hline 430929101104203 & 37N33W26AAAA3 & 121 & 10140203 & 121OGLL & 236 & 2,953 \\
\hline 430931100251801 & 37N26W20CDD & 121 & 10150006 & 122ARKR & 140 & 2,605 \\
\hline 430932100211701 & 37N26W23DDCD & 121 & 10150006 & 122ARKR & 140 & 2,538 \\
\hline 430932100262401 & 37N26W19DCC & 121 & 10150006 & 121OGLL & 160 & 2,655 \\
\hline 430932100390001 & $37 \mathrm{~N} 28 \mathrm{~W} 21 \mathrm{CCCC}$ & 121 & 10150006 & 121OGLL & 163 & 2,772 \\
\hline 430932100424901 & 37N29W23DACC & 121 & 10150006 & 121OGLL & 210 & 2,840 \\
\hline 430933100415401 & 37N29W24CDD & 121 & 10150006 & 121OGLL & 150 & 2,820 \\
\hline 430938101523101 & 37N39W24DDB & 7 & 10140203 & 122ARKR & 130 & 3,297 \\
\hline 430940100340501 & 37N28W24DDAA & 121 & 10150006 & 121OGLL & 80 & 2,559 \\
\hline 430940100364101 & $37 N 28 W 22 C$ & 121 & 10150006 & 121OGLL & -- & 2,740 \\
\hline 430940100373201 & 37N28W22CABB2 & 121 & 10150006 & 121OGLL & 214 & 2,732 \\
\hline 430941100394701 & $37 N 28 W 20 C$ & 121 & 10150006 & 1210GLL & -- & 2,750 \\
\hline 430941101433601 & $37 \mathrm{~N} 37 \mathrm{~W} 20 \mathrm{C}$ & 7 & 10140203 & $122 \mathrm{ARKR}$ & 260 & 3,240 \\
\hline 430943100572801 & $37 \mathrm{~N} 31 \mathrm{~W} 23 \mathrm{CB}$ & 121 & -- & $122 \mathrm{ARKR}$ & 440 & 3,000 \\
\hline 430943101192301 & $37 N 34 \mathrm{~W} 22 \mathrm{DBB}$ & 7 & 10140203 & 121OGLL & 370 & 2,965 \\
\hline 430943101551401 & 37N39W22DBCC & 7 & 10140203 & $122 \mathrm{ARKR}$ & 160 & 3,287 \\
\hline 430946102012401 & 37N40W23CAA & 7 & 10140203 & 122ARKR & 400 & 1,030 \\
\hline 430947101274801 & $37 N 35 \mathrm{~W} 21 \mathrm{DBB}$ & 7 & 10140203 & 1210GLL & 200 & 2,992 \\
\hline 430948101571601 & 37N39W20DABC & 7 & 10140203 & 122ARKR & 460 & 3,343 \\
\hline 430949100252301 & 37N26W20CAAA3 & 121 & 10150006 & 122ARKR & 130 & 2,559 \\
\hline 430949101292701 & 37N35W20CBBA & 7 & 10140203 & 1210GLL & 128 & 2,995 \\
\hline
\end{tabular}


Table 57. Selected descriptive data (from the U.S. Geological Survey database) for ground-water sites in the High Plains aquifer in South Dakota-Continued

[County code: $7=$ Bennett County, $53=$ Gregory County, $71=$ Jackson County, $95=$ Mellette County, $113=$ Shannon County, and $121=$ Todd County; Datum, National Geodetic Vertical Datum; 121OGLL, Ogallala Formation; 122ARKR, Arikaree Formation; --, no data]

\begin{tabular}{|c|c|c|c|c|c|c|}
\hline Site number & Local identifier & $\begin{array}{c}\text { County } \\
\text { code }\end{array}$ & Hydrologic unit & Geologic unit & $\begin{array}{c}\text { Depth of well, } \\
\text { total } \\
\text { (feet) }\end{array}$ & $\begin{array}{c}\text { Altitude of land } \\
\text { surface, datum } \\
\text { (feet) }\end{array}$ \\
\hline 430950101275401 & 37N35W21BDD & 7 & 10140203 & 122ARKR & 120 & 2,950 \\
\hline 430952100251701 & $37 \mathrm{~N} 26 \mathrm{~W} 20 \mathrm{CAA}$ & 121 & 10150006 & 122ARKR & 130 & 2,560 \\
\hline 430952100251702 & 37N26W20CAA2 & 121 & 10150006 & 122ARKR & 130 & 2,570 \\
\hline 430952100550201 & 37N30W19DDDA & 121 & 10140203 & 122ARKR & 393 & 2,996 \\
\hline 430952102231601 & $37 N 43 W 22 D A$ & 113 & 10140201 & 122ARKR & 120 & 3,210 \\
\hline 430953100200001 & 37N26W24DAA & 121 & 10150006 & 122ARKR & 90 & 2,451 \\
\hline 430954101551201 & 36N39W 9BAC & 7 & 10140203 & 122ARKR & 120 & 3,450 \\
\hline 430954102421801 & 37N46W24DBAD & 113 & 10140201 & 122ARKR & 355 & 2,998 \\
\hline 430955100402401 & $37 \mathrm{~N} 28 \mathrm{~W} 19 \mathrm{ADCC}$ & 121 & 10150006 & 1210GLL & -- & 2,758 \\
\hline 430955101233501 & 37N34W19CBB & 7 & 10140203 & 122ARKR & 120 & 2,970 \\
\hline 430955102324201 & $37 N 44 W 20 D A$ & 113 & 10140201 & $122 \mathrm{ARKR}$ & 400 & 3,500 \\
\hline 430956100402901 & 37N28W19ACDD & 121 & 10150006 & 121OGLL & 120 & 2,800 \\
\hline 430958100384001 & $37 \mathrm{~N} 28 \mathrm{~W} 21 \mathrm{BCDD}$ & 121 & 10150006 & 121OGLL & 205 & 2,742 \\
\hline 430959100444001 & $37 \mathrm{~N} 29 \mathrm{~W} 22 \mathrm{CCCC}$ & 121 & 10150006 & 122ARKR & 265 & 2,882 \\
\hline 431002101210001 & 37N34W21BBD2 & 7 & 10140203 & 121OGLL & 350 & 2,956 \\
\hline 431002101254501 & 37N35W23BBD & 7 & 10140203 & 121OGLL & 260 & 2,969 \\
\hline 431003100183201 & 37N25W19ADBC & 121 & 10150006 & 122ARKR & 130 & 2,438 \\
\hline 431003101091101 & $37 N 32 W 19 B$ & 121 & 10140203 & 1210GLL & 160 & 3,038 \\
\hline 431003101395901 & 37N37W23B & 7 & 10140203 & 122ARKR & 420 & 3,231 \\
\hline 431005100484401 & $37 \mathrm{~N} 30 \mathrm{~W} 24 \mathrm{~A}$ & 121 & 10140203 & 121OGLL & -- & 2,900 \\
\hline 431005100580402 & $37 \mathrm{~N} 31 \mathrm{~W} 22 \mathrm{ABCD} 3$ & 121 & 10140203 & 122ARKR & 450 & 2,943 \\
\hline 431005101553501 & $37 N 39 \mathrm{~W} 22 \mathrm{BBD}$ & 7 & 10140203 & 122ARKR & 480 & 3,330 \\
\hline 431005102225801 & $37 \mathrm{~N} 43 \mathrm{~W} 23 \mathrm{BC}$ & 113 & 10140201 & 122ARKR & 120 & 3,200 \\
\hline 431006100425001 & $37 N 29 W 23 A D B B$ & 121 & 10140203 & 121OGLL & 210 & 2,839 \\
\hline 431007101250601 & $37 N 35 W 23 A$ & 7 & 10140203 & 1210GLL & 470 & 2,978 \\
\hline 431007101435101 & $37 N 37 \mathrm{~W} 20 \mathrm{BBCC}$ & 7 & 10140203 & 122ARKR & -- & 3,264 \\
\hline 431008100423401 & 37N29W23AADD & 121 & 10150006 & 121OGLL & 150 & 2,864 \\
\hline 431008100443701 & 37N29W22BBDD & 121 & 10150006 & 1210GLL & 238 & 2,861 \\
\hline
\end{tabular}


Table 57. Selected descriptive data (from the U.S. Geological Survey database) for ground-water sites in the High Plains aquifer in South Dakota-Continued

[County code: 7 = Bennett County, $53=$ Gregory County, $71=$ Jackson County, $95=$ Mellette County, $113=$ Shannon County, and $121=$ Todd County; Datum, National Geodetic Vertical Datum; 1210GLL, Ogallala Formation; 122ARKR, Arikaree Formation; --, no data]

\begin{tabular}{|c|c|c|c|c|c|c|}
\hline Site number & Local identifier & $\begin{array}{l}\text { County } \\
\text { code }\end{array}$ & Hydrologic unit & Geologic unit & $\begin{array}{c}\text { Depth of well, } \\
\text { total } \\
\text { (feet) }\end{array}$ & $\begin{array}{l}\text { Altitude of land } \\
\text { surface, datum } \\
\text { (feet) }\end{array}$ \\
\hline 431009101274101 & $37 \mathrm{~N} 35 \mathrm{~W} 21 \mathrm{ABB}$ & 7 & 10140203 & 121OGLL & 390 & 3,002 \\
\hline 431010100362001 & $35 \mathrm{~N} 28 \mathrm{~W} 10 \mathrm{CCBC}$ & 121 & 10140203 & 122ARKR & 125 & 2,750 \\
\hline 431011100440501 & $37 \mathrm{~N} 29 \mathrm{~W} 22 \mathrm{ABAD}$ & 121 & 10150006 & 121OGLL & 180 & 2,832 \\
\hline 431011100595701 & $37 \mathrm{~N} 31 \mathrm{~W} 21 \mathrm{~B}$ & 121 & 10140203 & 122ARKR & 82 & 2,565 \\
\hline 431013100391501 & 37N28W20AACB & 121 & 10150006 & 121OGLL & 206 & 2,733 \\
\hline 431015102064601 & $37 \mathrm{~N} 41 \mathrm{~W} 24 \mathrm{AA}$ & 113 & 10140201 & 122ARKR & 200 & 3,461 \\
\hline 431015102111501 & $37 \mathrm{~N} 41 \mathrm{~W} 21 \mathrm{BB}$ & 113 & 10140201 & 122ARKR & 349 & 3,543 \\
\hline 431017102141401 & $37 \mathrm{~N} 42 \mathrm{~W} 24 \mathrm{AB}$ & 113 & 10140201 & 122ARKR & 250 & 3,300 \\
\hline 431018101132301 & 37N33W16DCDC & 121 & 10140203 & 121OGLL & 270 & 3,023 \\
\hline 431018101152001 & $37 \mathrm{~N} 33 \mathrm{~W} 17 \mathrm{CCCC}$ & 7 & 10140203 & 121OGLL & 181 & 2,998 \\
\hline 431018101212001 & 37N34W17DDDD & 7 & 10140203 & 122ARKR & 43.7 & 2,956 \\
\hline 431018102222301 & $37 \mathrm{~N} 43 \mathrm{~W} 23 \mathrm{AB}$ & 113 & 10140201 & 122ARKR & 380 & 3,360 \\
\hline 431018102224101 & 37N43W23BA & 113 & 10140201 & $122 \mathrm{ARKR}$ & 420 & 3,300 \\
\hline 431019100200001 & 37N26W24AAA & 121 & 10150006 & 122ARKR & 110 & 2,471 \\
\hline 431019100453201 & 37N29W21BAAA & 121 & 10140203 & 121OGLL & 154 & 2,885 \\
\hline 431020100243501 & 37N26W16CCBB & 121 & 10150006 & 122ARKR & -- & 2,530 \\
\hline 431021100384701 & $37 \mathrm{~N} 28 \mathrm{~W} 16 \mathrm{CCDD}$ & 121 & 10150006 & 121OGLL & 80 & 2,744 \\
\hline 431021100542401 & $37 \mathrm{~N} 30 \mathrm{~W} 17 \mathrm{CCCC}$ & 121 & 10140203 & 122ARKR & 332 & 2,995 \\
\hline 431021101094201 & 37N33W13DDCB & 121 & 10140203 & 121OGLL & 230 & 3,054 \\
\hline 431022100242001 & $37 \mathrm{~N} 26 \mathrm{~W} 22 \mathrm{CADC}$ & 121 & 10150006 & 121OGLL & 140 & 2,595 \\
\hline 431022100542301 & $37 \mathrm{~N} 30 \mathrm{~W} 17 \mathrm{CCCB}$ & 121 & 10140203 & 122ARKR & 386 & 2,995 \\
\hline 431022101555001 & 37N39W16DDDD & 7 & 10140203 & 122ARKR & 565 & 3,350 \\
\hline 431022101570101 & 37N39W17DDDD & 7 & 10140203 & 122ARKR & 305 & 3,337 \\
\hline 431025100541701 & $37 \mathrm{~N} 30 \mathrm{~W} 17 \mathrm{CC}$ & 121 & 10140203 & 122ARKR & 342 & 2,975 \\
\hline 431025101291501 & $37 \mathrm{~N} 35 \mathrm{~W} 19 \mathrm{C}$ & 7 & 10140203 & 122ARKR & 60 & 3,000 \\
\hline 431027100333001 & 37N27W18DDAB & 121 & 10150004 & 121OGLL & 53 & 2,609 \\
\hline 431027100403601 & 37N28W18DCDB & 121 & 10150006 & 121OGLL & 165 & 2,818 \\
\hline 431029101150401 & 37N33W17CBDD & 7 & 10140203 & 121OGLL & 300 & 2,992 \\
\hline 431029101400902 & 37N37W17CB2 & 7 & 10140203 & $122 \mathrm{ARKR}$ & 500 & 3,287 \\
\hline 431029101441601 & 37N37W18DCAB & 7 & 10140203 & 122ARKR & -- & 3,290 \\
\hline 431030101031201 & $37 \mathrm{~N} 32 \mathrm{~W} 13 \mathrm{CAD}$ & 121 & 10140203 & 122ARKR & 325 & 2,970 \\
\hline 431030101215001 & 37N34W17D & 7 & 10140203 & 122ARKR & 120 & 2,970 \\
\hline 431030101560001 & 37N39W16DDDD2 & 7 & 10140203 & 122ARKR & 565 & 3,350 \\
\hline 431031101431801 & 37N37W17CADD & 7 & 10140203 & 122ARKR & -- & 3,284 \\
\hline 431031101435201 & $37 \mathrm{~N} 37 \mathrm{~W} 17 \mathrm{CCBB}$ & 7 & 10140203 & 122ARKR & -- & 3,297 \\
\hline 431031101442201 & 37N37W 18DCBA & 7 & 10140203 & 122ARKR & -- & 3,287 \\
\hline 431032100231301 & $37 \mathrm{~N} 26 \mathrm{~W} 15 \mathrm{CC}$ & 121 & 10150006 & 122ARKR & 160 & 2,553 \\
\hline 431032100440201 & 37N29W15D & 121 & 10150006 & 121OGLL & -- & 2,852 \\
\hline
\end{tabular}


Table 57. Selected descriptive data (from the U.S. Geological Survey database) for ground-water sites in the High Plains aquifer in South Dakota-Continued

[County code: 7 = Bennett County, $53=$ Gregory County, $71=$ Jackson County, $95=$ Mellette County, $113=$ Shannon County, and $121=$ Todd County; Datum, National Geodetic Vertical Datum; 1210GLL, Ogallala Formation: 122ARKR, Arikaree Formation; --, no data]

\begin{tabular}{|c|c|c|c|c|c|c|}
\hline Site number & Local identifier & $\begin{array}{l}\text { County } \\
\text { code }\end{array}$ & Hydrologic unit & Geologic unit & $\begin{array}{c}\text { Depth of well, } \\
\text { total } \\
\text { (feet) }\end{array}$ & $\begin{array}{l}\text { Altitude of land } \\
\text { surface, datum } \\
\text { (feet) }\end{array}$ \\
\hline 431033100432601 & 37N29W14DDBB & 121 & 10150006 & 121OGLL & -- & 2,855 \\
\hline 431033100493201 & 37N30W13CBCD & 121 & 10140203 & 121OGLL & 60 & 2,783 \\
\hline 431034100443601 & $37 \mathrm{~N} 29 \mathrm{~W} 15 \mathrm{C}$ & 121 & 10150006 & 121OGLL & -- & 2,843 \\
\hline 431034100451201 & 37N29W16D & 121 & 10140203 & 121OGLL & -- & 2,858 \\
\hline 431034100454801 & 37N29W16CBDD & 121 & 10150006 & 121OGLL & 240 & 2,863 \\
\hline 431034101441101 & 37N37W18D & 7 & 10140203 & 122ARKR & 480 & 3,293 \\
\hline 431034101441102 & 37N37W18D2 & 7 & 10140203 & 122ARKR & 500 & 3,293 \\
\hline 431035100381701 & 37N28W17DDBB & 121 & 10150006 & 121OGLL & 210 & 2,725 \\
\hline 431036102192301 & $37 \mathrm{~N} 42 \mathrm{~W} 17 \mathrm{C}$ & 113 & 10140201 & 122ARKR & -- & 3,490 \\
\hline 431037100381801 & 37N28W17DBDD & 121 & 10150006 & 1210GLL & 193 & 2,719 \\
\hline 431038101433701 & $37 \mathrm{~N} 37 \mathrm{~W} 17 \mathrm{CB}$ & 7 & 10140203 & 122ARKR & 580 & 3,313 \\
\hline 431040100442801 & 37N29W15CABB & 121 & 10150006 & 1210GLL & 222 & 2,855 \\
\hline 431042100535601 & 37N30W17CAA & 121 & 10140203 & 122ARKR & 460 & 2,927 \\
\hline 431043102171001 & $37 \mathrm{~N} 42 \mathrm{~W} 15 \mathrm{CB}$ & 113 & 10140201 & 122ARKR & 312 & 3,460 \\
\hline 431044100252301 & $37 \mathrm{~N} 26 \mathrm{~W} 17 \mathrm{CAA}$ & 121 & 10150006 & 122ARKR & 160 & 2,515 \\
\hline 431044101431801 & 37N37W17CAAA & 7 & 10140203 & 122ARKR & -- & 3,208 \\
\hline 431044102363201 & 37N45W14DB & 113 & 10140201 & 122ARKR & 410 & 3,575 \\
\hline 431045101293701 & 37N35W18DAAA & 7 & 10140203 & 1210GLL & 27.1 & 2,995 \\
\hline 431047100445601 & 37N29W16DAAA & 121 & 10140203 & 1210GLL & 100 & 2,875 \\
\hline 431050100274501 & 37N27W13BDD & 121 & 10150006 & 122ARKR & 140 & 2,530 \\
\hline 431051100283801 & $37 \mathrm{~N} 27 \mathrm{~W} 14 \mathrm{ACD}$ & 121 & 10150006 & 122ARKR & 150 & 2,532 \\
\hline 431051100293101 & $37 \mathrm{~N} 27 \mathrm{~W} 15 \mathrm{ADD}$ & 121 & 10150006 & 122ARKR & 150 & 2,518 \\
\hline 431051100434701 & 37N29W15ADDA & 121 & 10150006 & 121OGLL & 150 & 2,849 \\
\hline 431054101154001 & 37N33W18AAC & 7 & 10140203 & 1210GLL & 225 & 3,005 \\
\hline 431057100220701 & $37 \mathrm{~N} 26 \mathrm{~W} 14 \mathrm{BCA}$ & 121 & 10150006 & 122ARKR & 165 & 2,515 \\
\hline 431058100462401 & $37 \mathrm{~N} 29 \mathrm{~W} 17 \mathrm{~A}$ & 121 & 10140203 & 1210GLL & -- & 2,850 \\
\hline 431100100305801 & 37N27W16A & 121 & 10150006 & 122ARKR & 115 & 2,547 \\
\hline 431100100461601 & 37N29W17AACD & 121 & 10140203 & 121OGLL & 180 & 2,845 \\
\hline 431100100481101 & $37 \mathrm{~N} 29 \mathrm{~W} 18 \mathrm{~A}$ & 121 & 10140203 & 121OGLL & 143 & 2,810 \\
\hline 431100101440801 & 37N37W18A & 7 & 10140203 & 122ARKR & 240 & 3,330 \\
\hline 431100102320701 & 37N44W16BD & 113 & 10140201 & 122ARKR & 276 & 3,400 \\
\hline 431102102155101 & $37 \mathrm{~N} 42 \mathrm{~W} 14 \mathrm{~B}$ & 113 & 10140201 & 122ARKR & -- & 3,290 \\
\hline 431106100581301 & $37 N 31 W 15 B$ & 121 & 10140203 & 122ARKR & 73 & 2,640 \\
\hline 431106101233001 & 37N34W17B & 7 & 10140203 & 122ARKR & 120 & 2,950 \\
\hline 431108100584601 & $37 \mathrm{~N} 31 \mathrm{~W} 10 \mathrm{CCC}$ & 121 & 10140203 & 122ARKR & 145 & 2,550 \\
\hline 431109100445901 & 37N29W16AAAA & 121 & 10140203 & 121OGLL & 183.8 & 2,852 \\
\hline 431115100360401 & 37 N28W14 & 121 & 10150006 & 122ARKR & 163 & 2,750 \\
\hline 431116100453901 & $37 \mathrm{~N} 29 \mathrm{~W} 9 \mathrm{CDCA}$ & 121 & 10140203 & 1210GLL & 100 & 2,831 \\
\hline
\end{tabular}


Table 57. Selected descriptive data (from the U.S. Geological Survey database) for ground-water sites in the High Plains aquifer in South Dakota-Continued

[County code: 7 = Bennett County, 53 = Gregory County, $71=$ Jackson County, $95=$ Mellette County, $113=$ Shannon County, and $121=$ Todd County; Datum, National Geodetic Vertical Datum; 1210GLL, Ogallala Formation; 122ARKR, Arikaree Formation; --, no data]

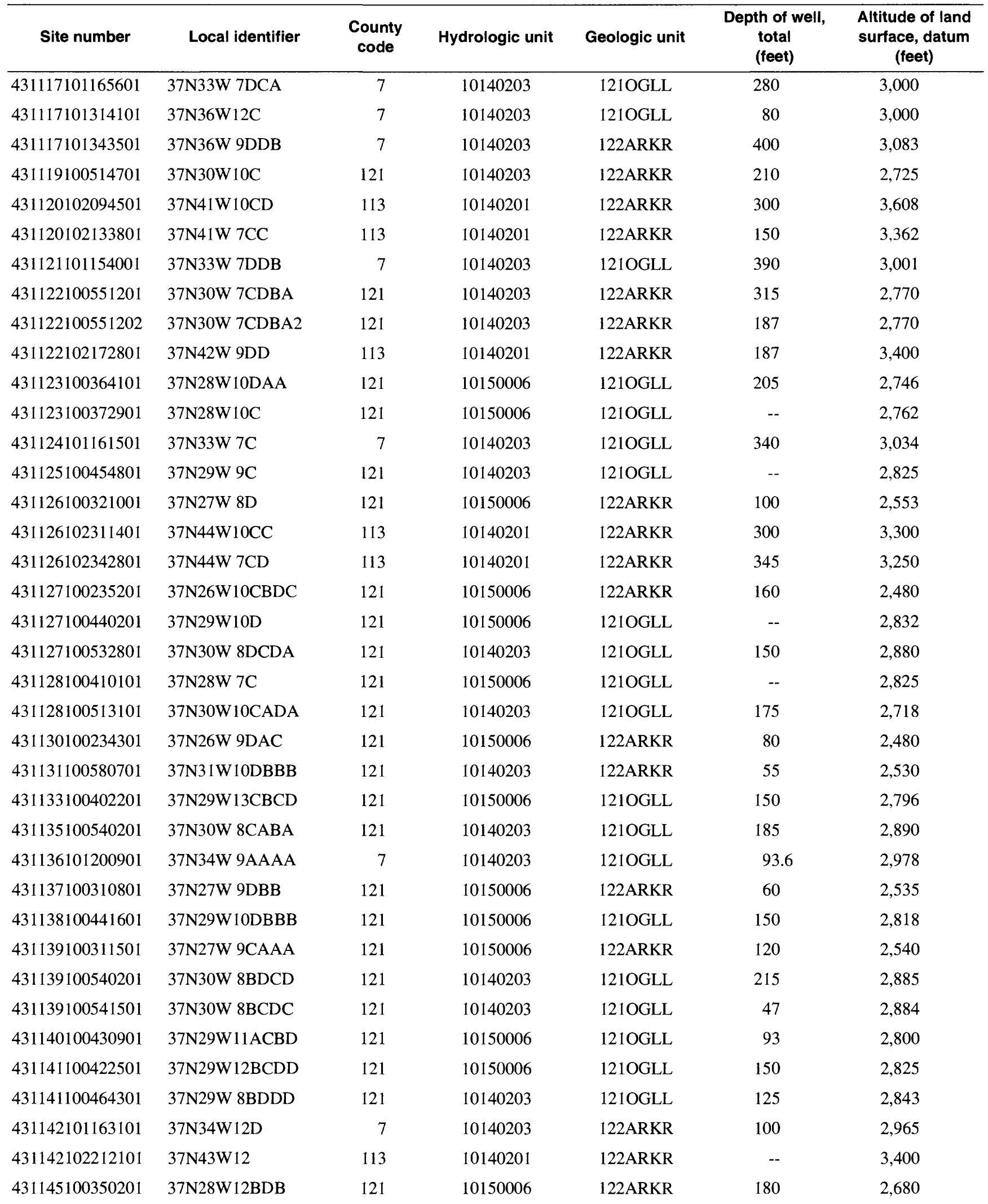


Table 57. Selected descriptive data (from the U.S. Geological Survey database) for ground-water sites in the High Plains aquifer in South Dakota-Continued

[County code: 7 = Bennett County, $53=$ Gregory County, $71=$ Jackson County, $95=$ Mellette County, $113=$ Shannon County, and $121=$ Todd County; Datum, National Geodetic Vertical Datum; 121OGLL, Ogallala Formation; 122ARKR, Arikaree Formation; --, no data]

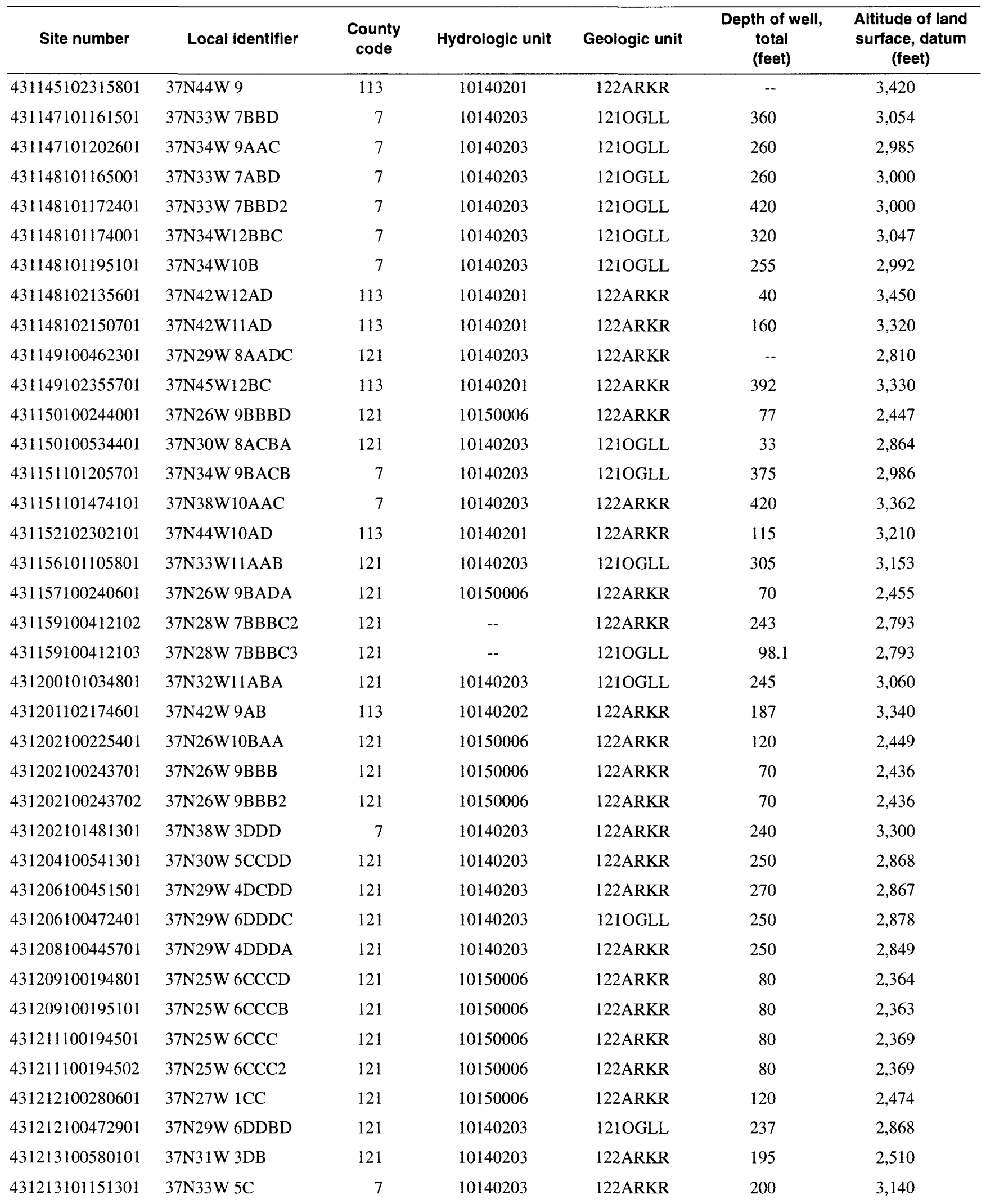


Table 57. Selected descriptive data (from the U.S. Geological Survey database) for ground-water sites in the High Plains aquifer in South Dakota-Continued

[County code: 7 = Bennett County, 53 = Gregory County, 71 = Jackson County, 95 = Mellette County, $113=$ Shannon County, and $121=$ Todd County; Datum, National Geodetic Vertical Datum; 121OGLL, Ogallala Formation; 122ARKR, Arikaree Formation; --, no data]

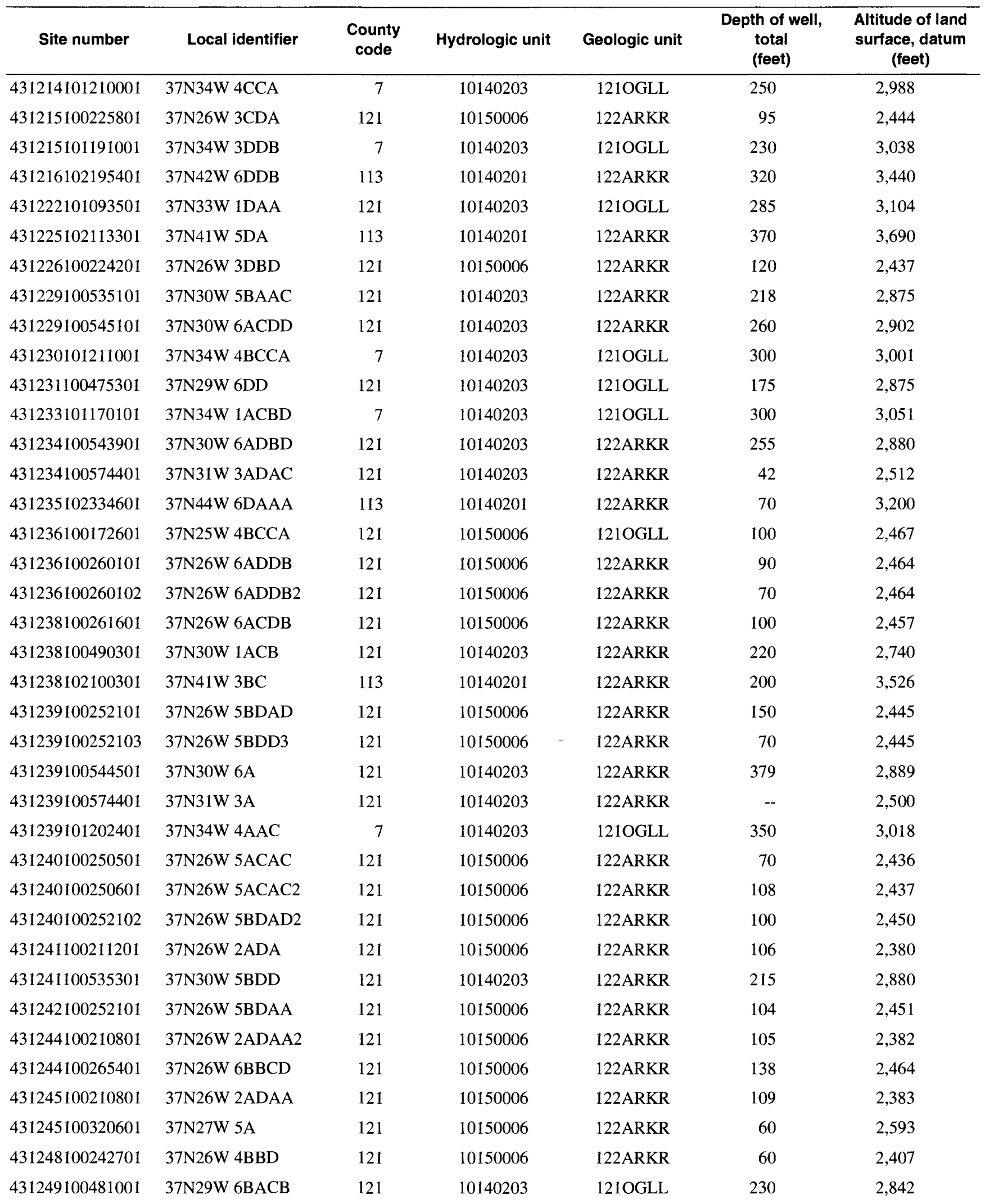


Table 57. Selected descriptive data (from the U.S. Geological Survey database) for ground-water sites in the High Plains aquifer in South Dakota-Continued

[County code: 7 = Bennett County, $53=$ Gregory County, $71=$ Jackson County, $95=$ Mellette County, $113=$ Shannon County, and $121=$ Todd County; Datum, National Geodetic Vertical Datum; 121OGLL, Ogallala Formation; 122ARKR, Arikaree Formation; --, no data]

\begin{tabular}{|c|c|c|c|c|c|c|}
\hline Site number & Local identifier & $\begin{array}{l}\text { County } \\
\text { code }\end{array}$ & Hydrologic unit & Geologic unit & $\begin{array}{l}\text { Depth of well, } \\
\text { total } \\
\text { (feet) }\end{array}$ & $\begin{array}{l}\text { Altitude of land } \\
\text { surface, datum } \\
\text { (feet) }\end{array}$ \\
\hline 431250100373701 & $37 \mathrm{~N} 28 \mathrm{~W} 3 \mathrm{BBD}$ & 121 & 10150006 & 121OGLL & 140 & 2,750 \\
\hline 431250100530101 & $37 \mathrm{~N} 30 \mathrm{~W} 4 \mathrm{BAA}$ & 121 & 10140203 & 122ARKR & 265 & 2,842 \\
\hline 431251100532801 & 37N30W 5AABA & 121 & 10140203 & 122ARKR & 195 & 2,878 \\
\hline 431251100532802 & 37N30W 5AABA2 & 121 & -- & 122ARKR & 180 & 2,878 \\
\hline 431251102074101 & $37 \mathrm{~N} 41 \mathrm{~W} 1 \mathrm{BBC}$ & 113 & 10140201 & 122ARKR & 280 & 3,500 \\
\hline 431251102074401 & $37 \mathrm{~N} 41 \mathrm{~W} 1 \mathrm{BBC} 2$ & 113 & 10140201 & 122ARKR & -- & 3,477 \\
\hline 431252100501801 & 38N30W35BDDB & 121 & 10140203 & 122ARKR & 219 & 2,708 \\
\hline 431252102162801 & $37 \mathrm{~N} 42 \mathrm{~W} 3 \mathrm{ABCA}$ & 113 & 10140201 & $122 \mathrm{ARKR}$ & 172 & 3,225 \\
\hline 431253100191001 & $37 \mathrm{~N} 25 \mathrm{~W} 6 \mathrm{ABA} 2$ & 121 & 10150006 & $122 \mathrm{ARKR}$ & 120 & 2,412 \\
\hline 431254100191001 & $37 \mathrm{~N} 25 \mathrm{~W} 6 \mathrm{ABA}$ & 121 & 10150006 & $122 \mathrm{ARKR}$ & 120 & 2,410 \\
\hline 431254102363201 & $37 \mathrm{~N} 45 \mathrm{~W} 2 \mathrm{AB}$ & 113 & 10140201 & $122 \mathrm{ARKR}$ & 280 & 3,150 \\
\hline 431254102391101 & $37 \mathrm{~N} 45 \mathrm{~W} 4 \mathrm{BA}$ & 113 & 10140201 & 122ARKR & 390 & 3,600 \\
\hline 431255100545701 & 38N30W31DCCD & 121 & 10140203 & $122 \mathrm{ARKR}$ & 250 & 2,872 \\
\hline 431255101212001 & 38N34W32DDDD & 7 & 10140203 & 121OGLL & 172 & 3,031 \\
\hline 431257100151901 & 37N25W 3AAAB & 121 & 10150006 & 1210GLL & 40 & 2,467 \\
\hline 431257101281501 & $38 \mathrm{~N} 35 \mathrm{~W} 33 \mathrm{CC}$ & 7 & 10140203 & 122ARKR & 96 & 2,969 \\
\hline 431259100574401 & 38N31W34DDAC & 121 & 10140203 & 122ARKR & 85 & 2502 \\
\hline 431259100574402 & 38N31W34DDAC2 & 121 & 10140203 & 122ARKR & 40 & 2,500 \\
\hline 431300100541901 & $38 \mathrm{~N} 30 \mathrm{~W} 32 \mathrm{CC}$ & 121 & 10140203 & 122ARKR & 245 & 2,860 \\
\hline 431300101020301 & 38N34W31DDD & 7 & 10140203 & 122ARKR & 60 & 3,185 \\
\hline 431301100251301 & $38 \mathrm{~N} 26 \mathrm{~W} 32 \mathrm{DCC}$ & 121 & 10150006 & 122ARKR & 80 & 2,440 \\
\hline 431303101075401 & $38 \mathrm{~N} 32 \mathrm{~W} 32 \mathrm{CDB}$ & 121 & 10140203 & 122ARKR & 285 & 3,032 \\
\hline 431304102124401 & 38N41W31DD & 113 & 10140201 & 122ARKR & 90 & 3,444 \\
\hline 431304102200701 & 38N42W31DCCD & 113 & 10140201 & 122ARKR & 313 & 3,423 \\
\hline 431305100490201 & 38N30W36DCB & 121 & 10140203 & 122ARKR & 225 & 2,818 \\
\hline 431305100512501 & 38N30W34DCB & 121 & 10140203 & 122ARKR & 130 & 2,685 \\
\hline 431305101091101 & $38 \mathrm{~N} 32 \mathrm{~W} 31 \mathrm{C}$ & 121 & 10140203 & 122ARKR & -- & 3,150 \\
\hline 431306102165301 & $38 \mathrm{~N} 42 \mathrm{~W} 34 \mathrm{CD}$ & 113 & 10140201 & $122 \mathrm{ARKR}$ & 165 & 3,230 \\
\hline 431307100522402 & 38N30W33D2 & 121 & 10140203 & 1210GLL & 48 & 2,840 \\
\hline 431307100522403 & 38N30W33D3 & 121 & 10140203 & $122 \mathrm{ARKR}$ & 325 & 2,840 \\
\hline 431308100223101 & $38 \mathrm{~N} 26 \mathrm{~W} 34 \mathrm{DDB}$ & 121 & 10150006 & $122 \mathrm{ARKR}$ & 100 & 2,417 \\
\hline 431310100490101 & $38 \mathrm{~N} 30 \mathrm{~W} 36 \mathrm{DBCC}$ & 121 & 10140203 & 122ARKR & 250 & 2,810 \\
\hline 431310102275901 & 38N44W36DD & 113 & 10140201 & 122ARKR & 161 & 3,100 \\
\hline 431310102313101 & 38N44W33DD & 113 & 10140201 & 122ARKR & 315 & 3,401 \\
\hline 431310102342801 & 38N44W31CD & 113 & 10140201 & $122 \mathrm{ARKR}$ & 310 & 3,030 \\
\hline 431311100193201 & $38 \mathrm{~N} 25 \mathrm{~W} 31 \mathrm{CDBA}$ & 121 & 10150006 & 122ARKR & 56 & 2,426 \\
\hline 431311100574302 & $38 \mathrm{~N} 31 \mathrm{~W} 34 \mathrm{D} 2$ & 121 & 10140203 & 122ARKR & 80 & 2,500 \\
\hline 431311100574303 & 38N31W34D3 & 121 & 10140203 & 122ARKR & 80 & 2,500 \\
\hline
\end{tabular}


Table 57. Selected descriptive data (from the U.S. Geological Survey database) for ground-water sites in the High Plains aquifer in South Dakota-Continued

[County code: 7 = Bennett County, 53 = Gregory County, 71 = Jackson County, $95=$ Mellette County, $113=$ Shannon County, and $121=$ Todd County; Datum, National Geodetic Vertical Datum; 121OGLL, Ogallala Formation; 122ARKR, Arikaree Formation; --, no data]

\begin{tabular}{|c|c|c|c|c|c|c|}
\hline Site number & Local identifier & $\begin{array}{l}\text { County } \\
\text { code }\end{array}$ & Hydrologic unit & Geologic unit & $\begin{array}{c}\text { Depth of well, } \\
\text { total } \\
\text { (feet) }\end{array}$ & $\begin{array}{l}\text { Altitude of land } \\
\text { surface, datum } \\
\text { (feet) }\end{array}$ \\
\hline 431315100491501 & 38N30W36CBAD & 121 & 10140203 & 122ARKR & 253 & 2,812 \\
\hline 431315100541301 & $38 \mathrm{~N} 30 \mathrm{~W} 32 \mathrm{CBD}$ & 121 & 10140203 & 122ARKR & 265 & 2,887 \\
\hline 431315101033501 & $38 \mathrm{~N} 40 \mathrm{~W} 33$ & 7 & 10140201 & 122ARKR & 230 & 3,500 \\
\hline 431318100532301 & 38N30W32DAAB & 121 & 10140203 & 1210GLL & 47 & 2,838 \\
\hline 431323100213501 & 38N26W35DBBA & 121 & 10150006 & 122ARKR & 100 & 2,403 \\
\hline 431325100324601 & $38 \mathrm{~N} 27 \mathrm{~W} 32 \mathrm{CBA}$ & 121 & 10150006 & 121OGLL & 135 & 2,680 \\
\hline 431326102175501 & $38 \mathrm{~N} 42 \mathrm{~W} 33$ & 113 & 10140201 & 122ARKR & 92 & 3,300 \\
\hline 431327100164501 & $38 \mathrm{~N} 25 \mathrm{~W} 33 \mathrm{ACD}$ & 121 & 10150006 & 121OGLL & 24 & 2,475 \\
\hline 431327100242701 & $38 \mathrm{~N} 26 \mathrm{~W} 33 \mathrm{BCC}$ & 121 & 10150006 & 122ARKR & 130 & 2,439 \\
\hline 431327100242901 & $38 \mathrm{~N} 26 \mathrm{~W} 33 \mathrm{BCDC}$ & 121 & 10150006 & 122ARKR & 110 & 2,442 \\
\hline 431328100240901 & $38 \mathrm{~N} 26 \mathrm{~W} 33 \mathrm{BDD}$ & 121 & 10150006 & 122ARKR & 70 & 2,424 \\
\hline 431328100503101 & $38 \mathrm{~N} 30 \mathrm{~W} 35 \mathrm{BDDB} 2$ & 121 & 10140203 & 121OGLL & 165 & 2,708 \\
\hline 431328101053801 & 38N32W34BCAD & 121 & 10140203 & 122ARKR & 280 & 2,987 \\
\hline 431332102191401 & $38 \mathrm{~N} 42 \mathrm{~W} 32 \mathrm{BD}$ & 113 & 10140201 & 122ARKR & 190 & 3,240 \\
\hline 431335100511101 & $38 \mathrm{~N} 30 \mathrm{~W} 34 \mathrm{~A}$ & 121 & 10140203 & 122ARKR & -- & 2,600 \\
\hline 431337100272201 & 38N27W36AACC & 121 & 10150006 & 122ARKR & 180 & 2,483 \\
\hline 431337100312101 & $38 \mathrm{~N} 27 \mathrm{~W} 33 \mathrm{BDA}$ & 121 & 10150006 & 122ARKR & 130 & 2,570 \\
\hline 431338100570901 & $38 \mathrm{~N} 31 \mathrm{~W} 35 \mathrm{BA}$ & 121 & 10140203 & 122ARKR & 60 & 2,485 \\
\hline 431340100253901 & $38 \mathrm{~N} 26 \mathrm{~W} 32 \mathrm{BBD}$ & 121 & 10150006 & 122ARKR & 85 & 2,452 \\
\hline 431340100253902 & 38N26W32BBD2 & 121 & 10150006 & 122ARKR & 85 & 2,452 \\
\hline 431340100430401 & $38 \mathrm{~N} 29 \mathrm{~W} 35 \mathrm{ACBB}$ & 121 & 10150006 & $122 \mathrm{ARKR}$ & 370 & 2,803 \\
\hline 431340100510201 & $38 \mathrm{~N} 30 \mathrm{~W} 34 \mathrm{AA}$ & 121 & 10140203 & 122ARKR & -- & 2,645 \\
\hline 431340100510202 & $38 \mathrm{~N} 30 \mathrm{~W} 34 \mathrm{AA} 2$ & 121 & 10140203 & 122ARKR & -- & 2,645 \\
\hline 431340100521401 & $38 \mathrm{~N} 30 \mathrm{~W} 33 \mathrm{AA}$ & 121 & 10140203 & 122ARKR & 255 & 2,840 \\
\hline 431340100521402 & $38 \mathrm{~N} 30 \mathrm{~W} 33 \mathrm{AA} 2$ & 121 & 10140203 & 1210GLL & - & 2,840 \\
\hline 431340100521405 & 38 N30W33AA5 & 121 & 10140203 & 122ARKR & 237.5 & 2,840 \\
\hline 431342100344101 & $38 \mathrm{~N} 28 \mathrm{~W} 36 \mathrm{ABCB}$ & 121 & 10150004 & 122ARKR & 73 & 2,620 \\
\hline 431343102113301 & $38 \mathrm{~N} 41 \mathrm{~W} 32 \mathrm{AA}$ & 113 & 10140201 & 122ARKR & 280 & 3,510 \\
\hline 431343102180101 & $38 \mathrm{~N} 42 \mathrm{~W} 33 \mathrm{BADC}$ & 113 & 10140201 & 122ARKR & 120 & 3,200 \\
\hline 431345102160001 & $38 \mathrm{~N} 42 \mathrm{~W} 35 \mathrm{BB}$ & 113 & 10140201 & 122ARKR & 290 & 3,345 \\
\hline 431346100410901 & $38 \mathrm{~N} 28 \mathrm{~W} 31 \mathrm{BBA}$ & 121 & 10150006 & 1210GLL & -- & 2,680 \\
\hline 431347100232501 & $38 \mathrm{~N} 26 \mathrm{~W} 34 \mathrm{BBB}$ & 121 & 10150006 & 122ARKR & 100 & 2,405 \\
\hline 431347100404901 & 38N28W31ABBB & 121 & 10150006 & 122ARKR & 120 & 2,666 \\
\hline 431349102291001 & $38 \mathrm{~N} 44 \mathrm{~W} 35 \mathrm{AA}$ & 113 & 10140201 & 122ARKR & 150 & 3,350 \\
\hline 431349102345701 & 38N45W36AAA & 113 & 10140201 & 122ARKR & 200 & 3,075 \\
\hline 431350100570501 & $38 \mathrm{~N} 31 \mathrm{~W} 26 \mathrm{CDDB}$ & 121 & 10140203 & 122ARKR & -- & 2,477 \\
\hline 431351100521501 & $38 \mathrm{~N} 30 \mathrm{~W} 28 \mathrm{DDCA}$ & 121 & 10140203 & 122ARKR & 265 & 2,758 \\
\hline 431352100231601 & $38 \mathrm{~N} 26 \mathrm{~W} 27 \mathrm{CCDC}$ & 121 & 10150006 & 122ARKR & 100 & 2,408 \\
\hline
\end{tabular}


Table 57. Selected descriptive data (from the U.S. Geological Survey database) for ground-water sites in the High Plains aquifer in South Dakota-Continued

[County code: 7 = Bennett County, $53=$ Gregory County, $71=$ Jackson County, $95=$ Mellette County, $113=$ Shannon County, and $121=$ Todd County; Datum, National Geodetic Vertical Datum; 1210GLL, Ogallala Formation; 122ARKR, Arikaree Formation; --, no data]

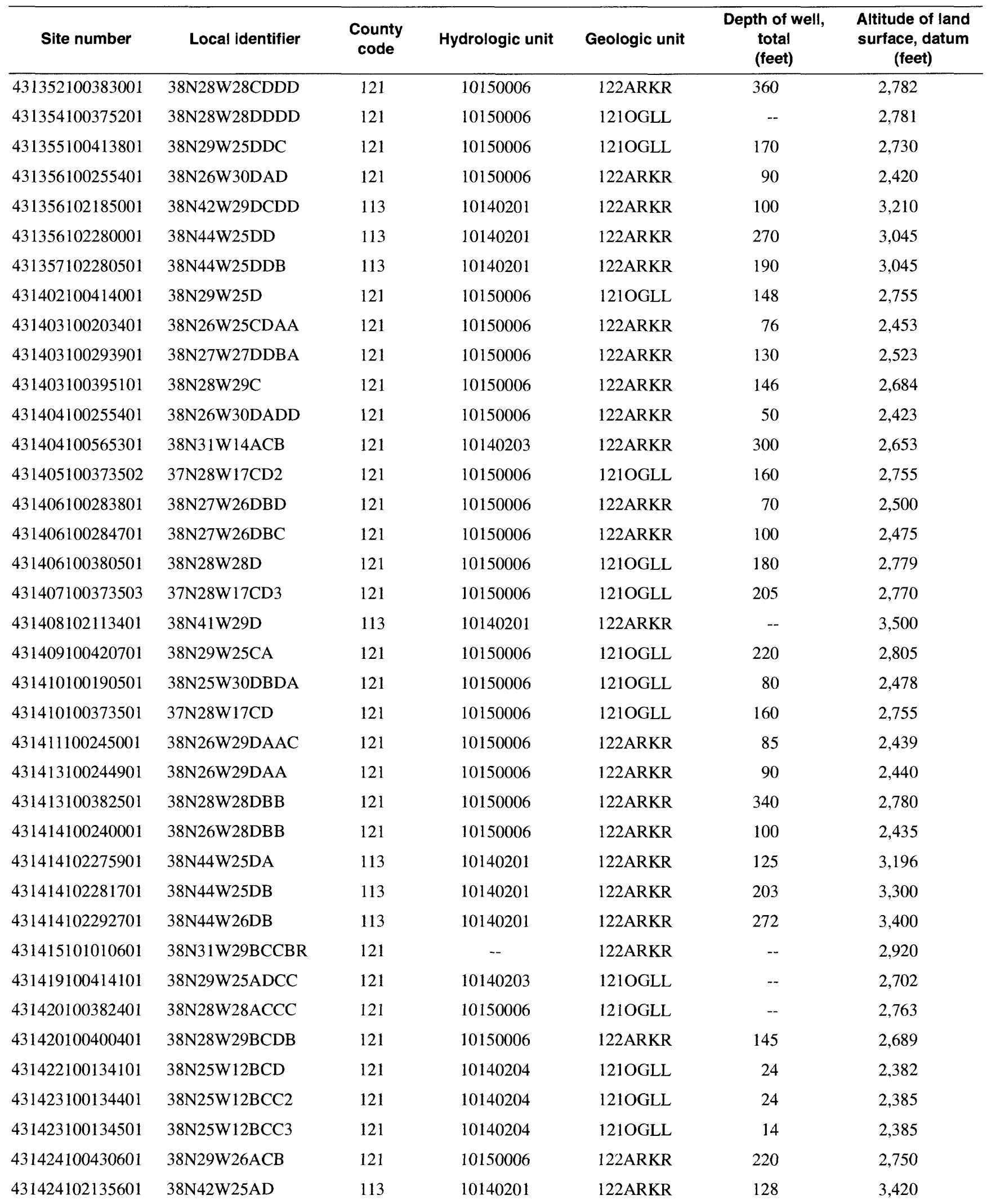


Table 57. Selected descriptive data (from the U.S. Geological Survey database) for ground-water sites in the High Plains aquifer in South Dakota-Continued

[County code: 7 = Bennett County, $53=$ Gregory County, $71=$ Jackson County, $95=$ Mellette County, $113=$ Shannon County, and $121=$ Todd County; Datum, National Geodetic Vertical Datum; 121OGLL, Ogallala Formation; 122ARKR, Arikaree Formation; --, no data]

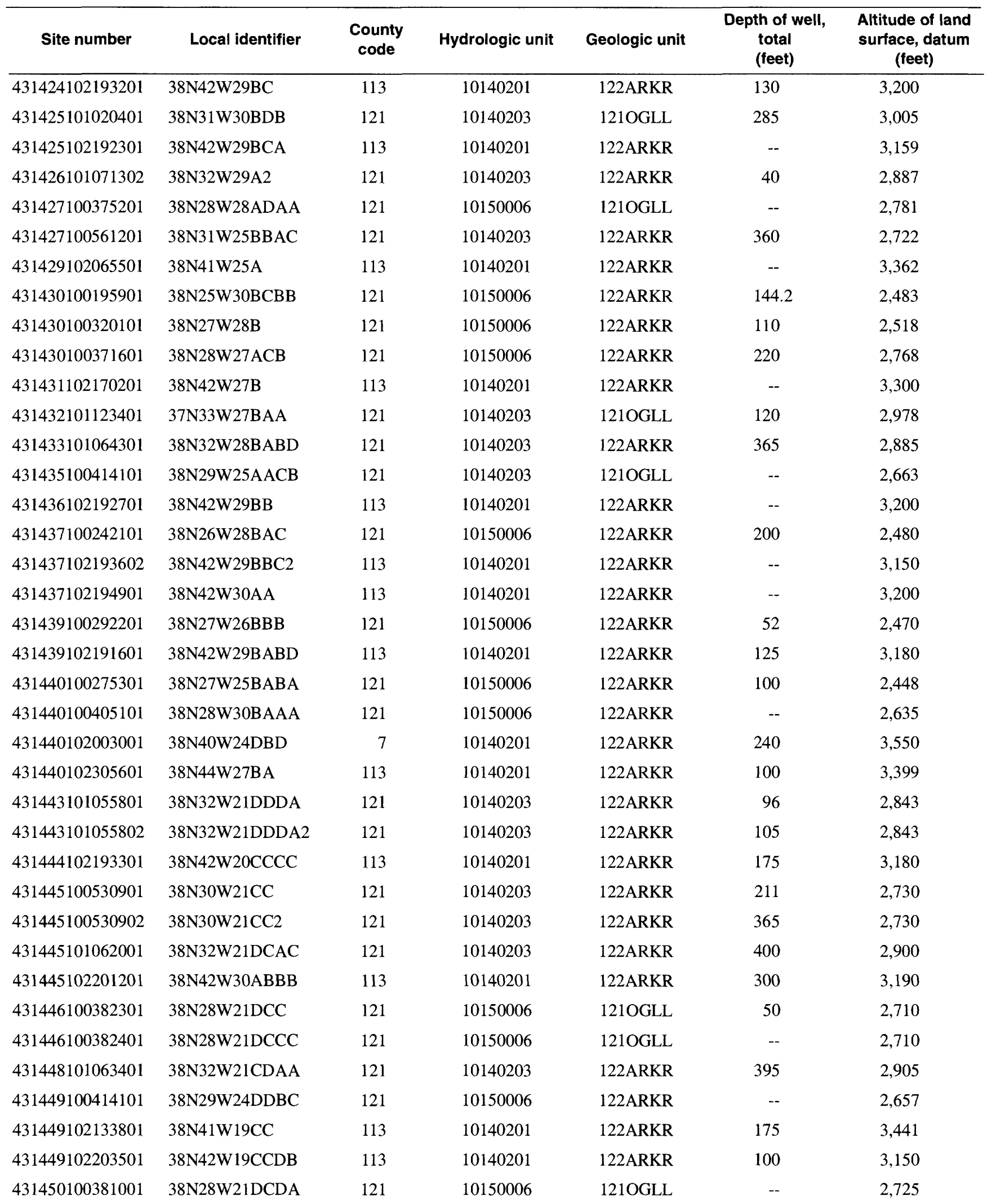


Table 57. Selected descriptive data (from the U.S. Geological Survey database) for ground-water sites in the High Plains aquifer in South Dakota-Continued

[County code: 7 = Bennett County, 53 = Gregory County, $71=$ Jackson County, 95 = Mellette County, $113=$ Shannon County, and $121=$ Todd County; Datum, National Geodetic Vertical Datum; 1210GLL, Ogallala Formation; 122ARKR, Arikaree Formation; --, no data]

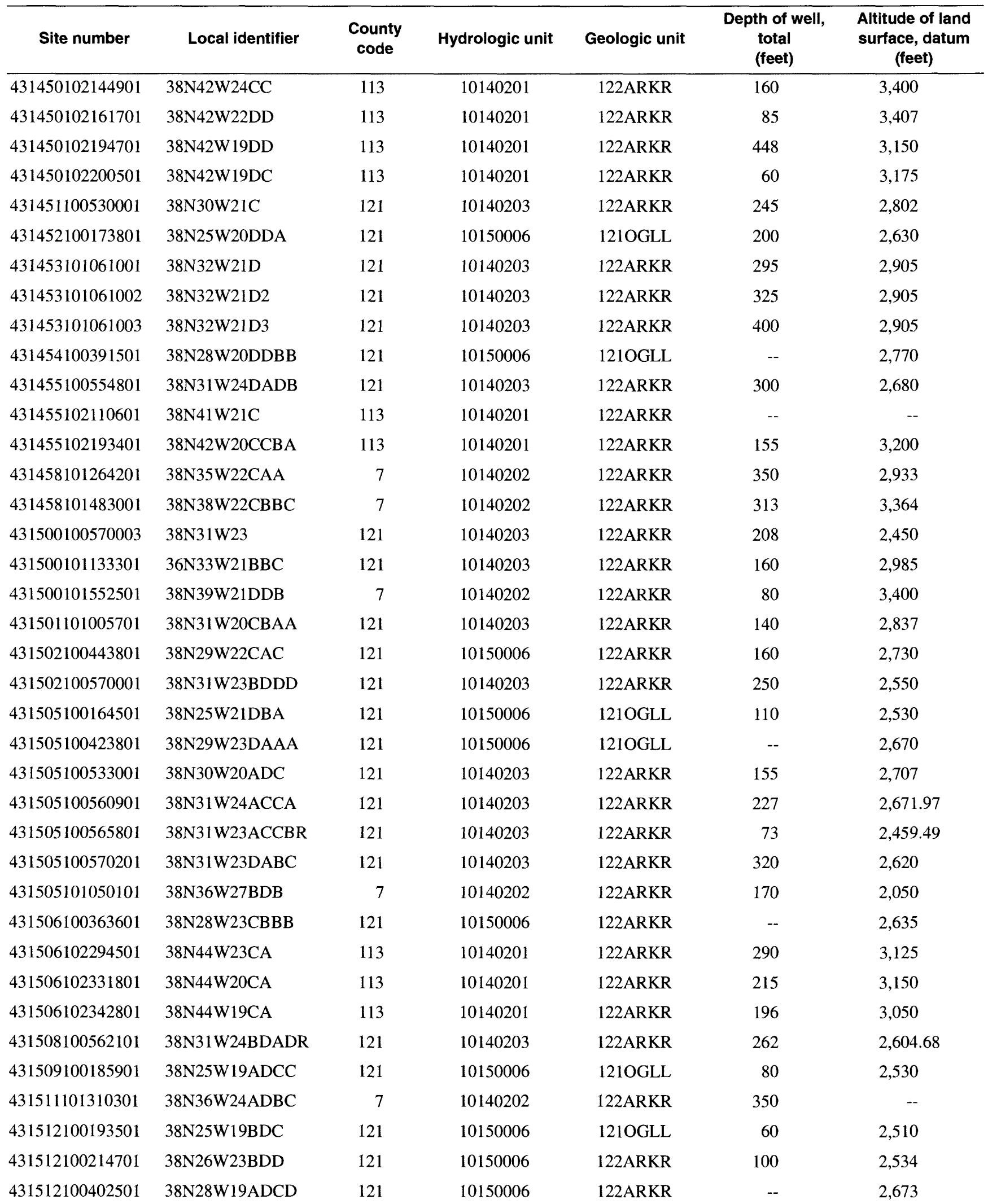


Table 57. Selected descriptive data (from the U.S. Geological Survey database) for ground-water sites in the High Plains aquifer in South Dakota-Continued

[County code: 7 = Bennett County, $53=$ Gregory County, 71 = Jackson County, $95=$ Mellette County, $113=$ Shannon County, and $121=$ Todd County; Datum, National Geodetic Vertical Datum; 12 lOGLL, Ogallala Formation; 122ARKR, Arikaree Formation; --, no data]

\begin{tabular}{|c|c|c|c|c|c|c|}
\hline Site number & Local identifier & $\begin{array}{l}\text { County } \\
\text { code }\end{array}$ & Hydrologic unit & Geologic unit & $\begin{array}{c}\text { Depth of well, } \\
\text { total } \\
\text { (feet) }\end{array}$ & $\begin{array}{l}\text { Altitude of land } \\
\text { surface, datum } \\
\text { (feet) }\end{array}$ \\
\hline 431514101061101 & $38 \mathrm{~N} 32 \mathrm{~W} 21 \mathrm{ADBB}$ & 121 & 10140203 & 122ARKR & 60 & 2,845 \\
\hline 431515100501701 & $38 \mathrm{~N} 30 \mathrm{~W} 23 \mathrm{ACBC}$ & 121 & 10140203 & 122ARKR & 258 & 2,720 \\
\hline 431515100553901 & 38N30W19BCBAR & 121 & 10140203 & 122ARKR & 274 & $2,659.35$ \\
\hline 431515100562701 & 38N31W24BDAC & 121 & 10140203 & 122ARKR & -- & 2,600 \\
\hline 431516100381001 & $38 \mathrm{~N} 28 \mathrm{~W} 21 \mathrm{ACAD}$ & 121 & 10150006 & 1210GLL & -- & 2,776 \\
\hline 431516100542901 & 38N30W19 & 121 & 10140203 & 122ARKR & -- & 2,508 \\
\hline 431517100562301 & $38 \mathrm{~N} 31 \mathrm{~W} 24 \mathrm{BDAC} 2 \mathrm{R}$ & 121 & 10140203 & $122 \mathrm{ARKR}$ & 242 & $2,573.99$ \\
\hline 431517101284201 & $38 \mathrm{~N} 35 \mathrm{~W} 20 \mathrm{AAC}$ & 7 & 10140202 & 122ARKR & 320 & 2,952 \\
\hline 431518100245401 & 38N26W20ADB & 121 & 10150006 & 122ARKR & 90 & 2,520 \\
\hline 431518102280501 & 38N44W24ADCC & 113 & 10140201 & 122ARKR & 95 & 3,280 \\
\hline 431520100593601 & 38N31W16DBAA & 121 & 10140203 & 122ARKR & 128 & 2,710 \\
\hline 431520101281201 & $38 \mathrm{~N} 35 \mathrm{~W} 21 \mathrm{BBDC}$ & 7 & 10140202 & 122ARKR & 330 & 2,942 \\
\hline 431521100500401 & 38N30W23ABDD & 121 & 10140203 & 121OGLL & 65 & 2,727 \\
\hline 431522100284302 & $38 \mathrm{~N} 27 \mathrm{~W} 23 \mathrm{ABCD} 2$ & 121 & 10150006 & 122ARKR & 90 & 2,450 \\
\hline 431522100284501 & 38N27W23ABCD & 121 & 10150006 & 122ARKR & 100 & 2,448 \\
\hline 431525100380601 & $38 \mathrm{~N} 28 \mathrm{~W} 21 \mathrm{AAC}$ & 121 & 10150006 & 121OGLL & -- & 2,765 \\
\hline 431525100502902 & 38N30W23BACA2 & 121 & 10140203 & 122ARKR & 230 & 2,717 \\
\hline 431525100563801 & 38N31W23ABAD2 & 121 & 10140203 & 122ARKR & -- & 2,446 \\
\hline 431526100314601 & $38 \mathrm{~N} 27 \mathrm{~W} 21 \mathrm{BBC} 2$ & 121 & 10150006 & 122ARKR & 100 & 2,500 \\
\hline 431526100563202 & 38N31W23AAAB2R & 121 & 10140203 & 122ARKR & 60 & $2,442.71$ \\
\hline 431527100502901 & $38 \mathrm{~N} 30 \mathrm{~W} 23 \mathrm{BABD}$ & 121 & 10140203 & 122ARKR & 264 & 2,716 \\
\hline 431527101265701 & $38 \mathrm{~N} 35 \mathrm{~W} 22 \mathrm{BBA}$ & 7 & 10140202 & 122ARKR & 280 & 2,936 \\
\hline 431528100314701 & $38 \mathrm{~N} 27 \mathrm{~W} 21 \mathrm{BBCB}$ & 121 & 10150006 & 122ARKR & 100 & 2,500 \\
\hline 431530101264101 & 38N35W23BAAA & 7 & 10140202 & 1210GLL & 261 & 2,933 \\
\hline 431531101282701 & $38 \mathrm{~N} 35 \mathrm{~W} 16 \mathrm{CCCC}$ & 7 & 10140202 & 1210GLL & 264 & 2,940 \\
\hline 431532100200101 & $38 \mathrm{~N} 26 \mathrm{~W} 24 \mathrm{AAAB}$ & 121 & 10150006 & 1210GLL & 85 & 2,503 \\
\hline 431532100332801 & 38N27W19ABA & 121 & 10150006 & 122ARKR & 80 & 2,504 \\
\hline 431533101080501 & $38 \mathrm{~N} 32 \mathrm{~W} 17 \mathrm{CCD}$ & 121 & 10140203 & 122ARKR & 154 & 3,059 \\
\hline 431534100503801 & 38N30W14CCDD & 121 & 10140203 & 122ARKR & 270 & 2,715 \\
\hline 431536100472201 & $38 \mathrm{~N} 29 \mathrm{~W} 17 \mathrm{BCDA}$ & 121 & 10140203 & 122ARKR & 100 & 2,687 \\
\hline 431536100543101 & 38N30W18DDAD & 121 & 10140203 & 122ARKR & 60 & $2,419.62$ \\
\hline 431536101274201 & 38 N35W16DCC & 7 & 10140202 & $122 \mathrm{ARKR}$ & 360 & 2,946 \\
\hline 431537100504701 & $38 \mathrm{~N} 30 \mathrm{~W} 14 \mathrm{CCCA}$ & 121 & 10140203 & 122ARKR & 280 & 2,723 \\
\hline 431538100334201 & $38 \mathrm{~N} 27 \mathrm{~W} 18 \mathrm{DCC}$ & 121 & 10150006 & 122ARKR & 120 & 2,498 \\
\hline 431539100352401 & $38 \mathrm{~N} 28 \mathrm{~W} 13 \mathrm{CCCC}$ & 121 & 10140204 & 122ARKR & -- & 2,577 \\
\hline 431540100154501 & 38N25W15DCCB & 121 & 10140204 & 1210GLL & 110 & 2,467 \\
\hline 431540100545801 & 38N30W18DCBD & 121 & 10140203 & 122ARKR & 27 & 2,425 \\
\hline 431540101062301 & $38 \mathrm{~N} 32 \mathrm{~W} 16 \mathrm{DCBA} 2$ & 121 & 10140203 & 122ARKR & 108 & 2,825 \\
\hline
\end{tabular}


Table 57. Selected descriptive data (from the U.S. Geological Survey database) for ground-water sites in the High Plains aquifer in South Dakota-Continued

[County code: 7 = Bennett County, $53=$ Gregory County, 71 = Jackson County, $95=$ Mellette County, $113=$ Shannon County, and $121=$ Todd County; Datum, National Geodetic Vertical Datum; 1210 GLL, Ogallala Formation; 122ARKR, Arikaree Formation; --, no data]

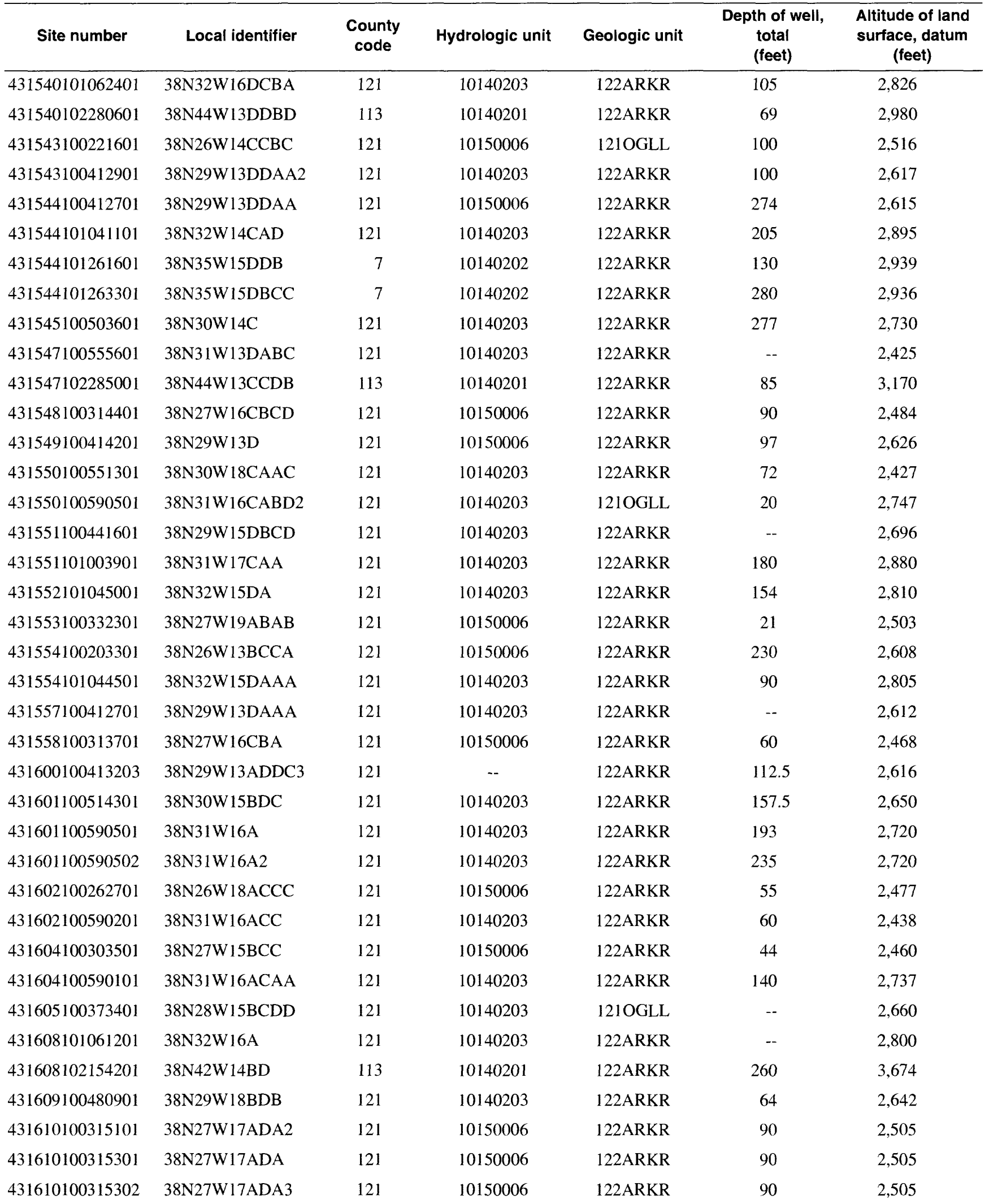


Table 57. Selected descriptive data (from the U.S. Geological Survey database) for ground-water sites in the High Plains aquifer in South Dakota-Continued

[County code: 7 = Bennett County, $53=$ Gregory County, $71=$ Jackson County, $95=$ Mellette County, $113=$ Shannon County, and $121=$ Todd County; Datum, National Geodetic Vertical Datum; 1210GLL, Ogallala Formation; 122ARKR, Arikaree Formation; --, no data]

\begin{tabular}{|c|c|c|c|c|c|c|}
\hline Site number & Local identifier & $\begin{array}{l}\text { County } \\
\text { code }\end{array}$ & Hydrologic unit & Geologic unit & $\begin{array}{l}\text { Depth of well, } \\
\text { total } \\
\text { (feet) }\end{array}$ & $\begin{array}{l}\text { Altitude of land } \\
\text { surface, datum } \\
\text { (feet) }\end{array}$ \\
\hline 431610100484701 & $38 \mathrm{~N} 30 \mathrm{~W} 13 \mathrm{ADBB}$ & 121 & 10140203 & 122ARKR & 119 & 2,645 \\
\hline 431610101005801 & $38 \mathrm{~N} 31 \mathrm{~W} 17 \mathrm{BBD}$ & 121 & 10140203 & 122ARKR & 300 & 2,808 \\
\hline 431612100444001 & $38 \mathrm{~N} 29 \mathrm{~W} 15 \mathrm{BAC}$ & 121 & 10150006 & 122ARKR & 180 & 2,760 \\
\hline 431612100551201 & 38N30W18BADB & 121 & 10140203 & 122ARKR & 245 & 2,550 \\
\hline 431613100375001 & $38 \mathrm{~N} 28 \mathrm{~W} 15 \mathrm{BCBB}$ & 121 & 10150006 & 121OGLL & -- & 2,632 \\
\hline 431615100390701 & 38N28W17AADD & 121 & 10150006 & 122ARKR & -- & 2,664 \\
\hline 431615102133001 & $38 \mathrm{~N} 41 \mathrm{~W} 18 \mathrm{~B}$ & 113 & 10140201 & 122ARKR & -- & 3,425 \\
\hline 431618100563901 & $38 \mathrm{~N} 31 \mathrm{~W} 14 \mathrm{AAB}$ & 121 & 10140203 & 122ARKR & 310 & 2,620 \\
\hline 431618101044001 & $38 \mathrm{~N} 32 \mathrm{~W} 14 \mathrm{BBBD}$ & 121 & 10140203 & 122ARKR & 383 & 2,820 \\
\hline 431620100560601 & 38N30W18BAAAR & 121 & -- & 122ARKR & -- & 2,562 \\
\hline 431620100581301 & 38N31W10DCCCR & 121 & - & 122ARKR & -- & 2,772 \\
\hline 431620101081901 & $38 \mathrm{~N} 32 \mathrm{~W} 18 \mathrm{AAAA}$ & 121 & 10140203 & 122ARKR & 264 & 2,920 \\
\hline 431620101081902 & 38N32W18AAAA2 & 121 & 10140203 & 122ARKR & 407 & 2,920 \\
\hline 431622101044801 & 38N32W10DDDD & 121 & 10140203 & 122ARKR & 90 & 2,792 \\
\hline 431623100232501 & $38 \mathrm{~N} 26 \mathrm{~W} 15 \mathrm{BBB}$ & 121 & 10150006 & 122ARKR & 100 & 2,507 \\
\hline 431623100355801 & $38 \mathrm{~N} 28 \mathrm{~W} 14 \mathrm{ABB}$ & 121 & 10150006 & 122ARKR & 165 & 2,578 \\
\hline 431623101010501 & $38 \mathrm{~N} 31 \mathrm{~W} 8 \mathrm{CCC}$ & 121 & 10140203 & 122ARKR & 92.5 & 2,787 \\
\hline 431623101102902 & $38 \mathrm{~N} 33 \mathrm{~W} 12 \mathrm{CCDC} 2$ & 121 & 10140203 & 122ARKR & 157 & 2,943 \\
\hline 431625100361301 & 38N28W14BAAB & 121 & 10150006 & 122ARKR & 115 & 2,589 \\
\hline 431625100411801 & $38 \mathrm{~N} 28 \mathrm{~W} 18 \mathrm{BBBA}$ & 121 & 10150006 & $122 \mathrm{ARKR}$ & -- & 2,596 \\
\hline 431625101032501 & $38 \mathrm{~N} 32 \mathrm{~W} 12 \mathrm{C}$ & 121 & 10140203 & 122ARKR & 155 & 2,755 \\
\hline 431625101103001 & $38 \mathrm{~N} 33 \mathrm{~W} 12 \mathrm{CCDC}$ & 121 & 10140202 & 122ARKR & 150 & 2,943 \\
\hline 431627101110501 & 38N33W11DC & 121 & 10140203 & 122ARKR & 145 & 3,045 \\
\hline 431628100301502 & $38 \mathrm{~N} 27 \mathrm{~W} 10 \mathrm{CDCD}$ & 121 & 10150006 & 122ARKR & 98 & 2,508 \\
\hline 431628100471801 & $38 \mathrm{~N} 28 \mathrm{~W} 8 \mathrm{CCC}$ & 121 & 10140203 & 122ARKR & 130 & 2,695 \\
\hline 431630100301703 & $38 \mathrm{~N} 27 \mathrm{~W} 10 \mathrm{CDC} 2$ & 121 & 10150006 & 122ARKR & 110 & 2,508 \\
\hline 431630100434501 & $38 \mathrm{~N} 29 \mathrm{~W} 11 \mathrm{CCCC}$ & 121 & 10150006 & $122 \mathrm{ARKR}$ & - & 2,678 \\
\hline 431630100570201 & $38 \mathrm{~N} 31 \mathrm{~W} 11 \mathrm{CDAA}$ & 121 & 10140203 & $122 \mathrm{ARKR}$ & 240 & 2,652 \\
\hline 431630101084701 & $38 N 32 W 7 D C B D$ & 121 & 10140203 & 122ARKR & 205 & 2,885 \\
\hline 431631100301501 & $38 \mathrm{~N} 27 \mathrm{~W} 10 \mathrm{CDC}$ & 121 & 10150006 & 122ARKR & 110 & 2,509 \\
\hline 431633100560601 & $38 \mathrm{~N} 31 \mathrm{~W} 12 \mathrm{C}$ & 121 & 10140203 & $122 \mathrm{ARKR}$ & 47 & 2,640 \\
\hline 431633101001801 & $38 \mathrm{~N} 31 \mathrm{~W} 8 \mathrm{DCAA}$ & 121 & 10140203 & $122 \mathrm{ARKR}$ & 238 & 2,748 \\
\hline 431633102090901 & 38N41W10DD & 113 & 10140201 & 122ARKR & 180 & 3,284 \\
\hline 431634101113201 & $38 \mathrm{~N} 33 \mathrm{~W} 11 \mathrm{C}$ & 121 & 10140203 & 122ARKR & 152 & 3,090 \\
\hline 431636100310101 & $38 \mathrm{~N} 27 \mathrm{~W}$ 9DDA & 121 & 10150006 & 122ARKR & 90 & 2,500 \\
\hline 431636100560201 & $38 \mathrm{~N} 31 \mathrm{~W} 12 \mathrm{CAC}$ & 121 & 10140203 & 122ARKR & 180 & 2,620 \\
\hline 431637100264101 & $38 \mathrm{~N} 26 \mathrm{~W} 7 \mathrm{CDB}$ & 121 & 10150006 & 122ARKR & 90 & 2,513 \\
\hline 431637101084801 & $38 \mathrm{~N} 32 \mathrm{~W} 7 \mathrm{DCAB}$ & 121 & 10140203 & 122ARKR & 162 & 2,890 \\
\hline
\end{tabular}


Table 57. Selected descriptive data (from the U.S. Geological Survey database) for ground-water sites in the High Plains aquifer in South Dakota-Continued

[County code: 7 = Bennett County, 53 = Gregory County, 71 = Jackson County, $95=$ Mellette County, $113=$ Shannon County, and $121=$ Todd County; Datum, National Geodetic Vertical Datum; 1210GLL, Ogallala Formation; 122ARKR, Arikaree Formation; --, no data]

\begin{tabular}{|c|c|c|c|c|c|c|}
\hline Site number & Local identifier & $\begin{array}{l}\text { County } \\
\text { code }\end{array}$ & Hydrologic unit & Geologic unit & $\begin{array}{c}\text { Depth of well, } \\
\text { total } \\
\text { (feet) }\end{array}$ & $\begin{array}{l}\text { Altitude of land } \\
\text { surface, datum } \\
\text { (feet) }\end{array}$ \\
\hline 431637101084802 & 38N32W 7DCAB2 & 121 & 10140203 & 122ARKR & 152 & 2,890 \\
\hline 431638101054201 & $38 \mathrm{~N} 32 \mathrm{~W} 10 \mathrm{CBDB} 2$ & 121 & 10140203 & 122ARKR & 135 & 2,766 \\
\hline 431638101084801 & 38N32W 7DBCC & 121 & 10140203 & 122ARKR & 162 & 2,880 \\
\hline 431639100481501 & $38 \mathrm{~N} 29 \mathrm{~W} 7 \mathrm{C}$ & 121 & 10140203 & 122ARKR & 115 & 2,660 \\
\hline 431639101043102 & 38N32W11CBD2 & 121 & 10140203 & 122ARKR & 225 & 2,785 \\
\hline 431639101054201 & $38 \mathrm{~N} 32 \mathrm{~W} 10 \mathrm{CBDB} 2$ & 121 & 10140203 & 122ARKR & 165 & 2,768 \\
\hline 431644100285501 & 38N27W11CAD & 121 & 10150006 & 122ARKR & 80 & 2,508 \\
\hline 431644102340201 & $38 \mathrm{~N} 44 \mathrm{~W} 7 \mathrm{D}$ & 113 & 10140201 & 122ARKR & -- & 3,000 \\
\hline 431645100184201 & $38 \mathrm{~N} 25 \mathrm{~W} 8 \mathrm{CBC}$ & 121 & 10140204 & 1210GLL & 90 & 2,470 \\
\hline 431646100465501 & $38 \mathrm{~N} 29 \mathrm{~W} 8 \mathrm{CA}$ & 121 & 10140203 & 122ARKR & 130 & 2,718 \\
\hline 431647102183901 & $38 \mathrm{~N} 42 \mathrm{~W} 8 \mathrm{DA}$ & 113 & 10140201 & 122ARKR & 320 & 3,349 \\
\hline 431648101285301 & $38 \mathrm{~N} 35 \mathrm{~W} 8 \mathrm{ACCC}$ & 7 & 10140202 & 122ARKR & 280 & 2,952 \\
\hline 431649100174801 & $38 \mathrm{~N} 25 \mathrm{~W} 8 \mathrm{DAB}$ & 121 & 10140204 & 121OGLL & 60 & 2,415 \\
\hline 431649100272701 & $38 \mathrm{~N} 27 \mathrm{~W} 12 \mathrm{DBA}$ & 121 & 10150006 & 122ARKR & 50 & 2,480 \\
\hline 431649101085201 & 38N32W 7ADD2 & 121 & 10140203 & 122ARKR & -- & 2,867 \\
\hline 431649101085301 & $38 \mathrm{~N} 32 \mathrm{~W} 7 \mathrm{~A}$ & 121 & 10140203 & 122ARKR & 65 & 2,867 \\
\hline 431650100283701 & $38 \mathrm{~N} 27 \mathrm{~W} 11 \mathrm{DBA}$ & 121 & 10150006 & 122ARKR & 90 & 2,516 \\
\hline 431650100330701 & 38N27W 7DAAB & 121 & 10150006 & 122ARKR & -- & 2,477 \\
\hline 431651100264401 & 38N26W 7CAB & 121 & 10150006 & 122ARKR & 160 & 2,523 \\
\hline 431652101050102 & $38 \mathrm{~N} 32 \mathrm{~W} 10 \mathrm{~A} 2$ & 121 & 10140203 & 122ARKR & 140 & 2,820 \\
\hline 431652101050103 & 38N32W10A3 & 121 & 10140203 & 122ARKR & 90 & 2,870 \\
\hline 431653100225201 & 38N26W10ACC & 121 & 10150006 & 121OGLL & 100 & 2,539 \\
\hline 431653102221501 & 38N43W11ADCC & 113 & 10140201 & 122ARKR & 390 & 3,200 \\
\hline 431654100574301 & 38N31W10ADAC & 121 & 10140203 & 122ARKR & 220 & 2,698 \\
\hline 431654100574302 & 38N31W10ADAC2 & 121 & 10140203 & 122ARKR & 210 & 2,698 \\
\hline 431654101552701 & $38 \mathrm{~N} 39 \mathrm{~W} 10 \mathrm{BC}$ & 7 & 10140202 & 122ARKR & 120 & 3,379 \\
\hline 431655100272901 & $38 \mathrm{~N} 27 \mathrm{~W} 12 \mathrm{ACDC}$ & 121 & 10150006 & 122ARKR & 90 & 2,510 \\
\hline 431655100574301 & 38N31W10ADBD & 121 & 10140203 & 122ARKR & 370 & 2,704 \\
\hline 431655100583401 & 38N31W10BBD & 121 & 10140203 & 122ARKR & 150 & 2,780 \\
\hline 431700101113201 & $38 \mathrm{~N} 33 \mathrm{~W} 11 \mathrm{~B}$ & 121 & 10140203 & 122ARKR & 115 & 3,040 \\
\hline 431700101113202 & 38N33W11B2 & 121 & 10140203 & 122ARKR & 250 & 3,040 \\
\hline 431701101061201 & $38 \mathrm{~N} 32 \mathrm{~W} 9 \mathrm{~A}$ & 121 & 10140203 & 122ARKR & 96 & 2,770 \\
\hline 431701101094401 & $38 \mathrm{~N} 33 \mathrm{~W} 12 \mathrm{~A}$ & 121 & 10140203 & 122ARKR & 252 & 2,980 \\
\hline 431702101010001 & $38 \mathrm{~N} 36 \mathrm{~W} 1 \mathrm{DDC}$ & 7 & 10140202 & 122ARKR & 80 & 2,086 \\
\hline 431703100314601 & $38 \mathrm{~N} 27 \mathrm{~W} 9 \mathrm{BCB}$ & 121 & 10150006 & 122ARKR & 125 & 2,510 \\
\hline 431703102342801 & $38 \mathrm{~N} 44 \mathrm{~W} 7 \mathrm{BD}$ & 113 & 10140201 & 122ARKR & 200 & 3,035 \\
\hline 431704100314502 & $38 \mathrm{~N} 27 \mathrm{~W} 9 \mathrm{BCBA}$ & 121 & 10150006 & 122ARKR & 100 & 2,510 \\
\hline 431704101042301 & $38 \mathrm{~N} 32 \mathrm{~W} 11 \mathrm{BCC}$ & 121 & 10140203 & 122ARKR & 284 & 2,738 \\
\hline
\end{tabular}


Table 57. Selected descriptive data (from the U.S. Geological Survey database) for ground-water sites in the High Plains aquifer in South Dakota-Continued

[County code: 7 = Bennett County, $53=$ Gregory County, 71 = Jackson County, $95=$ Mellette County, $113=$ Shannon County, and $121=$ Todd County; Datum, National Geodetic Vertical Datum; 1210GLL, Ogallala Formation; 122ARKR, Arikaree Formation; --, no data]

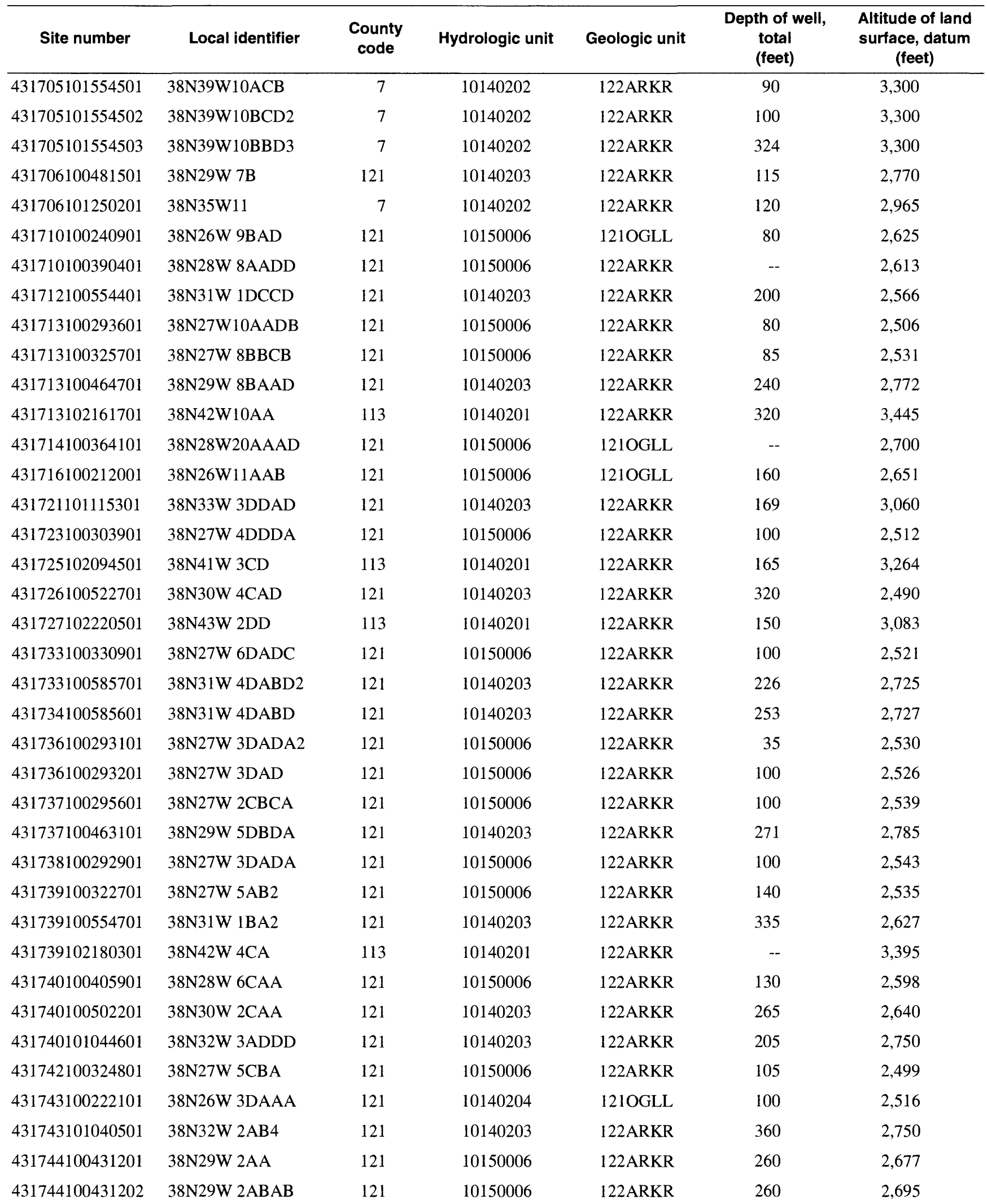


Table 57. Selected descriptive data (from the U.S. Geological Survey database) for ground-water sites in the High Plains aquifer in South Dakota-Continued

[County code: 7 = Bennett County, 53 = Gregory County, 71 = Jackson County, 95 = Mellette County, $113=$ Shannon County, and $121=$ Todd County; Datum, National Geodetic Vertical Datum; 121OGLL, Ogallala Formation; 122ARKR, Arikaree Formation; --, no data]

\begin{tabular}{|c|c|c|c|c|c|c|}
\hline Site number & Local identifier & $\begin{array}{l}\text { County } \\
\text { code }\end{array}$ & Hydrologic unit & Geologic unit & $\begin{array}{c}\text { Depth of well, } \\
\text { total } \\
\text { (feet) }\end{array}$ & $\begin{array}{c}\text { Altitude of land } \\
\text { surface, datum } \\
\text { (feet) }\end{array}$ \\
\hline 431744100464501 & $38 \mathrm{~N} 29 \mathrm{~W}$ 5AABB & 121 & 10140203 & 122ARKR & 260 & 2,740 \\
\hline 431744100561401 & $38 \mathrm{~N} 31 \mathrm{~W} 1 \mathrm{BACB} 2$ & 121 & 10140203 & 122ARKR & 160 & 2,600 \\
\hline 431744100583601 & $38 \mathrm{~N} 31 \mathrm{~W} 3 \mathrm{ABAB}$ & 121 & 10140203 & 122ARKR & 200 & 2,692 \\
\hline 431745100371401 & $38 \mathrm{~N} 28 \mathrm{~W} 3 \mathrm{BDA}$ & 121 & 10150006 & 122ARKR & 165 & 2,558 \\
\hline 431746100322801 & $38 \mathrm{~N} 27 \mathrm{~W} 5 \mathrm{AB} 2$ & 121 & 10150006 & 122ARKR & 140 & 2,535 \\
\hline 431746100562201 & $38 \mathrm{~N} 31 \mathrm{~W} 1 \mathrm{BCBC}$ & 121 & 10140203 & 122ARKR & 50 & 2,627 \\
\hline 431747100524401 & 38N30W 4BDD & 121 & 10140203 & 122ARKR & 340 & 2,420 \\
\hline 431748100412501 & $38 \mathrm{~N} 29 \mathrm{~W} 1 \mathrm{BCD}$ & 121 & 10140203 & 122ARKR & 165 & 2,680 \\
\hline 431751100560301 & $38 \mathrm{~N} 31 \mathrm{~W} 1 \mathrm{BACB}$ & 121 & 10140203 & 122ARKR & 152 & 2,600 \\
\hline 431755101033601 & $38 \mathrm{~N} 32 \mathrm{~W} 2 \mathrm{~A}$ & 121 & 10140203 & 122ARKR & 65 & 2,720 \\
\hline 431755101035801 & $38 \mathrm{~N} 32 \mathrm{~W} 2 \mathrm{AB} 3$ & 121 & 10140203 & 122ARKR & 364 & 2,747 \\
\hline 431757100323302 & $38 \mathrm{~N} 27 \mathrm{~W} 5 \mathrm{BDAB}$ & 121 & 10150006 & 122ARKR & 100 & 2,522 \\
\hline 431758100575301 & $38 \mathrm{~N} 31 \mathrm{~W} 3 \mathrm{~A}$ & 121 & 10140203 & 122ARKR & 190 & 2,690 \\
\hline 431759100324703 & 38N27W 5BBDD2 & 121 & 10150006 & 122ARKR & 100 & 2,523 \\
\hline 431800100324601 & 38N27W 5BBDD & 121 & 10150006 & 122ARKR & 100 & 2,523 \\
\hline 431800100392501 & $38 \mathrm{~N} 28 \mathrm{~W} 5 \mathrm{AAC}$ & 121 & 10150006 & 122ARKR & 136 & 2,567 \\
\hline 431800100450201 & $38 \mathrm{~N} 29 \mathrm{~W}$ 4DDAA & 121 & 10140203 & 122ARKR & 182 & 2,750 \\
\hline 431802101035601 & $38 \mathrm{~N} 32 \mathrm{~W} 2 \mathrm{AB}$ & 121 & 10140203 & 122ARKR & 354 & 2,650 \\
\hline 431802101035602 & $38 \mathrm{~N} 32 \mathrm{~W} 2 \mathrm{AB} 2$ & 121 & 10140203 & 122ARKR & 349 & 2,747 \\
\hline 431802102340201 & $38 \mathrm{~N} 44 \mathrm{~W} 6 \mathrm{~A}$ & 113 & 10140201 & 122ARKR & -- & 2,950 \\
\hline 431803100431101 & $38 \mathrm{~N} 29 \mathrm{~W} 2 \mathrm{ABCB}$ & 121 & 10150006 & 122ARKR & 227 & 2,708 \\
\hline 431803100460301 & $38 \mathrm{~N} 29 \mathrm{~W} 4 \mathrm{BB}$ & 121 & 10140203 & 122ARKR & 178 & 2,767 \\
\hline 431804100393501 & $38 \mathrm{~N} 28 \mathrm{~W} 5 \mathrm{AB}$ & 121 & 10150006 & 122ARKR & 160 & 2,565 \\
\hline 431804100393601 & $38 \mathrm{~N} 28 \mathrm{~W} 5 \mathrm{ABD}$ & 121 & 10150006 & 122ARKR & 135 & 2.575 \\
\hline 431804100431101 & $38 \mathrm{~N} 29 \mathrm{~W} 2 \mathrm{ABBC}$ & 121 & 10150006 & 122ARKR & 217 & 2,706 \\
\hline 431805100280501 & $38 \mathrm{~N} 27 \mathrm{~W} 1 \mathrm{BABB}$ & 121 & 10150006 & 122ARKR & 40 & 2.539 \\
\hline 431805100402001 & 39N28W32DDD & 121 & 10150006 & 122ARKR & 110 & 2,657 \\
\hline 431805100432501 & $38 \mathrm{~N} 29 \mathrm{~W} 2 \mathrm{BAB}$ & 121 & 10150006 & 122ARKR & 230 & 2,710 \\
\hline 431806100425801 & $38 \mathrm{~N} 29 \mathrm{~W} 2 \mathrm{ABAD}$ & 121 & 10150006 & 122ARKR & 230 & 2,687 \\
\hline 431808100312801 & $38 \mathrm{~N} 27 \mathrm{~W} 4 \mathrm{BAB}$ & 121 & 10150006 & 122ARKR & 100 & 2,550 \\
\hline 431808100430901 & $38 \mathrm{~N} 29 \mathrm{~W} 2 \mathrm{ABB}$ & 121 & 10150006 & 122ARKR & 260 & 2,707 \\
\hline 431809100312402 & $38 \mathrm{~N} 27 \mathrm{~W} 4 \mathrm{BABA}$ & 121 & 10150006 & 122ARKR & 144 & 2,565 \\
\hline 431809100483401 & 38N30W 1AAAA & 121 & 10140203 & 122ARKR & 180 & 2,763 \\
\hline 431809100483402 & $38 \mathrm{~N} 30 \mathrm{~W} 1 \mathrm{AAAA} 2$ & 121 & 10140203 & 122ARKR & 163 & 2,763 \\
\hline 431809100483403 & $38 \mathrm{~N} 30 \mathrm{~W} 1 \mathrm{AAAA} 3$ & 121 & 10140203 & 122ARKR & 380 & 2,760 \\
\hline 431810100295601 & $38 \mathrm{~N} 27 \mathrm{~W} 2 \mathrm{BABB}$ & 121 & 10150006 & 122ARKR & 100 & 2,539 \\
\hline 431810100394501 & $39 \mathrm{~N} 28 \mathrm{~W} 32 \mathrm{DCA}$ & 121 & 10150006 & 122ARKR & 150 & 2,582 \\
\hline 431810100402001 & $39 \mathrm{~N} 28 \mathrm{~W} 32 \mathrm{DDB}$ & 121 & 10150006 & 122ARKR & 135 & 2,582 \\
\hline
\end{tabular}


Table 57. Selected descriptive data (from the U.S. Geological Survey database) for ground-water sites in the High Plains aquifer in South Dakota-Continued

[County code: 7 = Bennett County, $53=$ Gregory County, $71=$ Jackson County, $95=$ Mellette County, $113=$ Shannon County, and $121=$ Todd County; Datum, National Geodetic Vertical Datum; 1210GLL, Ogallala Formation; 122ARKR, Arikaree Formation; --, no data]

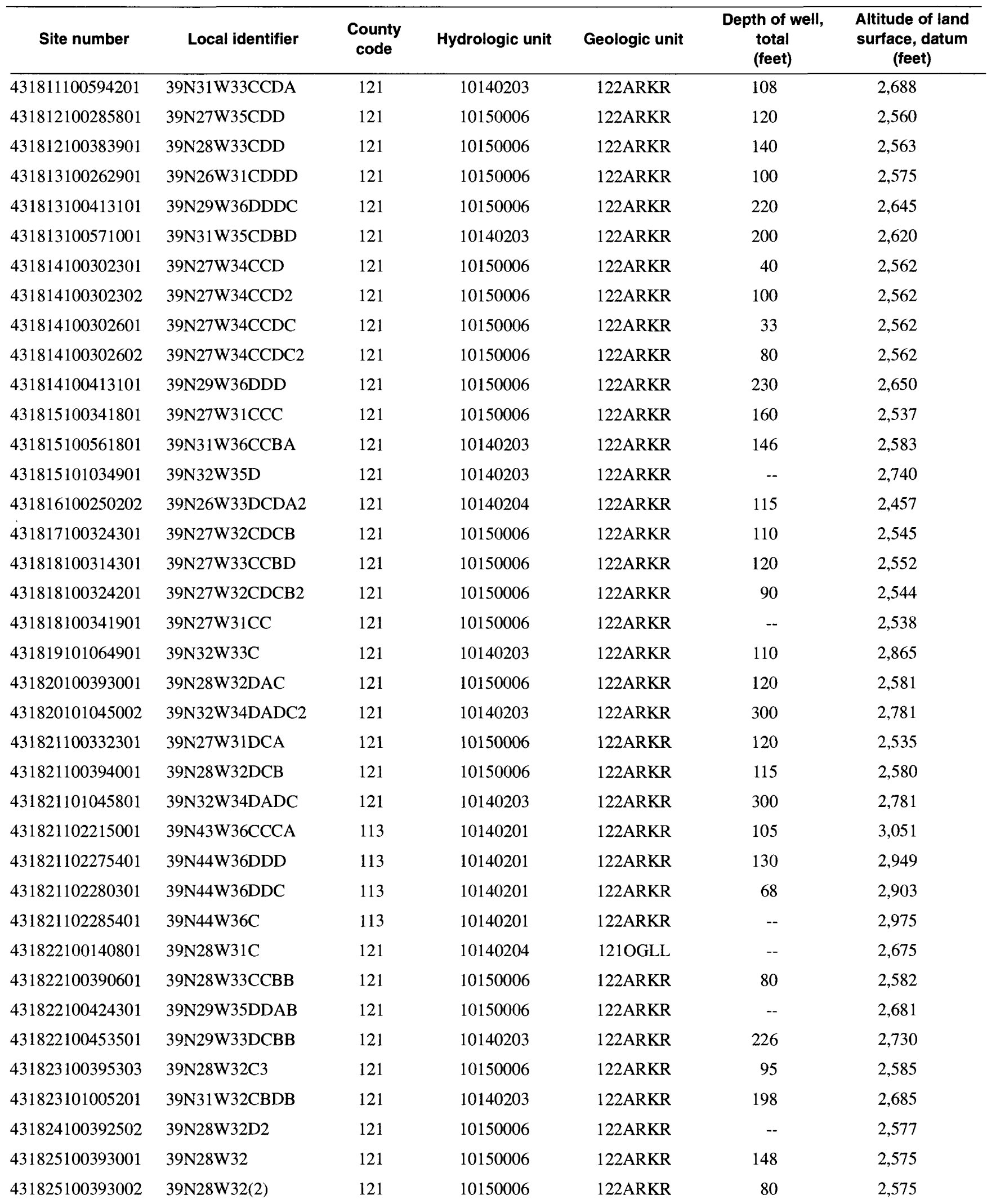


Table 57. Selected descriptive data (from the U.S. Geological Survey database) for ground-water sites in the High Plains aquifer in South Dakota-Continued

[County code: 7 = Bennett County, 53 = Gregory County, 71 = Jackson County, $95=$ Mellette County, $113=$ Shannon County, and $121=$ Todd County; Datum, National Geodetic Vertical Datum; 121OGLL, Ogallala Formation; 122ARKR, Arikaree Formation; --, no data]

\begin{tabular}{|c|c|c|c|c|c|c|}
\hline Site number & Local identifier & $\begin{array}{l}\text { County } \\
\text { code }\end{array}$ & Hydrologic unit & Geologic unit & $\begin{array}{c}\text { Depth of well, } \\
\text { total } \\
\text { (feet) }\end{array}$ & $\begin{array}{l}\text { Altitude of land } \\
\text { surface, datum } \\
\text { (feet) }\end{array}$ \\
\hline 431825100393003 & 39N28W32(3) & 121 & 10150006 & 122ARKR & 80 & 2,575 \\
\hline 431826100420601 & $39 \mathrm{~N} 29 \mathrm{~W} 36 \mathrm{CAD}$ & 121 & 10150006 & 122ARKR & 140 & 2,680 \\
\hline 431830100250201 & 39N26W32DCDA & 121 & 10140204 & 122ARKR & 153 & 2,533 \\
\hline 431830100432201 & $39 \mathrm{~N} 29 \mathrm{~W} 35 \mathrm{CA}$ & 121 & 10150006 & 122ARKR & 140 & 2,700 \\
\hline 431830100450901 & 39N29W33DA & 121 & 10140203 & 122ARKR & 140 & 2,657 \\
\hline 431830101553001 & 39N39W33CCC & 7 & 10140202 & 122ARKR & 200 & 3,400 \\
\hline 431833101035301 & 39N32W35ACDD & 121 & 10140203 & 122ARKR & 72 & 2,690 \\
\hline 431834100404001 & $39 \mathrm{~N} 28 \mathrm{~W} 31 \mathrm{DB}$ & 121 & 10150006 & 121OGLL & -- & 2,593 \\
\hline 431834100413001 & 39N29W36DAAA & 121 & 10150006 & 122ARKR & 210 & 2,657 \\
\hline 431835100412801 & 39N29W36DAAA2 & 121 & 10150006 & 122ARKR & 190 & 2,655 \\
\hline 431836102215201 & $39 \mathrm{~N} 43 \mathrm{~W} 36 \mathrm{CBB}$ & 113 & 10140201 & 122ARKR & -- & 3,041 \\
\hline 431836102340901 & 39N44W31DBDB & 113 & 10140201 & 122ARKR & -- & 2,900 \\
\hline 431837100431401 & 39N29W35ADAD & 121 & 10140203 & 122ARKR & 240 & 2,685 \\
\hline 431840100302501 & 39N27W34BCD & 121 & 10150006 & 122ARKR & 100 & 2,559 \\
\hline 431843100263301 & $39 \mathrm{~N} 26 \mathrm{~W} 31 \mathrm{BDA}$ & 121 & 10140204 & 122ARKR & 140 & 2,600 \\
\hline 431845100401501 & $39 \mathrm{~N} 28 \mathrm{~W} 32 \mathrm{BCB}$ & 121 & 10150006 & 122ARKR & 160 & 2,594 \\
\hline 431847100323901 & $39 \mathrm{~N} 27 \mathrm{~W} 32 \mathrm{BDB}$ & 121 & 10150006 & 122ARKR & 50 & 2,559 \\
\hline 431849100282301 & $39 \mathrm{~N} 27 \mathrm{~W} 35 \mathrm{ADAB}$ & 121 & 10150006 & 122ARKR & 140 & 2,582 \\
\hline 431849100311401 & 39N27W33ACBB & 121 & 10150006 & 122ARKR & 120 & 2,622 \\
\hline 431849100425501 & $39 \mathrm{~N} 29 \mathrm{~W} 35 \mathrm{~A}$ & 121 & 10140203 & 122ARKR & 203 & 2,650 \\
\hline 431852100565201 & $39 \mathrm{~N} 31 \mathrm{~W} 35 \mathrm{~A}$ & 121 & 10140203 & 122ARKR & 198 & 2,590 \\
\hline 431854100275701 & $39 \mathrm{~N} 27 \mathrm{~W} 36 \mathrm{BACB}$ & 121 & 10150006 & 122ARKR & 40 & 2,572 \\
\hline 431854100424001 & 39N29W35AADA & 121 & 10140203 & 122ARKR & 226 & 2,659 \\
\hline 431858102191401 & $39 \mathrm{~N} 42 \mathrm{~W} 32 \mathrm{BA}$ & 113 & 10140201 & 122ARKR & 400 & 3,444 \\
\hline 431900100424001 & $39 \mathrm{~N} 29 \mathrm{~W} 35 \mathrm{AAAA}$ & 121 & 10140203 & 122ARKR & 215 & 2,638 \\
\hline 431900102111901 & $39 \mathrm{~N} 41 \mathrm{~W} 33 \mathrm{BBB}$ & 113 & 10140201 & 122ARKR & -- & 3,182 \\
\hline 431900102114601 & $39 \mathrm{~N} 41 \mathrm{~W} 32 \mathrm{ABA}$ & 113 & 10140201 & 122ARKR & -- & 3,238 \\
\hline 431902100412301 & $39 \mathrm{~N} 28 \mathrm{~W} 31 \mathrm{BBBA}$ & 121 & 10150006 & 122ARKR & -- & 2,598 \\
\hline 431902100561502 & $39 \mathrm{~N} 31 \mathrm{~W} 25 \mathrm{CCCA} 2$ & 121 & 10140203 & 122ARKR & 155 & 2,558 \\
\hline 431902100561503 & $39 \mathrm{~N} 31 \mathrm{~W} 25 \mathrm{CCCA} 3$ & 121 & 10140203 & 122ARKR & 104 & 2,558 \\
\hline 431903100265201 & $39 \mathrm{~N} 26 \mathrm{~W} 31 \mathrm{BBAB}$ & 121 & 10140204 & 122ARKR & 100 & 2,598 \\
\hline 431903100282002 & 39N27W35AAAB2 & 121 & 10150006 & $122 \mathrm{ARKR}$ & 91 & 2,598 \\
\hline 431903100561701 & $39 \mathrm{~N} 31 \mathrm{~W} 25 \mathrm{CCCA}$ & 121 & 10140203 & 122ARKR & 170 & 2,558 \\
\hline 431903101090701 & $39 \mathrm{~N} 32 \mathrm{~W} 30 \mathrm{CDCB}$ & 121 & 10140203 & 122ARKR & -- & 2,820 \\
\hline 431905100402401 & 39N28W30DDDC & 121 & 10150006 & 122ARKR & 150 & 2,618 \\
\hline 431905101124501 & 39N33W27CCDA & 121 & 10140202 & 122ARKR & -- & 2,900 \\
\hline 431907100305501 & $39 \mathrm{~N} 27 \mathrm{~W} 27 \mathrm{CCD}$ & 121 & 10150006 & 122ARKR & 80 & 2,615 \\
\hline 431907100325701 & $39 \mathrm{~N} 27 \mathrm{~W} 29 \mathrm{CCC}$ & 121 & 10150006 & 122ARKR & 120 & 2,567 \\
\hline
\end{tabular}


Table 57. Selected descriptive data (from the U.S. Geological Survey database) for ground-water sites in the High Plains aquifer in South Dakota-Continued

[County code: 7 = Bennett County, $53=$ Gregory County, 71 = Jackson County, $95=$ Mellette County, $113=$ Shannon County, and $121=$ Todd County; Datum, National Geodetic Vertical Datum; 121OGLL, Ogallala Formation; 122ARKR, Arikaree Formation; --, no data]

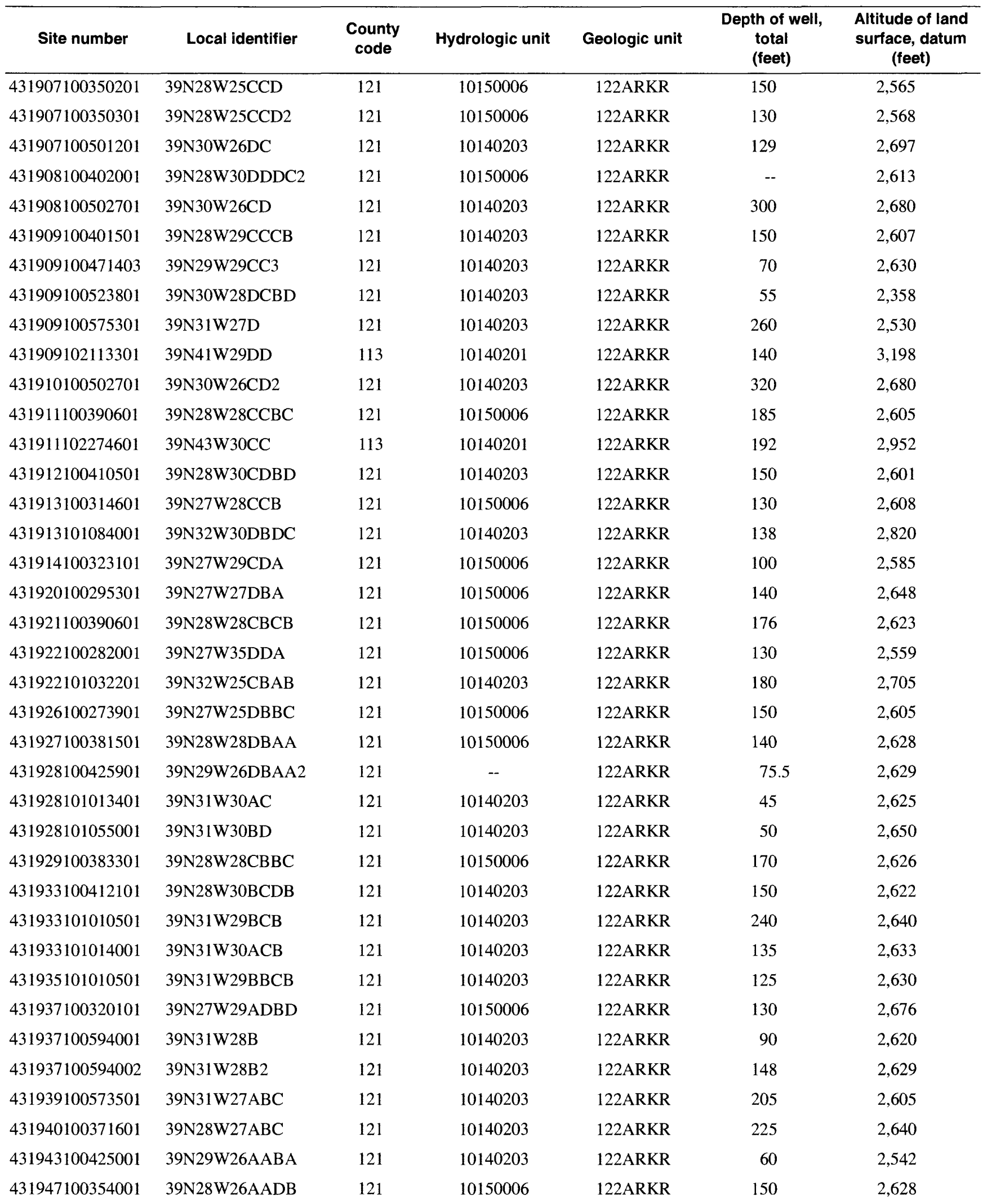


Table 57. Selected descriptive data (from the U.S. Geological Survey database) for ground-water sites in the High Plains aquifer in South Dakota-Continued

[County code: 7 = Bennett County, $53=$ Gregory County, $71=$ Jackson County. $95=$ Mellette County, $113=$ Shannon County, and $121=$ Todd County; Datum, National Geodetic Vertical Datum; 121OGLL, Ogallala Formation; 122ARKR, Arikaree Formation; --, no data]

\begin{tabular}{|c|c|c|c|c|c|c|}
\hline Site number & Local identifier & $\begin{array}{c}\text { County } \\
\text { code }\end{array}$ & Hydrologic unit & Geologic unit & $\begin{array}{c}\text { Depth of well, } \\
\text { total } \\
\text { (feet) }\end{array}$ & $\begin{array}{c}\text { Altitude of land } \\
\text { surface, datum } \\
\text { (feet) }\end{array}$ \\
\hline 431949100343101 & $39 \mathrm{~N} 28 \mathrm{~W} 25 \mathrm{AA}$ & 121 & 10150006 & 122ARKR & 160 & 2,622 \\
\hline 431949100392901 & $39 \mathrm{~N} 28 \mathrm{~W} 29 \mathrm{ABAD}$ & 121 & 10140203 & 122ARKR & 150 & 2,634 \\
\hline 431949100584602 & $39 \mathrm{~N} 31 \mathrm{~W} 27 \mathrm{BBBB} 2$ & 121 & 10140203 & 122ARKR & 95 & 2,622 \\
\hline 431951100232301 & 39N26W27BBD & 121 & 10140204 & 122ARKR & -- & 2,407 \\
\hline 431951100424302 & 39N29W26AAA & 121 & 10140203 & 122ARKR & 125 & 2,540 \\
\hline 431958101582101 & 39N39W19DDBC2 & 7 & 10140201 & 122ARKR & 295 & 3,320 \\
\hline 432002100432501 & $39 \mathrm{~N} 29 \mathrm{~W} 23 \mathrm{CDB}$ & 121 & 10140203 & 122ARKR & 100 & 2,520 \\
\hline 432002100571101 & $39 N 31 W 23 C$ & 121 & 10140203 & 122ARKR & 225 & 2,575 \\
\hline 432003100352501 & $39 \mathrm{~N} 28 \mathrm{~W} 24 \mathrm{CC}$ & 121 & 10140203 & 122ARKR & 150 & 2,675 \\
\hline 432003102165301 & $39 \mathrm{~N} 42 \mathrm{~W} 22 \mathrm{CD}$ & 113 & 10140201 & 122ARKR & 190 & 3,297 \\
\hline 432003102171001 & $39 \mathrm{~N} 42 \mathrm{~W} 22 \mathrm{CC}$ & 113 & 10140201 & $122 \mathrm{ARKR}$ & 170 & 3,320 \\
\hline 432006100300701 & $39 \mathrm{~N} 27 \mathrm{~W} 22 \mathrm{CDA}$ & 121 & 10150006 & 1210GLL & 120 & 2,741 \\
\hline 432006101044701 & $39 \mathrm{~N} 32 \mathrm{~W} 22 \mathrm{DAD}$ & 121 & 10140203 & 122ARKR & 125 & 2,659 \\
\hline 432007102281401 & 39N44W24DCAC & 113 & 10140201 & 122ARKR & 95 & 2,939 \\
\hline 432009100290301 & $39 \mathrm{~N} 27 \mathrm{~W} 23 \mathrm{CDBA}$ & 121 & 10140204 & 122ARKR & 130 & 2,651 \\
\hline 432009100344001 & 39N28W24D & 121 & 10150006 & 122ARKR & 150 & 2,660 \\
\hline 432014101014401 & 39N31W19CDD & 121 & 10140203 & 122ARKR & 230 & 2,650 \\
\hline 432015100294701 & 39N27W22DBDA & 121 & 10150006 & 1210GLL & 160 & 2,654 \\
\hline 432016102161701 & 39N42W22DA & 113 & 10140201 & 122ARKR & 50 & 3,211 \\
\hline 432017100473201 & 39N29W19DA & 121 & 10140203 & 122ARKR & -- & 2,490 \\
\hline 432018100393901 & 39N28W20DBB & 121 & 10140203 & 122ARKR & 150 & 2,727 \\
\hline 432020101010901 & 39N31W19ACDA2 & 121 & 10140203 & 122ARKR & 300 & 2,625 \\
\hline 432020101012704 & 39N31W19ACDA4 & 121 & 10140203 & 122ARKR & 135 & 2,626 \\
\hline 432021100484401 & 39N30W24ADCD & 121 & 10140203 & 122ARKR & 33 & 2,540 \\
\hline 432021102115901 & $-39 N 41 W 20$ & 113 & 10140201 & 122ARKR & 112 & 3,146 \\
\hline 432021102115902 & $39 N 41 W 20(2)$ & 113 & 10140201 & 122ARKR & 102 & 3,146 \\
\hline 432021102115903 & $39 \mathrm{~N} 41 \mathrm{~W} 20(3)$ & 113 & 10140201 & 122ARKR & 100 & 3,146 \\
\hline 432023102225001 & 39N43W23BDCC & 113 & 10140201 & 122ARKR & 50 & 3,000 \\
\hline 432025100321301 & 39N27W20ACD & 121 & 10150006 & 122ARKR & 100 & 2,700 \\
\hline 432025100434001 & 39N29W23BCD & 121 & 10140203 & 122ARKR & 112 & 2,500 \\
\hline 432025101223501 & 39N34W19AAAA & 7 & 10140202 & 122ARKR & 465 & 2,820 \\
\hline 432028102120801 & $39 \mathrm{~N} 41 \mathrm{~W} 20 \mathrm{BD}$ & 113 & 10140201 & 122ARKR & 96 & 3,231 \\
\hline 432033100305601 & $39 \mathrm{~N} 27 \mathrm{~W} 21 \mathrm{ADDA}$ & 121 & 10150006 & 122ARKR & 220 & 2,754 \\
\hline 432033100312301 & $39 \mathrm{~N} 27 \mathrm{~W} 21 \mathrm{BDAB}$ & 121 & 10150006 & 122ARKR & 120 & 2,714 \\
\hline 432035100315101 & 39N27W20ADAA & 121 & 10150006 & 122ARKR & 120 & 2,706 \\
\hline 432035102162601 & $39 \mathrm{~N} 42 \mathrm{~W} 22 \mathrm{~A}$ & 113 & 10140201 & 122ARKR & -- & 3,218 \\
\hline 432036102225001 & $39 N 43 W 23 B$ & 113 & 10140201 & 122ARKR & 75 & 2,956 \\
\hline 432038101223501 & 39N34W19AAAA2 & 7 & 10140202 & 122ARKR & 465 & 2,820 \\
\hline
\end{tabular}


Table 57. Selected descriptive data (from the U.S. Geological Survey database) for ground-water sites in the High Plains aquifer in South Dakota-Continued

[County code: 7 = Bennett County, $53=$ Gregory County, $71=$ Jackson County, $95=$ Mellette County, $113=$ Shannon County, and $121=$ Todd County; Datum, National Geodetic Vertical Datum; 1210GLL, Ogallala Formation; 122ARKR, Arikaree Formation; --, no data]

\begin{tabular}{|c|c|c|c|c|c|c|}
\hline Site number & Local identifier & $\begin{array}{c}\text { County } \\
\text { code }\end{array}$ & Hydrologic unit & Geologic unit & $\begin{array}{c}\text { Depth of well, } \\
\text { total } \\
\text { (feet) }\end{array}$ & $\begin{array}{c}\text { Altitude of land } \\
\text { surface, datum } \\
\text { (feet) }\end{array}$ \\
\hline 432040100441601 & $39 \mathrm{~N} 29 \mathrm{~W} 22 \mathrm{AB}$ & 121 & 10140203 & 122ARKR & 100 & 2,465 \\
\hline 432044101115201 & 39N33W15DDDD & 121 & 10140203 & 122ARKR & 360 & 2,800 \\
\hline 432048100392501 & 39N28W17DCD & 121 & 10140203 & 122ARKR & 233 & 2,680 \\
\hline 432049100415301 & $39 \mathrm{~N} 29 \mathrm{~W} 13 \mathrm{CC}$ & 121 & 10140203 & 122ARKR & 200 & 2,640 \\
\hline 432052100303401 & $39 \mathrm{~N} 27 \mathrm{~W} 15 \mathrm{CCC}$ & 121 & 10140204 & 122ARKR & 100 & 2,665 \\
\hline 432054100582701 & $39 \mathrm{~N} 31 \mathrm{~W} 15 \mathrm{C}$ & 121 & 10140203 & 122ARKR & 96 & 2,600 \\
\hline 432054102124401 & 39N41W18DD & 113 & 10140201 & 122ARKR & 94 & 3,080 \\
\hline 432055101134801 & 39N33W16D & 121 & 10140202 & 122ARKR & 129 & 2,825 \\
\hline 432055101134803 & $39 N 33 W 16 D 3$ & 121 & 10140202 & 122ARKR & 85 & 2,825 \\
\hline 432057102230501 & $39 \mathrm{~N} 43 \mathrm{~W} 14 \mathrm{CCBC}$ & 113 & 10140201 & 122ARKR & 100 & 2,952 \\
\hline 432102101185801 & 39N42W17ACD2 & 113 & 10140201 & 122ARKR & 300 & 3,200 \\
\hline 432108100400001 & $39 \mathrm{~N} 28 \mathrm{~W} 17 \mathrm{CBD}$ & 121 & 10140203 & 122ARKR & 210 & 2,683 \\
\hline 432108102231601 & 39N43W15DA & 113 & 10140201 & 122ARKR & 40 & 3,011 \\
\hline 432108102231602 & 39N43W15DA2 & 113 & 10140201 & 122ARKR & 90 & 3,011 \\
\hline 432113102115901 & $39 N 41 W 17$ & 113 & 10140201 & 122ARKR & -- & 3,215 \\
\hline 432115102234301 & $39 N 43 W 15$ & 113 & 10140201 & 122ARKR & 64 & 3,179 \\
\hline 432117100371601 & 39N28W15ACC & 121 & 10140203 & 122ARKR & 160 & 2,585 \\
\hline 432120101120001 & $39 \mathrm{~N} 41 \mathrm{~W} 17 \mathrm{BCCC}$ & 113 & 10140201 & 122ARKR & 220 & 3,077 \\
\hline 432120102124401 & 39N41W18AD & 113 & 10140201 & 122ARKR & 100 & 3,070 \\
\hline 432128102240001 & $39 N 43 W 15 B$ & 113 & 10140201 & 122ARKR & -- & 3,054 \\
\hline 432128102240002 & $39 \mathrm{~N} 43 \mathrm{~W} 15 \mathrm{~B} 2$ & 113 & 10140201 & 122ARKR & -- & 3,054 \\
\hline 432131101032701 & $39 \mathrm{~N} 32 \mathrm{~W} 13 \mathrm{BBBB}$ & 121 & 10140203 & 122ARKR & 125 & 2,633 \\
\hline 432131101034001 & $39 \mathrm{~N} 32 \mathrm{~W} 14 \mathrm{AAA}$ & 121 & 10140203 & 122ARKR & 125 & 2,634 \\
\hline 432131101053001 & $39 \mathrm{~N} 32 \mathrm{~W} 15 \mathrm{BAB}$ & 121 & 10140203 & 122ARKR & 200 & 2,678 \\
\hline 432134102214801 & $39 N 43 W 13 B B$ & 113 & 10140201 & 122ARKR & 110 & 3,001 \\
\hline 432149101005001 & 39N31W 8CACB & 121 & 10140203 & 122ARKR & 274 & 2,653 \\
\hline 432150100381401 & 39N28W 9DDBB & 121 & 10140203 & 122ARKR & 120 & 2,540 \\
\hline 432150100381701 & $39 \mathrm{~N} 28 \mathrm{~W} 9 \mathrm{DCA}$ & 121 & 10140203 & 122ARKR & 68 & 2,518 \\
\hline 432153100371201 & 39N28W10D & 121 & 10140203 & 122ARKR & 72 & 2,545 \\
\hline 432154102151501 & $39 N 42 W 11 D$ & 113 & 10140201 & 122ARKR & -- & 3,125 \\
\hline 432203100361301 & $39 \mathrm{~N} 28 \mathrm{~W} 11 \mathrm{CAA}$ & 121 & 10140203 & 122ARKR & 160 & 2,535 \\
\hline 432205100294301 & $39 \mathrm{~N} 27 \mathrm{~W} 10 \mathrm{DABB}$ & 121 & 10140204 & 122ARKR & 75 & 2,513 \\
\hline 432205101032201 & $39 \mathrm{~N} 32 \mathrm{~W} 11 \mathrm{ACDA}$ & 121 & 10140203 & 122ARKR & 125 & 2,595 \\
\hline 432209101012501 & 39N31W 7ACAA & 121 & 10140203 & 122ARKR & 110 & 2,538 \\
\hline 432213102224102 & 39N43W11BD2 & 113 & 10140201 & 122ARKR & 120 & 2,936 \\
\hline 432218100414401 & $39 \mathrm{~N} 29 \mathrm{~W} 12 \mathrm{~A}$ & 121 & 10140203 & 122ARKR & 35 & 2,500 \\
\hline 432218100414402 & $39 \mathrm{~N} 29 \mathrm{~W} 12 \mathrm{~A} 2$ & 121 & 10140203 & 122ARKR & 70 & 2,500 \\
\hline 432225102130201 & $39 \mathrm{~N} 41 \mathrm{~W} 7 \mathrm{AB}$ & 113 & 10140201 & 122ARKR & 110 & -- \\
\hline
\end{tabular}


Table 57. Selected descriptive data (from the U.S. Geological Survey database) for ground-water sites in the High Plains aquifer in South Dakota-Continued

[County code: 7 = Bennett County, $53=$ Gregory County, $71=$ Jackson County, $95=$ Mellette County, $113=$ Shannon County, and $121=$ Todd County; Datum, National Geodetic Vertical Datum; 1210GLL, Ogallala Formation; 122ARKR, Arikaree Formation; --, no data]

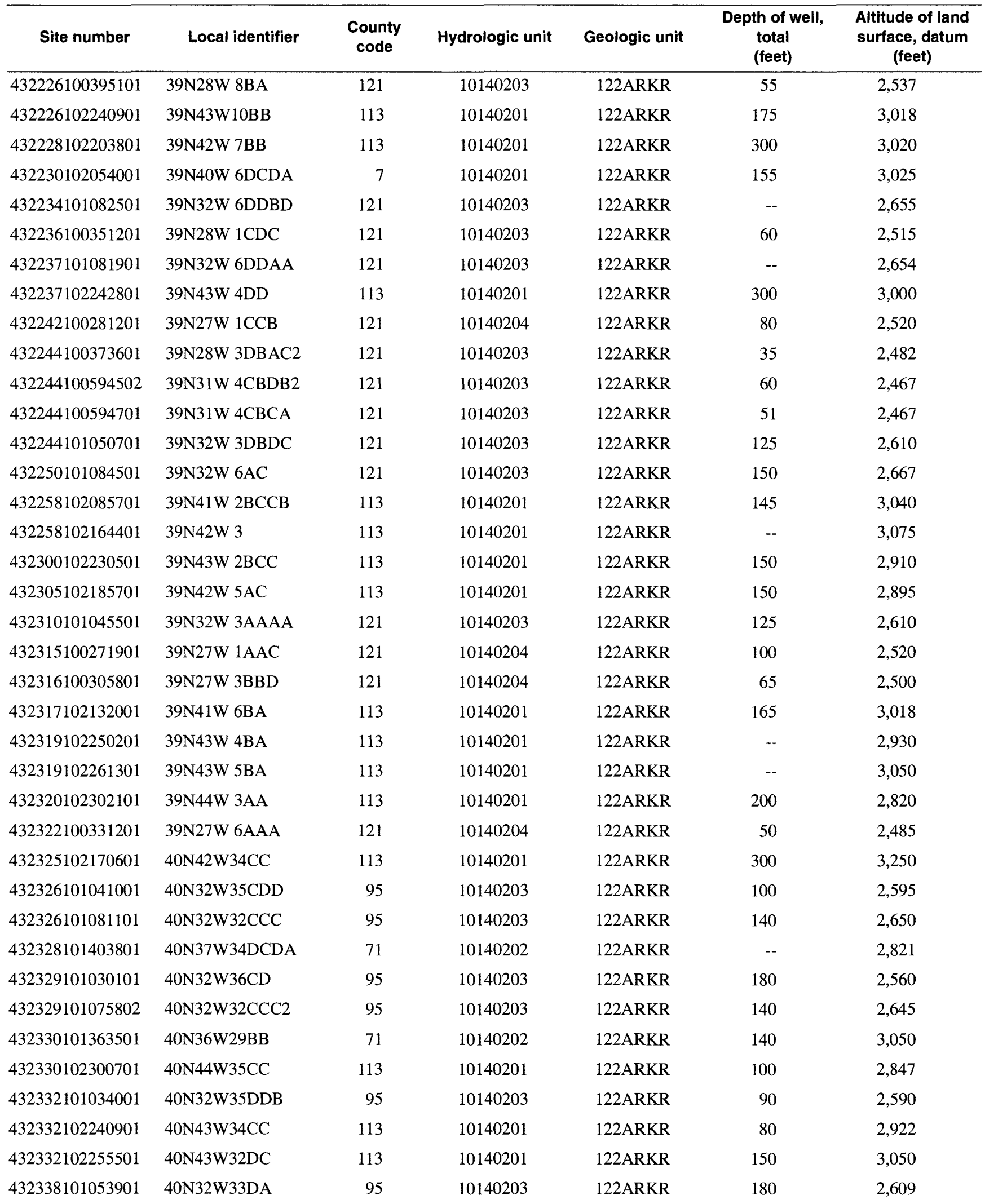


Table 57. Selected descriptive data (from the U.S. Geological Survey database) for ground-water sites in the High Plains aquifer in South Dakota-Continued

[County code: 7 = Bennett County, $53=$ Gregory County, $71=$ Jackson County, $95=$ Mellette County, $113=$ Shannon County, and $121=$ Todd County; Datum, National Geodetic Vertical Datum; 1210GLL, Ogallala Formation; 122ARKR, Arikaree Formation; --, no data]

\begin{tabular}{|c|c|c|c|c|c|c|}
\hline Site number & Local identifier & $\begin{array}{l}\text { County } \\
\text { code }\end{array}$ & Hydrologic unit & Geologic unit & $\begin{array}{c}\text { Depth of well, } \\
\text { total } \\
\text { (feet) }\end{array}$ & $\begin{array}{l}\text { Altitude of land } \\
\text { surface, datum } \\
\text { (feet) }\end{array}$ \\
\hline 432338101405701 & $40 \mathrm{~N} 37 \mathrm{~W} 34 \mathrm{CA}$ & 71 & 10140202 & 122ARKR & -- & 2,900 \\
\hline 432339101042801 & $40 \mathrm{~N} 32 \mathrm{~W} 35 \mathrm{CDB}$ & 95 & 10140203 & 122ARKR & 150 & 2,595 \\
\hline 432341101161001 & $40 \mathrm{~N} 33 \mathrm{~W} 31 \mathrm{DBDC}$ & 71 & 10140202 & 122ARKR & 325 & 2,776 \\
\hline 432344101102501 & $40 \mathrm{~N} 33 \mathrm{~W} 36 \mathrm{CBA}$ & 95 & 10140202 & 122ARKR & 125 & 2,660 \\
\hline 432345102231601 & 40N43W34DA & 113 & 10140201 & 122ARKR & 120 & 2,900 \\
\hline 432350101502701 & $40 \mathrm{~N} 38 \mathrm{~W} 32$ & 71 & 10140202 & 122ARKR & 265 & 2,857 \\
\hline 432354101251001 & $40 \mathrm{~N} 35 \mathrm{~W} 35 \mathrm{AC}$ & 71 & 10140202 & 122ARKR & 350 & 2,700 \\
\hline 432355101280001 & $40 \mathrm{~N} 35 \mathrm{~W} 33 \mathrm{BA}$ & 71 & 10140202 & 122ARKR & 150 & 2,700 \\
\hline 432356101550501 & $40 \mathrm{~N} 39 \mathrm{~W} 34 \mathrm{AC}$ & 71 & 10140202 & 122ARKR & -- & 3,103 \\
\hline 432357101145901 & 40N33W32BACD & 71 & 10140202 & $122 \mathrm{ARKR}$ & 300 & 2,730 \\
\hline 432358101045501 & $40 \mathrm{~N} 32 \mathrm{~W} 34 \mathrm{AC}$ & 95 & 10140203 & 122ARKR & 270 & 2,610 \\
\hline 432359101003501 & $40 \mathrm{~N} 31 \mathrm{~W} 32 \mathrm{AC}$ & 95 & 10140203 & 122ARKR & 220 & 2,540 \\
\hline 432403101553201 & $40 N 39 W 34 B$ & 71 & 10140202 & 122ARKR & -- & 3,080 \\
\hline 432404102240001 & $40 N 43 W 34 B$ & 113 & 10140201 & 122ARKR & 49 & 2,852 \\
\hline 432407101103001 & 40N33W36BB & 95 & 10140202 & 122ARKR & 120 & 2,660 \\
\hline 432407101131201 & $40 \mathrm{~N} 33 \mathrm{~W} 33 \mathrm{AAB}$ & 95 & 10140202 & 122ARKR & 225 & 2,742 \\
\hline 432408101250301 & $40 \mathrm{~N} 35 \mathrm{~W} 35 \mathrm{AA}$ & 71 & 10140202 & 122ARKR & -- & 2,739 \\
\hline 432408101253901 & 40N35W35BA & 71 & 10140202 & 122ARKR & -- & 2,700 \\
\hline 432408101281901 & 40N35W33BB & 71 & 10140202 & 122ARKR & 150 & 2,696 \\
\hline 432408101283701 & $40 \mathrm{~N} 35 \mathrm{~W} 32 \mathrm{AA}$ & 71 & 10140202 & 122ARKR & 55 & 2,690 \\
\hline 432409102235401 & 40N43W34BACD & 113 & 10140201 & 122ARKR & 45 & 2,850 \\
\hline 432410101294001 & 40N35W31AAAA & 71 & 10140203 & 122ARKR & 325 & 2,620 \\
\hline 432410101294002 & 40N35W31AAAA2 & 71 & 10140202 & 122ARKR & 318.5 & 2,732 \\
\hline 432411102231601 & 40N43W34AA & 113 & 10140201 & $122 \mathrm{ARKR}$ & 275 & 3,001 \\
\hline 432416100315601 & 40N27W32AAA & 95 & 10140204 & 122ARKR & 16 & 2,478 \\
\hline 432416100315602 & 40N27W32AAA2 & 95 & 10140204 & 122ARKR & 22 & 2,478 \\
\hline 432417102024501 & $40 \mathrm{~N} 40 \mathrm{~W} 27 \mathrm{CC}$ & 71 & 10140202 & 122ARKR & 370 & 3,225 \\
\hline 432420102155501 & $40 \mathrm{~N} 42 \mathrm{~W} 26 \mathrm{C}$ & 113 & 10140201 & 122ARKR & 130 & 3,075 \\
\hline 432422101350701 & $40 \mathrm{~N} 36 \mathrm{~W} 28 \mathrm{CD}$ & 71 & 10140202 & $122 \mathrm{ARKR}$ & 140 & 3,051 \\
\hline 432422101451401 & 40N38W25DD & 71 & 10140202 & 122ARKR & 65 & 2,719 \\
\hline 432425100315601 & 40N27W29DDD & 95 & 10140204 & 122ARKR & 40 & 2,470 \\
\hline 432425100315602 & 40N27W29DDD2 & 95 & 10140204 & 122ARKR & 40 & 2,480 \\
\hline 432427102100001 & $40 \mathrm{~N} 41 \mathrm{~W} 27 \mathrm{CAA}$ & 113 & 10140201 & $122 \mathrm{ARKR}$ & 200 & 2,932 \\
\hline 432428101181101 & 40N34W26DB & 71 & 10140202 & 122ARKR & -- & 2,660 \\
\hline 432428102211501 & 40N43W25DB & 113 & 10140201 & 122ARKR & 250 & 3,000 \\
\hline 432429102024201 & $40 \mathrm{~N} 40 \mathrm{~W} 27 \mathrm{C}$ & 71 & 10140202 & 122ARKR & -- & 3,116 \\
\hline 432430101094001 & 40N33W25DAC & 95 & 10140202 & 122ARKR & 125 & 2,635 \\
\hline 432430101094002 & 40N33W25DAC2 & 95 & 10140202 & $122 \mathrm{ARKR}$ & 125 & 2,540 \\
\hline
\end{tabular}


Table 57. Selected descriptive data (from the U.S. Geological Survey database) for ground-water sites in the High Plains aquifer in South Dakota-Continued

[County code: 7 = Bennett County, $53=$ Gregory County, $71=$ Jackson County, $95=$ Mellette County, $113=$ Shannon County, and $121=$ Todd County; Datum, National Geodetic Vertical Datum; 1210GLL, Ogallala Formation; 122ARKR, Arikaree Formation; --, no data]

\begin{tabular}{|c|c|c|c|c|c|c|}
\hline Site number & Local identifier & $\begin{array}{l}\text { County } \\
\text { code }\end{array}$ & Hydrologic unit & Geologic unit & $\begin{array}{c}\text { Depth of well, } \\
\text { total } \\
\text { (feet) }\end{array}$ & $\begin{array}{l}\text { Altitude of land } \\
\text { surface, datum } \\
\text { (feet) }\end{array}$ \\
\hline 432430101264001 & $40 \mathrm{~N} 35 \mathrm{~W} 27 \mathrm{CA}$ & 71 & 10140202 & 122ARKR & - & 2,675 \\
\hline 432434101291201 & $40 \mathrm{~N} 35 \mathrm{~W} 29 \mathrm{CA}$ & 71 & 10140202 & 122ARKR & -- & 2,703 \\
\hline 432435101380401 & $40 \mathrm{~N} 37 \mathrm{~W} 25 \mathrm{DA}$ & 71 & 10140202 & 122ARKR & -- & 3,034 \\
\hline 432439100341101 & $40 \mathrm{~N} 27 \mathrm{~W} 30 \mathrm{CBC}$ & 95 & 10140204 & 122ARKR & 120 & 2,415 \\
\hline 432441100343701 & 40N28W25DABB & 95 & 10140203 & 122ARKR & 65 & 2,455 \\
\hline 432441101431701 & 40 N37W29 & 71 & 10140202 & 122ARKR & -- & 2,808 \\
\hline 432442100333501 & $40 \mathrm{~N} 27 \mathrm{~W} 30 \mathrm{DBB}$ & 95 & 10140204 & 122ARKR & 30 & 2,353 \\
\hline 432442101584801 & 40 N39W30 & 71 & 10140202 & 122ARKR & -- & 3,021 \\
\hline 432442102071301 & $40 \mathrm{~N} 4 \mathrm{IW} 25$ & 113 & 10140201 & 122ARKR & -- & 2,969 \\
\hline 432446101131104 & $40 \mathrm{~N} 33 \mathrm{~W} 28 \mathrm{AD}$ & 95 & 10140202 & 122ARKR & 110 & 2,740 \\
\hline 432446101131105 & 40N33W28AD2 & 95 & 10140202 & 122ARKR & 60 & 2,740 \\
\hline 432446101203701 & $40 \mathrm{~N} 34 \mathrm{~W} 28 \mathrm{AC}$ & 71 & 10140202 & 122ARKR & -- & 2,709 \\
\hline 432447101253901 & $40 \mathrm{~N} 35 \mathrm{~W} 26 \mathrm{BD}$ & 71 & 10140202 & 122ARKR & -- & 2,677 \\
\hline 432448101330301 & $40 \mathrm{~N} 36 \mathrm{~W} 26 \mathrm{BC}$ & 71 & 10140202 & 122ARKR & -- & 3,087 \\
\hline 432450102213001 & $40 N 43 W 25 B D$ & 113 & 10140201 & 122ARKR & 100 & 2,915 \\
\hline 432450102261301 & 40N43W29BD & 113 & 10140201 & 122ARKR & 80 & 2,900 \\
\hline 432453101075601 & $40 \mathrm{~N} 32 \mathrm{~W} 29 \mathrm{BCAB}$ & 95 & 10140202 & 122ARKR & 240 & 2,618 \\
\hline 432454101530001 & 40N39W25BA & 71 & 10140202 & 122ARKR & -- & 3,200 \\
\hline 432455101201801 & 40N34W28AA & 71 & 10140202 & 122ARKR & -- & 2,710 \\
\hline 432455102065501 & 40N41W25A & 113 & 10140201 & 122ARKR & -- & 2,952 \\
\hline 432456102002501 & $40 \mathrm{~N} 40 \mathrm{~W} 25 \mathrm{BBB}$ & 71 & 10140202 & 122ARKR & -- & 3,100 \\
\hline 432456102213901 & 40N43W25B & 113 & 10140201 & 122ARKR & -- & 2,930 \\
\hline 432500101170401 & $40 \mathrm{~N} 34 \mathrm{~W} 25 \mathrm{AB}$ & 71 & 10140202 & 122ARKR & -- & 2,703 \\
\hline 432500101213001 & 40N34W29AA & 71 & 10140202 & 122ARKR & -- & 2,686 \\
\hline 432500102092001 & 40N41W27ABDD & 113 & 10140201 & 122ARKR & 53 & 2,959 \\
\hline 432502102094501 & $40 \mathrm{~N} 41 \mathrm{~W} 27 \mathrm{BA}$ & 113 & 10140201 & 122ARKR & -- & 2,936 \\
\hline 432502102182101 & $40 \mathrm{~N} 42 \mathrm{~W} 28 \mathrm{BB}$ & 113 & 10140201 & 122ARKR & 350 & 2,910 \\
\hline 432503102242701 & 40N43W28AA & 113 & 10140201 & 122ARKR & 82 & 2,870 \\
\hline 432505101502201 & $40 \mathrm{~N} 38 \mathrm{~W} 20 \mathrm{CD}$ & 71 & 10140202 & 122ARKR & -- & 2,800 \\
\hline 432510101562701 & $40 \mathrm{~N} 39 \mathrm{~W} 21 \mathrm{CD}$ & 71 & 10140202 & 122ARKR & -- & 3,100 \\
\hline 432512101145801 & $40 \mathrm{~N} 33 \mathrm{~W} 20 \mathrm{CDBC}$ & 71 & 10140202 & 122ARKR & -- & 2,707 \\
\hline 432512101151601 & $40 \mathrm{~N} 33 \mathrm{~W} 20 \mathrm{CDDC}$ & 71 & 10140202 & 122ARKR & -- & 2,662 \\
\hline 432512101161001 & 40N33W19CDDD & 71 & 10140202 & 122ARKR & -- & 2,720 \\
\hline 432513101252601 & $40 \mathrm{~N} 35 \mathrm{~W} 23 \mathrm{CC}$ & 71 & 10140202 & 122ARKR & 135 & 2,654 \\
\hline 432513101283701 & $40 \mathrm{~N} 35 \mathrm{~W} 20 \mathrm{DD}$ & 71 & 10140202 & 122ARKR & 170 & 2,755 \\
\hline 432514101434401 & $40 \mathrm{~N} 37 \mathrm{~W} 20 \mathrm{CC}$ & 71 & 10140202 & 122ARKR & -- & 2,795 \\
\hline 432514102250401 & 40N43W21CDCD & 113 & 10140201 & 122ARKR & 80 & 2,850 \\
\hline 432515101071701 & $40 \mathrm{~N} 32 \mathrm{~W} 20 \mathrm{DDBB}$ & 95 & 10140202 & 122ARKR & 220 & 2,597 \\
\hline
\end{tabular}


Table 57. Selected descriptive data (from the U.S. Geological Survey database) for ground-water sites in the High Plains aquifer in South Dakota-Continued

[County code: 7 = Bennett County, $53=$ Gregory County, $71=$ Jackson County, $95=$ Mellette County, $113=$ Shannon County, and $121=$ Todd County; Datum, National Geodetic Vertical Datum; 1210GLL, Ogallala Formation; 122ARKR, Arikaree Formation; --, no data]

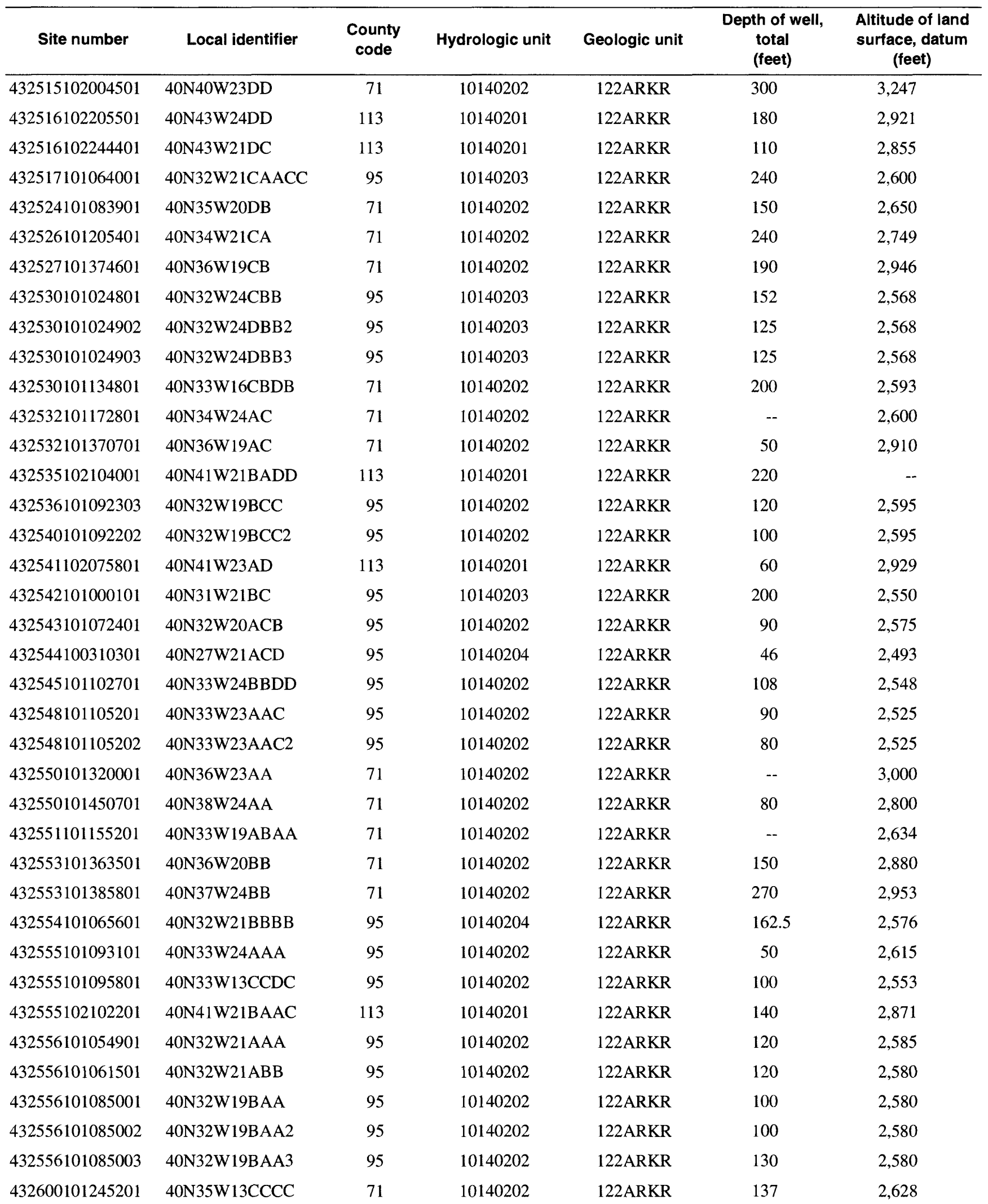


Table 57. Selected descriptive data (from the U.S. Geological Survey database) for ground-water sites in the High Plains aquifer in South Dakota-Continued

[County code: 7 = Bennett County, $53=$ Gregory County, 71 = Jackson County, $95=$ Mellette County, $113=$ Shannon County, and $121=$ Todd County; Datum, National Geodetic Vertical Datum; 121OGLL, Ogallala Formation; 122ARKR, Arikaree Formation; --, no data]

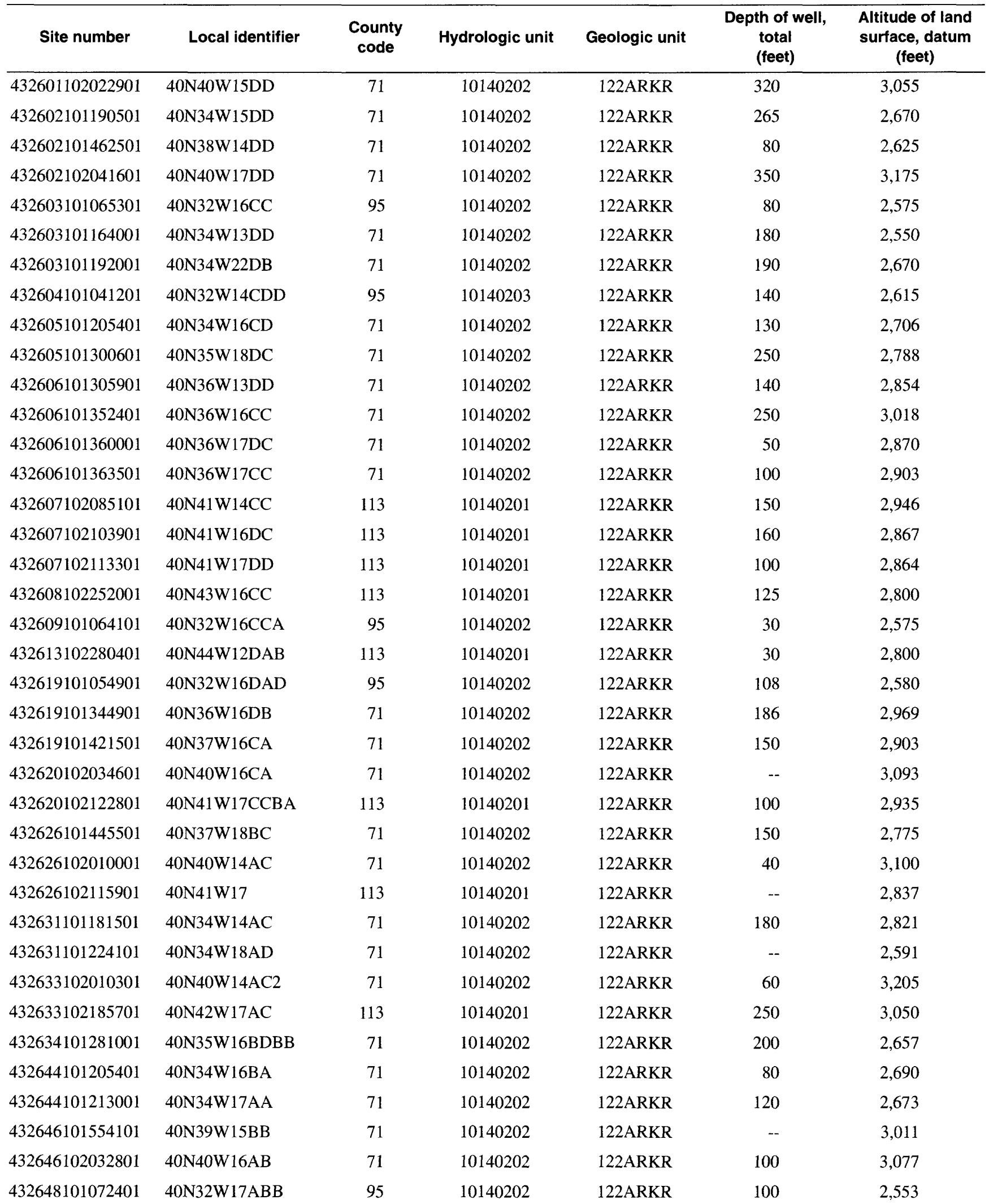


Table 57. Selected descriptive data (from the U.S. Geological Survey database) for ground-water sites in the High Plains aquifer in South Dakota-Continued

[County code: 7 = Bennett County, 53 = Gregory County, 71 = Jackson County, $95=$ Mellette County, $113=$ Shannon County, and $121=$ Todd County; Datum, National Geodetic Vertical Datum; 1210GLL, Ogallala Formation; 122ARKR, Arikaree Formation; --, no data]

\begin{tabular}{|c|c|c|c|c|c|c|}
\hline Site number & Local identifier & $\begin{array}{c}\text { County } \\
\text { code }\end{array}$ & Hydrologic unit & Geologic unit & $\begin{array}{c}\text { Depth of well, } \\
\text { total } \\
\text { (feet) }\end{array}$ & $\begin{array}{c}\text { Altitude of land } \\
\text { surface, datum } \\
\text { (feet) }\end{array}$ \\
\hline 432649101081802 & $40 \mathrm{~N} 32 \mathrm{~W} 17 \mathrm{BBBB} 2$ & 95 & - & 122ARKR & 112 & 2,546 \\
\hline 432654101094901 & $40 \mathrm{~N} 33 \mathrm{~W} 12 \mathrm{DCD}$ & 95 & 10140202 & 122ARKR & 40 & 2,540 \\
\hline 432654101130401 & 40N33W 9DDDA & 95 & 10140202 & 122ARKR & 120 & 2,570 \\
\hline 432654101130601 & 40N33W 9DDDA2 & 95 & 10140202 & 122ARKR & 90 & 2,575 \\
\hline 432656102024801 & $40 \mathrm{~N} 40 \mathrm{~W} 15 \mathrm{BB}$ & 71 & 10140201 & 122ARKR & 100 & 3,100 \\
\hline 432657101155201 & $40 \mathrm{~N} 33 \mathrm{~W} 7 \mathrm{CDCC}$ & 71 & 10140202 & 122ARKR & 200 & 2,667 \\
\hline 432657101213001 & $40 N 34 W$ 8DD & 71 & 10140202 & 122ARKR & 100 & 2,641 \\
\hline 432658101374601 & $40 \mathrm{~N} 36 \mathrm{~W} 7 \mathrm{CC}$ & 71 & 10140202 & 122ARKR & 250 & 2,919 \\
\hline 432659102053401 & 40N40W 7DD & 71 & 10140202 & 122ARKR & 308 & 3,136 \\
\hline 432659102200501 & 40N42W 7DC & 113 & 10140201 & 122ARKR & 300 & 3,060 \\
\hline 432700101120401 & 40N33W10DAB & 95 & 10140202 & 122ARKR & 35 & 2,540 \\
\hline 432704101232501 & $40 \mathrm{~N} 34 \mathrm{~W} 7 \mathrm{C}$ & 71 & 10140202 & 122ARKR & 190 & 2,604 \\
\hline 432705102054301 & 40N40W 7D & 71 & 10140202 & 122ARKR & -- & 3,192 \\
\hline 432711101302301 & $40 \mathrm{~N} 35 \mathrm{~W} 7 \mathrm{CA}$ & 71 & 10140202 & 122ARKR & 200 & 2,739 \\
\hline 432712102015701 & 40N40W10DA & 71 & 10140202 & 122ARKR & 102 & 3,113 \\
\hline 432715101040201 & $40 \mathrm{~N} 32 \mathrm{~W} 11 \mathrm{DBB}$ & 95 & 10140202 & 122ARKR & 80 & 2,640 \\
\hline 432716101051701 & $40 \mathrm{~N} 32 \mathrm{~W} 10$ & 95 & 10140202 & 122ARKR & 72 & 2,581 \\
\hline 432718102033701 & 40N40W 9 & 71 & 10140202 & 122ARKR & 237 & 3,005 \\
\hline 432720101281101 & $40 \mathrm{~N} 35 \mathrm{~W} 8 \mathrm{BD}$ & 71 & 10140202 & 122ARKR & 230 & 2,660 \\
\hline 432720101310701 & $40 \mathrm{~N} 36 \mathrm{~W} 12 \mathrm{AC}$ & 71 & 10140202 & 122ARKR & -- & 2,750 \\
\hline 432720102032301 & 40N40W 9AC & 71 & 10140202 & 122ARKR & 60 & 3,000 \\
\hline 432721102030601 & 40N40W 9BD & 71 & 10140202 & 122ARKR & 150 & 3,000 \\
\hline 432723101145801 & 40N33W 8BDDA & 71 & 10140202 & 122ARKR & 108 & 2,605 \\
\hline 432723101145802 & 40N33W 8BD2 & 71 & 10140202 & 122ARKR & 110 & 2,600 \\
\hline 432724101294801 & 40N35W 7AD & 71 & 10140202 & 122ARKR & 245 & 2,729 \\
\hline 432724101404501 & $40 \mathrm{~N} 37 \mathrm{~W} 10 \mathrm{AC}$ & 71 & 10140202 & 122ARKR & 150 & 2,920 \\
\hline 432725102031001 & 40N40W 9AD & 71 & 10140202 & 122ARKR & 87 & 3,018 \\
\hline 432725102100301 & $40 \mathrm{~N} 41 \mathrm{~W} 10 \mathrm{BC}$ & 113 & 10140201 & 122ARKR & 350 & 3,001 \\
\hline 432725102111501 & $40 \mathrm{~N} 41 \mathrm{~W} 9 \mathrm{BC}$ & 113 & 10140201 & 122ARKR & 360 & 2,870 \\
\hline 432725102152401 & $40 \mathrm{~N} 42 \mathrm{~W} 11 \mathrm{AC}$ & 113 & 10140201 & 122ARKR & 150 & 2,810 \\
\hline 432725102165301 & 40N42W10BD & 113 & 10140201 & 122ARKR & 220 & 2,985 \\
\hline 432732101261501 & $40 \mathrm{~N} 35 \mathrm{~W} 10 \mathrm{AB}$ & 71 & 10140202 & 122ARKR & 180 & 2,600 \\
\hline 432733101120401 & 40N33W10AAC & 95 & 10140202 & 122ARKR & 124 & 2,540 \\
\hline 432734101145801 & 40N33W 8BDD2 & 71 & 10140202 & 122ARKR & 110 & 2,604 \\
\hline 432737101300601 & $40 \mathrm{~N} 35 \mathrm{~W} 7 \mathrm{AB}$ & 71 & 10140202 & 122ARKR & 150 & 2,707 \\
\hline 432737101321001 & $40 \mathrm{~N} 36 \mathrm{~W} 11 \mathrm{AA}$ & 71 & 10140202 & 122ARKR & 270 & 2,778 \\
\hline 432737101352401 & $40 N 36 \mathrm{~W} 9 \mathrm{BB}$ & 71 & 10140202 & 122ARKR & 250 & 2,821 \\
\hline 432738102124401 & $40 \mathrm{~N} 41 \mathrm{~W} 7 \mathrm{AA}$ & 113 & 10140201 & 122ARKR & 110 & 2,814 \\
\hline
\end{tabular}


Table 57. Selected descriptive data (from the U.S. Geological Survey database) for ground-water sites in the High Plains aquifer in South Dakota-Continued

[County code: 7 = Bennett County, 53 = Gregory County, 71 = Jackson County. $95=$ Mellette County, $113=$ Shannon County, and $121=$ Todd County; Datum, National Geodetic Vertical Datum; 1210GLL, Ogallala Formation; 122ARKR, Arikaree Formation; --, no data]

\begin{tabular}{|c|c|c|c|c|c|c|}
\hline Site number & Local identifier & $\begin{array}{c}\text { County } \\
\text { code }\end{array}$ & Hydrologic unit & Geologic unit & $\begin{array}{c}\text { Depth of well, } \\
\text { total } \\
\text { (feet) }\end{array}$ & $\begin{array}{l}\text { Altitude of land } \\
\text { surface, datum } \\
\text { (feet) }\end{array}$ \\
\hline 432740101223501 & 40N34W 6DDDD & 71 & 10140202 & 122ARKR & 185 & 2,620 \\
\hline 432742101115802 & 40N33W 3DDDC2 & 95 & -- & 122ARKR & 116 & 2,526 \\
\hline 432744102051101 & $40 \mathrm{~N} 40 \mathrm{~W} 5 \mathrm{CC}$ & 71 & 10140202 & 122ARKR & 420 & 3,100 \\
\hline 432745101281001 & $40 \mathrm{~N} 35 \mathrm{~W} 4 \mathrm{CC}$ & 71 & 10140202 & 122ARKR & 150 & 2,610 \\
\hline 432746102013301 & 40N40W 2CCDD & 71 & 10140202 & 122ARKR & 345 & 3,116 \\
\hline 432750101281901 & $40 \mathrm{~N} 35 \mathrm{~W} 4 \mathrm{CC} 2$ & 71 & 10140202 & 122ARKR & 90 & 2,650 \\
\hline 432752101123101 & $40 N 33 \mathrm{~W} 3 \mathrm{CDA}$ & 95 & 10140202 & 122ARKR & 70 & 2,530 \\
\hline 432755101590501 & 40N39W 6CC & 71 & 10140202 & 122ARKR & 320 & 3,200 \\
\hline 432756101295701 & $40 N 35 W 6 D$ & 71 & 10140202 & 122ARKR & -- & 2,664 \\
\hline 432759101114601 & $40 \mathrm{~N} 33 \mathrm{~W} 2 \mathrm{CBC}$ & 95 & 10140202 & 122ARKR & 80 & 2,517 \\
\hline 432800101094201 & $40 \mathrm{~N} 35 \mathrm{~W} 6 \mathrm{DA}$ & 71 & 10140202 & 122ARKR & 160 & 2,600 \\
\hline 432802101115901 & $40 \mathrm{~N} 33 \mathrm{~W} 3 \mathrm{DA}$ & 95 & 10140202 & 122ARKR & 80 & 2,517 \\
\hline 432802101121701 & $40 \mathrm{~N} 33 \mathrm{~W} 3 \mathrm{DB}$ & 95 & 10140202 & 122ARKR & 110 & 2,520 \\
\hline 432808101012901 & $40 \mathrm{~N} 31 \mathrm{~W} 5 \mathrm{CBB}$ & 95 & 10140203 & 122ARKR & 65 & 2,605 \\
\hline 432808101012903 & $40 \mathrm{~N} 31 \mathrm{~W} 5 \mathrm{CBB} 3$ & 95 & 10140203 & 122ARKR & 15 & 2,605 \\
\hline 432811101361901 & 40N36W 6AD & 71 & 10140202 & 122ARKR & 265 & 3,000 \\
\hline 432811102071301 & $40 \mathrm{~N} 41 \mathrm{~W} 1$ & 113 & 10140201 & 122ARKR & 30 & 3,198 \\
\hline 432812101123101 & 40N33W 3BDDD & 95 & 10140202 & 122ARKR & 80 & 2,535 \\
\hline 432812101465501 & 40N38W 2BDDD & 71 & 10140202 & 122ARKR & 260 & 2,690 \\
\hline 432813101473001 & $40 N 38 W 3 A D$ & 71 & 10140202 & 122ARKR & 40 & 2,800 \\
\hline 432816101281901 & $40 \mathrm{~N} 35 \mathrm{~W} 4 \mathrm{BC}$ & 71 & 10140202 & 122ARKR & 125 & 2,634 \\
\hline 432816101344901 & $40 \mathrm{~N} 36 \mathrm{~W} 4 \mathrm{AC}$ & 71 & 10140202 & 122ARKR & 80 & 2,723 \\
\hline 432816101354201 & $40 \mathrm{~N} 36 \mathrm{~W} 5 \mathrm{AD}$ & 71 & 10140202 & 122ARKR & 300 & 2,785 \\
\hline 432816101363501 & $40 \mathrm{~N} 36 \mathrm{~W} 5 \mathrm{BC}$ & 71 & 10140202 & 122ARKR & 265 & 2,782 \\
\hline 432817102124401 & $40 \mathrm{~N} 41 \mathrm{~W} 6 \mathrm{AD}$ & 113 & 10140201 & 122ARKR & 165 & 2,788 \\
\hline 432821101504301 & 40N38W 5BDBB & 71 & 10140202 & $122 \mathrm{ARKR}$ & 200 & 2,782 \\
\hline 432821101504302 & 40N38W 5BDBB2 & 71 & 10140202 & 122ARKR & 49 & 2,782 \\
\hline 432825102013301 & $40 \mathrm{~N} 40 \mathrm{~W} 2 \mathrm{BB}$ & 71 & 10140202 & 122ARKR & 273 & 3,055 \\
\hline 432825102015201 & 40N40W 3AA & 71 & 10140202 & 122ARKR & 275 & 3,025 \\
\hline 432829101321001 & $40 \mathrm{~N} 36 \mathrm{~W} 2 \mathrm{AA}$ & 71 & 10140202 & 122ARKR & 170 & 2,736 \\
\hline 432830101561601 & $40 \mathrm{~N} 39 \mathrm{~W} 4 \mathrm{AB}$ & 71 & 10140202 & 122ARKR & 300 & 2,923 \\
\hline 432830102132001 & $40 \mathrm{~N} 41 \mathrm{~W} 6 \mathrm{BA}$ & 113 & 10140201 & $122 \mathrm{ARKR}$ & 80 & 2,791 \\
\hline 432833101075001 & $40 \mathrm{~N} 32 \mathrm{~W} 5 \mathrm{BBA}$ & 95 & 10140202 & $122 \mathrm{ARKR}$ & 19 & 2,580 \\
\hline 432844101563301 & 41N39W34DD & 71 & 10140202 & 122ARKR & 320 & 2,833 \\
\hline 432852101553101 & $41 N 39 W 35 D B$ & 71 & 10140202 & 122ARKR & 360 & 2,900 \\
\hline 432909101363101 & $41 N 36 \mathrm{~W} 33 \mathrm{AC}$ & 71 & 10140202 & 122ARKR & 200 & 2,854 \\
\hline 432912101264201 & $41 N 35 W 35 A$ & 71 & 10140202 & 122ARKR & 113 & 2,550 \\
\hline 432915102154201 & $41 \mathrm{~N} 42 \mathrm{~W} 36 \mathrm{~A}$ & 113 & 10140201 & 122ARKR & 62 & 2,925 \\
\hline
\end{tabular}


Table 57. Selected descriptive data (from the U.S. Geological Survey database) for ground-water sites in the High Plains aquifer in South Dakota-Continued

[County code: 7 = Bennett County, $53=$ Gregory County, $71=$ Jackson County, $95=$ Mellette County, $113=$ Shannon County, and $121=$ Todd County; Datum, National Geodetic Vertical Datum; 1210GLL, Ogallala Formation; 122ARKR, Arikaree Formation; --, no data]

\begin{tabular}{|c|c|c|c|c|c|c|}
\hline Site number & Local identifier & $\begin{array}{c}\text { County } \\
\text { code }\end{array}$ & Hydrologic unit & Geologic unit & $\begin{array}{c}\text { Depth of well, } \\
\text { total } \\
\text { (feet) }\end{array}$ & $\begin{array}{c}\text { Altitude of land } \\
\text { surface, datum } \\
\text { (feet) }\end{array}$ \\
\hline 432920101442801 & 41N37W29DD & 71 & 10140202 & 122ARKR & 180 & 2,820 \\
\hline 432920101573801 & 41N39W33AA & 71 & 10140202 & 122ARKR & 310 & 2,840 \\
\hline 432921101224301 & $41 N 34 \mathrm{~W} 33 \mathrm{BB}$ & 71 & 10140202 & 122ARKR & 30 & 2,568 \\
\hline 432921101333801 & 41N36W35AA & 71 & 10140202 & 122ARKR & 144 & 2,700 \\
\hline 432921101351301 & 41N36W34AB & 71 & 10140202 & 122ARKR & 210 & 2,700 \\
\hline 432921102130901 & $41 \mathrm{~N} 41 \mathrm{~W} 32 \mathrm{AACD}$ & 113 & 10140201 & 122ARKR & 40 & 2,772 \\
\hline 432921102160801 & 41N42W36BA & 113 & 10140201 & 122ARKR & 120 & 2,979 \\
\hline 432922101372501 & 41N36W32AA & 71 & 10140202 & 122ARKR & 250 & 2,828 \\
\hline 432923101594701 & $41 \mathrm{~N} 39 \mathrm{~W} 32 \mathrm{BB}$ & 71 & 10140202 & 122ARKR & 290 & 3,000 \\
\hline 432930101341501 & $41 \mathrm{~N} 36 \mathrm{~W} 26 \mathrm{CD}$ & 71 & 10140202 & 122ARKR & 100 & 2,625 \\
\hline 432934101235501 & $41 \mathrm{~N} 34 \mathrm{~W} 29 \mathrm{CC}$ & 71 & 10140202 & 122ARKR & -- & 2,509 \\
\hline 432934101573001 & $41 \mathrm{~N} 39 \mathrm{~W} 27 \mathrm{CC}$ & 71 & 10140202 & 122ARKR & 320 & 2,850 \\
\hline 432941102154201 & $41 N 42 W 25 D$ & 113 & 10140201 & 122ARKR & -- & 2,931 \\
\hline 432941102154202 & $41 N 42 W 25 D 2$ & 113 & 10140201 & 122ARKR & 38 & 2,931 \\
\hline 432948101380101 & $41 \mathrm{~N} 36 \mathrm{~W} 29 \mathrm{CA}$ & 71 & 10140202 & 122ARKR & 150 & 2,824 \\
\hline 432951101350901 & 41N36W27DA & 71 & 10140202 & 122ARKR & 260 & 2,500 \\
\hline 432954102182201 & $41 \mathrm{~N} 42 \mathrm{~W} 27$ & 113 & 10140201 & 122ARKR & -- & 2,948 \\
\hline 433000101354701 & $41 \mathrm{~N} 36 \mathrm{~W} 27 \mathrm{BC}$ & 71 & 10140202 & 122ARKR & 250 & 2,700 \\
\hline 433001101383701 & 41N36W30AD & 71 & 10140202 & 122ARKR & 150 & 2,837 \\
\hline 433001101435901 & $41 \mathrm{~N} 37 \mathrm{~W} 28 \mathrm{BD}$ & 71 & 10140202 & 122ARKR & -- & 2,850 \\
\hline 433002102015801 & 41N40W25BD & 71 & 10140202 & 122ARKR & -- & 2,950 \\
\hline 433002102115501 & 41N41W28AD & 113 & 10140201 & 122ARKR & 180 & 2,790 \\
\hline 433010101351401 & 41N36W27ABD & 71 & 10140202 & 122ARKR & 150 & 2,772 \\
\hline 433013101542801 & $41 \mathrm{~N} 39 \mathrm{~W} 25 \mathrm{AB}$ & 71 & 10140202 & 122ARKR & 100 & 2,755 \\
\hline 433014101451101 & $41 N 37$ W29BA & 71 & 10140202 & 122ARKR & 450 & 2,820 \\
\hline 433020101394201 & 41N37W24DDDD & 71 & 10140202 & 122ARKR & 305 & 2,855 \\
\hline 433026101303201 & $41 \mathrm{~N} 35 \mathrm{~W} 20 \mathrm{DC}$ & 71 & 10140202 & 122ARKR & 100 & 2,552 \\
\hline 433026102012001 & 41N40W24DD & 71 & 10140202 & 122ARKR & 140 & 2,950 \\
\hline 433027101393101 & $41 \mathrm{~N} 36 \mathrm{~W} 19 \mathrm{CC}$ & 71 & 10140202 & 122ARKR & 150 & 2,840 \\
\hline 433029101302001 & 41N35W20DC2 & 71 & 10140202 & 122ARKR & 100 & 2,530 \\
\hline 433029101550201 & $41 \mathrm{~N} 39 \mathrm{~W} 24 \mathrm{CC}$ & 71 & 10140202 & 122ARKR & 100 & 2,801 \\
\hline 433039101312601 & 41N35W19DA & 71 & 10140202 & 122ARKR & 115 & 2,483 \\
\hline 433045101270401 & $41 \mathrm{~N} 35 \mathrm{~W} 23$ & 71 & 10140202 & 122ARKR & -- & 2,542 \\
\hline 433047101450201 & 41 N37W20 & 71 & 10140202 & 122ARKR & 140 & 2,802 \\
\hline 433052101163601 & $41 N 33 W 20 C D B B$ & 71 & 10140202 & 122ARKR & 175 & 2,562 \\
\hline 433055101081901 & $41 \mathrm{~N} 32 \mathrm{~W} 21 \mathrm{BCBC}$ & 95 & 10140202 & 122ARKR & -- & 2,575 \\
\hline 433104101350001 & $41 \mathrm{~N} 36 \mathrm{~W} 22 \mathrm{AA}$ & 71 & 10140202 & 122ARKR & 240 & 2,800 \\
\hline 433104101452801 & 41N37W20BB & 71 & 10140202 & 122ARKR & 55 & 2,820 \\
\hline
\end{tabular}


Table 57. Selected descriptive data (from the U.S. Geological Survey database) for ground-water sites in the High Plains aquifer in South Dakota-Continued

[County code: 7 = Bennett County, $53=$ Gregory County, $71=$ Jackson County, $95=$ Mellette County, $113=$ Shannon County, and $121=$ Todd County; Datum, National Geodetic Vertical Datum; 1210GLL, Ogallala Formation; 122ARKR, Arikaree Formation; --, no data]

\begin{tabular}{|c|c|c|c|c|c|c|}
\hline Site number & Local identifier & $\begin{array}{c}\text { County } \\
\text { code }\end{array}$ & Hydrologic unit & Geologic unit & $\begin{array}{c}\text { Depth of well, } \\
\text { total } \\
\text { (feet) }\end{array}$ & $\begin{array}{l}\text { Altitude of land } \\
\text { surface, datum } \\
\text { (feet) }\end{array}$ \\
\hline 433105101214901 & 41N34W21AA & 71 & 10140202 & 122ARKR & 84 & 2,601 \\
\hline 433105101322001 & $41 N 35 W 19 B B$ & 71 & 10140202 & 122ARKR & 115 & 2,493 \\
\hline 433107102124901 & $41 \mathrm{~N} 41 \mathrm{~W} 21 \mathrm{BB}$ & 113 & 10140201 & 122ARKR & 340 & 2,741 \\
\hline 433108101563301 & $41 \mathrm{~N} 39 \mathrm{~W} 22 \mathrm{AA}$ & 71 & 10140202 & 122ARKR & 167 & 2,880 \\
\hline 433108101595201 & 41N39W20BB & 71 & 10140202 & 122ARKR & 196 & 2,915 \\
\hline 433108101595202 & $41 \mathrm{~N} 39 \mathrm{~W} 20 \mathrm{BB} 2$ & 71 & 10140202 & 122ARKR & 200 & 2,915 \\
\hline 433111102102201 & $41 \mathrm{~N} 41 \mathrm{~W} 23 \mathrm{BABB}$ & 113 & 10140201 & 122ARKR & 82 & 2,970 \\
\hline 433118101214901 & $41 \mathrm{~N} 34 \mathrm{~W} 16 \mathrm{DD}$ & 71 & 10140202 & 122ARKR & 110 & 2,585 \\
\hline 433118101261901 & $41 \mathrm{~N} 35 \mathrm{~W} 13 \mathrm{CC}$ & 71 & 10140202 & 122ARKR & 85 & 2,575 \\
\hline 433119101381901 & 41N36W17CC & 71 & 10140202 & 122ARKR & 130 & 2,750 \\
\hline 433119101432301 & 41N37W16DD & 71 & 10140202 & 122ARKR & 180 & 2,785 \\
\hline 433119101432302 & 41N37W16DD2 & 71 & 10140202 & 122ARKR & 100 & 2,785 \\
\hline 433119101452901 & $41 \mathrm{~N} 37 \mathrm{~W} 17 \mathrm{CC}$ & 71 & 10140202 & 122ARKR & 350 & 2,800 \\
\hline 433131101220701 & $41 \mathrm{~N} 34 \mathrm{~W} 16 \mathrm{DB}$ & 71 & 10140202 & 122ARKR & 95 & 2,565 \\
\hline 433132101454601 & $41 \mathrm{~N} 37 \mathrm{~W} 18 \mathrm{DA}$ & 71 & 10140202 & 122ARKR & 100 & 2,795 \\
\hline 433133102112701 & $41 \mathrm{~N} 41 \mathrm{~W} 15 \mathrm{CA}$ & 113 & 10140201 & 122ARKR & 500 & 2,830 \\
\hline 433139101243001 & 41N34W18DC & 71 & 10140202 & 122ARKR & 120 & 2,500 \\
\hline 433141101250001 & $41 \mathrm{~N} 34 \mathrm{~W} 18 \mathrm{BC}$ & 71 & 10140202 & 122ARKR & 100 & 2,470 \\
\hline 433150102133001 & 40N41W17AA & 113 & 10140201 & 122ARKR & 90 & 3,000 \\
\hline 433156101442801 & 41N37W16BB & 71 & 10140202 & 122ARKR & 234 & 2,715 \\
\hline 433157101462301 & $41 \mathrm{~N} 37 \mathrm{~W} 18 \mathrm{BA}$ & 71 & 10140202 & 122ARKR & 350 & 2,700 \\
\hline 433206101251601 & $41 \mathrm{~N} 35 \mathrm{~W} 12 \mathrm{DD}$ & 71 & 10140202 & 122ARKR & 100 & 2,500 \\
\hline 433211101572701 & $41 \mathrm{~N} 39 \mathrm{~W} 10 \mathrm{CC}$ & 71 & 10140202 & 122ARKR & -- & 2,940 \\
\hline 433218101431601 & 41N37W 9DA & 71 & 10140202 & 122ARKR & 180 & 2,730 \\
\hline 433236101181202 & $41 \mathrm{~N} 34 \mathrm{~W} 12 \mathrm{AD} 2$ & 71 & 10140202 & 122ARKR & 85 & 2,641 \\
\hline 433237101380101 & $41 \mathrm{~N} 36 \mathrm{~W} 8 \mathrm{BD}$ & 71 & 10140202 & 122ARKR & 160 & 2,775 \\
\hline 433250101361301 & 41N36W 9AA & 71 & 10140202 & 122ARKR & 200 & 2,783 \\
\hline 433255101384301 & $42 \mathrm{~N} 36 \mathrm{~W} 31 \mathrm{DD}$ & 71 & 10140202 & 122ARKR & 170 & 2,680 \\
\hline 433302101235501 & $41 \mathrm{~N} 34 \mathrm{~W} 5 \mathrm{CC}$ & 71 & 10140202 & 122ARKR & 80 & 2,542 \\
\hline 433303101460401 & $41 \mathrm{~N} 37 \mathrm{~W} 6 \mathrm{DC}$ & 71 & 10140202 & 122ARKR & 74 & 2,750 \\
\hline 433304101505101 & 41N38W 4DC & 71 & 10140202 & 122ARKR & 75 & 2,700 \\
\hline 433310101361001 & 41N36W 4DA & 71 & 10140202 & 122ARKR & 160 & 2,600 \\
\hline 433321101244001 & 41N34W 6 & 71 & 10140202 & 122ARKR & -- & 2,477 \\
\hline 433328101322001 & $41 \mathrm{~N} 35 \mathrm{~W} 6 \mathrm{BC}$ & 71 & 10140202 & 122ARKR & 120 & 2,627 \\
\hline 433335101180801 & $42 \mathrm{~N} 34 \mathrm{~W} 36 \mathrm{DD}$ & 71 & 10140202 & 122ARKR & 510 & 2,500 \\
\hline 433342101385501 & $41 \mathrm{~N} 36 \mathrm{~W} 6 \mathrm{AB}$ & 71 & 10140202 & 122ARKR & 160 & 2,670 \\
\hline 433342101415401 & $41 \mathrm{~N} 37 \mathrm{~W} 2 \mathrm{BB}$ & 71 & 10140202 & 122ARKR & -- & 2,725 \\
\hline 433355101364901 & $42 \mathrm{~N} 36 \mathrm{~W} 33 \mathrm{CD}$ & 71 & 10140202 & 122ARKR & 120 & 2,744 \\
\hline
\end{tabular}


Table 57. Selected descriptive data (from the U.S. Geological Survey database) for ground-water sites in the High Plains aquifer in South Dakota-Continued

[County code: 7 = Bennett County, $53=$ Gregory County, $71=$ Jackson County, $95=$ Mellette County, $113=$ Shannon County, and $121=$ Todd County; Datum, National Geodetic Vertical Datum; 121OGLL, Ogallala Formation; 122ARKR, Arikaree Formation; --, no data]

\begin{tabular}{|c|c|c|c|c|c|c|}
\hline Site number & Local identifier & $\begin{array}{c}\text { County } \\
\text { code }\end{array}$ & Hydrologic unit & Geologic unit & $\begin{array}{c}\text { Depth of well, } \\
\text { total } \\
\text { (feet) }\end{array}$ & $\begin{array}{l}\text { Altitude of land } \\
\text { surface, datum } \\
\text { (feet) }\end{array}$ \\
\hline 433356101462201 & $42 \mathrm{~N} 37 \mathrm{~W} 31 \mathrm{CD}$ & 71 & 10140202 & 122ARKR & 54 & 2,700 \\
\hline 433357101525401 & $42 \mathrm{~N} 38 \mathrm{~W} 32 \mathrm{CD}$ & 71 & 10140202 & 122ARKR & 100 & 2,800 \\
\hline 433357101550201 & $42 \mathrm{~N} 39 \mathrm{~W} 36 \mathrm{CC}$ & 71 & 10140202 & 122ARKR & 160 & 2,910 \\
\hline 433407101323601 & 42N36W36DA & 71 & 10140202 & 122ARKR & 80 & 2,789 \\
\hline 433407101380001 & $42 \mathrm{~N} 36 \mathrm{~W} 32 \mathrm{CA}$ & 71 & 10140202 & 122ARKR & 150 & 2,740 \\
\hline 433407101393101 & $42 \mathrm{~N} 36 \mathrm{~W} 31 \mathrm{CB}$ & 71 & 10140202 & 122ARKR & 245 & 2,650 \\
\hline 433410101535001 & $42 \mathrm{~N} 38 \mathrm{~W} 31 \mathrm{CB}$ & 71 & 10140202 & 122ARKR & 246 & 2,789 \\
\hline 433417101400001 & $42 \mathrm{~N} 37 \mathrm{~W} 36 \mathrm{ACDD}$ & 71 & 10140202 & 122ARKR & -- & 2,660 \\
\hline 433421101385501 & $42 \mathrm{~N} 36 \mathrm{~W} 31 \mathrm{AC}$ & 71 & 10140202 & 122ARKR & 90 & 2,650 \\
\hline 433422101394901 & 42N37W36AD & 71 & 10140202 & 122ARKR & -- & 2,600 \\
\hline 433422101394902 & $42 \mathrm{~N} 37 \mathrm{~W} 36 \mathrm{AD} 2$ & 71 & 10140202 & 122ARKR & -- & 2,600 \\
\hline 433422101421201 & 42N37W34AD & 71 & 10140202 & 122ARKR & 100 & 2,718 \\
\hline 433422101421202 & $42 \mathrm{~N} 37 \mathrm{~W} 34 \mathrm{AD} 2$ & 71 & 10140202 & 122ARKR & 98 & 2,718 \\
\hline 433422101443501 & $42 \mathrm{~N} 37 \mathrm{~W} 32 \mathrm{AD}$ & 71 & 10140202 & 122ARKR & 50 & 2,600 \\
\hline 433427102100501 & $42 \mathrm{~N} 41 \mathrm{~W} 35 \mathrm{ACBB}$ & 113 & 10140201 & 122ARKR & 22 & 2,898 \\
\hline 433434101363101 & $42 \mathrm{~N} 36 \mathrm{~W} 33 \mathrm{AB}$ & 71 & 10140202 & 122ARKR & 120 & 2,675 \\
\hline 433443101393201 & $42 \mathrm{~N} 36 \mathrm{~W} 30 \mathrm{CB}$ & 71 & 10140202 & 122ARKR & 90 & 2,650 \\
\hline 433445101321501 & $42 \mathrm{~N} 35 \mathrm{~W} 30 \mathrm{CC}$ & 71 & 10140202 & 122ARKR & 310 & 2,840 \\
\hline 433500101352001 & 42N36W27DB & 71 & 10140202 & $122 \mathrm{ARKR}$ & 140 & 2,500 \\
\hline 433501101330101 & $42 \mathrm{~N} 36 \mathrm{~W} 25 \mathrm{ACCC}$ & 71 & 10140202 & $122 \mathrm{ARKR}$ & -. & 2,650 \\
\hline 433505101390601 & $42 \mathrm{~N} 36 \mathrm{~W} 30 \mathrm{CAAA}$ & 71 & 10140202 & 122ARKR & 125 & 2,685 \\
\hline 433513101385501 & $42 \mathrm{~N} 36 \mathrm{~W} 30 \mathrm{AC}$ & 71 & 10140202 & 122ARKR & -- & 2,700 \\
\hline 433527101415401 & $42 \mathrm{~N} 37 \mathrm{~W} 26 \mathrm{BB}$ & 71 & 10140202 & 122ARKR & -- & 2,752 \\
\hline 433539101334801 & 42N36W23DD & 71 & 10140202 & 122ARKR & 134 & 2,575 \\
\hline 433539101334802 & $42 \mathrm{~N} 36 \mathrm{~W} 23 \mathrm{DD} 2$ & 71 & 10140202 & 122ARKR & 108 & 2,575 \\
\hline 433611102072601 & $42 \mathrm{~N} 40 \mathrm{~W} 19 \mathrm{~A}$ & 71 & 10140201 & 122ARKR & -- & 2,930 \\
\hline 433657101564201 & $42 \mathrm{~N} 39 \mathrm{~W} 15 \mathrm{AC}$ & 71 & 10140202 & 122ARKR & 110 & 2,750 \\
\hline 433659101565101 & $42 \mathrm{~N} 39 \mathrm{~W} 15 \mathrm{AC} 2$ & 71 & 10140202 & 122ARKR & 110 & 2,700 \\
\hline
\end{tabular}




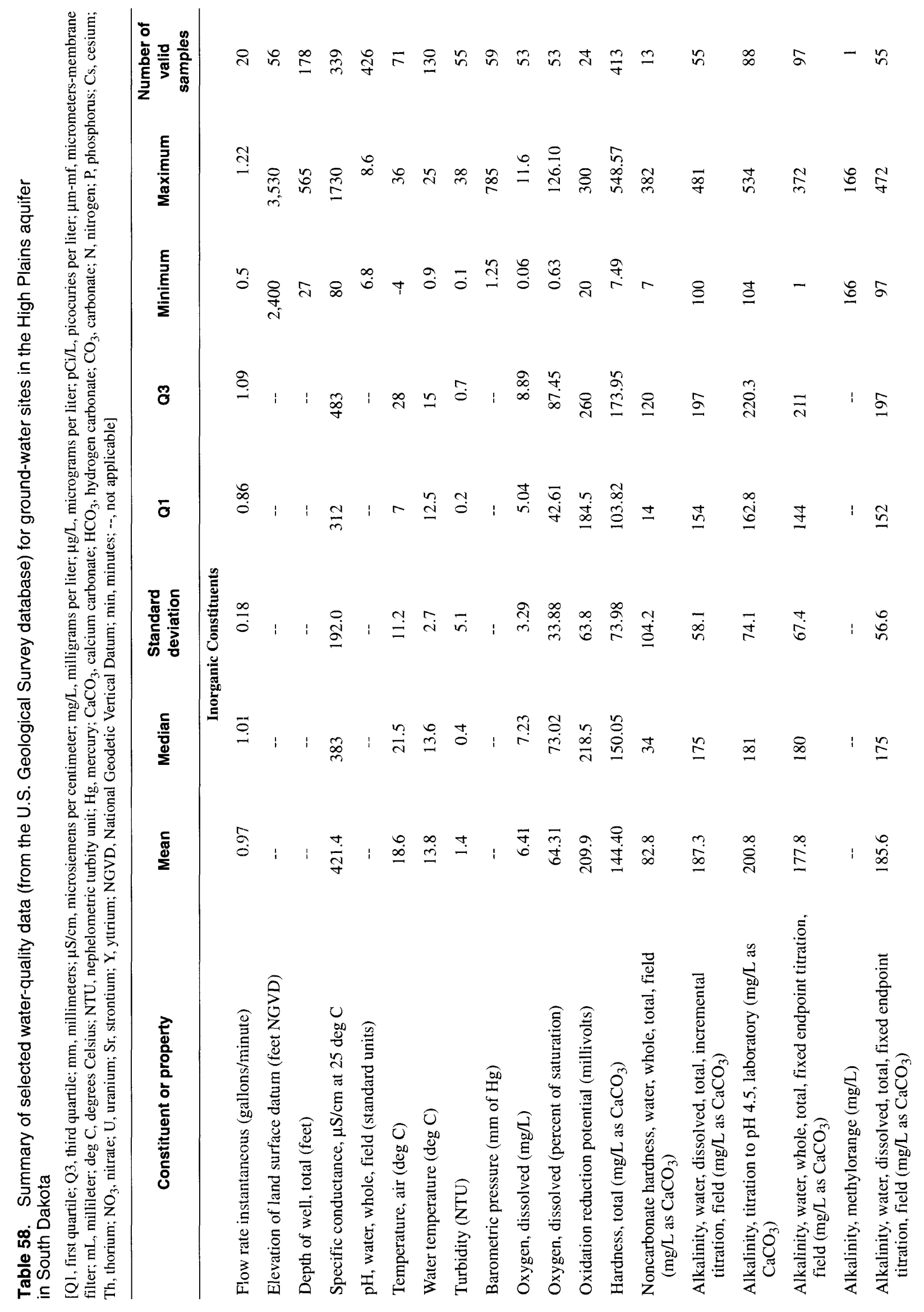




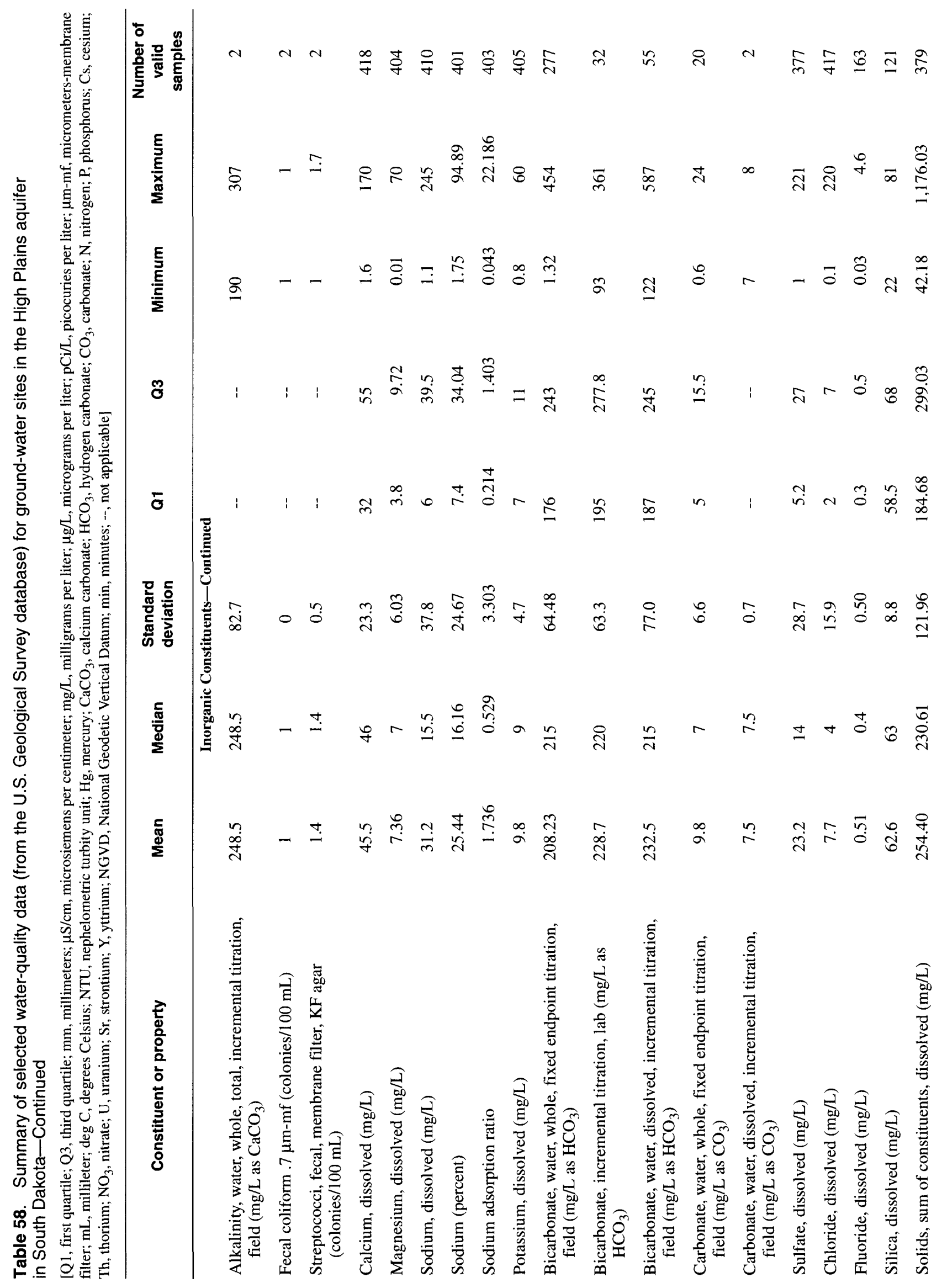




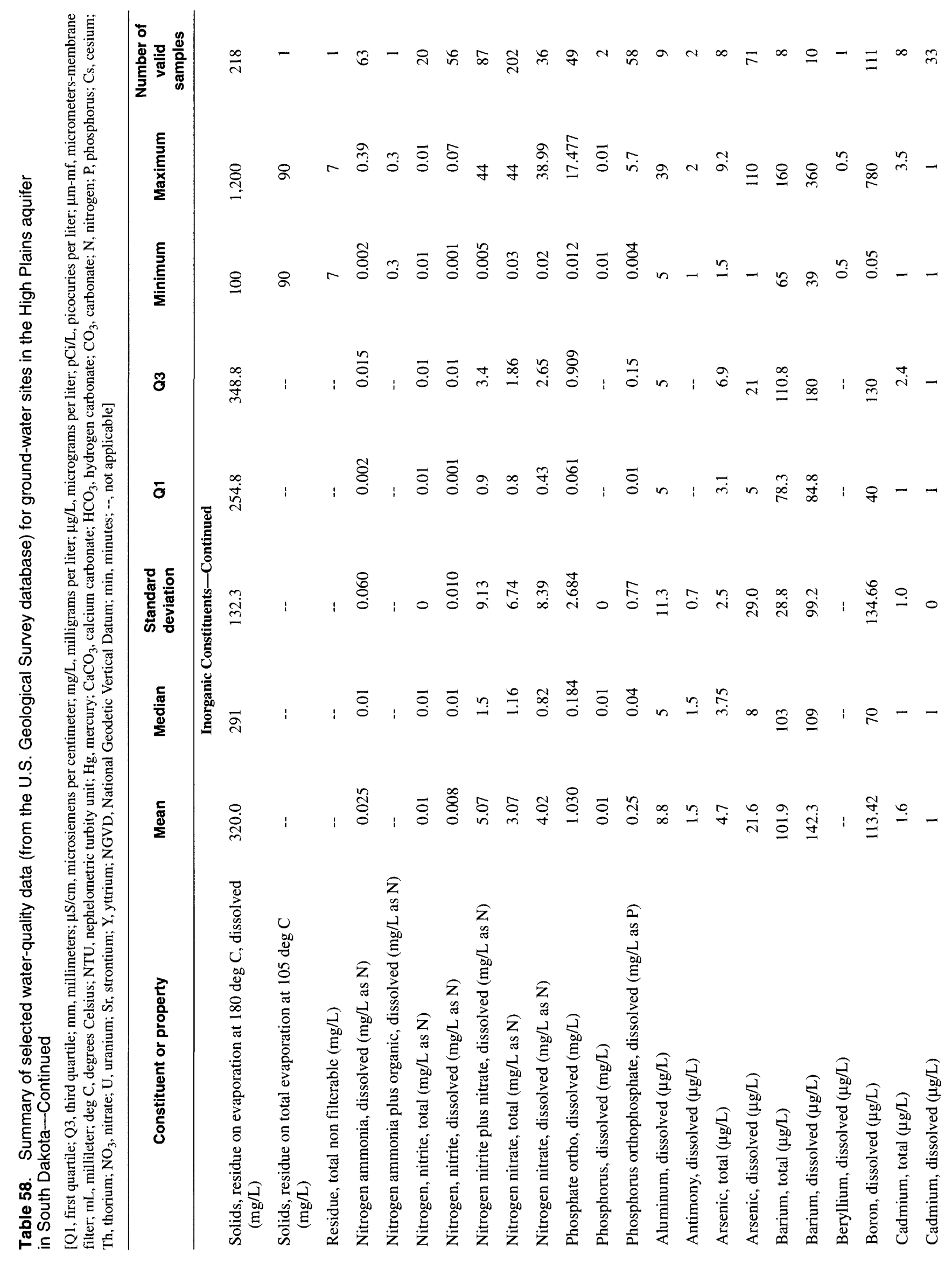




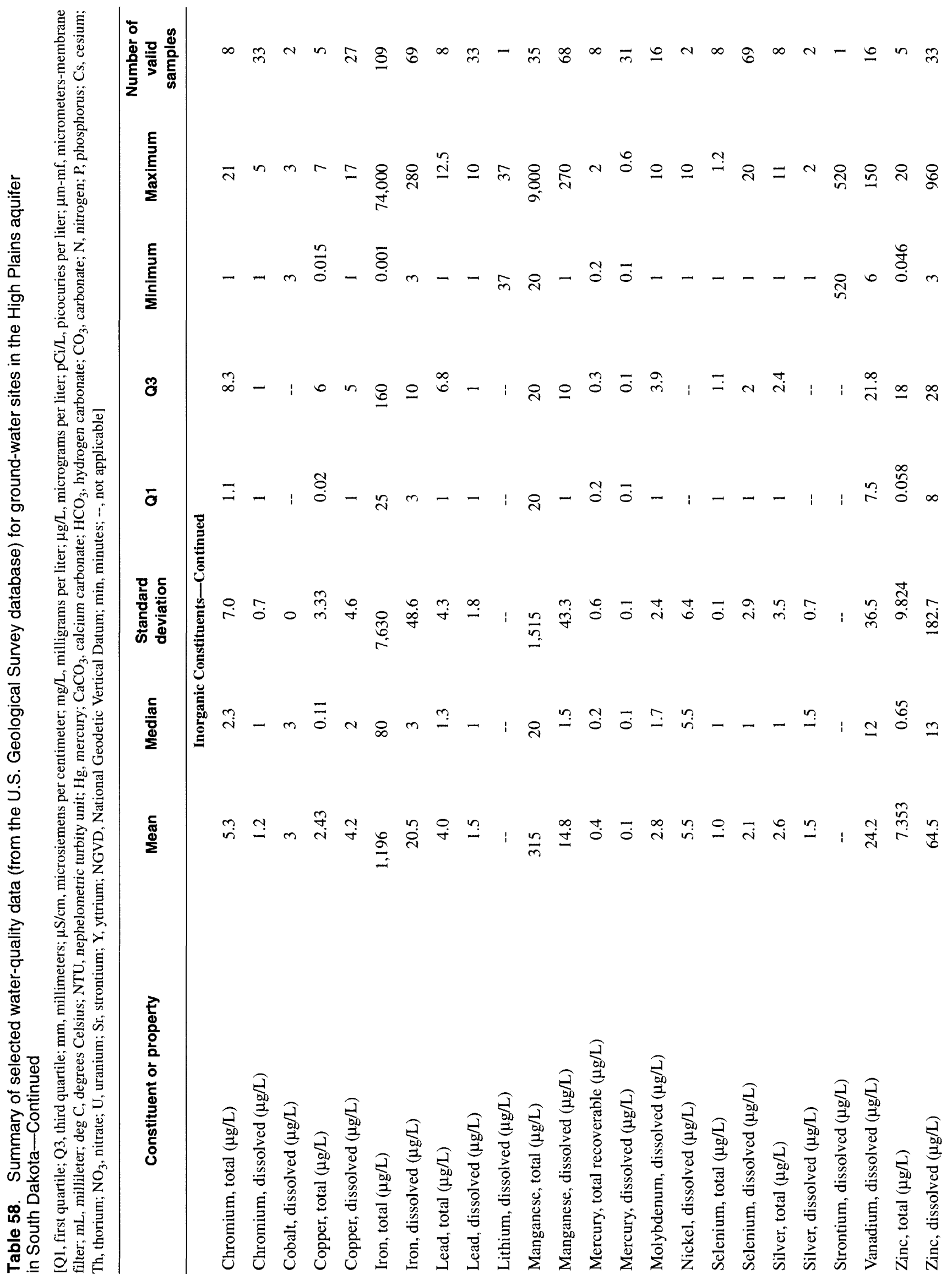




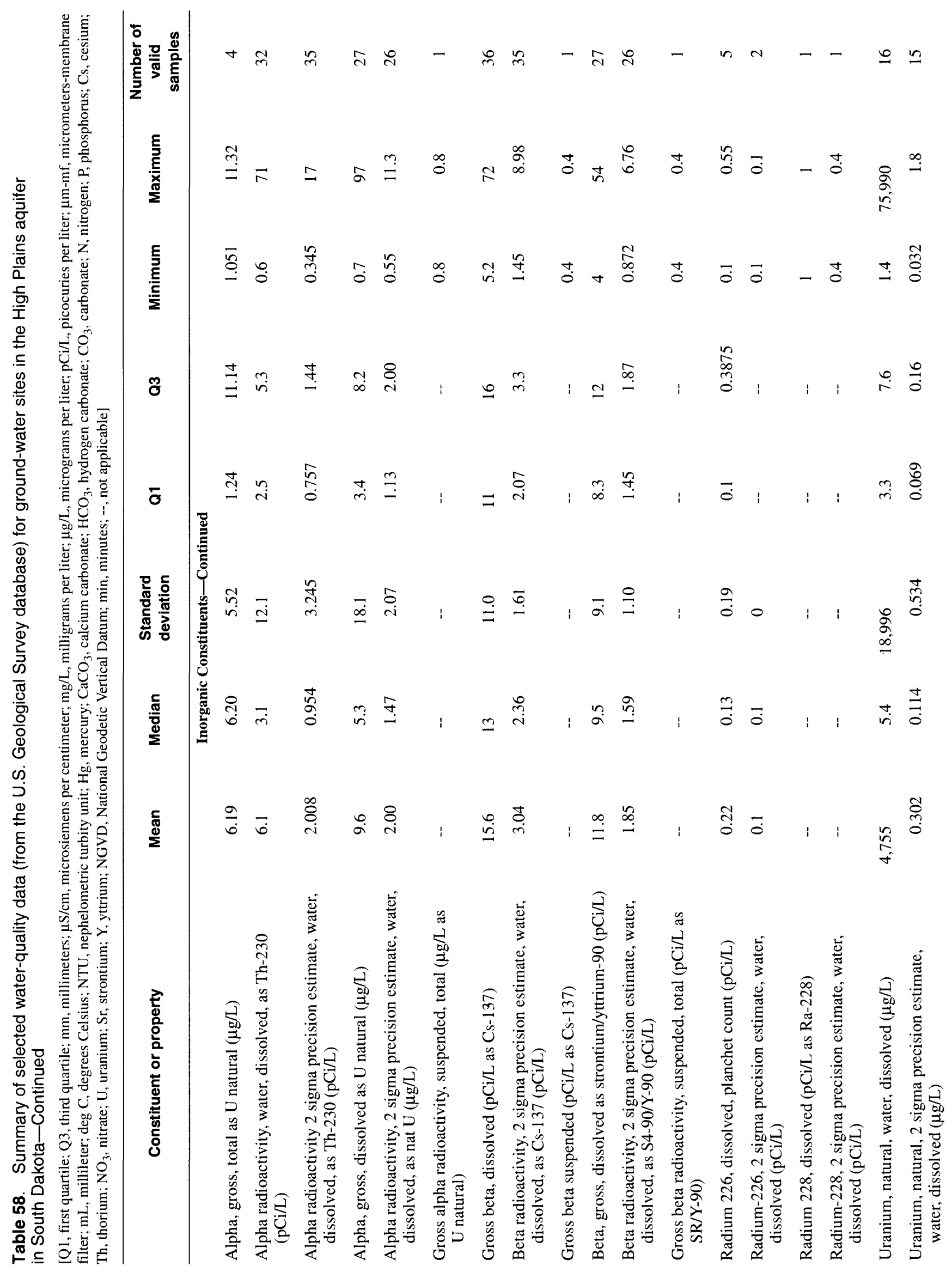




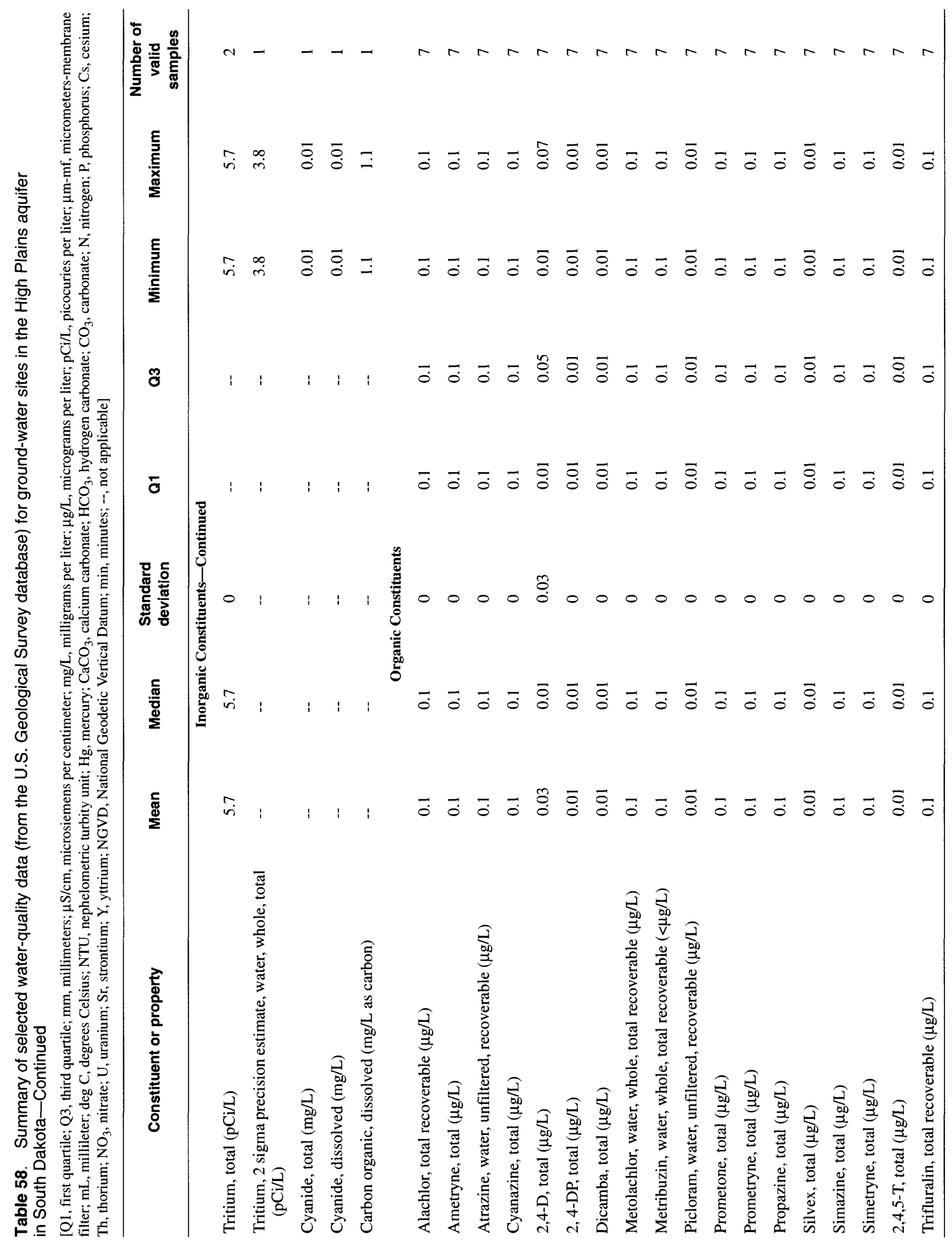


Table 59. Estimated total water use in 1995 within the Little White River Basin in South Dakota

\section{Description}

Total ground-water withdrawals, fresh

Total ground-water withdrawals, saline

Total withdrawals, ground water

Total surface-water withdrawals, fresh

Total surface-water withdrawals, saline

Total withdrawals, surface water

Total withdrawals, fresh

Total withdrawals, saline

Total withdrawals

Consumptive use, fresh

Consumptive use, saline

Consumptive use, total

Reclaimed wastewater

Conveyance losses
Quantity

(million gallons per day)

7.08

.00

7.08

.47

.00

.47

7.55

.00

7.55

5.96

.00

5.96

.00

.19 


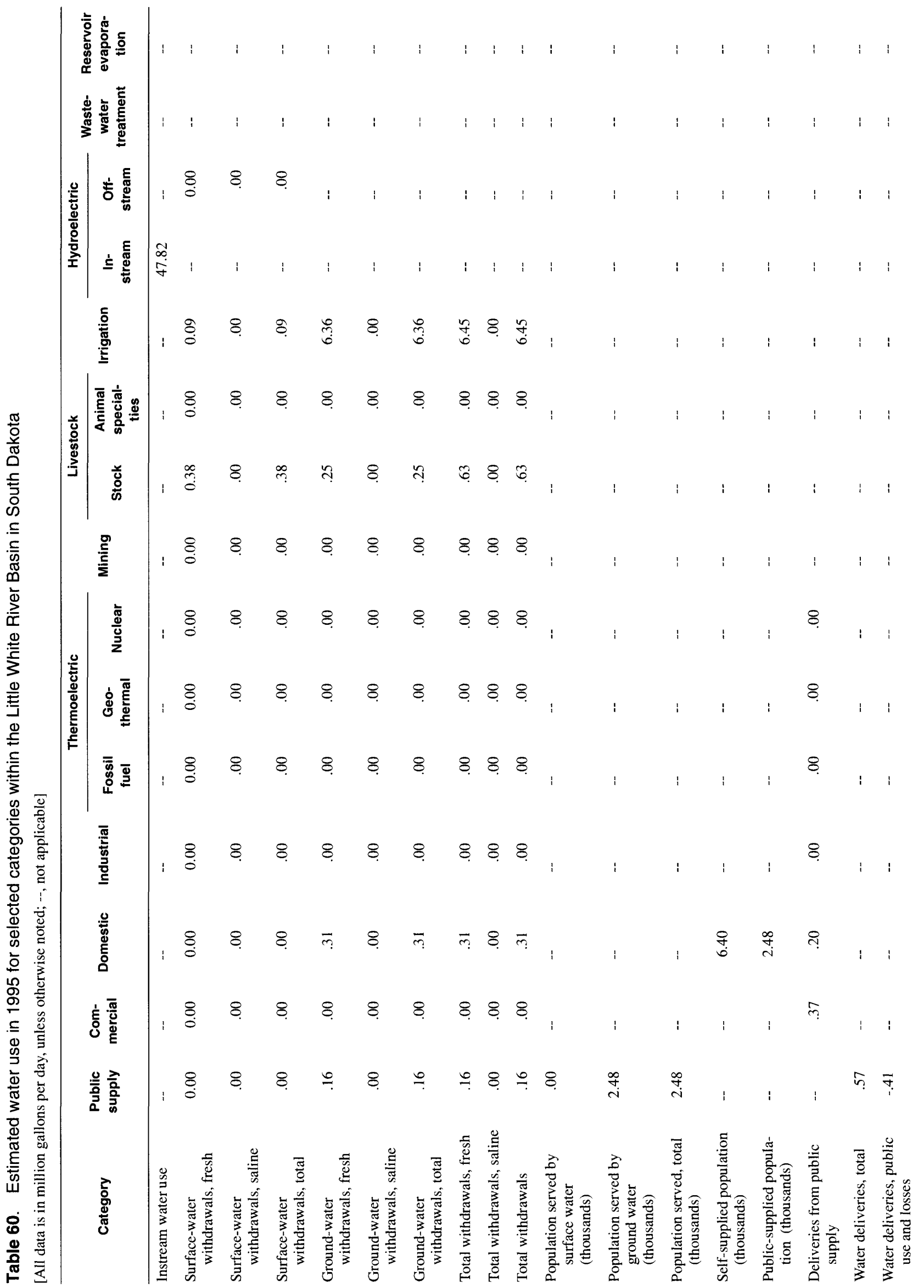




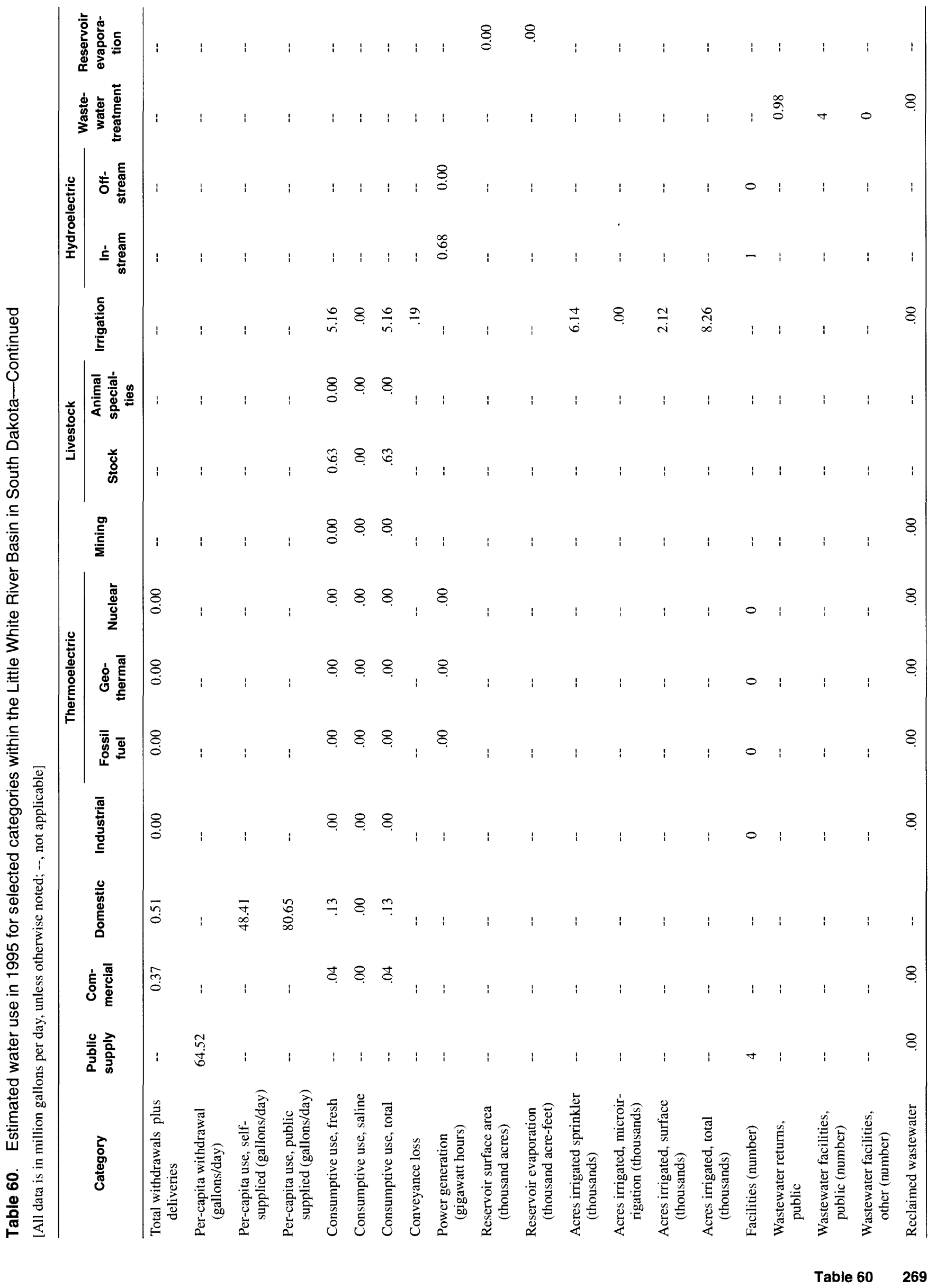


Table 61. Selected water-quality data (from the South Dakota Geological Survey) for ground-water sites in the Little White River Basin, South Dakota and Nebraska

[SDGS, South Dakota Geological Survey; dd, decimal degrees; milligrams per liter; $\mu \mathrm{S} / \mathrm{cm}$, microsiemens per centimeter; $\mu \mathrm{g} / \mathrm{L}$, micrograms per liter; deg $\mathrm{C}$, degrees Celsius; $\mathrm{CaCO}_{3}$, calcium carbonate; $\mathrm{PVC}, \mathrm{SCH} 40$, poly vinyl chloride, schedule 40; lab, laboratory; <, less than; --, no data]

\begin{tabular}{|c|c|c|c|c|c|}
\hline \multirow{2}{*}{ Description } & \multicolumn{5}{|c|}{ Sample number } \\
\hline & SWM-95-061 & SWM-95-150 & SWM-96-046 & SWM-95-062 & SWM-95-151 \\
\hline SDGS well name & R20-94-28 & R20-94-28 & R20-94-28 & R20-94-29 & R20-94-29 \\
\hline Owner & SDGS & SDGS & SDGS & SDGS & SDGS \\
\hline Latitude (dd) & 43.0128 & 43.0128 & 43.0128 & 43.0128 & 43.0128 \\
\hline Longitude (dd) & 101.2921 & 101.2921 & 101.2921 & 101.2921 & 101.2921 \\
\hline Aquifer & Sandhills & Sandhills & Sandhills & Sandhills & Sandhills \\
\hline Ground surface elevation (feet) & 3,228 & 3,228 & 3,228 & 3,228 & 3,228 \\
\hline Well depth (feet from casing top) & 41.9 & 41.9 & 41.9 & 23.8 & 23.8 \\
\hline Casing top elevation (feet) & $3,230.18$ & $3,230.18$ & $3,230.18$ & $3,230.24$ & $3,230.24$ \\
\hline Depth to water (feet from casing top) & 12.25 & 11.40 & 11.72 & 11.20 & 10.70 \\
\hline Ground-water elevation (feet) & $3,217.93$ & $3,218.78$ & $3,218.46$ & $3,219.04$ & $32,19.54$ \\
\hline Casing type & PVC, SCH.40 & PVC, SCH.40 & PVC, SCH.40 & PVC, SCH.40 & PVC, SCH.40 \\
\hline Collection date & $05 / 17 / 1995$ & $08 / 08 / 1995$ & $05 / 06 / 1996$ & $05 / 17 / 1995$ & 08/08/1995 \\
\hline $\begin{array}{l}\text { Lab specific conductance }(\mu \mathrm{S} / \mathrm{cm} \text { at } \\
25 \mathrm{deg} \text { C) }\end{array}$ & 147 & 142 & 131 & 504 & 360 \\
\hline Lab pH (standard units) & 6.71 & 6.66 & 6.86 & 7.74 & 7.73 \\
\hline Hardness $\left(\mathrm{mg} / \mathrm{L}\right.$ as $\mathrm{CaCO}_{3}$ ) & 47 & 44 & 44 & 150 & 92 \\
\hline Hydrogen carbonate $(\mathrm{mg} / \mathrm{L})$ & 78 & 74 & 70.2 & 140 & 151 \\
\hline Carbonate $(\mathrm{mg} / \mathrm{L})$ & -- & - & 0 & & -- \\
\hline Lab alkalinity $\left(\mathrm{mg} / \mathrm{L}\right.$ as $\left.\mathrm{CaCO}_{3}\right)$ & 64 & 61 & 57.5 & 115 & 124 \\
\hline Calcium (mg/L) & 15 & 14 & 13.5 & 51 & 32 \\
\hline Magnesium $(\mathrm{mg} / \mathrm{L})$ & 2.3 & 2.1 & 2.4 & 4.9 & 2.9 \\
\hline Sodium (mg/L) & 9.8 & 9.7 & 10.5 & 49 & 43 \\
\hline Potassium $(\mathrm{mg} / \mathrm{L})$ & 5.2 & 5.6 & 5.7 & 3.5 & 3.8 \\
\hline Sulfate $(\mathrm{mg} / \mathrm{L})$ & 5.3 & 5.8 & $<10.0$ & 127 & 55 \\
\hline Chloride (mg/L) & 0.7 & 0.6 & 0.8 & 1.7 & 0.8 \\
\hline Fluoride $(\mathrm{mg} / \mathrm{L})$ & 0.15 & 0.14 & 0.18 & 0.17 & 0.26 \\
\hline $\begin{array}{l}\text { Lab total dissolved solids }(\mathrm{mg} / \mathrm{L} \text { as } \\
\left.\mathrm{CaCO}_{3}\right)\end{array}$ & 115 & 112 & 113 & 329 & 226 \\
\hline Ammonia-nitrogen $(\mathrm{mg} / \mathrm{L})$ & $<0.05$ & $<0.05$ & $<0.02$ & $<0.05$ & $<0.05$ \\
\hline $\begin{array}{l}\text { Nitrate-nitrogen + nitrite-nitrogen } \\
\quad(\mathrm{mg} / \mathrm{L})\end{array}$ & 0.80 & 0.66 & 0.9 & 3.25 & 1.09 \\
\hline Total phosphorus (mg/L) & -- & -- & -- & -- & -- \\
\hline Antimony $(\mu \mathrm{g} / \mathrm{L})$ & $<0.01$ & -- & -- & $<0.01$ & -- \\
\hline Arsenic $(\mu \mathrm{g} / \mathrm{L})$ & $<0.01$ & -- & -- & $<0.01$ & -- \\
\hline Barium $(\mu \mathrm{g} / \mathrm{L})$ & 0.09 & -- & -- & 0.32 & -- \\
\hline Beryllium $(\mu \mathrm{g} / \mathrm{L})$ & $<0.002$ & -- & -- & $<0.002$ & -- \\
\hline
\end{tabular}


Table 61. Selected water-quality data (from the South Dakota Geological Survey) for ground-water sites in the Little White River Basin, South Dakota and Nebraska-Continued

[SDGS, South Dakota Geological Survey; dd, decimal degrees; milligrams per liter; $\mu \mathrm{S} / \mathrm{cm}$, microsiemens per centimeter; $\mu \mathrm{g} / \mathrm{L}$, micrograms per liter; $\operatorname{deg} \mathrm{C}$, degrees Celsius; $\mathrm{CaCO}_{3}$, calcium carbonate; $\mathrm{PVC}, \mathrm{SCH} 40$, poly vinyl chloride, schedule 40; lab, laboratory; <, less than; --, no data]

\begin{tabular}{|c|c|c|c|c|c|}
\hline & \multicolumn{5}{|c|}{ Sample number } \\
\hline & SWM-95-061 & SWM-95-150 & SWM-96-046 & SWM-95-062 & SWM-95-151 \\
\hline Boron $(\mu \mathrm{g} / \mathrm{L})$ & -- & -- & -- & -- & -- \\
\hline Cadmium $(\mu \mathrm{g} / \mathrm{L})$ & $<0.001$ & -- & -- & $<0.001$ & -- \\
\hline Chromium $(\mu \mathrm{g} / \mathrm{L})$ & $<0.01$ & -- & -- & $<0.01$ & -- \\
\hline Copper $(\mu \mathrm{g} / \mathrm{L})$ & $<0.01$ & -- & -- & $<0.01$ & -- \\
\hline Iron $(\mathrm{mg} / \mathrm{L})$ & $<0.05$ & $<0.05$ & $<0.06$ & $<0.05$ & $<0.05$ \\
\hline Lead $(\mu \mathrm{g} / \mathrm{L})$ & 0.001 & -- & -- & 0.002 & -- \\
\hline Manganese $(\mathrm{mg} / \mathrm{L})$ & $<0.05$ & $<0.05$ & 0.03 & $<0.05$ & $<0.05$ \\
\hline Mercury $(\mu \mathrm{g} / \mathrm{L})$ & $<0.001$ & -- & -- & $<0.001$ & -- \\
\hline Nickel $(\mu \mathrm{g} / \mathrm{L})$ & $<0.05$ & -- & -- & $<0.05$ & -- \\
\hline Selenium $(\mu \mathrm{g} / \mathrm{L})$ & $<0.01$ & -- & -- & $<0.01$ & -- \\
\hline Silver $(\mu \mathrm{g} / \mathrm{L})$ & -- & -- & -- & -- & -- \\
\hline Thallium $(\mu \mathrm{g} / \mathrm{L})$ & $<0.001$ & -- & -- & $<0.001$ & -- \\
\hline $\operatorname{Zinc}(\mu \mathrm{g} / \mathrm{L})$ & -- & -- & -- & -- & -- \\
\hline \multirow[t]{3}{*}{ Cyanide $(\mathrm{mg} / \mathrm{L})$} & $<0.01$ & -- & -- & $<0.01$ & -- \\
\hline & \multicolumn{5}{|c|}{ Sample number } \\
\hline & SWM-96-047 & GMA-95-001 & GMA-95-002 & SWM-95-049 & SWM-95-152 \\
\hline SDGS well name & R20-94-29 & -- & -- & R20-94-30 & R20-94-30 \\
\hline Owner & SDGS & -- & -- & SDGS & SDGS \\
\hline Latitude (dd) & 43.0128 & 43.1146 & 43.1146 & 43.1018 & 43.1018 \\
\hline Longitude (dd) & 101.2921 & 101.0022 & 101.0022 & 101.2122 & 101.2122 \\
\hline Aquifer & Sandhills & -- & -- & Ogallala & Ogallala \\
\hline Ground surface elevation (feet) & 3,228 & 2,860 & 2,680 & 2,962 & $2,962.00$ \\
\hline Well depth (feet from casing top) & 23.8 & -- & -- & 43.3 & 43.3 \\
\hline Casing top elevation (feet) & $3,230.24$ & -- & -- & $2,964.11$ & $2,964.11$ \\
\hline Depth to water (feet from casing top) & 11.28 & -- & -- & 27.14 & 26.20 \\
\hline Ground-water elevation (feet) & $3,218.96$ & -- & -- & $2,936.97$ & $2,937.91$ \\
\hline Casing type & PVC, SCH.40 & -- & -- & PVC, SCH.40 & PVC, SCH.40 \\
\hline Collection date & $05 / 06 / 1996$ & $01 / 18 / 1995$ & $01 / 18 / 1995$ & 05/08/1995 & 08/08/1995 \\
\hline $\begin{array}{l}\text { Lab specific conductance }(\mu \mathrm{S} / \mathrm{cm} \text { at } \\
25 \operatorname{deg} \mathrm{C})\end{array}$ & 303 & -- & -- & 528 & 516 \\
\hline Lab pH (standard units) & 7.80 & -- & -- & 7.47 & 7.53 \\
\hline Hardness $\left(\mathrm{mg} / \mathrm{L}\right.$ as $\left.\mathrm{CaCO}_{3}\right)$ & 122 & -- & -- & 150 & 150 \\
\hline Hydrogen carbonate $(\mathrm{mg} / \mathrm{L})$ & 189 & -- & -- & 284 & 275 \\
\hline Carbonate $(\mathrm{mg} / \mathrm{L})$ & 0 & -- & -- & -- & -- \\
\hline Lab alkalinity $\left(\mathrm{mg} / \mathrm{L}\right.$ as $\left.\mathrm{CaCO}_{3}\right)$ & 155 & -- & -- & 233 & 226 \\
\hline Calcium (mg/L) & 41.7 & -- & -- & 47 & 45 \\
\hline
\end{tabular}


Table 61. Selected water-quality data (from the South Dakota Geological Survey) for ground-water sites in the Little White River Basin, South Dakota and Nebraska-Continued

[SDGS, South Dakota Geological Survey; dd, decimal degrees; milligrams per liter; $\mu \mathrm{S} / \mathrm{cm}$, microsiemens per centimeter; $\mu \mathrm{g} / \mathrm{L}$, micrograms per liter; deg $\mathrm{C}$, degrees Celsius; $\mathrm{CaCO}_{3}$, calcium carbonate; $\mathrm{PVC}, \mathrm{SCH} 40$, poly vinyl chloride, schedule 40: lab, laboratory; <, less than; --, no data]

\begin{tabular}{|c|c|c|c|c|c|}
\hline & \multicolumn{5}{|c|}{ Sample number } \\
\hline & SWM-96-047 & GMA-95-001 & GMA-95-002 & SWM-95-049 & SWM-95-152 \\
\hline Magnesium $(\mathrm{mg} / \mathrm{L})$ & 4.4 & -- & -- & 8.8 & 8.3 \\
\hline Sodium (mg/L) & 27.0 & -- & -- & 35 & 35 \\
\hline Potassium (mg/L) & 3.4 & -- & -- & 36 & 38 \\
\hline Sulfate $(\mathrm{mg} / \mathrm{L})$ & 18.1 & -- & -- & 24 & 28 \\
\hline Chloride $(\mathrm{mg} / \mathrm{L})$ & 1.2 & -- & -- & 3.1 & 2.9 \\
\hline Fluoride $(\mathrm{mg} / \mathrm{L})$ & 0.19 & -- & -- & 0.92 & 0.92 \\
\hline $\begin{array}{l}\text { Lab total dissolved solids ( } \mathrm{mg} / \mathrm{L} \text { as } \\
\quad \mathrm{CaCO}_{3} \text { ) }\end{array}$ & 206 & -- & -- & 354 & 348 \\
\hline Ammonia-nitrogen $(\mathrm{mg} / \mathrm{L})$ & $<0.02$ & -- & -- & $<0.05$ & $<0.05$ \\
\hline $\begin{array}{l}\text { Nitrate-nitrogen + nitrite-nitrogen } \\
(\mathrm{mg} / \mathrm{L})\end{array}$ & 0.4 & -- & -- & 2.38 & 2.81 \\
\hline Total phosphorus (mg/L) & -- & -- & -- & -- & \\
\hline Antimony $(\mu \mathrm{g} / \mathrm{L})$ & -- & -- & -- & $<0.005$ & - \\
\hline Arsenic $(\mu \mathrm{g} / \mathrm{L})$ & -- & 3 & 3.0 & 0.02 & -- \\
\hline Barium $(\mu \mathrm{g} / \mathrm{L})$ & -- & -. & -- & 0.33 & -- \\
\hline Beryllium $(\mu \mathrm{g} / \mathrm{L})$ & -- & -- & -- & $<0.002$ & -- \\
\hline Boron $(\mu \mathrm{g} / \mathrm{L})$ & -- & -- & -- & -- & -- \\
\hline Cadmium $(\mu \mathrm{g} / \mathrm{L})$ & -- & -- & -- & $<0.001$ & -- \\
\hline Chromium $(\mu \mathrm{g} / \mathrm{L})$ & -- & -- & -- & $<0.01$ & -- \\
\hline Copper $(\mu \mathrm{g} / \mathrm{L})$ & -- & -- & -- & $<0.01$ & -- \\
\hline Iron $(\mathrm{mg} / \mathrm{L})$ & $<0.06$ & -- & -- & $<0.05$ & $<0.05$ \\
\hline Lead $(\mu \mathrm{g} / \mathrm{L})$ & -- & -- & -- & $<0.001$ & -- \\
\hline Manganese $(\mathrm{mg} / \mathrm{L})$ & $<0.02$ & -- & -- & 0.14 & 0.14 \\
\hline Mercury $(\mu \mathrm{g} / \mathrm{L})$ & -- & -- & -- & $<0.001$ & -- \\
\hline Nickel $(\mu \mathrm{g} / \mathrm{L})$ & -- & -- & -- & $<0.05$ & -- \\
\hline Selenium $(\mu \mathrm{g} / \mathrm{L})$ & -- & -- & -- & $<0.01$ & -- \\
\hline Silver $(\mu \mathrm{g} / \mathrm{L})$ & -- & -- & -- & -- & -- \\
\hline Thallium $(\mu \mathrm{g} / \mathrm{L})$ & -- & -- & -- & $<0.001$ & -- \\
\hline $\operatorname{Zinc}(\mu \mathrm{g} / \mathrm{L})$ & -- & -- & -- & -- & - \\
\hline Cyanide (mg/L) & -- & -- & -- & $<0.01$ & -- \\
\hline
\end{tabular}


Table 61. Selected water-quality data (from the South Dakota Geological Survey) for ground-water sites in the Little White River Basin, South Dakota and Nebraska-Continued

[SDGS, South Dakota Geological Survey; dd, decimal degrees; milligrams per liter; $\mu \mathrm{S} / \mathrm{cm}$, microsiemens per centimeter; $\mu \mathrm{g} / \mathrm{L}$, micrograms per liter; $\operatorname{deg} \mathrm{C}$, degrees Celsius; $\mathrm{CaCO}_{3}$, calcium carbonate; $\mathrm{PVC}$, $\mathrm{SCH} 40$, poly vinyl chloride, schedule 40; lab, laboratory; <, less than; --, no data]

\begin{tabular}{|c|c|c|c|c|}
\hline & \multicolumn{4}{|c|}{ Sample number } \\
\hline & SWM-96-048 & SWM-95-050 & SWM-95-153 & SWM-96-049 \\
\hline SDGS well name & R20-94-30 & R20-94-31 & R20-94-31 & R20-94-31 \\
\hline Owner & SDGS & SDGS & SDGS & SDGS \\
\hline Latitude (dd) & 43.1018 & 43.1018 & 43.1018 & 43.1018 \\
\hline Longitude (dd) & 101.2122 & 101.2122 & 101.2122 & 101.2122 \\
\hline Aquifer & Ogallala & Ogallala & Ogallala & Ogallala \\
\hline Ground surface elevation (feet) & 2,962 & 2,962 & $2,962.00$ & 2,962 \\
\hline Well depth (feet from casing top) & 43.3 & 33.1 & 33.1 & 33.1 \\
\hline Casing top elevation (feet) & $2,964.11$ & $2,964.11$ & $2,964.11$ & $2,964.11$ \\
\hline Depth to water (feet from casing top) & 25.96 & 27.72 & 26.75 & 26.53 \\
\hline Ground-water elevation (feet) & $2,938.15$ & $2,936.39$ & $2,937.36$ & $2,937.58$ \\
\hline Casing type & PVC, SCH.40 & PVC, SCH.40 & PVC, SCH.40 & PVC, SCH.40 \\
\hline Collection date & $05 / 06 / 1996$ & $05 / 08 / 1995$ & 08/08/1995 & $05 / 06 / 1996$ \\
\hline $\begin{array}{l}\text { Lab specific conductance }(\mu \mathrm{S} / \mathrm{cm} \text { at } \\
25 \mathrm{deg} C)\end{array}$ & 505 & 337 & 300 & 253 \\
\hline Lab pH (standard units) & 7.77 & 7.25 & 7.31 & 7.61 \\
\hline Hardness $\left(\mathrm{mg} / \mathrm{L}\right.$ as $\left.\mathrm{CaCO}_{3}\right)$ & 150 & 98 & 88 & 85 \\
\hline Hydrogen carbonate $(\mathrm{mg} / \mathrm{L})$ & 284 & 158 & 140 & 154 \\
\hline Carbonate $(\mathrm{mg} / \mathrm{L})$ & 0 & -- & -- & 0 \\
\hline Lab alkalinity $\left(\mathrm{mg} / \mathrm{L}\right.$ as $\left.\mathrm{CaCO}_{3}\right)$ & 233 & 130 & 115 & 126 \\
\hline Calcium (mg/L) & 45.4 & 28 & 25 & 23.5 \\
\hline Magnesium (mg/L) & 8.8 & 6.8 & 6.1 & 6.3 \\
\hline Sodium $(\mathrm{mg} / \mathrm{L})$ & 37.2 & 14 & 11 & 17.5 \\
\hline Potassium (mg/L) & 35.0 & 30 & 30 & 28.1 \\
\hline Sulfate $(\mathrm{mg} / \mathrm{L})$ & 12.2 & 20 & 19 & 12.2 \\
\hline Chloride (mg/L) & 4.3 & 2.5 & 2.3 & 2.4 \\
\hline Fluoride (mg/L) & 0.86 & 0.59 & 0.48 & 0.77 \\
\hline $\begin{array}{l}\text { Lab total dissolved solids }(\mathrm{mg} / \mathrm{L} \text { as } \\
\qquad \mathrm{CaCO}_{3} \text { ) }\end{array}$ & 353 & 235 & 215 & 224 \\
\hline Ammonia-nitrogen $(\mathrm{mg} / \mathrm{L})$ & $<0.02$ & $<0.05$ & $<0.05$ & $<0.02$ \\
\hline $\begin{array}{l}\text { Nitrate-nitrogen + nitrite-nitrogen } \\
\quad(\mathrm{mg} / \mathrm{L})\end{array}$ & 2.5 & 2.76 & 2.34 & 2.0 \\
\hline Total phosphorus (mg/L) & -- & -- & -- & -- \\
\hline Antimony $(\mu \mathrm{g} / \mathrm{L})$ & -- & $<0.005$ & -- & -- \\
\hline Arsenic $(\mu \mathrm{g} / \mathrm{L})$ & -- & $<0.01$ & -- & -- \\
\hline Barium $(\mu \mathrm{g} / \mathrm{L})$ & -- & 0.14 & -- & -- \\
\hline Beryllium $(\mu \mathrm{g} / \mathrm{L})$ & -- & $<0.002$ & -- & - \\
\hline Boron $(\mu \mathrm{g} / \mathrm{L})$ & -- & -- & -- & -- \\
\hline
\end{tabular}


Table 61. Selected water-quality data (from the South Dakota Geological Survey) for ground-water sites in the Little White River Basin, South Dakota and Nebraska-Continued

[SDGS, South Dakota Geological Survey; dd, decimal degrees; milligrams per liter; $\mu \mathrm{S} / \mathrm{cm}$, microsiemens per centimeter; $\mu \mathrm{g} / \mathrm{L}$, micrograms per liter; $\operatorname{deg} \mathrm{C}$, degrees Celsius; $\mathrm{CaCO}_{3}$, calcium carbonate; $\mathrm{PVC}, \mathrm{SCH} 40$, poly vinyl chloride, schedule 40 ; lab, laboratory; <, less than; --, no data]

\begin{tabular}{lcccc}
\hline & \multicolumn{5}{c}{ Sample number } \\
\cline { 2 - 5 } & SWM-96-048 & SWM-95-050 & SWM-95-153 & SWM-96-049 \\
\hline Cadmium $(\mu \mathrm{g} / \mathrm{L})$ & -- & $<0.001$ & -- & -- \\
Chromium $(\mu \mathrm{g} / \mathrm{L})$ & -- & $<0.01$ & - & - \\
Copper $(\mu \mathrm{g} / \mathrm{L})$ & -- & $<0.01$ & -- & - \\
Iron $(\mathrm{mg} / \mathrm{L})$ & $<0.06$ & $<0.05$ & $<0.05$ & $<0.06$ \\
Lead $(\mu \mathrm{g} / \mathrm{L})$ & -- & $<0.001$ & -- & -- \\
Manganese $(\mathrm{mg} / \mathrm{L})$ & 0.10 & $<0.05$ & $<0.05$ & $<0.02$ \\
Mercury $(\mu \mathrm{g} / \mathrm{L})$ & -- & $<0.001$ & -- & -- \\
Nickel $(\mu \mathrm{g} / \mathrm{L})$ & -- & $<0.05$ & -- & - \\
Selenium $(\mu \mathrm{g} / \mathrm{L})$ & -- & $<0.01$ & -- & - \\
Silver $(\mu \mathrm{g} / \mathrm{L})$ & -- & -- & -- & -- \\
Thallium $(\mu \mathrm{g} / \mathrm{L})$ & -- & $<0.001$ & -- & - \\
Zinc $(\mu \mathrm{g} / \mathrm{L})$ & -- & -- & -- & - \\
Cyanide $(\mathrm{mg} / \mathrm{L})$ & -- & $<0.01$ & & - \\
\hline
\end{tabular}


Table 62. Selected radionuclide data (from the South Dakota Geological Survey) for ground-water sites in the Little White River Basin, South Dakota and Nebraska

[SDGS, South Dakota Geological Survey; dd, decimal degrees; $\mu \mathrm{S} / \mathrm{cm}$, microsiemens per centimeter; $\mathrm{mg} / \mathrm{L}$, milligrams per liter; pCi/L, picocuries per liter; $\operatorname{deg} \mathrm{C}$, degrees Celsius; $\mathrm{CaCO}_{3}$, calcium carbonate; $\mathrm{PVC}, \mathrm{SCH} 40$, poly vinyl chloride, schedule 40; <, less than; --, no data]

\begin{tabular}{|c|c|c|c|c|}
\hline \multirow{2}{*}{ Description } & \multicolumn{4}{|c|}{ Sample number } \\
\hline & SWM-95-061 & SWM-95-062 & SWM-95-049 & SWM-95-050 \\
\hline SDGS well name & R20-94-28 & R20-94-29 & R20-94-30 & R20-94-31 \\
\hline Owner & SDGS & SDGS & SDGS & SDGS \\
\hline Latitude (dd) & 43.0128 & 43.0128 & 43.1018 & 43.1018 \\
\hline Longitude (dd) & 101.2921 & 101.2921 & 101.2122 & 101.2122 \\
\hline Aquifer & Sand Hills & Sand Hills & Ogallala & Ogallala \\
\hline Ground surface elevation (feet) & 3,228 & 3,228 & 2,962 & 2,962 \\
\hline Well depth (feet from casing top) & 41.9 & 23.8 & 43.3 & 33.1 \\
\hline Casing top elevation (feet) & $3,230.18$ & $3,230.24$ & $2,964.11$ & $2,964.11$ \\
\hline Depth to water (feet from casing top) & 12.25 & 11.20 & 27.14 & 27.72 \\
\hline Ground-water elevation (feet) & $3,217.93$ & $3,219.04$ & $2,936.97$ & $2,936.39$ \\
\hline Casing type & PVC, SCH. 40 & PVC, SCH. 40 & PVC, SCH. 40 & PVC, SCH. 40 \\
\hline Collection date & $05 / 17 / 1995$ & 05/17/1995 & 05/08/1995 & $05 / 08 / 1995$ \\
\hline \multicolumn{5}{|c|}{ Field parameters } \\
\hline $\begin{array}{l}\text { Specific conductance, } \mu \mathrm{S} / \mathrm{cm} \text { at } \\
25 \text { deg } \mathrm{C} \text { (unfiltered) }\end{array}$ & 125 & 375 & 425 & 250 \\
\hline $\begin{array}{l}\text { Specific conductance, } \mu \mathrm{S} / \mathrm{cm} \text { at } \\
25 \mathrm{deg} \text { C (filtered) }\end{array}$ & 125 & 375 & 425 & 250 \\
\hline $\mathrm{pH}$ (unfiltered, standard units) & 6.40 & 7.42 & 7.45 & 7.30 \\
\hline $\mathrm{pH}$ (filtered, standard units) & 6.57 & 7.61 & 7.50 & 7.28 \\
\hline Temperature, deg C (unfiltered) & 11.0 & 9.5 & 11.5 & 11.5 \\
\hline Alkalinity $\mathrm{mg} / \mathrm{L}$ as $\mathrm{CaCO}_{3}$ (filtered) & 66 & 117 & 228 & 124 \\
\hline \multicolumn{5}{|c|}{ Radionuclide information } \\
\hline Analyzing laboratory & $\begin{array}{l}\text { University of Iowa } \\
\text { Hygienic Laboratory }\end{array}$ & $\begin{array}{l}\text { University of Iowa } \\
\text { Hygienic Laboratory }\end{array}$ & $\begin{array}{l}\text { University of Iowa } \\
\text { Hygienic Laboratory }\end{array}$ & $\begin{array}{l}\text { University of Iowa } \\
\text { Hygienic Laboratory }\end{array}$ \\
\hline Gross alpha $(\mathrm{pCi} / \mathrm{L})$ & $<0.6+/-0.4$ & $3.7+/-0.9$ & $1.9+/-0.7$ & $<0.8$ \\
\hline Radium $226(\mathrm{pCi} / \mathrm{L})$ & -- & $<0.5$ & -- & -- \\
\hline Radium $228(\mathrm{pCi} / \mathrm{L})$ & -- & $<0.7$ & -- & -- \\
\hline Radium $226+228(\mathrm{pCi} / \mathrm{L})$ & -- & $<1.2$ & -- & -- \\
\hline
\end{tabular}


Table 63. Selected pesticide data (from the South Dakota Geological Survey) for ground-water sites in the Little White River Basin, South Dakota and Nebraska

[SDGS, South Dakota Geological Survey; dd, decimal degrees; $\mu \mathrm{S} / \mathrm{cm}$, microsiemens per centimeter; mg/L, milligrams per liter; $\mu \mathrm{g} / \mathrm{L}$, micrograms per liter; deg $\mathrm{C}$, degrees Celsius; $\mathrm{CaCO}_{3}$, calcium carbonate; $\mathrm{PVC}, \mathrm{SCH} 40$, poly vinyl chloride, schedule 40; <, less than; --, no data]

\begin{tabular}{lcccc}
\hline \multirow{2}{*}{ Description } & \multicolumn{4}{c}{ Sample number } \\
\cline { 2 - 5 } & SWM-95-061 & SWM-96-046 & SWM-95-062 & SWM-96-047 \\
\hline SDGS well name & R20-94-28 & R20-94-28 & R20-94-29 & R20-94-29 \\
Owner & SDGS & SDGS & SDGS & SDGS \\
Latitude (dd) & 43.0128 & 43.0128 & 43.0128 & 43.0128 \\
Longitude (dd) & 101.2921 & 101.2921 & 101.2921 & 101.2921 \\
Aquifer & Sandhills & Sandhills & Sandhills & Sandhills \\
Ground surface elevation (feet) & 3,228 & 3,228 & 3,228 & 3,228 \\
Well depth (feet from casing top) & 41.9 & 41.9 & 23.8 & 23.8 \\
Casing top elevation (feet) & $3,230.18$ & $3,230.18$ & $3,230.24$ & $3,230.24$ \\
Depth to water (feet from casing top) & 12.25 & 11.72 & 11.20 & 11.28 \\
Ground-water elevation (feet) & $3,217.93$ & $3,218.46$ & $3,219.04$ & $3,218.96$ \\
Casing type & PVC, SCH. 40 & PVC,SCH.40 & PVC,SCH.40 & PVC,SCH.40 \\
Collection date & $05 / 17 / 1995$ & $05 / 06 / 1996$ & $05 / 17 / 1995$ & $05 / 06 / 1996$
\end{tabular}

Field parameters

\begin{tabular}{llccc}
\hline $\begin{array}{l}\text { Specific conductance, } \mu \mathrm{S} / \mathrm{cm} \text { at } 25 \\
\quad \text { deg } \mathrm{C} \text { (unfiltered) }\end{array}$ & 125 & 125 & 375 & 275 \\
$\begin{array}{l}\text { Specific conductance, } \mu \mathrm{S} / \mathrm{cm} \text { at } 25 \mathrm{deg} \\
\mathrm{C} \text { (filtered) }\end{array}$ & 125 & 125 & 375 & 275 \\
$\mathrm{pH}$ (unfiltered, standard units) & 6.40 & 6.72 & 7.42 & 7.56 \\
$\mathrm{pH}$ (filtered, standard units) & 6.57 & 6.74 & 7.61 & 7.74 \\
Temperature, deg C (unfiltered) & 11.0 & 11 & 9.5 & 9.5 \\
Alkalinity mg/L as $\mathrm{CaCO}_{3}$ (filtered) & 66 & 60 & 117 & 160
\end{tabular}

\begin{tabular}{lcccc}
\hline & & Pesticide results & \\
\hline Acetochlor $(\mu \mathrm{g} / \mathrm{L})$ & $<0.1$ & $<0.5$ & $<0.1$ & $<0.5$ \\
Alachlor $(\mu \mathrm{g} / \mathrm{L})$ & $<0.1$ & $<0.5$ & $<0.1$ & $<0.5$ \\
Atrazine $(\mu \mathrm{g} / \mathrm{L})$ & $<0.1$ & $<0.5$ & $<0.1$ & $<0.5$ \\
Deethyl atrazine $(\mu \mathrm{g} / \mathrm{L})$ & $<0.1$ & $<0.5$ & $<0.1$ & $<0.5$ \\
Deisopropyl atrazine $(\mu \mathrm{g} / \mathrm{L})$ & $<0.1$ & $<0.25$ & $<0.1$ & $<0.25$ \\
Bromoxynil $(\mu \mathrm{g} / \mathrm{L})$ & - & $<2.0$ & & $<2.0$ \\
Chlorpyrifos $(\mu \mathrm{g} / \mathrm{L})$ & $<0.1$ & $<0.5$ & $<0.5$ \\
Cyanazine $(\mu \mathrm{g} / \mathrm{L})$ & $<0.1$ & $<0.5$ & $<0.1$ & $<2.0$ \\
$2,4-\mathrm{D}(\mu \mathrm{g} / \mathrm{L})$ & $<0.2$ & $<2.0$ & $<0.1$ & $<2.0$ \\
Dicamba $(\mu \mathrm{g} / \mathrm{L})$ & $<0.2$ & $<2.0$ & $<0.2$ & $<0.5$ \\
EPTC $(\mu \mathrm{g} / \mathrm{L})$ & -- & $<0.5$ & $<0.2$ & $<-.5$ \\
MCPA $(\mu \mathrm{g} / \mathrm{L})$ & $<200$ & $<0.5$ & $<200$ & $<0.5$ \\
Metolachlor $(\mu \mathrm{g} / \mathrm{L})$ & $<0.1$ & $<0.5$ & $<0.1$ &
\end{tabular}


Table 63. Selected pesticide data (from the South Dakota Geological Survey) for ground-water sites in the Little White River Basin, South Dakota and Nebraska-Continued

[SDGS, South Dakota Geological Survey; dd, decimal degrees; $\mu \mathrm{S} / \mathrm{cm}$, microsiemens per centimeter; $\mathrm{mg} / \mathrm{L}$, milligrams per liter; $\mu \mathrm{g} / \mathrm{L}, \mathrm{micrograms}$ per liter; $\operatorname{deg} \mathrm{C}$, degrees Celsius; $\mathrm{CaCO}_{3}$, calcium carbonate; $\mathrm{PVC}, \mathrm{SCH} 40$, poly vinyl chloride, schedule 40 ; <, less than; --, no data]

\begin{tabular}{|c|c|c|c|c|}
\hline & \multicolumn{4}{|c|}{ Sample number } \\
\hline & SWM-95-061 & SWM-96-046 & SWM-95-062 & SWM-96-047 \\
\hline \multicolumn{5}{|c|}{ Pesticide results-Continued } \\
\hline Metribuzin $(\mu \mathrm{g} / \mathrm{L})$ & -- & $<0.500$ & -- & $<0.500$ \\
\hline Parathion $(\mu \mathrm{g} / \mathrm{L})$ & $<0.1$ & $<0.500$ & $<0.1$ & $<0.500$ \\
\hline Picloram $(\mu \mathrm{g} / \mathrm{L})$ & $<0.2$ & $<2.00$ & $<0.2$ & $<2.00$ \\
\hline Simazine $(\mu \mathrm{g} / \mathrm{L})$ & $<0.1$ & $<0.500$ & $<0.1$ & $<0.500$ \\
\hline Trifluralin $(\mu \mathrm{g} / \mathrm{L})$ & -- & $<0.500$ & -- & $<0.500$ \\
\hline
\end{tabular}

\begin{tabular}{lcccc}
\hline & \multicolumn{3}{c}{ Sample number } \\
\cline { 2 - 5 } & SWM-95-049 & SWM-96-048 & SWM-95-050 & SWM-96-049 \\
\hline SDGS well name & R20-94-30 & R20-94-30 & R20-94-31 & R20-94-31 \\
Owner & SDGS & SDGS & SDGS & SDGS \\
Latitude (dd) & 43.1018 & 43.1018 & 43.1018 & 43.1018 \\
Longitude (dd) & 101.2122 & 101.2122 & 101.2122 & 101.2122 \\
Aquifer & Ogallala & Ogallala & Ogallala & Ogallala \\
Ground surface elevation (feet) & 2,962 & 2,962 & 2,962 & 2,962 \\
Well depth (feet from casing top) & 43.3 & 43.3 & 33.1 & 33.1 \\
Casing top elevation (feet) & $2,964.11$ & $2,964.11$ & $2,964.11$ & $2,964.11$ \\
Depth to water (feet from casing top) & 27.14 & 25.96 & 27.72 & 26.53 \\
Ground-water elevation (feet) & $2,936.97$ & $2,938.15$ & $2,936.39$ & $2,937.58$ \\
Casing type & PVC,SCH.40 & PVC,SCH.40 & PVC,SCH.40 & PVC,SCH.40 \\
Collection date & $05 / 08 / 1995$ & $05 / 06 / 1996$ & $05 / 08 / 1995$ & $05 / 06 / 1996$
\end{tabular}

\begin{tabular}{lcccc}
\hline & \multicolumn{3}{c}{ Field parameters } & \\
\hline $\begin{array}{l}\text { Specific conductance, uS/cm at 25 deg } \\
\quad \text { C (unfiltered) }\end{array}$ & 425 & 450 & 250 & 225 \\
$\begin{array}{l}\text { Specific conductance, uS/cm at 25 deg } \\
\quad \text { (filtered) }\end{array}$ & 425 & 450 & 250 & 225 \\
$\mathrm{pH}$ (unfiltered, standard units) & 7.45 & 7.59 & 7.30 & 7.36 \\
$\mathrm{pH}$ (filtered, standard units) & 7.50 & 7.65 & 7.28 & 7.42 \\
Temperature, deg C (unfiltered) & 11.5 & 11.5 & 11.5 & 11.0 \\
Alkalinity mg/L as CaCO3 (filtered) & 228 & 236 & 124 & 124 \\
& & & & $<0.5$ \\
& $<0.1$ & Pesticide results & $<0.1$ & $<0.5$ \\
\hline Acetochlor $(\mu \mathrm{g} / \mathrm{L})$ & $<0.5$ & $<0.1$ & $<0.5$ \\
Alachlor $(\mu \mathrm{g} / \mathrm{L})$ & $<0.1$ & $<0.5$ & $<0.1$ & $<0.5$ \\
Atrazine $(\mu \mathrm{g} / \mathrm{L})$ & $<0.1$ & $<0.5$ & $<0.1$ & \\
Deethyl atrazine $(\mu \mathrm{g} / \mathrm{L})$ & $<0.1$ & $<0.5$ & & \\
\hline
\end{tabular}


Table 63. Selected pesticide data (from the South Dakota Geological Survey) for ground-water sites in the Little White River Basin, South Dakota and Nebraska-Continued

[SDGS, South Dakota Geological Survey; dd, decimal degrees; $\mu \mathrm{S} / \mathrm{cm}$, microsiemens per centimeter; mg/L, milligrams per liter; $\mu \mathrm{g} / \mathrm{L}$, micrograms per liter; $\operatorname{deg} \mathrm{C}$, degrees Celsius; $\mathrm{CaCO}_{3}$, calcium carbonate; $\mathrm{PVC}, \mathrm{SCH} 40$, poly vinyl chloride, schedule 40 ; <, less than; --, no data]

\begin{tabular}{lcccc}
\hline & \multicolumn{4}{c}{ Sample number } \\
\cline { 2 - 4 } & \multicolumn{1}{c}{ PWM-95-049 } & SWM-96-048 & SWM-95-050 & SWM-96-049 \\
\hline Deisopropyl atrazine $(\mu \mathrm{g} / \mathrm{L})$ & $<0.1$ & $<0.25$ & $<0.1$ & $<0.25$ \\
Bromoxynil $(\mu \mathrm{g} / \mathrm{L})$ & -- & $<2.0$ & -- & $<2.0$ \\
Chlorpyrifos $(\mu \mathrm{g} / \mathrm{L})$ & $<0.1$ & $<0.5$ & $<0.1$ & $<0.5$ \\
Cyanazine $(\mu \mathrm{g} / \mathrm{L})$ & $<0.1$ & $<0.5$ & $<0.5$ \\
$2,4-\mathrm{D}(\mu \mathrm{g} / \mathrm{L})$ & $<0.2$ & $<2.0$ & $<0.1$ & $<2.0$ \\
Dicamba $(\mu \mathrm{g} / \mathrm{L})$ & $<0.2$ & $<2.0$ & $<0.2$ & $<0.5$ \\
EPTC $(\mu \mathrm{g} / \mathrm{L})$ & -- & $<0.5$ & $<0.2$ & $<0.5$ \\
MCPA $(\mu \mathrm{g} / \mathrm{L})$ & $<200$ & $<0.5$ & -- & $<0.5$ \\
Metolachlor $(\mu \mathrm{g} / \mathrm{L})$ & $<0.1$ & $<0.5$ & $<200$ & $<0.5$ \\
Metribuzin $(\mu \mathrm{g} / \mathrm{L})$ & -- & $<0.5$ & $<0.1$ & $<0.5$ \\
Parathion $(\mu \mathrm{g} / \mathrm{L})$ & $<0.1$ & $<0.5$ & - & $<2.0$ \\
Picloram $(\mu \mathrm{g} / \mathrm{L})$ & $<0.2$ & $<2.0$ & $<0.1$ & $<0.5$ \\
Simazine $(\mu \mathrm{g} / \mathrm{L})$ & $<0.1$ & $<0.5$ & $<0.2$ & $<0.5$ \\
Trifluralin $(\mu \mathrm{g} / \mathrm{L})$ & -- & $<0.5$ & $<0.1$ & - \\
\hline
\end{tabular}




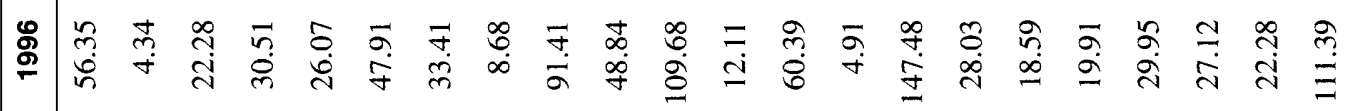

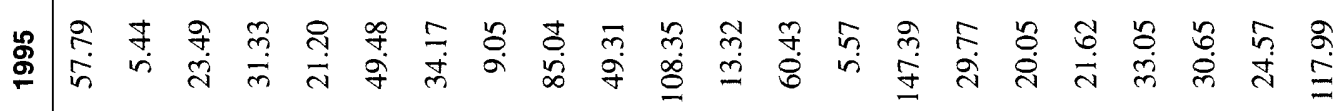

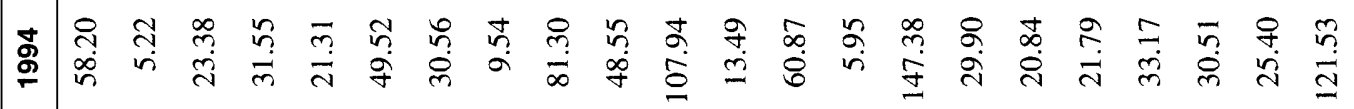

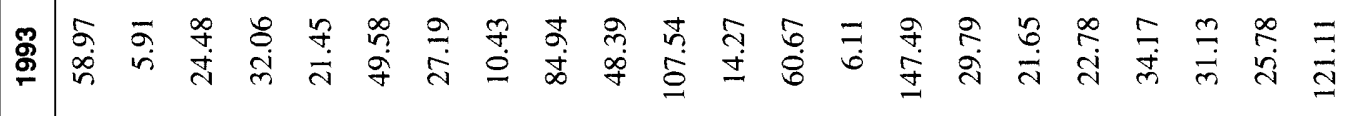

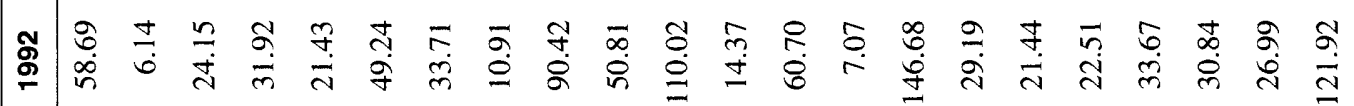

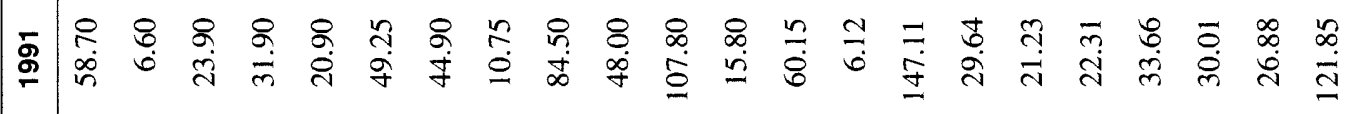

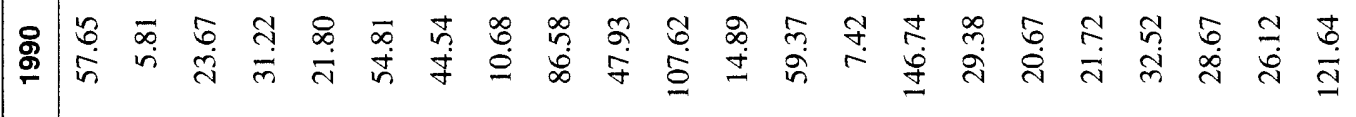

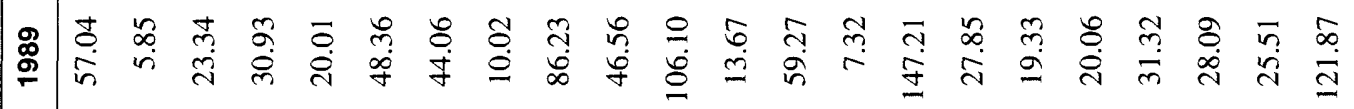

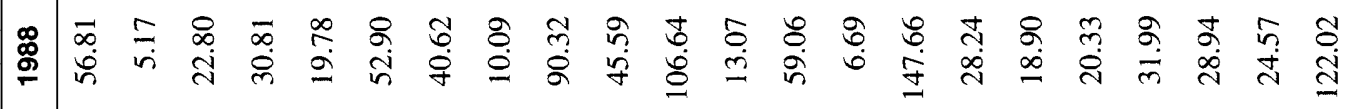

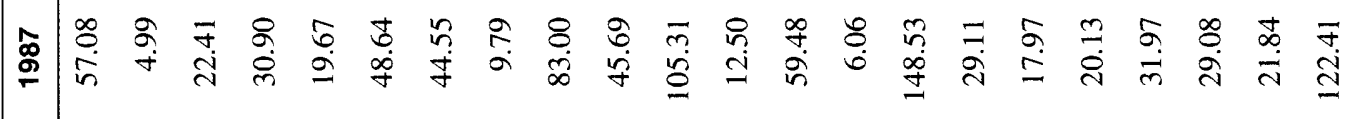

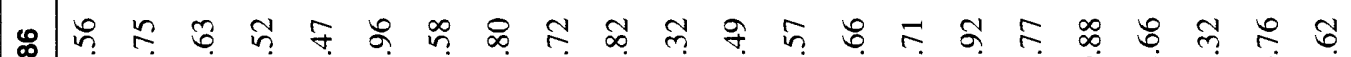
$\stackrel{\circ}{\circ}$ i

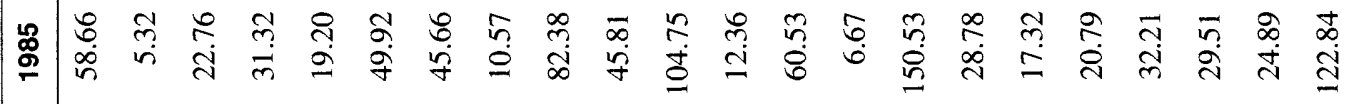

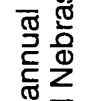

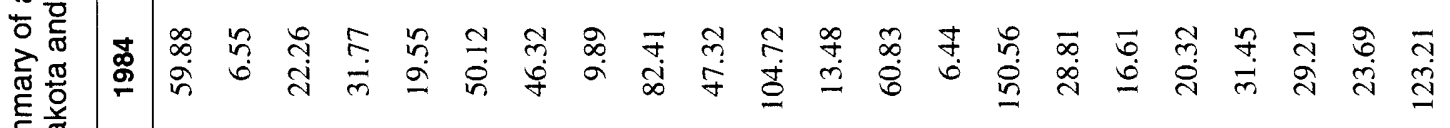

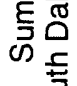

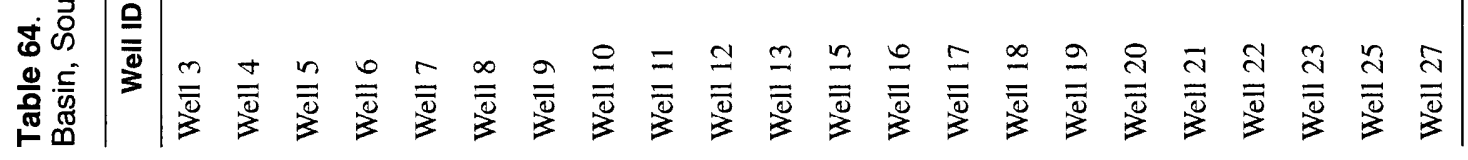


Table 65. Summary of selected water-quality data (from the Rosebud Sioux Tribe) for sites (surface and/or ground water) in the Little White River Basin, South Dakota and Nebraska

[Q1, first quartile; Q3, third quartile; $\mu \mathrm{S} / \mathrm{cm}$, microsiemens per centimeter; mg/L, milligrams per liter; deg C, degrees Celsius; NTU, nephelometric turbidity unit]

\begin{tabular}{|c|c|c|c|c|c|c|c|c|}
\hline $\begin{array}{l}\text { Constituent or } \\
\text { property }\end{array}$ & Mean & Median & $\begin{array}{l}\text { Standard } \\
\text { deviation }\end{array}$ & Q1 & Q3 & Minimum & Maximum & $\begin{array}{l}\text { Number of } \\
\text { valid } \\
\text { samples }\end{array}$ \\
\hline $\begin{array}{l}\text { Specific } \\
\text { conductance } \\
(\mu \mathrm{S} / \mathrm{cm} \text { at } 25 \\
\operatorname{deg} \mathrm{C})\end{array}$ & 564.7 & 381.5 & 702.3 & 306.3 & 598.8 & 0 & 4,590 & 92 \\
\hline $\mathrm{pH}$ (standard units) & 7.66 & 7.61 & 0.78 & 7.4 & 8 & 0 & 13 & 232 \\
\hline Turbidity (NTU) & 0.8 & 0.2 & 2.3 & 0 & 0.4 & 0 & 19 & 90 \\
\hline $\begin{array}{l}\text { Dissolved oxygen } \\
(\mathrm{mg} / \mathrm{L})\end{array}$ & 7.0 & 6.9 & 3.1 & 5.9 & 8.2 & 0 & 15.5 & 20 \\
\hline Hardness (mg/L) & 202.0 & 132.5 & 274.1 & 88.5 & 190 & 0 & 1,800 & 140 \\
\hline Total solids (mg/L) & 603.9 & 250 & 978.5 & 195 & 405 & 144 & 3,754 & 26 \\
\hline $\begin{array}{l}\text { Dissolved solids } \\
\quad(\mathrm{mg} / \mathrm{L})\end{array}$ & 473.0 & 282 & 723.3 & 229.9 & 418.5 & 0 & 5,200 & 98 \\
\hline $\begin{array}{l}\text { Suspended solids } \\
(\mathrm{mg} / \mathrm{L})\end{array}$ & 306.4 & 192 & 383.9 & 0 & 528 & 0 & 1,152 & 15 \\
\hline
\end{tabular}




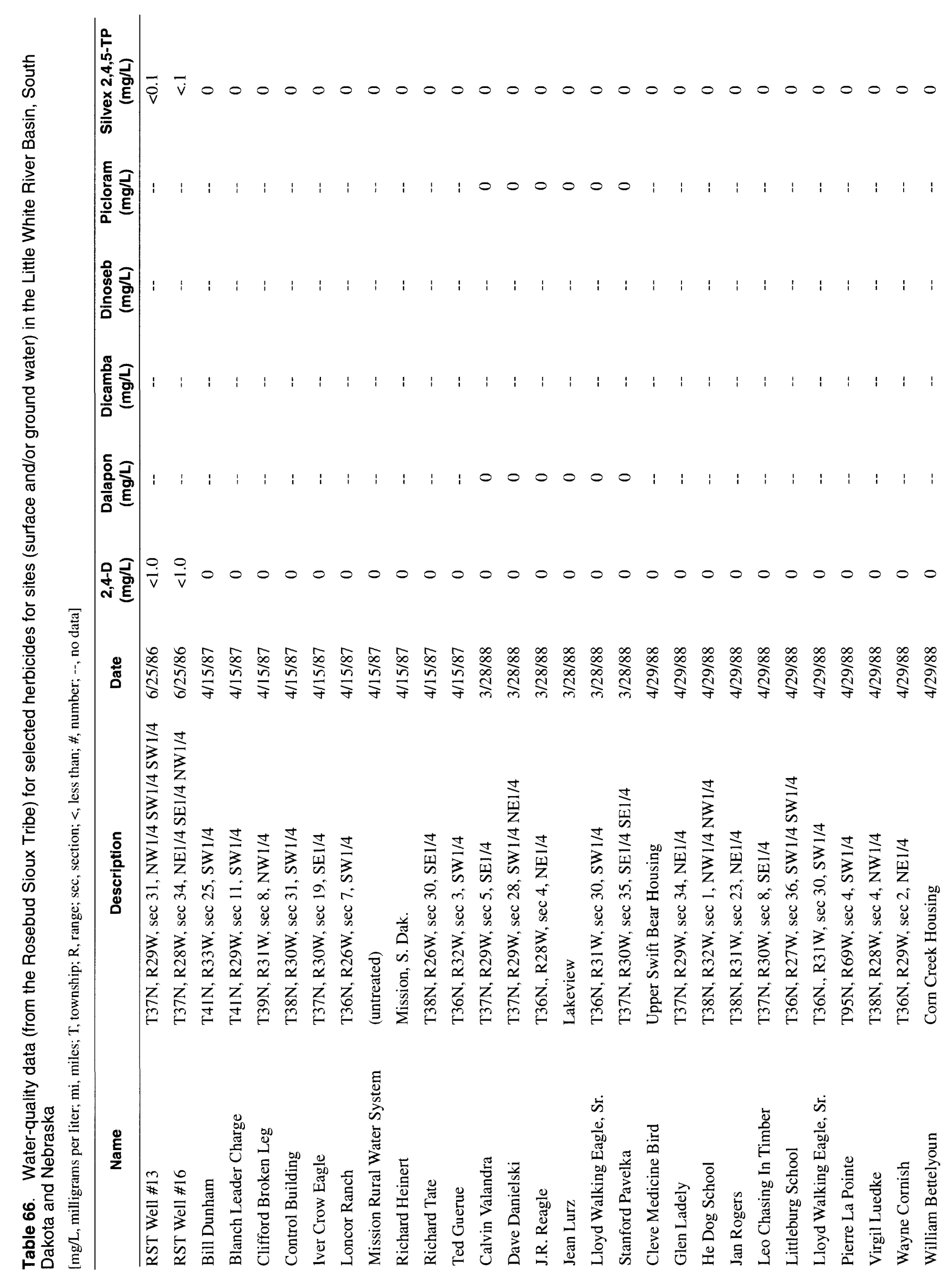




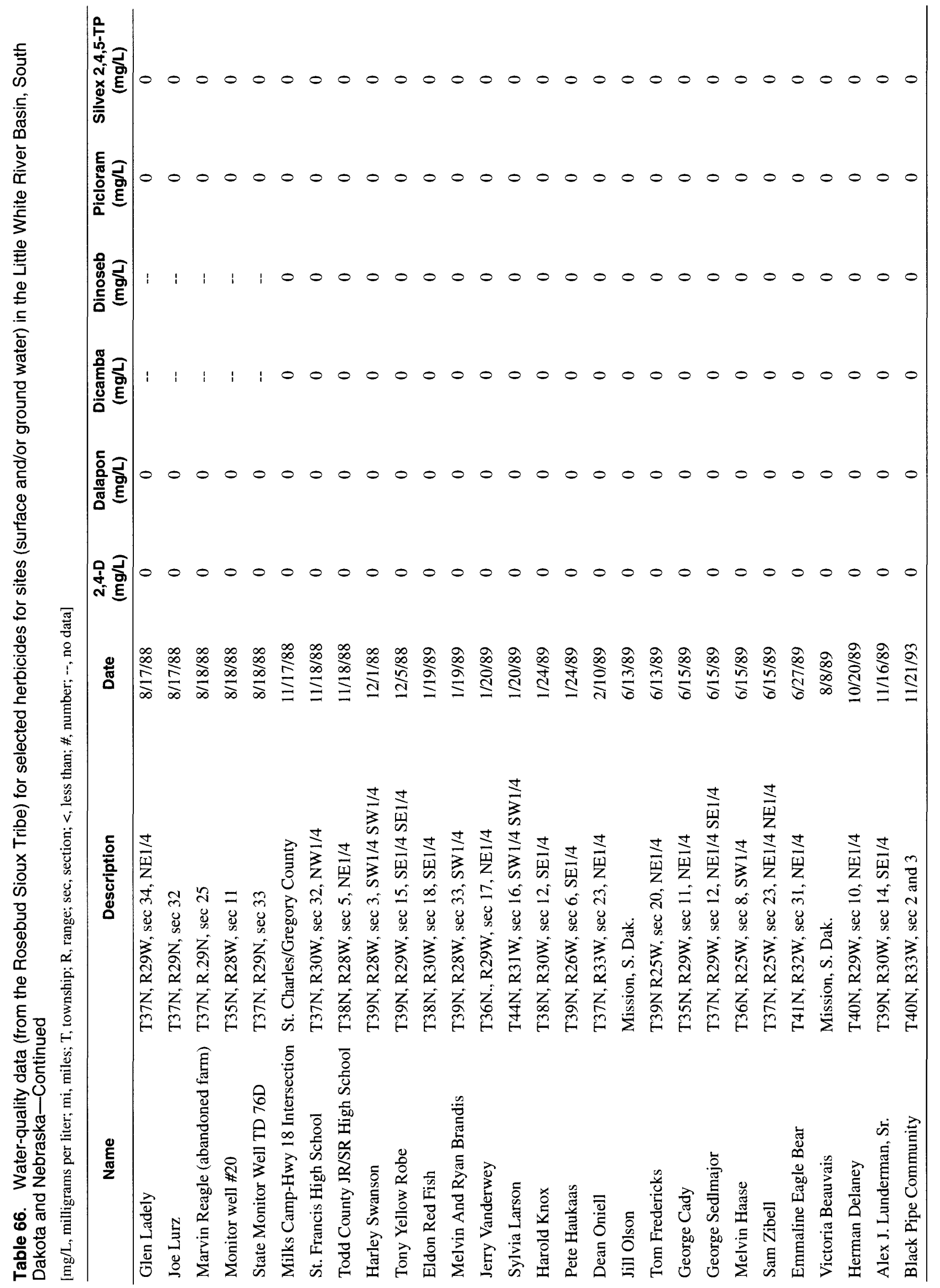


Table 67. Summary of water-quality data (from the Rosebud Sioux Tribe) for selected metals for sites (surface and/or ground water) in the Little White River Basin, South Dakota and Nebraska

[Q1, first quartile; Q3, third quartile; mg/L, milligrams per liter; $\mu \mathrm{g} / \mathrm{L}$, micrograms per liter; --, not applicable]

\begin{tabular}{|c|c|c|c|c|c|c|c|c|}
\hline $\begin{array}{l}\text { Constituent or } \\
\text { property }\end{array}$ & Mean & Median & $\begin{array}{l}\text { Standard } \\
\text { deviation }\end{array}$ & Q1 & Q3 & Minimum & Maximum & $\begin{array}{c}\text { Number of } \\
\text { valid } \\
\text { samples }\end{array}$ \\
\hline Calcium $(\mathrm{mg} / \mathrm{L})$ & 186.3 & 40.2 & 565.8 & 23.2 & 62.2 & 0 & 2,812 & 44 \\
\hline Magnesium (mg/L) & 22.6 & 6.3 & 49.4 & 2.4 & 10.9 & 0 & 239 & 35 \\
\hline Sodium (mg/L) & 73.0 & 35 & 122.8 & 7.9 & 78.3 & 0 & 920 & 112 \\
\hline Potassium (mg/L) & 11.1 & 8.8 & 9.6 & 6 & 10.2 & 0 & 48 & 34 \\
\hline Aluminum $(\mu \mathrm{g} / \mathrm{L})$ & 0 & 0 & 0 & 0 & 0 & 0 & 0 & 5 \\
\hline Antimony $(\mathrm{mg} / \mathrm{L})$ & 0 & 0 & 0 & 0 & 0 & 0 & 0 & 13 \\
\hline Arsenic (mg/L) & 2.5 & 0.006 & 6.8 & 0.001 & 0.1 & 0 & 47.9 & 143 \\
\hline Barium (mg/L) & 16.5 & 0 & 84.4 & 0 & 0.1 & 0 & 530 & 115 \\
\hline Beryllium (mg/L) & 0.02 & 0 & 0.02 & 0 & 0.05 & 0 & 0.05 & 23 \\
\hline Boron $(\mathrm{mg} / \mathrm{L})$ & 0 & 0 & 0 & 0 & 0 & 0 & 0 & 5 \\
\hline Cadmium (mg/L) & 0.7 & 0.001 & 6.6 & 0 & 0.004 & 0 & 75.5 & 133 \\
\hline Chromium (mg/L) & 2.0 & 0 & 16.2 & 0 & 0.002 & 0 & 185 & 131 \\
\hline Cobalt $(\mathrm{mg} / \mathrm{L})$ & 0 & 0 & 0 & 0 & 0 & 0 & 0 & 5 \\
\hline Copper (mg/L) & 0.08 & 0.02 & 0.22 & 0.01 & 0.05 & 0 & 2.03 & 224 \\
\hline Gold (mg/L) & 0 & 0 & 0 & 0 & 0 & 0 & 0 & 5 \\
\hline Iron $(\mathrm{mg} / \mathrm{L})$ & 20.7 & 0.1 & 151.3 & 0.02 & 0.1 & 0 & 1,360 & 120 \\
\hline Lead $(\mu \mathrm{g} / \mathrm{L})$ & 2.3 & 0.1 & 14.3 & 0.001 & 0.8 & 0 & 200 & 251 \\
\hline Lithium (mg/L) & 0 & 0 & 0 & 0 & 0 & 0 & 0 & 5 \\
\hline Manganese (mg/L) & 0.6 & 0.02 & 3.8 & 0.001 & 0.1 & 0 & 35 & 120 \\
\hline Manganese chart & -- & -- & -- & -- & -- & 0.05 & 0.05 & 8 \\
\hline Mercury (mg/L) & 0.1 & 0 & 0.3 & 0 & 0.0002 & 0 & 3.6 & 133 \\
\hline $\begin{array}{l}\text { Molybdenum } \\
(\mathrm{mg} / \mathrm{L})\end{array}$ & 0 & 0 & 0 & 0 & 0 & 0 & 0 & 5 \\
\hline Nickel (mg/L) & 0.5 & 0 & 1.5 & 0 & 0.005 & 0 & 6.7 & 124 \\
\hline Selenium (mg/L) & 0.2 & 0 & 1.3 & 0 & 0.005 & 0 & 10.7 & 112 \\
\hline Silicon $(\mathrm{mg} / \mathrm{L})$ & 0 & 0 & 0 & 0 & 0 & 0 & 0 & 5 \\
\hline Silver $(\mu \mathrm{g} / \mathrm{L})$ & 0.06 & 0 & 0.21 & 0 & 0.001 & 0 & 1 & 107 \\
\hline Thallium (mg/L) & -- & -- & -- & -- & -- & 0 & 0 & 1 \\
\hline Strontium (mg/L) & 0 & 0 & 0 & 0 & 0 & 0 & 0 & 13 \\
\hline Vanadium $(\mathrm{mg} / \mathrm{L})$ & 0 & 0 . & 0 & 0 & 0 & 0 & 0 & 5 \\
\hline Zinc $(m g / L)$ & 6.3 & 0.1 & 16.8 & 0.02 & 0.1 & 0 & 58 & 115 \\
\hline
\end{tabular}


Table 68. Summary of water-quality data (from the Rosebud Sioux Tribe) for selected nonmetallic and inorganic parameters for sites (surface and/or ground water) in the Little White River Basin, South Dakota and Nebraska

[Q1, first quartile; Q3, third quartile; Alkalinity-M, alkalinity-methylorange: Alkalinity-P; alkalinity-phenophthalein; mg/L, milligrams per liter]

\begin{tabular}{|c|c|c|c|c|c|c|c|c|}
\hline $\begin{array}{l}\text { Constituent or } \\
\text { property }\end{array}$ & Mean & Median & $\begin{array}{l}\text { Standard } \\
\text { deviation }\end{array}$ & Q1 & Q3 & Minimum & Maximum & $\begin{array}{c}\text { Number of } \\
\text { valid } \\
\text { samples }\end{array}$ \\
\hline Alkalinity-M (mg/L) & 194.2 & 172 & 98.8 & 144.5 & 215.3 & 0 & 600 & 122 \\
\hline Alkalinity-P (mg/L) & 0 & 0 & 0 & 0 & 0 & 0 & 0 & 12 \\
\hline Bicarbonate (mg/L) & 251.6 & 216 & 130.2 & 194.3 & 262.5 & 0 & 732 & 32 \\
\hline Carbonate $(\mathrm{mg} / \mathrm{L})$ & 0 & 0 & 0 & 0 & 0 & 0 & 0 & 6 \\
\hline Sulfate $(\mathrm{mg} / \mathrm{L})$ & 128.8 & 10 & 435.7 & 0.1 & 32.1 & 0 & 2,696 & 133 \\
\hline Chloride (mg/L) & 14.8 & 4 & 31.3 & 0 & 18 & 0 & 250 & 129 \\
\hline Fluoride (mg/L) & 2.2 & 0.29 & 11.8 & 0 & 0.8 & 0 & 102 & 101 \\
\hline Silica (mg/L) & 11.92 & 10 & 15.31 & 0.95 & 10 & 0.35 & 80 & 38 \\
\hline $\begin{array}{l}\text { Nitrogen, ammonia } \\
(\mathrm{mg} / \mathrm{L})\end{array}$ & 0.6 & 1 & 0.5 & 0.02 & 1 & 0 & 1 & 63 \\
\hline $\begin{array}{l}\text { Nitrogen, nitrite } \\
\quad(\mathrm{mg} / \mathrm{L})\end{array}$ & 0.2 & 0 & 1.1 & 0 & 0.01 & 0 & 8.3 & 60 \\
\hline $\begin{array}{l}\text { Nitrogen, nitrate } \\
\quad(\mathrm{mg} / \mathrm{L})\end{array}$ & 2.0 & 0.4 & 7.4 & 0.1 & 1.1 & 0 & 100 & 254 \\
\hline Phosphate (mg/L) & 1.2 & 0.1 & 7.6 & 0.05 & 0.4 & 0 & 71.9 & 93 \\
\hline Total cyanide $(\mathrm{mg} / \mathrm{L})$ & 0.002 & 0 & 0.004 & 0 & 0.003 & 0 & 0.01 & 6 \\
\hline Free cyanide $(\mathrm{mg} / \mathrm{L})$ & 0 & 0 & 0 & 0 & 0 & 0 & 0 & 5 \\
\hline
\end{tabular}




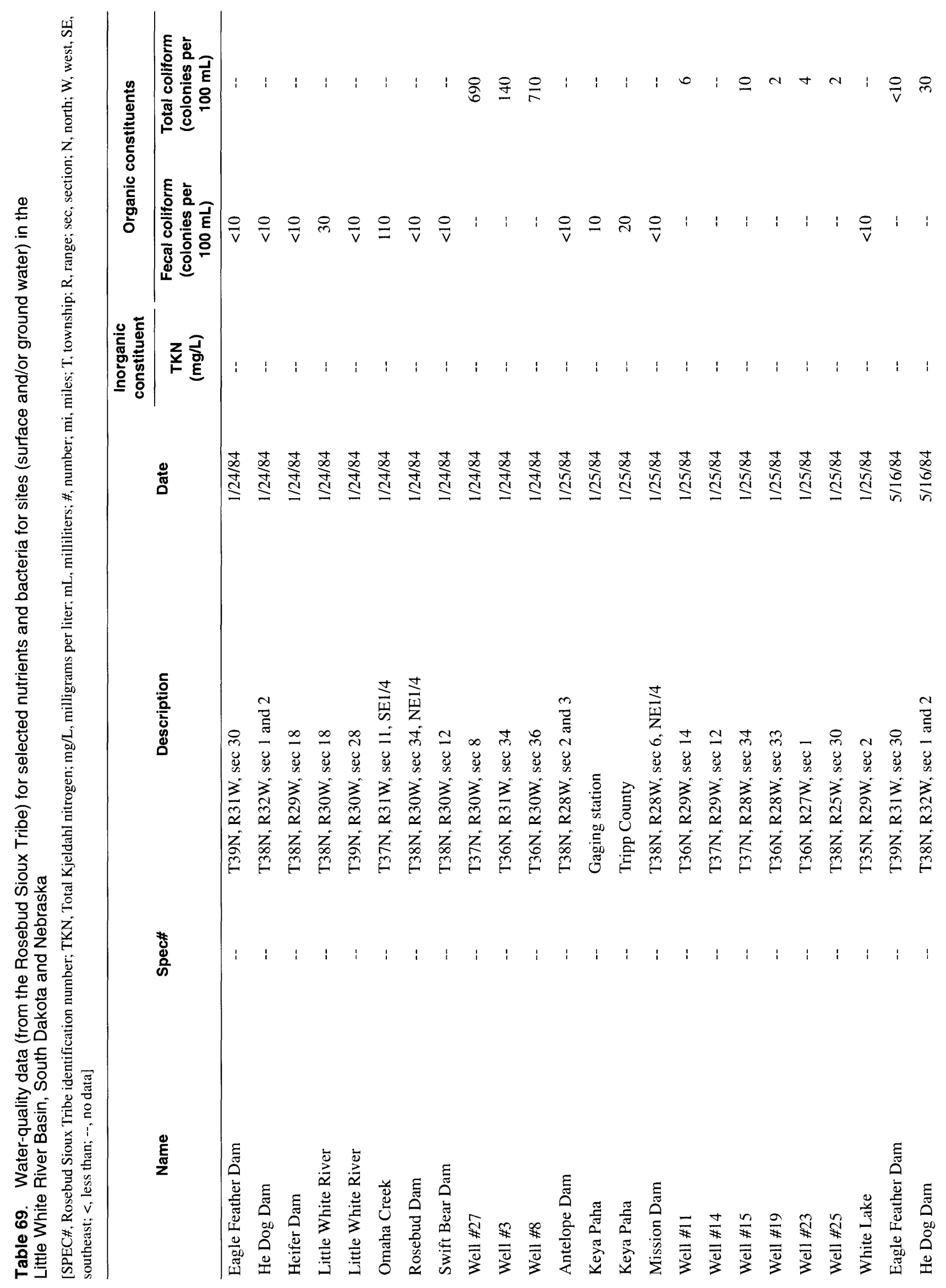




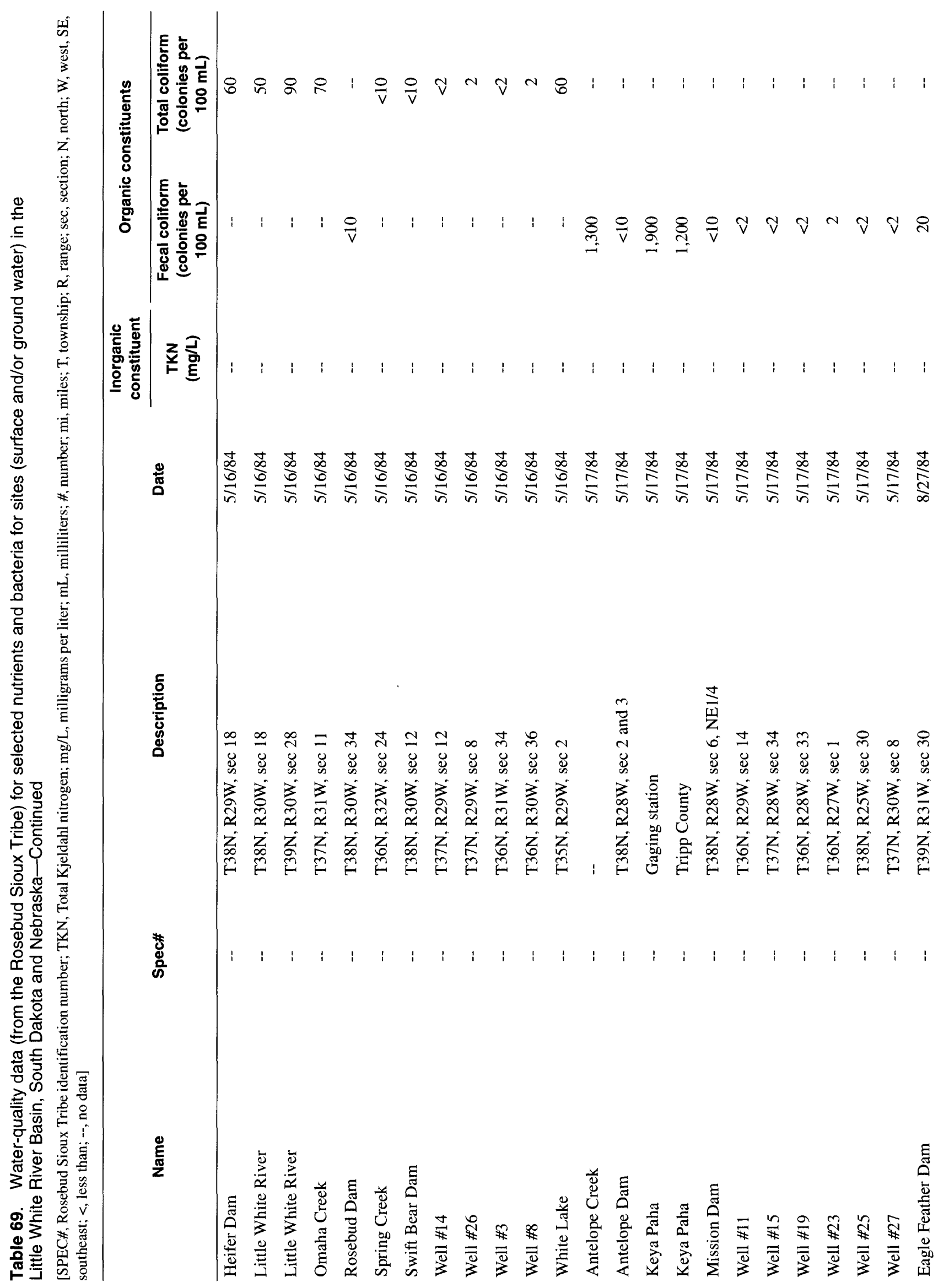




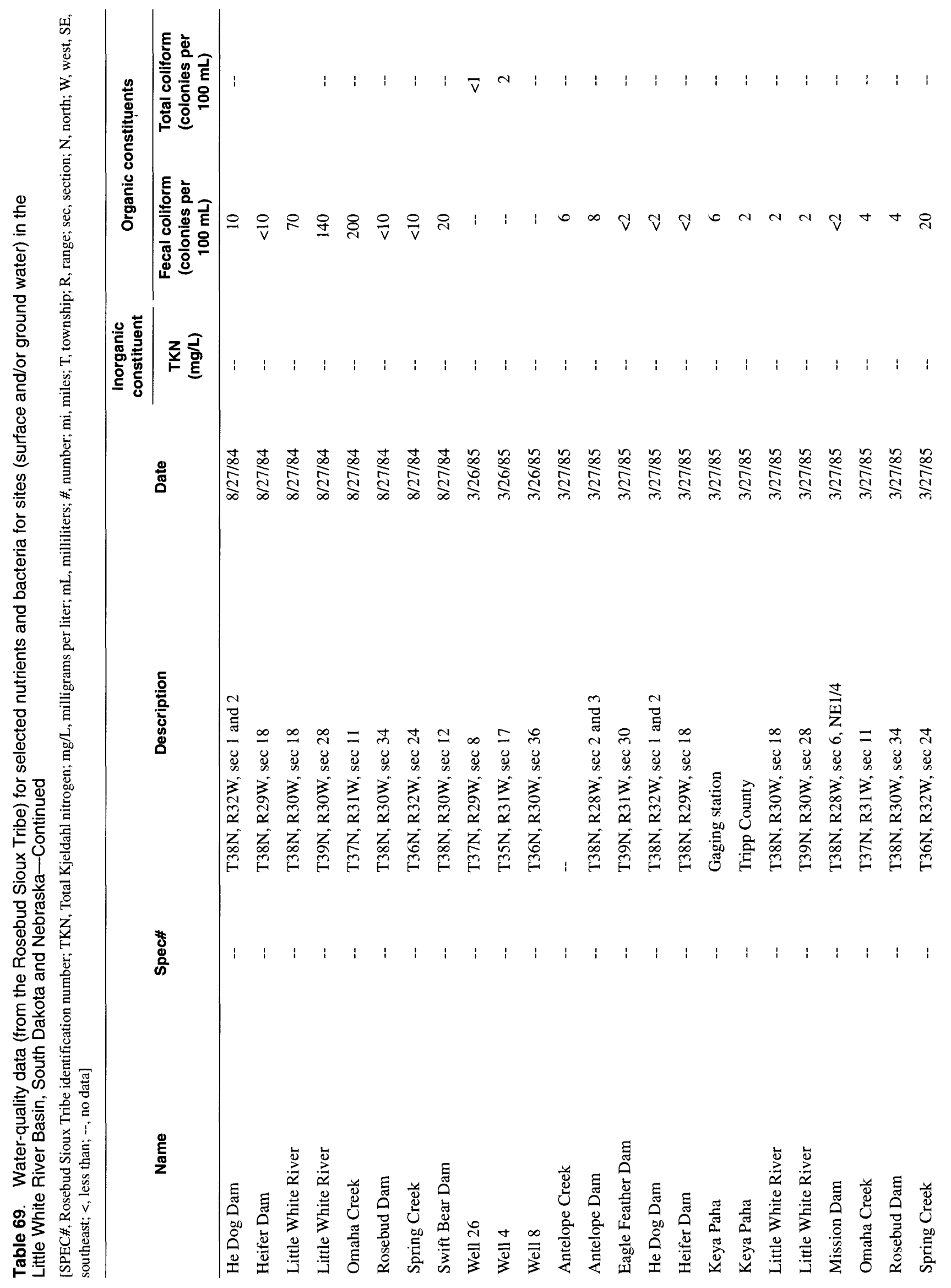




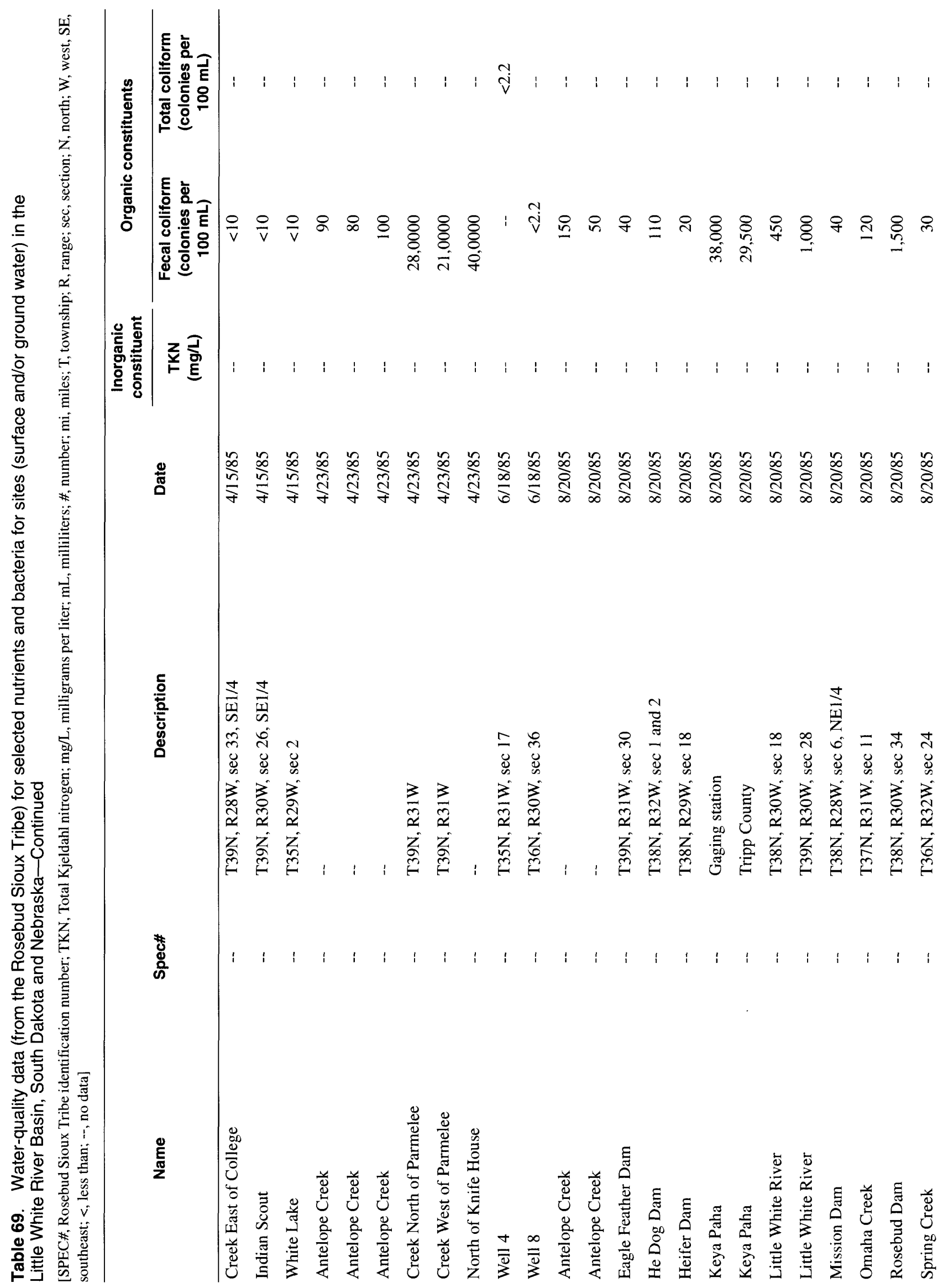




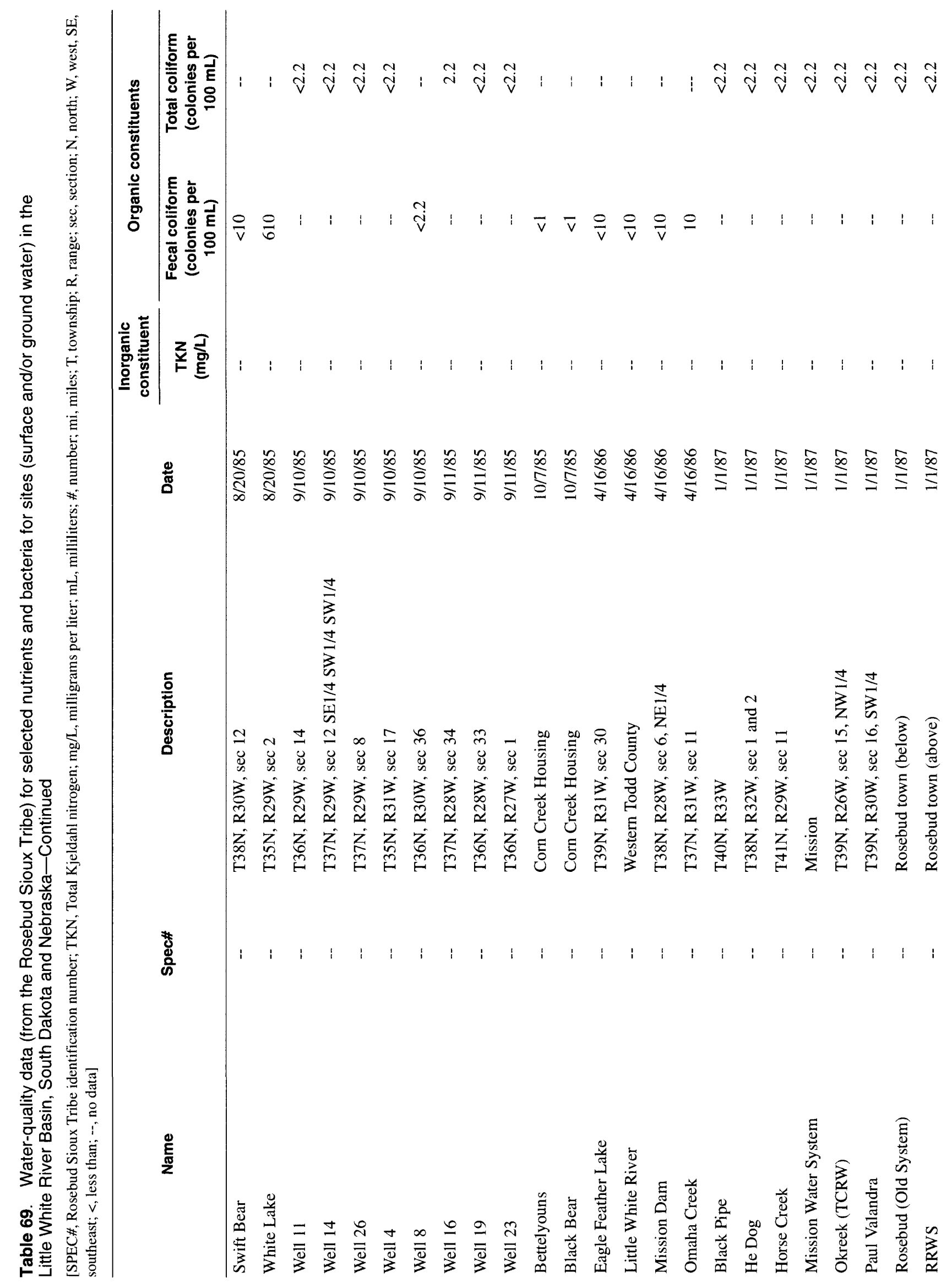




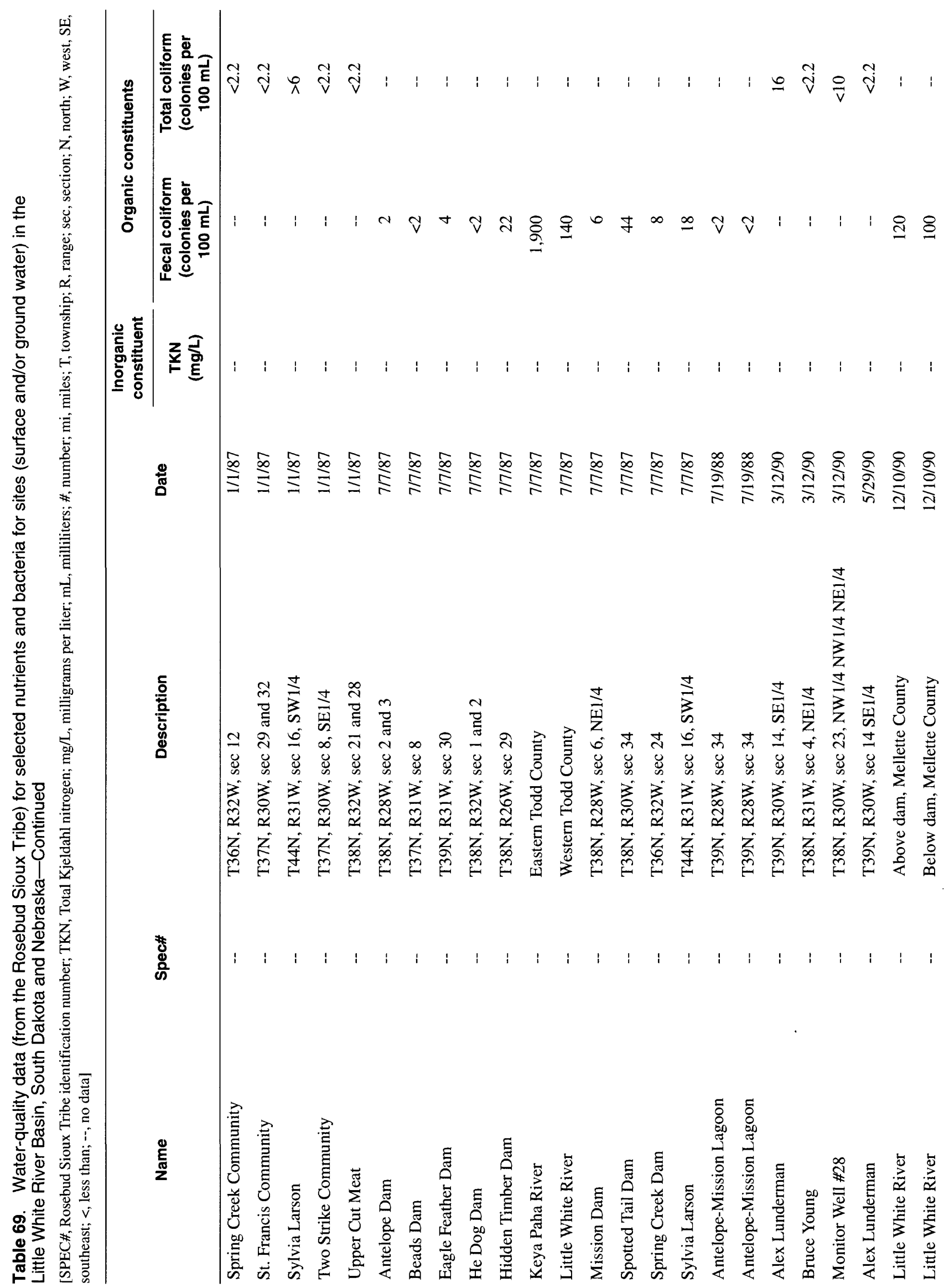




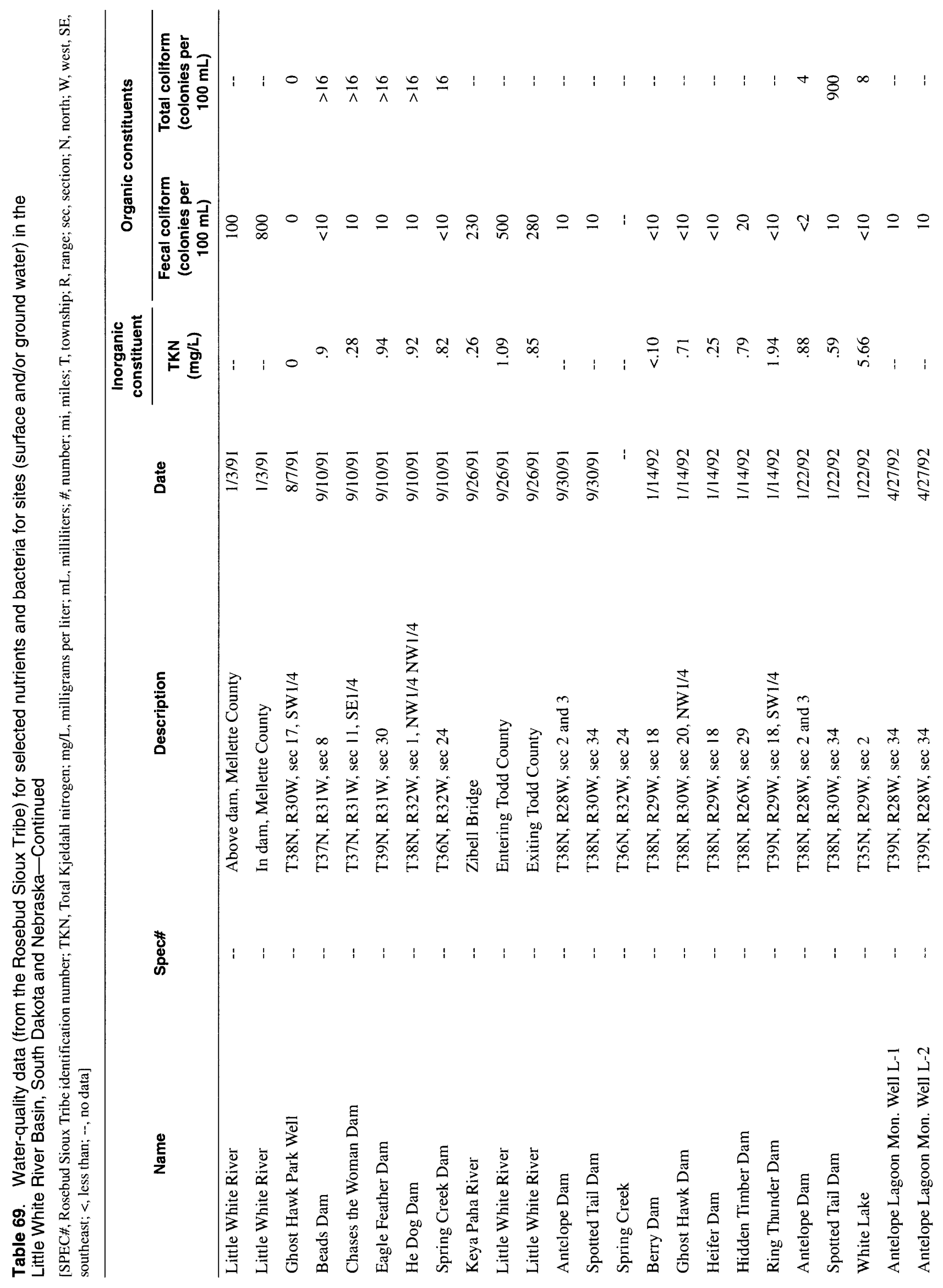




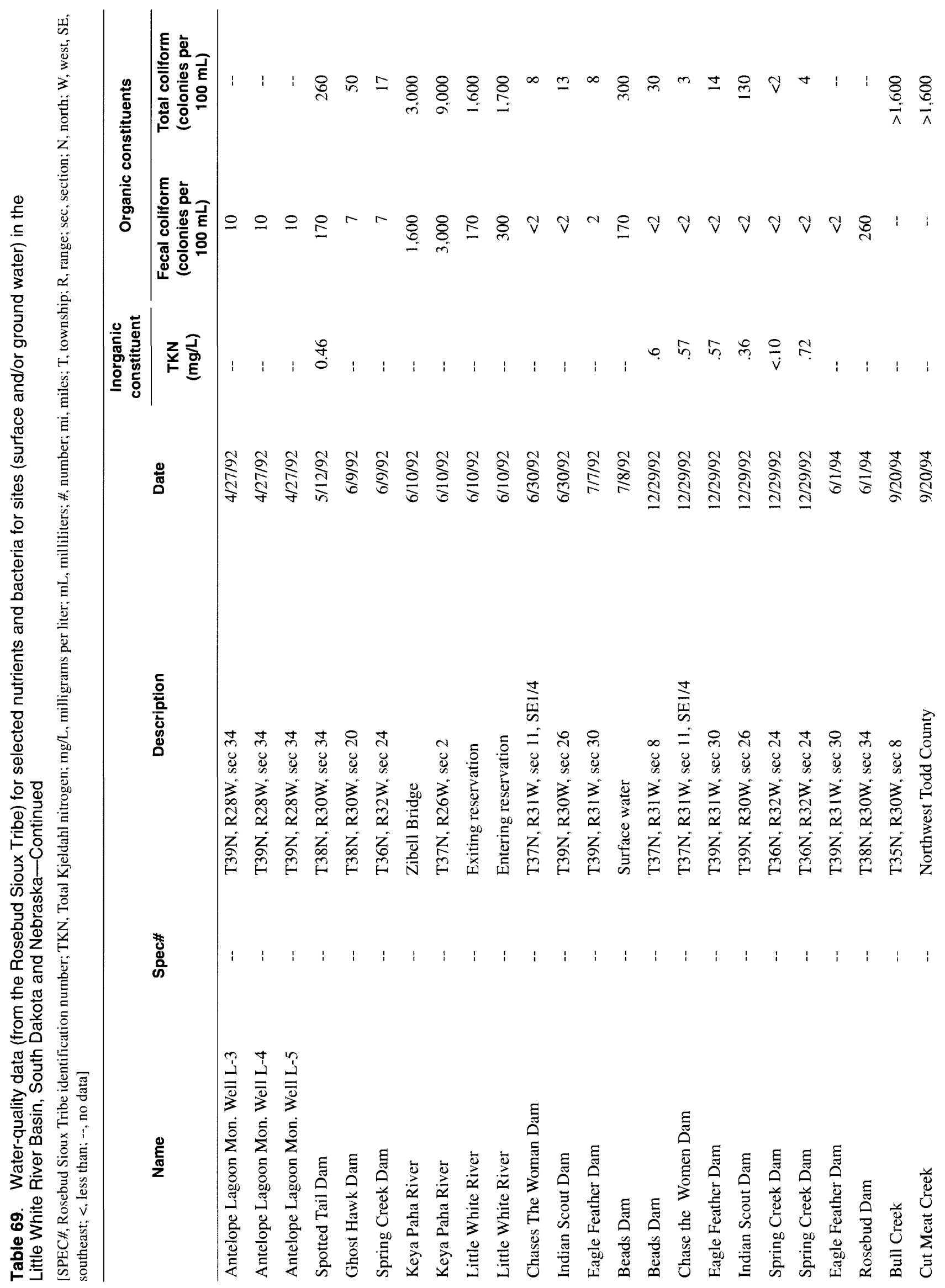




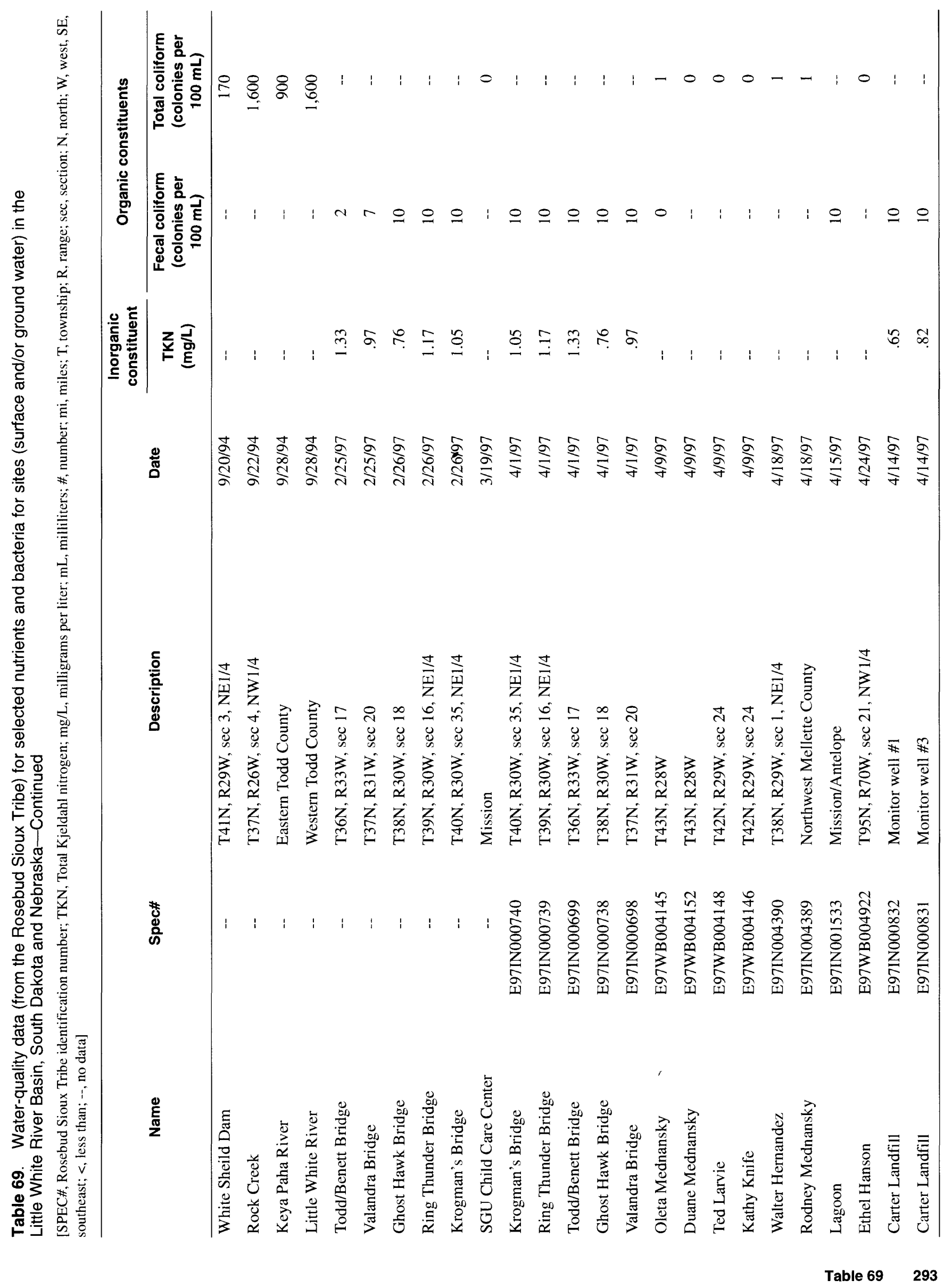




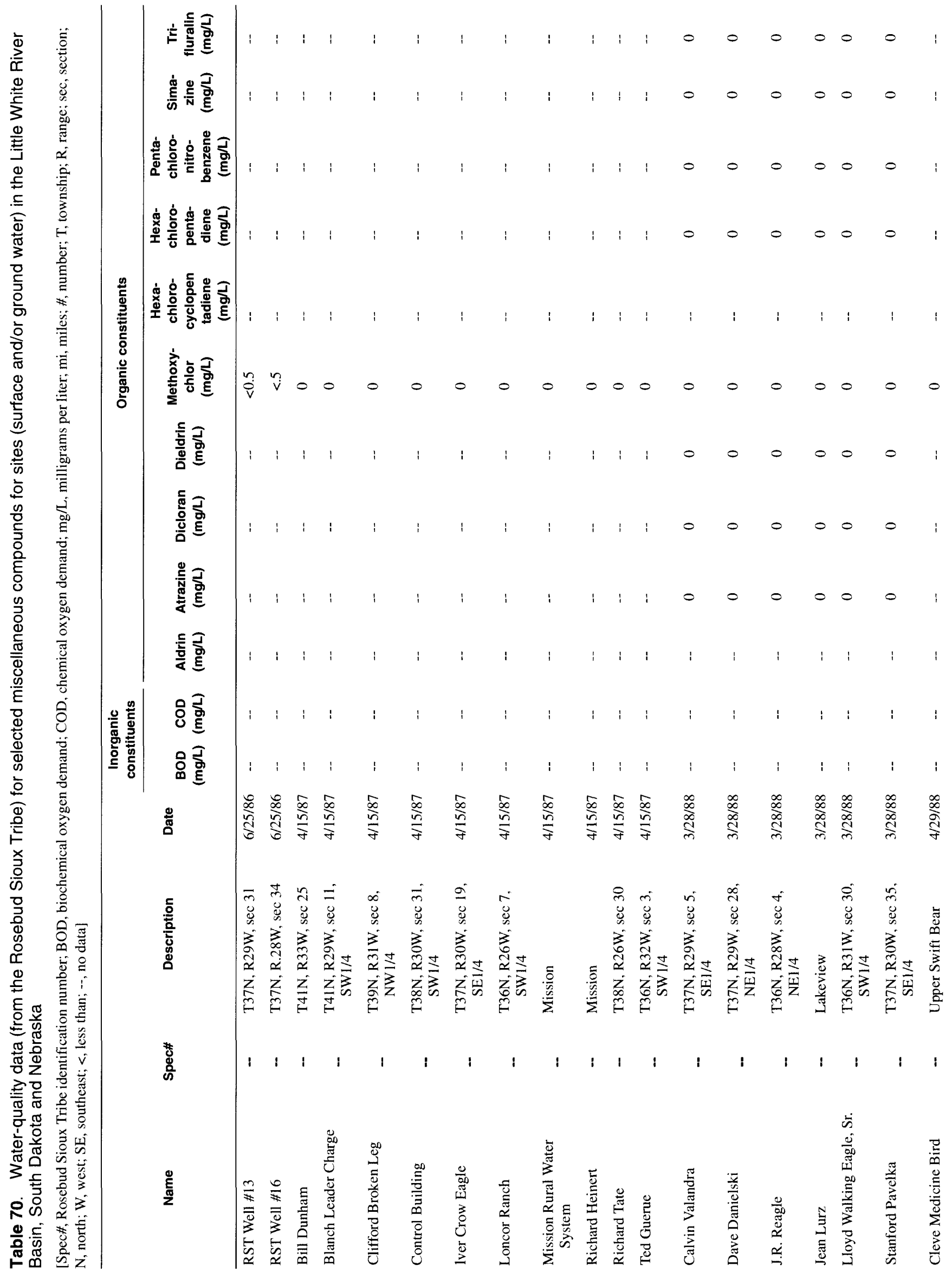




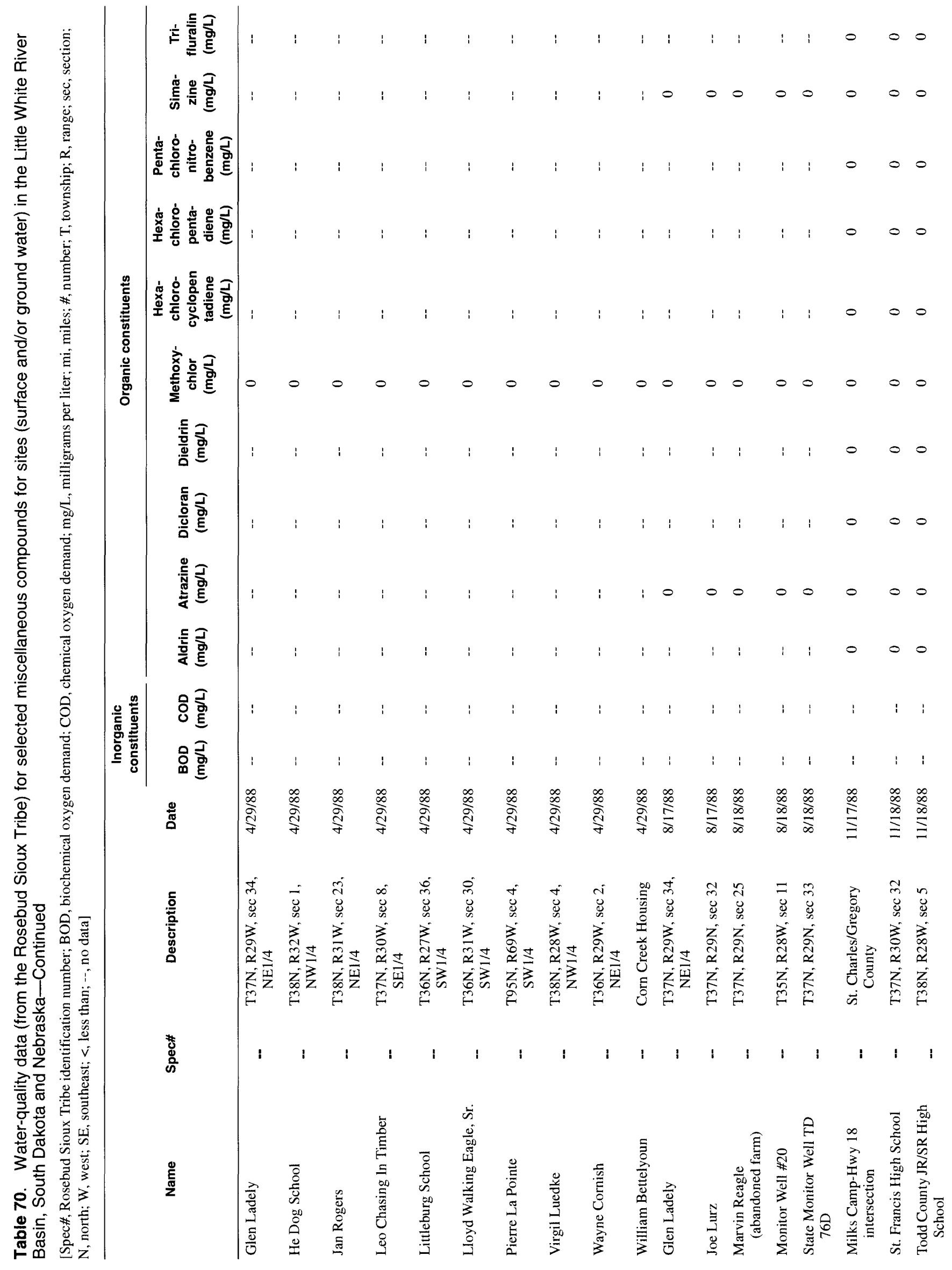




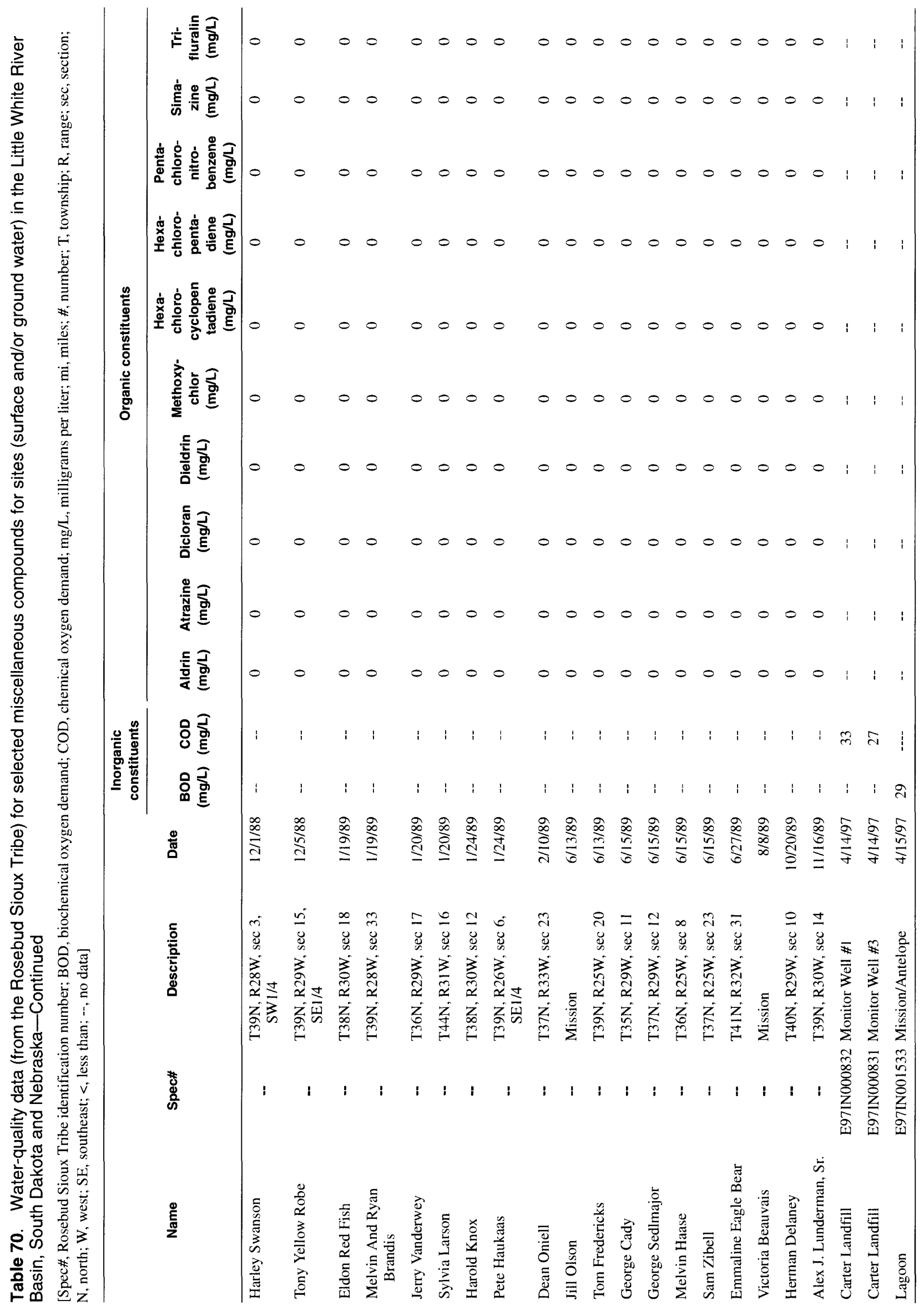


Table 71. Water-quality data (from the Rosebud Sioux Tribe) for PCB's for sites (surface and/or ground water) in the Little White River Basin, South Dakota and Nebraska

[mg/L, milligrams per liter; mi, miles; T, township; R, range; sec, section; N, north; W, west; SE, southeast; \#, number; --, no data]

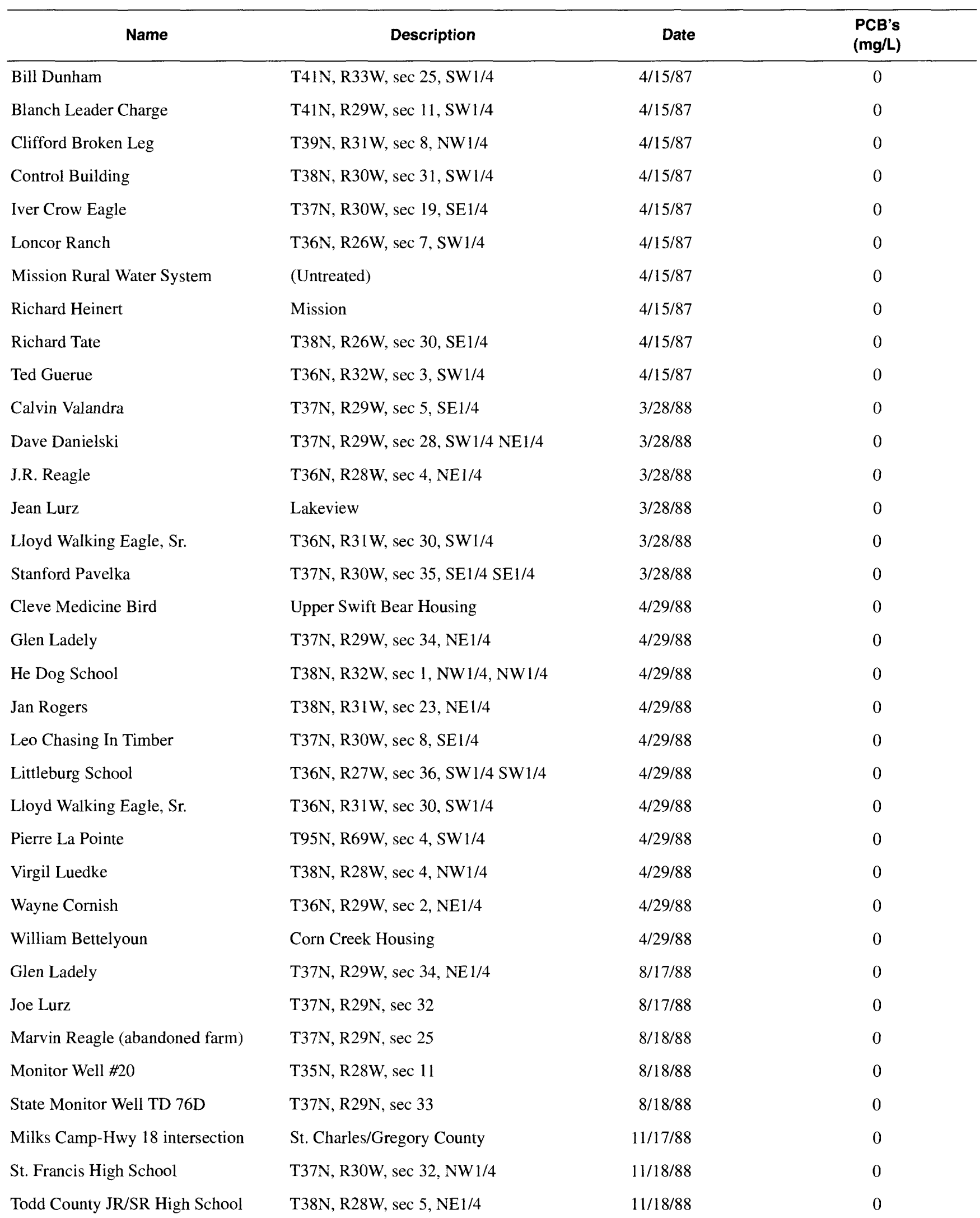


Table 71. Water-quality data (from the Rosebud Sioux Tribe) for PCB's for sites (surface and/or ground water) in the Little White River Basin, South Dakota and Nebraska-Continued

[mg/L, milligrams per liter; mi, miles; T, township; R, range; sec, section; N, north; W, west; SE, southeast; \#, number; --, no data]

\begin{tabular}{lllc}
\hline \multicolumn{1}{c}{ Name } & \multicolumn{1}{c}{ Description } & Date & $\begin{array}{c}\text { PCB's } \\
\text { (mg/L) }\end{array}$ \\
\hline Harley Swanson & T39N, R28W, sec 3, SW1/4 SW1/4 & $12 / 1 / 88$ & 0 \\
Tony Yellow Robe & T39N, R29W, sec 15, SE1/4 SE1/4 & $12 / 5 / 88$ & 0 \\
Eldon Red Fish & T38N, R30W, sec 18, SE1/4 & $1 / 19 / 89$ & 0 \\
Melvin And Ryan Brandis & T39N, R28W, sec 33, SW1/4 & $1 / 19 / 89$ & 0 \\
Jerry Vanderwey & T36N, R29W, sec 17, NE1/4 & $1 / 20 / 89$ & 0 \\
Sylvia Larson & T44N, R31W, sec 16, SW1/4 SW1/4 & $1 / 20 / 89$ & 0 \\
Harold Knox & T38N, R30W, sec 12, SE1/4 & $1 / 24 / 89$ & 0 \\
Pete Haukaas & T39N, R26W, sec 6, SE1/4 & $1 / 24 / 89$ & 0 \\
Dean Oniell & T37N, R33W, sec 23, NE1/4 & $2 / 10 / 89$ & 0 \\
Jill Olson & Mission & $6 / 13 / 89$ & 0 \\
Tom Fredericks & T39N, R25W, sec 20, NE1/4 & $6 / 13 / 89$ & 0 \\
George Cady & T35N, R29W, sec 11, NE1/4 & $6 / 15 / 89$ & 0 \\
George Sedlmajor & T37N, R29W, sec 12, NE1/4 SE1/4 & $6 / 15 / 89$ & 0 \\
Melvin Haase & T36N, R25W, sec 8, SW1/4 & $6 / 15 / 89$ & 0 \\
Sam Zibell & T37N, R25W, sec 23, NE1/4 NE1/4 & $6 / 15 / 89$ & 0 \\
Emmaline Eagle Bear & T41N, R32W, sec 31, NE1/4 & $6 / 27 / 89$ & 0 \\
Victoria Beauvais & Mission & $8 / 8 / 89$ & 0 \\
Herman Delaney & T40N, R29W, sec 10, NE1/4 & $11 / 16 / 89$ & 0 \\
Alex J. Lunderman, Sr. & T39N, R30W, sec 14, SE1/4 & 0 \\
\hline
\end{tabular}




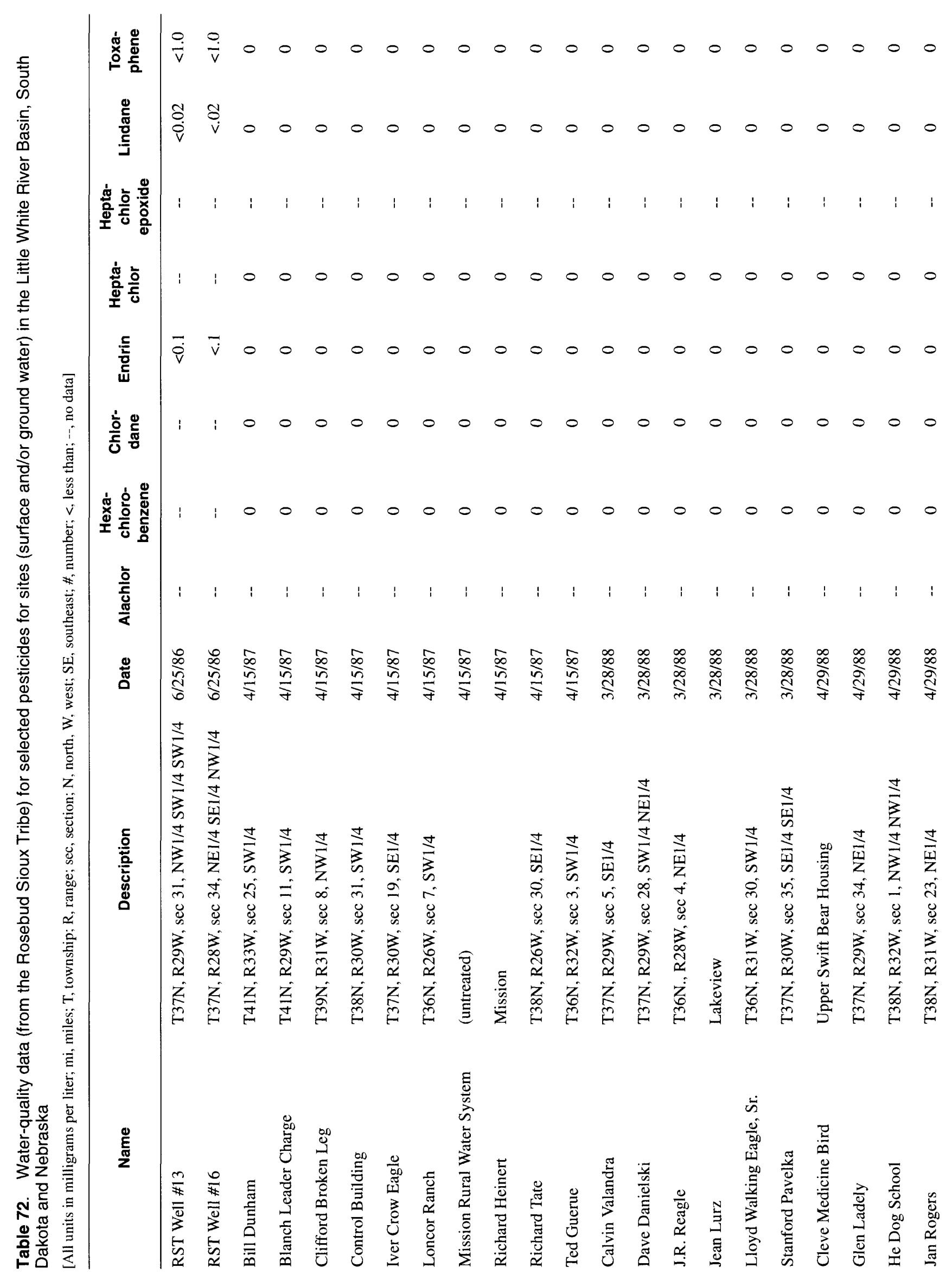


跑竞

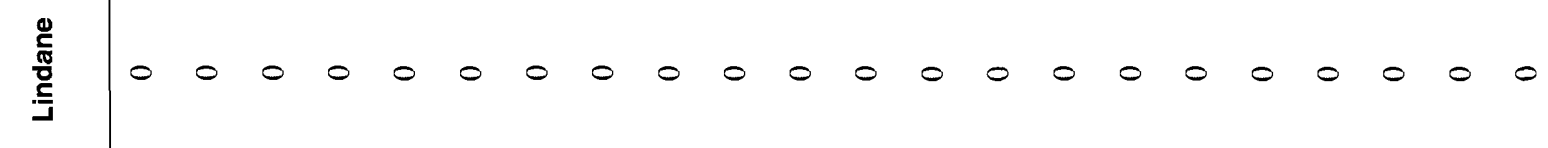

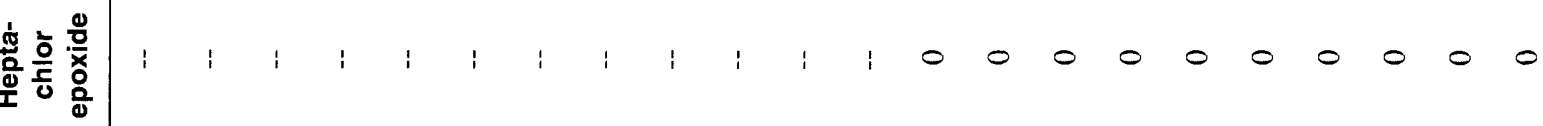

要喜

重

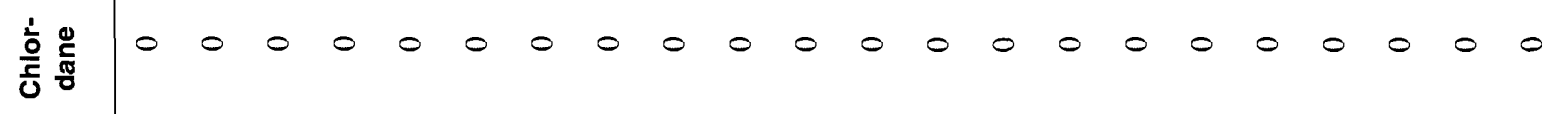

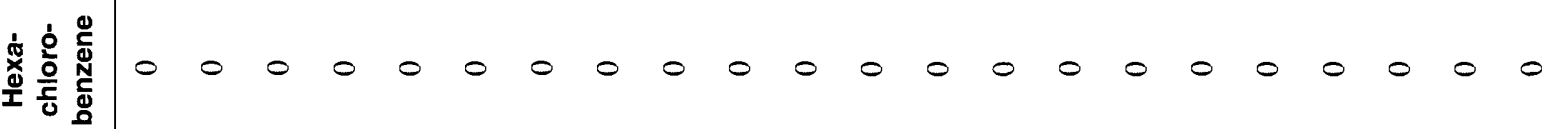

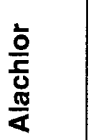

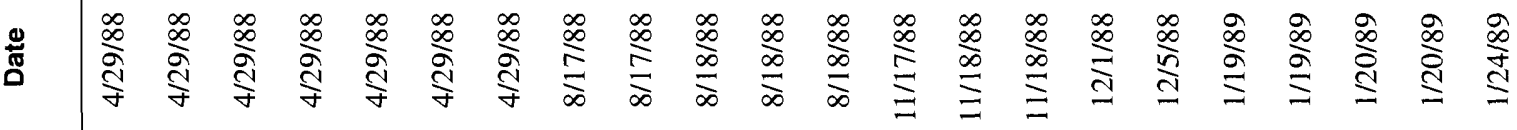




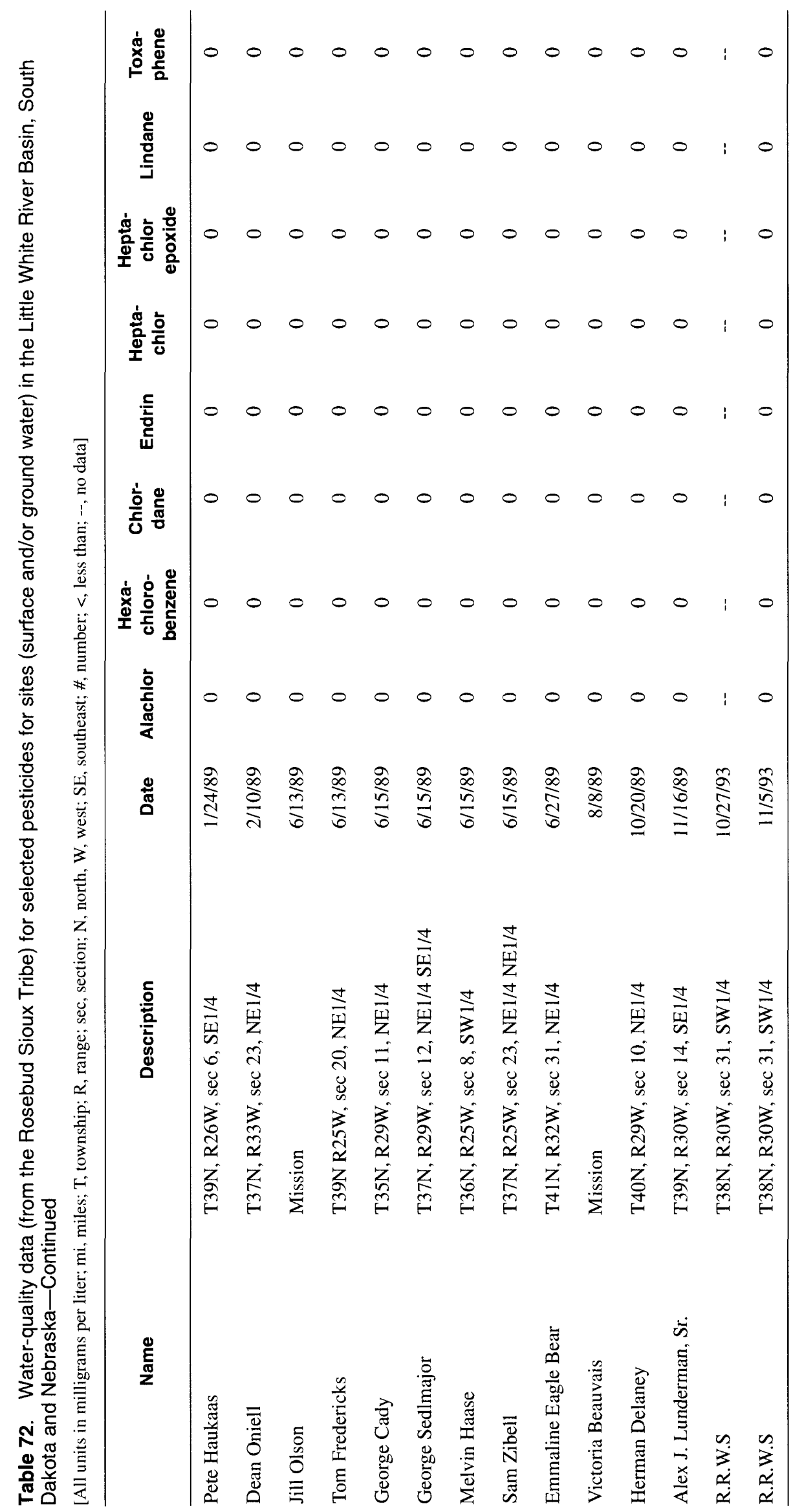




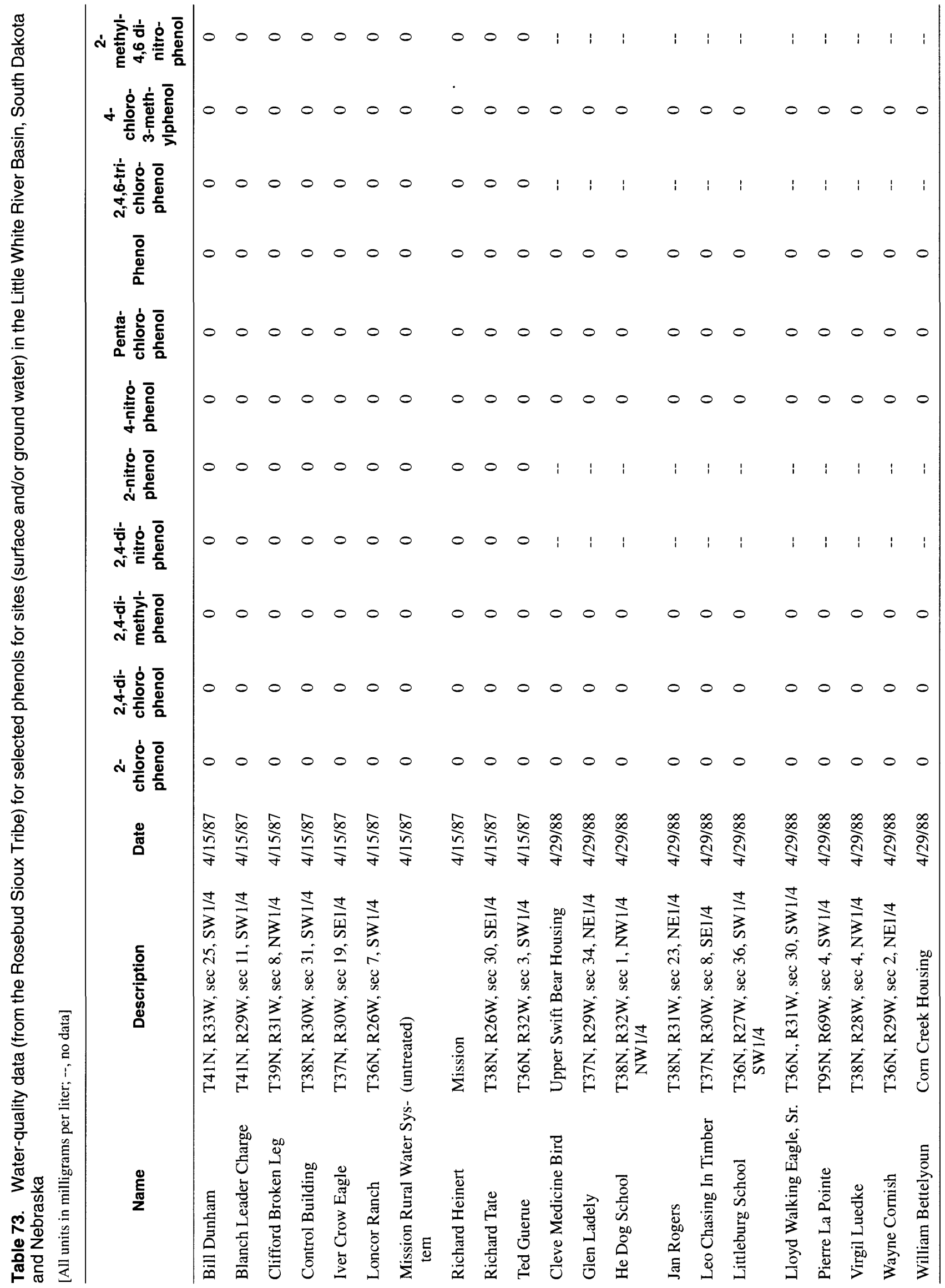


Table 74. Water-quality data (from the Rosebud Sioux Tribe) for selected radionuclides for sites (surface and/or ground water) in the Little White River Basin, South Dakota and Nebraska

[RST, Rosebud Sioux Tribe; pCi/L; picocuries per liter; \#, number; --, no data]

\begin{tabular}{|c|c|c|c|c|c|c|}
\hline Name & Description & Date & $\begin{array}{l}\text { Radon } \\
\text { (pCi/L) }\end{array}$ & $\begin{array}{c}\text { Gross } \\
\text { alpha } 15 \\
\text { (pCi/L) }\end{array}$ & $\begin{array}{l}\text { Gross beta } \\
(\mathrm{pCi} / \mathrm{L})\end{array}$ & $\begin{array}{l}\text { Radium } 226 \\
\quad(\mathrm{pCi} / \mathrm{L})\end{array}$ \\
\hline House Well & Stock well & $5 / 21 / 85$ & -- & $2.7+2.0$ & -- & -- \\
\hline Monitor Well \#1 & $\begin{array}{l}\text { T38N, R31W, sec 30, SE1/4 } \\
\text { NW1/4 SW1/4 }\end{array}$ & $5 / 21 / 85$ & -- & $1.9+1.6$ & -- & -- \\
\hline Monitor Well \#24 & $\begin{array}{l}\text { T37N, R25W, sec } 1, \text { SE1/4 } \\
\text { NW1/4 NW1/4 }\end{array}$ & $5 / 21 / 85$ & -- & $2.3+1.4$ & -- & -- \\
\hline Monitor Wel1 \#27 & $\begin{array}{l}\text { T37N, R30W, sec 9, NW1/4 } \\
\text { SE1/4 SE1/4 }\end{array}$ & $5 / 21 / 85$ & -- & $3.9+2.6$ & -- & -- \\
\hline Monitor Well \#4 & $\begin{array}{l}\text { T35N, R31W, sec } 17 \text { SW } 1 / 4 \\
\text { SE1/4 SW1/4 }\end{array}$ & $5 / 21 / 85$ & -- & $0.9+1.3$ & -- & -- \\
\hline Stock Well & -- & $5 / 21 / 85$ & -- & $1.6+1.4$ & -- & -- \\
\hline Antelope Senior Citizen Center & T39N, R28W, sec 34 & $7 / 30 / 91$ & -- & $3.8+2.1$ & -- & -- \\
\hline Milks Camp House \#474 & T95N, R69W, sec 4 & $7 / 30 / 91$ & -- & $6.5+2.6$ & -- & $0.2+0.2$ \\
\hline Old Rosebud System & Lower Rosebud town & $7 / 30 / 91$ & -- & $3.2+1.8$ & -- & -- \\
\hline RRWS Control Building & T38N, R30W, sec 31 & $7 / 30 / 91$ & -- & $4.3+2.1$ & $10.6+1.8$ & -- \\
\hline Two Strike House \#232 & R37N, R30W, sec 8 & $7 / 30 / 91$ & -- & $2.1+2.7$ & $10.0+2.2$ & -- \\
\hline Grass Mountain House \#364 & T38N, R31W, sec 23 & $8 / 1 / 91$ & -- & $9.9+4.5$ & $6.5+2.5$ & $0.04+0.1$ \\
\hline Spring Creek Duplex & T36N, R32W, sec 12 & $8 / 1 / 91$ & -- & $7.2+3.0$ & $12.3+2.2$ & $0.3+0.1$ \\
\hline Upper Cut Meat House \#141 & T38N, R32W, sec 28 & $8 / 1 / 91$ & -- & $5.8+3.5$ & $6.8+2.5$ & $0.1+0.1$ \\
\hline Black Pipe Community Well & T40N, R33W & $8 / 2 / 91$ & -- & $10.4+4.4$ & $8.0+2.7$ & $0+0.1$ \\
\hline Horse Creek Community & T41N, R29W, sec 11 & $8 / 2 / 91$ & -- & $1.8+3.3$ & $12.0+2.8$ & -- \\
\hline RST/ Eagle Bear & T41N, R32W, sec 31, NE1/4 & $2 / 24 / 93$ & 983.31 & -- & -- & -- \\
\hline RST/ Furrey & T38N, R25W, sec 5, NE1/4 & $2 / 24 / 93$ & 568.95 & -- & -- & -- \\
\hline RST/ Heinert, Ann & $\mathrm{T} 39 \mathrm{~N}, \mathrm{R} 32 \mathrm{~W}$ & $2 / 24 / 93$ & 764.64 & -- & -- & -- \\
\hline RST/ Hunger & Mission, private well & $2 / 24 / 93$ & $1,163.57$ & -- & -- & -- \\
\hline RST/ Joseph & T40N, R27W, sec 35, NW1/4 & $2 / 24 / 93$ & 226.12 & -- & -- & -- \\
\hline RST/ Loncors & T36N, R26W, sec 7, SW1/4 & $2 / 24 / 93$ & 937.12 & -- & -- & -- \\
\hline RST/ Lunderman, Alex & T39N, R30W, sec 14 & $2 / 24 / 93$ & 799.76 & -- & -- & -- \\
\hline RST/ Lurz & T36N, R29W, sec 4 & $2 / 24 / 93$ & 332.85 & -- & -- & -- \\
\hline RST/ Valandra & T37N, R29W, sec 5, SE1/4 & $2 / 24 / 93$ & 132.55 & -- & -- & -- \\
\hline RST/ Walking Eagle & $\mathrm{T} 36 \mathrm{~N}, \mathrm{R} 31 \mathrm{~W}, \sec 30$ & $2 / 24 / 93$ & 572.02 & -- & -- & -- \\
\hline RST/ Whipple & T39N, R32W, sec 30, SE1/4 & $2 / 24 / 93$ & 352.45 & -- & -- & -- \\
\hline RST/browning & T38N, R27W, sec 5, NE1/4 & $2 / 24 / 93$ & 630.56 & -- & -- & -- \\
\hline RST/ Boyles, John & T43 and 44N, R28W, sec 10 & $2 / 25 / 93$ & 454.11 & -- & -- & -- \\
\hline RST/ Fuerst, Roy & T42N, R31W, sec 33 & $2 / 25 / 93$ & 180.96 & -- & -- & -- \\
\hline RST/ Horsley, Ted & T42N, R27W, sec 9 & $2 / 25 / 93$ & 509.76 & -- & -- & -- \\
\hline RST/Larvie, Ted & T42N, R29W, sec 24 & $2 / 25 / 93$ & 227.72 & -- & -- & -- \\
\hline RST/ Schoppeert, D.E. & T41N, R28W, sec 6 & $2 / 25 / 93$ & 791.61 & -- & -- & -- \\
\hline RST/ Sherwood, Howard & T42N, R27W, sec 1 & $2 / 25 / 93$ & 551.25 & -- & -- & -- \\
\hline RST/ Sayler, Arthur & T43N, R28W, sec 18 & $2 / 25 / 93$ & 132.22 & -- & -- & -- \\
\hline RST/ Waldron, Mike & T43, 44N., R28W, sec 24 & $2 / 25 / 93$ & 912.87 & -- & -- & -- \\
\hline
\end{tabular}




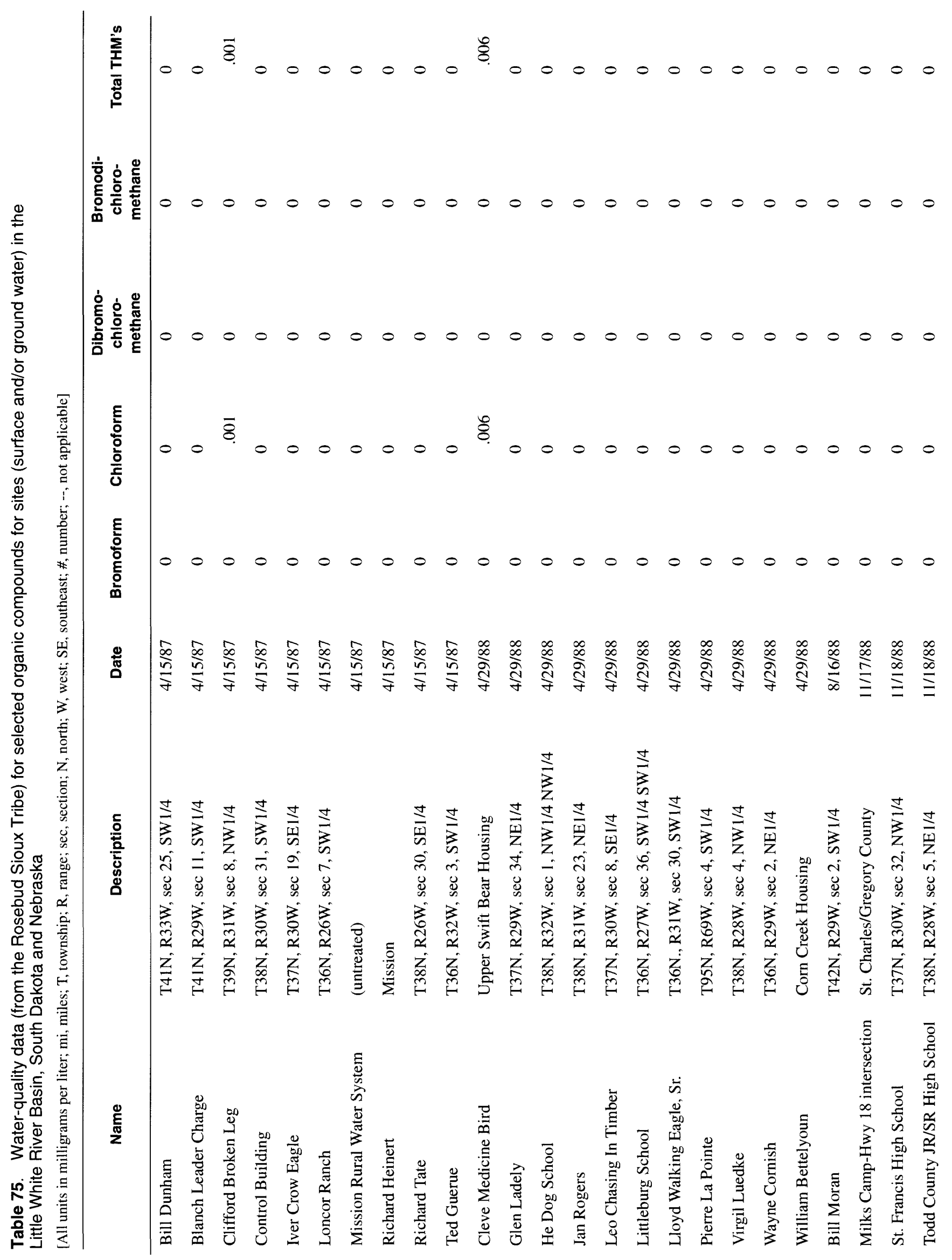




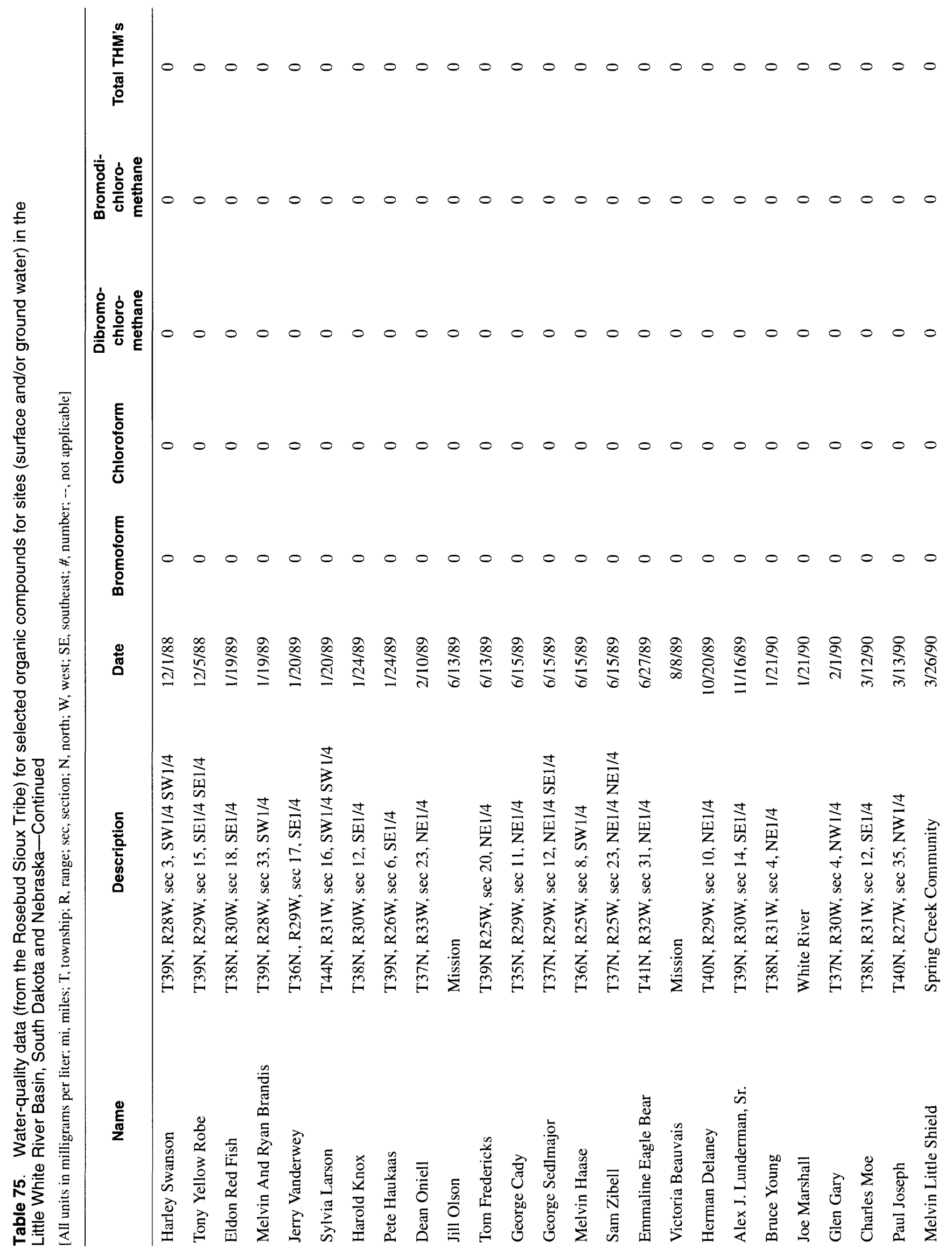




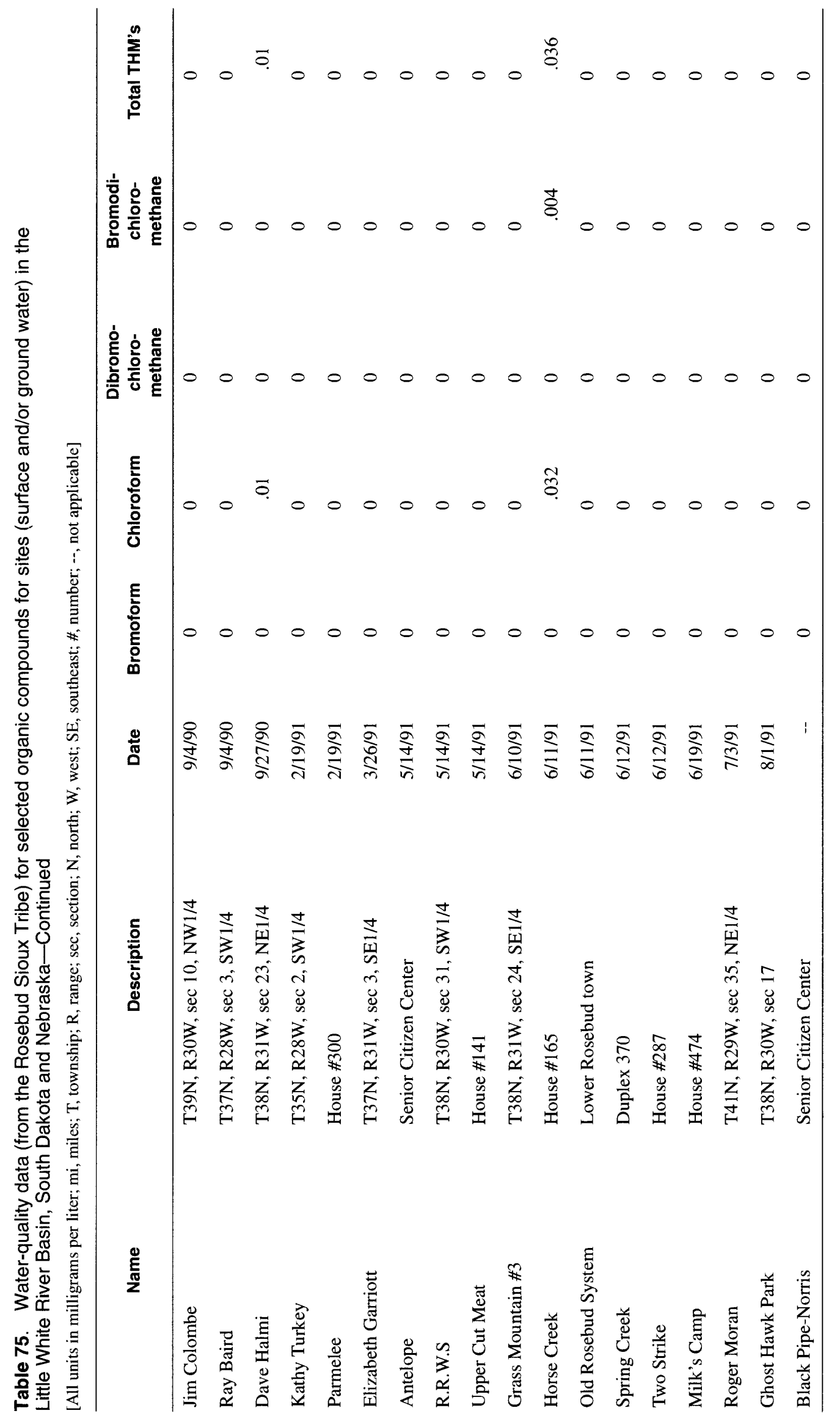

